\title{
Experimental and Numerical Study on Bolted/Bonded Composite Joints for Aircraft
}

\author{
by \\ Pedro Lopez Cruz, B. Eng \& M. Sc.
}

A thesis submitted to the Faculty of Graduate and Postdoctoral Affairs in partial fulfillment of the requirements for the degree of

\author{
Doctor of Philosophy \\ in \\ Aerospace Engineering
}

Carleton University

Ottawa, Ontario

(C) 2016, Pedro Lopez Cruz 


\section{Abstract}

Bolted and bonded joining technologies in composite structures have been widely studied since the 1970s. Although every technology has advantages and disadvantages, bonded joint technology has the potential to reduce the weight and manufacturing cost of advanced aircraft structures. However, bolted joining is still generally required for certification and regulatory compliance. An alternative to these technologies is the use of hybrid bolted/bonded joints combining elements from both technologies.

When compared with bolted and bonded technology in composite structures, hybrid joining technology is at an early stage of development, very few journal papers have been written on this topic as of the time of this writing.

Previously reported research efforts have been focused on the load sharing and strength improvement. The load sharing has been analyzed experimentally using instrumented bolts and numerically using finite element modelling. The strength analysis has been studied experimentally.

The present research was proposed to study hybrid bolted/bonded joints experimentally using a "design of experiments" approach. The aim was to investigate the effect of several factors on the joint strength and load sharing in bolted/bonded hybrid joints. In addition to this, finite element modelling was successfully applied to predict the load sharing and strength, and the results were compared with the experiments with good agreement.

The instrumented bolt technique is limited due to the bolt size. To surpass this limitation with the proposed design of experiments, a different approach was needed. Along with the design of experiments and numerical analysis, a novel technique using 
digital image correlation to measure the shear strains at the adhesive edge was applied to measure the load sharing. Also, the load sharing was computed in all the joint configurations, not only a single sample joint configuration, as was typically reported in other studies. The numerical results in terms load sharing showed a very good agreement with the experiments. On the other hand, using cohesive zone modelling, the strength was predicted with a good agreement compared with the experimental results. Finally, the analysis of variance from the design of experiments quantified the effect of the proposed factors in the joint strength. 


\section{Acknowledgements}

I would like to express my recognition and gratitude to my supervisor, Dr. Jeremy Laliberté for giving me the opportunity to pursue this $\mathrm{PhD}$ research. In addition to this, I would like to thank Dr. Laliberté for his guidance and support during this research project.

Thanks to the Consortium for Research and Innovation in Aerospace in Quebec (CRIAQ) and the Natural Sciences and Engineering Research Council of Canada for their financial support for this project.

My gratitude to the partners of the CRIAQ COMP 506 project for their support during the project: Bombardier Aerospace, Delastek, L3Com, McGill University, Ecole Polytechnique de Montreal and National Research Council Canada Aerospace Portfolio.

Many thanks to Mr. Rick Cole and Mr. Matthieu Harrison for their guidance and support during the manufacturing of the testing coupons at the National Research Council Canada.

I would also like to thank Carleton University staff, especially Mr. Steve Truttmann for his invaluable help and guidance using the MTS testing equipment, Mr. Alex Proctor for helping out at the machine shop and Neil McFadyen for setting up Abaqus ${ }^{\circledR}$ along with FORTRAN compiler in the MAAE computing cluster.

I would like to express my gratitude to the Programa de Mejoramiento del Profesorado (Promep), Mexico, for granting me a scholarship to pursue my $\mathrm{PhD}$ studies.

Lastly, I would like to thank my wife, Selene, for her invaluable support and motivation. 


\section{Table of Contents}

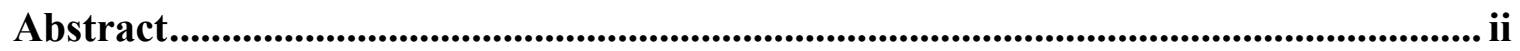

Acknowledgements ........................................................................................................................ iv

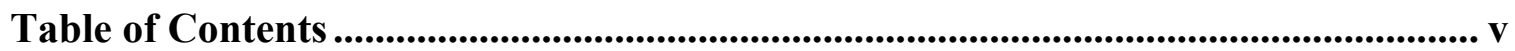

List of Tables .............................................................................................................................. ix

List of Illustrations.....................................................................................................

List of Symbols and Acronyms .......................................................................................... Xxv

List of Appendices...................................................................................................................... xxvii

Chapter 1: Introduction ..................................................................................................... 1

$1.1 \quad$ Composites in Aircraft Structural Design ................................................................. 1

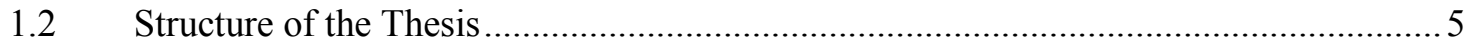

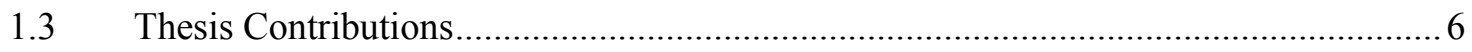

Chapter 2: Background and Literature Review ............................................................ 8

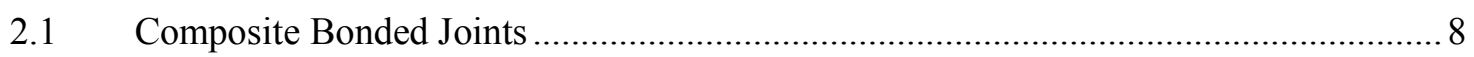

$2.2 \quad$ Composite Bolted Joints ............................................................................... 12

2.3 Bolted/Bonded Joints in Composites................................................................... 16

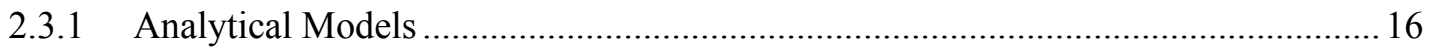

2.3.2 Experimental and Numerical Studies ...............................................................2

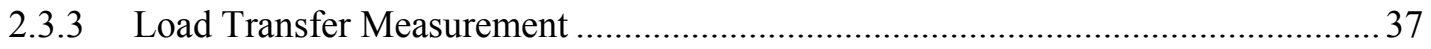

2.4 Cohesive Zone Modelling ......................................................................................... 39

2.4.1 CZM on Bonded Joints .................................................................................. 44

2.4.2 CZM studies on Bolted/Bonded Joints................................................................4 48

2.5 Damage and Failure in Composites ………………………………………………...4

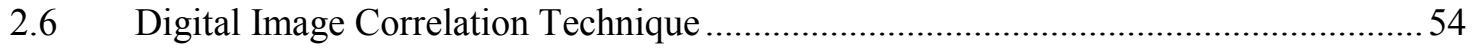




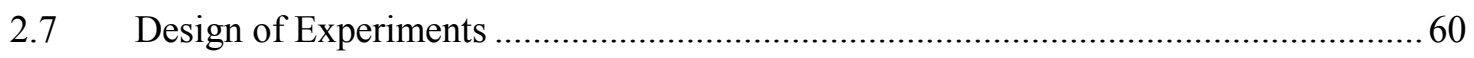

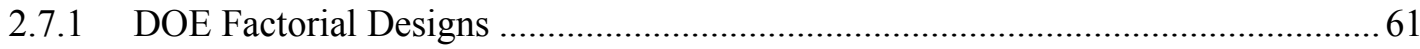

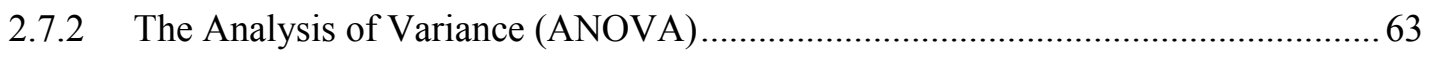

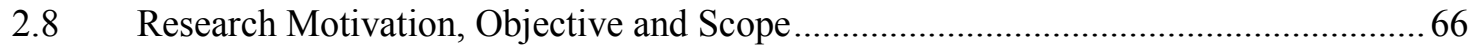

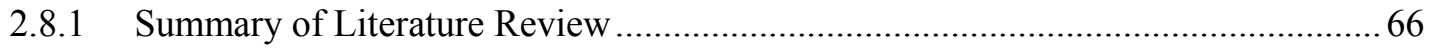

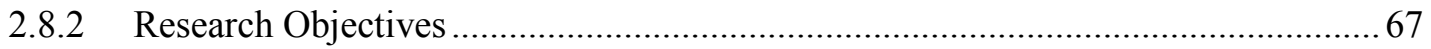

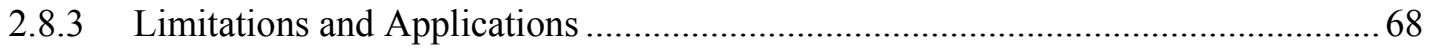

Chapter 3: Methodology......................................................................................................... 69

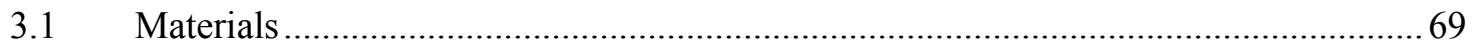

3.2 Specimen for Bolted/Bonded Joints .............................................................. 71

3.3 Design of the Experiment Using Factorial Designs .................................................. 74

3.3.1 Selection of the DOE Factors and Response Variables ........................................... 74

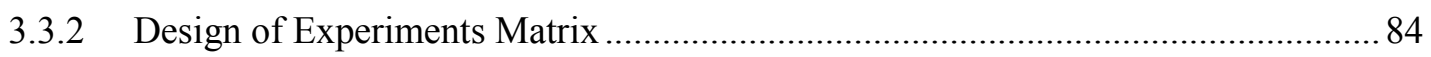

3.3.3 Associated Risk of Using DOE for the Experimental Study ................................. 85

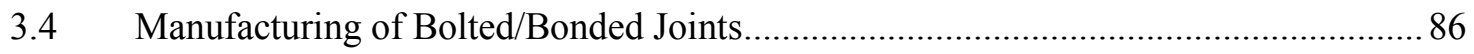

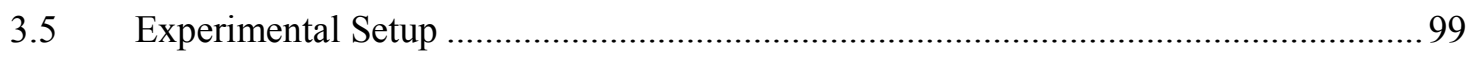

3.6 Measurements Using Digital Image Correlation .................................................. 102

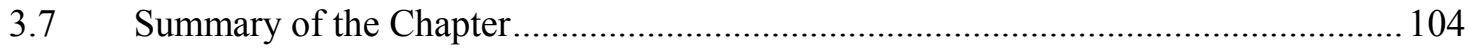

Chapter 4: Experimental Results ................................................................................ 106

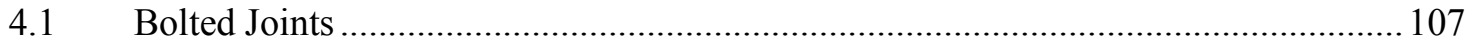

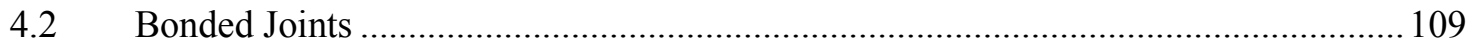

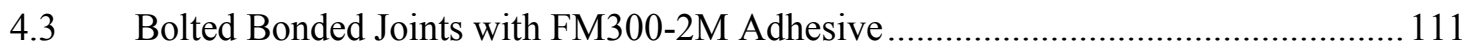

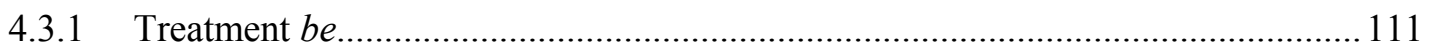

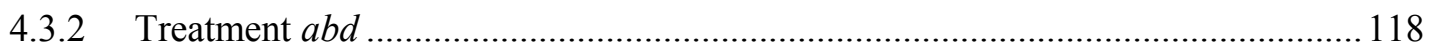

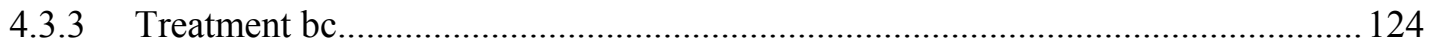

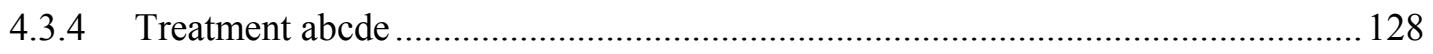




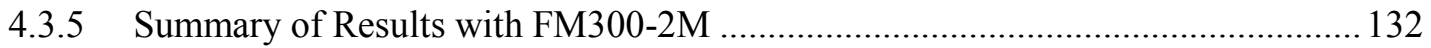

4.4 Bolted Bonded Joints with EA9361 Adhesive ........................................................... 134

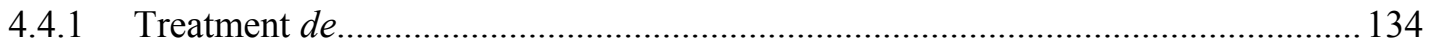

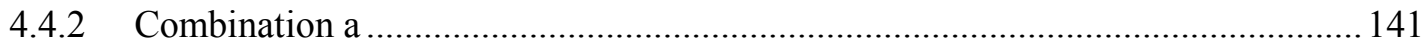

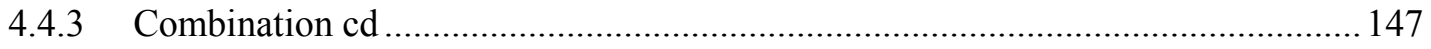

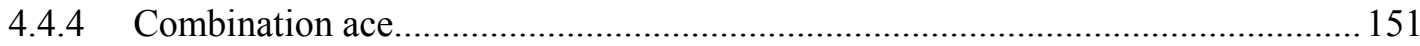

4.4.5 Summary of Results for Joint with EA9361 Adhesive ........................................ 157

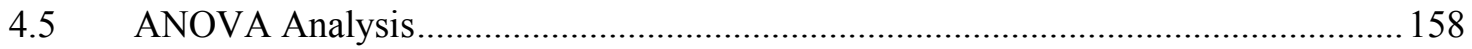

Chapter 5: Finite Element Analysis of Bolted/Bonded Composite Joints ................ 164

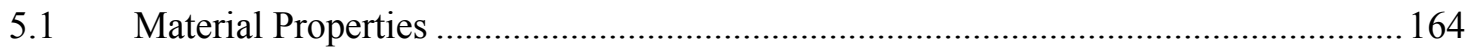

5.1.1 Carbon Fibre Unidirectional Prepreg Properties ................................................... 164

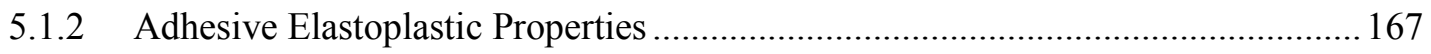

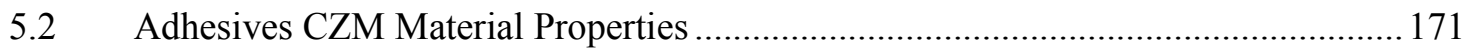

5.2.1 FM 300-2M Adhesive CZM Properties .......................................................... 172

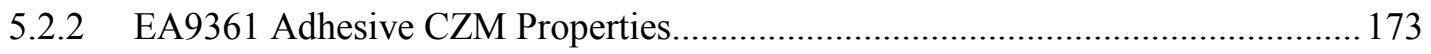

5.2.3 Summary of CZM Parameters for FM300-2M and EA9361 Adhesives................ 175

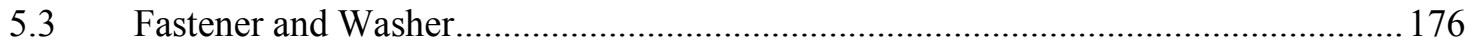

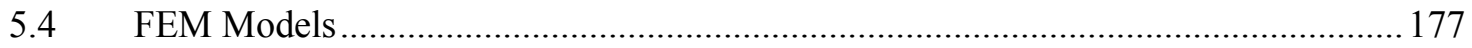

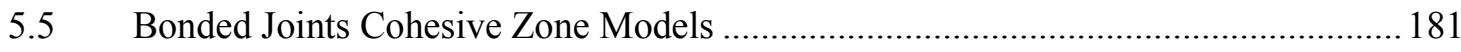

5.5.1 CZM Bonded Joints With FM300-2 Adhesive ................................................... 183

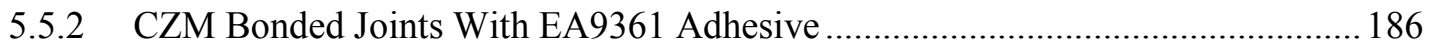

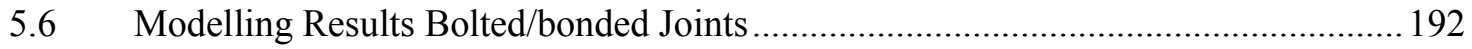

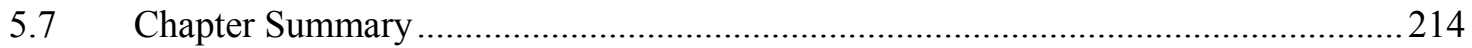

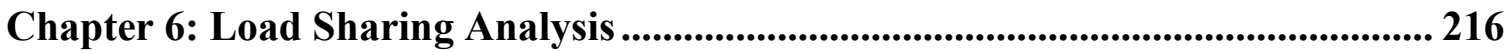

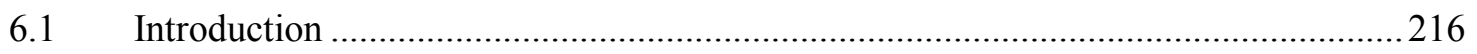

6.2 Load Sharing Analysis in Joints with FM300-2M Adhesive ..................................220 


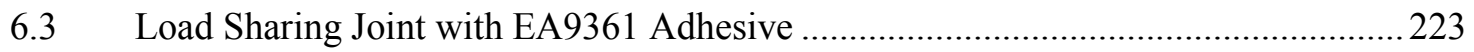

6.4 Bolted/bonded Joints with Interference Fit Assembly........................................... 233

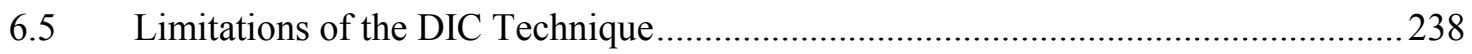

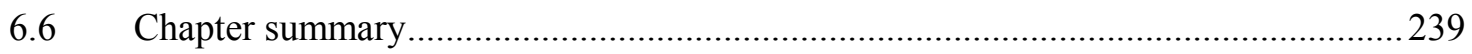

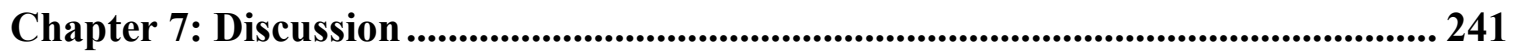

Chapter 8: Conclusions and Recommendations ...................................................... 246

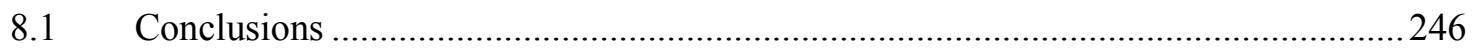

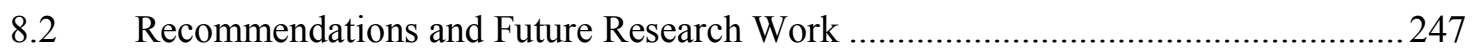

Bibliography ........................................................................................................................ 249

Appendix A: Procedure to Adjust the Stroke Displacement due to the Test Machine

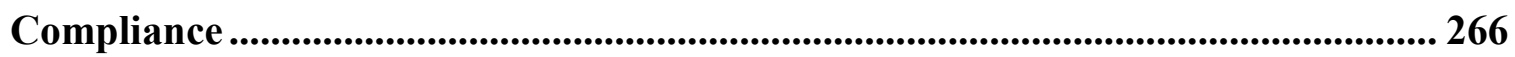

Appendix B: Procedure to Measure Shear Strain at the Adhesive Edge ................. 268

Appendix C: ANOVA Results.................................................................................... 274

Appendix D: FORTRAN USDFLD Subroutine....................................................... 278

Appendix E: Adhesive Thickness. Bolted/bonded joints............................................ 282

Appendix F: Typical Failure Modes in Tested Coupons....................................... 283

F.1 Bolted/bonded joints with FM300-2M adhesive ...................................................283

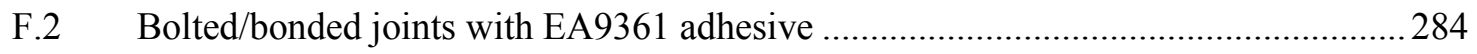

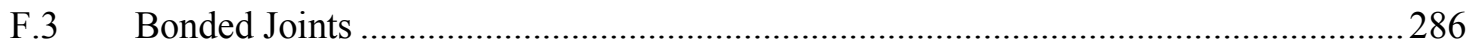




\section{List of Tables}

Table 2.1 Effect of several factors in the load transferred to the bolt on hybrid bolted/bonded joint according to Paroissien [51] ………………................................ 23

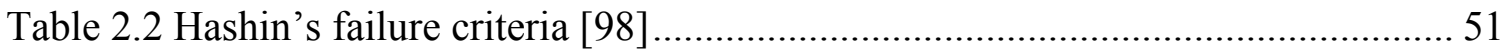

Table 2.3 Stiffness degradation parameters according to McCarthy [104] ...................... 53

Table 2.4 Number of runs required for 2k experimental design.................................... 63

Table 2.5 Analysis of variance table for a three-factor model. Adapted from [117]....... 65

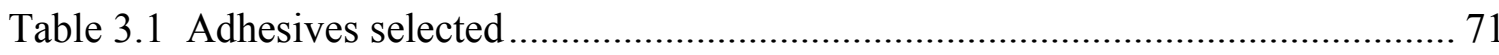

Table 3.2 Factors selected for the DOE and their levels.............................................. 79

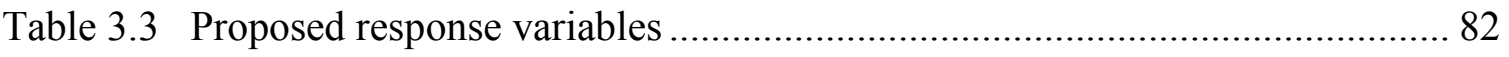

Table 3.4 "Held Constant" Factors in the DOE .................................................................. 83

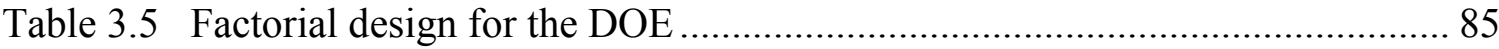

Table 3.6 Cytec Cycom 5230 recommended curing cycles [120] ................................ 89

Table 4.1 Experimental treatments with FM300-2 adhesive ........................................ 111

Table 4.2 Summary of results. Force versus displacement for treatment be ................. 114

Table 4.3 Summary of results. Force versus displacement for treatment $a b d$............... 120

Table 4.4 Summary of results. Force versus displacement for treatment $b c \ldots \ldots \ldots \ldots \ldots \ldots . . . .125$

Table 4.5 Summary of results. Force versus displacement for treatment abcde ............ 130

Table 4.6 Experimental treatments with EA9361 adhesive........................................... 134

Table 4.7 Summary of results. Force versus displacement for treatment de ................. 136

Table 4.8 Summary of results. Force versus displacement for treatment $a \ldots \ldots \ldots \ldots \ldots \ldots \ldots . . .142$

Table 4.9 Summary of results. Force versus displacement for treatment $c d$................. 148

Table 4.10 Summary of results. Force versus displacement for treatment ace .............. 152 
Table 4.11 Effect of each factor in the joint strength. First failure point, total strength vs

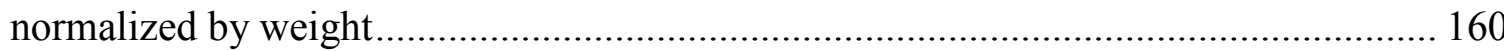

Table 4.12 Effect of each factor in the maximum strength. Total strength vs normalized by weight 163

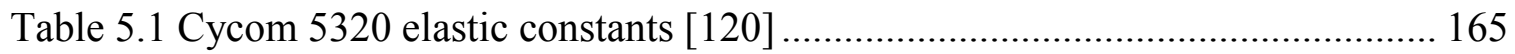

Table 5.2 Cycom 5320 elastic constants. Adapted from [120]................................. 165

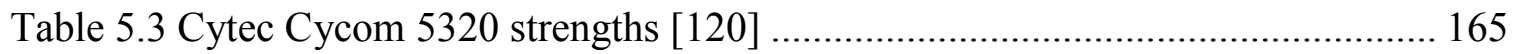

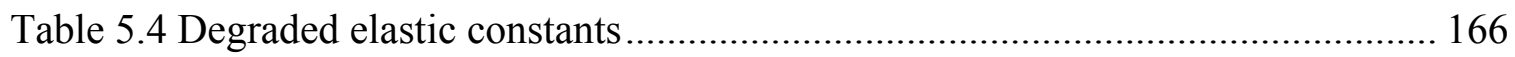

Table 5.5 Comparison between mechanical properties FM300 and FM300-2M adhesives.

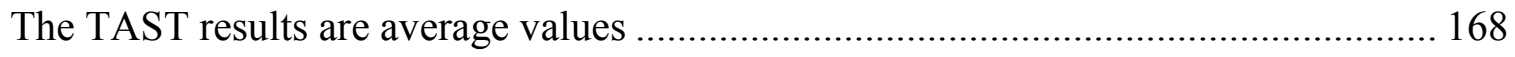

Table 5.6 TAST data EA9361 adhesive. Average values.......................................... 169

Table 5.7 Summary of CZM data for FM300 adhesive from literature review............. 175

Table 5.8 Summary of CZM data for EA9361 adhesive from literature review ........... 175

Table 5.9 FM300-2M and EA9361 adhesives. CZM parameters used in the cohesive

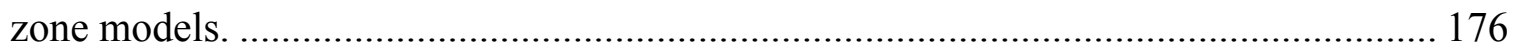

Table 5.10 Experimental treatments with FM300-2 adhesive .................................. 193

Table 5.11 Bolted/bonded joints. Predicted failure load using CZM and comparison with

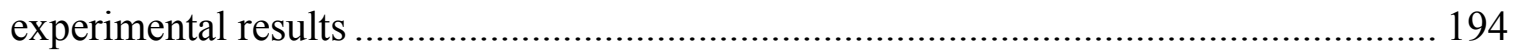

Table 5.12 Experimental Treatments with EA9361 Adhesive .................................. 198

Table 6.1 Predicted force in the bolt. Treatments with FM300-2M adhesive .............. 222

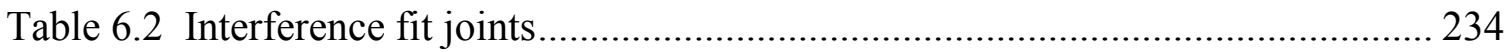

Table B.1 Extracted displacements from the DIC software ................................... 271

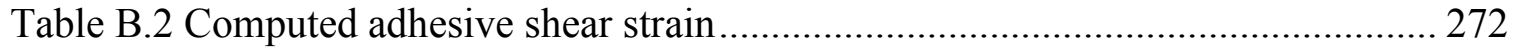


Table C.1 ANOVA results. First failure point, total strength.

Table C.2 ANOVA results. First failure point, strength normalized by weight ............ 275

Table C.3 ANOVA results. Maximum strength. ................................................. 276

Table C.4 ANOVA results. Maximum strength, normalized by weight ..................... 277

Table E.1 Adhesive thickness in bolted/bonded joints ........................................... 282 


\section{List of Illustrations}

Figure 1.1 Composite materials used in the Boeing aircraft family showing the composites increase rate over the time as well as the current 787 Dreamliner [6]........... 2

Figure 2.1 Typical stress distribution in single-lap bonded joint, Volkersen model .......... 9

Figure 2.2 A typical stress distribution in single-lap bonded joint, Goland and Reissner

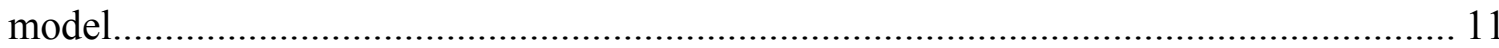

Figure 2.3 Fastener flexibility in a single-lap joint configuration .................................. 13

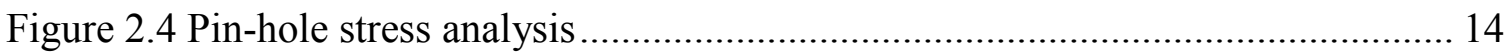

Figure 2.5 Failure modes in composite bolted joints [12] ......................................... 15

Figure 2.6 Composite Bolted/bonded joint............................................................... 17

Figure 2.7 Through-the thickness stress variation in a bolted composite joint ................ 17

Figure 2.8 Graphical description of load paths on bolted/bonded joints according to Yamaguchi et al. (Reprinted from [42] with permission from Elsevier).......................... 19

Figure 2.9 Riveted-bonded joint analyzed by Gomez showing and the proposed model and the bond-graph diagram. (Reprinted from [50] with permission from Elsevier)....... 20 Figure 2.10 Force-elongation results according to Gomez. (Reprinted from [50] with

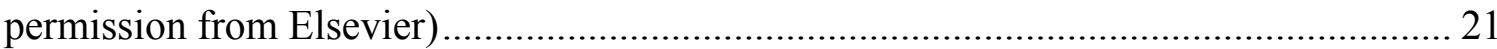

Figure 2.11 Hybrid bolted-bonded joint model proposed and analyzed by Paroissien [51]

Figure 2.12 Effect of the adhesive modulus on the bolt load transfer according to

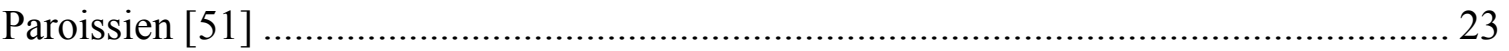

Figure 2.13 Maximum principal strain in bolted/bonded joint measured with DIC.

(Reprinted from [66] with permission from Springer) ……......................................... 32 
Figure 2.14 Instrumented bolt configuration for shear force measurement. (Reprinted

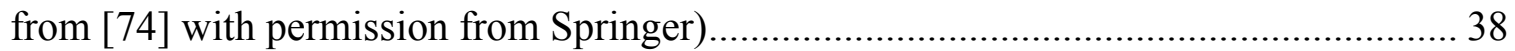

Figure 2.15 Cohesive zone model, triangular traction-separation law shape ................. 42

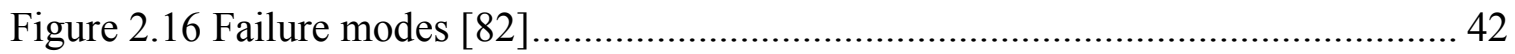

Figure 2.17 Cohesive zone model using continuum approach with a single row of

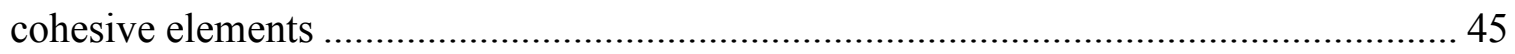

Figure 2.18 Cohesive zone model using local approach with zero-thickness cohesive

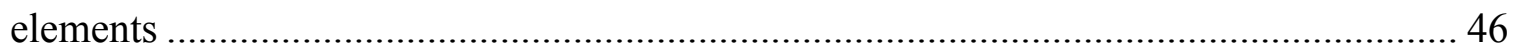

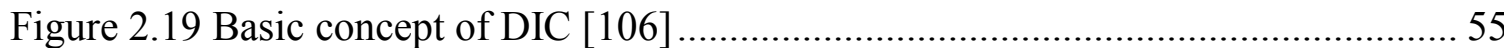

Figure 2.20 Strain calculation using DIC performed by Lemmen et al. (Reprinted from [114] with kind permission from Dr. Lemmen) ..................................................... 59

Figure 2.21 Relationship between factors interaction; a) Linear; b) Exponential ........... 62

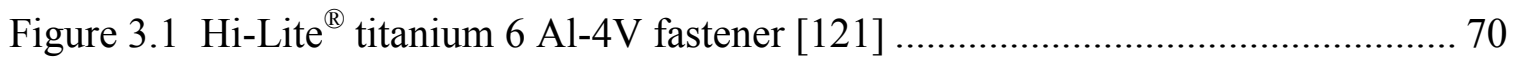

Figure 3.2 Bonded joint specimen according to ASTM D-3165. Adapted from [122] ... 72 Figure 3.3 Bolted joint specimen from ASTM D5961/D5961M-10. Adapted from [123] 73

Figure 3.4 Bolted/bonded joint specimen design................................................ 73

Figure 3.5 Washer dimensions, mm (in). a) NAS1149-C0363B aerospace grade washer;

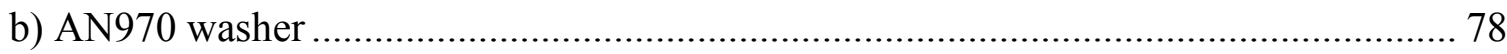

Figure 3.6 Bagging process. a) Prepreg and edge dams; b) Caul plates and teflon layer at the top of the laminates; c) Final bag showing the breather and thermocouples; d)

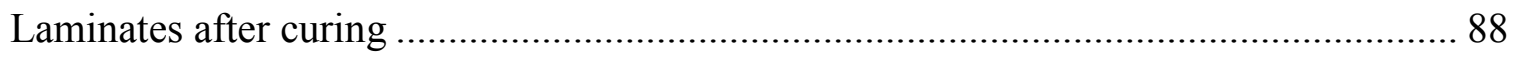

Figure 3.7 Curing process Cytec Cycom 5320 unidirectional prepreg laminates .......... 89 
Figure 3.8 Piano wires installation for the bonding procedure, units $\mathrm{mm}$.................... 91

Figure 3.9 Curing process for FM300-2M adhesive............................................... 91

Figure 3.10 EA936 paste adhesive application on plates with piano wires .................... 92

Figure 3.11 Transverse location of piano wires to bond EA9361 paste adhesive. The

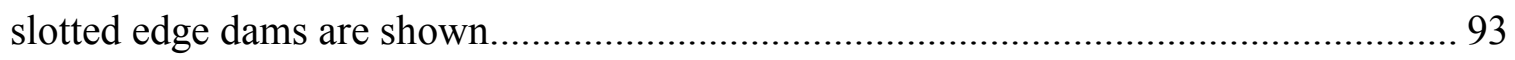

Figure 3.12 Gaertner traveling microscope used to measure the adhesive thickness ....... 94

Figure 3.13 Cutting specifications for plates with longitudinally located piano wires

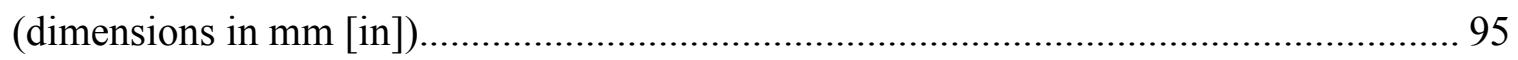

Figure 3.14 Cutting specifications for plates with transversally located piano wires ...... 95

Figure 3.15 Hole diameter measurement ........................................................... 96

Figure 3.16 Bolt hole: a) surface at tool entrance; b) surface at tool exit....................... 97

Figure 3.17 Notch details indicating the cut for two adhesive thicknesses; a) $0.5 \mathrm{~mm}$; b)

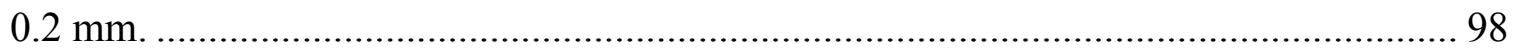

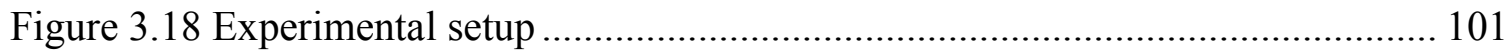

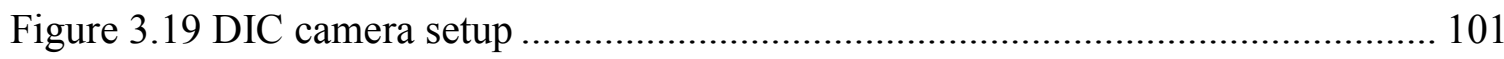

Figure 3.20 Local displacement measurement. Selected lengths, mm. ...................... 103

Figure 3.21 Selected points to measure the adhesive's shear strain inside the bondline 104 Figure 4.1 Bolted joints. a) 3.29mm adherend thickness; b) $5.48 \mathrm{~mm}$ adherend thickness

Figure 4.2 Experimental results for bolted joints. Force versus displacement .............. 108

Figure 4.3 Failure modes in bolted joints. a) Joint with $3.29 \mathrm{~mm}$ adherend thickness, b)

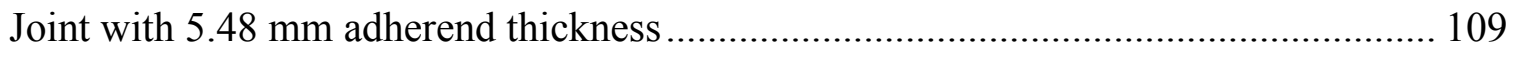


Figure 4.4 Bonded joints, force versus displacement results. a) adherend thickness 3.29 $\mathrm{mm}$ and adhesive thickness $0.2 \mathrm{~mm}\left(\mathrm{de} \_01\right)$ and $0.5 \mathrm{~mm}\left(\mathrm{~cd} \_01\right)$; b) adherend thickness $5.48 \mathrm{~mm}$ and adhesive thickness $0.2 \mathrm{~mm}$ (a_01) and $0.5 \mathrm{~mm}$ (ace_02) ....................... 110

Figure 4.5 Treatment be, force versus displacement results .................................... 112

Figure 4.6 Load versus displacement in sample be_01 depicting the failure points ...... 113

Figure 4.7 Sample be_01. Force versus relative displacement using $35 \mathrm{~mm}$ reference length. The experimental data is compared against FEM models, elastoplastic and

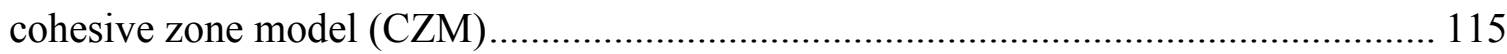

Figure 4.8 Sample be_01. Adhesive shear strain at $1 \mathrm{~mm}$ distance from the notch........ 116 Figure 4.9 Sample be_02. Force versus relative displacement using $17.5 \mathrm{~mm}$ reference length 117

Figure 4.10 Sample be_02. Adhesive shear strain at 1mm distance from the notch ...... 117

Figure 4.11 Sample be_03. Adhesive shear strain at 1mm distance from notch ........... 118

Figure 4.12 Combination $a b d$, force versus displacement results .............................. 119

Figure 4.13 Combination $a b d$, force versus displacement after displacement correction

Figure 4.14 Sample $a b d \_02$, failure at point A.................................................... 120

Figure 4.15 Sample $a b d \_01$ and $a b d \_02$. Adhesive shear strain at $1 \mathrm{~mm}$ from the notch

Figure 4.16 Sample $a b d \_02$. Force versus relative displacement using $17.5 \mathrm{~mm}$ reference length 122

Figure 4.17 Sample $a b d \_02$. Adhesive shear strain at $1 \mathrm{~mm}$ distance from the notch ... 122 
Figure 4.18 Sample abd_03. Force versus relative displacement using $35 \mathrm{~mm}$ reference length 123

Figure 4.19 Samples $a b d \_01$ and $a b d \_02$. Comparison between shear strains at $1 \mathrm{~mm}$ distance from the notch. 124

Figure 4.20 Combination $b c$, force versus displacement results 125

Figure 4.21 Sample $b c \_01$. Force versus displacement and failure points 126

Figure 4.22 Sample $b c \_01$ and sample $b c \_03$. Force versus relative displacement using $35 \mathrm{~mm}$ reference length 127

Figure 4.23 Sample $b c \_02$. Shear strain at $3 \mathrm{~mm}$ distance from the notch. 128

Figure 4.24 Combination abcde. Force versus displacement results 129

Figure 4.25 Combination abcde. Corrected force versus displacement results 130

Figure 4.26 Sample abcde_01. Adhesive shear strain at $3 \mathrm{~mm}$ distance from the notch 131 Figure 4.27 Sample abcde_02. Force versus relative displacement using $35 \mathrm{~mm}$ reference length 132

Figure 4.28 Combination de. Force versus displacement results. 135

Figure 4.29 Treatment $d e$ and comparison with bolted and bonded joints. Force versus displacement results 137

Figure 4.30 Reference DIC image for coupons de_01, de_02 and de_03 138 Figure 4.31 Samples de_01, de_02 and de_03. Adhesive shear strain at $1 \mathrm{~mm}$ and $2 \mathrm{~mm}$ from the notch 139 Figure 4.32 Sample de_02. Adhesive shear strain at different distances from the notch 139

Figure 4.33 Joint failure sequence, treatment de, sample de_01. 140 
Figure 4.34 Treatment $a$. Force versus displacement results. 142

Figure 4.35 Treatment $a$ and comparison between bonded and bolted joints. Force

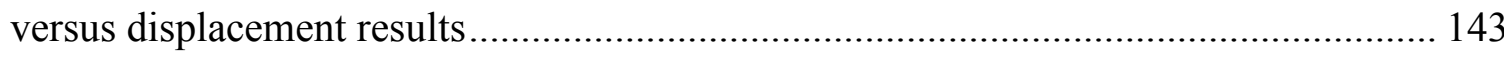

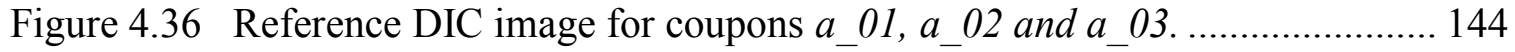

Figure 4.37 Samples $a \_01, a \_02$ and $a \_03$. Adhesive $s$ hear strain at $1 \mathrm{~mm}$ and $2 \mathrm{~mm}$ from

the notch. 145

Figure 4.38 Failure process in the bolted bonded joint compared with the bonded joints.

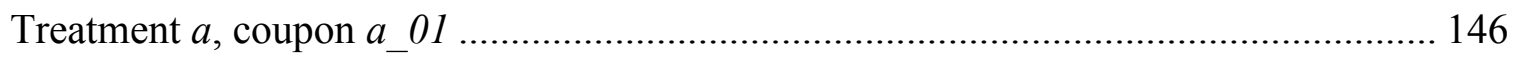

Figure 4.39 Treatment $c d$. Force versus displacement results................................ 147

Figure 4.40 Samples $c d \_01, c d \_02$ and cd_03. Adhesive $s$ hear strain at $1 \mathrm{~mm}$ and $2 \mathrm{~mm}$

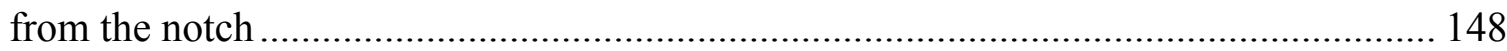

Figure 4.41 Sample cd_03. Adhesive shear strain at different distances from the notch

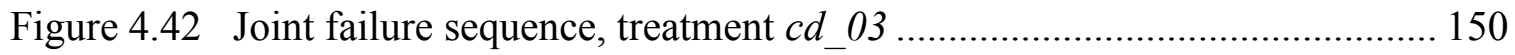

Figure 4.43 Force versus displacement results. Treatment ace ............................... 151

Figure 4.44 Force versus displacement results after displacement correction. Treatment

ace

Figure 4.45 Sample ace_02. Force versus relative displacement using $35 \mathrm{~mm}$ reference

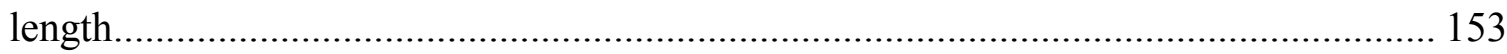

Figure 4.46 Repetition test, treatment ace_04. Force versus displacement results. ....... 154

Figure 4.47 Sample ace_rep_01. Adhesive shear strain at different distances from the

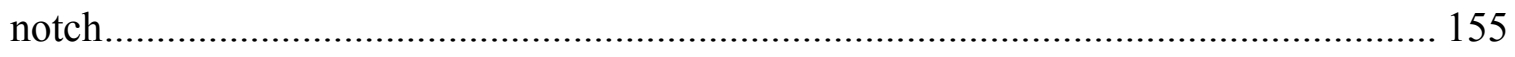

Figure 4.48 Joint failure sequence, treatment ace .................................................. 156 
Figure 4.49 Experimental results, stength at first failure point. a) Total force; b) Total force per weight

Figure 4.50 ANOVA results, main factors on the strength at first failure point. a) Total force; b) Total force per weight 160 Figure 4.51 Experimental results, maximum strength. a) Total force; b) Total force per weight. 162 Figure 4.52 ANOVA results, main factors effect in the total maximum strength. a) Total force; b) Total force per weight 162 Figure 5.1 Laminate quasi-isotropic Young's modulus and Poisson's ratio. Experimental results 167

Figure 5.2 Shear stress versus strain data from TAST for EA9361 and FM300-2 adhesives 168

Figure 5.3 EA9361 adhesive. Shear strain-stress properties from TAST, and converted tensile strain-stress properties 170 Figure 5.4 FM300-2M adhesive. Shear strain-stress properties from TAST, and converted tensile strain-stress properties 171

Figure 5.5 EA9361 adhesive. Thickness-dependent cohesive parameters; a) In tension; b) In Shear ( Reprinted from [140] with permission from Elsevier). 174 Figure 5.6 FEM model geometry 177 Figure 5.7 Laminate with 24 plies, $3.29 \mathrm{~mm}$ thickness and stacking sequence [0/45/90/$45]_{3 \mathrm{~s}}$ 179

Figure 5.8 Laminate with 40 plies, $5.48 \mathrm{~mm}$ thickness and stacking sequence [0/45/90/$45]_{5 \mathrm{~s}}$ 179 
Figure 5.9 Finite element models. a) Adherend thickness $3.29 \mathrm{~mm}$, adhesive thickness 0.5 mm, small washer; b) Adherend thickness $5.48 \mathrm{~mm}$, adhesive thickness $0.5 \mathrm{~mm}$, large washer 180

Figure 5.10 Bonded joints with FM300-2M adhesive. Force versus displacement CZM results and comparison with experimental results.

Figure 5.11 Bonded joints with FM300-2M adhesive. CZM results. The adhesive degradation is shown at different force values. 184

Figure 5.12 Bonded joints with FM300-2M adhesive. Predicted strength using CZM.. 185 Figure 5.13 Bonded joint with FM300-2M adhesive. CZM results showing the damage in the adhesive at maximum strength of $11.6 \mathrm{kN}$ 186

Figure 5.14 Bonded joint with EA9361 adhesive. Load versus displacement response from cohesive zone modelling results. Adherend thickness $3.29 \mathrm{~mm}$ and adhesive thickness $0.2 \mathrm{~mm}$ 187 Figure 5.15 Bonded joint with EA9361 adhesive. Damage in the adhesive at maximum strength of $17.84 \mathrm{kN}$ using CZM continuum approach. Adherend thickness $3.29 \mathrm{~mm}$ and adhesive thickness $0.2 \mathrm{~mm}$ 188 Figure 5.16 Bonded joint with EA9361 adhesive. Damage progression in the adhesive using CZM local approach: a) at maximum strength of $17.84 \mathrm{kN}, \mathrm{b}$ ) at $15.52 \mathrm{kN}$, after the maximum strength. Adherend thickness $3.29 \mathrm{~mm}$ and adhesive thickness $0.2 \mathrm{~mm}$ 189 Figure 5.17 Bonded joint with EA9361 adhesive. Load versus displacement from cohesive zone modelling results. Adherend thickness $5.48 \mathrm{~mm}$ and adhesive thickness 0.2 $\mathrm{mm}$ 190 
Figure 5.18 Bonded joint with EA9361 adhesive. Load versus displacement from cohesive zone modelling results. Adherend thickness $3.29 \mathrm{~mm}$ and adhesive thickness 0.5

$\mathrm{mm}$

Figure 5.19 Bolted/bonded joints with FM300-2M adhesive. Force versus displacement finite element results 193

Figure 5.20 Bolted/bonded joints with FM300-2M adhesive. CZM strength prediction and comparison with elastoplastic models 195

Figure 5.21 Bolted/Bonded joint,combination $b c$. Failure progress in the adhesive layer using CZM analysis. a) Maximum force of $15.2 \mathrm{kN}$, b) After maximum force, at $13.7 \mathrm{kN}$

Figure 5.22 Bolted/bonded joints with FM300-2M adhesive. Predicted load at the bolts from finite element analysis 196

Figure 5.23 Bolted/bonded joints with FM300-2M adhesive. Predicted adhesive shear strain from finite element analysis

Figure 5.24 Bolted/bonded joint, model bc. Predicted shear strain comparison. Model with progressive failure in the composite vs model without damage in the composite 198 Figure 5.25 Bolted/bonded joints with EA9361 adhesive. Force versus displacement finite element results 200 Figure 5.26 Bolted/bonded joints with EA9361 adhesive. Total predicted load at the bolts from finite element analysis 200 Figure 5.27 Bolted/bonded joints with EA9361 adhesive. Percent of load at the bolts from finite element analysis. 201 
Figure 5.28 Bolted/bonded joints with EA9361 adhesive. Predicted adhesive shear strain from finite element analysis 202

Figure 5.29 Bolted/bonded joints with EA9361 adhesive. Adhesive shear strain comparison. Shear strain at $3 \mathrm{~mm}$ distance from the notch 203

Figure 5.30 Bolted/bonded joint, model $F E_{-} c d$. Effect of the composite damage in the joint performance. a) Load taken by the bolt; b) Adhesive shear strain 204 Figure 5.31 Bolted/bonded joint, model $F E \_c d$. Predicted failure modes in the composite at $18 \mathrm{kN}$ of applied force 205 Figure 5.32 Bolted/bonded joint, model $c d$ with EA9361 adhesive. CZM strength prediction 206 Figure 5.33 Bolted/Bonded joint, combination $c d$. Failure progress in the adhesive layer using CZM analysis 207

Figure 5.34 Bolted/Bonded joint, combination $c d$. Predicted composite damage after adhesive failure using CZM 208

Figure 5.35 Bolted/bonded joint, model $c d$. Load transfer comparison CZM vs FEM elastoplastic 210

Figure 5.36 Bolted/bonded joint, model ace with EA9361 adhesive. CZM strength prediction 211

Figure 5.37 Bolted/bonded joints, model ace. Strength prediction using CZM local approach and comparison with experimental results and FEM elastoplastic 212 Figure 5.38 Bolted/bonded joint, model ace. Adhesive damage at $20 \mathrm{kN}$ of applied force at the surface of the cohesive elements located at the top interface between the adhesive and the composite adherend. 213 
Figure 6.1 Bolted/bonded joint, FE model $c d$. Comparison between the extracted shear strain from the nodes and the shear strain computed using the 3 point method. Distance 1

$\mathrm{mm}$ from the notch

Figure 6.2 Bolted/bonded joint, FE model de. Comparison between the extracted shear strain from the nodes and the shear strain computed using the 3 point method. Distance 1

$\mathrm{mm}$ from the notch

Figure 6.3 Bolted/bonded joint, treatment be_02 with FM300-2M adhesive. Predicted force in the bolt comparing experimental and numerical adhesive shear strains 220 Figure 6.4 Bolted/bonded joint, treatment be_01 with FM300-2M adhesive. Predicted force in the bolt comparing experimental and numerical relative displacements. Figure 6.5 Bolted/bonded joint, treatment de. Bolt force analysis using shear strains at 1 $\mathrm{mm}$ form the notch 225

Figure 6.6 Bolted/bonded joint, treatment de. Bolt force analysis and comparison with a joint with neat fit assembly 227

Figure 6.7 Bolted/Bonded joint, treatment $a$. Bolt force analysis using shear strains at a distance $3 \mathrm{~mm}$ from the notch. 228 Figure 6.8 Bolted/bonded joint, treatment cd. Bolt force analysis using shear strains at a distance $1 \mathrm{~mm}$ from the notch. 230 Figure 6.9 Bolted/bonded joint, treatment ace_rep_01. Bolt force analysis using shear strains at a distance $1 \mathrm{~mm}$ from the notch. 232 Figure 6.10 Bolted/bonded joints with EA9361 adhesive and interference fit. Force versus displacement results. 235 
Figure 6.11. Bolted/bonded joint with EA9361 adhesive and interference fit assembly. Adhesive shear strain at $1 \mathrm{~mm}$ distance from the notch 235

Figure 6.12 Bolted/bonded joints with FM300-2M adhesive and interference fit. Force versus displacement results. 236

Figure 6.13 Bolted/bonded joint with FM300-2M adhesive and interference fit assembly.

Adhesive shear strain at $1 \mathrm{~mm}$ distance from the notch 237

Figure 7.1 Bonded, bolted and bolted bonded joint comparison with stiff adhesive

FM300-2M. 242

Figure 7.2 Bonded, bolted and bolted bonded joint comparison with ductile adhesive EA9361. 244

Figure A.1 Test machine compliance displacement 266

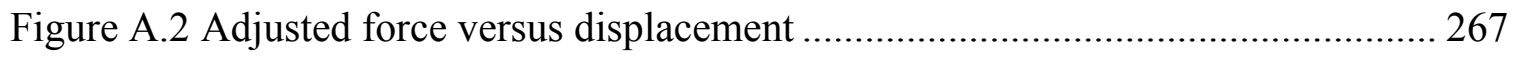

Figure B.1 Adhesive shear strain analysis. Points before and after applied force........ 268

Figure B.2 Adhesive shear strain analysis. Final position of points $l, r$, and $b$ 269

Figure B.3 Adhesive shear strain measurement. Initial image and reference points ...... 270 Figure B.4 Adhesive shear strain measurement. Deformed image number 142, time $=70.5$

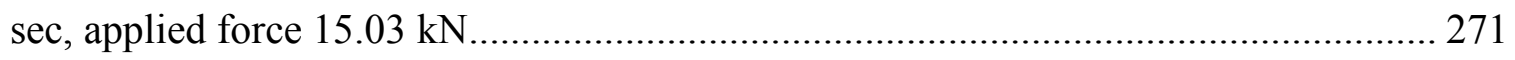

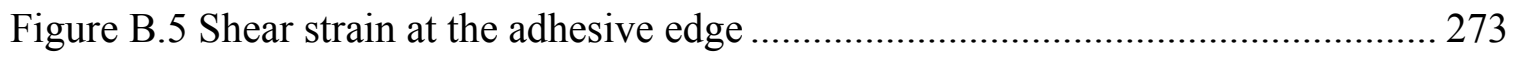
Figure C.1 Strength at first failure point. a) Normal probability plot; b) Normal plot of standardized effect 274

Figure C.2 Interaction plots strength at first failure point 274 Figure C.3 Strength normalized at first failure point. a) Normal probability plot; b) Normal plot of standardized effect 275 
Figure C.4 Interaction plots strength at first failure point. Normalized by weight

Figure C.5 Maximum strength. a) Normal probability plot; b) Normal plot of

standardized effect

Figure C.6 Interaction plots at maximum strength

276

Figure C.7 Maximum strength normalized. a) Normal probability plot; b) Normal plot of standardized effect 277

Figure C.8 Interaction plots maximum strength.Normalized by weight 277 


\section{List of Symbols and Acronyms}

$\tau$

$\sigma$

G

$\mathrm{G}_{\mathrm{a}}$

$\gamma$

$v$

E

$\mathrm{t}$

$\mathrm{t}_{\mathrm{a}}$

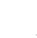

1 Length of the adhesive layer

b Bonded area

P Applied force

$\bar{P} \quad$ Applied force per unit width

k Bending moment factor

$k^{\prime} \quad$ Transverse force factor

f Fastener flexibility

$\delta \quad$ Fastener displacement

$t_{n} \quad$ CZM nominal traction normal direction

$t_{s} \quad$ CZM nominal traction shear direction

$\mathrm{k}_{\mathrm{n}} \quad$ CZM nominal stiffness normal direction

$\mathrm{k}_{\mathrm{s}} \quad \mathrm{CZM}$ nominal stiffness shear direction

$\mathrm{G}_{\mathrm{Ic}} \quad$ Critical energy release normal direction

$\mathrm{G}_{\text {IIc }} \quad$ Critical energy release shear direction

$\mathrm{P}_{\mathrm{b}} \quad$ Load transferred to the bolt

$\mathrm{P}_{\mathrm{a}} \quad$ Load transferred to the adhesive 


$\begin{array}{ll}\text { ANOVA } & \text { Analysis of Variance } \\ \text { CFRP } & \text { Carbon Fibre Reinforced Plastic } \\ \text { CZM } & \text { Cohesive Zone Modelling } \\ \text { DIC } & \text { Digital Image Correlation } \\ \text { DCB } & \text { Double Cantilever Beam } \\ \text { DOE } & \text { Design of Experiments } \\ \text { ENF } & \text { End Notched Flexure } \\ \text { GFRP } & \text { Glass Fibre Reinforced Polymer } \\ \text { FEM } & \text { Finite Element Modelling } \\ \text { FOV } & \text { Field of View } \\ \text { MS } & \text { Mean square (ANOVA) } \\ \text { SS } & \text { Sum of squares (ANOVA) } \\ \text { SPR } & \text { Self-Piercing Riveting }\end{array}$




\section{List of Appendices}

Appendix A: Procedure to Adjust the Stroke Displacement due to the Test Machine

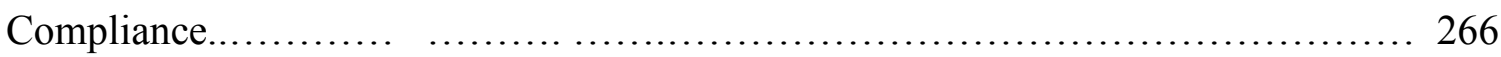

Appendix B: Procedure to Measure Shear Strain at the Adhesive Edge ............. 268

Appendix C: ANOVA results........................................ 274

Appendix D: FORTRAN USDFLD Subroutine............................ 278

Appendix E: Adhesive Thickness. Bolted/bonded Joints.................... 282

Appendix F: Typical Failure Modes in Tested Coupons..................... 283

F.1: $\quad$ Bolted/bonded joints with FM300-2M Adhesive..........................283

F.2: $\quad$ Bolted/bonded joints with EA9361Adhesive..........................284

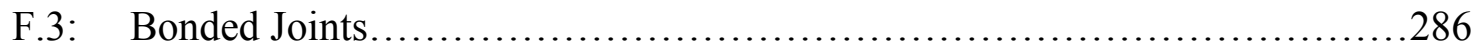




\section{Chapter 1: Introduction}

\subsection{Composites in Aircraft Structural Design}

Traditional materials and manufacturing processes used in the aerospace industry are changing dramatically since the past years. Increases in oil prices, tightening environmental regulations and a steady increase in the aircraft traffic have been the main drivers for these changes [1]. Therefore, the need for weight reductions to improve overall efficiency has been a goal for both aircraft manufacturers and operators.

To address this required reduction in weight, advanced composite materials have been increasingly used in secondary and primary aircraft structures. Historically, both military aircraft and rotorcraft manufacturers were the first to recognize the potential use of composites materials followed by general aviation manufacturers, whose efforts led to the development of the first all-composite aircraft [2] in the 1970s. For example, the Vari-Eze and the Long EZ designed by Burt Rutan. However, these aircraft were more often used for recreational and general aviation purposes.

Until recently, transport aircraft manufacturers have been using advanced composite materials in primary aircraft structures in a small scale. In the 1970s, due to the oil crisis, the NASA launched the Aircraft Energy Efficiency project (ACEE) [3]. Between the six main programs of the ACEE, the Composite Primary Aircraft Structures Program was proposed with the aim of developing the technology to use composites in commercial aircraft, and the main objective was to reduce the weight of the aircraft by 25 percent [3]. However, several factors have delayed large-scale applications, mainly high manufacturing costs compared with similar structures made of metals, lack of data about 
durability and maintenance [3], and the high certification cost for new components [4]. Notwithstanding this, their use has been increasing steadily until 2011 when the new Boeing 787 Dreamliner was certified [5]. This aircraft has 50\% of its structural weight consisting of fibre reinforced polymer composites, a significant increase compared with 11\% found in the Boeing 777 model as shown in Figure 1.1 [6]. Similarly, the new Airbus A350 XWB was designed using 53\% composites on the overall airframe structure [7].

These achievements have been made possible due to the new automated manufacturing techniques and the new generation of composite materials. Traditionally, fibres pre-impregnated with initiated resin (prepreg) [8], and autoclave processing have been the standard for aerospace structures. However, out-of-autoclave (OOA) prepregs are becoming attractive to the aircraft manufacturers because of their potential to reduce high capital, raw material and operational costs during manufacturing and their increasing performance which, in some cases, match the performance of autoclave-cured prepregs [9].
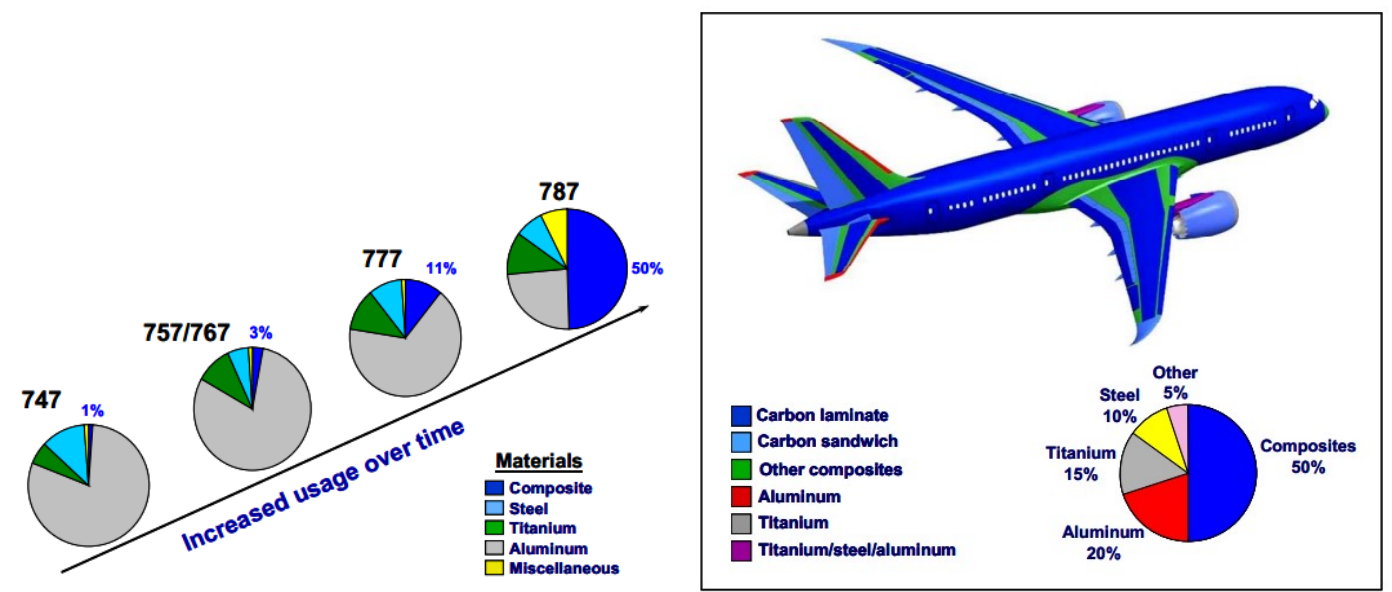

Figure 1.1 Composite materials used in the Boeing aircraft family showing the composites increase rate over the time as well as the current 787 Dreamliner [6] 
The most important composite material used in aircraft structures is the carbon fibre in an epoxy resin matrix, also referred as carbon/epoxy, graphite epoxy or Carbon Fibre Reinforced Plastic (CFRP). The high strength of carbon fibre improves both the high specific strength (strength/density) and specific modulus (modulus/density), even stronger than comparable metal alloys, including the fatigue strength [10]. On the other hand, because there are no fibres oriented in the through-the-thickness direction, the mechanical performance is poor in that direction. Absorption of environmental moisture and temperature also affect the composite performance. In general aviation, these conditions are dictated by the environment during flight and parking conditions [11]. The matrix is prone to moisture absorption from the humid air reducing the mechanical properties [4]. Additionally, these materials are susceptible to impact damage and delamination.

Another issue with the application of composite materials in structures is the diversity in the material systems combinations, resulting in a wide variety of structural data for every laminate configuration. Moreover, there is no consensus on analytical failure prediction tools in laminated composites $[12,13]$. The former affects the analysis of composite laminates in aircraft structures, and bolted and bonded composite joints are no exception. Adhesively bonded and mechanically-fastened composite joints have been widely studied for more than thirty years, and extensive government-funded programs have already produced much information on this topic [14-19]. The joining using adhesive secondary bonding can reduce the weight and manufacturing cost significantly [20]. However, the bonded joint technology is still not widely applied, mainly due to the difficulty to meet the certification requirements [21]. The requirements for substantiating 
the primary composite airframe structures are specified in the federal code of regulations title 14 [22]. For bonded joints, the requirements specify that the limit load capacity in the bonded joints must be substantiated by using one of the following methods [21, 22]:

a) Minimize the disbonds using "design features",

b) Proof testing on each production article, and:

c) Ensure the strength of each joint using non-destructive inspection.

Gardiner [23] pointed out that full-scale proof testing for aircraft structures is prohibitively expensive and the current non-destructive techniques are not capable of measuring bond strength. On the other hand, minimizing the disbond using design features requires the addition of crack arresters. Therefore, the addition of bolts is the most widely used "design feature" to meet the current substantiation requirements for bonded joints in primary aircraft composite structures. By adding fasteners in the bonded joint, the two joining technologies are combined to create a hybrid bolted/bonded joint.

However, hybrid bolted/bonded joint technology is currently in the early stages of research and development and will require extensive research to reach the same level of knowledge as compared to bonded and bolted joint technologies.

Currently, CRIAQ (Consortium for Research and Innovation in Aerospace in Quebec) and the Natural Sciences and Engineering Research Council (NSERC) are cosponsoring a Collaborative Research and Development program on bolted/bonded joints in composite materials. This program, denominated COMP 506, is being carried out with academic and industrial partners including Bombardier Aerospace, Delastek, L3Com, the National Research Council Canada Aerospace Portfolio, McGill University, and Carleton University. 


\subsection{Structure of the Thesis}

The following section describes the literature review, methodology, results, and discussions presented in this thesis.

Chapter 1 provides a general overview on the use of composite materials in aircraft and joining technologies. The structure of the thesis, objectives and contributions are also explained.

Chapter 2 presents the literature review on joining technologies in bonded, bolted and bolted/bonded composite joints. Additionally, a review of the tools and technologies used in this research to achieve the objectives is presented, including: design of experiments, modelling composite joints using cohesive zone modelling, digital image correlation technique and progressive failure modelling in composites. Finally, the research motivation and objectives are presented based on the literature review analysis.

Chapter 3 presents all of the steps carried out during the experimental work, starting with the procedure to define the experimental design, design of the final coupons, manufacturing procedures, the experimental setup and methods to collect data from the testing.

Chapter 4 describes the results from the experiments in bolted, bonded and bolted/bonded joints. A summary of the experimental results using ANOVA was carried out on the proposed response parameters.

Chapter 5 covers the numerical models for bolted/bonded joints and the modelling results. First, results from the numerical models using an elastoplastic approach in the adhesive, stresses, strains and load sharing between adhesive and bolt are described. 
Following this, the joint strength analysis results using the cohesive zone model (CZM) approach is also analyzed.

Chapter 6 presents the analysis of load sharing in bolted/bonded joints using the proposed method of measuring the shear strains at the adhesive edge. The procedure was carried out by comparing the shear strains in bonded and bolted/bonded configuration. Also, the experimental shear strains were compared with the numerical results.

Chapter 7 summarizes the results from the experiments and numerical models.

Chapter 8 presents the conclusions drawn from this research work and the recommendations for future research work.

\subsection{Thesis Contributions}

During the present research the following contributions to expand the knowledge on composite bolted/bonded joints were achieved:

1. The application of the design of experiments methodology to quantify the effect of several variables on the bolted/bonded joint strength. The selected variables were identified from the literature review. The effect of the clearance between the bolt and the hole, which was not reported in the literature, was incorporated in the design of experiments

2. The novel application of the digital image correlation strain measurement method as an alternative load transfer measurement technique, as opposed to using instrumented bolts, to measure the strains in the adhesive edge on bonded and bolted/bonded joints. Using this information, the load taken by the bolt during the full load path until failure for several joint was computed. This was 
complemented with 3D FEM modelling to successfully compare against the experimental results.

3. The application of the cohesive zone modelling to predict the strength of composite bolted/bonded joints and, including in the FEM model, progressive failure in the layered composite in some joint configurations.

4. The material properties to be entered into the FEM commercial software are of great importance to achieve accurate calculations and good agreement with the experimental results. Therefore, an experimental adhesive characterization was needed for this research program. Adhesive characterization and FEM models including data from this characterization were carried out to analyze the composite bolted/bonded joints performance. This study was carried out jointly with other participants and a journal paper manuscript was written. Currently, the manuscript is in review at the National Research Council Canada prior to submission to a peer-reviewed journal. 


\section{Chapter 2: Background and Literature Review}

\subsection{Composite Bonded Joints}

Adhesive bonding technology encompasses several fields of science and engineering such as chemistry, surface physics and mechanics [24]. This joining technology has been widely used in the aircraft industry because of their advantages [25] including weight reduction, good fatigue life, good external finishing, capability to join thin substrates, ability to join different substrates and good environmental sealing. On the other hand, disassembly generally is not possible. Moreover, adhesively bonded joints are susceptible to manufacturing process quality problems such as surface preparation and environmental conditions that can negatively affect the joint.

Structural adhesives commonly used in the aerospace industry include phenolic, phenolic-epoxy, bismaeleimide, cyanate ester, acrylic and polyurethane adhesives. Among these kinds of adhesives, epoxy is the most widely used in the aerospace industry $[26]$.

The main function of the adhesive is to transfer load between substrates, mainly due shear loading. This function can be carried out in several joint configurations, namely single-lap, double-lap, single strap, double trap, tapered double strap, scarf and step lap joints.

Analytical models have been used along with extensive experimentation on adhesive joints to assess the joint performance. An early attempt to analyze analytically bonded joints was conducted by Volkersen [27]. This model computes the shear stress in the adhesive, but it does not include peel stress due to secondary bending. 
The shear stress distribution along the bondline shown in Figure 2.1 is given by:

$$
\tau=\frac{P \omega}{2 b} \frac{\cosh (\omega x)}{\sinh \left(\frac{\omega l}{2}\right)}+\left(\frac{t_{t}-t_{b}}{t_{t}+t_{b}}\right)\left(\frac{\omega l}{2}\right) \frac{\sinh (\omega x)}{\cosh \left(\frac{\omega l}{2}\right)}
$$

Where:

$$
\omega=\sqrt{\frac{G_{a}}{E t_{t} t_{a}}\left(1+\frac{t_{t}}{t_{a}}\right)}
$$

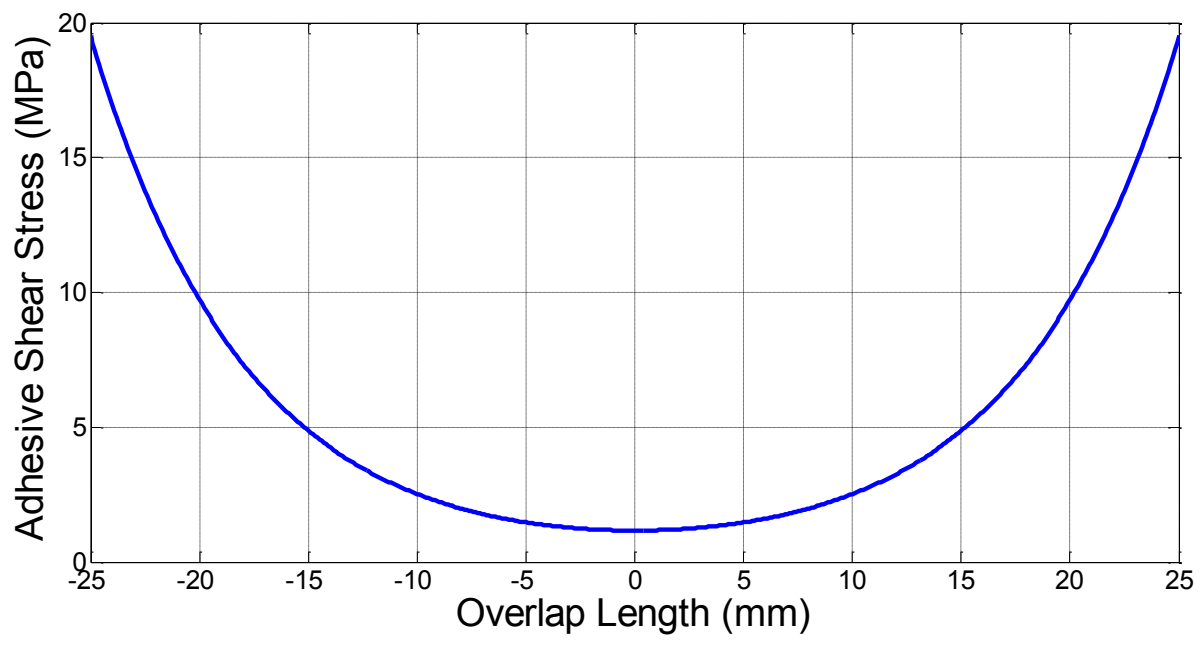

Figure 2.1 Typical stress distribution in single-lap bonded joint, Volkersen model

Goland and Reissner [28] developed a model including the bending moment created by the eccentric load in the single-lap joint. Therefore, the model was able to predict the shear stress distribution along with the peel stress in the bondline as shown in Figure 2.2. The shear stress along the overlap is given by: 


$$
\tau=-\frac{1}{8} \frac{\bar{P}}{c}\left\{\frac{\beta c}{t}(1+3 k) \frac{\cosh \left(\frac{\beta c}{t} \frac{x}{c}\right)}{\sinh (\beta c / t}+3(1-k)\right\}
$$

Where:

$$
k=\frac{\cosh \left(u_{2} c\right)}{\cosh \left(u_{2} c\right)+2 \sqrt{2} \sinh \left(u_{2} c\right)}
$$

And

$$
\begin{gathered}
\mathrm{u}_{2}=\sqrt{\frac{3\left(1-\mathrm{v}^{2}\right)}{2} \frac{1}{\mathrm{t}} \sqrt{\frac{\overline{\mathrm{P}}}{\mathrm{tE}}}} \\
\beta^{2}=8 \frac{\mathrm{G}_{\mathrm{a}}}{\mathrm{E}} \frac{\mathrm{t}}{\mathrm{t}_{\mathrm{a}}}
\end{gathered}
$$

The peel stress is given by:

$$
\begin{aligned}
\sigma= & \frac{1}{\Delta} \frac{\overline{\mathrm{P}} \mathrm{t}}{\mathrm{c}^{2}}\left[\left(\mathrm{R}_{2} \lambda^{2} \frac{\mathrm{k}}{2}+\lambda \mathrm{k}^{\prime} \cosh (\lambda) \cos (\lambda)\right) \cosh \left(\frac{\lambda \mathrm{x}}{\mathrm{c}}\right) \cos \left(\frac{\lambda \mathrm{x}}{\mathrm{c}}\right)\right. \\
& \left.+\left(\mathrm{R}_{1} \lambda^{2} \frac{\mathrm{k}}{2}+\lambda \mathrm{k}^{\prime} \sinh (\lambda) \sin (\lambda)\right) \sinh \left(\frac{\lambda \mathrm{x}}{\mathrm{c}}\right) \sin \left(\frac{\lambda \mathrm{x}}{\mathrm{c}}\right)\right]
\end{aligned}
$$

Where:

$$
\begin{gathered}
\mathrm{k}^{\prime}=\frac{\mathrm{kc}}{\mathrm{t}} \sqrt{3\left(1-\mathrm{v}^{2} \frac{\overline{\mathrm{P}}}{\mathrm{tE}}\right.} \\
\lambda=\gamma \frac{\mathrm{c}}{\mathrm{t}} \\
\gamma^{4}=6 \frac{E_{a}}{E} \frac{t}{t_{a}} ; \Delta=\frac{1}{2}(\sin (2 \lambda)+\sinh (2 \lambda)) \\
R_{1}=\cosh (\lambda) \sin (\lambda)+\sinh (\lambda) \cos (\lambda) \\
R_{12}=-\cosh (\lambda) \sin (\lambda)+\sinh (\lambda) \cos (\lambda)
\end{gathered}
$$




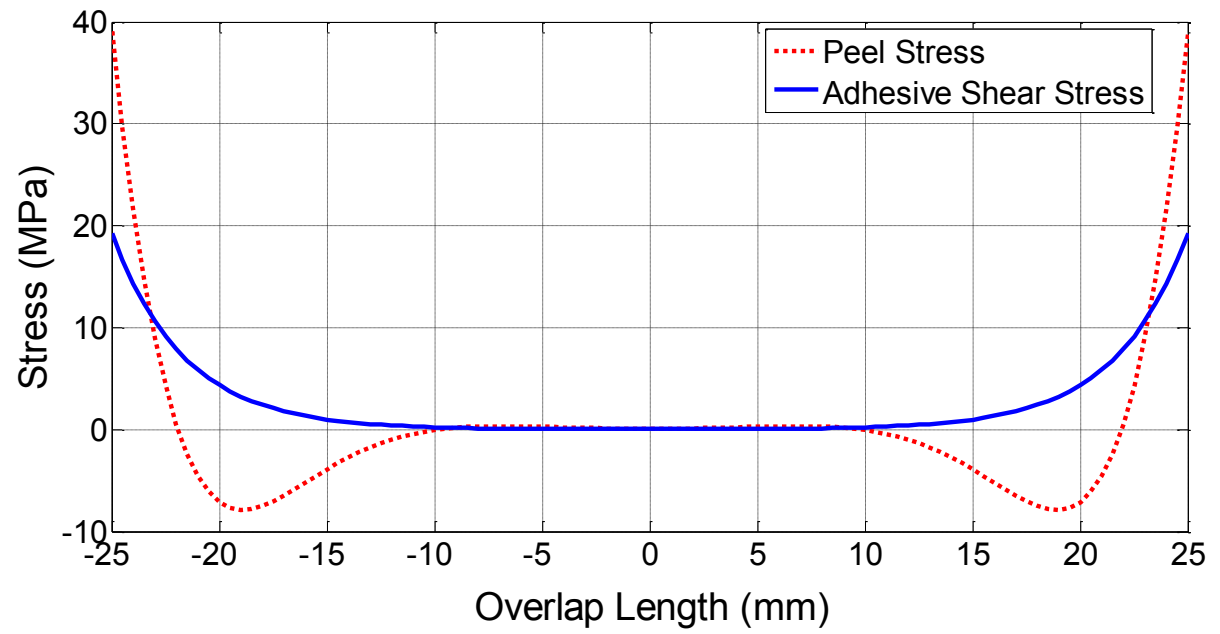

Figure 2.2 A typical stress distribution in single-lap bonded joint, Goland and Reissner model

The classical Volkersen and Goland and Reissner analyses are limited because the formulation of the adhesive model does not take into account the plastic strain and stress through the thickness. The stress distribution is for the mid-line thickness on the overlap. Additionally, there is no stress calculation at the interface between the adhesive and the adherend, which is important in composite laminates. Lastly, these models do not take into account the free-stress condition at the end of the overlap and, as a result, give a consevative load prediction [29]. This issue was later solved by Ojalvo and Eidinoff [30]; their model takes into account the effect of the adhesive thickness on the joint stresses calculation.

In the late 1970s Dr. Hart-Smith developed and published his seminal work on adhesively bonded composite joints, summarizing the results in [15]. For bonded joints, Hart-Smith developed several analytical models for various joints configurations, including single-lap joints [31], double-lap joints [32] and scarf and stepped-lap joints [33]. These analytical models, compared with previous models, take into account the elasto-plastic properties of the adhesive as well as mechanical properties for composite 
adherends for both balanced and unbalanced joints. According to Hart-Smith, the adhesive plasticity is the most important factor for and adequate agreement between theory and experimentation [31]. As the adhesive reaches plastic strain regime, the shear stress reaches its maximum value and, if the load increases, the zone with the maximum shear stress in the overlap also increases until the joint fails because the adhesive in the overlap length has reached the maximum allowable shear plastic strain.

The research performed by Hart-Smith on composite bonded joints is rich and extensive. Factors such as thermal mismatch, adherend thickness, adhesive thickness, adhesive modulus, layup sequence, and many others were analyzed. Design charts including these factors in the design of bonded joints were developed using computer codes [31-33].

There are three potential failure modes in the composite bonded joints: failure in the laminate outside the joint, adhesive failure due excesive shear strain and interlaminar failure in the adherend due to excesive peel stress at the end of the overlap. The latter is important in composite laminates and, should be prevented to improve the joint efficiency.

Many other researchers have proposed both analytical and numerical models for bonded joints. A thorough comparison of these models can be found in a recent literature review performed by Da Silva et al [29, 34].

\subsection{Composite Bolted Joints}

The structural analysis of bolted joints starts with the calculation of individual loads in the fasteners. The flexibility of the fastener plays an important role in the load 
transfer mechanism and, consequently, in the stress. The flexibility depends on the adherend material, fastener material, joint geometry and squeeze force [35]. The fastener flexibility (f) is defined as the displacement $(\delta)$ respect to the load transferred to the bolt $\left(P_{b}\right)$, and is also referred as the inverse of the rigidity constant. This displacement contributes to the total change in length in the joint due to the applied force as shown in Figure 2.3

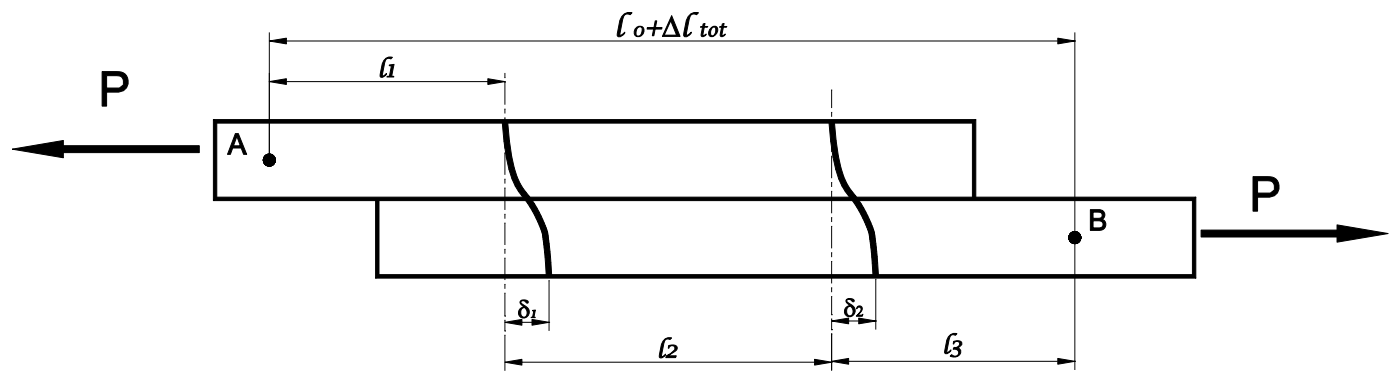

Figure 2.3 Fastener flexibility in a single-lap joint configuration

Mathematically:

$$
\mathrm{f}=\frac{1}{\mathrm{k}}=\frac{\delta}{\mathrm{P}_{b}}
$$

Several approaches have been developed to calculate the load taken by individual bolts for different configurations, including those described by Tate and Ronsfeld [36], Barrois [37], Huth [38] and Swift [39] just to mention a few.

Once the individual loads in the bolt are computed, stress analysis is performed in the pin-hole as shown in Figure 2.4. De Jong [40] developed an analytical solution on pin-hole based on complex function method developed by Muskhelishvili and adapted to orthotropic materials by Lekhnitskii considering a rigid pin, frictionless and uniform load distribution. 
Further analysis done by Zhang [41] takes into account the effect of friction in the pin-hole boundary demonstrated that the stress distribution is affected by both the friction and anisotropy of the laminate.

Later, Zhang extended their original analysis including arbitrary load direction in the joint. Hyer [42] considered the effect of pin elasticity and hole clearance and concluded that both the clearance and friction have a strong effect on the load distribution and maximum stress. Conversely, according to the author, the pin elasticity was not as important as the clearance.

Aluko and Whitworth [43] analyzed the effect of the hole deformation due to the pin load considering the hole as an ellipse instead circular form. The authors concluded that friction has a significant influence on the radial hoop and shear stresses.

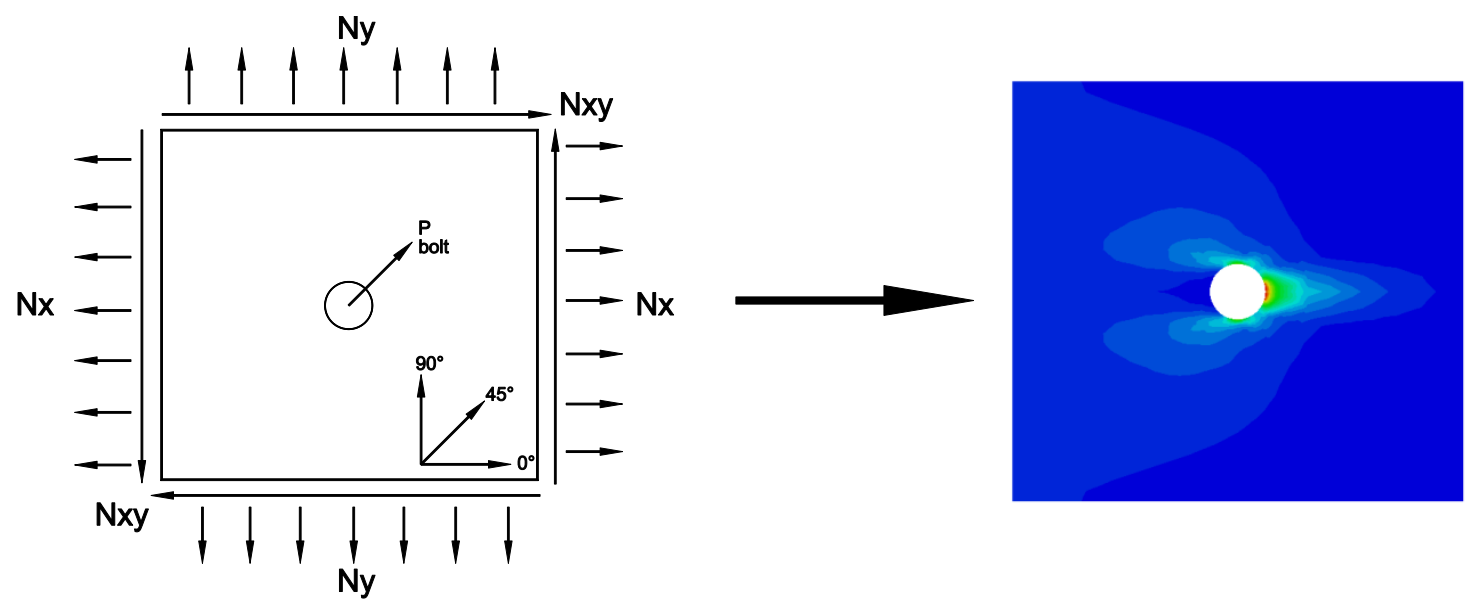

Figure 2.4 Pin-hole stress analysis

The previously described analytical procedure to calculate the stress distribution along the pin-hole are quite complex and do not capture the 3D behaviour. Hence, extensive research has been done by using finite element analysis taking into account pin- 
hole friction, clearance, friction between holes and laminates, out-of-plane strain, preload, linear and non-linear material properties and damage [44].

Such complexity has been the main driving factor in the research on composite mechanically-fastened joints experimentally rather than analytically, analyzing the influence of geometrical factors in the joint strength, and the traditional procedure is to vary the factor of interest and keeping the remaining factors constant [45].

The basic failure modes in mechanically fastened joints are net-tension, shear-out, bearing, cleavage and pull-through as shown in Figure 2.5. These failures modes are associated with the joint geometry, width (w), edge distance (e), hole diameter and laminate thickness [12]. Geometrically, the composite bolted joint design is more sensitive to $e / d$ and $w / d$ distances [46].

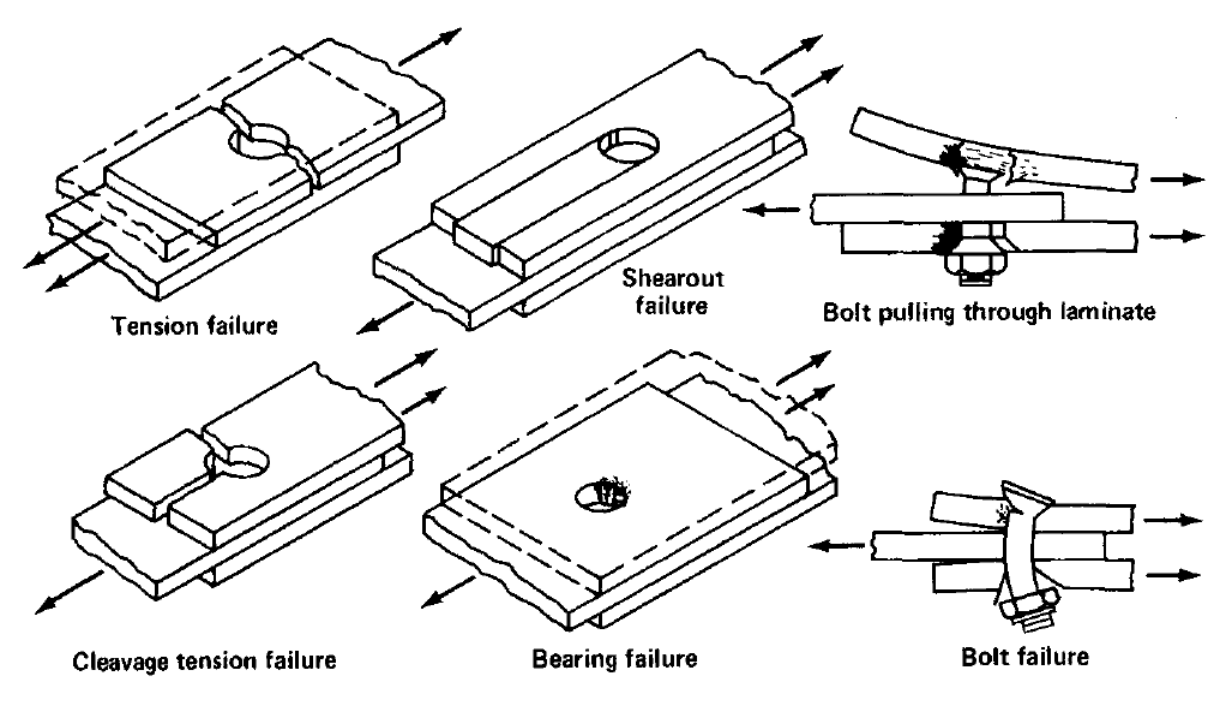

Figure 2.5 Failure modes in composite bolted joints [12] 


\subsection{Bolted/Bonded Joints in Composites}

\subsubsection{Analytical Models}

Closed-form solutions for stress analysis in bolted/bonded joints are not welldeveloped today. The complexity of the problem arises from the combination of two joining technologies, and the large number of variables involved. For the bonded joint technology, the typical variables are the adhesive properties, adhesive thickness, lap length, joint type, adherend properties, and adherend thickness. The traditional associated response variables are peel stress, shear stress and shear strain distribution along the bondline. In the case of bolted joint technology, the main variables are bolt size, bolt stiffness, number of bolts, bolt pattern, hole clearance, contact between bolt and hole, contact between bolt and adherends and preload. The main response variables are load sharing between bolts, stress concentration factor in the hole, stress in the bolt and stress distribution in the hole.

Along with this high number of variables, the nature of composite adherends increases the complexity in the joint analysis as shown in Figure 2.6. Laminae properties, as well as layup sequence play an important role in the design of the joint. Furthermore, the response variables are tri-dimensional as shown in Figure 2.7. Shear and peel stresses are in the $\mathrm{XY}$ plane, and the stress distribution in the hole is located in the $\mathrm{XZ}$ plane and varies through the thickness (XY) in the adherend. Therefore, a full $3 \mathrm{D}$ analysis is required to capture the whole joint behavior.

The earliest attempt to develop an analytical model for bolted-bonded joints was described by Hart-Smith [47]. The author developed a computer program called A4EK 
which is capable of computing the load transfer in a titanium-to-graphite-epoxy adhesive bolted/bonded joint. The aim of the study was to compare the effect on the load transfer by adding fasteners in the adhesively bonded joint whith flaws. The model included elasto-plastic adhesive, linear elastic composite, preload and clearance effects in the bolts, nonlinear effects for $\pm 45^{\circ}$ laminates and bearing/bypass load interaction in the bolts.

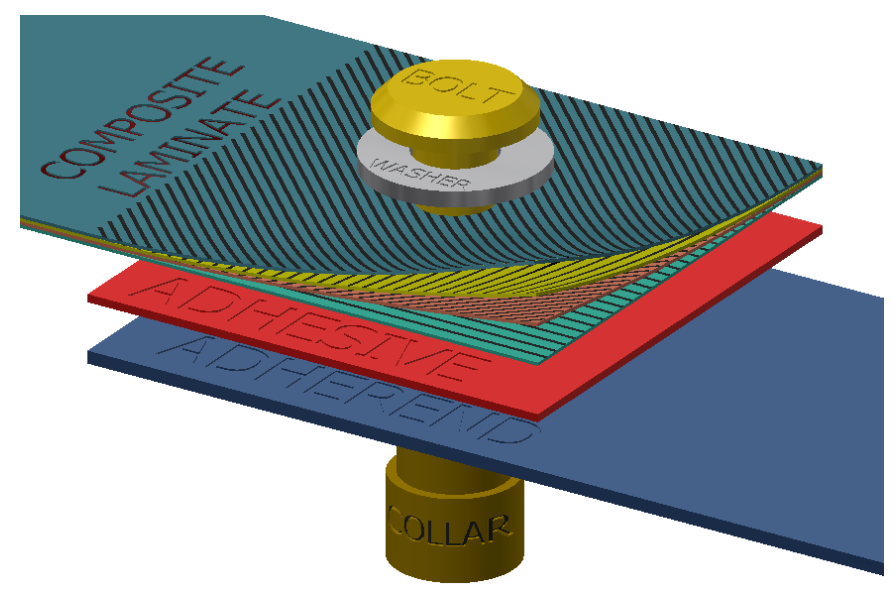

Figure 2.6 Composite Bolted/bonded joint

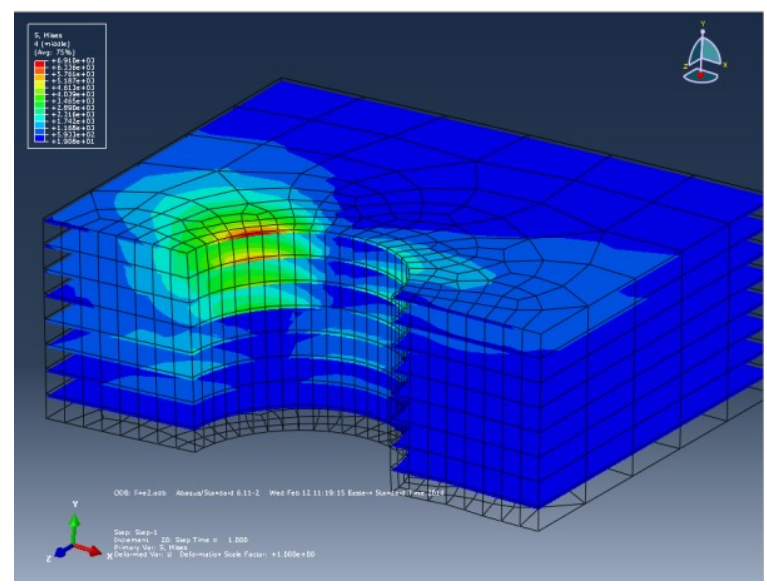

Figure 2.7 Through-the thickness stress variation in a bolted composite joint 
The adhesive shear strength was approximately 70,000 psi (480 MPa), the elastic shear strain was 0.0778 , and its shear modulus, $\underline{G}$, was equal to 90,000 psi (620 MPa-0.6 GPa). According to the author, the load taken by the bolt was approximately $1 \%$ of the total applied load [47]. However, despite this low load taken by the bolts, this joint can be used in repair locally damaged bonds. By doing this in a non-adhesive zone, the load transferred to the bolt can increase up to $14 \%$ and can also relieve the adjacent critical locations in the adhesive bond.

Yamaguchi and Amano [48] analyzed a bolted/bonded shear joint with steel as adherends. The effect of bolt diameter, lap length and number of bolts on the joint performance was analyzed. A simplified analytical model was used to calculate the average shear stress in the adhesive; this model included the nominal area in the lap joint minus the area in the hole diameter. Figure 2.8. a shows the theoretical behaviour of bolted/bonded joints. Three lines $P, P_{a}$ and $P_{b}$ depict the strain-load behaviour for bolted bonded, bonded and bolted joints respectively. For the case in which the adhesive shear strain is larger than the bolt shear strain, initially, the load is taken by both adhesive and bolt until the bolt fails at shear strain $\gamma_{1}$ (label $P_{l}$ in line $P$ ). Due to this, the load drops an amount $P_{b}$ at reaches the adhesive load path $P_{a}$ at $P_{2}$. The remaining load is taken by the adhesive until its final shear strain $\gamma_{2}\left(\right.$ label $\mathrm{P}_{3}$ on line $\left.\mathrm{P}_{\mathrm{a}}\right)$.

On the other hand, when the bolt shear strain capability is bigger than that of the adhesive, the load is taken by both elements until the adhesive fails at shear strain $\gamma_{2}$, producing a drop force equals to $P_{a}$, reaching the bolt load path $P_{b}$ at point $\mathrm{P}_{1}$. Afterwards, the load is taken entirely by the bolt until the shear strain $\gamma_{3}$, labeled $Q_{3}$. 
According to the author, the experimental results are in agreement with the theoretical calculations.

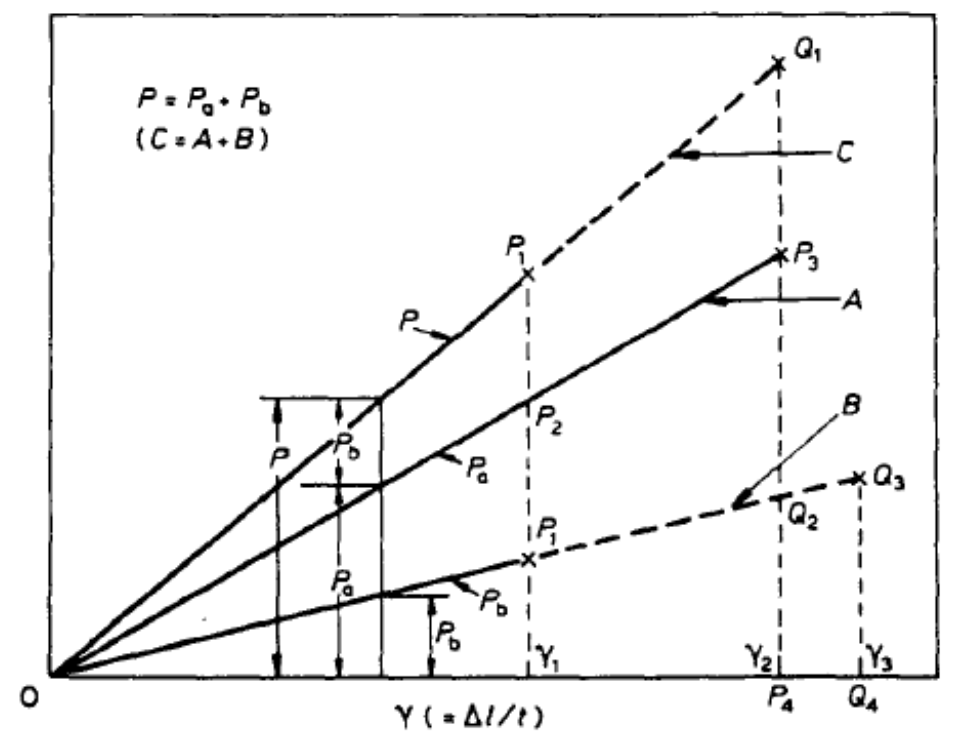

Figure 2.8 Graphical description of load paths on bolted/bonded joints according to Yamaguchi et al. (Reprinted from [42] with permission from Elsevier)

Sawa and Kobayashi [49] analyzed a T-type butt joint combining both adhesive and bolts and testing the joint subjected to bending moment. The objective of this study was to analyze the combined effect of adhesives with bolts on the joint strength, stress distribution in the adhesive layer and variation in the axial force in the bolts. Although the studied joint was a T-type butt joint with steel adherends, the synergistic effect due the two joint technologies was demonstrated improving the joint strength. This conclusion was demonstrated by using both an analytical model based on twodimensional theory of elasticity and further validation with experiments.

Gomez [50] proposed a simple mechanical model by using springs and dampers to analyze a riveted-bonded joint as shown in Figure 2.9. $K_{l}$ and $R$ represent the 
behaviour of the mechanical joint, $K_{2}$ and $R_{2}$ represents the adhesive joint and the elongations of each spring and damper are $x_{1}, x_{2}, x_{3}$ and $x_{4}$ respectively. The speed test $v$ is given by $v=\frac{d x}{d t}$ and is constant. The bond-graph technique was used to derive the equations of the system. The speeds are given by $v_{1}, v_{2}, v_{3}$ and $v_{4}$ respectively and the forces in the elements are the total joint force $\left(F=e_{1}\right)$, rivet force $\left(F_{1}=e_{2}\right)$ and adhesive force $\left(F_{2}=e_{3}\right)$.

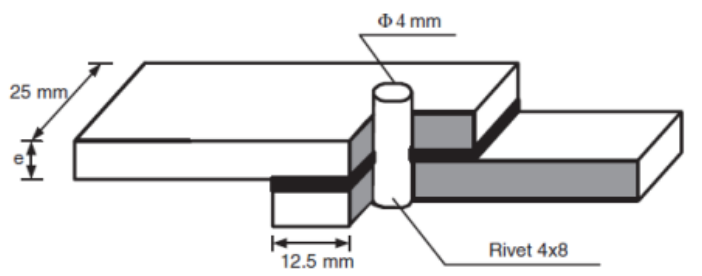

Joint
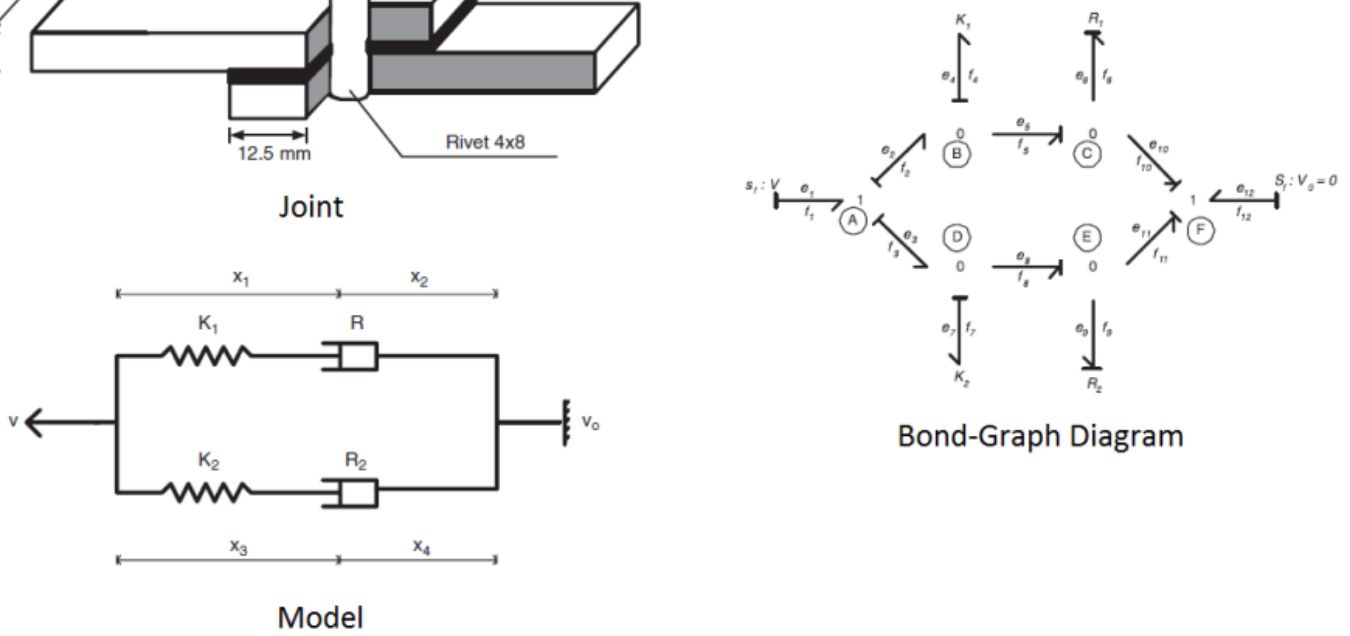

Figure 2.9 Riveted-bonded joint analyzed by Gomez showing and the proposed model and the bond-graph diagram. (Reprinted from [50] with permission from Elsevier)

According to the technique, in type 1 nodes the sum of the incoming elongations must be the sum of the outgoing elongation, and the flow is maintained. On the other hand, for type 0 nodes the elongations and incoming flow must be equal to the sum of the outgoing flow. Analyzing each node, grouping and deriving, the system of differential equations is obtained, and once the system is solved, the force in three rivets and adhesives is computed. The solutions are given by: 


$$
\begin{gathered}
F_{1}(x)=R_{1} v\left[1-e^{\left(K_{1} / R_{1} v\right) x}\right] \\
F_{2}(x)=R_{2} v\left[1-e^{\left(K_{2} / R_{2} v\right) x}\right] \\
F=F_{1}+F_{2}=F_{1}(x)=R_{1} v\left[1-e^{\left(K_{1} / R_{1} v\right) x}\right]+R_{2} v\left[1-e^{\left(K_{2} / R_{2} v\right) x}\right]
\end{gathered}
$$

This analytical model is useful when the parameters $K_{l}, K_{2}, R_{1}$ and $R_{2}$ are known. The authors determined these parameters from the experimental results and were input into the equations. The final results were compared against the experiments with good agreement as shown in Figure 2.10.

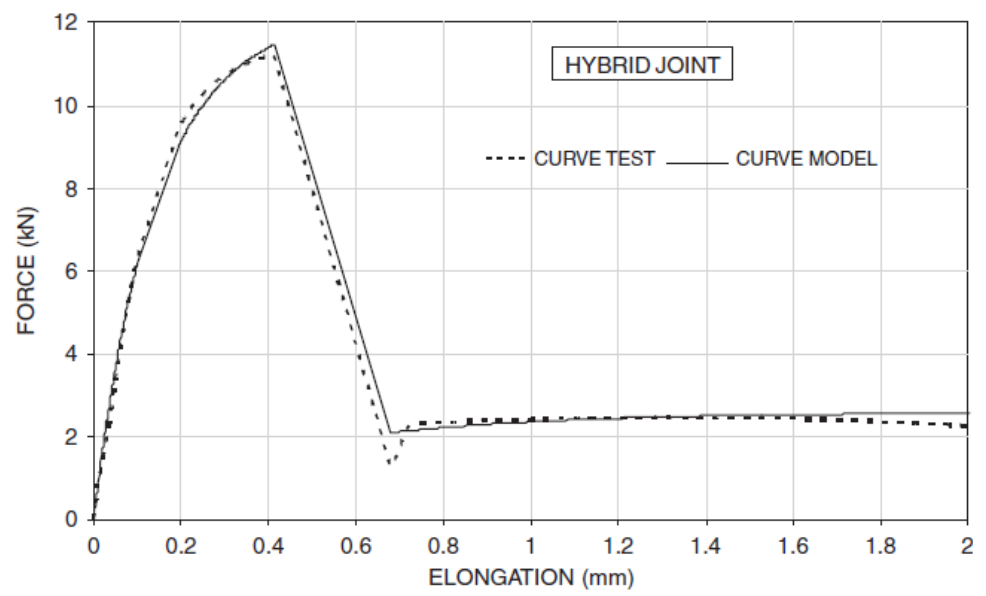

Figure 2.10 Force-elongation results according to Gomez. (Reprinted from [50] with permission from Elsevier)

Paroissien [51] proposed a parametric analytical two-dimensional model based on finite element method to analyze balanced single-lap joints. The model showed in Figure 2.11 included beam elements for the adherend outside the overlap, rigid body elements for the fasteners and "bonded beams" elements to represent both the adhesive and the adherend at the same time in the overlap. The model was able to predict the load taken by the bolts, normal force, shear force, bending moment due eccentric load and 
adhesive shear and peel stress. However, the mechanical properties of the adherends, adhesive and bolts remain in the linear elastic range. Moreover, it does not take into account the effect of friction between the bolts and both adhesive and adherends.

Using this model, the author performed an influence study to determine the effect in the load transfer due to the several factors involved in the joint ( Table 2.1), and the effect of the adhesive modulus (as seen in Figure 2.12) on the load transferred to the bolt for a balanced hybrid single-lap joint with fasteners. Finally, the model was compared against experimental results measuring the load taken by the bolts using an instrumented bolt and 3D FEM analysis.

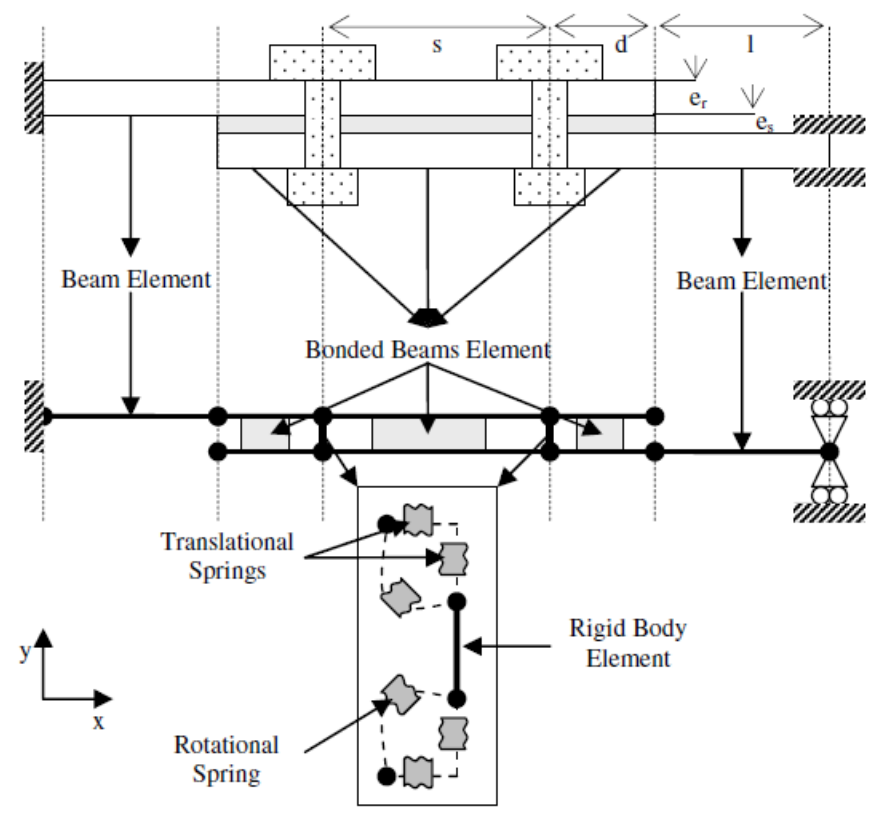

Figure 2.11 Hybrid bolted-bonded joint model proposed and analyzed by Paroissien [51] 


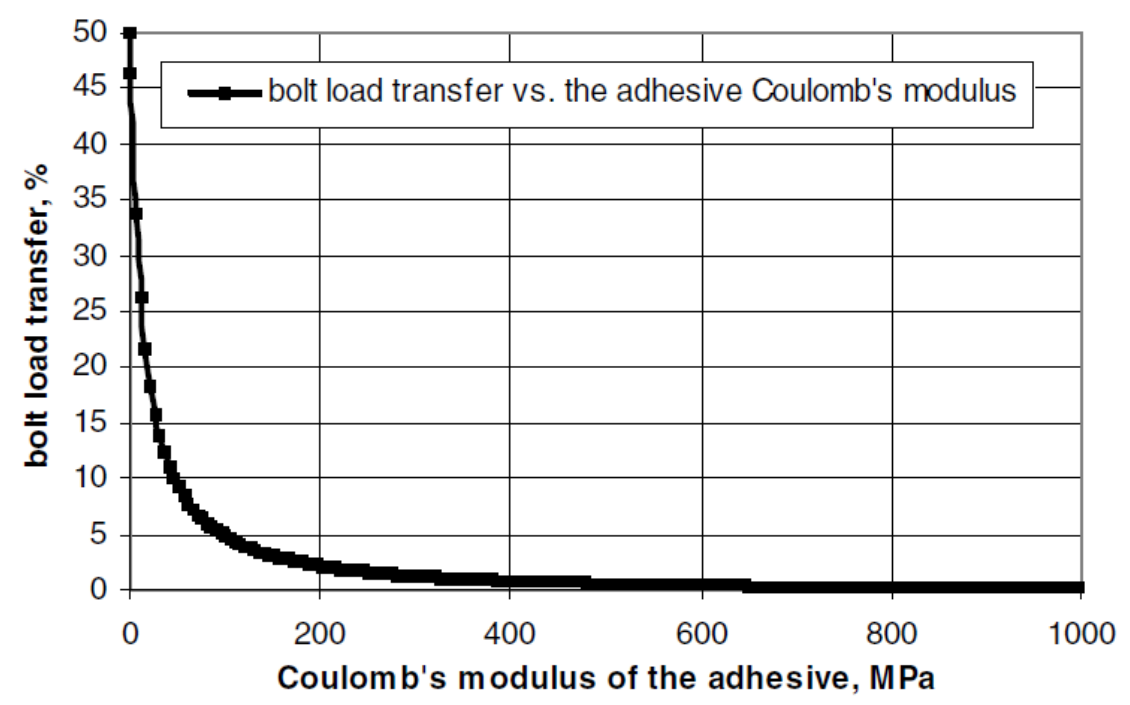

Figure 2.12 Effect of the adhesive modulus on the bolt load transfer according to Paroissien

Table 2.1 Effect of several factors in the load transferred to the bolt on hybrid bolted/bonded joint according to Paroissien [51]

\begin{tabular}{|l|l|}
\hline $\begin{array}{l}\text { Load transferred to the bolt increases when } \\
\text { the following factors decrease }\end{array}$ & $\begin{array}{l}\text { Load transferred to the bolt increases } \\
\text { when the following factors increases }\end{array}$ \\
\hline - The length of the overlap & - The Young's modulus of the \\
- Coulomb modulus of the adhesive & $\begin{array}{l}\text { adherends } \\
\text { - Longitudinal pitch between } \\
\text { fasteners }\end{array}$ \\
- Width of the adherends & - The thickness of the adherends \\
- The edge distance & \\
\hline
\end{tabular}

A semi-analytical model to analyze single-lap composite bolted/bonded joints for a general load case was developed by Oterkus [52]. The model included transverse surface loads, traction load and moments. The formulation of the model was carried out using the principle of virtual work and small deflection theory and was capable to determine displacements and stresses at the overlap region and load distribution between 
the adhesive and bolts. The adherends were modeled as composite laminates with isotropic transverse properties in the longitudinal and transverse directions, and both the layer thickness and ply angle can be arbitrary. Additionally, length and radius of the bolt, preload, outer radius of the clamped region, clearance and contact conditions between the bolt and the hole were also included. The material properties of the adhesive were linear elastic and failure criterion was not included to predict the joint strength.

Unfortunately, with all these features the model was very complicated and a closed-form solution was impossible to derive. Instead, a semi-analytical solution was achieved; however, this solution requiresd programming to solve it.

An attempt to include nonlinear (elastoplastic) adhesive properties and failure criteria for bolted/bonded joints with composite adherends was carried out by Bois et al [53]. Although the authors developed a 1D model for a double lap bolted-bonded joint including multi-criteria for joint strength prediction, the model is not able to predict the stress in the joint, i.e., shear and peel stress in the bondline and stress in the hole. The failure criteria for the adhesive was maximum shear stress, and bearing and net section failure criteria is based on Hart-Smith model to calculate the stress concentration factors in the composite part. Elasto-plastic properties in the adhesive were included to predict the load transferred into the bolt up to failure. The model's results were compared against FEM analysis and the strength compared against experimental results with good agreement. 


\subsubsection{Experimental and Numerical Studies}

The proposed models to analyze bolted/bonded joints have shown good agreement compared against the experimental results. However, a closed-form solution able to describe the full 3D nature of the joint has not been achieved. Therefore, experimental studies and fully 3D finite element analysis have been carried out to analyze the joint behavior. These studies generally compare bolted/bonded joints against bonded and bolted joints by varying some factors and assessing their effect in the strength as well as the load transferred to the bolts. Since the experimental procedure to measure the joint strength is relatively standardized, easier and cheaper, most of the previous experimental studies have been carried out to measure this response. Conversely, measuring the load transfer experimentally requires a complicated experimental setup and it has been mainly carried out using instrumented bolts. Due to this, numerical methods along with small experimental samples for validation have been used for load transfer analysis.

A testing program to investigate the efficiency of single-lap bolted, bonded and bolted/bonded joints was performed by Tan [19]. Two and four bolt configurations were tested for the bolted/bonded joints. The adherend material was graphite/PEEK AS4/APC2 unidirectional prepreg with stacking sequences $[0 / 90 / \pm 45]_{2 \mathrm{~s}}$ and $[02 / / \pm 45]_{2 \mathrm{~s}}$ and the adhesive used was FM-400. The bolt hardness was $20 \mathrm{RC}$ and washers with inside diameter 0.275 in $(6.9 \mathrm{~mm})$ and outside diameter 0.55 in $(13.97 \mathrm{~mm})$ were placed on both sides of the joint. A clamping force of $100 \mathrm{lb}-\mathrm{in}(11.3 \mathrm{~N}-\mathrm{m})$ was applied. It was found that the ultimate load required to debond the adhesive increases when the number of bolts increases. The authors also pointed out that the ultimate joint strength is slightly affected by the presence of the adhesive due to the relatively low force required to debond it. 
The damage initiation, stiffness, average load and average strain at failure in repairs, was evaluated by Stewart [54] testing single-lap composite bonded, bolted and bonded/bolted joints. The joint geometry and materials remaining constant except the layup sequence in the parent laminate for bonded, bolted and bolted/bonded configuration. The parent laminate was IM6/3501-6 grade 145 Gr/Ep Tape, and the repair laminate was ASA/3501-6 Gr/Ep Tape. The two-part paste adhesive Magnolia 6363 was used and the bolts were composi-lok MBF 2110S-6 150.

The bolted/bonded joints were 50\% stronger than bolted joints and 16\% stronger than bonded joints. The analysis of damage initiation and propagation showed that bonded and bolted/bonded joints behave similarly. The main difference was the crack propagation rate because the presence of the bolt delays the crack propagation and allows a higher final failure load.

Another investigation in bolted/bonded joints in composites for structural repair was performed by Chan and Vedhagari [55]. The experimental and numerical study was focused in the stress distribution and load transfer in bonded, bolted and bolted/bonded repaired joints. The FEM models included linear elastic isotropic adhesive, lamina properties for the adherends, stacking sequence for parent and repair laminates and frictionless contact between the bolt and laminate. For the parent laminate two stacking sequences were used, $[+45 /-45 / 0 /+45 /-45 /-45 /+45 /-45 /+45]$ and $[-45 /+45 /-45 /+45 /+45 /-$ $45 / 0 /+45 /-45]$ respectively. The stacking sequence for the repair was [+45/0/0/-45].

The results demonstrated that there is no load taken by the bolt, the entire load is taken by the adhesive. On the other hand, the axial stress near to the overlap is reduced due to the presence of the bolts [55]. 
The effect of the clamping area on the peel stress in the adhesive in bolted/bonded joints was analyzed by Fu and Mallick [56] who performed static and fatigue experiments on bonded/bolted joints with glass-fibre composite adherents using different combinations of washer sizes, washer shapes and adhesive thicknesses. Although the conclusion was that presence of clamping prevents or delays the maximum peel stress, the clamping force remained constant for all joint configurations. Therefore, there is no quantitative relationship between the clamping force and the reduction in peel stress. Another conclusion was that the bolted/bonded joints have better performance under both static and fatigue loading cases.

Lees and Makarov [57] carried out an experimental program to analyze the behaviour of pinned/bonded glass fibre reinforced polymer (GFRP) used in pipelines. Longitudinal pieces taken directly from cylindrical pipes were used to manufacture bolted, bonded and bolted bonded joints. The influence of the pin configuration, pin material and pin diameter was analyzed in the bonded/bolted joints. The pins were made with steel and GRFP rods with several diameters. The authors concluded that for a welldesigned pinned/bonded joint, the strength can be as high as the CRFP alone. In addition, the strength is higher than either the bonded and bolted joints alone.

A parametric study on hybrid bolted/bonded joints using FEM was carried out by Kelly [58]. The analyzed factors in the parametric numerical study included adherend thickness, adhesive thickness, overlap length, bolt pitch distance and adhesive modulus. The geometry of the bolt was the same for each analysis and the clamping force was $5 \mathrm{kN}$. The material for the numerical model was carbon fibre/epoxy T700/epicote 828LV with stacking sequence $[0 / 45 / 90 /-45]_{\mathrm{sn}}$. The effect of the adhesive modulus was studied using 
three different adhesives: pliogrip 7400/7410 with $\mathrm{E}=0.6 \mathrm{GPa}$, Betamate $1496-\mathrm{V}$ with $\mathrm{E}=$ 1.6 GPa, and betamate 1040 with $\mathrm{E}=2.9 \mathrm{GPa}$. The results for the parametric study can be summarized as follows [58]:

- Increasing the adherend thickness increases the load transferred to the bolt;

- Decreasing the adhesive thickness increases the load transferred to the bolt;

- Increasing the overlap length decreases the load transferred to the bolt;

- Decreasing the relationship w/d increases the load transferred to the bolt; and

- Decreasing the adhesive modulus increases the load transferred to the bolt.

The numerical analysis was experimentally validated with a bolted/bonded joint with special instrumented bolts to measure the load taken by the bolt. The adherend material was prepreg HTA/6376 from Hexcel composites with stacking sequence $\left[( \pm 45 / 0 / 90)_{4}\right]_{32 s}$. and the adherend thickness was $4.16 \mathrm{~mm}$. The overlap length and width were 20 and $25 \mathrm{~mm}$ respectively. The adhesive used was polyurethane Pliogrip $7400 / 7410$ with $0.5 \mathrm{~mm}$ thickness. According to the author, the experimental configuration was restricted by the diameter of the instrumented bolt, $6 \mathrm{~mm}$ in this case.

In a subsequent study, Kelly [59] also investigated the strength and fatigue life in both bonded and bonded/bolted composite single-lap joints. For the experimental study carbon fibre tow yarn with epoxy resin was used with two stacking sequences [0/45/90/45]s $(1.6 \mathrm{~mm})$ and [0/45/90/-45]s2 (3.2mm). The adhesive used was Pilogrip 7400 (low modulus) and Epibond 1590 A/B (high modulus). The adhesive thickness remained constant $(\mathrm{t}=0.5 \mathrm{~mm})$ and the overlap length and width were $25 \mathrm{~mm}$. The bolts used were stainless steel lock bolts with $6.35 \mathrm{~mm}$ diameter. The fatigue testing was carried out following the ASTMD 638-02a standard using stress ratio equal to 0.1 at $5 \mathrm{~Hz}$ frequency. 
In the static load, the failure in the bolted/bonded joint started in one adhesive corner, and the fracture propagated until the next adherend. This was followed by the total failure of the remaining bolted joint. Net-section and bearing mode failures were detected, and it was found that the failure mode depended on the adherend thickness. The ultimate strength of the hybrid joint was between $11 \%$ and $22 \%$ higher than bonded joints; this value was also highly dependent on the adherend thickness.

For fatigue load, the bolted/bonded joints have higher fatigue strength than bonded joints and the failure modes differ depending on the joint configuration. The failure started in the adhesive and then propagates through the bondline, at the same time, the load taken by the bolt increases and, finally, the adhesive failed completely so that the joint performed as bolted joint system until failure. According to the author, there is an improvement in load transfer mechanism, static strength and fatigue strength using hybrid bolted/bonded joints. However, these improvements are strongly affected by the adhesive modulus and, according to the results, low modulus adhesives are better than stiffer adhesives.

A parametric numerical study on two-bolted single-lap hybrid bolted-bonded joints was performed by Ganji [60]. The parametric study included three different composite adherend materials, three different adherend thicknesses, three different overlap lengths, three different bolt diameters, and friction between the bolt and holes and preload. The adhesive used was Pliogrip $7400 / 7410$ with $\mathrm{E}=0.6 \mathrm{GPa}$. According to the author the study was validated by using an instrumented bolt to measure the shear load. However, according to the author, "the dimensions of the joint were restricted by the design of the instrumented bolt" [60]. Due to this, just one configuration was used for 
validation as was done by Kelly [58]. The agreement between experimental and FEM model was very good in the linear elastic regime, after that point, there is a slight discrepancy.

Kweon et al [61] carried out an experimental study to investigate the effect on the strength of bonded joints by adding fasteners in a composite-to-aluminum joint double lap joint. To compare the strength, the testing program included bonded, bolted and bolted/bonded coupons with carbon/epoxy prepreg laminate and aluminum as adherends. Two different adhesives were used, paste and film adhesive with different elastic modulus. Despite the number of coupons tested, there is no statistical information about the influence of the parameters on the joint strength because the main focus was to measure the final strength regardless the variation on the parameters. The conclusion was that the bolted/bonded joints have better performance than bonded joints when the mechanical fastener is stronger than the adhesive alone. Otherwise, its contribution to the joint strength is low.

Matsuzaky [62] investigated the strength in a bolted/co-cured hybrid joint with glass fibre/epoxy and aluminum adherents. Although the author compared the strength between co-cured, bolted and hybrid joints, the experimental configuration was unique, without variables. A specific characteristic in this joint was the special manufacturing process, placing the bolts carefully between the glass fibre to avoid damage before the curing process. According to this study, for static loading the hybrid joints behaves as the bolted joint and have 1.84 times higher maximum shear stress compared with the bonded (co-cured) joint. For fatigue behavior, the bolted/bonded joints have much higher fatigue strength than that bolted joint. 
Kumar [63] proposed a new bonded/bolted joint configuration using attachments. The configuration was designed to activate the bolts to carry load bearing before the adhesive fails. Tensile tests were carried out in bonded, bolted, bonded/bolted configuration, and the new proposed bonded/bolted configuration with all the factors in the same configuration. The proposed joint had better performance in both maximum load and maximum displacement. However, the alternate load paths given by attachments were also bonded to the adherends and, as a result, the bonded area was larger. Moreover, because the analysis was focused on the joint strength, there is no information about load transferred to the bolt in the proposed configuration.

Moroni [64] performed an experimental analysis and comparison on the strength, stiffness and energy absorption of single lap joints made with aluminum alloy and galvanized steel, widely used in the automotive industry. The joining processes investigated were bonded, resistance spot welding, clinching, self-pierce-riveting and their combinations. The analysis was performed using the Design of Experiments methodology to evaluate the influence of 6 factors and the results were analyzed statistically to detect the most influential factors and their interactions. The adequacy of the model was also analyzed using analysis of variance. Adhesive thickness, pitch, ageing and two welding temperatures were selected as factors to analyze. Finally, the selected factors and interactions levels were used to proposed a new joint with enhanced behavior and both material and manufacturing operation cost savings.

Lee et al [65] evaluated and compared the strengths of bonded, bolted and bolted/bonded composite joints in different configurations. The strength of the hybrid joints was analyzed numerically using the FAI method failure criterion for the bolted 
joints and the damage zone method for the adhesive joint. The predictions were compared against the experimental results. The experimental DOE included different width-todiameter ratios $(\mathrm{w} / \mathrm{d})$, edge-to-diameter ratios $(\mathrm{e} / \mathrm{d})$ and adhered thicknesses. The adhesive used was FM73 M film adhesive from Cytec. The bolts were made of steel with $9.53 \mathrm{~mm}$ diameter and were installed without clamping force in the joint. The experimental results showed that failure loads of the bolted/bonded joints were nearly identical to those of the adhesive joints and were at least twice as large as those of the bolted joints. The adhesive failure in the bolted/bonded joints occurred before the failure of the bolted joints. The authors recommended further study of the dynamic characteristics of hybrid joints, such as fatigue life and crack propagation.

Backman and $\mathrm{Li}[66]$ applied the digital image correlation technique to measure the strain distribution in bonded and bolted/bonded composite butt joints (Figure 2.13). Using this technique, an accurate strain distribution in the bondline was obtained and it was compared against the results from FE analysis. According to the authors, the measurements and simulations results were in good agreement.

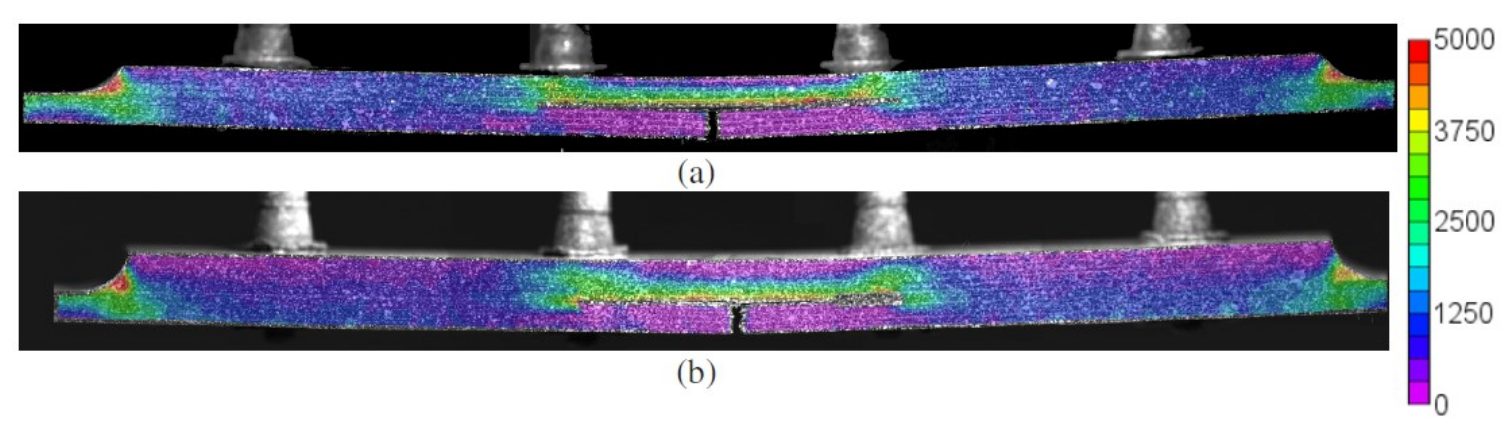

Figure 2.13 Maximum principal strain in bolted/bonded joint measured with DIC. (Reprinted from [66] with permission from Springer) 
Duc Hai and Mutsuyoshi [67] performed an experimental and numerical study on double-lap joints of steel plates bolted/bonded to hybrid composite laminated used in construction. The laminates were taken from an I-beam made with a combination of carbon and glass fibre to lowering the manufacturing cost and keeping the mechanical strength for the flanges and webs respectively. The large scale study included 45 test coupons and 6 large-scale beam specimens with both bolted and bonded/bolted joints configuration. The aim of the study was to analyze the effect of several factors on the joint strength. The following parameters were constant during the experiment: adherend width, adherend thickness, bolt-side distance, hole spacing, width to bolt diameter ratio $(w / d)$, bolt-side distance to diameter ratio $(s / d)$, pitch to bolt-diameter ratio $(p / d)$, bolt diameter to splice plate thickness ratio $\left(d / t_{s p}\right)$, washer diameter- to-bolt diameter ratio $\left(d_{w} / d\right)$, hole clearance, bolt diameter and bolt material.

The variables investigated were flat and v-notched splice plates, two or six bolt arrays, three torque values, three adhesive thicknesses and three bolt-end distance ratios. The effect of every variable on the joint strength and failure mode was analyzed one at the time in the design of experiments.

Although the aim was to apply the results for construction design, interesting conclusions were observed [67]:

- Increasing the adhesive thickness does not have significant effect on the joint strength;

- The v-notched plate specimens are stiffer than flat plates. Also, the debonding load is $14 \%$ higher. Finally, for both specimens, the identified failure mode was bolt shearing; 
- The bolt-end distance ratio has a significant effect in the bolted joint, but it has a minor effect on the bolted/bonded joints because the joint strength depends mainly on the adhesive strength. The recommended value for the bolt-end distance is $3 \mathrm{~d}$; and

- The bolt torque has a negligible effect in the joints. The torque simply fixes the plates during the adhesive curing process.

According to the authors, the adhesive carried most of the load until debonding and, thereafter, the load is transferred to the bolt. Therefore, the final failure load on bolted bonded joints is governed by the bolted part of the joint. The load sharing between adhesive and bolts was not investigated.

A finite element analysis with a two-bolt configuration was carried out to verify the experimental results. The composite laminate material was assigned engineering constants, the plates and bolts were defined as isotropic. For the contact between the bolts and plates a friction coefficient of 0.2 was assumed, and the clamping torque was set at $20 \mathrm{~N}-\mathrm{m}$. A feature in this model was the use of cohesive elements in the adhesive. The damage initiation criteria selected was the quadratic nominal stress and the damage evolution law was fracture energy. The results showed good agreement with the experiments.

Li et al [68] performed tension test on single butt joint with doublers and compared bonded, bolted and bonded/bolted joints by using a single strap joint, focusing on the failure mode and joint strength. The factors on the joints were constants with two different angle ply configurations for bonded joints. However, due the limited amount of material, the second configuration was not used for bolted and bonded/bolted joints. The 
nominal thickness for 16-ply laminate was $2.24 \mathrm{~mm}$. The adhesive was Cytec FM300K with $0.17 \mathrm{~mm}$ thickness and titanium bolts with $130^{\circ}$ countersunk head were used for the experiments. The authors concluded that the bonded/bolted joint was $52 \%$ stronger that the bonded joint and $127 \%$ stronger than bolted joint.

Di Franco et al [69] performed static tension test on bonded/SPR (Self-Piercing Riveting) joints with particular interest on the tensile strength, stiffness, failure energy and the effect of different curing processes. The joint with glass fibre/epoxy and aluminum adherends was analyzed on two different curing processes and three angle ply configurations in the composite adherent. The remaining features in the joint remained constant.

The comparison between the different joints showed that bonded/SPR joints have a synergy between the two coexisting joint technologies because the resistance level was dominated by the bonding and the failure energy was dominated by SPR riveting. The angle-ply laminates have improvements of tensile strength and energy absorption than bonded/SPR with cross-ply.

The effect of curing time on the performance of "aluminum to GFRP composite SPR/co-cured bonded joints" was analyzed by Fiore et al [70]. The main objective of this study was to analyze the effect of the required time to insert the rivet on the joint performance. The GFRP was unidirectional and 5 layers were chosen to get the required thickness, the epoxy resin (SP 106) was also used as adhesive (co-cured). The selected rivets were made with austenitic steel with $3.9 \mathrm{~mm}$ diameter with a protective zinc layer, and inserted at different times after the joint started curing in the middle of the $50 \mathrm{~mm}$ lap length. Because the manufacturing process required a specialized tool for inserting the 
rivet, both the pressure and cycle time were also considered in the design of experiments as variables. The design of experiments was designed with 3 levels for each curing time and combining each of them with three inserting pressures: 180, 220 and 240 bars for 2 hrs curing time; 220, 240 and 280 bars for 5 hrs curing time and 280, 300, and 340 bars for 24 hrs curing time.

An analysis of variance (ANOVA) was carried out for a three level factorial DOE using pressure and time as variables, and the joint strength as the response. The factors levels were low, medium, and high for the rivet inserting pressure and $2 \mathrm{hrs}, 5 \mathrm{hrs}$ and 24 hrs for curing time. The ANOVA showed that the effect on the joint strength was significant for both variables and their interaction.

The effect of flexible adhesives and it effect on the load transfer was analyzed by Ngoc and Paroissien [71] performing a fully 3D FEM analysis on bonded and bonded/bolted single lap joints. The model included hyperelastic properties for the mechanical behavior of the adhesive. The study was only numerical in nature without experiments to confirm the results. Moreover, the mechanical properties chosen for the adhesives were not based on any commercial adhesive because the study was done only to understand the differences in the behavior between stiff and flexible adhesives. The hybrid bonded/bolted joint was modeled with aluminum adherends with $\mathrm{E}=72,000 \mathrm{MPa}$ and $v=0.33$ and the bolt was titanium isotropic linear elastic with $E=110,000 \mathrm{MPa}$ and $v=0.33$. The novelty in the analysis was the use of hyperelastic adhesives modeled with Mooney-Rivlin elastic potential with two parameters $\mathrm{C}_{10}=0.8, \mathrm{C}_{01}=5000 \mathrm{MPa}$ and $v=0.5$. The load transfer rates were 0.337 for the first fastener, and 0.339 for the second fastener. The stress concentration factor in the fasteners was also calculated and compared against 
bolted joint only. According to the analysis hybrid bonded/bolted joints generate lower stress concentration factors than bolted joints, allowing a better fatigue performance.

Recently, Bodjona et al $[72,73]$ performed a sensitivity analysis to estimate the load sharing in a single lap single bolt bolted/bonded joint. The analysis was carried out using finite element analysis modelling the laminates with shell elements, the bolt as beam element and the adhesive as isotropic continuum solid. The results showed that the adhesive overlap must be fully plasticized to achieve load sharing higher than $10 \%$. In addition to this, the load sharing is governed by the adhesive's yield stress, edge distance ratio $(\mathrm{E} / \mathrm{D})$ and the hardening slope of the adhesive, with $0.89,0.37$ and 0.37 of effect respectively.

\subsubsection{Load Transfer Measurement}

The traditional way to analyze the synergistic effect between the adhesive and the bolt using hybrid joints is by testing the hybrid joint until failure and comparing the strength with bolted and bonded joints. However, this approach is limited because it does not assess the load sharing along the full loading path. To achieve such comparison, it is necessary to measure the load at least in one of the load carrying elements along the full loading path. Very few experimental studies have been carried out to identify the load sharing using bolts instrumented with strain gauges. These studies were limited to a few configurations, mainly due to complexity of installing strain gauges in small bolts.

The basic instrumented bolt configuration includes a pair of $45^{\circ}$ strain gauges located on a machined flat surface located immediately above and below the shear plane 
as shown in Figure 2.14. A detailed explanation including the geometrical details and calibration can be found in [74] and [75].

Another configuration with an axial stain gauge plus the pair of $45^{\circ}$ strain gauges has been used to measure the effect of the clearance [76] and pre-load [77] in the load distribution in composite bolted joints.

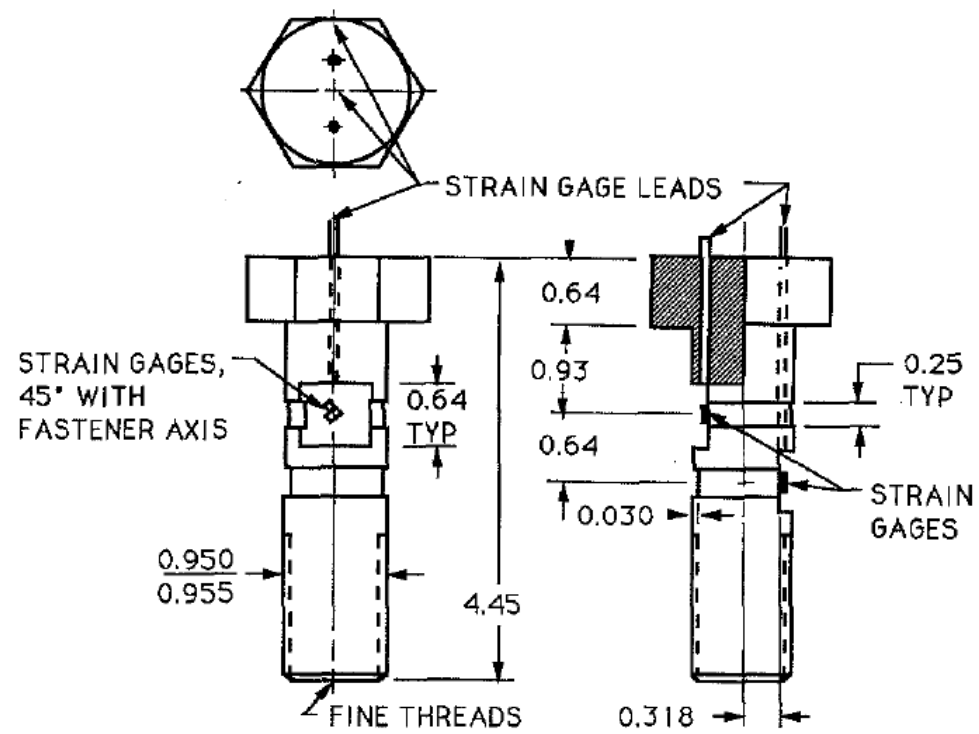

Figure 2.14 Instrumented bolt configuration for shear force measurement. (Reprinted from [74] with permission from Springer)

By using the same technique, Kelly [58] carried out load transfer measurements in composite bolted/bonded joints with one $6 \mathrm{~mm}$ diameter titanium instrumented bolt. The installation was carried out using hand tightening to avoid the effect of lateral clamping. The author compared the experimental results against FEA and found that the clearance affected significantly the numerical results. However, there is no report about the holeclearance in the experimental analysis. 
Paroissien [51] performed load transfer measurements on bolted/bonded joints with aluminum adherends and two $9.5 \mathrm{~mm}$ diameter titanium bolts tightened with $1 \mathrm{~N}-\mathrm{m}$ torque. According to the author, the summation of shear strain in the two instrumented bolts accounted for a $100 \%$ load transfer.

Despite the fact that the measurement device is located directly in the bolt, the effect of friction when clamping force is applied can lead to errors during summation of forces. By using the same technique in bolted composite joints, Sawyer [74] found that increasing the torque in the fasteners the summation of forces does not match the applied load, and the missing load is transferred by friction.

Another issue with this technique is that it is limited to the size of the bolt. As mentioned in the previous section, bolts sizes were $6 \mathrm{~mm}$ diameter or higher. Installing strain gauges in smaller bolts can be very complicated.

\subsection{Cohesive Zone Modelling}

One of the main efforts in bolted/bonded joints research has been to analyze the load shared between adhesive and joints as well as the analysis of the joint strength. Finite element models, with elastic or elastoplastic constitutive relationships in the adhesive have been shown to be suitable to compute the displacementes, strains and stresses with relatively high acuracy. Additionally, the forces in all the structural elements of the joint can be determined and, therefore, the load shared between the adhesive and the bolt can be calculated. Conversely, these models do not allow for the computation of the ultimate strengh in the joint or to compute the failure of one of the joining elements. 
Normally, the strength is predicted using a failure criterion in the adhesive and comparing it against the adhesive's properties. However, these failure criteria do not allow for the analysis of strength during and after the adhesive failure process. To achieve this, the adhesive has to be analyzed using a fracture mechanics approach, allowing to compute the strength of the joint during the whole process, starting with the load been carried by the joining elements acting togheter and computing the strength when one of them begins the failure process. Lastly, capturing the final strength of the joint with the remaining joining element.

In some cases, depending on the joint design, the failure can start in the composite adherends. This failure mode can also be determined using numerical analysis. A 3D finite element model that include all the failure modes in the joint elements will be very complicated. It will have several issues such as convergence, time stepping, and required computing power. Additionally, the lack of reliable material properties for all the components involved could limit the accuracy. However, it will be useful to stimate the joint strength numerically prior to the final design to save time and cost associated with experimentation.

The adhesive fracture can be modelled using a fracture mechanics approach. In recent years, the most used technique to model failure in composties and adhesives has been the cohesive zone modelling.

Cohesive zone modelling is mathematical formulation between interfacial elements used to model crack initiaton and propagtion. This technique has been widely applied to analyze fracture mechanics problems in metals and composites. Very few authors have reported using CZM to analyze chemical interfaces [78]. The concept was 
initially proposed by Dugdale [79] and Barrenblatt [80] and it is based on a damage mechanics approach using a constitutive relationship between stresses-displacement between points of an element. Therefore, the cohesive zone modelling can avoid the common problems with linear elastic fracture mechanics approach such as [81]:

a) The presence of a pre-cracked surface

b) Use of re-meshing techniques to follow the crack path

c) Infinite stress-strain values near the crack tip

The technique introduces a damage parameter into the element as follows:

$$
\sigma=D \delta
$$

where $\delta$ represents the relative displacement between points of the element, $D$ the damage parameter and $\sigma$ the applied stress. The damage initiation and damage propagation depends on the cohesive law that relates the damage parameter as a function of the relative displacement. For the triangular cohesive constitutive law showed in Figure 2.15 the initial slope represents the displacement in the element as a function of the applied traction. The damage begins at the displacement onset, $\delta_{o}$, and maximum traction value, $t_{n}$; reaching its maximum value at $\delta_{s}$, separation, where the failure occurs. The area under the traction-separation law is the energy release rate $G$. Although the former triangular cohesive law shape is the most widely used, other laws can be used, i.e., triangular with exponential propagation and trapezoidal. 


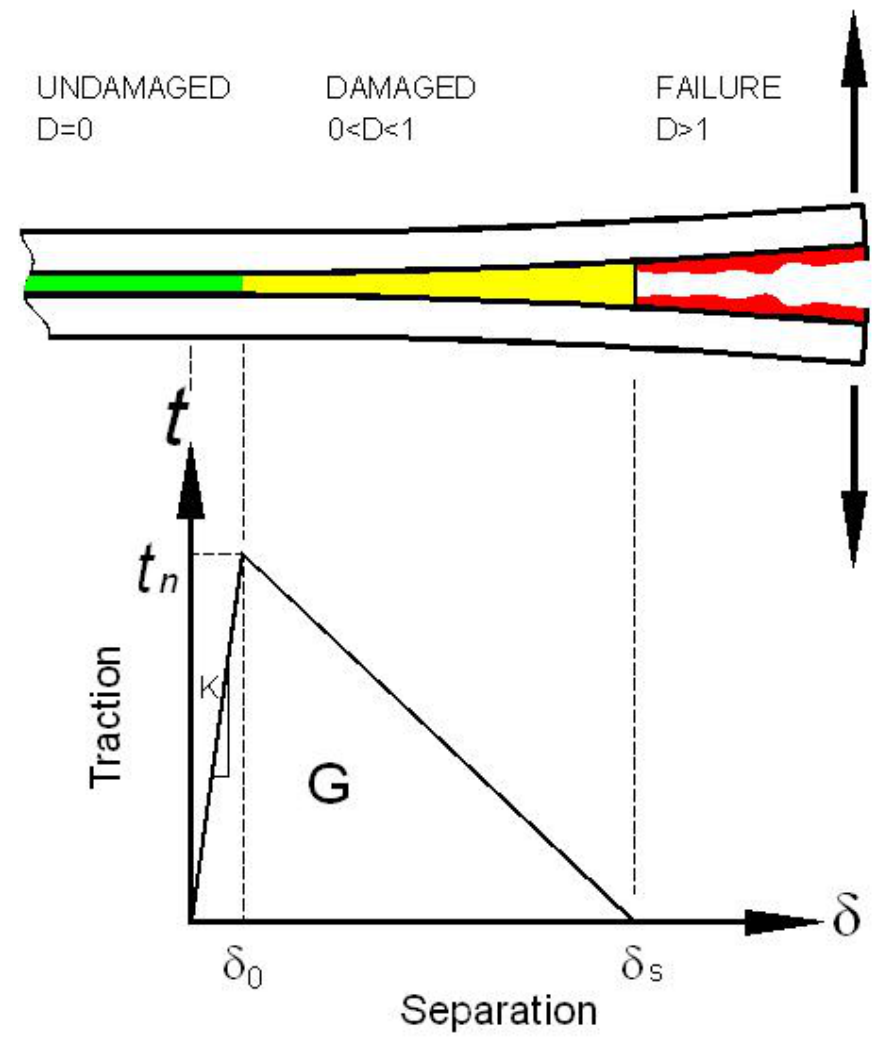

Figure 2.15 Cohesive zone model, triangular traction-separation law shape

The crack can be propagated in different directions or modes as shown in Figure 2.16. Mode I is when the force is applied normal to the crack; in mode II, in plane-shear, the force is applied parallel to the plane of the crack and the surfaces above the crack tend to slide. Finally, in mode III the surfaces above the crack tend to slide perpendicularly each other.

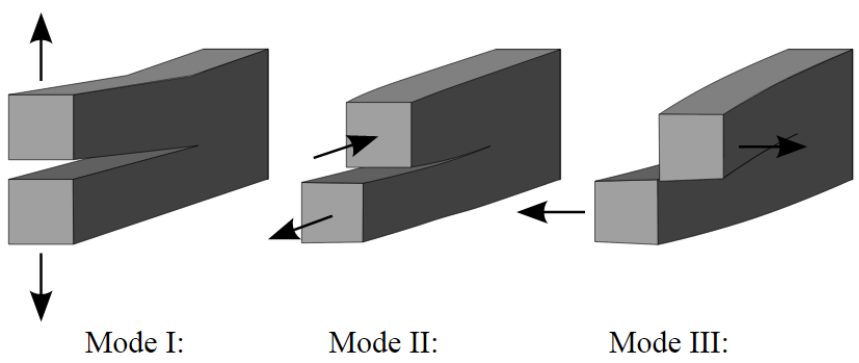

Figure 2.16 Failure modes [82] 
The damage initiation can be defined using different criteria [83]: maximum nominal stress, maximum nominal strain, quadratic nominal stress and quadratic nominal strain. The most widely used is the quadratic nominal stress criterion. This criterion is given by:

$$
\left\{\frac{\left\langle t_{n}\right\rangle}{t_{n}^{0}}\right\}^{2}+\left\{\frac{t_{s}}{t_{s}^{0}}\right\}^{2}+\left\{\frac{t_{t}}{t_{t}^{0}}\right\}^{2}
$$

where $n, s$, and $t$, represents the failure modes in the normal, first and second shear directions respectively. The superscript "o" represents the onset when the material starts degradation with its respective peak values: $t_{n o}, t_{s o}$ and $t_{t o}$. The values $t_{n}, t_{s}$ and $t_{t}$ are the components of force tractions in the normal and shear directions respectively. The former equation represents the criteria for a mixed mode, when two or more failure modes are presented in the analyzed element.

Once the degradation has been reached, a damage evolution law is needed to define the rate of stiffness degradation according to the applied traction forces and displacements. This law can be based on effective displacements or energy. The damage evolution law based on energy is described by:

$$
\left\{\frac{G_{s}}{G_{n}^{C}}\right\}^{\propto}+\left\{\frac{G_{s}}{G_{s}^{C}}\right\}^{\propto}+\left\{\frac{G_{t}}{G_{t}^{C}}\right\}^{\propto}
$$

where $G_{c}$ is the critical total energy release in the material in the normal, $n$, and shear directions, $s$ and $t$, respectively. The terms $G_{s}, G_{n}$ and $G_{t}$ represent the work done 
by the normal and shear tractions. These critical energy release rates are obtained experimentally from standardized ENF and DCB test.

The use of cohesive zone models may cause convergence issues produced by several factors: CZM law, adhesive plasticity, adherend stiffness and orthotropic nature of composite materials [84]. Models with trapezoidal cohesive laws are more prone to convergence problems. Brittle adhesives produce an abrupt change in the softening process increasing the aforementioned difficulties [84].

Another source of convergence problems is the mesh size. This parameter is also linked to the optimal cohesive length in the cohesive zone to accurately predict the fracture process. This parameter, still not well stablished yet, has been mostly studied in delamination problems rather than bonded joining. Unfortunately, there is no a standard solution to define the optimal number of elements. Solutions using between 2 and 10 elements have been satisfactorily applied [85, 86]. In addition, Turon et al [87] pointed out that, in some cases, to achieve the minimum required number of elements in the cohesive zone requires meshes under a half millimetre length, and such a small mesh may be intractable to solve large structures.

\subsubsection{CZM on Bonded Joints}

Cohesive zone modelling has been widely used to determine the strength of bonded joints mostly using 2D FEM analysis. Two different modelling techniques using cohesive elements on bonded joints can be used depending on the way that the cohesive zone elements are defined in the adhesive layer. The continuum approach is carried out using a single row of cohesive elements in the adhesive interface as shown in Figure 
2.17. Usually, this single layer of elements is given a triangular cohesive law shape. However, this cohesive law implies an elastic behaviour in the adhesive, and some authors have pointed out that plastic behaviour of the adhesive is not taken into account, leading in a loss of the physical meaning of the fracture process [84]. However, the aim of using CZM is to determine the strength in the joint, not the strain and stress distribution in the joint.

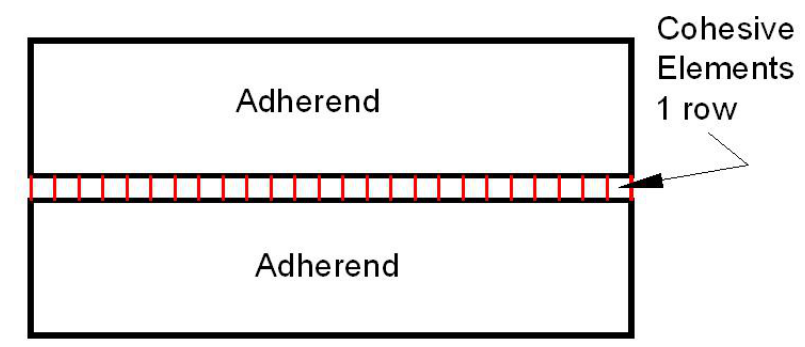

Figure 2.17 Cohesive zone model using continuum approach with a single row of cohesive elements

Using the local approach, the adhesive is modelled as a continuum with solid elements with embedded zero-thickness cohesive elements [84]. The number of solid elements used in the adhesive is not restricted to one row, and the material properties are not limited; therefore, the elastoplastic model can applied to these elements. Additionally, the location of the zero-thickness layers of cohesive elements is not restricted; many layers can be placed in the adhesive region. For instance, Figure 2.18 shows an adhesive layer modelled with solid elements and two rows of zero-thickness cohesive elements, each of them located in the interface between the adhesive and the adherend. 


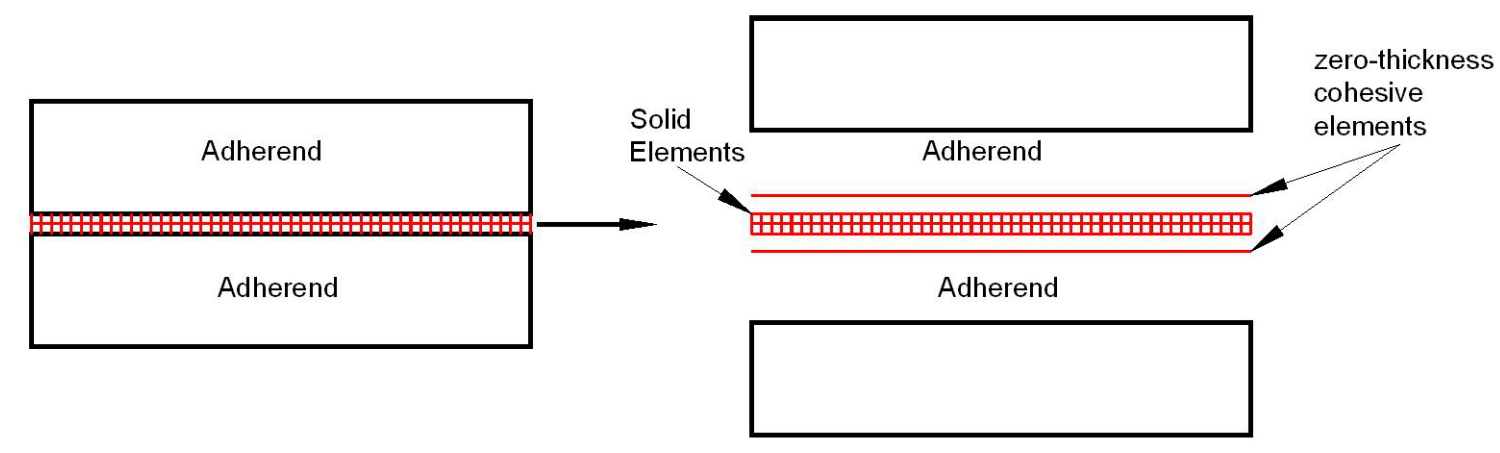

Figure 2.18 Cohesive zone model using local approach with zero-thickness cohesive elements

Due to its relative simplicity compared with the local approach, the continuum approach is the most widely used. The advantage of this approach is the relatively simple implementation and the solution can be achieved without significant convergence issues and less computational resources.

The amount of research on this topic is wide and has been mostly done to determine the strength of single lap joints and scarf joints $[88,89]$. Additionally, this technique has been also used to compare the effect of the adhesive properties on the joint strength. In some cases, parametric studies have been carried out to determine the effect of cohesive zone model parameters on the joint strength [90-92]

Analysis using the local approach are more challenging because convergence issues; due to this, few cases have been studied. Da Silva et al [93] analyzed the effect of the adhesive type and thickness on single lap joints. Three different adhesives were chosen, Araldite, AV138/HV998 and Hysol EA9361. Three different adhesive thicknesses were also chosen for the study, and the DOE was designed using Taguchi's 
method. The experimental study included heat treated steel adherends to keep them in the elastic regime, and the overlap length and width were $25 \mathrm{~mm}$.

A 2D plane-strain FEM analysis was carried out to compare against the experimental results using cohesive interface elements located at the interface adhesiveadherend. A triangular cohesive law with quadratic stress criterion for damage initiation and linear energetic criterion for damage propagation was used. The damage initiation parameter $t_{n}$ was assumed to be equal to the yielding stress of the adhesive and typical strain-energy release rates were chosen from literature.

For the elements in the adhesive layer, plane elements were used with DrukerPrager elastoplastic criterion. The stress-displacement was compared using the stroke displacement and, according to the authors, numerical and experimental values were not in good agreement.

Campilho et al [94] analyzed single-strap repairs using interface cohesive elements using a trapezoidal cohesive zone law. The model included several locations for the adhesive failure as well as for intralaminar failure with failure modes I and II respectively. The adhesive used was Araldite 420, and the traction separation parameters in mode I were defined from the adhesive's stress-strain bulk data. Additionally, the traction separation parameters in mode II were assumed using a von Mises yield criterion. The critical fracture energies were obtained from DCB and ENF test respectively. The composite adherends were analyzed using lamina properties. The model included several rows of cohesive elements in the adhesive as well as the adherends to include failure due to delamination. Using this model, a sensitivity analysis was carried out to analyze the effect of the different parameters of the trapezoidal cohesive law in the joint strength. 
From this sensitivity analysis, it was concluded that mode II properties have a higher effect on the failure load because the repair is loaded in shear.

\subsubsection{CZM studies on Bolted/Bonded Joints}

Very few studies using CZM modeling have been performed on joints with two different joining technologies. The 3D nature of the bolted/bonded joints makes difficult to apply this technique compared with bonded joints on which, traditionally, a 2D approach is used.

Sadowski [95] performed an experimental and numerical study on adhesively bonded aluminum plates with rivets. The model was developed using commercial software ABAQUS using an explicit scheme. The joint was a double lap with $2 \mathrm{~mm}$ aluminum plates for the top adherends and $4 \mathrm{~mm}$ for the middle adherend. The bondline width and length were $40 \mathrm{~mm}$ and five rivets were installed in the overlap. The adherends as well as the rivets were modelled with elastic properties only. The adhesive chosen was polyurethane Montagefix with thicknesses 0.1 and $0.2 \mathrm{~mm}$ and was discretized using a single row of 3D cohesive elements. The cohesive law chosen was a mixed mode triangular with quadratic nominal stress criterion for damage initiation and energetic power law for damage propagation, using an exponent value equal to 2 . The traction forces, $t_{n}$ and $t_{s}$, were asummed to be $4 \mathrm{MPa}$ and the fracture energies equal to $8.3 \mathrm{~N} / \mathrm{m}$ for modes I,II and III, and were assumed from the experimental results.

Good agreement on load-stroke results was found between experimental and FEM analysis for bonded, riveted and bonded/riveted joints. Additionally, the author concluded 
that the hybrid joints have best strength performance that bonded and riveted joints, with $11 \%$ and $130 \%$ higher strength respectively.

Campilho et al [96] performed a study to assess the advantage of spotwelded/bonded single lap joints compared with spot-welded and bonded joints technologies. The aim of the study was to compare the effect of the bondline length on the joint strength, using $\mathrm{L}_{\mathrm{o}}=15,30,45$ and $60 \mathrm{~mm}$. The adherend material chosen was steel and the adhesive was Araldite 2015 and it was characterized in bulk as well as in shear using TAST. The joint width chosen was $25 \mathrm{~mm}$ and the adhesive thickness was $0.2 \mathrm{~mm}$. A mixed-mode triangular CZM law was defined for the adhesive as well as the welded spot. Quadratic nominal stress criterion for damage initiation and energetic linear law for damage propagation was defined. For the adhesive, $t_{n}$ and $t_{s}$ values were chosen as the failure strengths from the bulk and TAST data, and $G_{n}$ and $G_{s}$ were characterized experimentally. The 3D FEM model included a single row of 3D cohesive elements. The results showed an improvement in the joint strength using a bondline length $\mathrm{L}_{0}=30 \mathrm{~mm}$. For larger values of bondline, there is no improvement compared with bonded joints alone.

In summary, although CZM technique is widely used for crack propagation analysis on materials and bonded joints, the application on bonded/bolted joining technologies is at very early stages.

\subsection{Damage and Failure in Composites}

Because of its layered construction, failure in composite materials cannot be analyzed using techniques for isotropic materials, i.e., metals. Usually, the failure starts at 
a point of a lamina, known as first ply failure. After this failure, this section of the lamina is not able to keep carrying load and this is re-distributed between the remaining layers of the composite part. This process continues until all the laminae are completely fail. Unfortunately, there are no failure theories capable to predict the complete failure in the composite laminate. The developed failure theories for layered composite materials are capable of predict the failure at lamina level only.

When the failure load on a composite structure is required, the progressive failure analysis approach has to be used and it has been widely implemented using finite element modelling. Typically, the load in the composite to be analyzed is applied in small increments; at each increment the stresses or strains are computed over the laminae and compared against a failure criterion. This failure criterion is a mathematical formulation that compares stresses or strains against the mechanical properties of the material and, thus, capable of defining the onset of the failure. Several failure criteria have been widely developed and tested for different configurations and materials. A comparison between several failure theories was carried out in the World Wide Failure Exercise I and II [12, $13,97]$

If failure is found at the elements of a lamina, their mechanical properties are degraded on these elements or section according to a degradation rule. The procedure continues until the full failure in the composite laminated is reached. Therefore, to perform a progressive failure analysis, a failure theory and a set of degradations rules are required.

Hashin's failure criterion [98] was chosen to be used in this research because is based on laminae strength, these strengths are easily obtained either from manufacturer's 
datasheets or standard mechanical testing. This criterion predicts the onset of damage for tensile fibre failure, compressive fibre failure, tensile matrix failure and compressive matrix failure as shown in Table 2.2.

Table 2.2 Hashin's failure criteria [98]

\begin{tabular}{|l|c|c|}
\hline \multicolumn{1}{|c|}{ Failure mode } & $\left(\frac{\sigma_{11}}{X_{T}}\right)^{2}+\left(\frac{\sigma_{12}^{2}+\sigma_{13}^{2}}{S_{12}^{2}}\right)=1$ & $\sigma_{11}>0$ \\
\hline Tensile fibre & $\left(\frac{\sigma_{11}}{X_{C}}\right)^{2}=1$ & $\sigma_{11}<0$ \\
\hline Compressive fibre & $\left(\frac{\sigma_{22}^{2}+\sigma_{33}^{2}}{Y_{T}^{2}}\right)+\frac{\sigma_{12}^{2}+\sigma_{13}^{2}+\sigma_{23}^{2} \sigma_{22} \sigma_{33}}{S_{12}^{2}}=1$ & $\sigma_{22}+\sigma_{33}>0$ \\
\hline Tensile matrix & $\frac{1}{Y_{C}}\left[\left(\frac{Y_{C}}{2 S_{12}}\right)-1\right]\left(\sigma_{22}+\sigma_{33}\right)+\left(\frac{\sigma_{22}^{2}+\sigma_{33}^{2}}{4 S_{12}^{2}}\right)^{2}$ & $\sigma_{22}+\sigma_{33}<0$ \\
& $+\frac{\sigma_{12}^{2}+\sigma_{13}^{2}+\sigma_{23-}^{2} \sigma_{22} \sigma_{33}}{S_{12}^{2}}=1$ & \\
\hline & & \\
\hline
\end{tabular}

Where $X_{T}$ and $X_{C}$ are the tensile and compressive strengths in the longitudinal direction, $Y_{T}$ and $Y_{C}$ are the tensile and compressive strengths in the transverse and $S_{12}$ the shear strength. $\sigma_{11}, \sigma_{22}$ and $\sigma_{33}$ are the stress components in the directions 1,2 , and 3 respectively.

Failure criterion along with progressive failure analysis has been widely used in the commercial FE software Abaqus for research on composites. The standard implementation of Hashin's failure criteria in Abaqus can predict the onset of failure on 2D shell elements only. However, after failure is predicted at the laminae, the software 
does not reduce the mechanical properties accordingly using degradation rules. A user subroutine has to be programmed and linked with the software during the analysis. The way to implement this degradation effect in the software is to reduce the desired elastic properties by a factor according a degradation rule. The number of proposed degradation rules is vast and, unfortunately, there is no a standard guide to choose the best rule for a specific application.

Throughout the years, several researchers have proposed degradation rules. A thorough review on this topic can be found in [99].

Chang [100] proposed a degradation model for notched composites under tensile load. To perform the analysis, the author developed a nonlinear FEM code to compute the stresses, apply a failure criterion and finally, if failure was predicted, the materials properties were degraded before the next load increment. For matrix cracking, the transverse modulus $E_{y}$ and Poisson's ratio $v_{\mathrm{y}}$ were reduced to zero. For fibre brakeage and/or fibre matrix shearing, $E_{y y}$ and $v_{y}$ were reduced to zero and $E_{x}$ and $G_{x y}$ were reduced using a Weibull distribution as a function of the predicted damaged area.

Tan [101] performed a progressive failure analysis in composites with openings using the Tsai-Wu failure criterion. The author proposed several stiffness degradation parameters: $\mathrm{D}_{1}$ for stiffness degradation on the fibre direction due to fiber breakage, $\mathrm{D}_{2}$ and $\mathrm{D}_{6}$ for transverse and shear stiffness for matrix failure. After comparing the FEM results against the experimental data, the authors concluded that the best agreement was with $\mathrm{D}_{1}=0.07$ and $\mathrm{D}_{2}=\mathrm{D}_{6}=0.2$. 
Camanho and Lewis [102] extended Tan's work to three dimensions by adding a degradation parameter to take into account the degradation of $G_{23}$ by using the same degradation parameter used for $G_{12}, \mathrm{D}=0.2$

Tserpes et al [103] proposed a progressive damage model for composite bolted joints. The model was developed using the ANSYS commercial FE software applying Hashin's failure criterion with the following failure criteria: matrix tensile and compressive, fibre tensile and compressive, fibre-matrix shear out and delamination. For matrix tensile and compressive, $E_{y y}$ and $v_{x y}$ were degraded to zero. For fibre tensile and compressive, all the elastic constants were degraded to zero.

McCarthy [104] performed a progressive damage analysis on multi bolted composite joints. Hashin's failure criterion was chosen for the analysis and some elastic constants were reduced to $10 \%$ of their original values ( 0.1 degradation parameter) according to the failure mode as shown in Table 2.3. The remaining elastic constants were unaffected by the failure mode.

Table 2.3 Stiffness degradation parameters according to McCarthy [104]

\begin{tabular}{|l|c|c|c|c|c|c|c|c|c|}
\hline \multicolumn{1}{|c|}{ Failure mode } & $\mathrm{E}_{11}$ & $\mathrm{E}_{22}$ & $\mathrm{E}_{33}$ & $\mathrm{G}_{12}$ & $\mathrm{G}_{23}$ & $\mathrm{G}_{13}$ & $v_{12}$ & $v_{23}$ & $v_{13}$ \\
\hline Tensile matrix mode & & 0.1 & 0.1 & & 0.1 & & & 0.1 & \\
\hline Tensile fibre mode & 0.1 & & & 0.1 & & 0.1 & 0.1 & & 0.1 \\
\hline Compressive matrix mode & & 0.1 & 0.1 & & 0.1 & & & 0.1 & \\
\hline Compressive fibre mode & 0.1 & & & 0.1 & 0.1 & 0.1 & 0.1 & & 0.1 \\
\hline More than one failure mode & 0.1 & 0.1 & 0.1 & 0.1 & 0.1 & 0.1 & 0.1 & 0.1 & 0.1 \\
\hline
\end{tabular}

Implementing a progressive damage model into the commercial software Abaqus requires choosing both the failure theory and degradation model. Unfortunately, as 
pointed out previously, there is no guidance to select between the many available options. The choice remains in the final user.

Several other studies have been carried out using different degradation rules. However, for this research, the rules proposed by McCarthy were chosen. Although some degraded parameters are lower compared with others, i.e., 0.2 for transverse and shear matrix failure, the difference is $10 \%$. Consequently, these values account for a more conservative model. Finally, all the elastic constants to be degraded according to the failure mode are determined in the failure matrix.

\subsection{Digital Image Correlation Technique}

Digital image correlation (DIC) is a well-stablished and proven optical technique to measure displacements and strains. The technique is based on the optical detection of motion of a set of points, subset, of the material being tested. Usually, a random speckle pattern is used to maximize the contrast between points. The motion is captured using digital cameras at a specific frame rate, and the change in position or displacement of small regions or subsets is mapped using a correlation criterion as shown in Figure 2.19. This correlation criteria evaluate the similarity between the deformed state and the reference state [105]. The best match is when the correlation algorithm reaches the peak value. 

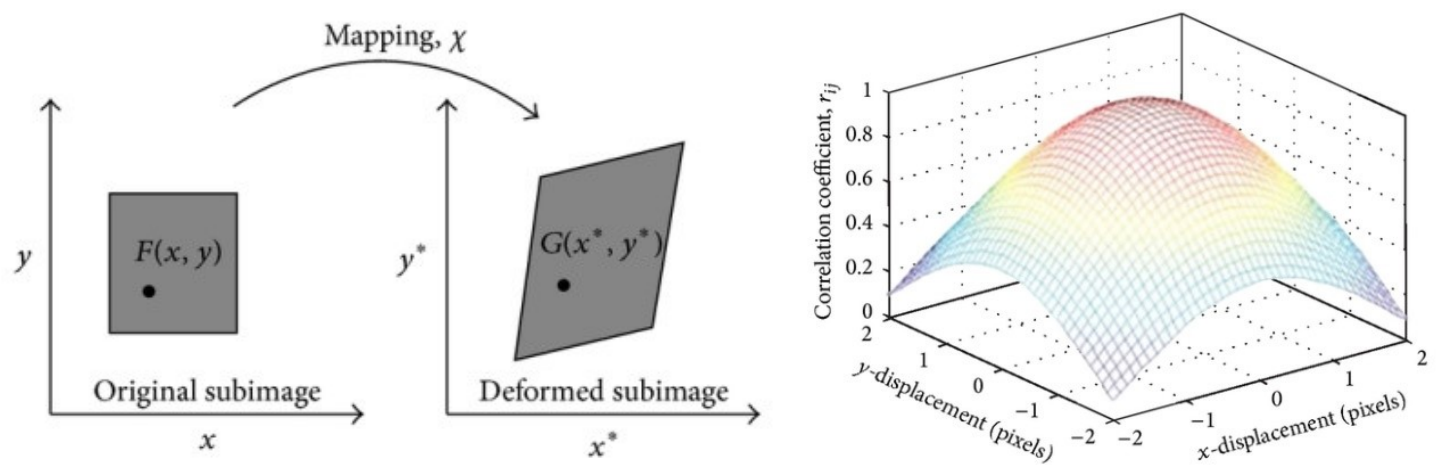

Figure 2.19 Basic concept of DIC [106]

The correlation algorithm can use different criteria to assess the similarity between subsets. These algorithms can be divided into cross-correlation criteria (CC) algorithms and summation of the squared differences (SSD) algorithms [105]. From the first method, cross-correlation (CC), normalized cross-correlation (NCC) and zeronormalized cross-correlation (ZNCC), are the most commonly used algorithms. On the other hand, for the second method, sum of the squared differences (SSD), normalized sum of squared differences (NSS), and zero-normalized sum of the squared differences are the most commonly used algorithms.

However, in real measurements, a perfect correlation is hard to achieve. There is some uncertainty due to several sources of error such as lighting, lens distortion, quality of the CCD cameras, out-of-plane displacements, speckle pattern and the correlation algorithm [107].

The applicability of this technique is wide, including materials characterization, fracture mechanics and analysis of strain in structures. As with every measurement technique, it has its advantages and disadvantages. 
Advantages:

- A surface or volume can be analyzed. Compared with traditional strain gauges where a single point is be measured;

- Versatility to measure different field of view (FOV), from square meters to microscopic sizes;

- There is no need to attach mechanical devices to the specimen; and

- The capability of the software to analyze either displacements or strain.

Disadvantages:

- The need of digital cameras and lenses. The quality of these devices affects the results;

- The commercial software is expensive;

- The speckle pattern can affect the results;

- Some cases, the rigid body motion cannot be properly isolated. Depends on the software algorithm; and

- There are sources of error.

This technique has been applied in bonded joints in very few cases, mostly using commercial software. One of the aspects that need to be carefully managed during the analysis to measure strains in adhesively bonded joints using 2D DIC is the effect of the rotation due to secondary bending moment.

Colavito et al [108, 109] performed DIC measurements of strains on double lap joints using commercial software ADASIM. A high resolution camera with telecentric lenses were used and the area of analysis was $1.828 \times 1.828 \mathrm{~mm}$. The frame capturing procedure was manual, a force was applied at the desired level, and the testing machine 
was stopped to take the picture frames fifteen times. The authors pointed out that even one degree in rotation could significantly affect the results. Although the software ADASIM had the capability to remove the rigid body translations, it could not remove the rigid body rotations. Therefore, the rotation was computed and removed from the DIC data using further post processing.

Kumar et al [110] performed an experimental analysis on composite single lap joints using DIC and compared the results against FEM. The bondline thickness in the joint was $0.76 \mathrm{~mm}$ and the area of analysis was $25.4 \times 25.4 \mathrm{~mm}$. The 16 MPixel camera along with the lenses allowed a spatial resolution of 38pixel $/ \mathrm{mm}$. The authors measured both shear strain as well as peel strains in the joints. However, there is no information about the effect of the rotation in the measurements as well as the capability of the software to remove it.

Bao Quiao et al [111] preformed shear strain measurements on single lap joints to determine the shear modulus in an adhesive using high magnification DIC analysis. Up to $500 \mathrm{X}$ magnification were achieved using a microscope CCD camera to capture the images. The testing equipment included a loading stage controlled manually and instrumented with a load cell. For the shear strain measurement, the authors claim that the removal of rigid body rotation was not needed.

Comer et al performed 2D and 3D DIC analysis on composite single lap joints. Two part epoxy adhesive was used to join the carbon fibre composite adherends. The aim of the research was to use $2 \mathrm{D}$ and $3 \mathrm{D}$ to evaluate the evolution of the joints before failure. The 2D DIC measurement was carried out at a working distance of $86 \mathrm{~mm}$ with magnifications from 0.58 to $7 \mathrm{X}$, and the spatial resolution was $4 \mathrm{~nm} /$ pixel. The analysis 
was performed using Strainmaster ${ }^{\circledR}$ software using a fast transform subpixel using a bicubic interpolation scheme. The analysis using 2D DIC was carried out on the adhesive fillet region. The authors found a maximum principal strain of 3\% in the adhesive at $95 \%$ of failure load. Although the adhesive used is not explicitly mentioned, they claimed that these strain values are near to these obtained in the bulk adhesive from previous research where the adhesive used was and epoxy EA9380 from Henkel [112]. The authors did not mention the effect of the joint rotation in the analysis.

Crammond et al [113] used 3D-DIC to analyze the damage and load transfer in composite double strap joints. The DIC system included a 5 MP camera with Canon lens to achieve three spatial resolutions: $30.8,250$ and 726 pixels $/ \mathrm{mm}$. The analysis was performed using LaVision Davis 7.4 software employing a correlation algorithm using inverse fast Fourier transform. The 3D measurements were carried out on the front face of the joint and the 2D DIC measurements were carried out at the edge of the joint at very high magnification. Because the joint was not prone to rotation, there was no need to make corrections due to this in the analysis. However, the author pointed out that during the 3D DIC measurement, an out-of-plane motion of $0.2 \mathrm{~mm}$ was detected at the beginning at the test and it was difficult to eliminate. This motion affected the experiment during the 2D measurements because the edge rotated at the beginning of the test, between 0 to $200 \mathrm{~N}$. This issue was solved by using a micro stage to align the camera with the specimen edge.

Lemmen et al [114] performed DIC strain measurements on several experiments including a TAST specimens at very high magnifications. The shear strain was computed analyzing the images by choosing four points as shown in Figure 2.20, two on each 
adhesive substrate interface. The shear angle between points was computed and the rotation due to bending was subtracted. The shear strain, the tangent of the angle due to relative displacement between two points in the adhesive, was finally computed by dividing this relative displacement by the adhesive thickness. It was also found that at very small strains, the error in the strain calculation can be significant if rigid body rotation is not removed, near the same order of magnitude of computed strain.

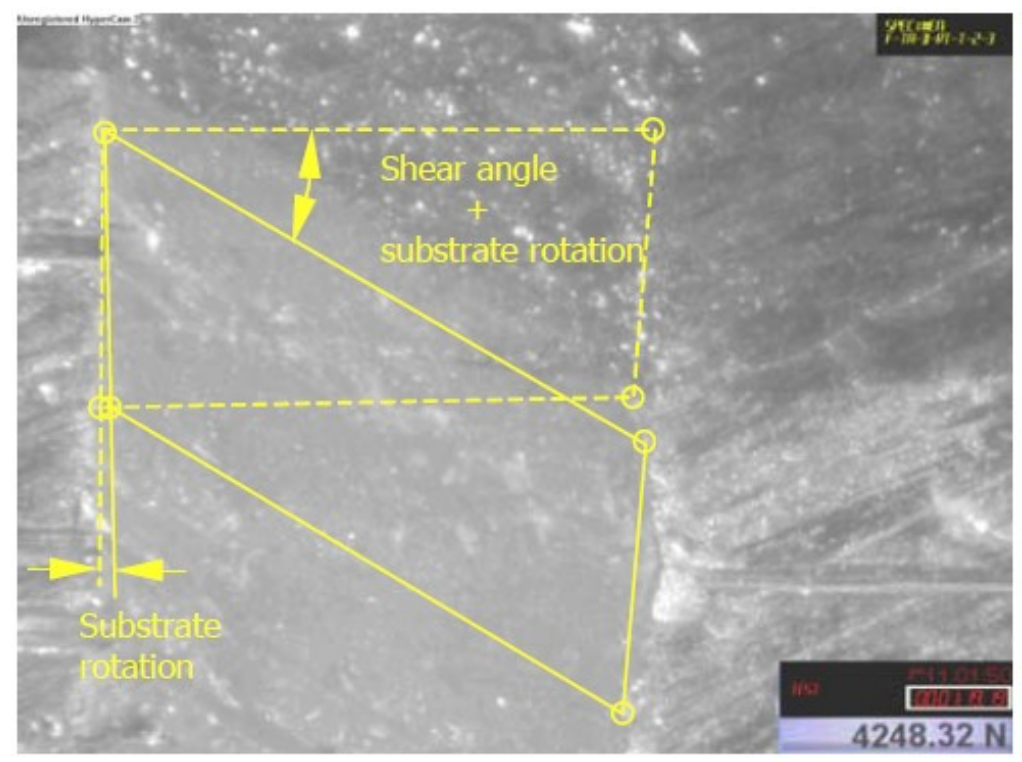

Figure 2.20 Strain calculation using DIC performed by Lemmen et al. (Reprinted from [114] with kind permission from Dr. Lemmen)

Van Blitterswyk et al [115] performed DIC strain measurements on TAST specimens. The objective of the research was to compare the shear strain measurement using the optical technique and the standard procedure using a KGR extensometer. The authors also pointed out the negative effect of the rotation in the strain results using the DIC technique. Although the commercial software used was capable of eliminating rigid body rotation, they also measured the strains by tracking the displacements of three 
points in the edge of the joint, in a similar way that the KGR extensometers measures the shear strain. Once the displacements in these three points were extracted, the shear strain was computed by measuring the relative displacement between two points in the substrates, correcting due to rotation and, finally, dividing by the adhesive thickness. The authors concluded that the most reliable and repeatable DIC procedure is by measuring the displacements between adherends on points adjacent to the bondline. Additionally, selecting the extraction points near to the bondline minimize the influence of the adherends deformations and, therefore, eliminating the need for correction due to adherend deformation according to the ASTM D5656-10 procedure. In contrast, using the software to extract directly the shear strain from the bondline could produce some problems to the algorithm during the analysis.

\subsection{Design of Experiments}

As discussed in the previous sections, the studies carried out on composite bolted/bonded joints are in the early stages. Therefore, the amount of data is limited compared with the huge amount of available information for bonded and bolted joints. To identify the effect of the several variables in the joint performance, the traditional method of choosing a single variable at a time is not recommended. Additionally, information about interaction between several variables cannot be obtained. If the effects of several variables at the same time and their interactions have to be assessed, a different approach has to be used. Alternatives such as Taguchi's method and design of experiments can be applied for this purpose. 
Taguchi's method is an experimental design approach widely used for the design of products and the improvement of processes [116]. It is mainly applied to reduce the variability around a target value or factor, on the desired response. The method separates the variables or factors in two groups, control factors and noise factors and the experiment is designed using these factors in a so-called orthogonal array on which, the factor's levels are represented as integers. Some authors [117] consider some of these orthogonal arrays as factorial designs. For instance, a L8 array with seven variables is equivalent to a factorial fractional design $2^{7-3}$ with resolution III. An L8 design with 4 factors is a $2^{4-1}$ factorial design. One of the main disadvantages of the Taguchi's method is the lack of information about the interaction between factors.

Another approach to analyze the effect of several variables in the joints performance can be using a DOE. The DOE is a systematic approach to analyze the response as a function of the factors involved [118]. A designed experiment using DOE can be applied for several purposes: comparison, screening, modelling and optimization [119].

\subsubsection{DOE Factorial Designs}

In design of experiments terminology, a factorial design is an experiment to identify the effect of several factors on a desired response. The values of the factors or variables to explore are defined in several levels during the experiment to identify their effect and their interactions on the response value at the same time. This approach contrasts with the traditional experimental approach of varying one factor at a time. The 
number of levels in which each factor varies is not restricted; however, the number of required tests increases exponentially with each added level.

For screening purposes, when the effects of many factors need to be assessed in the desired response, the suggestion is to define $k$ factors at two levels each. Additionally, if the number of resources is limited, a single-replicated strategy is recommended. Of course, if the resources are available, many replicates can give better results.

One disadvantage of using only 2 levels, is that the response and the interactions between factors will be linear over the range analyzed (Figure 2.21a). However, this assumption is reasonable in early stages of the DOE.

After the analysis of the data, a new design of experiments with more levels and replicates can be designed including only few specific factors of interest at more levels. This new approach usually gives better response data, including exponential behaviuor (Figure 2.21b).
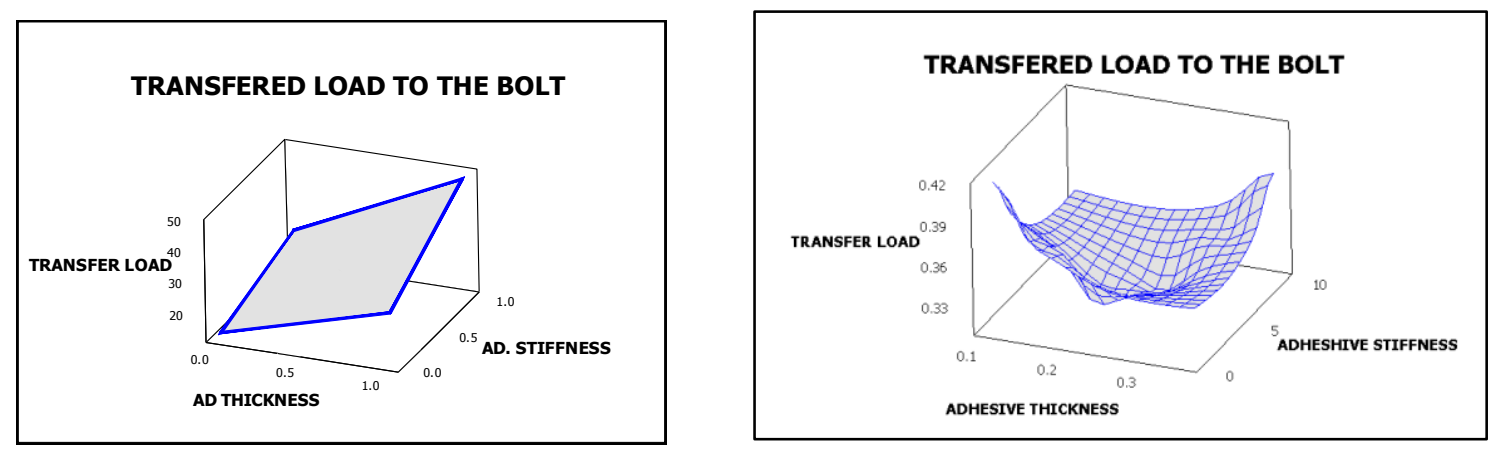

Figure 2.21 Relationship between factors interaction; a) Linear; b) Exponential

The appropriate number of factors to run the design has to be carefully selected because the number of required tests increases exponentially as the number of factors increases as shown in Table 2.4. 
Table 2.4 Number of runs required for $2 \mathrm{k}$ experimental design

\begin{tabular}{|c|c|}
\hline $\begin{array}{c}\text { Factors } \\
\text { At two levels each }\end{array}$ & $\begin{array}{c}\text { Number of runs } \\
\text { 1 Replicate }\end{array}$ \\
\hline 2 & 4 \\
\hline 3 & 8 \\
\hline 4 & 16 \\
\hline 5 & 32 \\
\hline 6 & 64 \\
\hline
\end{tabular}

Alternative designs, running only a fraction of the complete factorial experiment are widely used to reduce the number of test based on the assumption that certain highorder interactions between factors are negligible. The fractional design derived can be one-half, one-quarter, one-eight or more fractions of the original factorial design. Nevertheless, when a smaller fraction is chosen, there is an associate risk of losing data because some factors' effect and its interactions on the response can be aliased with others [117]. The degree of aliasing between factors effects is defined by the resolution of the design, and the most common designs are resolution III, IV and V. The lower the resolution, the greater the level of aliasing between the factor effects.

There are several possibilities to select the fraction from the full design; this fraction is defined by the so-called generator. Normally, these generators are standardized. A detailed table of factorial designs and generators can be found in [117].

\subsubsection{The Analysis of Variance (ANOVA)}

After the experiment is carried out using a factorial design, the effect of the factors and their interactions on the response is analyzed using the analysis of variance (ANOVA). This statistical tool can be applied to several fields to detect variability by 
evaluating the means in the population or sample. For instance, in quality control, the input can be the variables in the process and the output (response) the number of defects; in materials properties, the input can be the processed batch and the output (response) the variation in strength. The application of ANOVA in design of experiments is to estimate the effect of several factors on the response values. The total variability is partitioned into its component parts [117]:

$$
\mathrm{SS}_{\mathrm{T}}=\mathrm{SS}_{\text {Treatments }}+\mathrm{SS}_{\mathrm{E}}
$$

In expanded form using the sum of the squares:

$$
\sum_{i=1}^{a} \sum_{j=1}^{n}\left(y_{i j}-\bar{y}_{. .}\right)^{2}=n \sum_{i=1}^{a}\left(\bar{y}_{i .-} \bar{y}_{. .}\right)^{2}+\sum_{i=1}^{a} \sum_{j=1}^{n}\left(y_{i j}-\bar{y}_{.}\right)^{2}
$$

where:

$a$ is the number of treatments and $b$ is the number of observations under the ith treatment. $y_{i}$ is the total of the observations under the ith treatment:

$$
y_{i .}=\sum_{j=1}^{n_{i}} y_{i j}
$$

$\bar{y}_{i .}$ is the average of the observations under the $i t h$ treatment:

$$
\bar{y}_{i .}=\frac{y_{i}}{n}
$$

$y_{. .}$is the sum of all observations:

$$
y_{. .}=\sum_{i=1}^{a} \sum_{j=1}^{n} y_{i j}
$$

$\bar{y}_{. .} \quad$ is the average of all observations $(\mathrm{N})$ :

$$
y_{. .}=\frac{y_{. .}}{N}
$$




$$
N=a b
$$

$\mathrm{SS}_{\mathrm{T}}$ is the total sum of the squares and represents the total variability in the data.

$\mathrm{SS}_{\text {Treatments }}$ is the sum of the squares between treatments and $\mathrm{SS}_{\mathrm{E}}$ is the sum of the squares due to error. Another way to refer this is that the total variability is partitioned between the treatment averages $\left(\mathrm{SS}_{\text {Treatmens }}\right)$ and within the treatments $\left(\mathrm{SS}_{\mathrm{E}}\right)$.

The previous definition can be generalized to a factorial design with several factors and levels. For instance, an ANOVA table for a three-factor design is shown in Table 2.5; where, $a, b$ and $c$, are the levels of the factors $\mathrm{A}, \mathrm{B}$, and $\mathrm{C}$ respectively. In a 2 levels design, the levels $a, b$ and $c$ should be the same and equal to 2 . The mean square (MS) is the sum of the squares divided by its degrees of freedom. Therefore, the SS and MS values are the same.

Table 2.5 Analysis of variance table for a three-factor model. Adapted from [117]

\begin{tabular}{|c|c|c|c|c|}
\hline $\begin{array}{c}\text { Source of } \\
\text { variability } \\
\text { (Factor) }\end{array}$ & $\begin{array}{c}\text { Degrees of } \\
\text { freedom }\end{array}$ & $\begin{array}{c}\text { Sum of } \\
\text { Squares } \\
(\mathrm{SS})\end{array}$ & $\begin{array}{c}\text { Mean Square } \\
(\mathrm{MS})\end{array}$ & $\mathrm{F}_{\mathrm{O}}$ \\
\hline $\mathrm{A}$ & $\mathrm{a}-1$ & $\mathrm{SS}_{\mathrm{A}}$ & $\mathrm{MS}_{\mathrm{A}}$ & $\mathrm{MS}_{\mathrm{A}} / \mathrm{MS}_{\mathrm{E}}$ \\
\hline $\mathrm{B}$ & $\mathrm{b}-1$ & $\mathrm{SS}_{\mathrm{B}}$ & $\mathrm{MS}_{\mathrm{B}}$ & $\mathrm{MS}_{\mathrm{B}} / \mathrm{MS}_{\mathrm{E}}$ \\
\hline $\mathrm{C}$ & $\mathrm{c}-1$ & $\mathrm{SS}_{\mathrm{C}}$ & $\mathrm{MS}_{\mathrm{C}}$ & $\mathrm{MS}_{\mathrm{C}} / \mathrm{MS}_{\mathrm{E}}$ \\
\hline $\mathrm{AB}$ & $(\mathrm{a}-1)(\mathrm{b}-1)$ & $\mathrm{SS}_{\mathrm{AB}}$ & $\mathrm{MS}_{\mathrm{AB}}$ & $\mathrm{MS}_{\mathrm{AB}} / \mathrm{MS}_{\mathrm{E}}$ \\
\hline $\mathrm{AC}$ & $(\mathrm{a}-1)(\mathrm{c}-1)$ & $\mathrm{SS}_{\mathrm{AC}}$ & $\mathrm{MS}_{\mathrm{AC}}$ & $\mathrm{MS}_{\mathrm{AC}} / \mathrm{MS}_{\mathrm{E}}$ \\
\hline ABC & $(\mathrm{b}-1)(\mathrm{c}-1)$ & $\mathrm{SS}_{\mathrm{BC}}$ & $\mathrm{MS}_{\mathrm{BC}}$ & $\mathrm{MS}_{\mathrm{BC}} / \mathrm{MS}_{\mathrm{E}}$ \\
\hline Error & $\mathrm{abc}(\mathrm{n}-1)(\mathrm{b}-1)(\mathrm{c}-1)$ & $\mathrm{SS}_{\mathrm{ABC}}$ & $\mathrm{MS}_{\mathrm{ABC}}$ & $\mathrm{MS}_{\mathrm{ABC}}$ \\
\hline Total & $\mathrm{abcn}-1$ & $\mathrm{SS}_{\mathrm{E}}$ & $\mathrm{MS}_{\mathrm{E}}$ & \\
\hline
\end{tabular}


Usually, a large $F_{o}$ value indicates a strong evidence against the hypothesis that the means are equal [117]. Therefore, this is evidence of a strong effect of the factor on the total variability in the analyzed response.

\subsection{Research Motivation, Objective and Scope}

\subsubsection{Summary of Literature Review}

The prior research described in literature to understand the behavior of bolted/bonded joints in composites is in relatively early stages. Very few publications are available on this topic.

Most of the experimental research has been focused on the measurement of the static strength of the joints. It has been found that the strength of the bolted/bonded joints, if properly designed, is better compared with bolted or bonded alone.

The load sharing analysis between the adhesive and bolt has been performed mostly numerically, with very limited experiments to validate the results using instrumented bolts in a limited number of configurations. Nevertheless, it has been found that the load sharing is mainly affected by the adhesive stiffness, adhesive thickness, and lap length.

Few analytical models have been proposed to understand the behavior of bolted/bonded joints in composites. However, these models do not capture the fully $3 \mathrm{D}$ complex behavior of the joint which includes peel and shear stress in the adhesive, stress distribution in the hole, stress due contact between bolt and adherend, preload, clearance 
and failure criteria for both the adhesive and the adherends and elastoplastic properties on the adhesive.

In summary, several gaps in the knowledge of hybrid bolted/bonded joints in composites have been identified, including:

- A lack of a well-supported experimental and statistical analysis on the effect of the variables on the joint behaviour. Most of the variables have been tested varying one factor at the time;

- A lack of extensive measurement of load sharing experimentally; and

- A lack numerical studies on damage mechanisms and damage propagation in the joint, including degradation of both the adhesive and composite laminate

\subsubsection{Research Objectives}

Based on the above review of literature, the present research has the following objectives:

- To carry out an experimental program on composite bolted/bonded joints using Design of Experiments methodology; to assess the effect of several variables on the joint performance;

- To measure the load sharing using a different approach. Instead of measuring the load in the bolt, measuring the strain in the adhesive using Digital Image Correlation technique;

- To perform numerical analysis including cohesive zone models in the adhesive to compute the joint strength in bolted/bonded joints with several configurations. 


\subsubsection{Limitations and Applications}

For this research, the following limitations for the experimental and numerical study are defined:

- Experiments will be carried out at coupon size level

- Experiments will be carried out at room temperature conditions

- Environmental factors are not included

The present research was supported by CRIAQ COMP 506. The results will be transferred to the academic and industrial partners. The final applicability of the results from the present study in actual aircraft for design purposes or repairs will depend on the industrial partners. 


\section{Chapter 3: Methodology}

The first task to designing an experimental study is to allocate the appropriate resources, facilities, materials, time, and budget. This is always a constraint for experimental projects as resources and time are limited and dictated by the budget. The second task is to define the geometrical design of the testing coupons and determine their capacity to be manufactured in the specified quantities and accuracy for the DOE. Lastly, with the previous information, the number of factors and levels to test can be defined using a specific DOE; during this process the number of coupons is determined and compared against the available resources and iterated as needed.

\subsection{Materials}

The current study was conducted for applications in the aerospace industry; therefore, commonly used aircraft composite materials, adhesives and fasteners were selected. The test coupons were manufactured using carbon fibre reinforced plastic (CFRP). The chosen material was Cycom 5320 T650 33/145 from Cytec - an out-ofautoclave CFRP composite. This particular CFRP utilizes an epoxy resin system developed to be used in primary aircraft structure for its mechanical performance, which is equivalent to that of the autoclave-cured carbon fibre epoxy systems [120].

The selected fastener was a titanium bolt from LISI Aerospace, formerly Hi-Shear Corporation. This particular bolt was chosen on a recommendation from the industrial partners for its importance and regular use in composite aircraft structures. A standard diameter of 3/16 in $(4.76 \mathrm{~mm})$ with a protruding tension head and nominal shank was 
chosen. A schematic of this bolt can be seen in Figure 3.1. The bolt material is titanium 6-Al-4V as per AMS4928 or AMS4967 with minimum strengths of $620 \mathrm{MPa}(95,000$ psi) and $1100 \mathrm{MPa}(160,000 \mathrm{psi})$ in shear and tensile respectively [121].

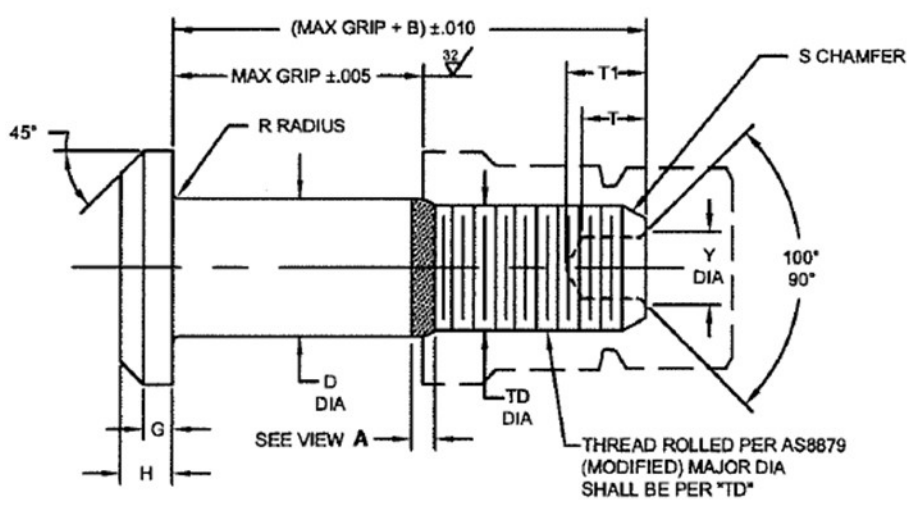

Figure 3.1 Hi-Lite ${ }^{\circledR}$ titanium 6 Al-4V fastener [121]

The adhesives were selected to obtain a range of low to high shear modulus. Additionally, the application for aircraft was taken into account. After a survey of available aerospace-grade adhesives, three systems were selected; FM300-2M (epoxy), EA9361 (high elongation epoxy) and PR1665 (polyurethane). The details of the adhesive systems are presented in Table 3.1

These adhesives were characterized in shear using the TAST method as described in Table 5.5 and Table 5.6 in Chapter 5. 
Table 3.1 Adhesives selected

\begin{tabular}{llll}
\hline Adhesive & Manufacturer & Type & $\begin{array}{l}\text { Service } \\
\text { temperatures }\left({ }^{\circ} \mathrm{C}\right)^{*}\end{array}$ \\
\hline FM300-2M & Cytec & Epoxy Film & -55 to 149 \\
EA9361 & Henkel & Epoxy Paste & -196 to 60 \\
PR1665 & PPG & Polyurethane & -196 to 121 \\
& & Paste & \\
\hline
\end{tabular}

* From manufacturer's data sheets

During the preliminary FEM analysis, it was found that the polyurethane adhesive (PPG) had a lower force versus displacement performance when compared to FM300-2M and EA9361 adhesives. As a result, it was decided to use the FM300-2M and EA9361 adhesives for the experimental work. This decision to include only high modulus (FM300-2M) and low modulus (EA9361) adhesives could seem contradictory if we consider that, according to the literature review the load sharing in joints using high modulus adhesives is expected to be very low. However, the testing program described in this thesis was performed not only to analyze load sharing capabilities but also the joint strength prior to failure while simultaneously monitoring other parameters. Therefore, a strong and stiff adhesive must be included in the experimental DOE.

\subsection{Specimen for Bolted/Bonded Joints}

There is no standard method for testing hybrid bolted/bonded joints; previous research work described in the literature has been carried out using varying joint designs. For this study, it was decided to use a joint design based on that described in standardized tests. The design options were analyzed from existing ASTM standards used to test 
composite bonded or bolted joints. Firstly, the bonded joint design from the ASTM D$3165-07$ [122] has a total length of $177.8 \mathrm{~mm}(7 \mathrm{in})$ plus the length of the overlap; the recommended overlap value is $12.7 \mathrm{~mm}(0.5 \mathrm{in})$ as shown in Figure 3.2. This overlap length is not suitable for a bolted/bonded joint for the reason that the length is too short to add a bolt in the middle. However, the joint width seems to be reasonable to be used with a $3 / 16$ in $(4.76 \mathrm{~mm})$ bolt.
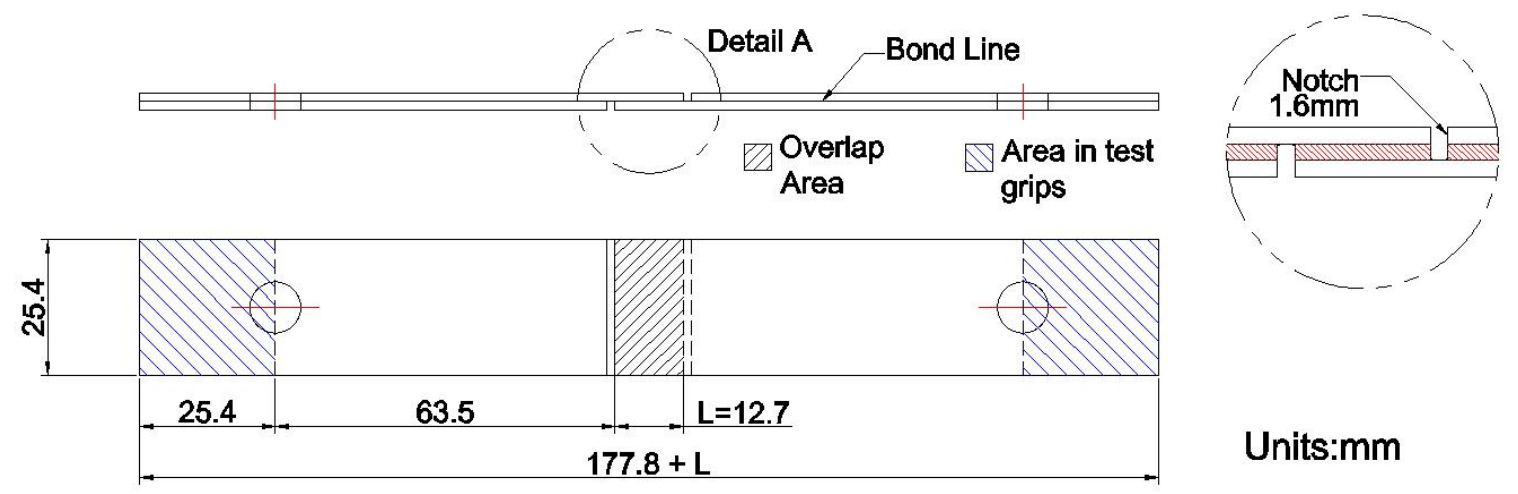

Figure 3.2 Bonded joint specimen according to ASTM D-3165. Adapted from [122]

The standard test ASTM D5961D/D5961M-10 [123], may be used to test the bearing response of composites using several configurations. There are configurations with one or two bolts in single shear. Figure 3.3 shows the configuration for a single bolt; the total length is $234 \mathrm{~mm}$ (9.2 in), and the edge-to-diameter ( $e / d)$ ratio is equal to 3 using the recommended $3 / 16$ in $(4.76 \mathrm{~mm})$ diameter bolt. Additionally, the $36 \mathrm{~mm}$ width give a width-to-diameter $(w / d)$ ratio equal to 6. 


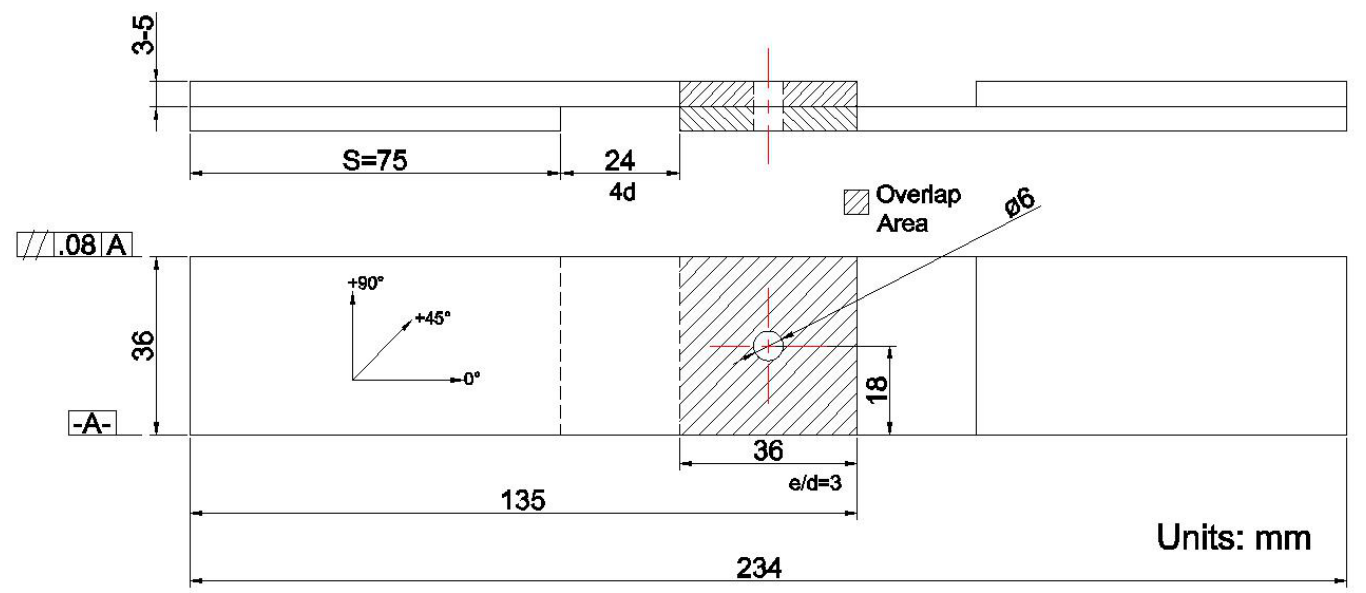

Figure 3.3 Bolted joint specimen from ASTM D5961/D5961M-10. Adapted from [123]

For the final design of the hybrid bolted/bonded coupon, the guidelines from the ASTM standards were taken into account. A few preliminary designs were defined and finally, ratios of $e / d=3$ and $w / d=3$ were chosen. The notch and the total length proportions were selected from the ASTM D3165-07, and the $e / d$ and $w / d$ ratios were chosen from the ASTM D5961/D5961M-10. The final hybrid coupons design was defined as shown in Figure 3.4.
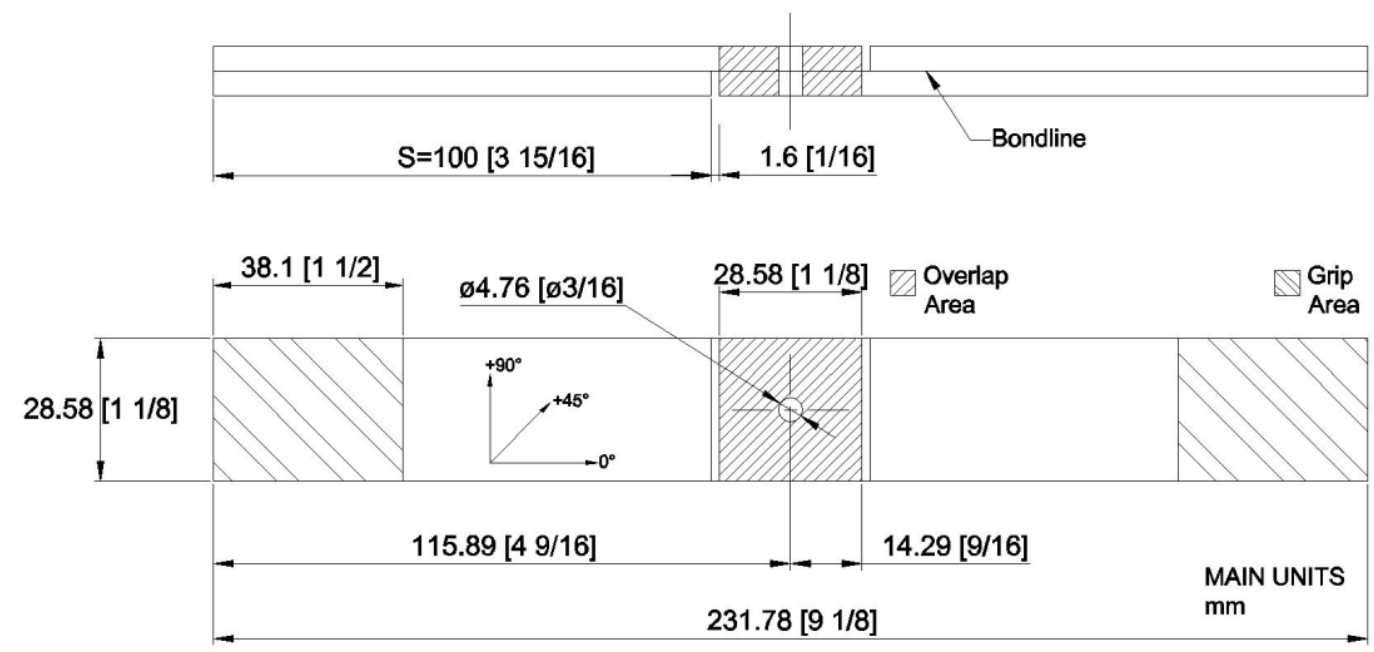

Figure 3.4 Bolted/bonded joint specimen design 


\subsection{Design of the Experiment Using Factorial Designs}

\subsubsection{Selection of the DOE Factors and Response Variables}

The process to select the factors for the DOE was carried out by analyzing the information from the literature review as well as the discussions with the CRIAQ project partners. As determined by the literature review described in Chapter 2, the most significant factors affecting the joint performance, load transfer and strength, are the adherend thickness, adhesive modulus, and adhesive thickness. These parameters have been analyzed in a qualitative sense; however a study to analyze and quantify the effect of these factors on the joint strength has not yet been carried out. The conclusions are given in a general sense, for instance, "increasing the adherend thickness increases the load transferred to the bolt"; or, "bolted/bonded joints are stronger than bonded and bolted joints alone”. Therefore, a DOE along with an ANOVA can be used to quantify the effect of these factors and their relative interactions in the bolted/bonded joint response.

The adherend thickness has a positive impact on the load transferred to the bolt; however, there is no a quantitative analysis to quantify this positive impact. Conversely, increasing the thickness of the adherend would have an adverse effect on the joint weight. therefore, the adherend thickness is a critical factor that needs to be included in the DOE in order to analyze and quantify its effect in the bolted/bonded joint strength and load transfer. Additionally, this analysis must include the effect of the strength-to-weight relationship on the response factors. 
By far, the most widely studied factor on bolted/bonded joints has been the effect of adhesive modulus; and the most important conclusion is that low modulus adhesives allow more load transfer to the bolts as compared to high modulus adhesives [51, 58, 60 , 71]. Additionally, while the effect of modulus has been the studied, the full range of elasto-plastic properties have not been examined in detail. Therefore, the effect the adhesive properties beyond the linear-elastic range need to be studied experimentally.

The effect of the adhesive thickness has been investigated numerically showing a positive effect on the load transfer when the thickness increases [58]. Very few experimental studies have been carried out using different joint thicknesses. However, in these studies, the thickness variation was not properly defined using a DOE [56] or the sample preparation was not accurate enough to get a good bondline thickness control [67]. Therefore, a thickness variation in the adhesive is a factor that needs to be included in the DOE.

The analysis of the bolt and its associated variables in the bolted/bonded joints has not been investigated experimentally. Variables such as material and bolt diameter could be included in the proposed DOE study. However, in aircraft applications, the weight must be minimized. This has led to the widespread use of titanium bolts instead of steel in aircraft structures. Therefore, titanium bolts have been selected for the proposed DOE study.

Regarding the bolt size, in previous experimental studies the bolt diameter has been kept constant; only a single numerical study has shown that increasing the bolt diameter increases the load transfer [60]. In addition to this, using different bolt sizes would have an great impact on the geometrical ratios in the joint design, $e / d$ and $w / d$. 
Therefore, it was decided to keep the bolt diameter in this study as a constant. The same rationale applies to the width and overlap length; these values will remain as constants in this study.

Continuing with the analysis of the variables associated with the bolt, the preload can also be considered as another factor to be analyzed. However, the chosen bolt has a torque locking feature; which limits the installation torque to between 3.95-5.08 N-m for the $4.76 \mathrm{~mm}$ (3/16 in) bolt. This design limits the choice of several torque values to be included in the DOE. Therefore, the torque is also going to be a constant during this DOE.

Notwithstanding this limitation, the clamping area is another possible variable to be explored. According to a previous experimental study [56], this factor has a positive effect on the joint strength. On the other hand, a numerical study [52] claims that clamping force reduces the peel stresses in a region near to the hole, but beyond this region, the peel stresses are unaffected by the clamping force. Therefore, the effect of the clamping area is not conclusive and should be included in the present DOE study.

The impact of the diameter clearance between the bolt and the hole has not been studied experimentally or numerically. However, Kelly [58] pointed out that the clearance affected the experimental results of load transfer using instrumented bolts; therefore, the author's FEM models had to be redefined and corrected for the clearance effect to achieve good agreement. Along with this, the input from the CRIAQ industrial partners suggested that the clearance effect should be studied in more depth. Thus, this variable will be included in the proposed DOE study. The challenge, once this factor is chosen, is the highly accurate manufacturing process need to achieve repeatability and 
precision; this situation could increase the manufacturing cost as well as the required time to manufacture the coupons.

In summary, five factors were selected for this experimental study as summarized in Table 3.2.

The proposed adherend is symmetric and balanced with layup sequences $[0 / 45 / 90 /-45]_{3 \mathrm{~s}}$ and $[0 / 45 / 90 /-45]_{5 \mathrm{~s}}$ for the low and high adherend thickness, respectively. The thicknesses were computed using the number of plies and the ply thickness given by the manufacturer. These proposed layup sequences would give theoretical thicknesses of $3.29 \mathrm{~mm}$ and $5.48 \mathrm{~mm}$ respectively.

The levels for the adhesives modulus or strengths are implicitly defined by the chosen adhesives, in this case, the lower level is for the low modulus adhesive EA9361, and the high level is for the stiffer adhesive FM300-2M.

The adhesive thicknesses were selected using the input from CRIAQ's project partners. Bondline thicknesses larger than $0.5 \mathrm{~mm}$ are unusual in aircraft applications. Using this value as a reference for the high level, the lower level for the adhesive thickness was chosen as $0.2 \mathrm{~mm}$. A challenging part for this factor, is to get a constant and accurate thickness along the entire coupon. Therefore, these levels were carefully chosen to be manufactured with the required precision. A uniform bondline thickness of $0.2 \mathrm{~mm}$ is still very challenging to manufacture.

Standard size washers with two different outer diameters were chosen to obtain different clamping areas. For the low level, a NAS1149-C0363B aerospace-grade washer was selected. The inner diameter is equal to 0.217 in $(5.516 \mathrm{~mm})$, and the outer diameter is equal to $7 / 16$ in $(11.125 \mathrm{~mm})$, giving a clamping area of $0.150 \mathrm{in}^{2}\left(97.2 \mathrm{~mm}^{2}\right)$. For the 
higher level, a standard AN970 washer was chosen. Although this is not an aerospacegrade washer, it was one of the few standard washers availables with larger clamping area for the required inner diameter. This washer has and inner diameter of 0.217 in $(5.516 \mathrm{~mm})$, an outer diameter equal to $7 / 8$ in $(22.225 \mathrm{~mm})$, and a clamping area equal to $0.601 \mathrm{in}^{2}\left(387.9 \mathrm{~mm}^{2}\right)$. Figure 3.5 shows the geometries of the selected washers.
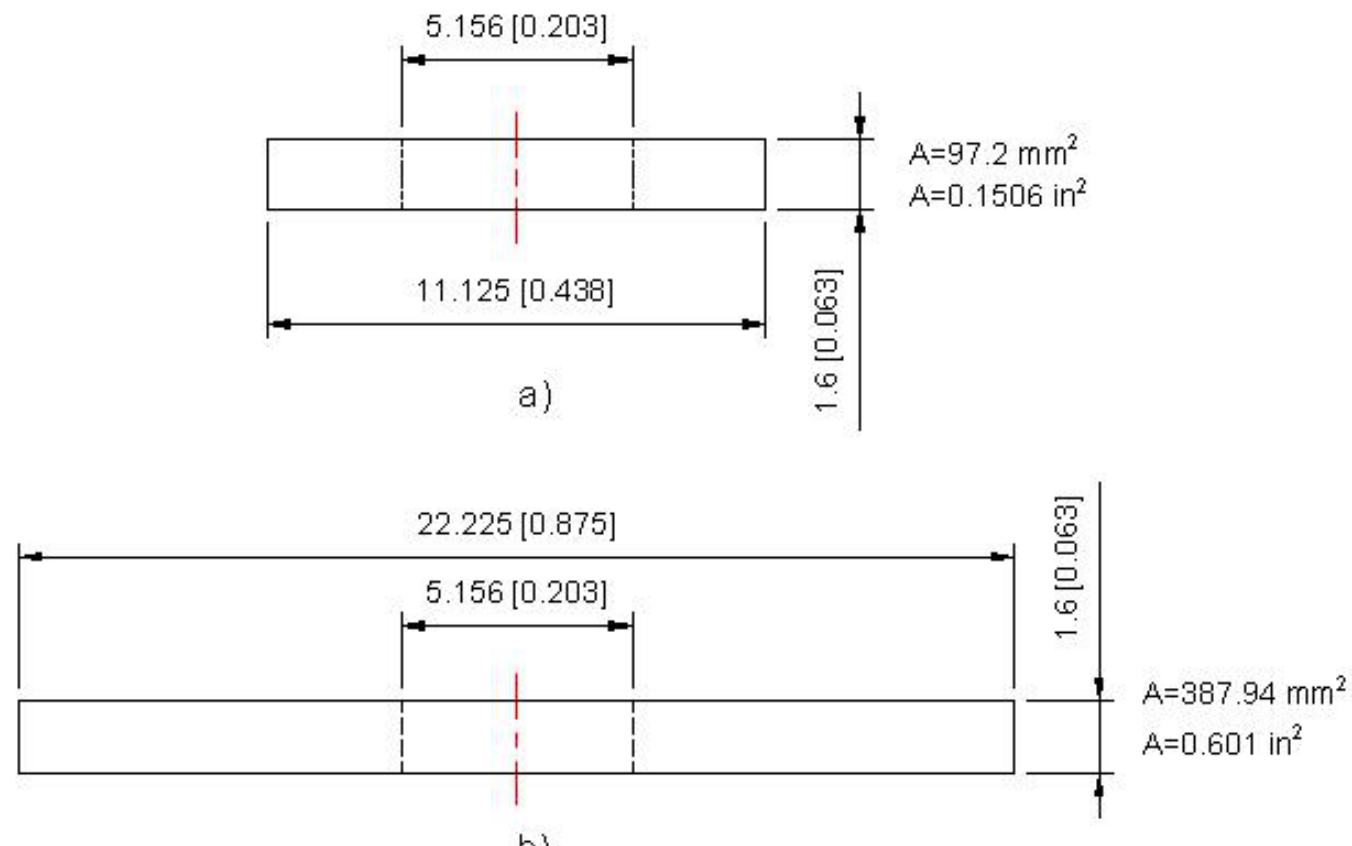

b)

Figure 3.5 Washer dimensions, mm (in). a) NAS1149-C0363B aerospace grade washer; b)

AN970 washer

Finally, the hole clearance has to be defined. The obvious choice for the low level clearance is net fit or zero clearance. On the other hand, the high level values must be chosen carefully. Firstly, the drilling procedures might require several tools and an accurate drilling process; thus, increasing the manufacturing costs. Additionally, the clearance gap should be in a range so that its effect could be detected during the testing before the adhesive failure. Unfortunately, there is no previous experimental study on this 
topic to use as a reference value. The selected clearance value was chosen to be $0.1 \mathrm{~mm}$ (0.004 inch).

The summary of the selected factors and their lower and high levels is shown in Table 3.2 .

Table 3.2 Factors selected for the DOE and their levels

\begin{tabular}{|c|c|c|c|c|}
\hline FACTOR & $\begin{array}{l}\text { Control } \\
\text { variable } \\
\text { (units) }\end{array}$ & $\begin{array}{l}\text { Normal level } \\
\text { \& range }\end{array}$ & $\begin{array}{l}\text { Measured } \\
\text { precision }\end{array}$ & Levels \\
\hline $\begin{array}{l}\text { A } \\
\text { Adherend } \\
\text { Thickness }\end{array}$ & $\begin{array}{l}\text { Number of } \\
\text { plies or mm. }\end{array}$ & $\begin{array}{l}\text { Variable } \\
\text { Depends on } \\
\text { the ply } \\
\text { thickness and } \\
\text { layup }\end{array}$ & $\begin{array}{l}0.05 \mathrm{~mm} \\
\text { Vernier caliper }\end{array}$ & $\begin{array}{l}\text { A1) } 3.29 \mathrm{~mm} \\
\text { (24 plies) } \\
\text { A2) } 5.48 \mathrm{~mm} \\
\text { (40 plies) }\end{array}$ \\
\hline $\begin{array}{l}\text { B } \\
\text { Adhesive } \\
\text { Modulus }\end{array}$ & $\begin{array}{l}\text { Stress-Strain } \\
\text { data }\end{array}$ & Variable & $\begin{array}{l}\text { Adhesive in- } \\
\text { house testing }\end{array}$ & $\begin{array}{l}\text { B1) EA9361 } \\
G=180 \mathrm{MPa} \\
\text { B2) FM-300 } \\
\mathrm{G}=880 \mathrm{MPa}\end{array}$ \\
\hline $\begin{array}{l}\text { C } \\
\text { Adhesive } \\
\text { Thickness }\end{array}$ & $\mathrm{mm}$ & $\begin{array}{l}\text { Variable } \\
\text { From } 0.1 \mathrm{~mm} \\
\text { to } 1.5 \mathrm{~mm}\end{array}$ & $\begin{array}{l}0.0127 \mathrm{~mm} \\
(0.0005 \%) \\
\text { Microscope } \\
\text { measurement }\end{array}$ & $\begin{array}{l}\text { C1) } 0.2 \mathrm{~mm} \\
\left(0.008^{\prime \prime}\right) \\
\text { C2) } 0.5 \mathrm{~mm} \\
(0.020 ”)\end{array}$ \\
\hline $\begin{array}{l}\text { D } \\
\text { Clamped Area } \\
\text { by the Washer }\end{array}$ & $\mathrm{mm}^{2}$ & $\begin{array}{l}\text { Unknown } \\
\text { Depends on } \\
\text { the bolt size } \\
\text { and standard } \\
\text { washer sizes }\end{array}$ & & $\begin{array}{l}\text { D1) } 97.2 \mathrm{~mm}^{2} \\
\text { D2) } 387.9 \mathrm{~mm}^{2}\end{array}$ \\
\hline $\begin{array}{l}\mathbf{E} \\
\text { Hole clearance }\end{array}$ & $\mathrm{mm}$ & $\begin{array}{l}\text { a) Neat fit } \\
\text { b) Unknown }\end{array}$ & Micrometer & $\begin{array}{l}\text { E1) Net fit } \\
\text { E2) } 0.1 \mathrm{~mm} \\
(0.004 \mathrm{in})\end{array}$ \\
\hline
\end{tabular}

Once the factors for the DOE have been selected, the next step is to define the response variables and the methods to measure them. From the literature review, the 
typical response values are the load force and stroke displacement. More information can be extracted from the tested coupons. However, more instrumentation would be needed. For instance, strain gauges, extensometers or digital image correlation equipment. Additionally, the choice of the response values depends on the availability of experimental data-acquisition systems to capture the joint response data synchronized with the load versus stroke signals.

The first choice is the load and stroke displacements at maximum strength. Moreover, if some interesting points on load-stroke are detected after the analysis, these points can be defined as new response factors. For instance, from literature review, it has been observed that some joints have a point where there is sudden drop in the strength, however, after this failure point, the adhesive is still capable of supporting load with the bolt until the maximum joint strength is reached. Therefore, this second strength point is a response of interest to explore. Lastly, after the complete failure in the adhesive, the final load is taken by the bolt until ultimate failure; this last load taken by the bolt can also be analyzed as a response of interest.

The joint stiffness can be assessed by measuring local displacements right outside the overlap length. This measurement can be carried out using extensometers or optical devices. Therefore, this local displacement could be another response factor.

The ultimate goal is to assess the load sharing between the adhesive and the bolt. The instrumented bolt technique is not viable for this research for several reasons. Firstly, this experimental study is designed to interrogate the performance of the bolted/bonded joints until total failure, including failure in the bolt. Therefore, adding instrumented bolts on each single test in not economically or technically viable. Second, the small size of the 
bolt makes it extremely difficult to install the strain gauges on it. Finally, it will not be possible to tighten the bolt because previous studies have been done using hand tight [58] or a very low torque value for a $9.5 \mathrm{~mm}$ bolt [51]. The main reason is to avoid damaging the instrumentation inside the bolt. These limitations suggest that this technique cannot be used in this study. Therefore, an alternate method has to be used or developed if the load sharing needs to be assessed. Otherwise, the only available information would be load stroke.

The proposed method, as described in section 2.8, is to use digital image correlation to assess the adhesive strain behaviour instead the bolt shear strain. Since this method is suitable to measure displacements and strains, it can be used to assess both, the local displacement outside the overlap length as well as the strains in the bondline. In addition to this, numerical analysis could be carried out to compare against the experimental results, to complement the load sharing study.

On the other hand, by choosing this technique, the required time to carry out the experimental study will be increased significantly. This will require a more sophisticated experimental setup and, therefore, more time to post process and analyze the data.

In this study, data will be extracted from the aforementioned techniques: loadstroke, strain gauge measurements and digital image correlation.

The final choice of response values is summarized in Table 3.3. Additionally; this table includes the proposed way to measure these values and, finally, the relationship of these response factors on the aim of the study. 
Table 3.3 Proposed response variables

\begin{tabular}{|l|l|l|l|}
\hline $\begin{array}{l}\text { Response } \\
\text { variable } \\
\text { (units) }\end{array}$ & $\begin{array}{l}\text { Normal } \\
\text { operating level } \\
\text { \& range }\end{array}$ & $\begin{array}{l}\text { Meas. precision, } \\
\text { accuracy } \\
\text { How known? }\end{array}$ & $\begin{array}{l}\text { Relationship of } \\
\text { response variable to } \\
\text { objective }\end{array}$ \\
\hline $\begin{array}{l}\text { Maximum load } \\
\text { before one joint } \\
\text { element fails } \\
\text { a) Adhesive } \\
\text { b) Bolt }\end{array}$ & Unknown & MTS Load Stroke & $\begin{array}{l}\text { Assess the effect of the } \\
\text { factors on the joint } \\
\text { strength }\end{array}$ \\
\hline $\begin{array}{l}\text { Maximum load at } \\
\text { total failure }\end{array}$ & Unknown & MTS Load Stroke & $\begin{array}{l}\text { Assess the effect of the } \\
\text { factors on the joint } \\
\text { strength }\end{array}$ \\
\hline Stiffness & $\begin{array}{l}\text { Unknown } \\
\text { Can be predicted } \\
\text { numerically }\end{array}$ & $\begin{array}{l}\text { Local displacement } \\
\text { measurement } \\
\text { Extensometer and/or } \\
\text { DIC measurements }\end{array}$ & $\begin{array}{l}\text { Assess the effect of the } \\
\text { factors on the joint } \\
\text { strength. } \\
\text { Infer the load } \\
\text { transferred to the bolt } \\
\text { comparing the results } \\
\text { against FEM }\end{array}$ \\
\hline Load transfer to bolt & $\begin{array}{l}\text { Unknown } \\
\text { Can be predicted } \\
\text { numerically }\end{array}$ & $\begin{array}{l}\text { Infer the value by } \\
\text { measuring the shear } \\
\text { strains at the } \\
\text { adhesive edge using } \\
\text { DIC. Comparing } \\
\text { these results against } \\
\text { FEM analysis }\end{array}$ & $\begin{array}{l}\text { Infer the load } \\
\text { transferred to the bolt }\end{array}$ \\
\hline
\end{tabular}

The factors that will remain in one single level or unchanged are given in Table 3.4. These values, in DOE terminology, are referred as held constant factors. 
Table 3.4 "Held Constant" Factors in the DOE

\begin{tabular}{|l|l|}
\hline \multicolumn{1}{|c|}{ Factor (units) } & \multicolumn{1}{c|}{ Desired experimental level } \\
\hline Joint Design & Single Lap \\
\hline Composite Material & $\begin{array}{l}\text { Cytec Cycom } 5320 \text { unidirectional } \\
\text { prepreg. Out of autoclave }\end{array}$ \\
\hline Bolt Size $(\mathrm{mm})$ & $3 / 16$ in $(4.76 \mathrm{~mm})$ \\
\hline Bolt Type & Hi-Lite $^{\circledR}$ \\
\hline $\begin{array}{l}\text { Bolt Configuration } \\
(\text { Pitch Distance } \mathrm{mm})\end{array}$ & 1 Bolt \\
\hline Bolt Material & Titanium 6 Al-4V \\
\hline Tightening $($ Preload $)$ & $2.82 \mathrm{~N}-\mathrm{m}(25 \mathrm{lb}-\mathrm{in})$ \\
\hline Lap Length $(\mathrm{mm})$ & Constant. $28.5 \mathrm{~mm}$ \\
\hline Lap Width $(\mathrm{mm})$ & Constant. $28.5 \mathrm{~mm}$ \\
\hline Temperature $\left({ }^{\circ} \mathrm{C}\right)$ & Room temperature \\
\hline
\end{tabular}

Finally, in some cases during the experimentation, some sources of noise or nuisance factors could produce slightly different results. For this DOE, these nuisance factors should be taken into account, particularly with composite materials where the plates are manufactured separately in different curing batches. Another possible source of nuisance could be during the testing process. For instance, temperature changes, use of different testing tools and accessories, and changing the test parameters. To minimize this effect, all the manufacturing and testing parameters have to be properly documented. 


\subsubsection{Design of Experiments Matrix}

Once the DOE factors were selected, the next step is to define the factorial design. The first point to be defined is the number of replicates per treatment; this is necessary to assess the capability to carry out the experiment with the available resources. The ASTM D3165-07 [122] standard for bonded joints requires at least 20 specimens to be tested, representing four different joints. That means that five replicates per joint configuration have to be tested. Similarly, the ASTM D5961/D5961M-10 [123] requires testing "at least five specimens per test condition unless valid results can be gained through the use of fewer specimens, as in the case of designed experiment."

The foregoing indicates that at least five specimens per condition are required. However, as pointed out on the ASTM D5961/D5961M-10 Standard, we could define fewer repetitions if the repeatability is good enough. Therefore, the initial DOE is defined to be carried out using three repetitions per joint combination.

With this information, the next step is to choose a suitable DOE to obtain enough information from the DOE using the least number of tests. Firstly, a full factorial using five factors requires 32 joint combinations and 96 tests. This number of coupons is definitely outside of the capability of this research. However, there are still two options to carry out the same experiment using fractions; the half fraction design will require 16 joint configurations and 48 tests.

The last option is to run the one quarter fraction $\left(2^{5-2}\right)$ which requires eight joint combinations with a total of 24 coupons. This design is a resolution III where some high order interactions are confounded. However, this option can be chosen and run at the beginning. If the results are not conclusive, there is an option to "defold" the design, 
which means that the second one-quarter design can be tested and integrated to the previous one-quarter design to improve the results.

Due to this, the factorial design $2^{5-2}$ resolution III has been defined for this DOE. The number of treatments, coded names, and levels are summarized in Table 3.5. A,B,C,D,E are the coded names for the factors, and the labels -1 and 1 indicate the lower and upper levels of the factors respectively. Finally, the treatment label is, according to the DOE terminology, the standard way to refer to the treatment or test combination. These coded names will be referred during the experiment procedures as well as the results.

Table 3.5 Factorial design for the DOE

\begin{tabular}{|c|c|c|c|c|c|c|c|c|c|c|}
\hline & $\begin{array}{l}\text { Std. } \\
\text { Order }\end{array}$ & Block & $\mathbf{A}$ & B & C & D & $\mathbf{E}$ & $\begin{array}{l}\text { Treatment } \\
\text { Combination }\end{array}$ & GENERATORS & \\
\hline \multirow{8}{*}{4} & 1 & 1 & -1 & -1 & -1 & 1 & 1 & de & \multirow{8}{*}{$\begin{array}{l}D=A B \\
E=A C\end{array}$} & \multirow{8}{*}{$\begin{array}{ll}\text { R } & \\
\text { E } & \\
\text { S } & \\
\text { O } & \\
\text { L } & \text { I } \\
\text { U } & \text { I } \\
\text { T } & \text { I } \\
\text { I } & \\
\text { O } & \\
\text { N } & \end{array}$} \\
\hline & 2 & 1 & 1 & -1 & -1 & -1 & -1 & $a$ & & \\
\hline & 3 & 1 & -1 & 1 & -1 & -1 & 1 & be & & \\
\hline & 4 & 1 & 1 & 1 & -1 & 1 & -1 & $a b d$ & & \\
\hline & 5 & 1 & -1 & -1 & 1 & 1 & -1 & $\mathrm{~cd}$ & & \\
\hline & 6 & 1 & 1 & -1 & 1 & -1 & 1 & ace & & \\
\hline & 7 & 1 & -1 & 1 & 1 & -1 & -1 & $b c$ & & \\
\hline & 8 & 1 & 1 & 1 & 1 & 1 & 1 & abcde & & \\
\hline
\end{tabular}

\subsubsection{Associated Risk of Using DOE for the Experimental Study}

From the literature review in section 2.7, it has been highlighted the advantage of using DOE methodology for this research: the analysis of several factors at the same time and to quantify their effect and interactions on the response variables.

However, there are negative scenarios that could be possible during this process: 
- The DOE cannot be finished as planned. In this case, the ANOVA cannot be carried out and the final objective to quantify the effects of the several factors on the selected responses would not be possible. According to the DOE methodology, at least one test for each treatment is required;

- The results from the ANOVA are not conclusive. This situation could be due to several reasons; for instance, poor DOE planning or a poor experimental execution. This could lead to inaccurate test data on some tests and the ANOVA could not be carried out.

Both scenarios should be anticipated to avoid the loss of data and the effort to carry out the experimental study.

Therefore, if some of the scenarios above described would be present in this experimental program, the way to analyze the data should be comparing and contrasting the individual results from each combination. In addition to this, every combination can be used to be compared against FEM models individually to assess the load transferred and strength.

\subsection{Manufacturing of Bolted/Bonded Joints}

Manufacturing accurate coupons is an essential part of the experimental study to ensure repeatability and accuracy with the response variables. The proper manufacturing process begins by controlling the quality of the manufactured composite plates following the manufacturer's specifications. These carbon fibre composite plates, as discussed previously, were made using and out of autoclave material, Cytec Cycom 5320 prepreg. 
The technical specifications suggest that better quality and low porosity can be achieved using a vacuum bagging procedure.

The prepreg roll used had a width of $0.61 \mathrm{~m}$ (24 in). Plates of $280 \mathrm{~mm}$ x $280 \mathrm{~mm}$ (11 $x 11$ inch) were manufactured to maximize the use of the material by minimizing the scrap due to the cuts at $45^{\circ}$ degrees. Using this plate size ensured a maximum use without compromising the final size of the coupons.

Several plates were manufactured following the bagging and curing procedure recommended by the manufacturer shown in Figure 3.6. According to the manufacturer specifications, debulking is only necessary to eliminate wrinkles. Therefore, to avoid them, the layup was carried out adding consecutive layers and debulking for 5 minutes after each layer was added. Once the plates were ready to be cured, they were covered with aluminum caul plates on the top to ensure flatness and thickness. In the top surface, a teflon layer and breather cloth were added. Thermocouples were installed in several locations on the aluminum tool plate as well as the composite laminates to ensure a better temperature control during the curing process. Figure 3.6 shows the process. 


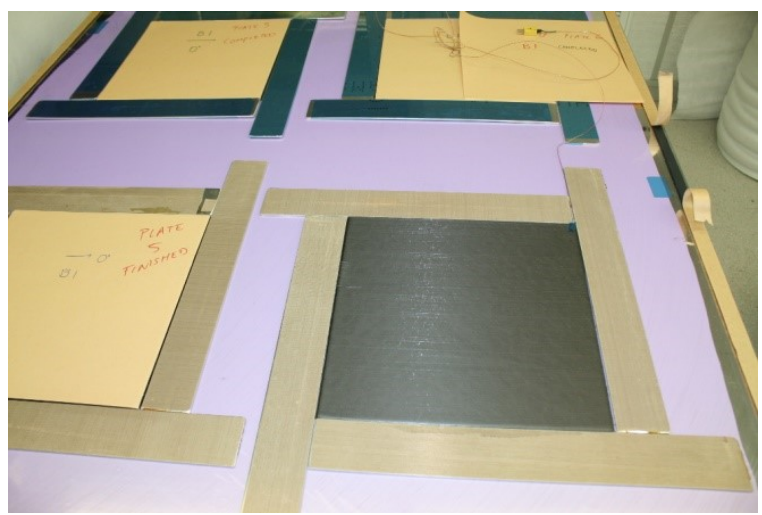

a)

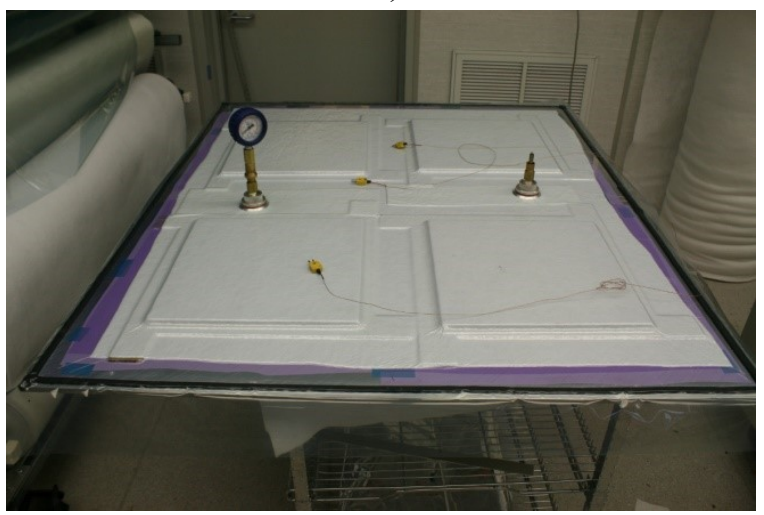

c)

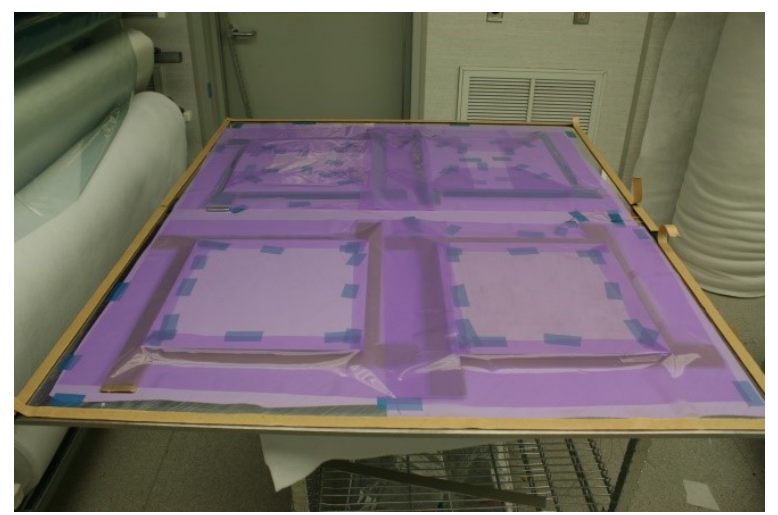

b)

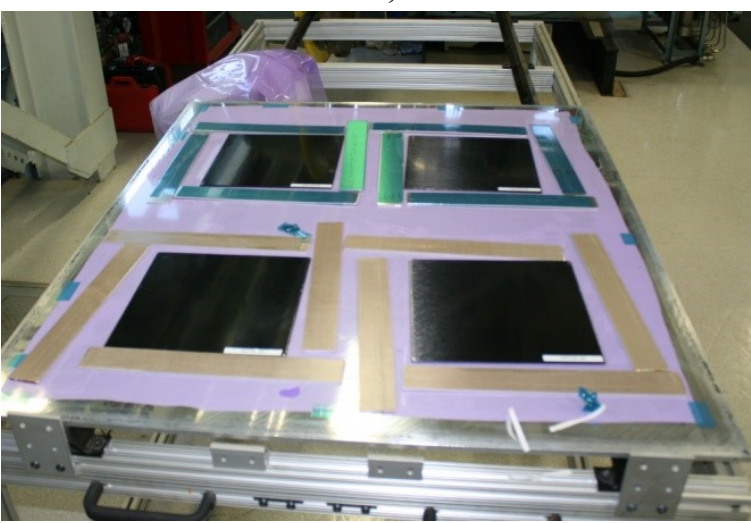

d)

Figure 3.6 Bagging process. a) Prepreg and edge dams; b) Caul plates and teflon layer at the top of the laminates; c) Final bag showing the breather and thermocouples; d) Laminates after curing

There were two options to carry out the curing cycle, twelve hours at $93^{\circ} \mathrm{C}$ or 3 hours at $121^{\circ} \mathrm{C}$ as shown in Table 3.6. Due to timing requirements, the cure cycle $\mathrm{B}, 3$ hours at $121^{\circ} \mathrm{C}$ was selected. 
Table 3.6 Cytec Cycom 5230 recommended curing cycles [120]

\begin{tabular}{|l|l|l|}
\hline Ramp Rate & Cure Cycle A & Cure Cycle B \\
\hline Cure Temperature & $\begin{array}{c}1-3^{\circ} \mathrm{F} / \text { minute } \\
\left(0.6-1.7^{\circ} \mathrm{C} / \text { minute }\right)\end{array}$ & $\begin{array}{c}1-5^{\circ} \mathrm{F} / \text { minute } \\
\left(0.6-2.8^{\circ} \mathrm{C} / \text { minute }\right)\end{array}$ \\
\hline Cure Time & $200 \pm 10^{\circ} \mathrm{F}\left(93 \pm 6^{\circ} \mathrm{C}\right)$ & $250 \pm 10^{\circ} \mathrm{F}\left(121 \pm 6^{\circ} \mathrm{C}\right)$ \\
\hline Free-Standing Post-Cure & 2 hours & 3 hours \\
\hline
\end{tabular}

The curing was carried out using a temperature ramp of $2.8^{\circ} \mathrm{C}$ per minute until $121^{\circ} \mathrm{C}$, holding that temperature for three hours and cooling down at $2.8^{\circ} \mathrm{C}$ per minute until room temperature was reached as shown in Figure 3.7. Finally, the plates were post cured at $177^{\circ} \mathrm{C}$ for two hours.

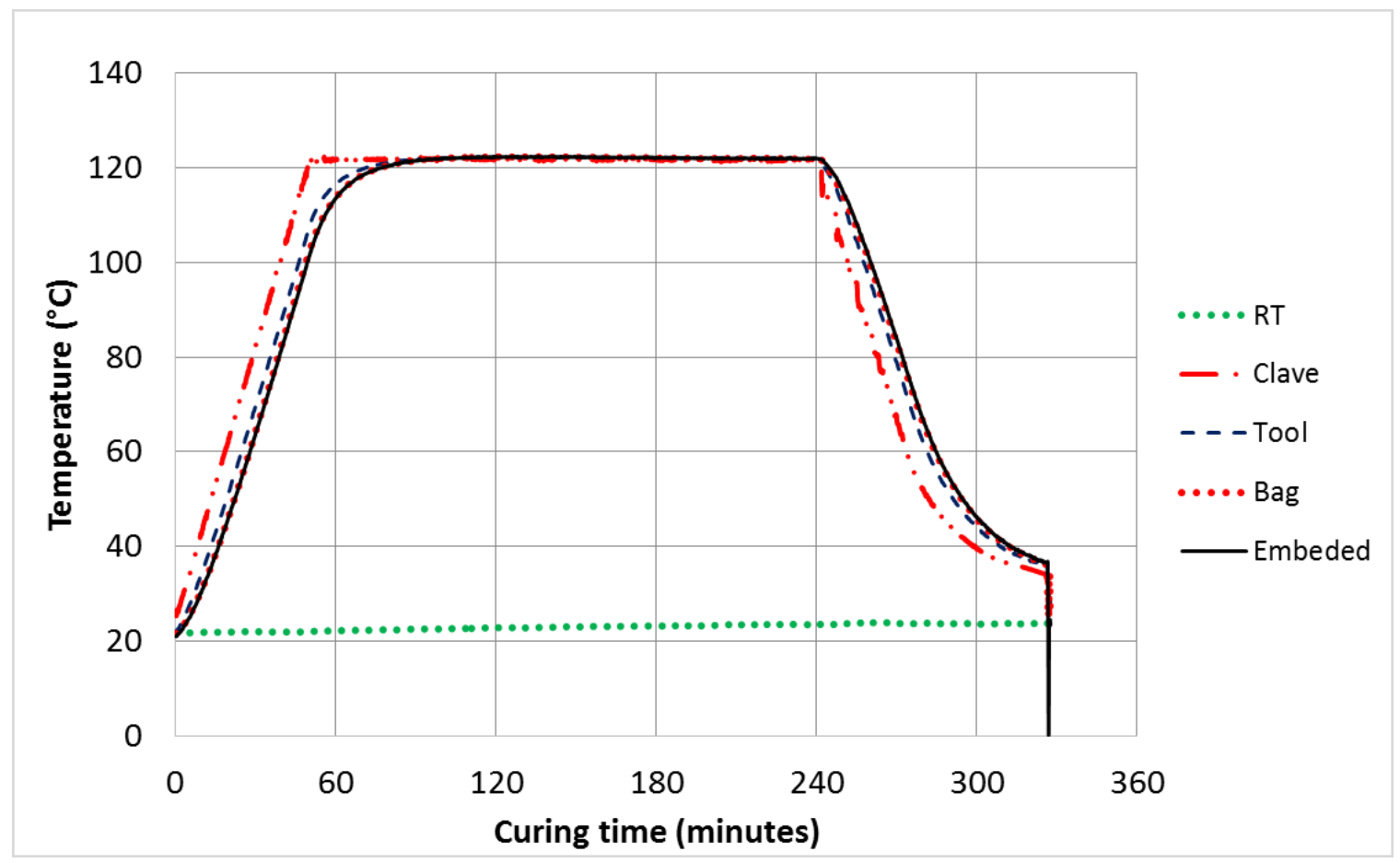

Figure 3.7 Curing process Cytec Cycom 5320 unidirectional prepreg laminates 
The next procedure was to cut these plates into halves to make the bonded joints folding the two halves and joining them. Following this procedure, each plate was used to get a full set of coupons (three) for each required combination. The cutting was carried out using a diamond blade.

The following processes were critical, complex and time consuming in order to achieve the desired coupon specifications: joining, drilling and notching.

The bonding process required a very accurate bondline thickness control to obtain the $0.2 \mathrm{~mm}$ and $0.5 \mathrm{~mm}$ uniform bondline thicknesses. Firstly, the plates were cleaned using methyl ethyl ketone (MEK) to eliminate any trace of dirt. After this, the surfaces were grit blasted and cleaned with nitrogen. Once the surfaces were activated with the grit blasting procedure, piano wires were installed to control the bondline thickness on the plates to be bonded. The first sets of plates were joined installing the piano wires following the longitudinal direction of the plates as shown in Figure 3.8. This procedure was effective for the film FM300-2M adhesive getting good bondline thickness along the plate. Edge dams were incorporated to minimize bondline thinning at the edges of the bonded plates. Four sets of bonded plates with FM300-2M adhesive were cured at $121^{\circ} \mathrm{C}$ for two hours with a pressure of $0.55 \mathrm{MPa}$ as shown in Figure 3.9. 


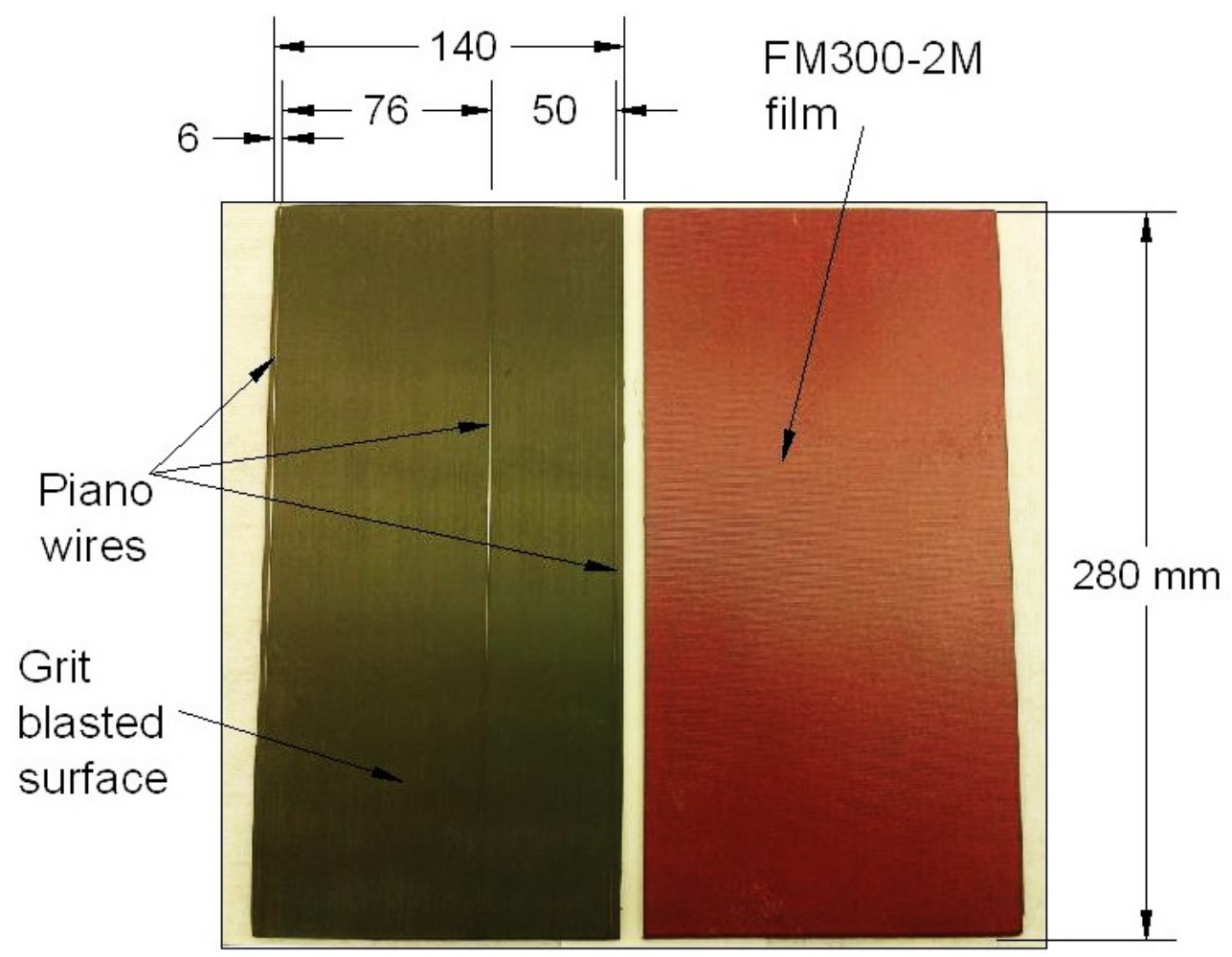

Figure 3.8 Piano wires installation for the bonding procedure, units $\mathrm{mm}$.

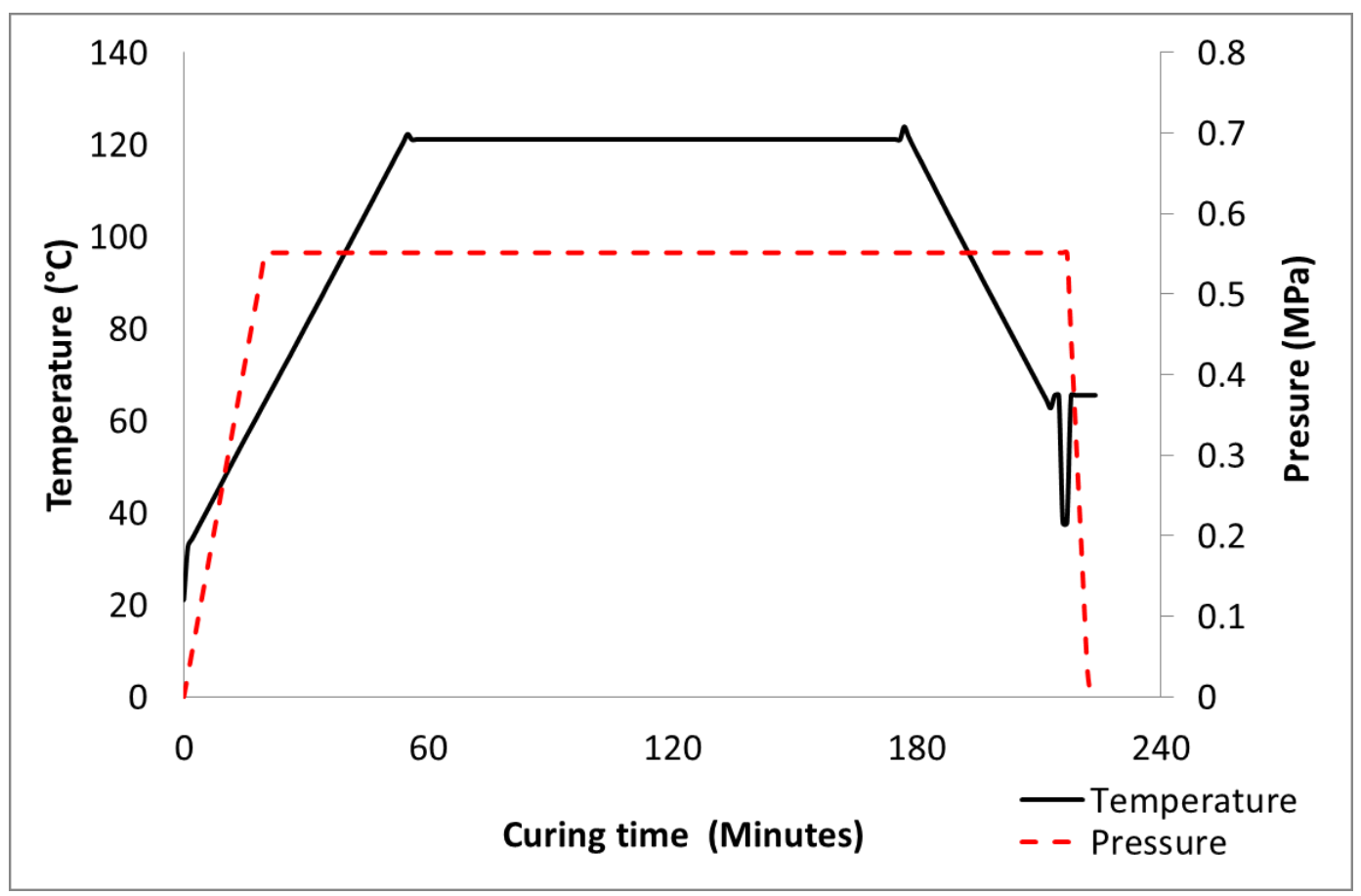

Figure 3.9 Curing process for FM300-2M adhesive 
The same geometrical configuration of the piano wires was used to bond the plates with EA9361 paste adhesive (Figure 3.10). The mixing was carried out using a Thinky ${ }^{\circledR}$ mixer for 3 minutes at 2000 RPM in mixing mode followed by 2 minutes at 2000 RPM in deformation mode. Similarly to the joint with FM300-2M adhesive, edge dams were incorporated at the edges of the bonded plates. The chosen procedure to apply pressure to the bonded plates was vacuum bagging. Initially, a full vacuum pressure of $0.095 \mathrm{MPa}(28 \mathrm{in} \mathrm{Hg}$ ) was applied. However, it was found, even with the edge dams, that the bondline thickness was non-uniform and very thick, it was observed that approximately $1.5 \mathrm{~mm}$ accumulated near the center of the plate.

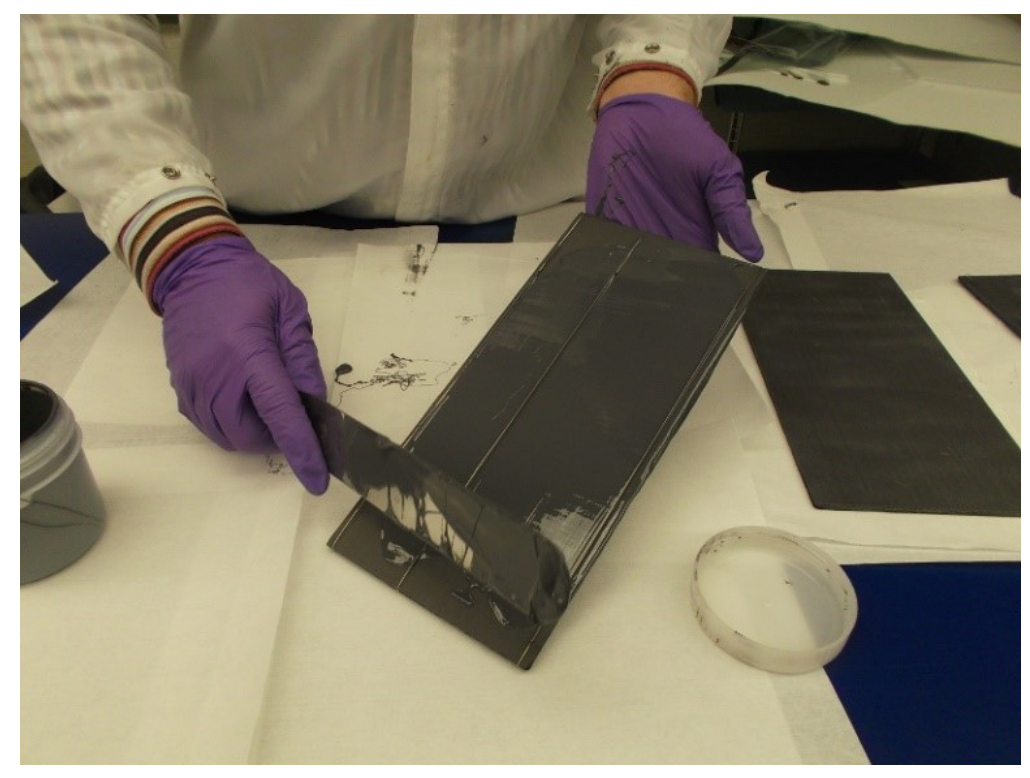

Figure 3.10 EA936 paste adhesive application on plates with piano wires

Due to this, several trials with different bonding conditions were conducted to achieve the desired thickness results to solve this issue. These attempts were carried out by adding a thick aluminum plate on the top to increase the stiffness and changing the 
piano wires for thickness gauges. However, it was found that the flow in the longitudinal direction was still insufficient to get uniform adhesive thickness on the bonded plates. The position of the wires was changed, locating them transversely instead longitudinally to promote the flow towards the shorter path of the plate as shown in Figure 3.11. This new configuration was carefully defined to keep enough distance between the central wires to get enough bondline length. This was necessary to avoid keeping part of the piano wires inside the desired bondline. Slots were incorporated in the edge dams to allow a free flow between the edge dams and the tool plate in the transverse direction.

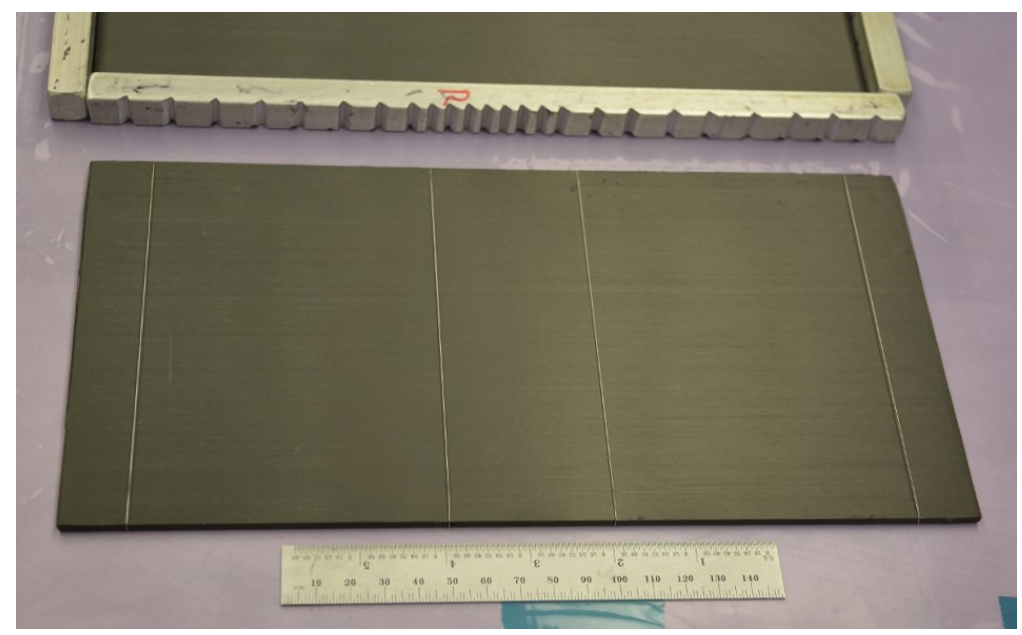

Figure 3.11 Transverse location of piano wires to bond EA9361 paste adhesive. The slotted edge dams are shown.

After several attempts, it was found that using full vacuum pressure, the thickness variation was significant and the centre of the plates after bonding were still bulged. A possible explanation could be that the pressure was high enough to bend the top plate outwards from the edges, producing a non-uniform bond layer, thinner near the edges and thicker at the centre. Another set of trials were conducted by reducing the pressure 
gradually. It was found that the best vacuum pressure to get very accurate bondline thicknesses was between $0.040-0.044 \mathrm{MPa}(12-13 \mathrm{in} \mathrm{Hg})$. With this pressure, the variation in the bondline thickness was $\pm 0.012 \mathrm{~mm}(0.0005$ in). The thickness was measured using a Gaertner traveling microscope with a resolution of 0.0002 in $(0.005$ $\mathrm{mm}$ ) as shown in Figure 3.12. The results are shown in Appendix E.

Once the plates were joined, the following step was to cut them into strips using a saw with a diamond tipped blade. The process was carried assuring that at least $1 \mathrm{~cm}$ from the exterior edges from the manufactured composite plates was discarded from the plate. For the bonded joints with the piano wires located longitudinally, up to three coupons were obtained as shown in Figure 3.13. For the plates with transversely located piano wires, four coupons were obtained (Figure 3.14). This last cutting procedure was carried out carefully to avoid having piano wires in the bondline.

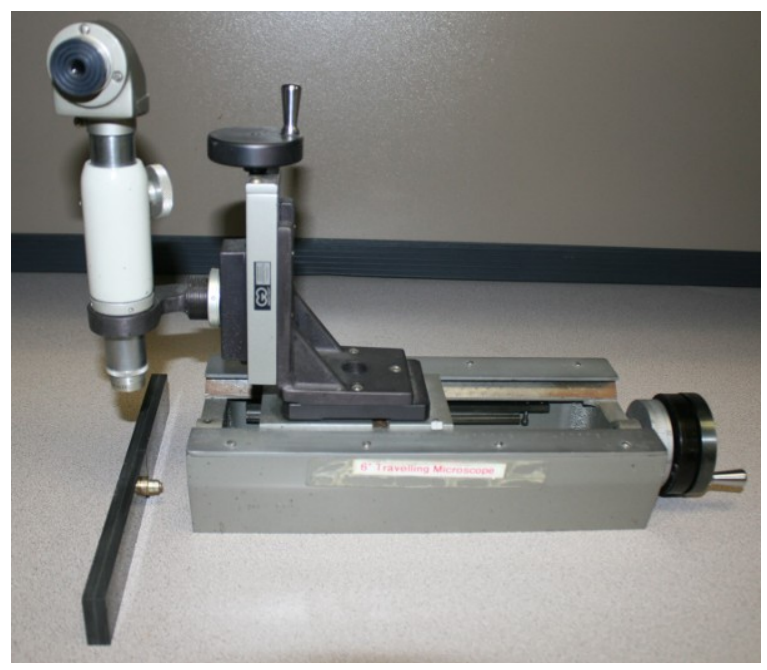

Figure 3.12 Gaertner traveling microscope used to measure the adhesive thickness 


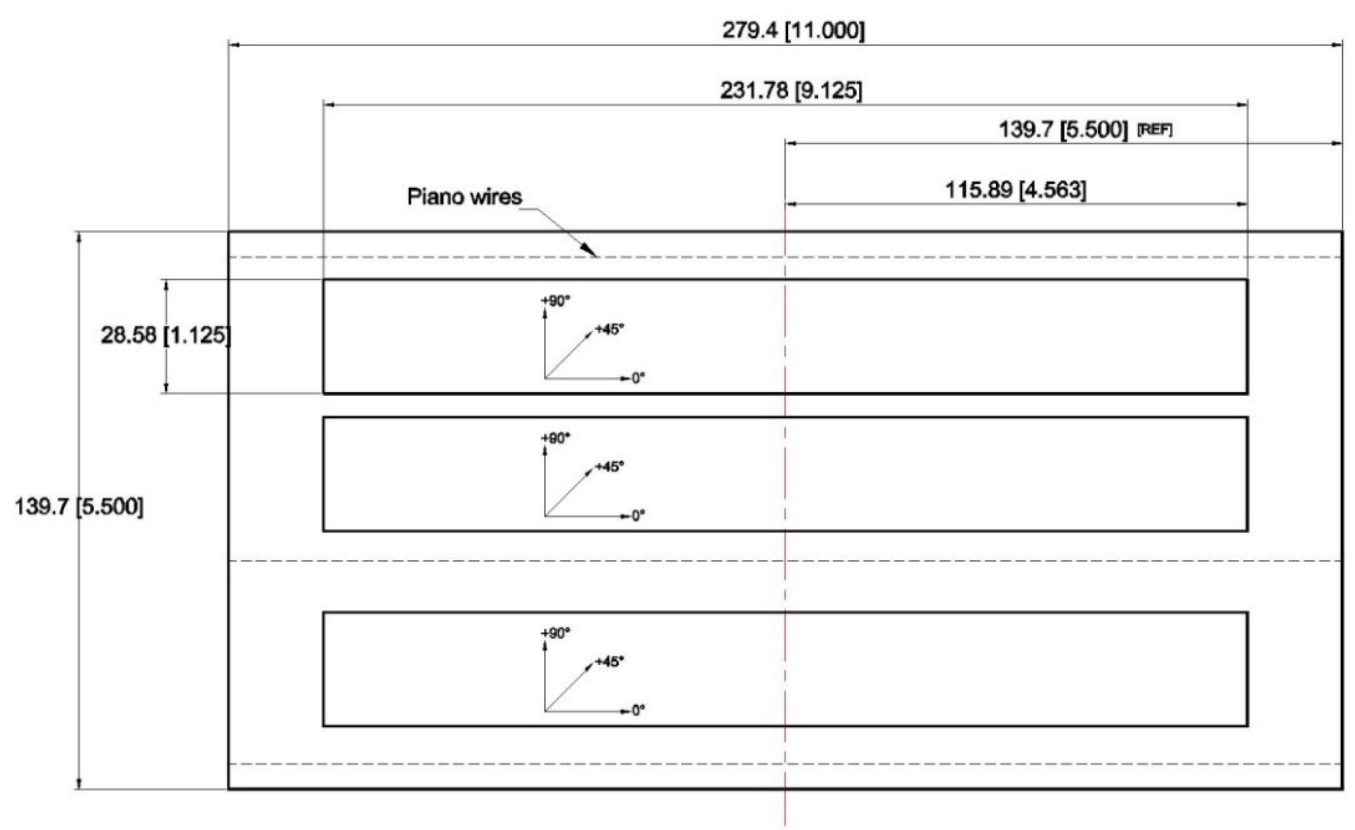

Figure 3.13 Cutting specifications for plates with longitudinally located piano wires (dimensions in $\mathrm{mm}[\mathrm{in}])$.

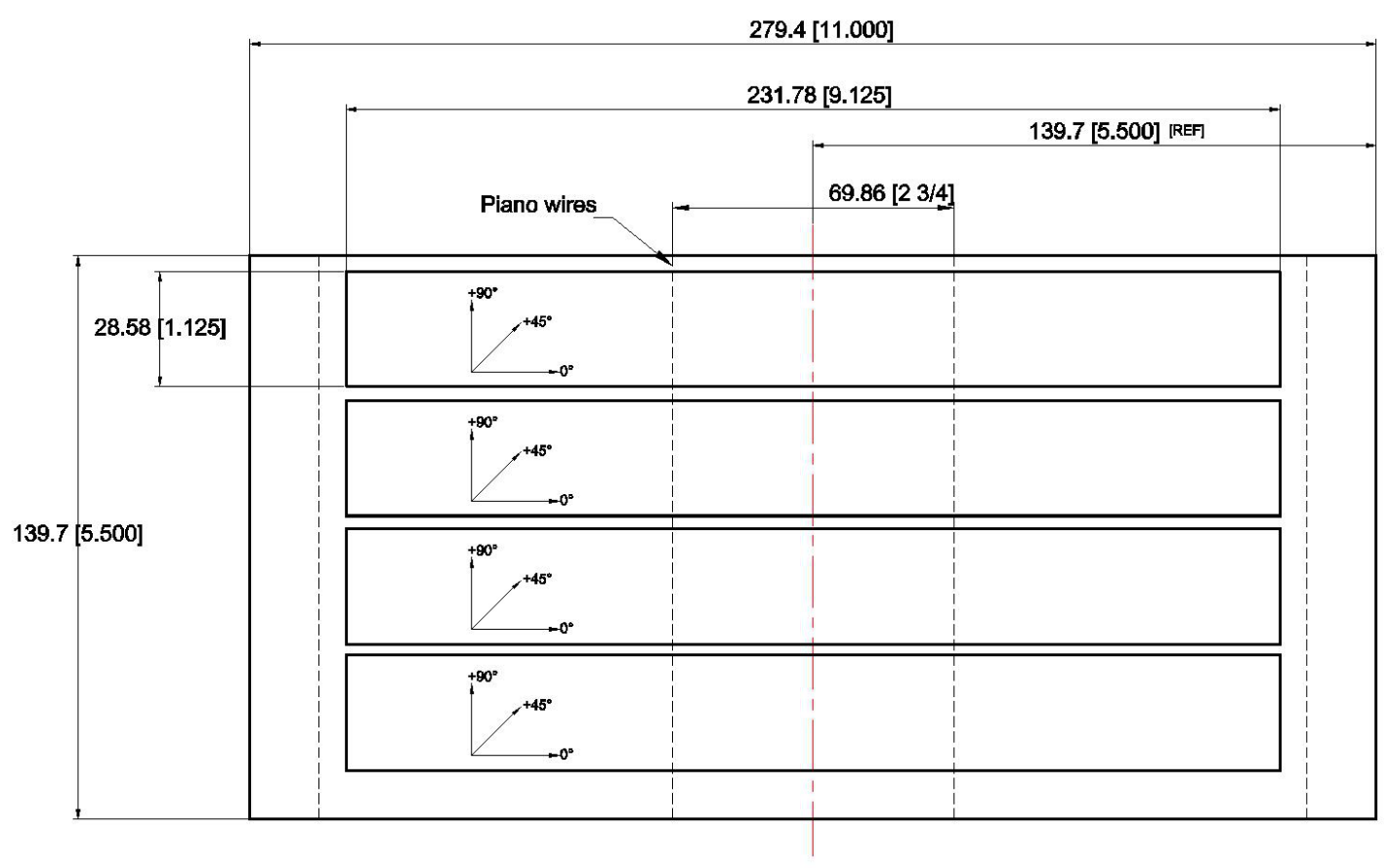

Figure 3.14 Cutting specifications for plates with transversally located piano wires 
It was decided to drill the holes before the notching process to avoid twisting or damaging the joint in the bondline area due to the drilling torque force. The drilling was carried out at an external machine shop. The first drilling step was carried out using a $135^{\circ}$ split point drill, the second step was drilling at 800 RPM using a $\# 15$ drill bit. Finally, the holes were reamed at 550 RPM using a set of solid carbide reamers with $0.1890 \mathrm{in}(4.80 \mathrm{~mm})$ and $0.193 \mathrm{in}(4.902 \mathrm{~mm})$ diameters, respectively. All the holes were measured using an inside diameter micrometer with a resolution of 0.0005 in $(0.012 \mathrm{~mm})$ at eight positions throughout the hole as shown in Figure 3.15. The measured results were very accurate, matching the reamer diameters, $4.8 \mathrm{~mm}(0.1890 \mathrm{in})$, and $4.902 \mathrm{~mm}(0.193$ in). Delamination was not detected after the drilling, just small scratches at the hole edge as shown in Figure 3.16

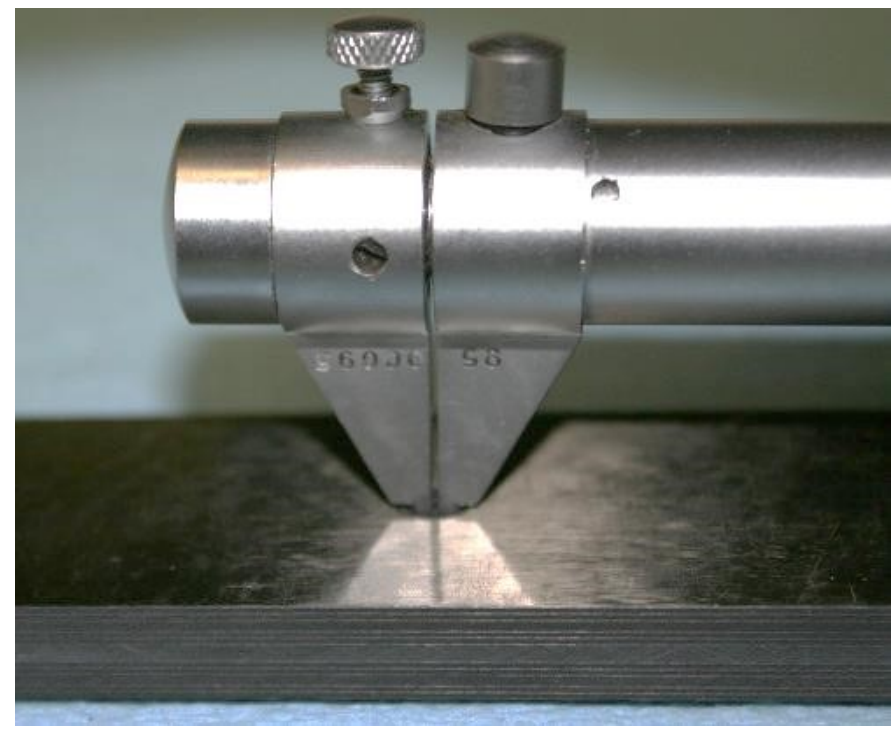

Figure 3.15 Hole diameter measurement 


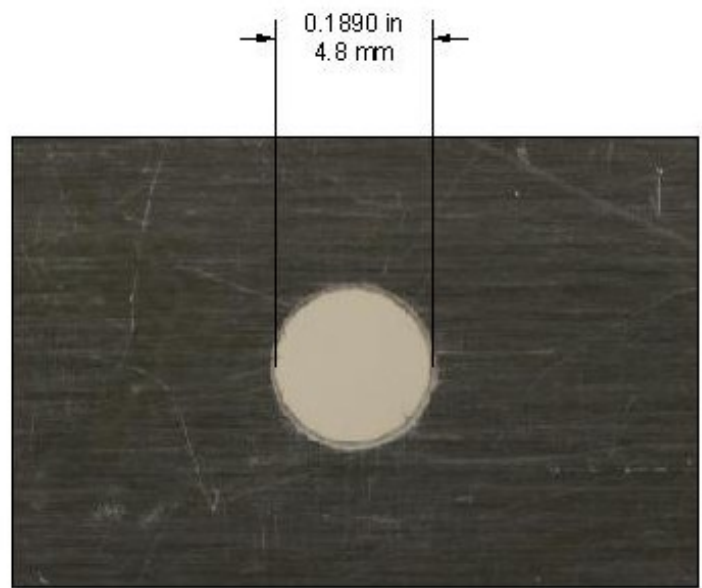

a

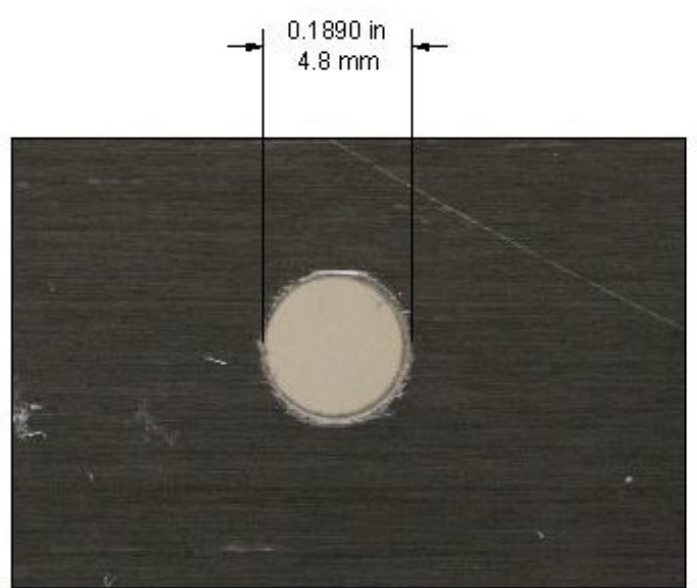

b

Figure 3.16 Bolt hole: a) surface at tool entrance; b) surface at tool exit

Two batches of twenty four bolts, each with grip lengths of $3 / 8$ in $(4.76 \mathrm{~mm})$ and $5 / 8$ in $(15.87 \mathrm{~mm})$ respectively were used for the experimental study. To ensure the best matching between the bolts and the holes to get the closest neat fit and clearance respectively, all the bolts were measured along the shank at six different positions and labeled to be used with a specific coupon. The average diameters were $0.18885 \pm$ 0.00026 in $(4.796 \pm 0.0066 \mathrm{~mm})$ for the bolts with $3 / 8$ in $(4.76 \mathrm{~mm})$ of grip length. For the bolts with grip length of 5/8 in $(15.87 \mathrm{~mm})$, the average bolt diameter was $0.1889 \pm$ 0.00018 in $(4.798 \pm 0.0045 \mathrm{~mm})$.

The notching was carried out using a diamond blade with a thickness of $1.7 \mathrm{~mm}$ (0.067 inch). This process was executed with great care to avoid touching the opposite adherend surface. Usually, the upper adherend was removed followed by the removal of the adhesive layer. An inspection was carried out using a traveling microscope to assure that the underneath surface was not damaged as well as to ensure the adhesive layer was removed as shown in Figure 3.17 

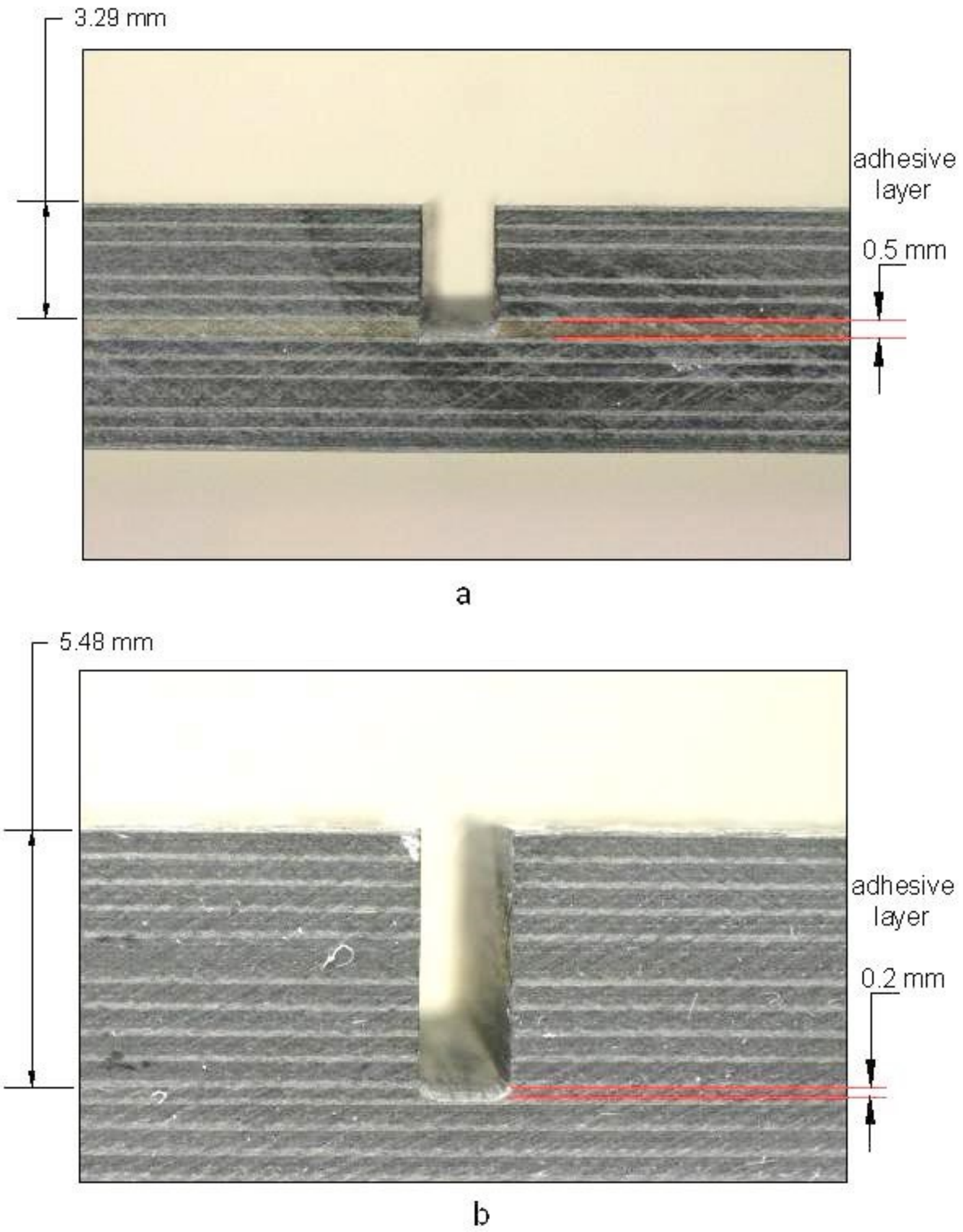

Figure 3.17 Notch details indicating the cut for two adhesive thicknesses; a) $0.5 \mathrm{~mm}$; b) 0.2 $\mathrm{mm}$. 


\subsection{Experimental Setup}

The specimens were tested using a servo-hydraulic MTS tensile testing machine model 318.10 with $100 \mathrm{kN}$ capacity. All the tests were carried out using displacement control at $1.27 \mathrm{~mm} / \mathrm{min}$. Due to the variety of treatments and thicknesses, two set of grips were required to clamp the specimens. Initially, the grip pressure was tested to avoid damage in the grip zone. A grip pressure of approximately $8.96 \mathrm{MPa}(1300 \mathrm{Psi})$ was sufficient to achieve a proper clamping force without apparent damage in the composite. Fifteen specimens were tested at the clamping pressure. Unfortunately, after post processing the data, it was found that the MTS machine had a conditioned maximum force depending on the grip pressure. At the selected grip pressure, the stroke undergoes rigid body motion at approximately $15.5 \mathrm{kN}$ and keeping this force meanwhile the stroke continued its displacement. This displacement continued for approximately $13 \mathrm{~mm}$ when suddenly, the force increased again until the total adhesive failure and total joint failure. Unfortunately, this condition in the MTS machine was not known to the technician in charge, and due to the short time available for the testing, several specimens exhibited the same phenomena, mainly the coupons with thicker adherends.

In order to correct the data for the machine compliance, a tensile test with a very stiff coupon was carried out using the same testing parameters. After comparing the response between the stiffer reference sample and the tested composite specimens, the rigid body motion and machine compliance was measured and removed from the load versus stroke data. One repetition test with the joint configuration labeled ace was carried and the stroke displacement compared with the corrected results was in good agreement. The procedure is described in Appendix A. 
To avoid the same problem for the remaining specimens, the next specimens were tested increasing the grip pressure up to $13.78 \mathrm{MPa}$ (2000 Psi).

A schematic of the DIC setup is shown in Figure 3.18. The camera was a scientific-cooled camera Sensicam ${ }^{\circledR}$ with 1 megapixel resolution and $16.93 \mathrm{~mm}(2 / 3 \mathrm{in})$ sensor size. The system included a CPU with the controlling software to trigger the camera and to store the images. A BNC cable was connected to send the triggering signal from the MTS to the desktop CPU and another set of BNC connectors was used to send the triggering signal from the CPU to the camera. Once the camera was triggered, the picture capturing process started at a programmed frame rate as needed.

A strain measuring device P3 was also connected and synchronized with the MTS. The signal from the strain gauge is sent to the $\mathrm{P} 3$ to obtain the strain value in voltage. This strain results in voltage, are sent to the MTS using the analog input channel and captured at the same rate as the displacement and load.

The MTS machine was programmed using the MTS Testware ${ }^{\circledR}$ control software to send the triggering signal to the camera and to capture the analog signal from the P3 strain gauge analyzer to ensure that the acquired data are synchronized with the captured DIC images.

The grips were rotated $45^{\circ}$ relative to the test frame's vertical to allow the measurement in the specimen's edge, and the camera was located at a varying distance from the joint's edge as shown in Figure 3.19. A Canon $50 \mathrm{~mm}$ lens was installed in the camera. In some cases, tube spacers were used to increase the magnification as required. 


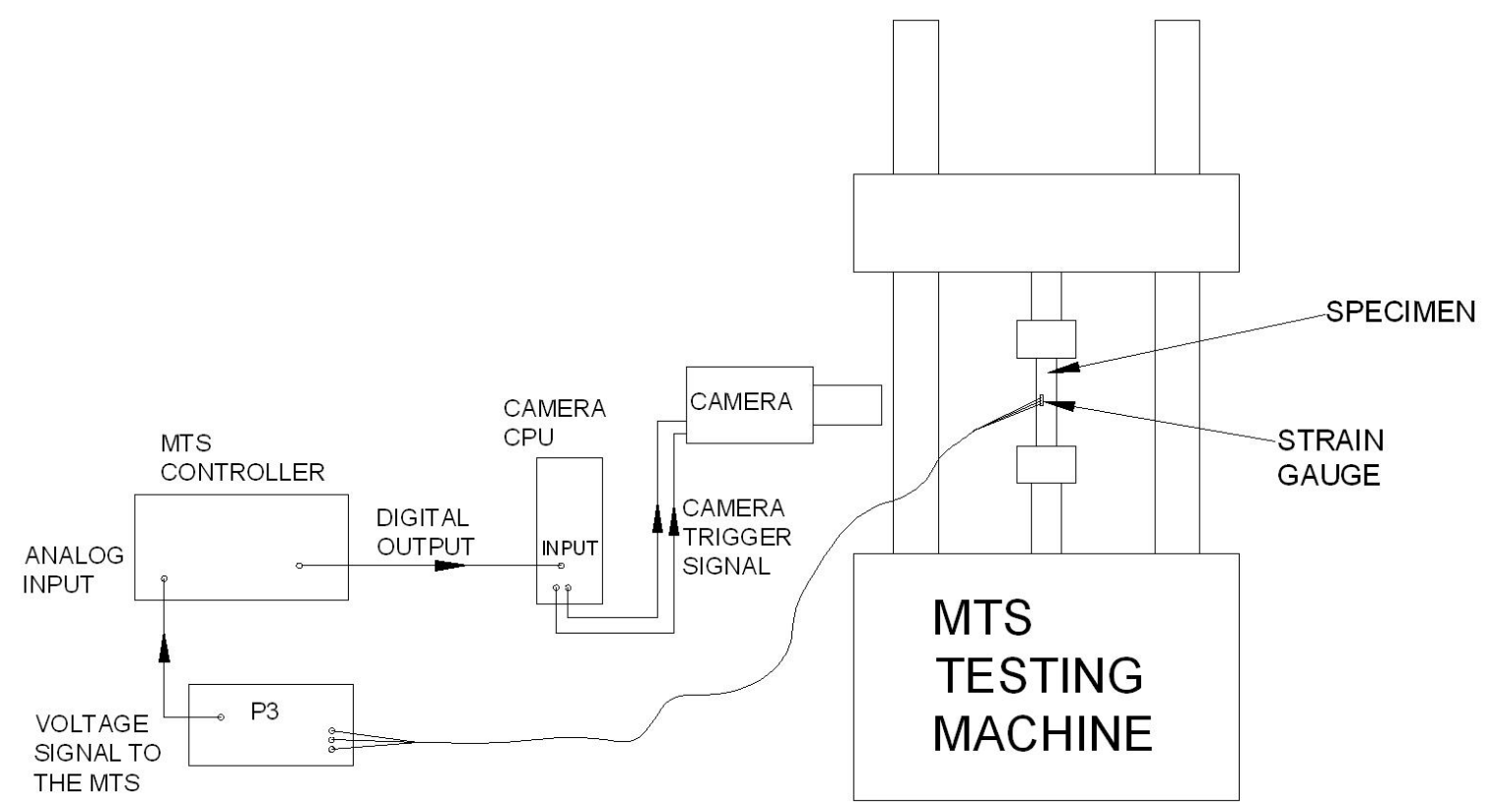

Figure 3.18 Experimental setup

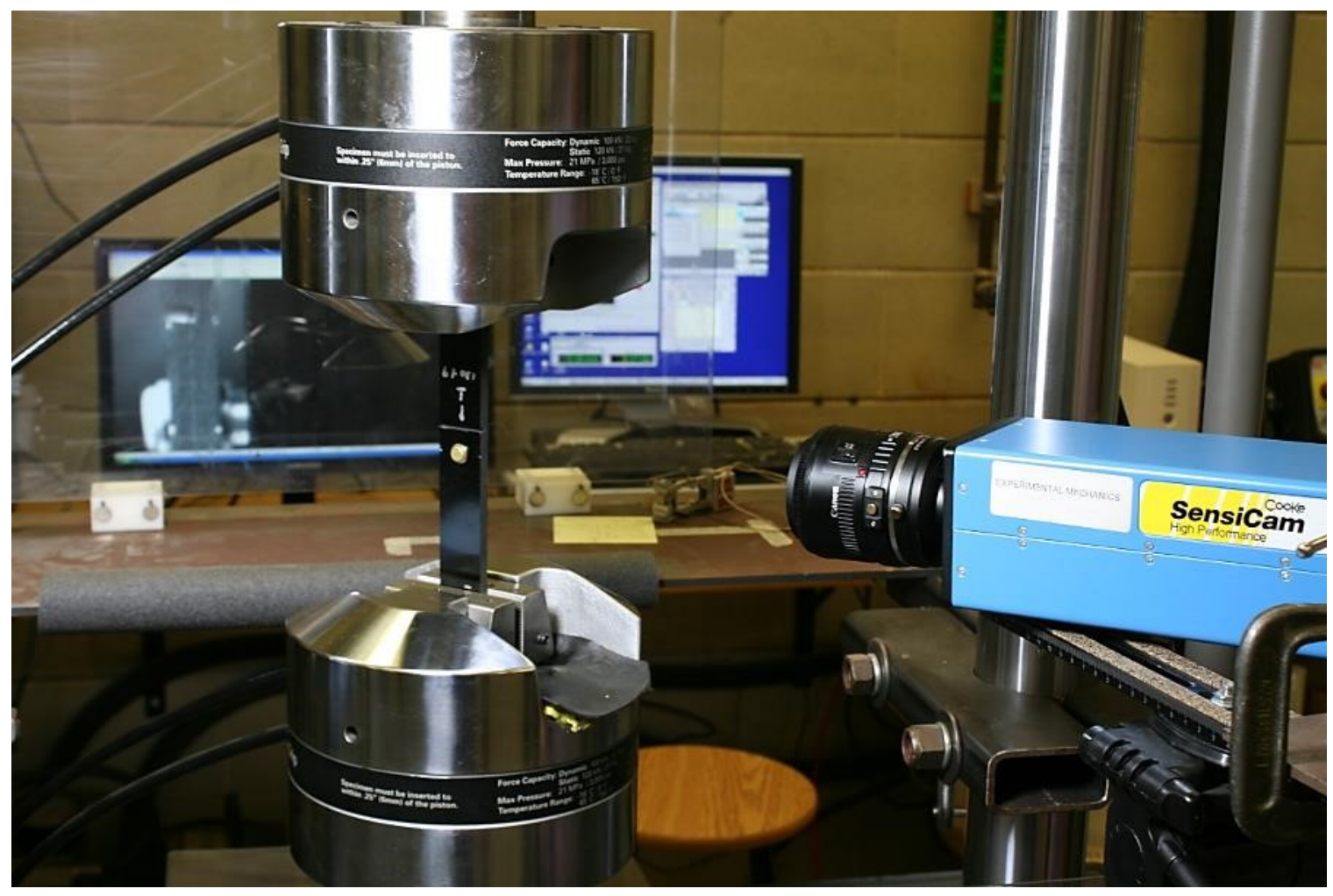

Figure 3.19 DIC camera setup 


\subsection{Measurements Using Digital Image Correlation}

For this research, the commercial 2D DIC software, MatchID, was used to analyze the images and to calculate the displacements and strains. Although the software can compute the shear strains directly, the algorithm does not compensate for any rigid body motion in real time; this has to be completed during post processing.

The local stiffness assessment requires measuring the adherend's displacement locally outside the bondline. This measurement is typically recorded from bolted joints using extensometers [123]. However, in this joint design, this is not possible because the gap between the adherends and the doublers is small. The wedge of the extensometer would be in contact with both the adherend and the doubler and, due to the secondary bending moment, the rotation could produce slippage between them. The consequence could be inadequate displacement readings. A couple of treatments were tested using the extensometer, and this issue was confirmed.

The advantage of using the DIC technique is the capability to measure displacements. In this case, the DIC was also applied to measure the local displacement right outside the bondline. Two reference distances were used, 35 and $17.5 \mathrm{~mm}$ respectively as shown in Figure 3.20. The chosen length depends on the magnification and the field of view of the images. 

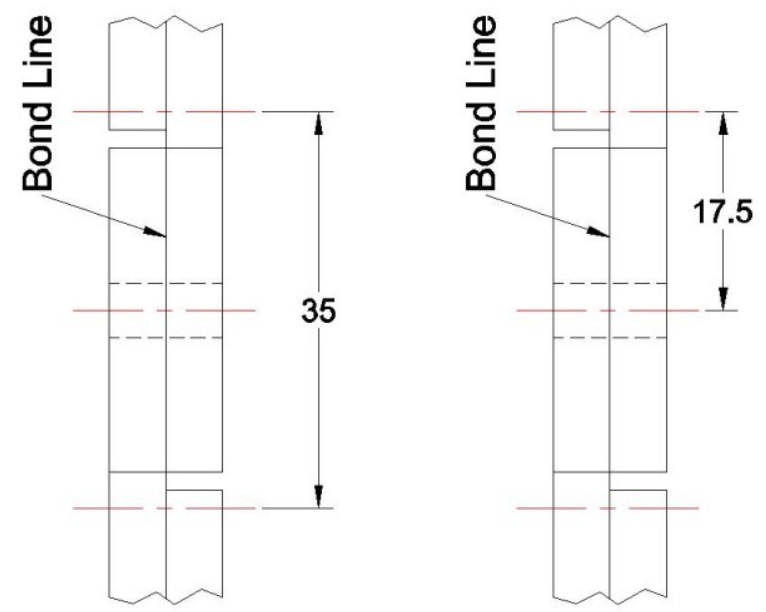

Figure 3.20 Local displacement measurement. Selected lengths, $\mathrm{mm}$.

In some cases, when high magnification was required to compute the strains with higher resolution in pixels $/ \mathrm{mm}$, it was not possible to measure the local displacements outside the bondline. Instead, the relative displacement between adherend was measured. This approach was carried in previous research [59] using an extensometer located between the adherends. However, by using optical measurements reference points near to the adhesive bondline can be selected to avoid further corrections due to the adherend deformation. Another advantage of measuring this local displacement is the possibility of measuring the shear strain by choosing the third point and applying the procedure previously discussed in section 2.6. and explained in Appendix B.

When DIC strain measurement is carried out on the adhesive edge, the usual choice is a full field of view of the bondline. However, this choice diminishes the resolution in pixels $/ \mathrm{mm}$ and the strain calculations may be less accurate. In this case, the resolution of the camera, 1 Megapixel, does not allow us to analyze the entire bondline 
with high resolution. The position of the points shown in Figure 3.21 was selected near to the notch to get a reference point to measure the distances.

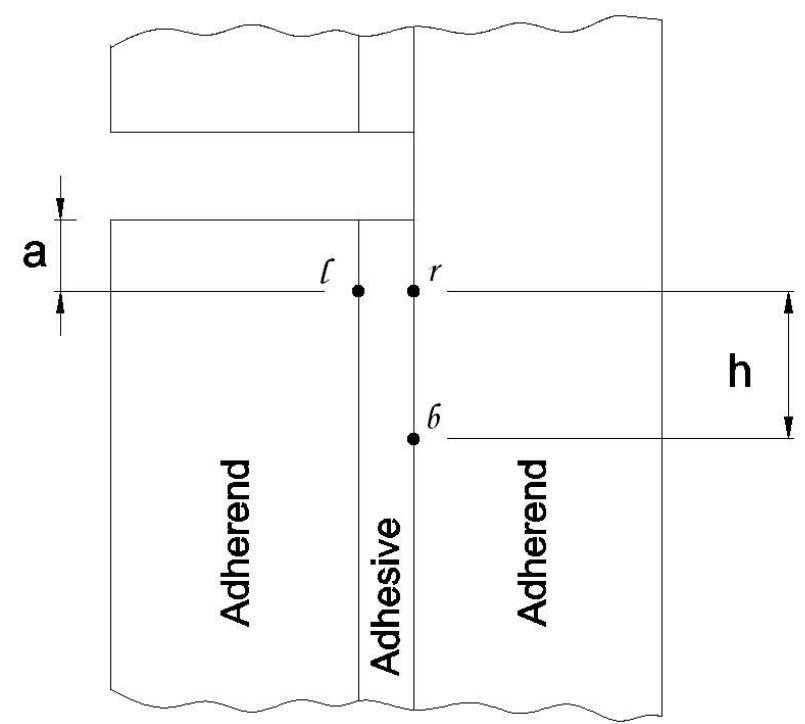

Figure 3.21 Selected points to measure the adhesive's shear strain inside the bondline

Choosing the point $l$ and $r$ very close to the adhesive edge, distance $a$, could affect the measurements due to the stress concentration. Therefore, the displacements were measured at several distances, $\mathrm{a}=1 \mathrm{~mm}, 2 \mathrm{~mm}$ and $3 \mathrm{~mm}$. Unfortunately, due to the higher magnification setup, measuring the strain at longer distances was not possible. After the calculation, the values were compared against the FEM results.

\subsection{Summary of the Chapter}

This chapter described the steps to carry out the experiments.

First, the chosen materials to manufacture the joints were described in section 3.1. The selected materials were: 
- Composite: Out-of-autoclave CFRP Cytec 5320 unidirectional prepreg from Cycom

- Adhesives:

○ FM300-2M. Epoxy

○ EA9361 (high elongation epoxy)

- Fastener: $3 / 16$ in $(4.76 \mathrm{~mm})$ diameter Hi-Lite ${ }^{\circledR}$ titanium 6 Al-4V from Hi-Shear Corporation.

- Washers:

○ NAS1149-CO363B

○ AN970

The section 3.2 described the procedure to design the bolted/bonded coupon and the final design was shown in Figure 3.4.

Similarly, from the literature review and the input from the CRIAQ partners, in section 3.3, the factor and levels were defined for the DOE. The final DOE was a $2^{5-2}$ factorial design with three replicates, as shown in Table 3.5.

The manufacturing process was described in section 3.4., starting from the composite manufacturing, cutting, joining, drilling and notching. Special attention was highlighted in the accuracy of the notching and drilling.

Finally, the experimental setup was described in section 3.5. In this section, the required instrumentation and connections to extract synchronized data: loads, displacements and images for the DIC analysis was described. 


\section{Chapter 4: Experimental Results}

Although randomization is an important aspect in the DOE, due to manufacturing issues, the sequence of the testing was not fully randomized in the present study. Controlling the bondline thickness was a key factor to start the testing program. The FM300-2M adhesive was supplied in a film form, therefore, controlling the bondline thickness was easier compared with the paste adhesive EA9361. Conversely, bonded plates with EA9361 were hard to manufacture, because the bondline thickness was not uniform during the first joining attempts. Several trials were performed to achieve good bondline thickness; therefore, the specimens with this adhesive were tested late in this program.

In parallel with the manufacturing process, it was possible to prepare the DIC setup for the first coupons. These first coupons were tested, and the results were satisfactory using the initial DIC setup. The extracted data was useful to compare against the finite element models. Further improvements to the DIC setup were possible by using a finer speckle pattern and higher magnifications. Fortunately, such an improvement was possible before the samples with EA9361 were tested. Therefore, the adhesive shear strain in these coupons was accurately analyzed due to the improved DIC setup.

The results are separated in two groups according to the adhesive type to allow a better comparison because the characteristic failure sequence is strongly dependent on this factor. 
It is important to have a reference point to assess the bolted/bonded joints' performance. For that reason, bolted and bonded joints were tested to compare against them. Unfortunately, due to the limited quantity of material, bonded joints with FM300$2 \mathrm{M}$ film adhesive were not tested initially. Only a set of tests were carried out late in the program. On the other hand, bonded joints with EA9361 were tested using a single replicate and, in some cases using two coupons. Fortunately, the extracted data was accurate enough to compare against hybrid bolted/bonded joints due to the good agreement between the experimental and numerical models.

\subsection{Bolted Joints}

To begin with, bolted joints with thinner and thicker adherends were tested (Figure 4.1). The thinner joints were manufactured with 24 plies of composite material and the final thickness was $3.29 \mathrm{~mm}$. The thicker joints were 40 plies with $5.48 \mathrm{~mm}$. The bolts were installed using the same torque specification as the bolted/bonded joints. The tests were carried out using displacement control at $1.27 \mathrm{~mm} / \mathrm{min}$. Figure 4.2 shows the results.

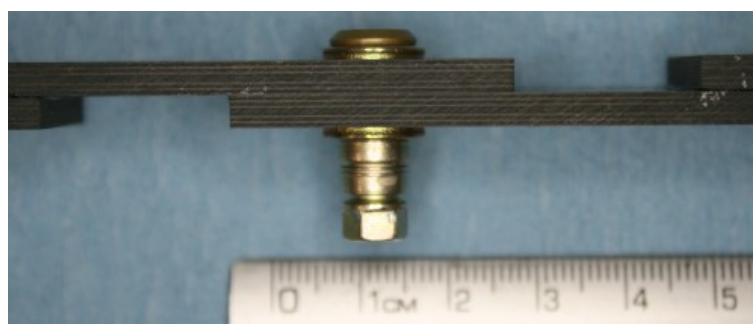

a)

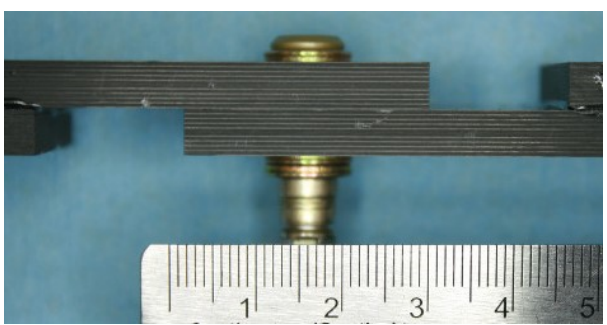

b)

Figure 4.1 Bolted joints. a) $3.29 \mathrm{~mm}$ adherend thickness; b) $5.48 \mathrm{~mm}$ adherend thickness 
The maximum strengths for bolted joints with $5.48 \mathrm{~mm}$ thickness were 15.68 and $15.55 \mathrm{kN}$ respectively, averaging $15.61 \mathrm{kN}$. At these force values, the failure was catastrophic in the bolts and bearing failure in the composite adherend.

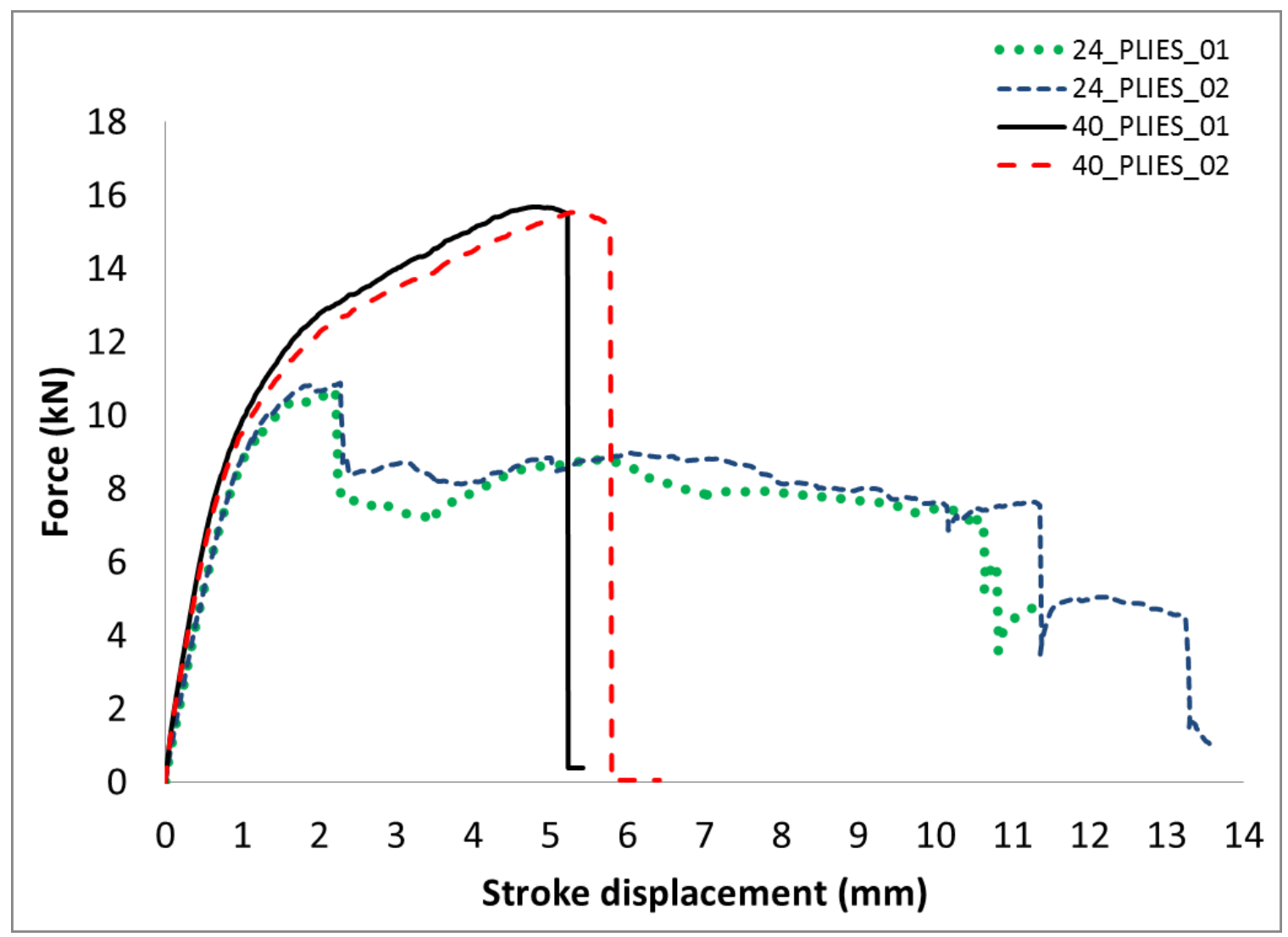

Figure 4.2 Experimental results for bolted joints. Force versus displacement

The maximum strength for joints with a $3.29 \mathrm{~mm}$ thickness were 10.71 and 10.87 $\mathrm{kN}$ respectively, averaging $10.79 \mathrm{kN}$. After these force peaks, there were a sudden drop in the force, but the bolt did not fail. The force dropped until 8.92 and $9.2 \mathrm{kN}$ at a stroke displacement of $2.19 \mathrm{~mm}$ and $2.28 \mathrm{~mm}$ respectively. The force was sustained averaging 7.9 and $8.29 \mathrm{kN}$ respectively until a second drop due to tear-out failure in the composite joint at $10.64 \mathrm{~mm}$ and $11.37 \mathrm{~mm}$ stroke displacements. Although only two repetitions 
were tested, the repeatability in the results was confirmed with good agreement. The failure modes are shown in Figure 4.3.

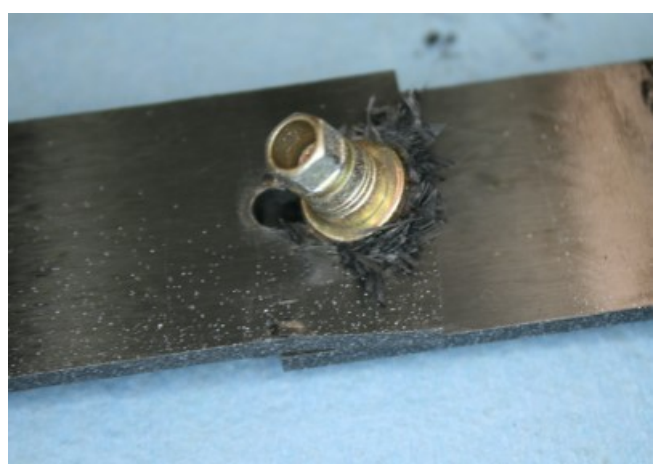

a)
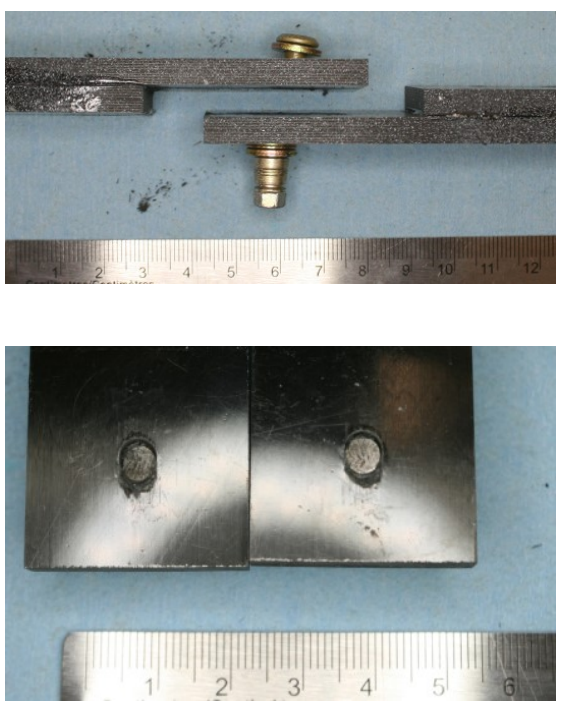

b)

Figure 4.3 Failure modes in bolted joints. a) Joint with $3.29 \mathrm{~mm}$ adherend thickness, b) Joint with $5.48 \mathrm{~mm}$ adherend thickness

\subsection{Bonded Joints}

Due to the number of combinations using different adhered thicknesses, adhesives and adhesive thicknesses, several tests were carried out using one of two repetitions depending of the material available. The load versus stroke results, for joints with EA9361 adhesive are shown in Figure 4.4.

The nomenclature is the same as the equivalent bolted/bonded joint treatment. 


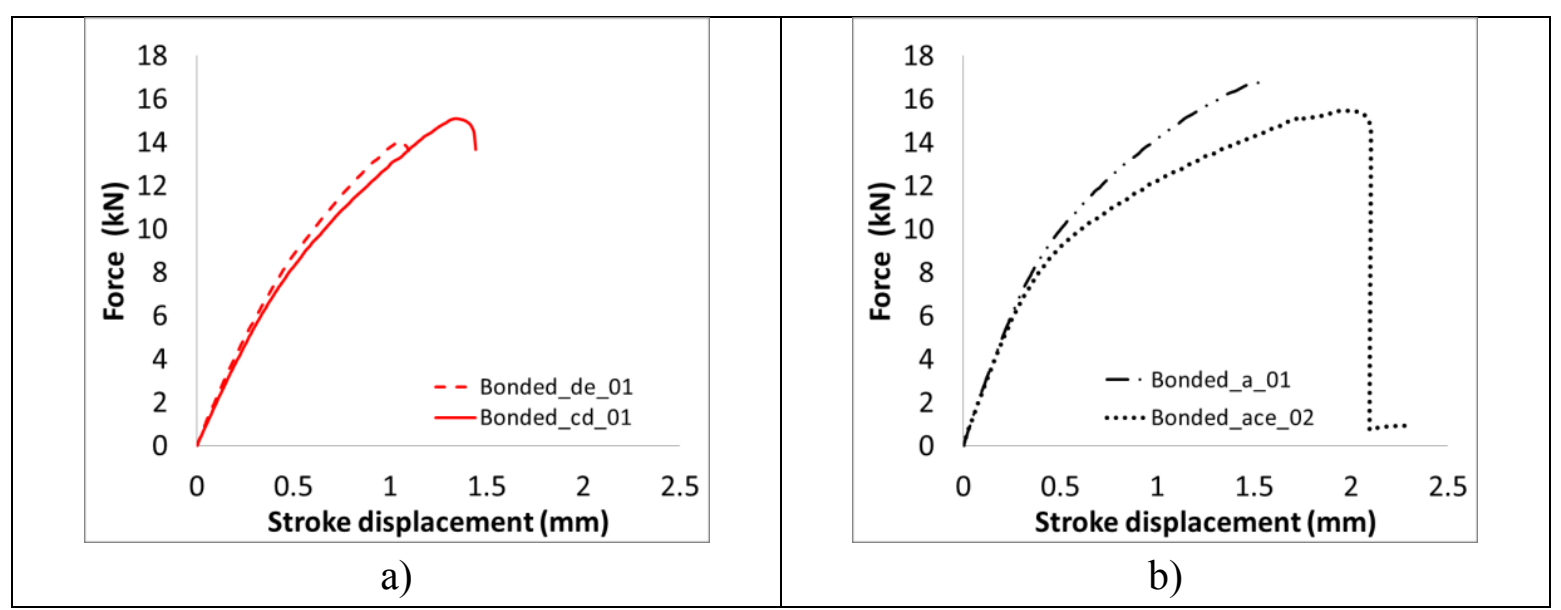

Figure 4.4 Bonded joints, force versus displacement results. a) adherend thickness 3.29 $\mathrm{mm}$ and adhesive thickness $0.2 \mathrm{~mm}$ (de_01) and $0.5 \mathrm{~mm}$ (cd_01); b) adherend thickness $5.48 \mathrm{~mm}$ and adhesive thickness $0.2 \mathrm{~mm}$ (a_01) and $0.5 \mathrm{~mm}$ (ace_02)

DIC measurements were carried out using a $50 \mathrm{~mm}$ lens and camera tube spacers to increase the magnification near to the adhesive bondline. The joints with thinner adherends $(3.29 \mathrm{~mm})$ were testes using the 13 and $31 \mathrm{~mm}$ spacers, with a resolution of $0.0067 \mathrm{~mm} /$ pixel. The joints with thicker $(5.29 \mathrm{~mm})$ adherends were tested using the 31 and $13 \mathrm{~mm}$ spacers. With this configuration, a resolution of $0.0073 \mathrm{~m} / \mathrm{pixel}$ ( 7 microns) was achieved. The shear strains were extracted at several distances from the notch, these strains were used to compare the joint performance against bolted/bonded joints to assess the load sharing. The plots of these results will be presented in Chapter 6 along with its comparison against the strains in hybrid joints with similar adherend thickness and adhesive thickness. 


\subsection{Bolted Bonded Joints with FM300-2M Adhesive}

The joints manufactured with the stiff adhesive FM300-2M correspond to treatments $b e, a b d, b c$ and $a b c d e$, respectively. To keep track of every joint and their factors levels, a summary of these treatments is shown in Table 4.1.

Table 4.1 Experimental treatments with FM300-2 adhesive

\begin{tabular}{|c|c|c|c|c|c|c|}
\hline \multirow{2}{*}{\multicolumn{2}{|c|}{ Treatment }} & \multicolumn{5}{|c|}{ FACTORS } \\
\hline & & A & B & C & D & $E$ \\
\hline \multicolumn{2}{|c|}{ number } & Adherend & Adhocivo & Adhesive & Clamped & Clearance \\
\hline 3 & be & 3.29 & FM300-2M & 0.2 & 97 & 0.1 \\
\hline 4 & $\mathrm{abd}$ & 5.48 & FM300-2M & 0.2 & 387 & 0 \\
\hline 7 & $\mathrm{bc}$ & 3.29 & FM300-2M & 0.5 & 97 & 0 \\
\hline 8 & abcde & 5.48 & FM300-2M & 0.5 & 387 & 0.1 \\
\hline
\end{tabular}

\subsubsection{Treatment be}

The load stroke results for treatment be are shown in Figure 4.5. It can be observed that the failure in the joints occurs in three stages. This process is depicted on Figure 4.6 using the specimen be_01. The joint supports an initial load of $13.58 \mathrm{kN}$ without sign of failure; at this point, the adhesive fails due to cracking near to the edge, and this crack propagates a few millimeters only. This failure produced the first drop in the joint force, point A; however, the adhesive is still capable of withstanding further loading until its final failure, point $\mathrm{B}$, at $14.54 \mathrm{kN}$. Due to this, after this point, the joints behave as a bolted joint and it is capable of supporting more load. The final failure is due to shearout in the adherend. A similar trend is observed for the joints be_02 and be_03. 


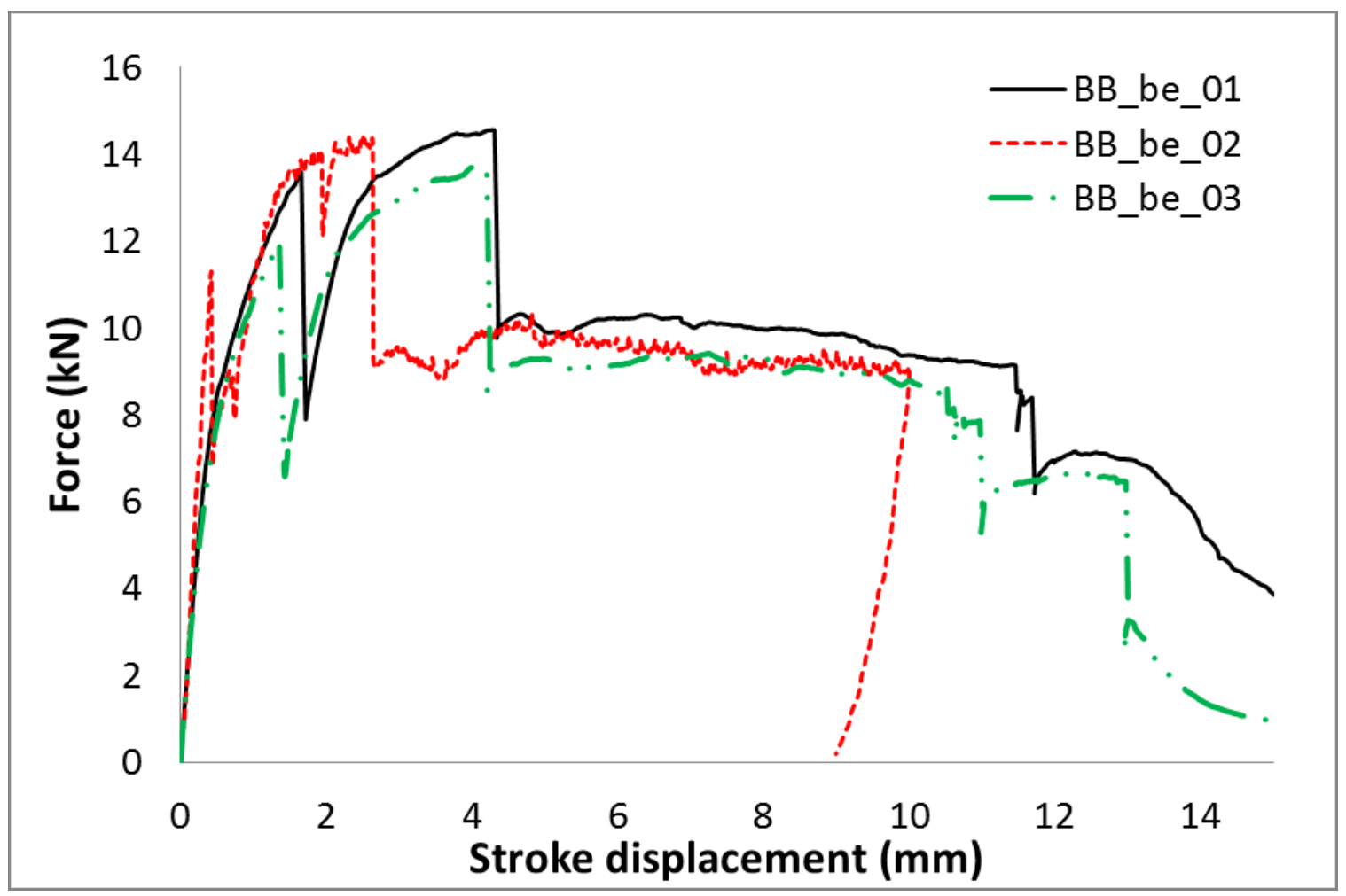

Figure 4.5 Treatment be, force versus displacement results 


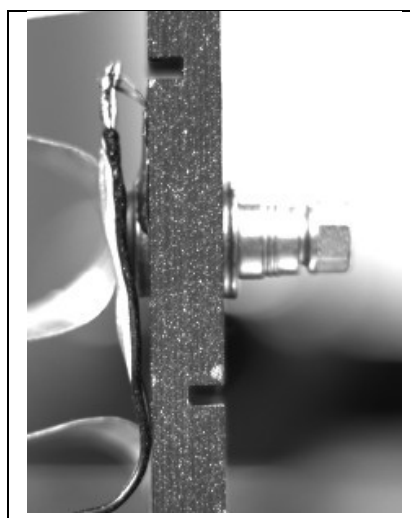

Initial time, reference
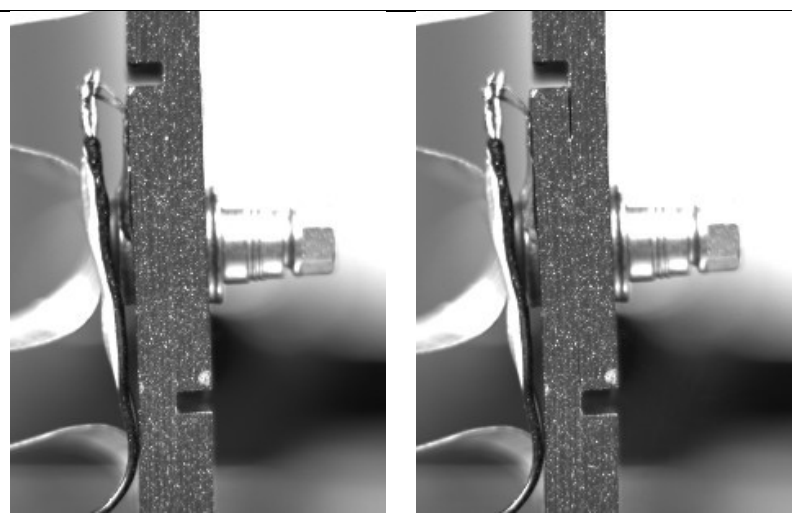

A) First crack at the adhesive, $(13.58 \mathrm{kN}, 78 \mathrm{sec})$
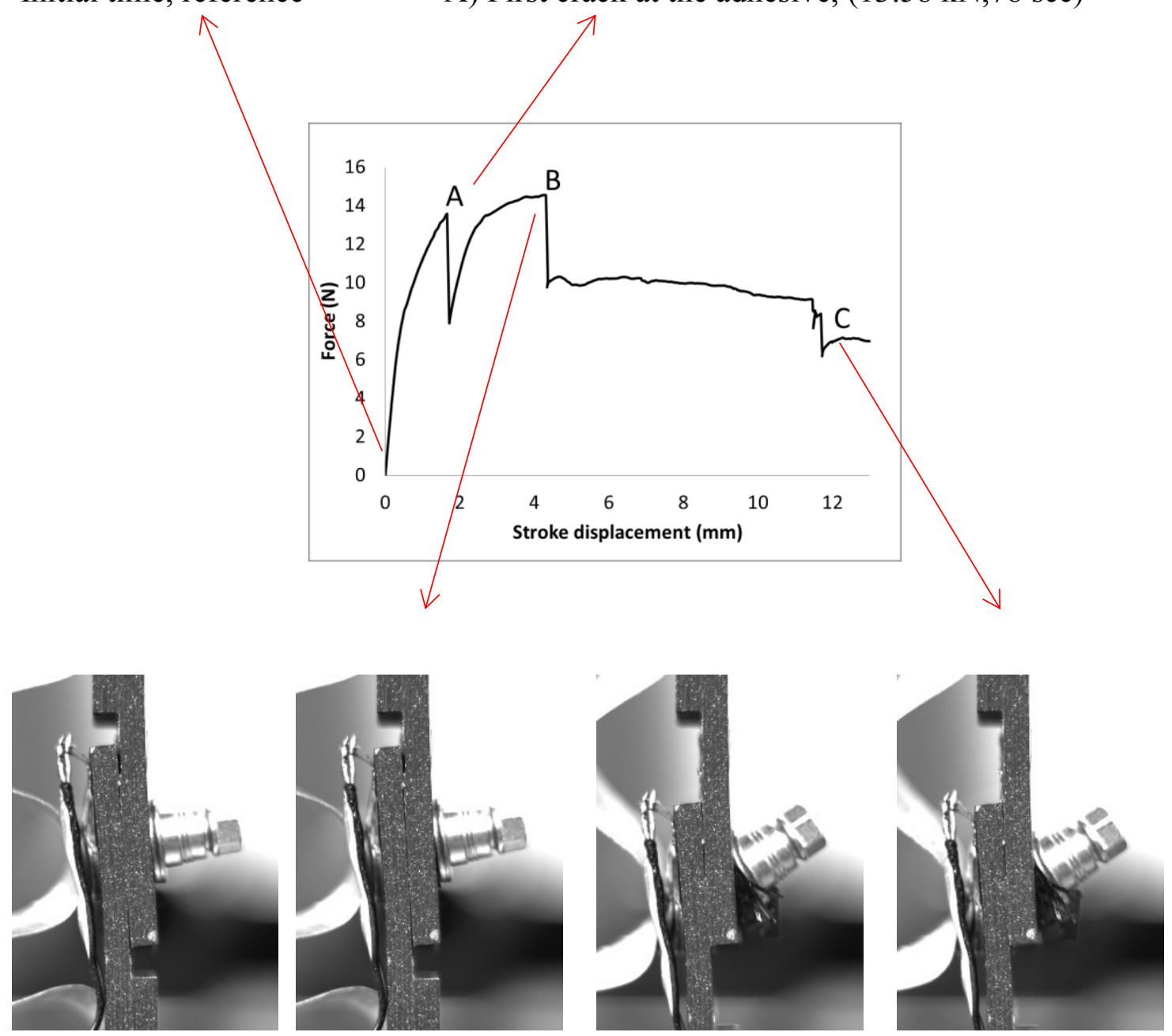

B) Second crack in the adhesive, $(14.54 \mathrm{kN}, 204 \mathrm{sec})$

$\mathrm{C}(8.4 \mathrm{kN}, 552 \mathrm{sec})$ 
The sample be_02 was tested in a different MTS testing machine model 318.25B01 with a $250 \mathrm{kN}$ capacity. Although this sample has the same trend in force capability, the stroke displacement is smaller because the $250 \mathrm{kN}$ MTS machine is stiffer than the $100 \mathrm{kN}$ MTS machine. The load stroke data is summarized in Table 4.2.

Table 4.2 Summary of results. Force versus displacement for treatment be

\begin{tabular}{|c|c|c|c|c|c|c|c|c|c|c|}
\hline \multirow{2}{*}{} & \multicolumn{2}{|c|}{$\begin{array}{c}\text { Sample } \\
\text { be_01 }\end{array}$} & \multicolumn{2}{c|}{$\begin{array}{c}\text { Sample } \\
\text { be_02 }\end{array}$} & \multicolumn{2}{c|}{$\begin{array}{c}\text { Sample } \\
\text { be_03 }\end{array}$} & \multicolumn{2}{c|}{$\begin{array}{c}\text { Force } \\
(\mathrm{kN})\end{array}$} & \multicolumn{2}{c|}{$\begin{array}{c}\text { *Displacement } \\
(\mathrm{mm})\end{array}$} \\
\hline \multirow{2}{*}{ POINT } & $\begin{array}{c}\text { Disp. } \\
(\mathrm{mm})\end{array}$ & $\begin{array}{c}\text { Force } \\
(\mathrm{kN})\end{array}$ & $\begin{array}{c}\text { Disp. } \\
(\mathrm{mm})\end{array}$ & $\begin{array}{c}\text { Force } \\
(\mathrm{kN})\end{array}$ & $\begin{array}{c}\text { Disp. } \\
(\mathrm{mm})\end{array}$ & $\begin{array}{r}\text { Force } \\
(\mathrm{kN})\end{array}$ & Avg & $\begin{array}{r}\text { Sdt. } \\
\text { dev }\end{array}$ & Avg & $\begin{array}{r}\text { Sdt. } \\
\text { dev }\end{array}$ \\
\hline A & 1.66 & 13.58 & 0.42 & 11.29 & 1.35 & 11.97 & 12.28 & 1.17 & 1.51 & - \\
\hline B & 4.31 & 14.54 & 2.64 & 14.28 & 4.20 & 13.67 & 14.16 & 0.45 & 4.25 & - \\
\hline C & 11.46 & 9.13 & 9.35 & 9.34 & 10.52 & 8.59 & 9.02 & 0.39 & 10.99 & - \\
\hline
\end{tabular}

* Samples be_02 and be_03 only.

The DIC analysis was carried out to extract enough data at different magnifications; lower magnification to extract the displacements and strains outside the bondline and higher magnification to measure the displacements and strains inside the bondline thickness. The speckle pattern was applied using standard spray paint, and some samples did not have the speckle. The paint produced a very coarse speckle in the bondline. It was believed according to previous work [114] that the gray scale was enough to allow a DIC analysis. Coupon be_01 was analyzed using a $50 \mathrm{~mm}$ lens only, as a result, the resolution was $0.0449 \mathrm{~mm} /$ pixel. Coupons be_02 and be_03 were tested using a $13 \mathrm{~mm}$ spacer to increase the magnification and the resolution per pixel was increased to $0.022 \mathrm{~mm} / \mathrm{pixel}$ (22 microns/pixel). 
Using the configuration without spacers, sample be_01, the relative displacements were measured using the $35 \mathrm{~mm}$ reference length according to the procedure described in section 3.6. Figure 4.7 shows the results, including the results from the finite element models, elastoplastic (EP) and cohesive zone model. In the label, TQ and CLC indicate that the model included the initial torque in the bolt and clearance. It can be observed a linear relationship between the displacement and the force until the adhesive failure; however, it is not conclusive if the adhesive's strain remains elastic. This requires a further analysis into the adhesive to extract the shear strain versus force. Unfortunately, due to the low magnification setup, the strain results data was scattered (Figure 4.8) and the results were not conclusive.

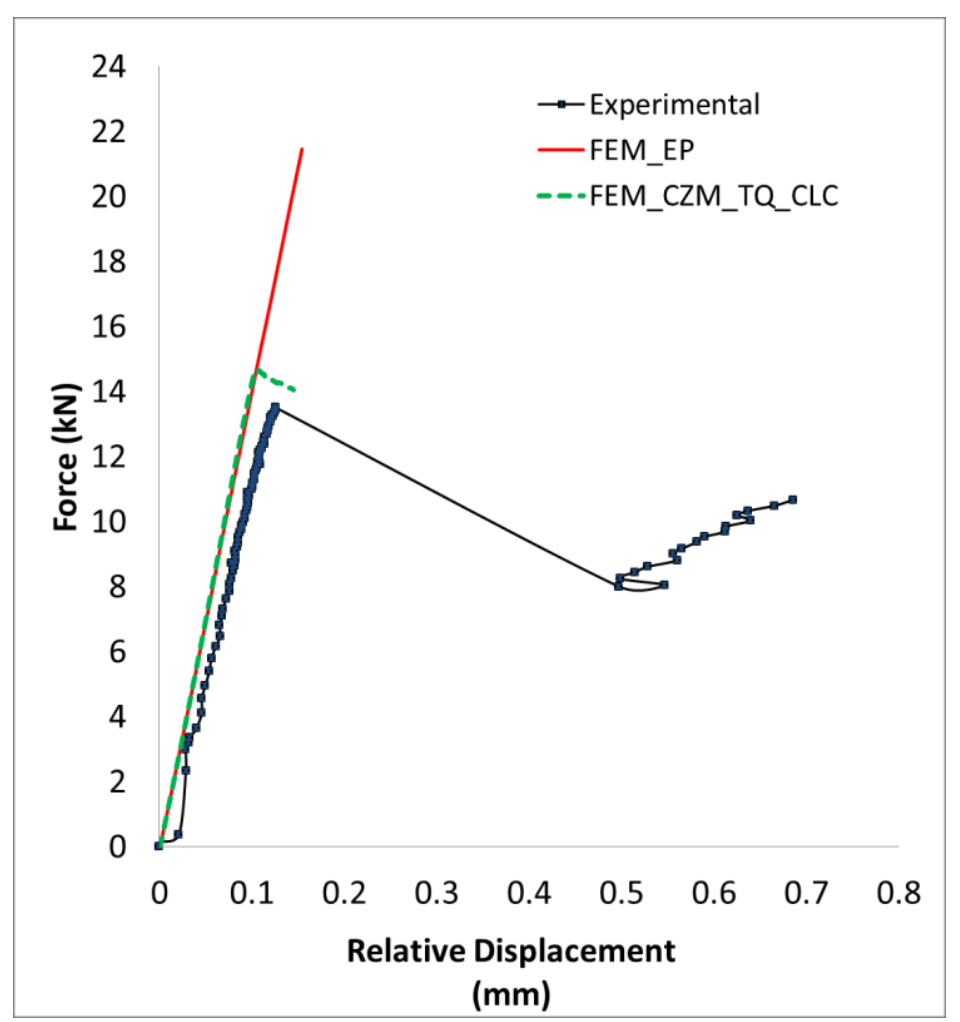

Figure 4.7 Sample be_01. Force versus relative displacement using $35 \mathrm{~mm}$ reference length. The experimental data is compared against FEM models, elastoplastic and cohesive zone model (CZM) 

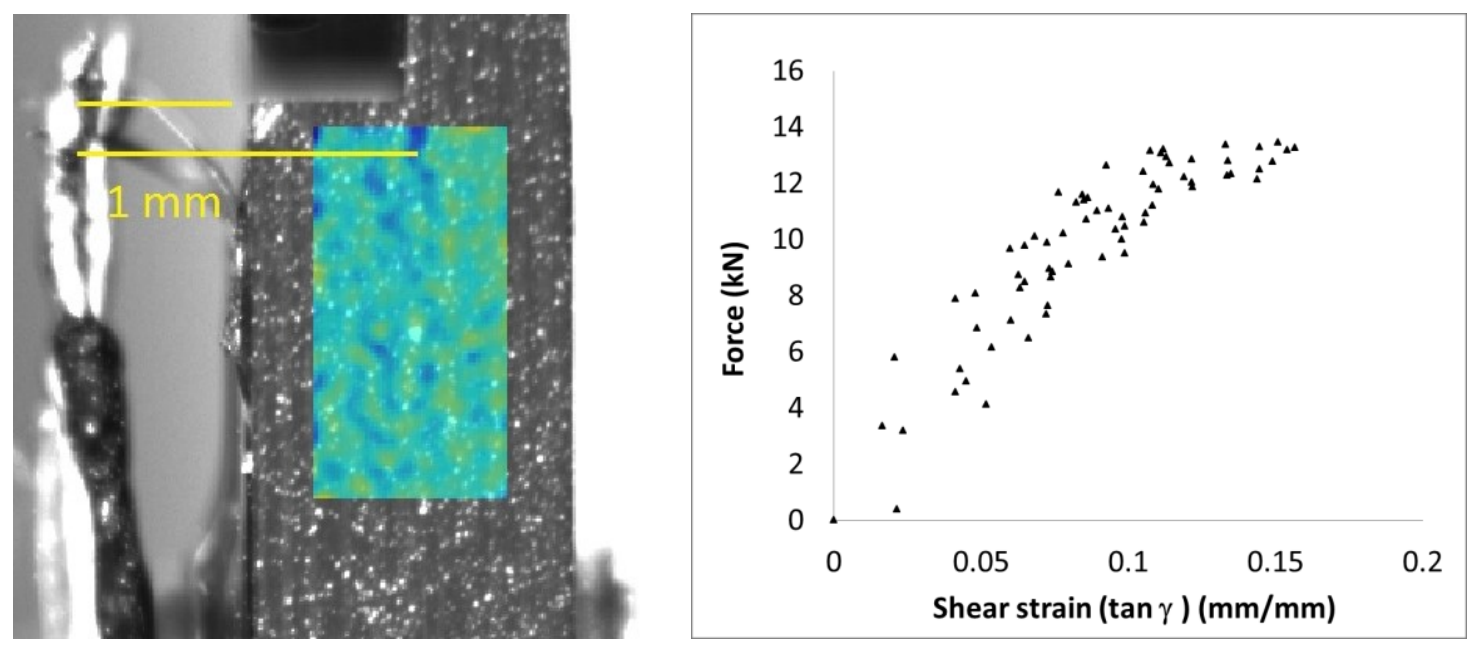

Figure 4.8 Sample be_01. Adhesive shear strain at $1 \mathrm{~mm}$ distance from the notch

The sample be_02 was the first tested coupon from the entire DOE, and it was analyzed using a $13 \mathrm{~mm}$ spacer along with the $50 \mathrm{~mm}$ lens. With this setup, the resolution was estimated at $0.02568 \mathrm{~mm} / \mathrm{pixel}$. After the data was analyzed, it was found that the image capturing was not perfectly synchronized with the MTS data. The issue was due to the programming in the camera setup. This issue was corrected for the next tests, and the pictures were very well synchronized with the MTS data. Even so, it was possible to analyze 11 time-synchronized data points. The displacement shown in Figure 4.9 was measured using a $17.5 \mathrm{~mm}$ distance from the centre to the outside of the notch. The same analysis was carried out for the shear strain at a distance of $1 \mathrm{~mm}$ from the notch as shown in Figure 4.10. 


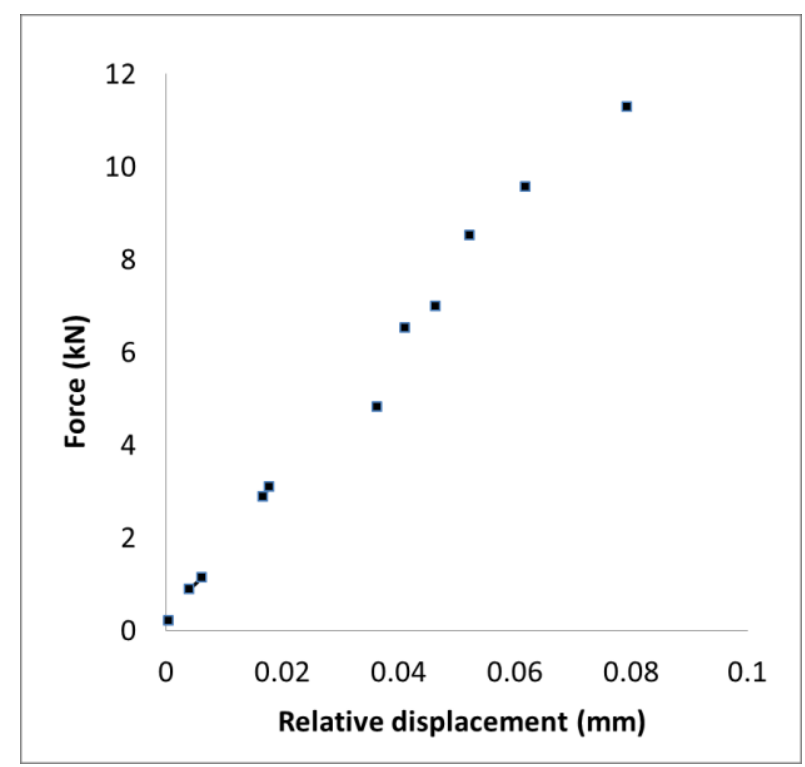

Figure 4.9 Sample be_02. Force versus relative displacement using $17.5 \mathrm{~mm}$ reference length.
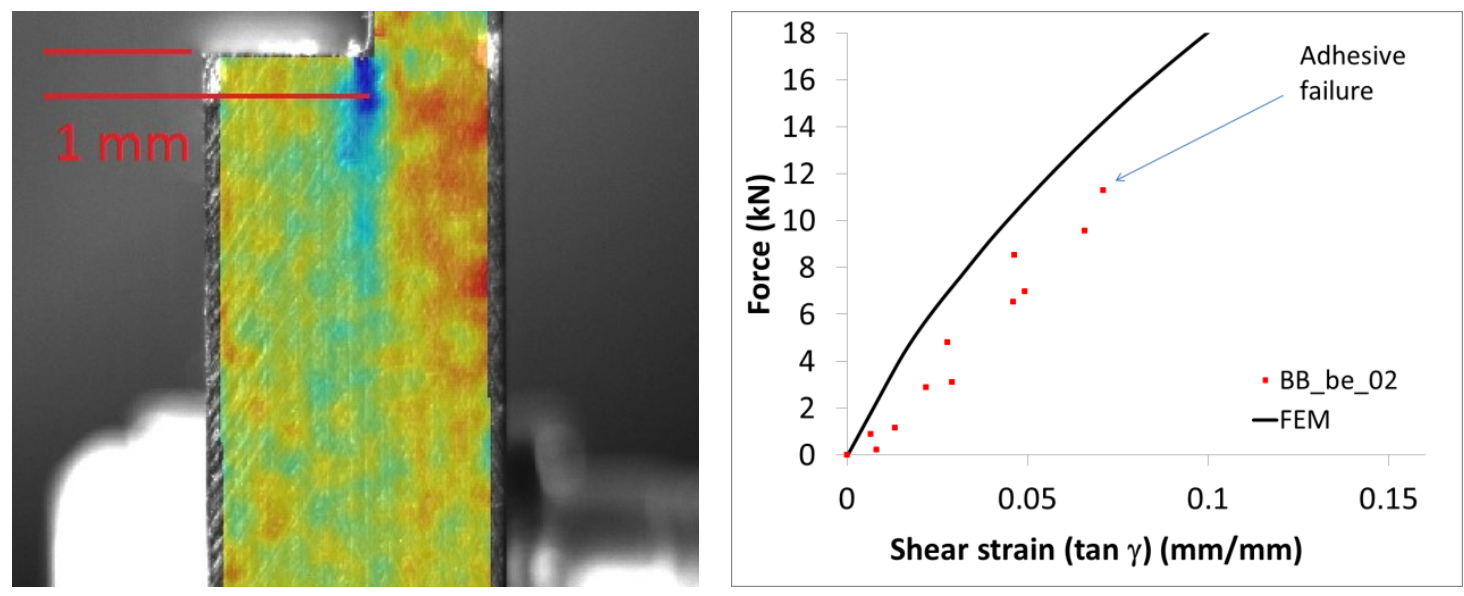

Figure 4.10 Sample be_02. Adhesive shear strain at $1 \mathrm{~mm}$ distance from the notch

The specimen be_03 was analyzed using the same setup used for the sample be_02. The aim of this setup was to analyze the shear strain rather than the joint stiffness. Therefore, the initial image for the DIC capturing process was adjusted to be right below the notch. The shear strain extracted from the sample be_03 was compared against the 
FEM model and shown in Figure 4.11. The agreement with the sample be_03 is good until approximately $8 \mathrm{kN}$ and a discrepancy of approximately $25 \%$ was found at maximum shear strain.
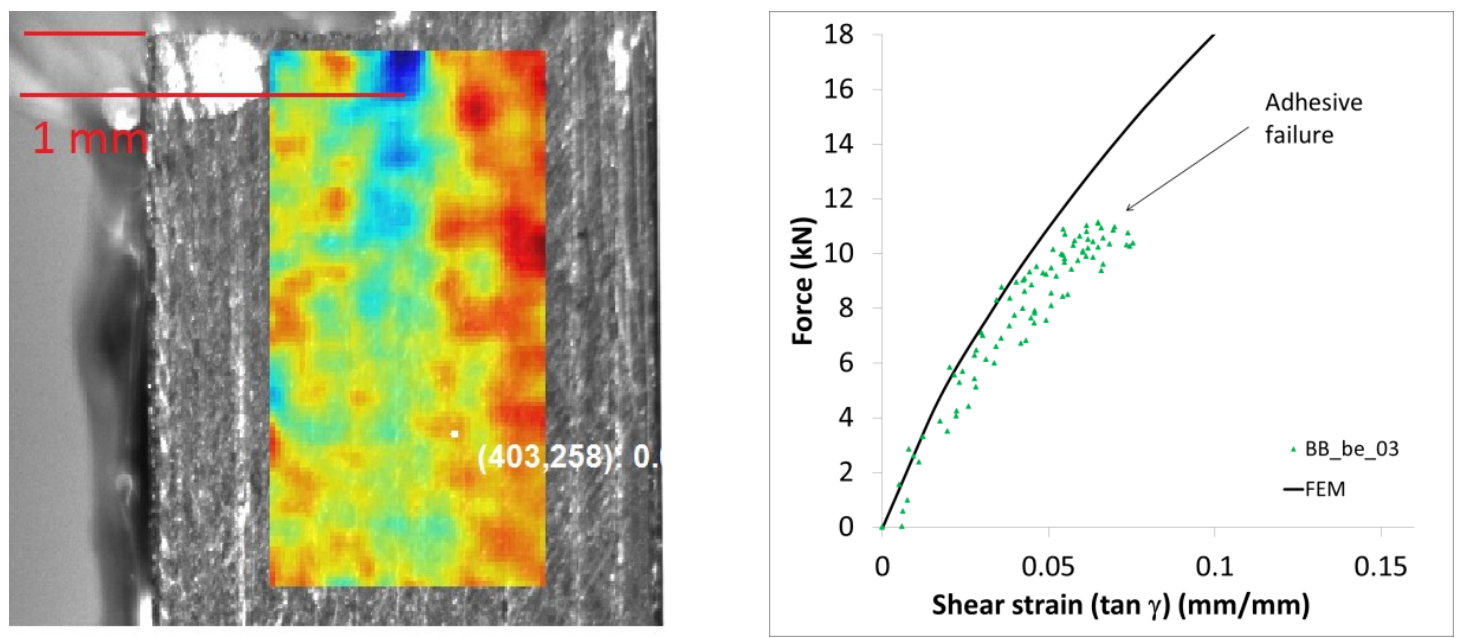

Figure 4.11 Sample be_03. Adhesive shear strain at $1 \mathrm{~mm}$ distance from notch

\subsubsection{Treatment $a b d$}

The force versus displacement plots for this treatment are shown in Figure 4.12. The coupon abd_02 failed before the MTS had undergone rigid body motion, therefore, we can use this sample as a reference to identify the quality of the correction in both displacement and force as described in section 3.5. The final results are shown in Figure 4.13. The average force at the first failure point was $16.58 \mathrm{kN}$ at an average stroke displacement of $1.94 \mathrm{~mm}$. After this point, the joints were still capable of supporting more load until an average failure load of $16.26 \mathrm{kN}$ and displacement of $5.39 \mathrm{~mm}$. At the last point, the bolt fails completely due to shear. The summary of these values is shown in Table 4.3. 


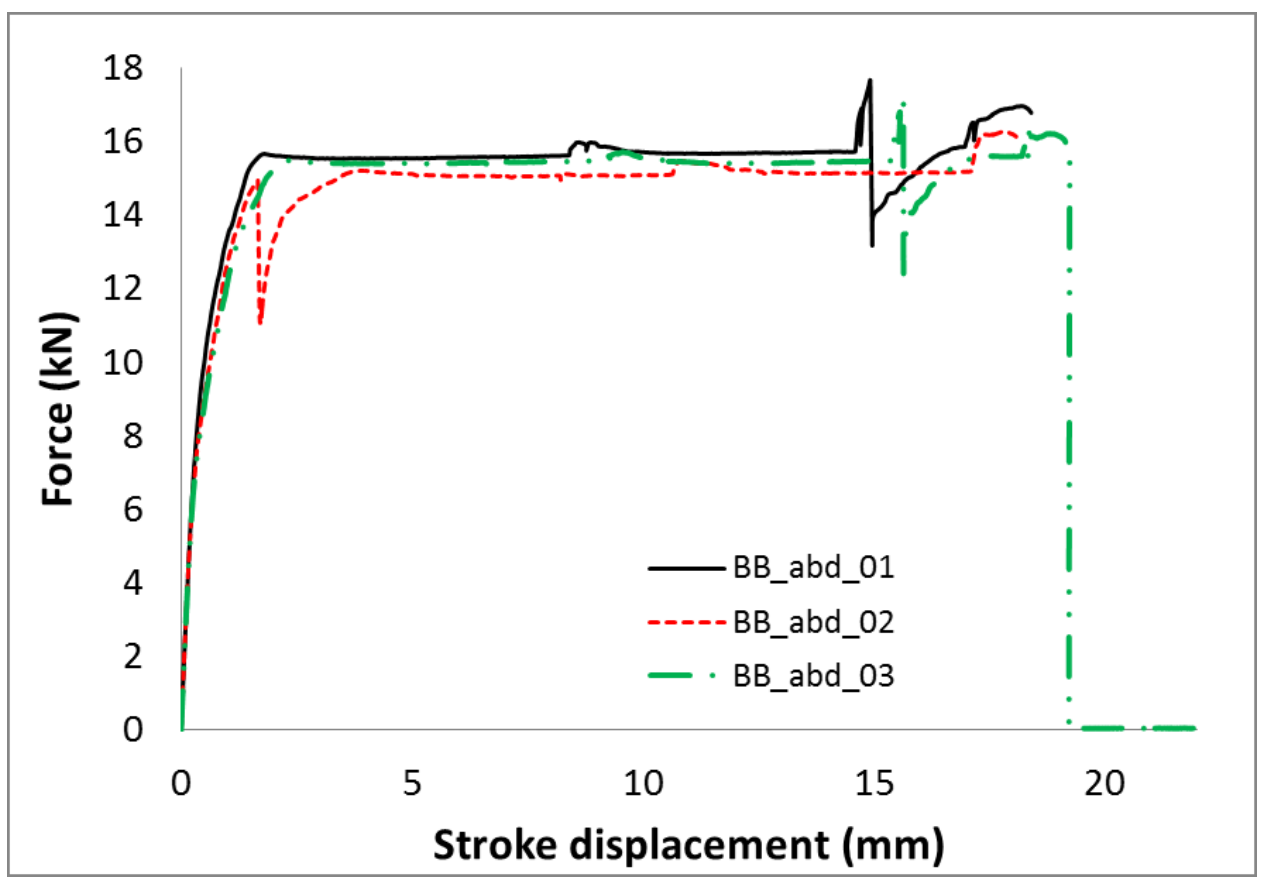

Figure 4.12 Combination abd, force versus displacement results

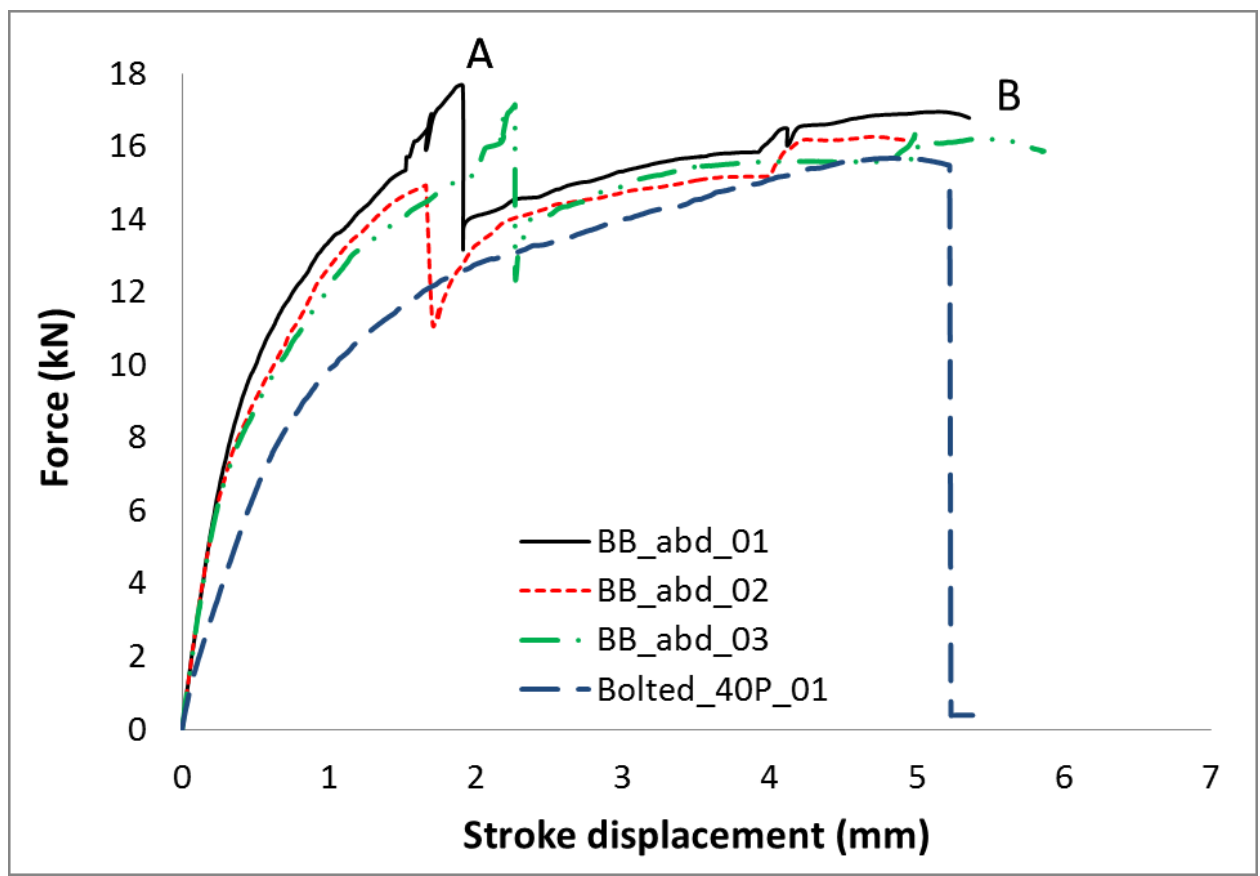

Figure 4.13 Combination abd, force versus displacement after displacement correction 
Compared with the joints with thinner adherends where the failure in the adhesive layer was in steps, these joints presented a sudden failure of the adhesive layer, point A in Figure 4.13, after this point, the joint behaves as a bolted joint. The force dropped until a value similar compared with the bolted joint and the strength increased steadily until bolt failure, point B. Figure 4.14 shows the adhesive fracture at point $\mathrm{A}$ for the coupon abd_02.

Table 4.3 Summary of results. Force versus displacement for treatment abd

\begin{tabular}{|c|c|c|c|c|c|c|c|c|c|c|}
\hline & \multicolumn{2}{|c|}{$\begin{array}{c}\text { Sample } \\
\text { abd_01 }\end{array}$} & \multicolumn{2}{c|}{$\begin{array}{c}\text { Sample } \\
\text { abd_02 }\end{array}$} & \multicolumn{2}{c|}{$\begin{array}{c}\text { Sample } \\
\text { abd_03 }\end{array}$} & \multicolumn{2}{c|}{$\begin{array}{c}\text { Force } \\
\text { (kN) }\end{array}$} & \multicolumn{2}{c|}{$\begin{array}{c}\text { Displacement } \\
\text { (mm) }\end{array}$} \\
\hline \multirow{2}{*}{ POINT } & $\begin{array}{c}\text { Disp. } \\
(\mathrm{mm})\end{array}$ & $\begin{array}{c}\text { Force } \\
(\mathrm{kN})\end{array}$ & $\begin{array}{c}\text { Disp. } \\
(\mathrm{mm})\end{array}$ & $\begin{array}{c}\text { Force } \\
(\mathrm{kN})\end{array}$ & $\begin{array}{c}\text { Disp. } \\
(\mathrm{mm})\end{array}$ & $\begin{array}{r}\text { Force } \\
(\mathrm{kN})\end{array}$ & Avg & $\begin{array}{r}\text { Sdt. } \\
\text { dev }\end{array}$ & Avg & $\begin{array}{r}\text { Sdt. } \\
\text { dev }\end{array}$ \\
\hline A & 1.91 & 17.66 & 1.66 & 14.94 & 2.26 & 17.15 & 16.58 & 1.45 & 1.94 & 0.30 \\
\hline B & 5.35 & 16.79 & 4.96 & 16.13 & 5.85 & 15.86 & 16.26 & 0.47 & 5.39 & 0.44 \\
\hline
\end{tabular}

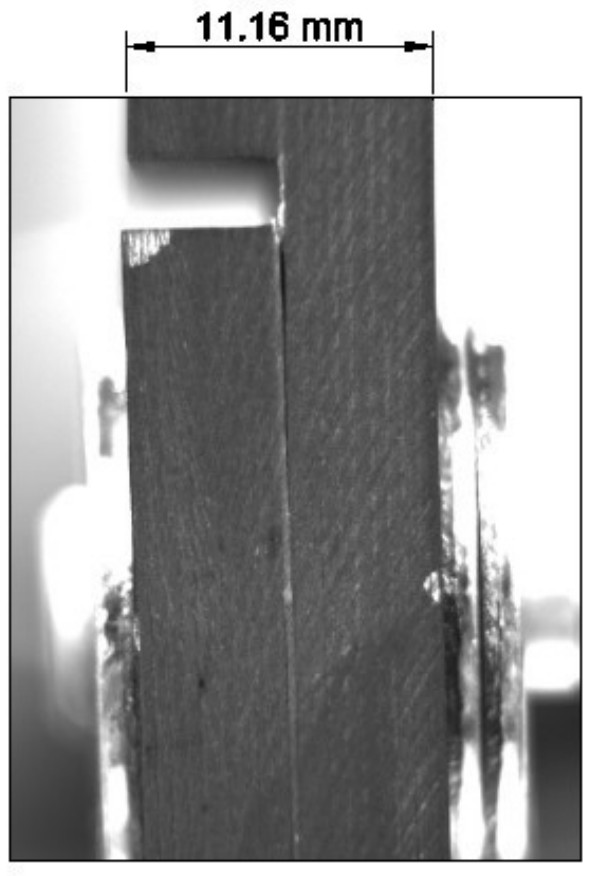

Figure 4.14 Sample abd_02, failure at point A 
The DIC analysis for the samples $a b d \_01$ and $a b d \_02$ was carried out using the $13 \mathrm{~mm}$ tube spacer, and the final resolution was $0.02208 \mathrm{~mm} /$ pixel. The shear strain at 1 $\mathrm{mm}$ distance from the notch from the samples is shown in Figure 4.15
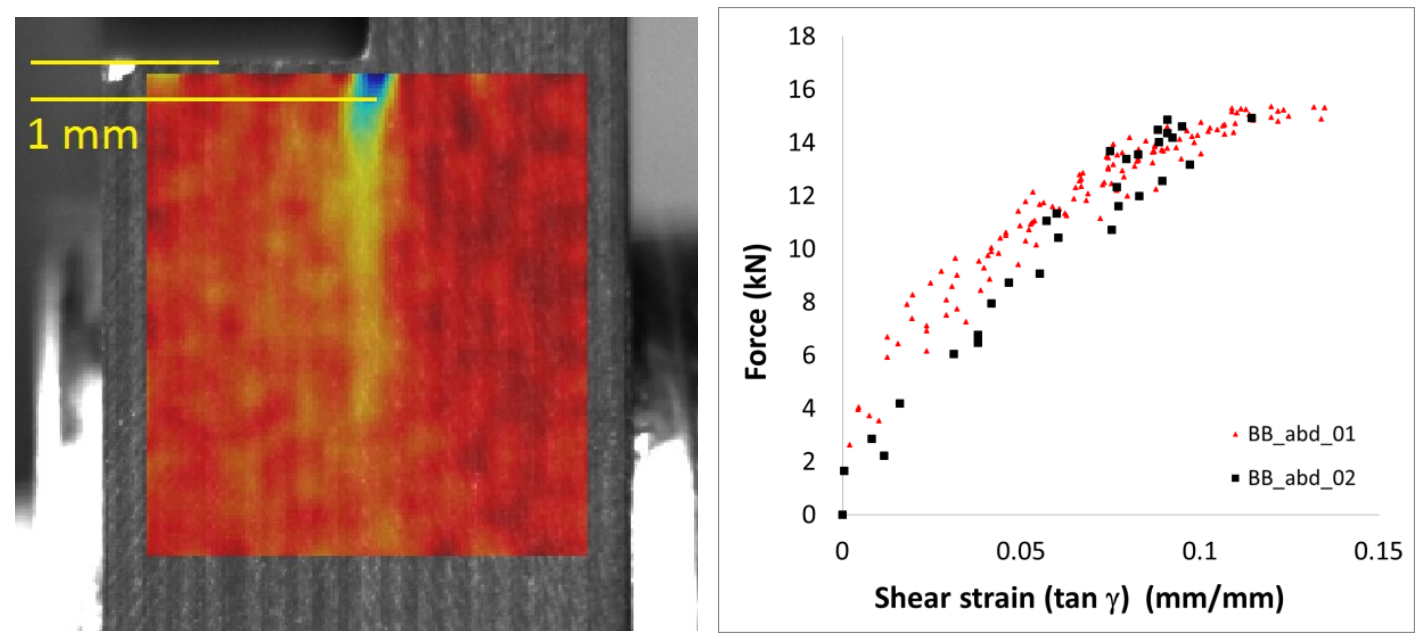

Figure 4.15 Sample abd_01and abd_02. Adhesive shear strain at $1 \mathrm{~mm}$ from the notch

From the sample $a b d \_02$, the relative displacement between adherends from the centre of the joint to outside the notch, at $17.5 \mathrm{~mm}$ distance is shown in Figure 4.16. For comparison purposes, the results from FE are also included; FEM elastoplastic and FEM CZM. 


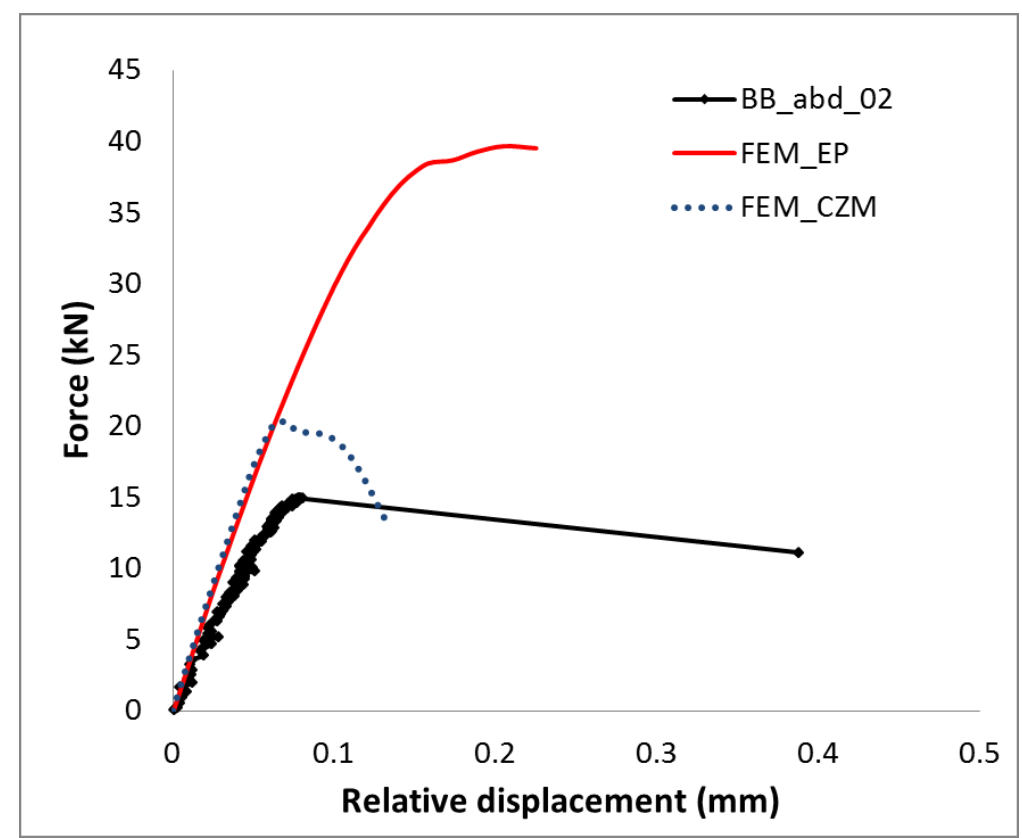

Figure 4.16 Sample abd_02. Force versus relative displacement using $17.5 \mathrm{~mm}$ reference length

The shear strain measurements for the sample $a b d \_02$ are shown in Figure 4.17. It can be observed that a higher scatter of the results occurs after $12 \mathrm{kN}$. This could be due to the instability produced by the fracture initiation in the adhesive layer.
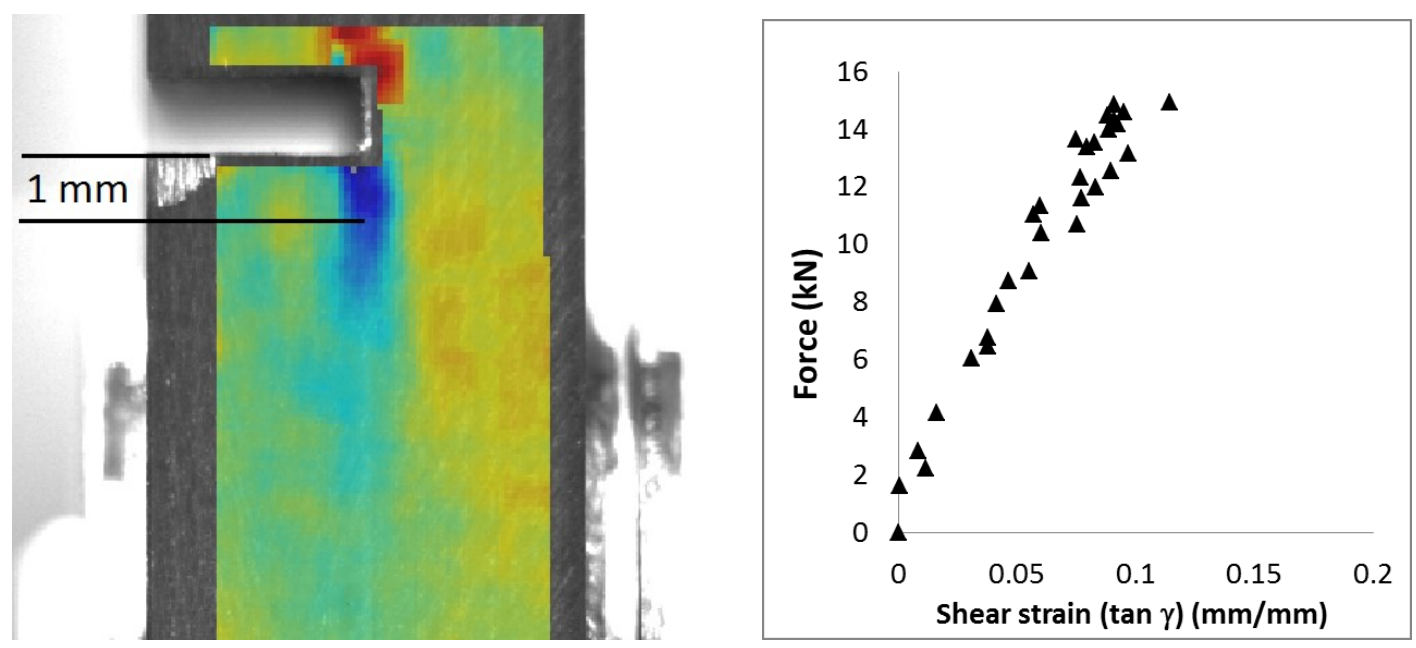

Figure 4.17 Sample abd_02. Adhesive shear strain at $1 \mathrm{~mm}$ distance from the notch 
The relative displacement for the sample $a b d \_03$ is shown in Figure 4.18, the displacements at lower forces were not captured because the adhesive was very stiff, and at the tested displacement rate, higher forces were reached in a short time, thus, the number of frames was limited at this stage of the test. However, a linear trend can be observed until $14.9 \mathrm{kN}$, where the adhesive failed.

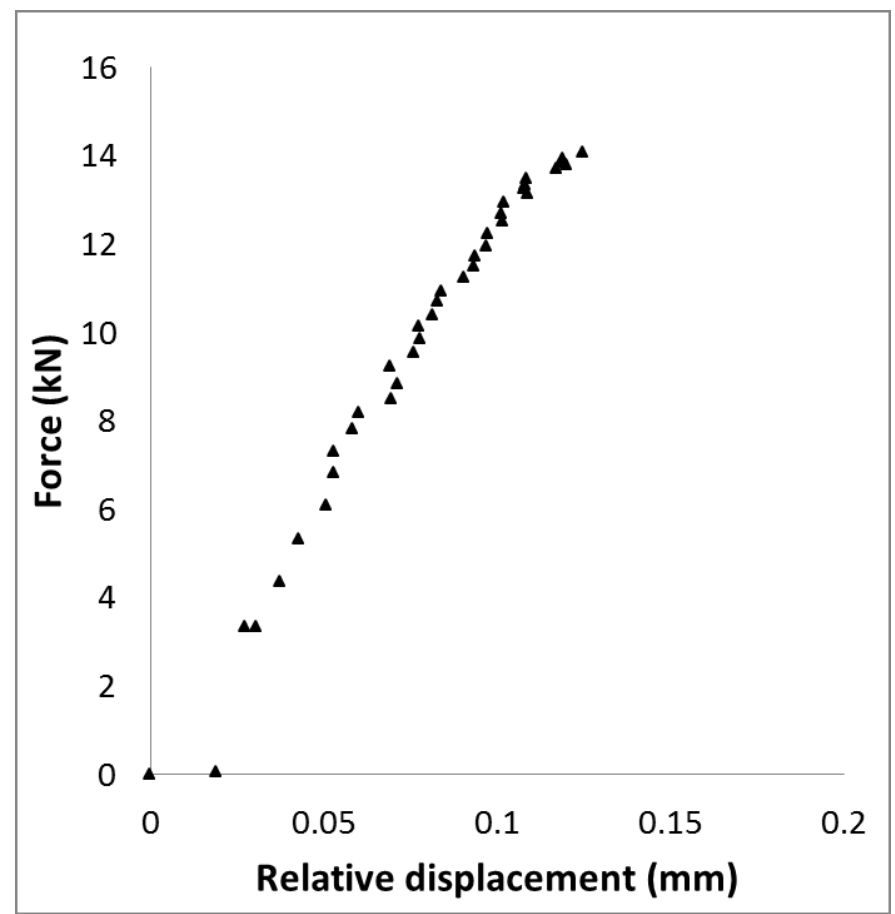

Figure 4.18 Sample abd_03. Force versus relative displacement using $35 \mathrm{~mm}$ reference length

It was not possible to extract the shear strain from the test of sample $a b d \_03$ due to the lower magnification and poor contrast. The test was carried out using a $50 \mathrm{~mm}$ lens without spacers, achieving a resolution of $0.0449 \mathrm{~mm} /$ pixel. Attempts to extract usable strain data were carried out, but the data was highly scattered. Finally, a comparison between shear strains on samples $a b d \_01$ and $a b d \_02$ are shown in Figure 4.19. 


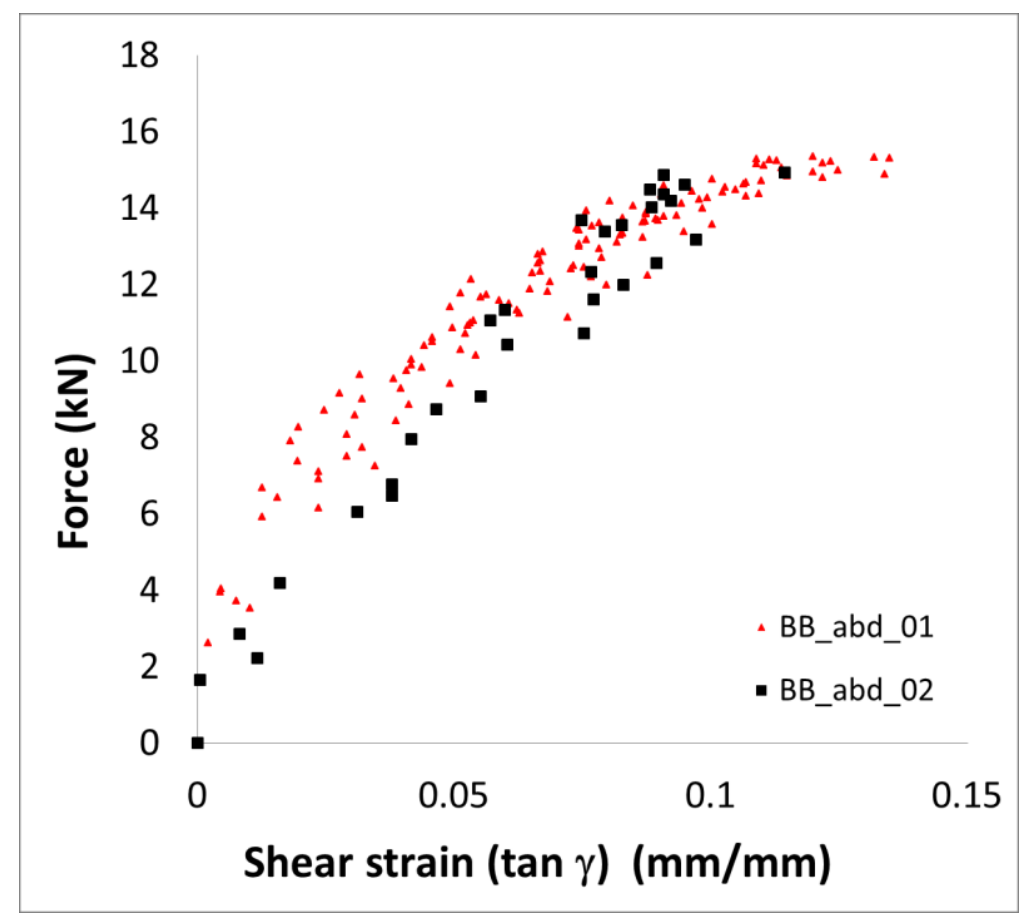

Figure 4.19 Samples abd_01 and abd_02. Comparison between shear strains at $1 \mathrm{~mm}$ distance from the notch

\subsubsection{Treatment bc}

The stroke versus force plots are shown in Figure 4.20. Similarly to the coupons from combination be, the adhesive fails in two stages. The first one is at an average value of $12.67 \mathrm{kN}$ of force and a $1.12 \mathrm{~mm}$ displacement. After this failure, the joint is stil capable of supporting increasing loading until the adhesive layer completely fails at an average value of $15.46 \mathrm{kN}$ of force and a $3.72 \mathrm{~mm}$ stroke displacement. At this point, the strength drops until an average value of approximately $11 \mathrm{kN}$ of force is reached, indicating that the joint has started to behave as a bolted joint. This process is depicted in coupon $b b \_01$ in Figure 4.21. Table 4.4 summarizes the stroke displacement results for all coupons of this treatment. 


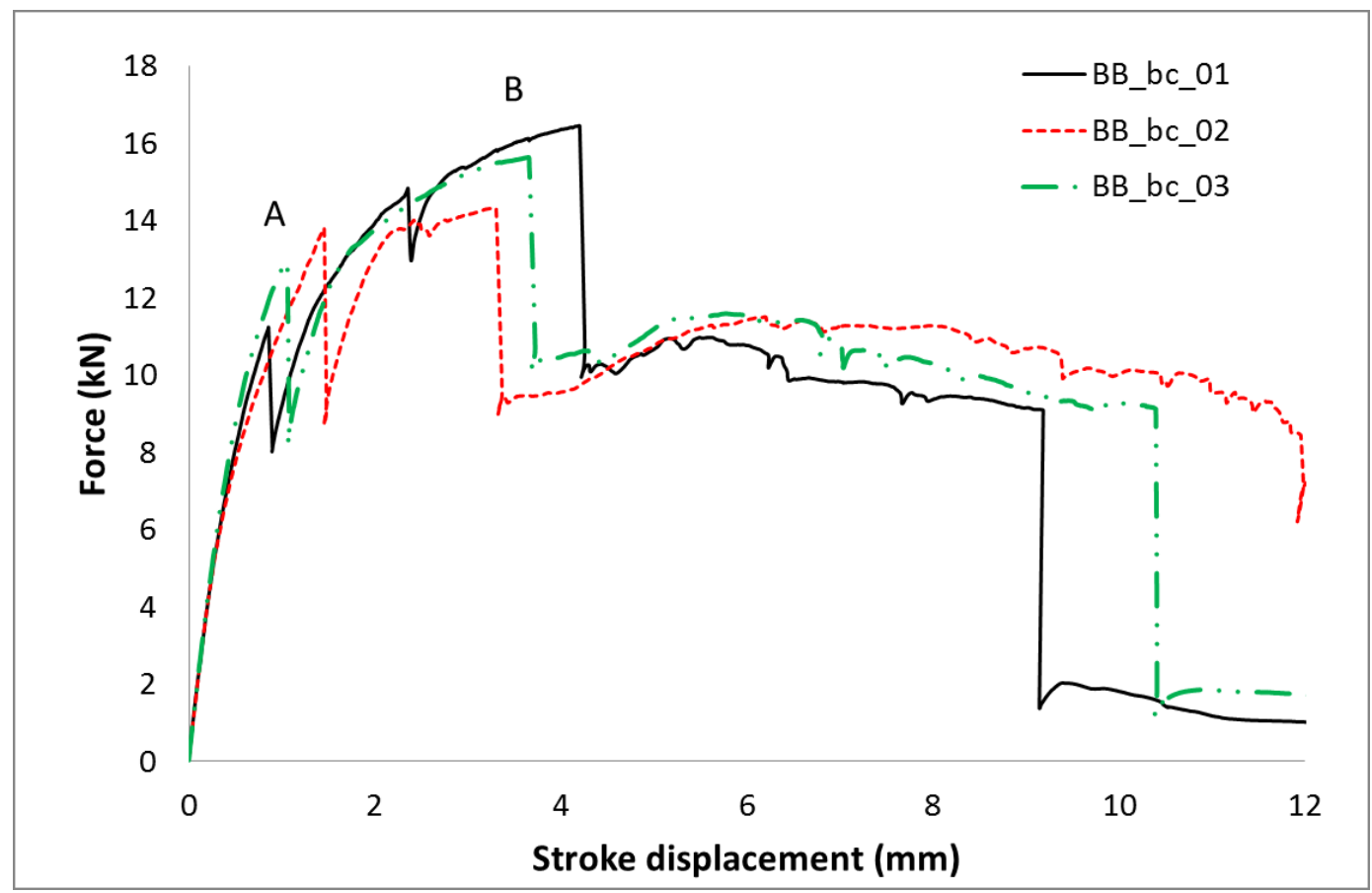

Figure 4.20 Combination $b c$, force versus displacement results

Table 4.4 Summary of results. Force versus displacement for treatment $b c$

\begin{tabular}{|c|c|c|c|c|c|c|c|c|c|c|}
\hline & \multicolumn{2}{|c|}{$\begin{array}{l}\text { Sample } \\
\text { bc_01 }\end{array}$} & \multicolumn{2}{|c|}{$\begin{array}{c}\text { Sample } \\
\text { bc_02 }\end{array}$} & \multicolumn{2}{|c|}{$\begin{array}{c}\text { Sample } \\
\text { bc_03 }\end{array}$} & \multicolumn{2}{|c|}{$\begin{array}{l}\text { Force } \\
\text { (kN) }\end{array}$} & \multicolumn{2}{|c|}{$\begin{array}{c}\text { Displacement } \\
\text { (mm) }\end{array}$} \\
\hline POINT & $\begin{array}{l}\text { Disp. } \\
(\mathrm{mm})\end{array}$ & $\begin{array}{l}\text { Force } \\
(\mathrm{kN})\end{array}$ & $\begin{array}{l}\text { Disp. } \\
(\mathrm{mm})\end{array}$ & $\begin{array}{l}\text { Force } \\
(\mathrm{kN})\end{array}$ & $\begin{array}{l}\text { Disp. } \\
\text { (mm) }\end{array}$ & $\begin{array}{l}\text { Force } \\
(\mathrm{kN})\end{array}$ & Avg. & $\begin{array}{l}\text { Sdt. } \\
\text { dev }\end{array}$ & Avg. & $\begin{array}{l}\text { Sdt. } \\
\text { dev }\end{array}$ \\
\hline A & 0.86 & 11.24 & 1.45 & 13.81 & 1.06 & 12.93 & 12.67 & 1.30 & 1.12 & 0.30 \\
\hline B & 4.20 & 16.46 & 3.30 & 14.30 & 3.66 & 15.61 & 15.46 & 1.08 & 3.72 & 0.45 \\
\hline
\end{tabular}




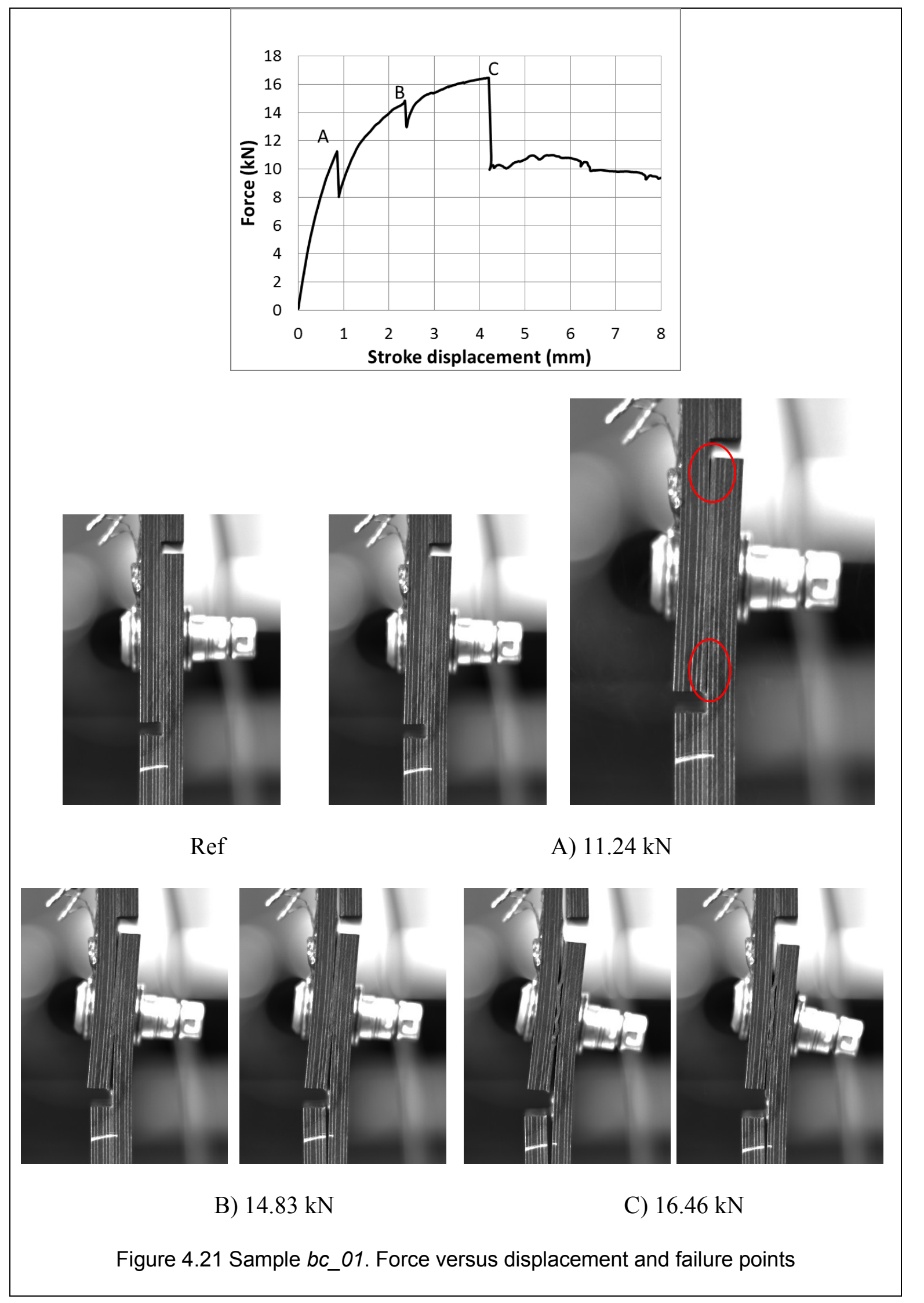


The DIC shear strain analysis on samples $b c \_01$ and $b c \_03$ was not carried out due to the poor resolution and contrast. From these samples, the relative displacement between adherends outside the notch at a $35 \mathrm{~mm}$ distance between points was measured, and the results are shown in Figure 4.22.

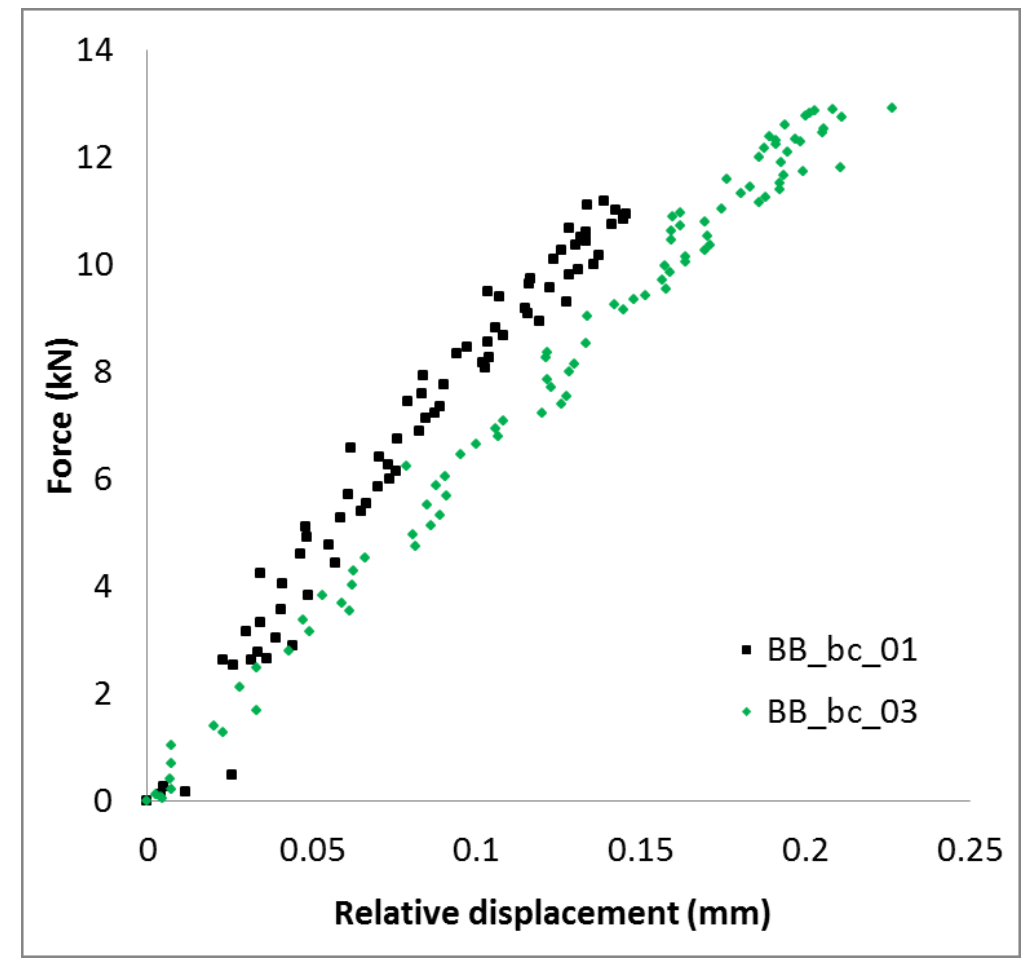

Figure 4.22 Sample bc_01 and sample bc_03. Force versus relative displacement using 35 $\mathrm{mm}$ reference length

For the sample $b c \_02$, the shear strain at $3 \mathrm{~mm}$ distance from the notch is shown along with the FEM results, in Figure 4.23. The maximum force that the joint is capable of supporting within the linear elastic limit (from TAST data) is about $3.5 \mathrm{kN}$; however, due to the scattered data this values is not fully conclusive. On the other hand, the joint is still capable of sustaining a shear strain of $0.1 \mathrm{~mm} / \mathrm{mm}$ before failure. 


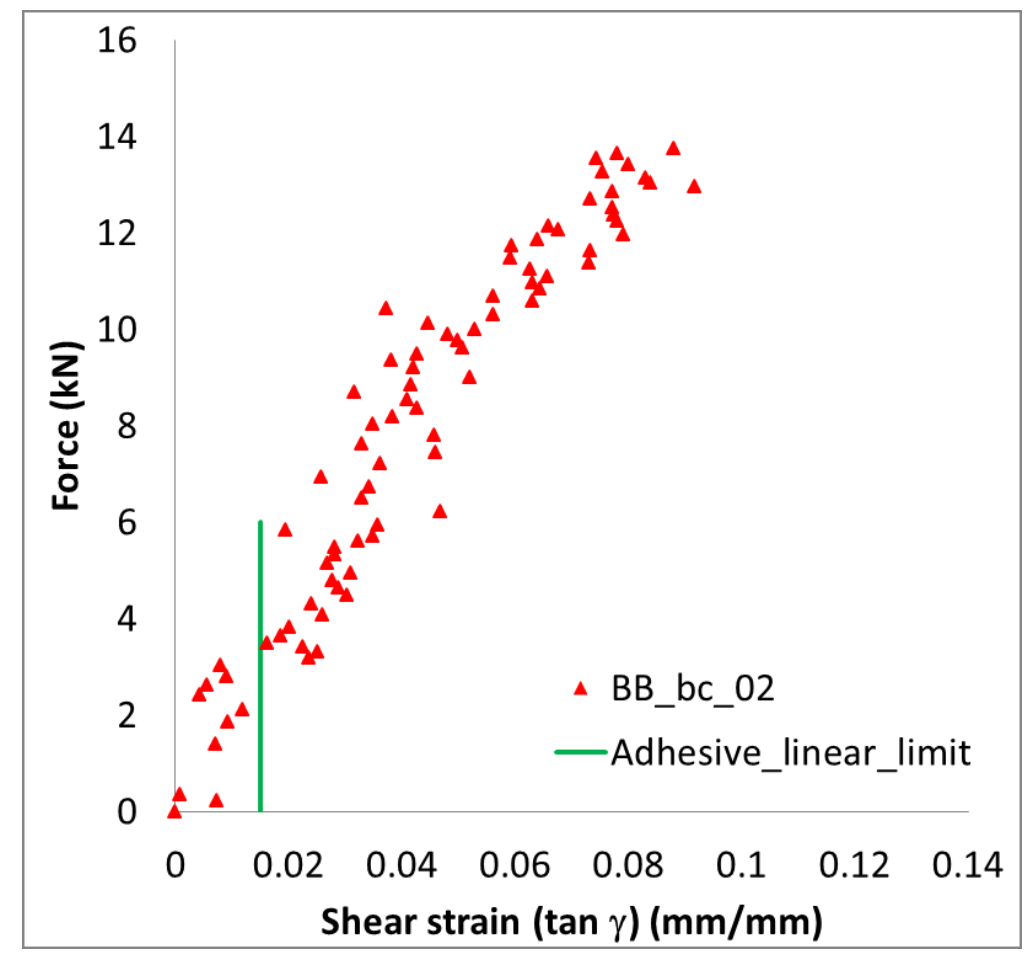

Figure 4.23 Sample bc_02. Shear strain at $3 \mathrm{~mm}$ distance from the notch

\subsubsection{Treatment abcde}

The testing on these specimens was affected by the test machine compliance as explained in section 3.6. Thus, further post-processing to remove the displacements due to this was carried out. Figure 4.24 shows the results of force versus displacement. It can be observed that in samples $a b c d e \_01$ and $a b c d e \_02$, the adhesive failed at $14.02 \mathrm{kN}$ and 14.75 $\mathrm{kN}$ respectively, after these forces, the load was taken by the bolts. At approximately $15 \mathrm{kN}$ of force, the rigid body motion appeared. On the other hand, the sample abcde_03 was able to support up to $15.8 \mathrm{kN}$ of force without failure, after this force the rigid body motion due to test machine compliance was acting in the coupon. However, the maximum strength was reached at $17.60 \mathrm{kN}$. At this point the adhesive 
failed with a subsequent drop in the strength, followed by a steady increment due the action of the bolt until its failure at $16.47 \mathrm{kN}$.

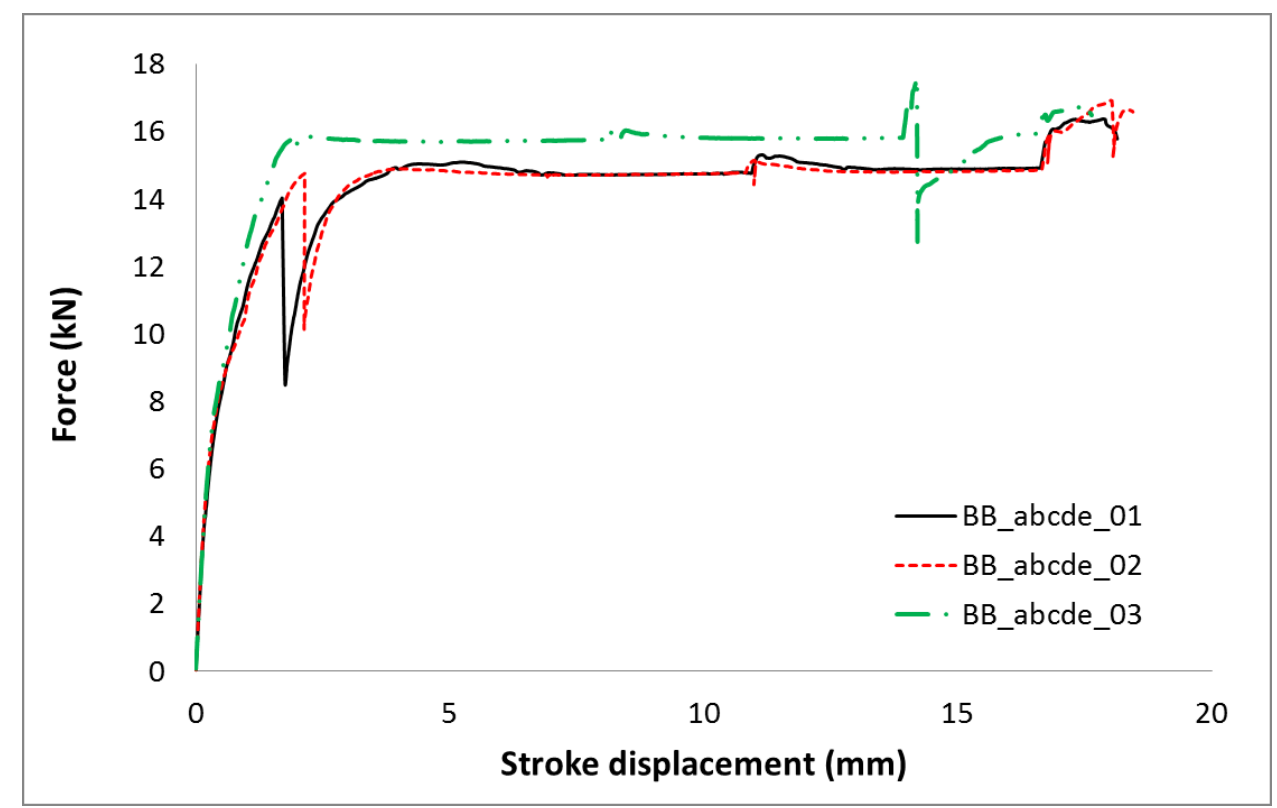

Figure 4.24 Combination abcde. Force versus displacement results

After correcting the displacements, the three samples seemed to have a similar behaviour as shown in Figure 4.25. For all the specimens, the failure starts in the adhesive, and afterwards, the bolt carries all the applied force until failure. There is no sign that the failure in the adhesive occurred in stages. The stroke displacements at failure were between 5.04 and $5.34 \mathrm{~mm}$.

The summary of the data about failure forces and stroke displacements is shown in Table 4.5. 


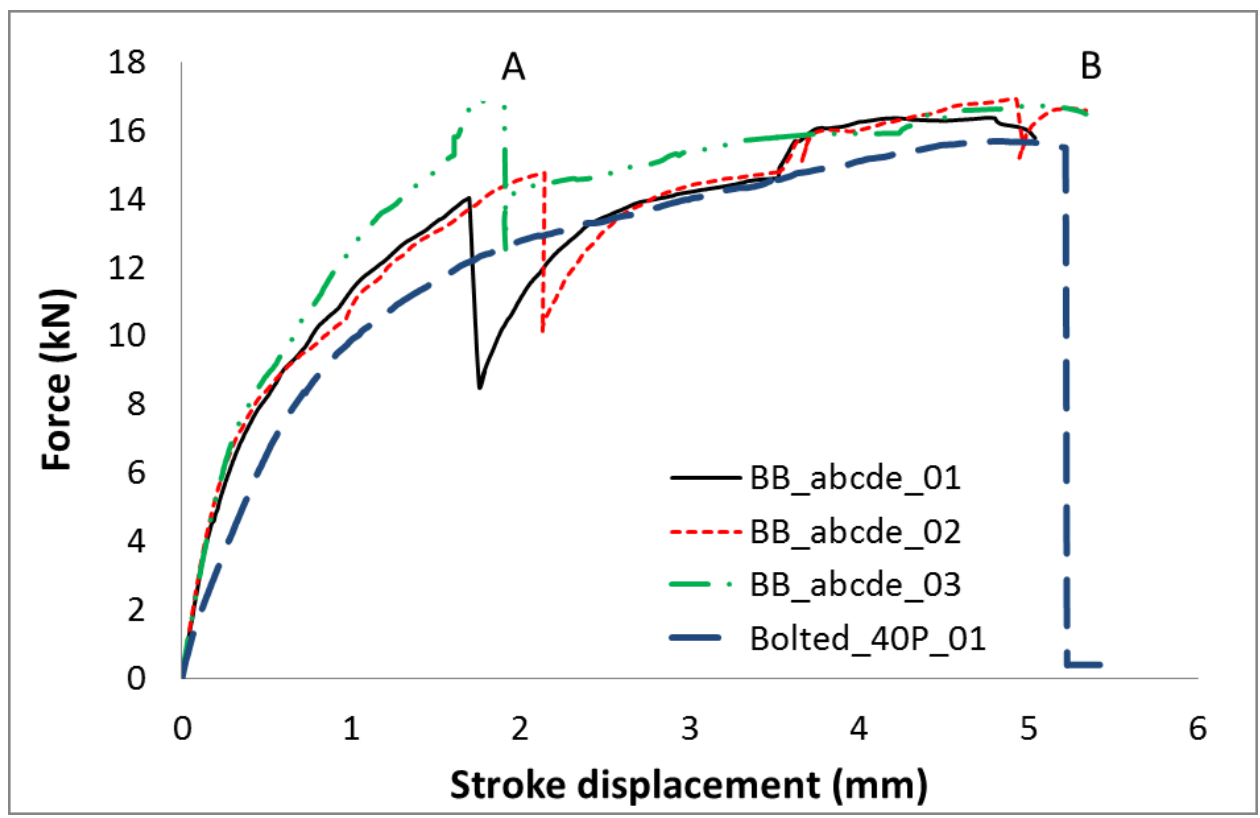

Figure 4.25 Combination abcde. Corrected force versus displacement results

Table 4.5 Summary of results. Force versus displacement for treatment abcde

\begin{tabular}{|c|c|c|c|c|c|c|c|c|c|c|}
\hline & \multicolumn{2}{|c|}{$\begin{array}{c}\text { Sample } \\
\text { abcde_01 }\end{array}$} & \multicolumn{2}{|c|}{$\begin{array}{c}\text { Sample } \\
\text { abcde_02 }\end{array}$} & \multicolumn{2}{|c|}{$\begin{array}{c}\text { Sample } \\
\text { abcde_03 }\end{array}$} & \multicolumn{2}{|c|}{$\begin{array}{l}\text { Force } \\
(\mathrm{kN})\end{array}$} & \multicolumn{2}{|c|}{$\begin{array}{c}\text { Displacement } \\
\text { (kN) }\end{array}$} \\
\hline POINT & $\begin{array}{l}\text { Disp. } \\
(\mathrm{mm})\end{array}$ & $\begin{array}{l}\text { Force } \\
(\mathrm{kN})\end{array}$ & $\begin{array}{l}\text { Disp. } \\
\text { (mm) }\end{array}$ & $\begin{array}{c}\text { Force } \\
(\mathrm{kN})\end{array}$ & $\begin{array}{l}\text { Disp. } \\
(\mathrm{mm})\end{array}$ & $\begin{array}{l}\text { Force } \\
\text { (kN) }\end{array}$ & Avg. & $\begin{array}{l}\text { Sdt. } \\
\text { dev }\end{array}$ & Avg. & $\begin{array}{l}\text { Sdt. } \\
\text { dev }\end{array}$ \\
\hline A & 1.70 & 14.02 & 2.14 & 14.75 & 1.90 & 17.60 & 15.46 & 1.88 & 1.91 & 0.22 \\
\hline B & 5.04 & 15.78 & 5.34 & 16.57 & 5.34 & 16.47 & 16.27 & 0.43 & 5.24 & 0.17 \\
\hline
\end{tabular}

The digital image correlation analysis was performed in different ways. For the specimen abcde_01, the shear strain was computed at several distances from the notch. Figure 4.26 shows the adhesive shear strain versus applied force at $3 \mathrm{~mm}$ from the notch. It can be observed that the failure occurred at a strain value of $0.038 \mathrm{~mm} / \mathrm{mm}$ and 14.02 $\mathrm{kN}$ of applied force. On the other hand, the adhesive behaviour remained elastic until approximately $7.3 \mathrm{kN}$. Along with the experimental results, the FEM results are plotted. 
It can be observed a very good agreement, approximately $95 \%$ at adhesive failure between experimental and numerical results.

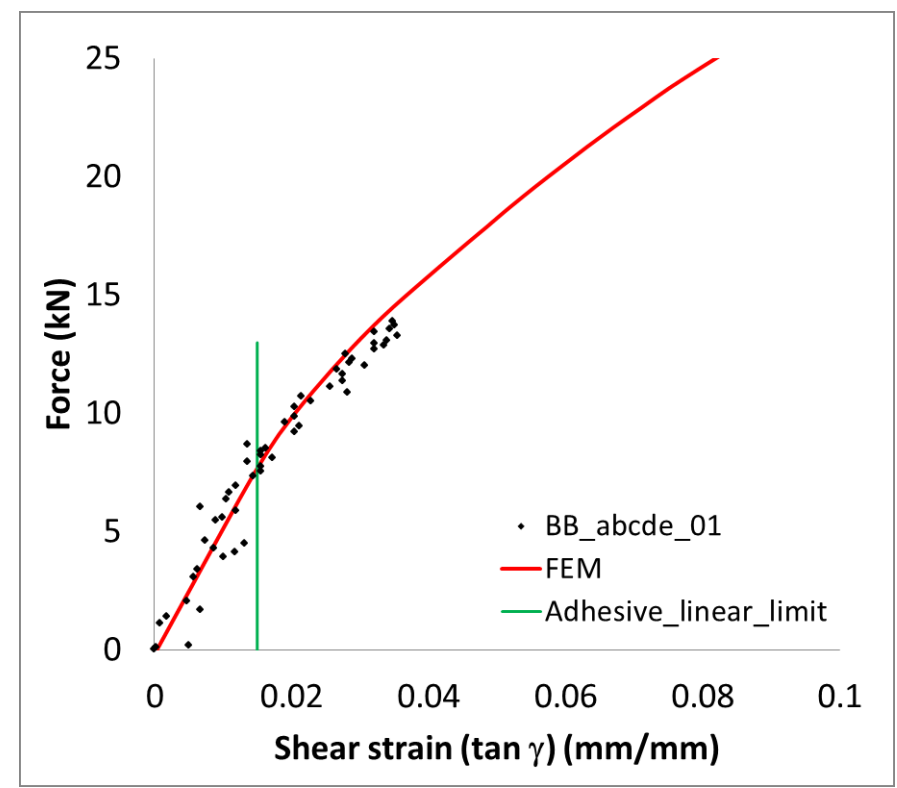

Figure 4.26 Sample abcde_01. Adhesive shear strain at $3 \mathrm{~mm}$ distance from the notch

In sample abcde_02, a different magnification was chosen in order to measure the local displacements at $35 \mathrm{~mm}$ reference length. The resolution was $0.046 \mathrm{~mm} / \mathrm{pixel}$. The Figure 4.27 shows the experimental measurements along with the FEM result. It can be observed that the jump in relative displacement is due to the adhesive fracture at 14.75 $\mathrm{kN}$. 


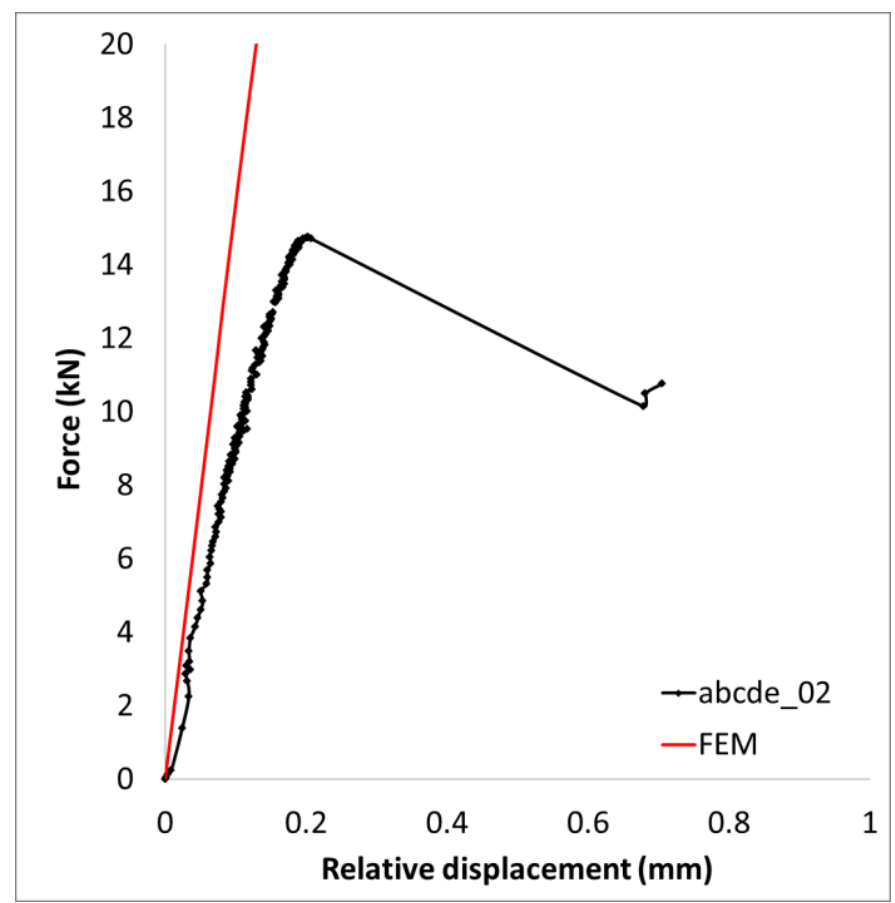

Figure 4.27 Sample abcde_02. Force versus relative displacement using $35 \mathrm{~mm}$ reference length

Finally, the preparation of sample abcde_03 was incorrect in terms of contrast. Due to this, the results from the DIC analysis showed excessive scatter and, therefore, they could not be used to assess neither the relative displacements nor the strains.

\subsubsection{Summary of Results with FM300-2M}

In terms of applied load and stroke displacement, it seems that joints with thicker adherends; 40 plies, are stronger, averaging $16.58 \mathrm{kN}$ and $16.27 \mathrm{kN}$ for treatments $a b d$ and abcde respectively.

The joints with thinner adherends; 24 plies, presented several drops in the strength before the total adhesive failure, and a maximum strength averaging $14.16 \mathrm{kN}$ and 15.46 $\mathrm{kN}$ respectively. By comparing these maximum strengths against the stronger joint with thicker adherend, the differences are $14.6 \%$ and $6.7 \%$ respectively. However, 
considering that the thicker joints are $60 \%$ heavier than the thinner joints, there is no net gain in specific strength or strength-to-weight. This is discussed in the ANOVA analysis.

The failure mode observed for thicker joints was due to bolt shear, and the maximum stroke displacements at failure were 5.39 and $5.24 \mathrm{~mm}$ respectively. In the joints with thinner adherends, the failure mode were due to shearout and cleavage. After the adhesive failure, the remaining average force was $9.02 \mathrm{kN}$ and $10.3 \mathrm{kN}$ respectively.

The effect of adhesive thickness, clearance and clamping areas cannot be assessed by simple comparison. These effects will be assessed using the ANOVA.

The analysis using DIC shows good data for displacement analysis. Conversely, the shear strain measurements are scattered due to the poor magnification chosen and the lack of contrast. The aim of the analysis for this adhesive, was to measure the relative displacement between adherends to compare against FEM models.

Finally, the discrepancies between the experiments are FEM models were between $15-25 \%$. In the case of the treatment abcde, the agreement was very good, approximately $95 \%$ at adhesive failure.

In order to improve these results, this setup was changed to measure the joints with EA9361adhesive. This was of particular interest because preliminary FEM analysis showed that load sharing up to $25 \%$ can be achieved for these joints. 


\subsection{Bolted Bonded Joints with EA9361 Adhesive}

The DIC setup was improved adding a very fine speckle pattern, and increasing the magnification to achieve resolutions up to 6 microns per pixel $(0.006 \mathrm{~mm} / \mathrm{pixel})$. The setup was also improved by increasing the number of frames per second to $4 \mathrm{~Hz}$.

The joints manufactured with the adhesive EA9361 correspond to treatments de, $a b d, b c$ and $a b c d e$ respectively. A summary of the factors and levels for these treatments is shown in Table 4.6.

Table 4.6 Experimental treatments with EA9361 adhesive

\begin{tabular}{|c|c|c|c|c|c|c|}
\hline \multirow{2}{*}{\multicolumn{2}{|c|}{ Treatment }} & \multicolumn{5}{|c|}{ FACTORS } \\
\hline & & $\mathbf{A}$ & B & C & D & E \\
\hline \multicolumn{2}{|c|}{ number } & Adherend & & Adhesive & Clamped & Clearance \\
\hline 1 & $\mathrm{de}$ & 3.29 & EA9361 & 0.2 & 387 & 0.1 \\
\hline 2 & $\bar{a}$ & 5.48 & EA9361 & 0.2 & 97 & 0 \\
\hline 5 & $\mathrm{~cd}$ & 3.29 & EA9361 & 0.5 & 387 & 0 \\
\hline 6 & ace & 5.48 & EA9361 & 0.5 & 97 & 0.1 \\
\hline
\end{tabular}

\subsubsection{Treatment $d e$}

The load versus stroke results for the treatment de are shown in Figure 4.28.

There are three points of interest; the first one labeled as A, is the first failure points, in this treatment; these points are also the maximum strength points. The maximum loads for specimens de_01 and de_02 were $14.19 \mathrm{kN}$ and $14.77 \mathrm{kN}$ respectively. After these points, there is a sudden drop in the strength, but the adhesive is still capable of sustaining a steadily increasing load along with the bolt. At point B, the adhesive fails 
completely and the remaining load is taken by the bolt. The forces are $13.06 \mathrm{kN}$ and $12.64 \mathrm{kN}$ respectively. The sample $d e \_03$ behaved similarly except that the maximum strength was at point B with $14.24 \mathrm{kN}$, approximately $1 \mathrm{kN}$ higher compared with the strength at point A, $13.52 \mathrm{kN}$. In addition, this coupon presented a higher displacement at the same point B.

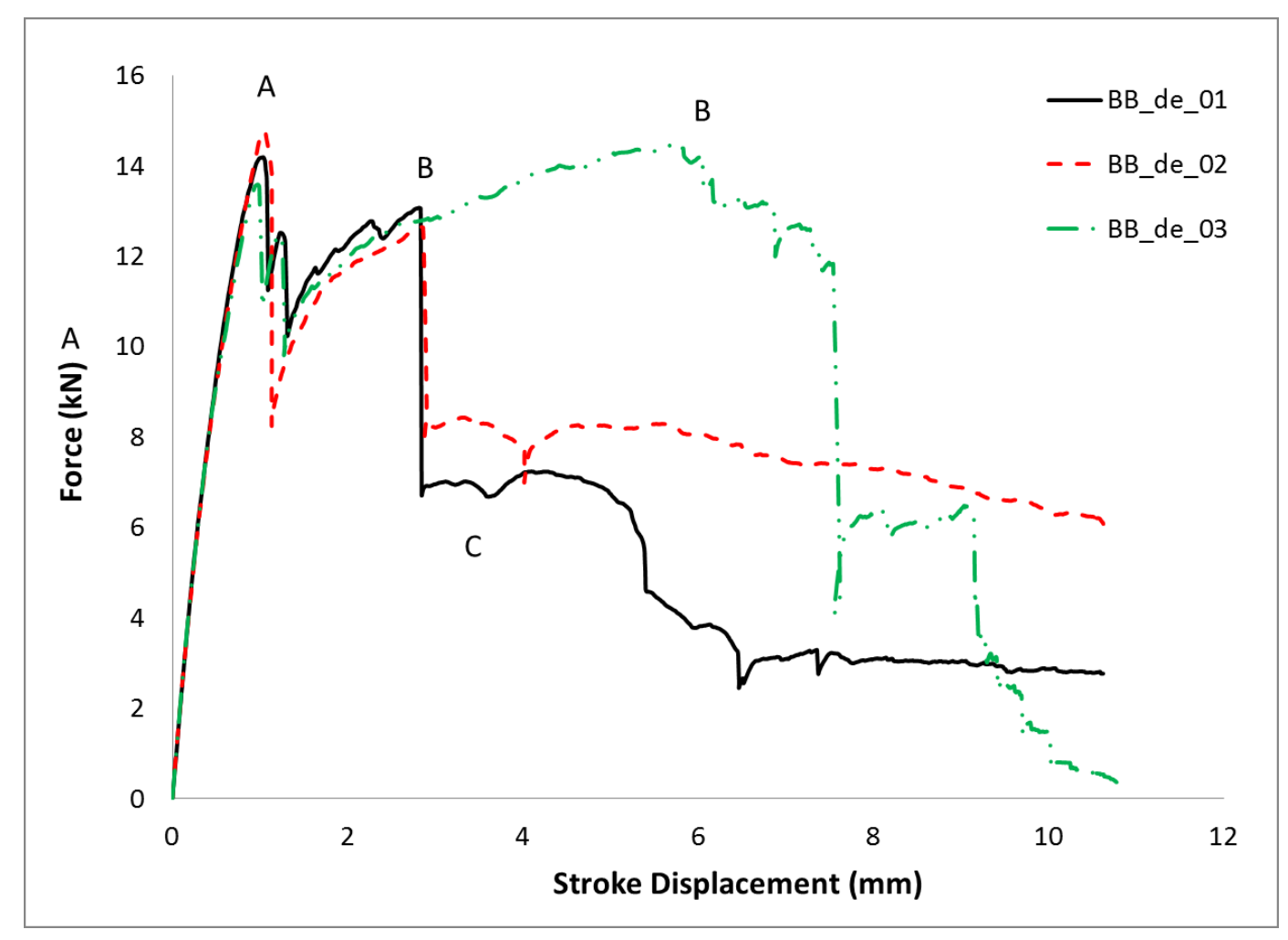

Figure 4.28 Combination de. Force versus displacement results

Finally, for all the coupons, the bolt carries the remaining load after point $C$, with averaging forces equal to $6.90,8.20$ and $6.12 \mathrm{kN}$, for the aforementioned specimens respectively. A summary of these points with their respective values is given in Table 4.7. 
Table 4.7 Summary of results. Force versus displacement for treatment de

\begin{tabular}{|c|c|c|c|c|c|c|c|c|c|c|}
\hline & \multicolumn{2}{c}{$\begin{array}{c}\text { Sample } \\
\text { de_01 }\end{array}$} & \multicolumn{2}{c|}{$\begin{array}{c}\text { Sample } \\
\text { de_02 }\end{array}$} & \multicolumn{2}{c|}{$\begin{array}{c}\text { Sample } \\
\text { de_03 }\end{array}$} & \multicolumn{2}{c|}{$\begin{array}{c}\text { Force } \\
(\mathrm{kN})\end{array}$} & \multicolumn{2}{c|}{$\begin{array}{c}\text { Displacement } \\
(\mathrm{mm})\end{array}$} \\
\hline POINT & $\begin{array}{c}\text { Disp. } \\
(\mathrm{mm})\end{array}$ & $\begin{array}{c}\text { Force } \\
(\mathrm{kN})\end{array}$ & $\begin{array}{c}\text { Disp. } \\
(\mathrm{mm})\end{array}$ & $\begin{array}{c}\text { Force } \\
(\mathrm{kN})\end{array}$ & $\begin{array}{c}\text { Disp. } \\
(\mathrm{mm})\end{array}$ & $\begin{array}{c}\text { Force } \\
(\mathrm{kN})\end{array}$ & $\begin{array}{c}\text { Avg. } \\
\text { Sdt. } \\
\text { dev }\end{array}$ & $\begin{array}{c}\text { Avg. } \\
\begin{array}{c}\text { Sdt. } \\
\text { dev }\end{array}\end{array}$ \\
\hline A & 1.04 & 14.19 & 1.05 & 14.77 & 0.99 & 13.52 & 14.16 & 0.63 & 1.03 & 0.031 \\
\hline B & 2.83 & 13.06 & 2.87 & 12.64 & 6.05 & 14.24 & 13.31 & 0.83 & 3.92 & 1.85 \\
\hline C & & 6.90 & & 8.20 & & 6.12 & 7.07 & 1.05 & & \\
\hline
\end{tabular}

A load versus stroke displacement comparison between bonded, bolted, and bolted bonded joints for this treatment is shown in Figure 4.29. For simplicity, just a repetition per test is shown. The hybrid joints behave similarly to the bonded joint until approximately $10 \mathrm{kN}$, after this point, and until the point of maximum strength, the synergistic effect between the adhesive and the bolt is minimal. The increase in strength between the bonded and bolted/bonded joint is very low, reaching a maximum difference of approximately $0.7 \mathrm{kN}$ at maximum strength. However, after the first drop in strength due to adhesive failure, it seems that the bolt started to carry a considerable amount of force along with the adhesive. This can be deducted due to the fact that the load dropped at approximately the same value as the bolted joint. At this point, the adhesive and bolt are supporting an increasing load, until a higher value compared with the bolted joint alone. Moreover, the increase in displacement, compared with bolted joint is also evident. Finally, the second drop in the force indicates that the adhesive failed completely and the joint starts to behave as a bolted joint. It can be observed that both bolted and bolted/bonded curves, match from this point until failure. 


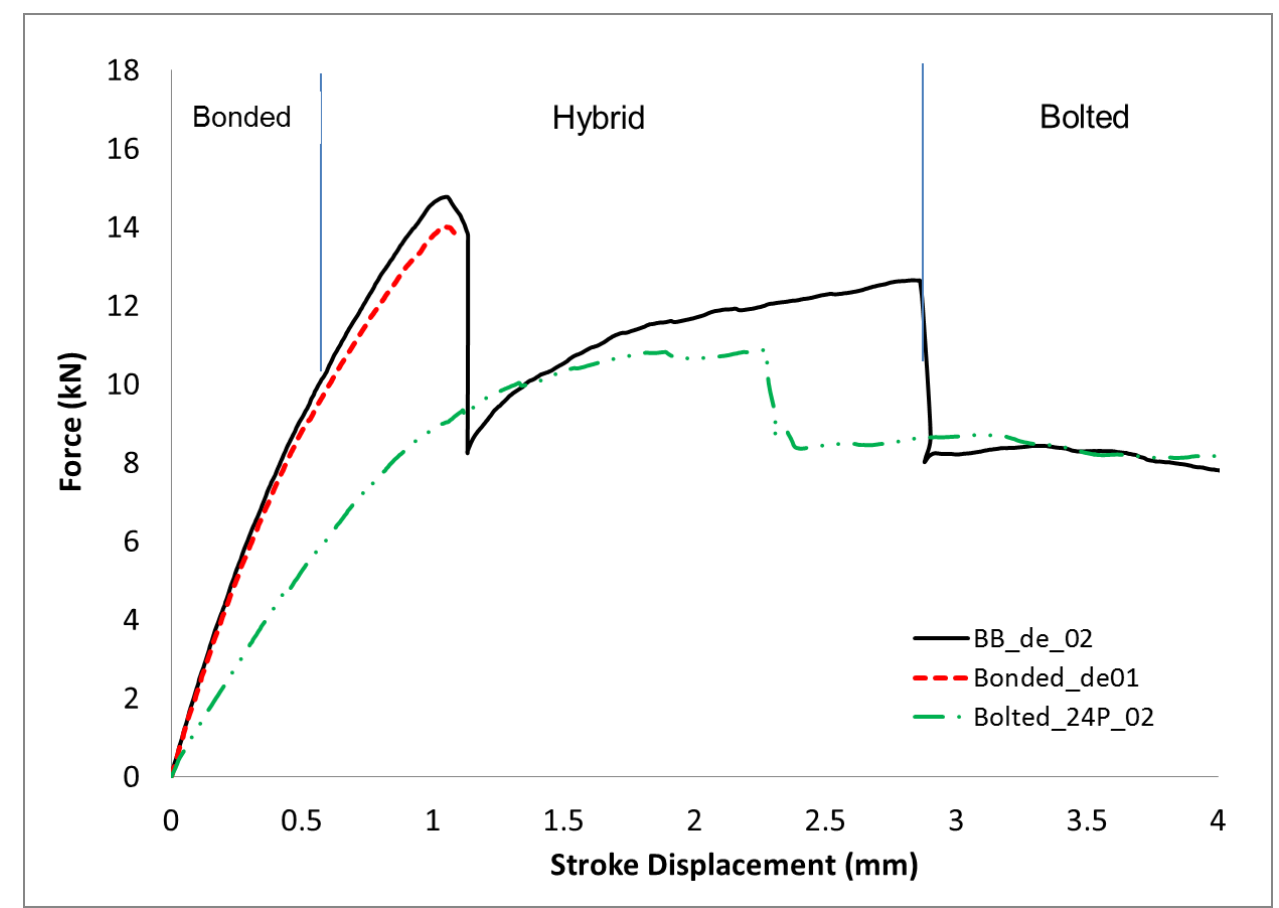

Figure 4.29 Treatment de and comparison with bolted and bonded joints. Force versus displacement results

For these specimens, the DIC analysis was carried out at high magnification near to the adhesive edge. However, the magnification was limited for two reasons. Firstly, the need of a large FOV to extract the displacements at several positions along the bondline length limits the magnification. Secondly, the need of a reference point to allow the software to convert the number of pixels into $\mathrm{mm}$ and vice versa. In this case, the reference length is the joint width. Figure 4.30 shows the reference images for DIC analysis for specimens de_01, de_02 and de_03 respectively. The speckle pattern was painted using an airbrush to allow a very fine speckle size. 


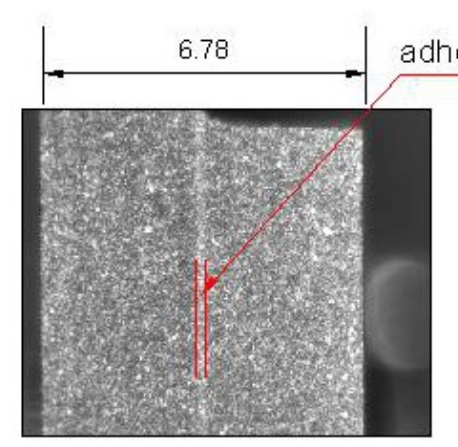

de_01

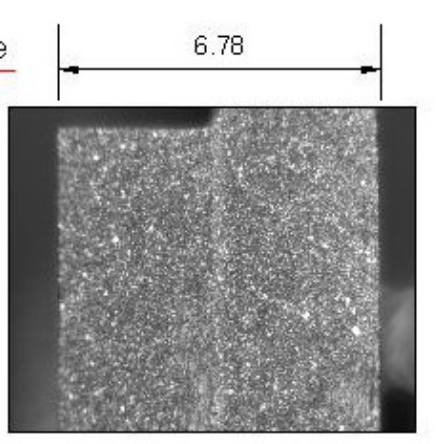

de_02

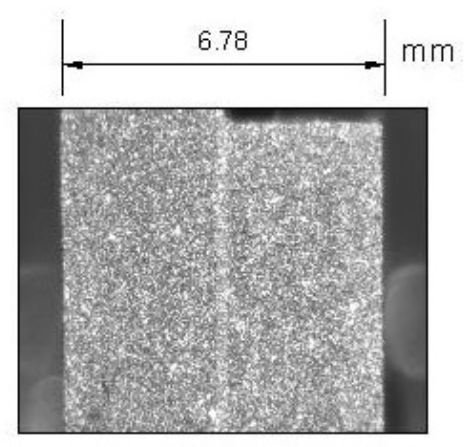

de_03

Figure 4.30 Reference DIC image for coupons de_01, de_02 and de_03

This configuration was achieved using a $50 \mathrm{~mm}$ camera with two camera tube spacers of 31 and $21 \mathrm{~mm}$ length, respectively. The resolution was $0.0067 \mathrm{~mm} /$ pixel or 6.7 microns per pixel. Along the bondline thickness of $0.2 \mathrm{~mm}$, there were approximately 30 pixels. Thus, the relative displacement between points at the interface between the adhesive and adherends has a very good resolution.

Images were taken every $0.25 \operatorname{secs}(4 \mathrm{~Hz})$, the same as the acquisition rate from the MTS test frame, allowing synchronization of data points with the captured images. The camera system memory allocation was limited to 361 images at full resolution (1024 $x$ 768). Thus, the number of frames for each specimen was limited to this quantity. The processed images were used to compute the shear strain at 1,2 and $3 \mathrm{~mm}$ from the notch. Figure 4.31 and Figure 4.32 shows the results. 

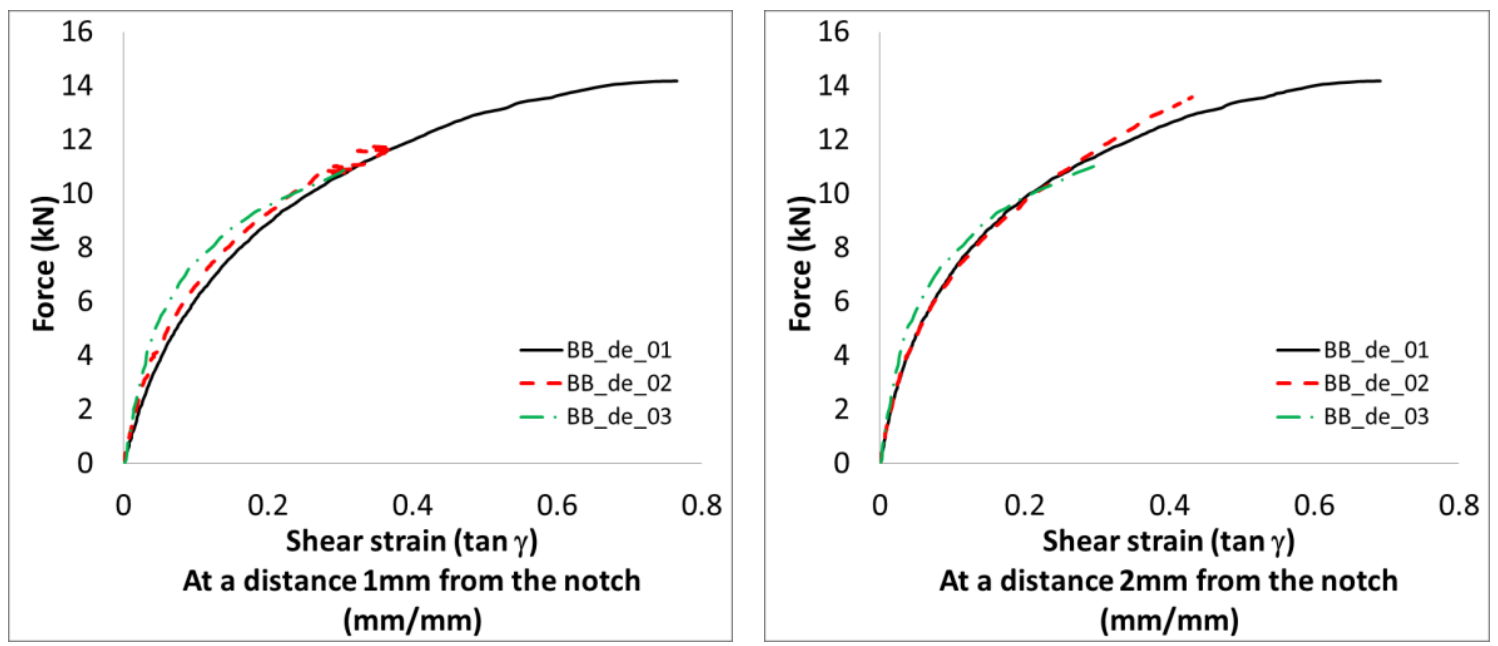

Figure 4.31 Samples de_01, de_02 and de_03. Adhesive shear strain at $1 \mathrm{~mm}$ and $2 \mathrm{~mm}$ from the notch

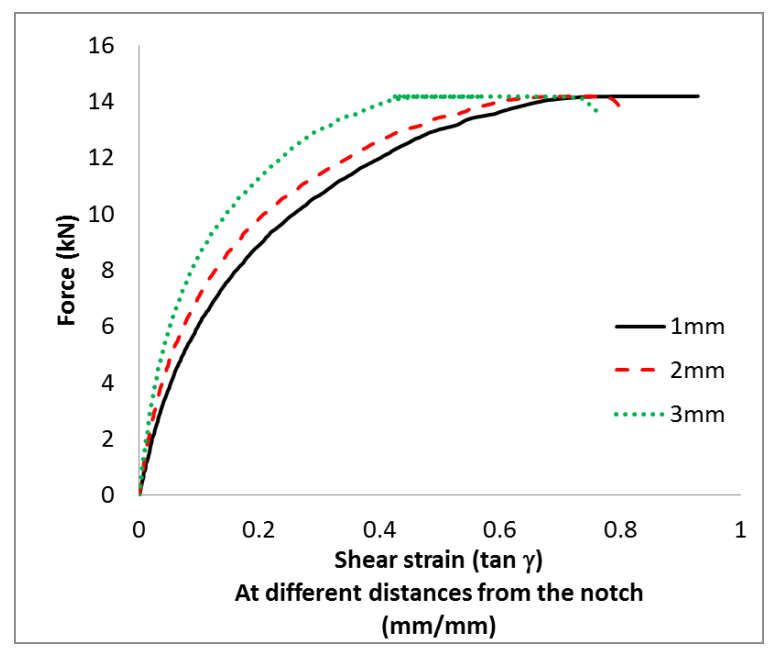

Figure 4.32 Sample de_02. Adhesive shear strain at different distances from the notch

Although the maximum strengths were reached at $14.19,14.77$, and $13.52 \mathrm{kN}$ for the tested specimens respectively, it was found, from the captured images, that the adhesive fracture process began before the maximum load was reached. For illustration purposes, only treatment de_01 is shown in Figure 4.33. It could be observed that the maximum strength is far from the point where the adhesive started to fracture. 


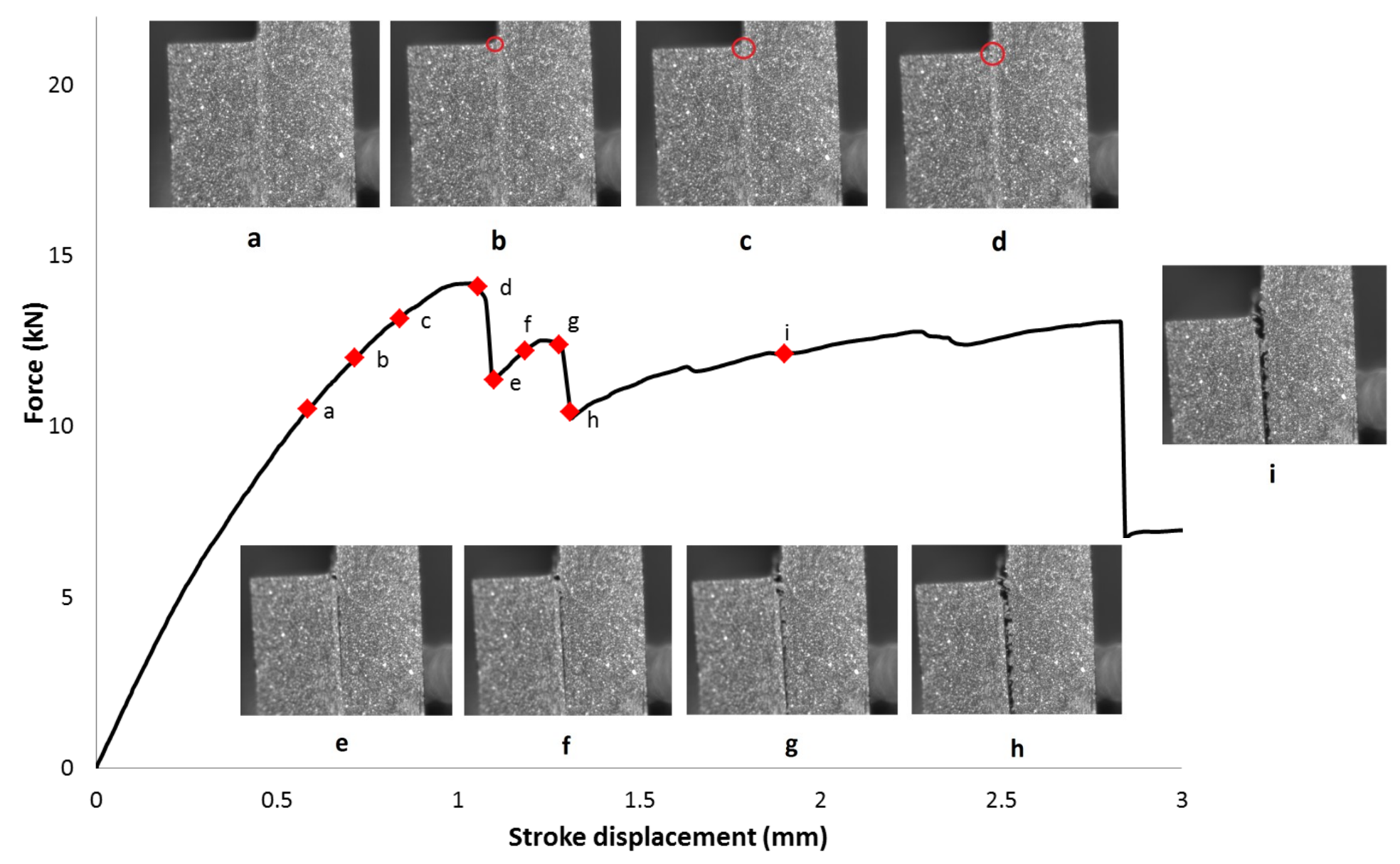

Figure 4.33 Joint failure sequence, treatment de, sample de_01. 
This situation was also evident when the shear strains at $1 \mathrm{~mm}$ distance from the notch were extracted; as shown in Figure 4.31, it was difficult to extract the shear strain for samples de_02 and de_03 after approximately $11 \mathrm{kN}$. Although the sample de_01 was capable of supporting higher strains, a change in the slope can be observed at $12 \mathrm{kN}$, evidencing that the fracture process started.

\subsubsection{Combination a}

Figure 4.34 shows the load versus stroke results for combination $a$. The three coupons $a \_01, a \_02$, and $a \_03$ presented the first failure point at 20.74, 21.06 and 19.24 $\mathrm{kN}$ of applied force respectively, with and average value of $20.34 \mathrm{kN}$. This failure point is also the maximum strength point. After reaching these values, the force dropped until $13.58,13.65$ and $12.73 \mathrm{kN}$ respectively, with and average value of $13.32 \mathrm{kN}$. The strength raised steadily after these points until 17.06 and17.57 kN for coupons $a \_01$ and a_02 respectively, when the joint failed suddenly. Coupon $a \_03$ presented a second drop in strength from $17.27 \mathrm{kN}$ to $13.47 \mathrm{kN}$, but it was still capable to sustain force, increasing the strength until $15.32 \mathrm{kN}$ where the sudden failure was reached. The summary of the maximum failure points is shown in Table 4.8. 


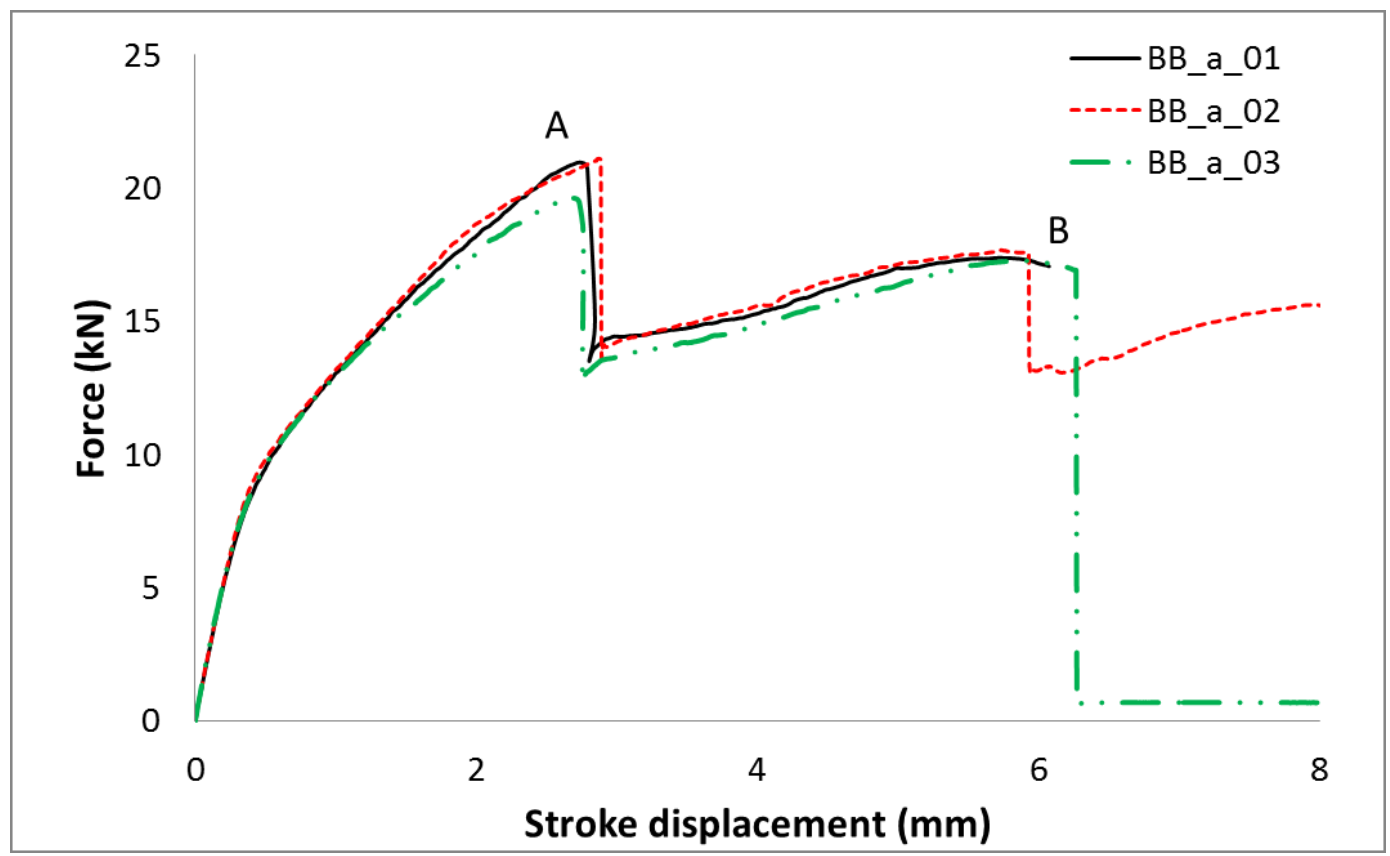

Figure 4.34 Treatment a. Force versus displacement results.

Table 4.8 Summary of results. Force versus displacement for treatment $a$

\begin{tabular}{|c|c|c|c|c|c|c|c|c|c|c|}
\hline & \multicolumn{2}{|c|}{$\begin{array}{c}\text { Sample } \\
\text { a_01 }\end{array}$} & \multicolumn{2}{|c|}{$\begin{array}{c}\text { Sample } \\
\text { a_02 }\end{array}$} & \multicolumn{2}{|c|}{$\begin{array}{c}\text { Sample } \\
\text { a_03 }\end{array}$} & \multicolumn{2}{|c|}{$\begin{array}{l}\text { Force } \\
\text { (kN) }\end{array}$} & \multicolumn{2}{|c|}{$\begin{array}{c}\text { Displacement } \\
\text { (mm) }\end{array}$} \\
\hline POINT & $\begin{array}{l}\text { Disp. } \\
\text { (mm) }\end{array}$ & $\begin{array}{l}\text { Force } \\
\text { (kN) }\end{array}$ & $\begin{array}{l}\text { Disp. } \\
(\mathrm{mm})\end{array}$ & $\begin{array}{l}\text { Force } \\
(\mathrm{kN})\end{array}$ & $\begin{array}{l}\text { Disp. } \\
(\mathrm{mm})\end{array}$ & $\begin{array}{l}\text { Force } \\
(\mathrm{kN})\end{array}$ & Avg. & $\begin{array}{l}\text { Sdt. } \\
\text { dev }\end{array}$ & Avg. & $\begin{array}{l}\text { Sdt. } \\
\text { dev }\end{array}$ \\
\hline A & 2.79 & 20.74 & 2.89 & 21.06 & 2.74 & 19.24 & 20.34 & 0.97 & 2.81 & 0.074 \\
\hline B & 6.08 & 17.06 & 5.93 & 17.57 & 5.88 & 17.27 & 17.30 & 0.25 & 5.96 & 0.100 \\
\hline
\end{tabular}

Similarly to the previous treatment tests, a comparison between bonded, bolted and bolted/bonded joints is shown in Figure 4.35. The hybrid joint behaves very similar to the bonded joint until approximately $8 \mathrm{kN}$. After this point, the bonded joint is stronger than the hybrid joint, with a load of $16.52 \mathrm{kN}$ at a displacement of $1.57 \mathrm{~mm}$. This point is approximately half the displacement of the hybrid joint at maximum strength, averaging $2.81 \mathrm{~mm}$ at $20.34 \mathrm{kN}$. It can be stated that the benefit of having a bolt is negative until 
this point; however, this situation could be incorrect. The stroke readings could be prone to small slippage because the grips used for these specimens were customized to fit the joint width. Due to the very small differences in force and local displacements between the bonded and hybrid joints, the load taken by the bolt is not accurate measured using the load stroke data.

Following the discussion, using the force versus displacement analysis, after the adhesive failure, the load is taken by the bolt.

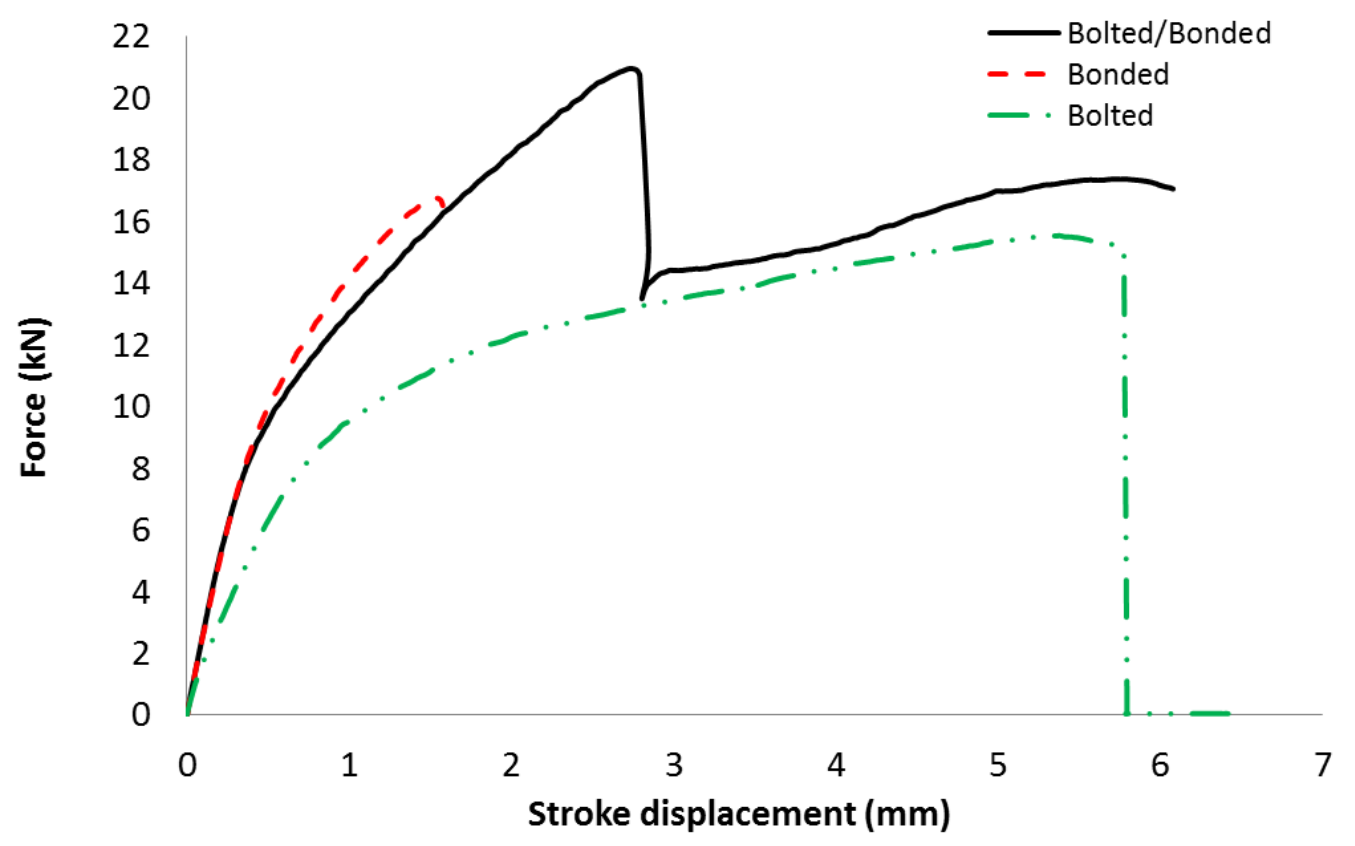

Figure 4.35 Treatment $a$ and comparison between bonded and bolted joints. Force versus displacement results

The DIC analysis was carried out using a $50 \mathrm{~mm}$ lens with two camera spacers, 31 and $13 \mathrm{~mm}$. With this configuration, a resolution of $0.0073 \mathrm{~mm} /$ pixel (7 microns) was achieved. The images were captured at four frames per second and synchronized with the MTS. The full FOV was used with 1024x768 pixels available and the total amount of available memory permitted the capture of 360 pictures. Compared with the coupons with 
$3.29 \mathrm{~mm}$ of thickness, these specimens were captured using a portion of the full thickness. The reason for this was the necessity to obtain both higher magnifications and points to define the reference length to compute the pixels $/ \mathrm{mm}$ during the DIC analysis. Therefore, the references were the edges and the notch and the adhesive bondline as shown in Figure 4.36. Similarly to the coupons from treatment de, the processed images were used to compute the shear strains at 1,2 and $3 \mathrm{~mm}$ distance from the notch.

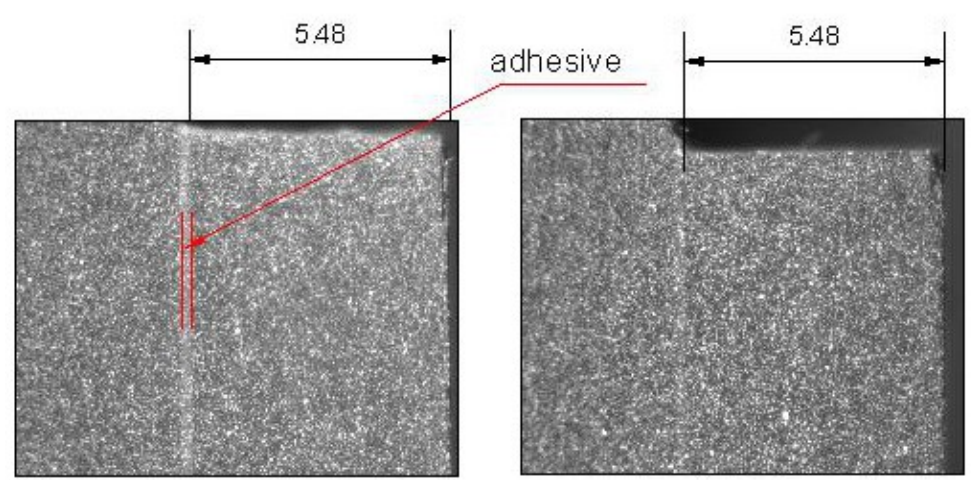

a_01 a_02

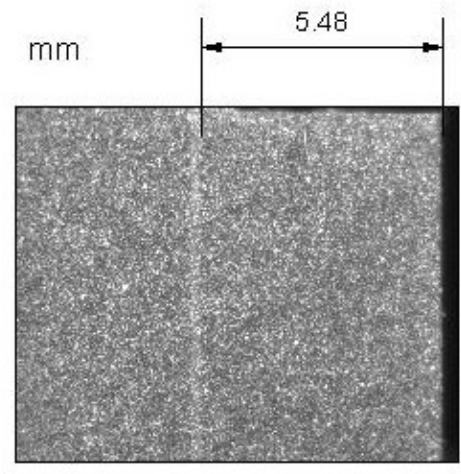

a_03

Figure 4.36 Reference DIC image for coupons a_01, a_02 and a_03.

From the DIC analysis, the shear strains are shown in Figure 4.37. A zone where the readings were unstable can be observed after $0.4-0.5 \mathrm{~mm} / \mathrm{mm}$ of shear strain and between $13 \mathrm{kN}-15.5 \mathrm{kN}$. Although at these regions the shear strain was unstable, the explanation is that the fracture process in the adhesive affected the measurements, this process produced jumps in the displacement field, and therefore, in the strains. 

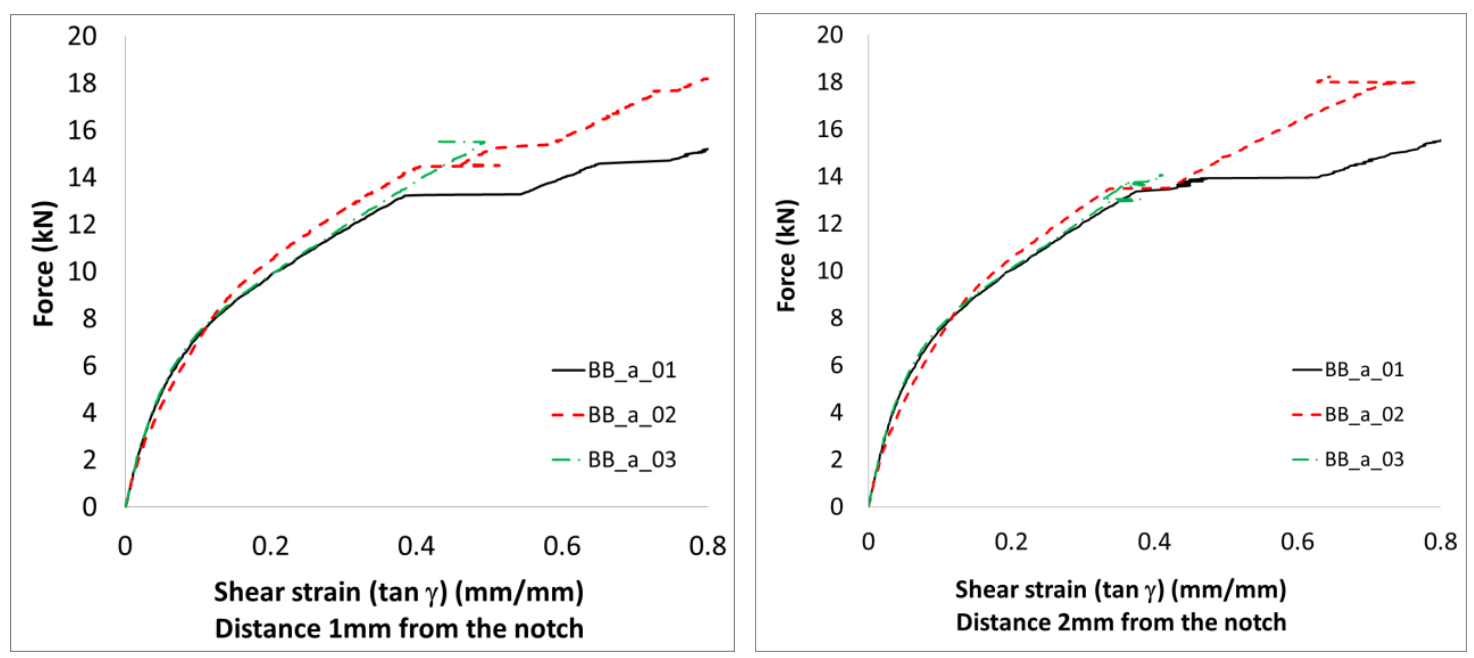

Figure 4.37 Samples a_01, a_02 and a_03. Adhesive shear strain at $1 \mathrm{~mm}$ and $2 \mathrm{~mm}$ from the notch

Figure 4.38 depicts the failure process in bonded and bolted bonded joints for treatment $a$. Again, due to the good repeatability in the measurements, only one coupon is used for comparison purposes. It can be observed that the failure starts almost at the same force values; $12.64 \mathrm{kN}$ and $13.04 \mathrm{kN}$ for the bonded and bolted/bonded joints respectively. In the case of the bonded joint, the crack grows steadily until it fails suddenly at $16.5 \mathrm{kN}$. The same pattern is observed for the bolted/bonded joint, however, the total failure is not presented until $20.7 \mathrm{kN}$ of force is reached. The average values for the treatment is $20.34 \mathrm{kN}$. From these results it is obvious that the effect of the bolt in the joint performance is to support the extra strength compared with the bonded joint. However, the bolted/bonded joint is not $100 \%$ healthy anymore because the adhesive started the fracture process. The region where the bolted/bonded joint is completely undamaged is far below the maximum strength value. Moreover, the strain analysis and FE results suggest a load sharing of $10 \%$ at $12 \mathrm{kN}$. Therefore, the joint is $90 \%$ adhesively bonded joint and the advantage of the hybridization is almost negligible. At $8 \mathrm{kN}$ of force the predicted load sharing is barely $2 \%$. 


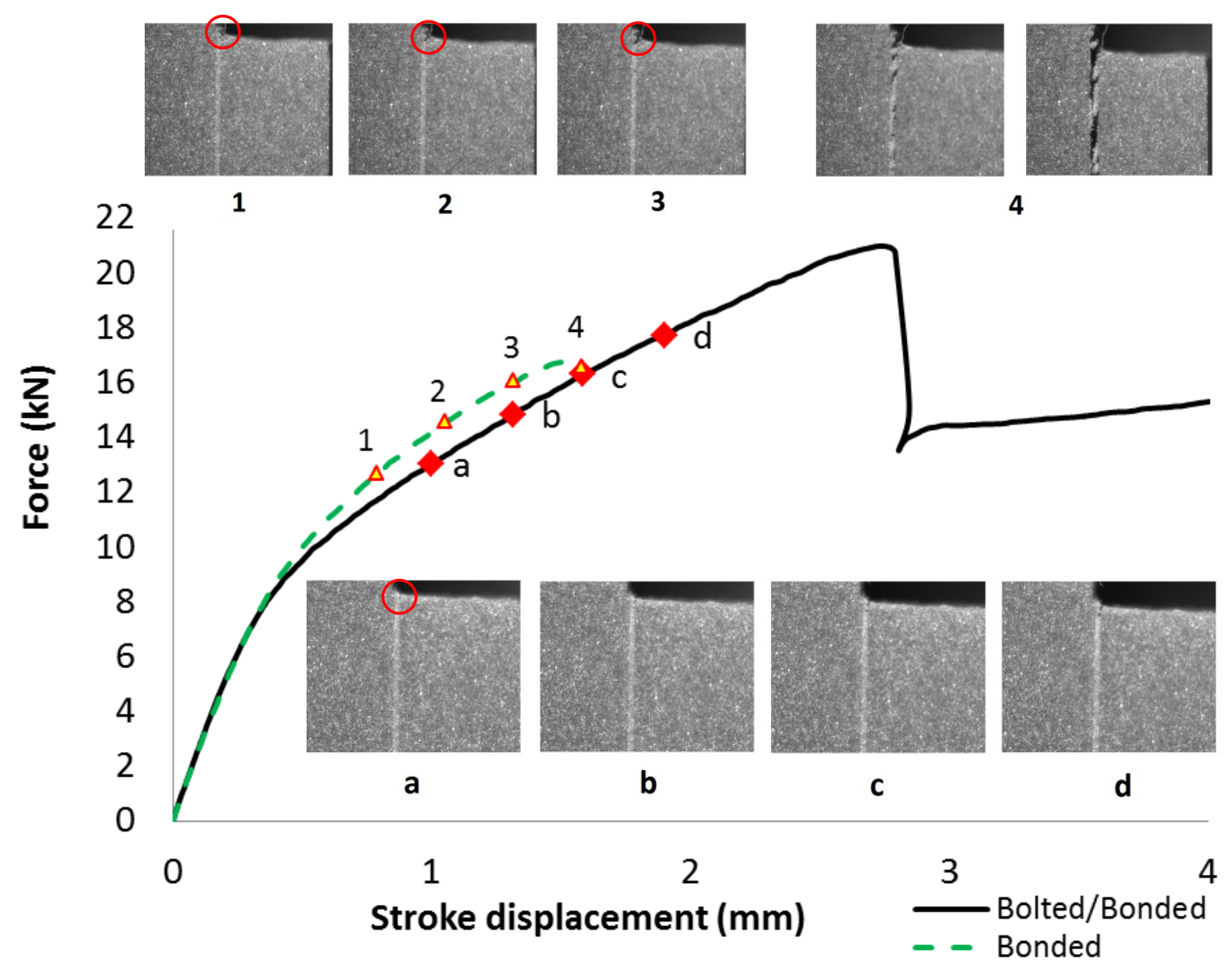

Figure 4.38 Failure process in the bolted bonded joint compared with the bonded joints.

Treatment a, coupon a_01 


\subsubsection{Combination cd}

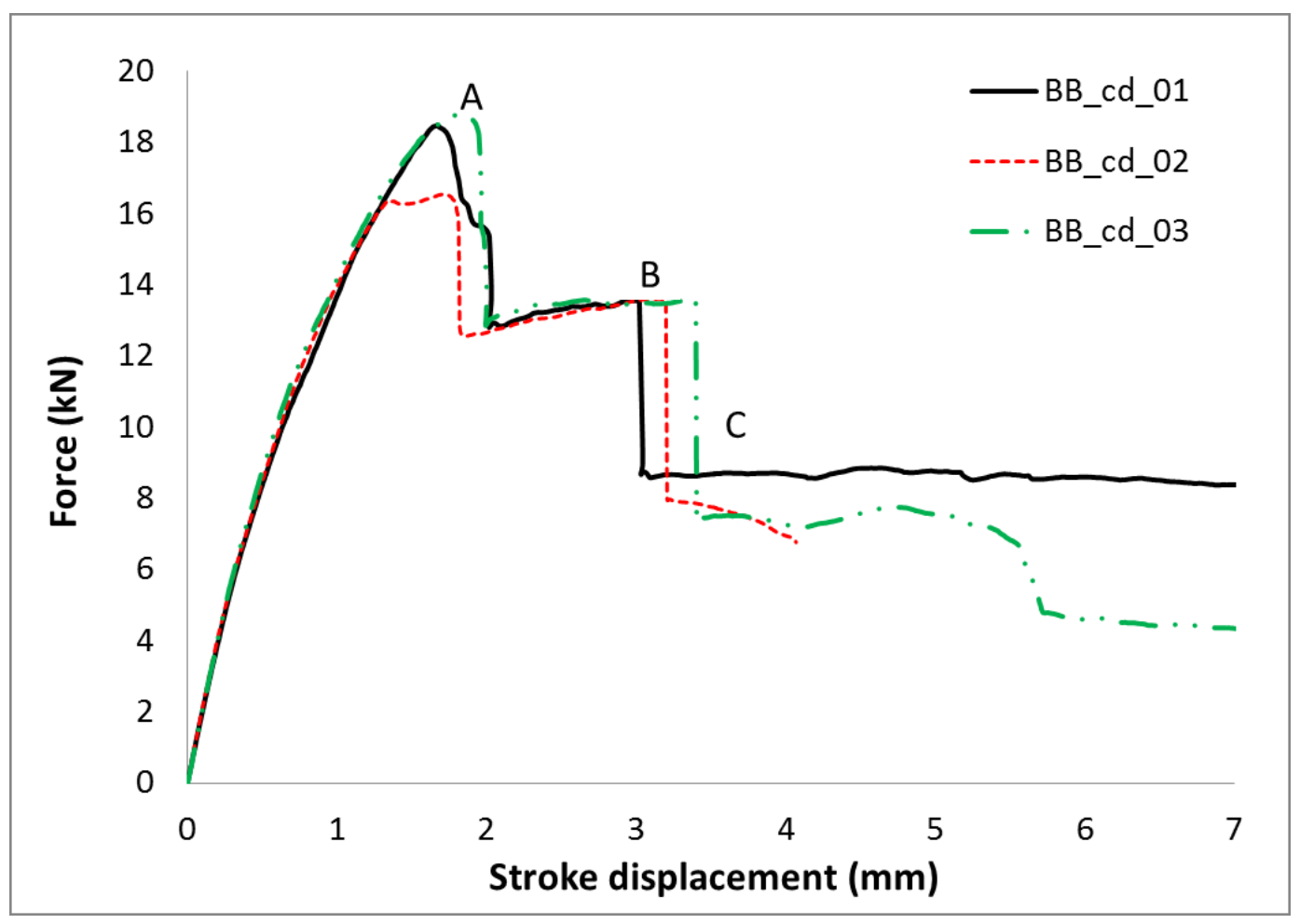

Figure 4.39 Treatment $c d$. Force versus displacement results.

In this combination, there are also three points of interest as shown in Figure 4.39. The first one labeled as $\mathrm{A}$, is the maximum load for specimens $c d_{-} 01, c d \_02$ and cd_03 with forces of $18.48 \mathrm{kN}, 16.54 \mathrm{kN}$ and $18.78 \mathrm{kN}$ respectively. At these points, it seems that the adhesive starts the failure process; however, it is still capable of sustaining load along with the bolts. At point B, it fails completely, and the remaining load is taken by the bolt. The forces are $13.53 \mathrm{kN}, 13.53 \mathrm{kN}$ and $13.57 \mathrm{kN}$ for the three specimens respectively. Finally, the bolt is carrying the remaining load after point $\mathrm{C}$, with forces equal to $8.73 \mathrm{kN}, 7.96 \mathrm{kN}$ and $7.38 \mathrm{kN}$ for the aforementioned specimens respectively. A summary of these points with their respective values is given in Table 4.9. 
Table 4.9 Summary of results. Force versus displacement for treatment $c d$

\begin{tabular}{|c|c|c|c|c|c|c|c|c|c|c|}
\hline & \multicolumn{2}{|c|}{$\begin{array}{c}\text { Sample } \\
\text { cd_01 }\end{array}$} & \multicolumn{2}{c|}{$\begin{array}{c}\text { Sample } \\
\text { cd_02 }\end{array}$} & \multicolumn{2}{c|}{$\begin{array}{c}\text { Sample } \\
\text { cd_03 }\end{array}$} & \multicolumn{2}{c|}{$\begin{array}{c}\text { Force } \\
(\mathrm{kN})\end{array}$} & \multicolumn{2}{c|}{$\begin{array}{c}\text { Displacement } \\
(\mathrm{mm})\end{array}$} \\
\hline POINT & $\begin{array}{c}\text { Disp. } \\
(\mathrm{mm})\end{array}$ & $\begin{array}{c}\text { Force } \\
(\mathrm{kN})\end{array}$ & $\begin{array}{c}\text { Disp. } \\
(\mathrm{mm})\end{array}$ & $\begin{array}{c}\text { Force } \\
(\mathrm{kN})\end{array}$ & $\begin{array}{c}\text { Disp. } \\
(\mathrm{mm})\end{array}$ & $\begin{array}{c}\text { Force } \\
(\mathrm{kN})\end{array}$ & Avg. & $\begin{array}{c}\text { Std. } \\
\text { dev. }\end{array}$ & Avg. & $\begin{array}{c}\text { Std. } \\
\text { dev. }\end{array}$ \\
\hline A & 1.67 & 18.48 & 1.72 & 16.54 & 1.83 & 18.78 & 17.93 & 1.22 & 1.74 & 0.08 \\
\hline B & 3.02 & 13.53 & 3.20 & 13.53 & 3.40 & 13.57 & 13.54 & 0.03 & 3.20 & 0.19 \\
\hline C & 3.04 & 8.73 & 3.21 & 7.96 & 3.40 & 7.38 & 8.02 & 0.67 & 3.22 & 0.18 \\
\hline
\end{tabular}

The DIC analysis was carried out using a $50 \mathrm{~mm}$ lens with two tube spacers with 31 and $21 \mathrm{~mm}$ lengths respectively. The resolution was $0.0066 \mathrm{~mm} /$ pixel or $6.7 \mathrm{microns}$ per pixel. Along the bondline thickness of $0.5 \mathrm{~mm}$, there were approximately 75 pixels. Samples sample $c d \_01$, and cd_03 were processed using 360 images. On the other hand, sample $c d \_02$ was processed using 250 images because the adhesive started the fracture process at $16.24 \mathrm{kN}$. This force is coincident with the acquisition time recorded.

The strain at several distances from the notch was computed after the capturing process as shown in Figure 4.40 and Figure 4.41.
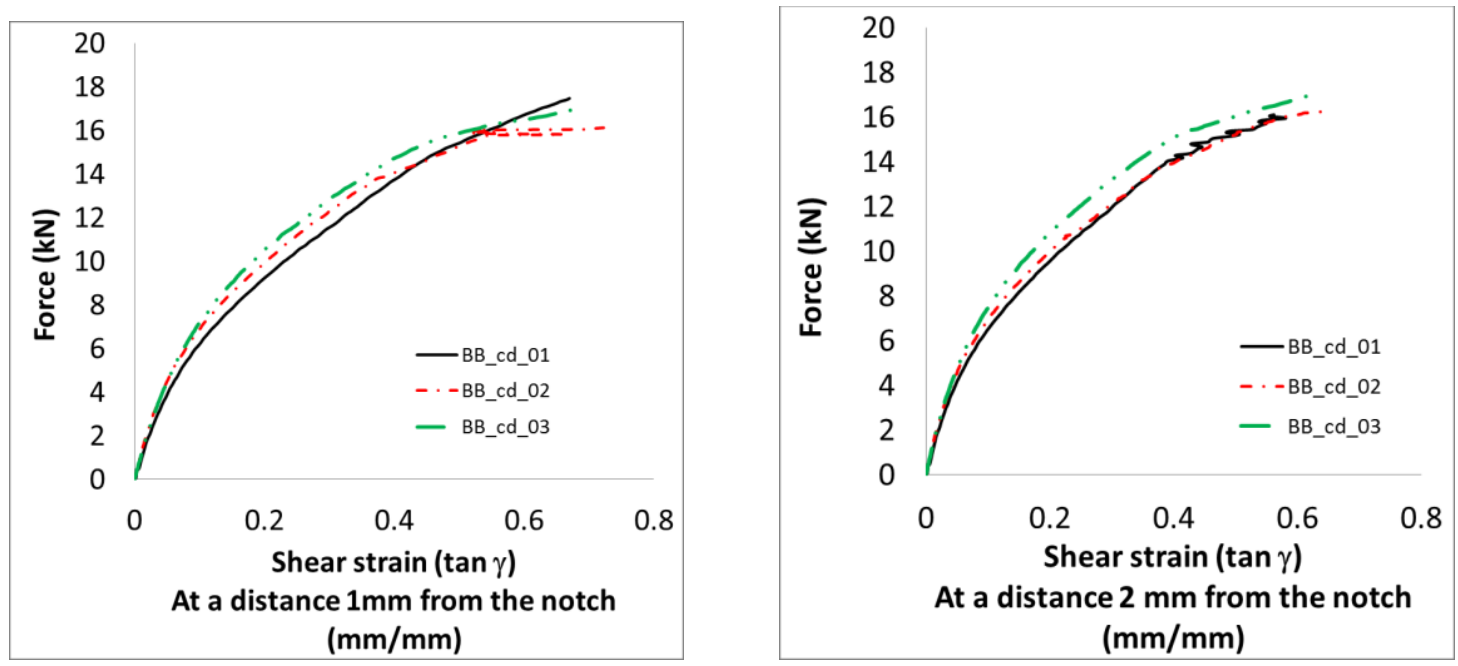

Figure 4.40 Samples cd_01,cd_02 and cd_03. Adhesive shear strain at $1 \mathrm{~mm}$ and $2 \mathrm{~mm}$ from the notch 


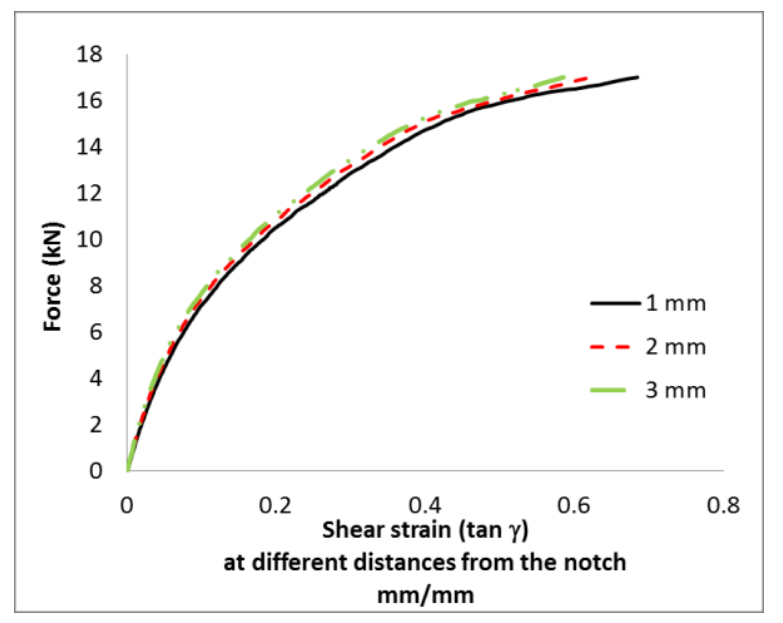

Figure 4.41 Sample cd_03. Adhesive shear strain at different distances from the notch

Although there is no drop in the load until the maximum measured value for each coupon, during the image processing it was noticed that the fracture process started prematurely. This failure process is depicted for coupon $c d$ _03 in Figure 4.42. At 14.04 $\mathrm{kN}$, as shown in the failure sequence, labeled a, the adhesive presented a very small flaw. This flaw was growing steadily until the maximum strength was reached, $18.78 \mathrm{kN}$. This failure process is compared with the bonded joint, labels 1 to 3 . It can be observed that the failure is almost sudden compared with the hybrid joint. Using the same rationale for the hybrid joint, it would be expected that a sudden drop in the force is due to the adhesive fracture. However, it seems that the load is redistributed between the remaining adhesive and the bolt, and due to the high adhesive flexibility, the joint can support more load. Unfortunately, this could not be verified experimentally because the FOV was not large enough to capture the entire fracture process in the whole bondline. When the adhesive failed completely, the strength of the joint dropped until an average force of 7.8 $\mathrm{kN}$ was reached, almost the same as the bolted joint in which the average strength was $8.09 \mathrm{kN}$. 

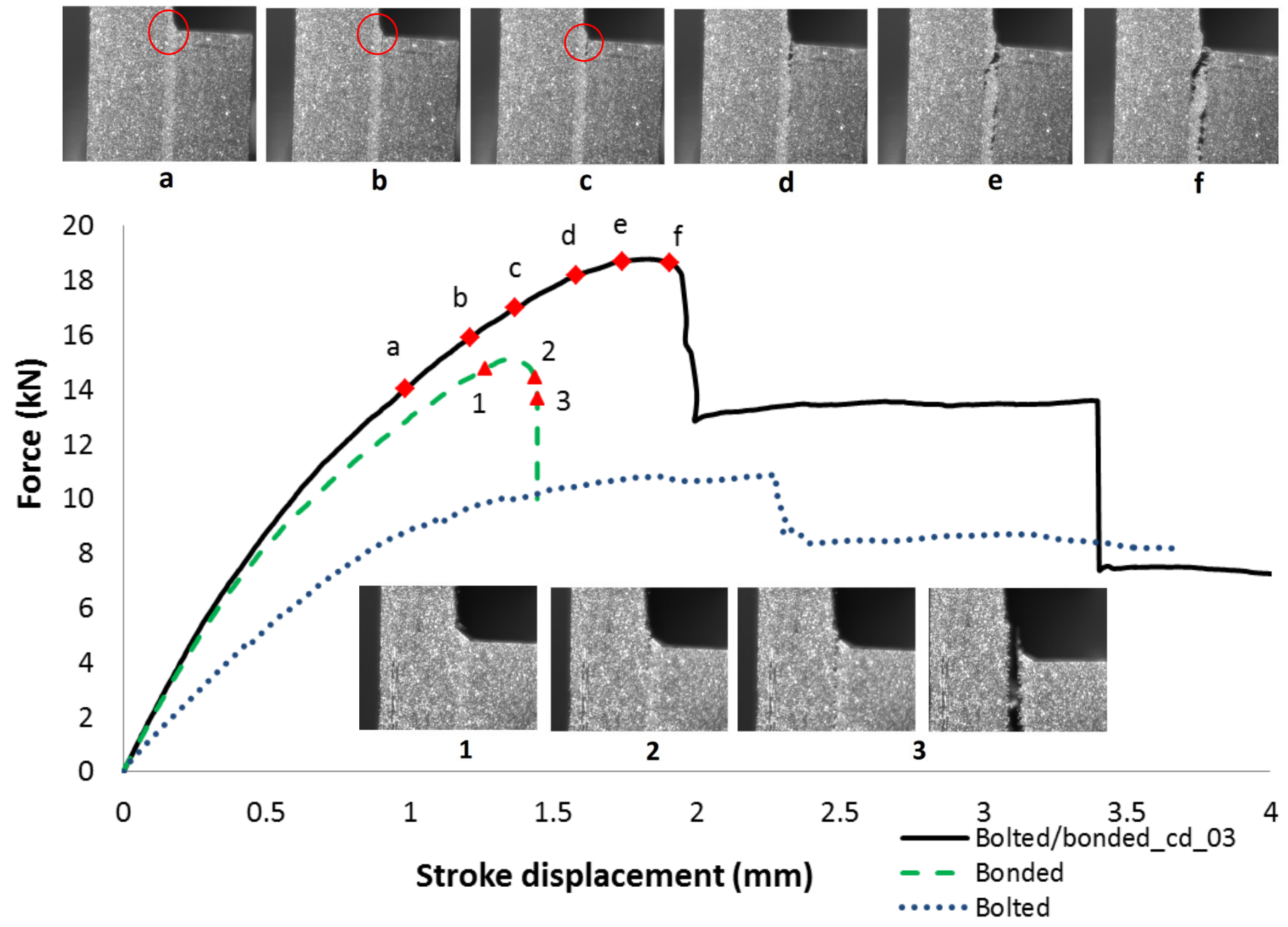

Figure 4.42 Joint failure sequence, treatment $c d \_03$ 


\subsubsection{Combination ace}

The coupons from this treatment were tested using a grip pressure of $8.96 \mathrm{MPa}$ (1300 psi). Thus, the displacement due to the test machine compliance was observed at $14 \mathrm{kN}$ on all coupons, similarly to the treatments $a b d$ and abcde. The procedure to subtract this displacement was carried out on all the coupons from this treatment. The final results are shown in Figure 4.43 and Figure 4.44.

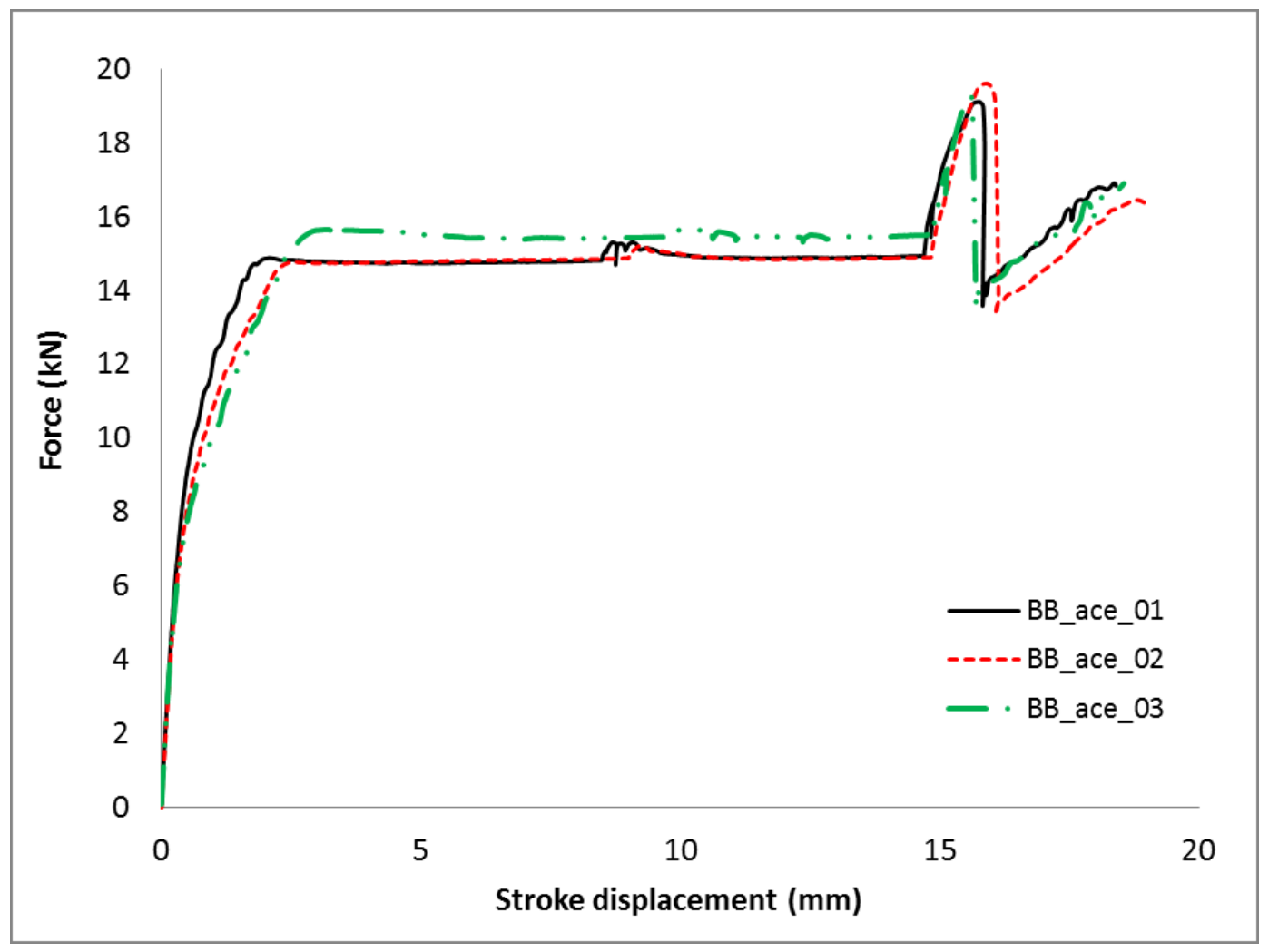

Figure 4.43 Force versus displacement results. Treatment ace.

The average failure strength for this combination was $19.34 \mathrm{kN}$ and the average strength after the adhesive failure was $16.6 \mathrm{kN}$. The failure strengths and displacements are shown in Table 4.10. 
Table 4.10 Summary of results. Force versus displacement for treatment ace

\begin{tabular}{|c|c|c|c|c|c|c|c|c|c|c|}
\hline & \multicolumn{2}{|c|}{$\begin{array}{l}\text { Sample } \\
\text { ace_01 }\end{array}$} & \multicolumn{2}{|c|}{$\begin{array}{l}\text { Sample } \\
\text { ace_02 }\end{array}$} & \multicolumn{2}{|c|}{$\begin{array}{l}\text { Sample } \\
\text { ace_03 }\end{array}$} & \multicolumn{2}{|c|}{$\begin{array}{l}\text { Force } \\
(\mathrm{kN})\end{array}$} & \multicolumn{2}{|c|}{$\begin{array}{c}\text { Displacement } \\
(\mathrm{mm})\end{array}$} \\
\hline POINT & $\begin{array}{l}\text { Disp. } \\
(\mathrm{mm})\end{array}$ & $\begin{array}{l}\text { Force } \\
(\mathrm{kN})\end{array}$ & $\begin{array}{l}\text { Disp. } \\
\text { (mm) }\end{array}$ & $\begin{array}{l}\text { Force } \\
(\mathrm{kN})\end{array}$ & $\begin{array}{l}\text { Disp. } \\
\text { (mm) }\end{array}$ & $\begin{array}{l}\text { Force } \\
(\mathrm{kN})\end{array}$ & Avg & $\begin{array}{l}\text { Sdt. } \\
\text { dev }\end{array}$ & Avg & $\begin{array}{l}\text { Sdt. } \\
\text { dev }\end{array}$ \\
\hline A & 3.06 & 19.12 & 3.36 & 19.61 & 3.51 & 19.28 & 19.34 & 0.25 & 3.31 & 0.23 \\
\hline B & 5.70 & 16.89 & 6.46 & 16.31 & 7.00 & 16.61 & 16.60 & 0.03 & 6.39 & 0.65 \\
\hline
\end{tabular}

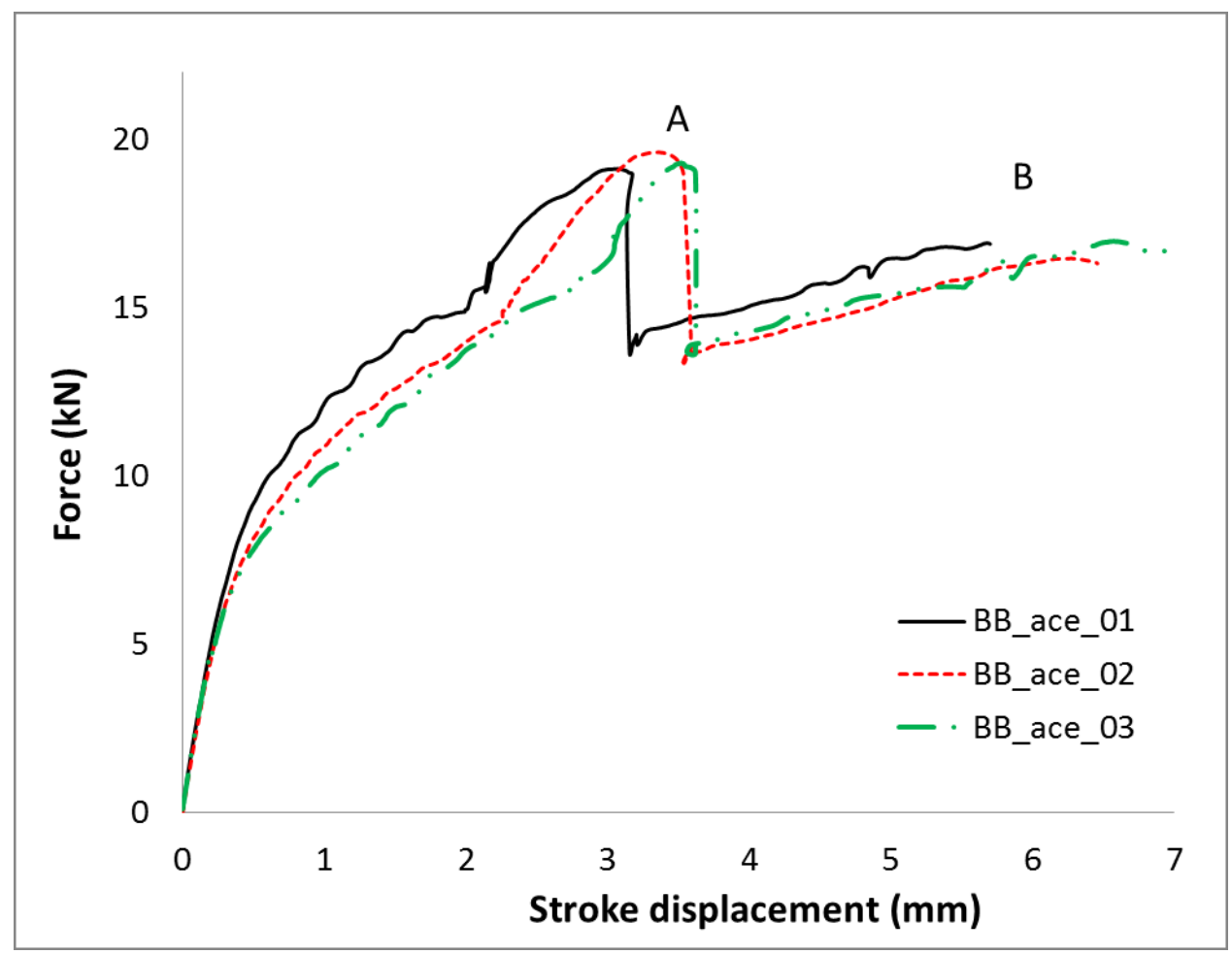

Figure 4.44 Force versus displacement results after displacement correction. Treatment ace

The DIC analysis was carried out at two magnifications, sample ace_01 was tested using a $13 \mathrm{~mm}$ length spacer, giving a resolution of $0.0268 \mathrm{~mm} /$ pixel. The aim of this setup was to measure the shear strain in the adhesive layer. On the other hand, samples ace_02 and ace_03 were tested without spacers, and the resolution was 0.046 
$\mathrm{mm} / \mathrm{pixel}$. The aim of this setup was to measure the local stiffness using the procedure 3 described in 3.6 , at $35 \mathrm{~mm}$ distance between points in the bondline. The image capturing frequency was $2 \mathrm{~Hz}$ for all the coupons.

The sample ace_02 was painted using a spray paint. The application to the speckle pattern enhanced the contrast, giving better results during the DIC analysis. With this, the relative displacement was measured as show in Figure 4.45 and compared against the results from the FE analysis. The experimental results are shown only until 14 $\mathrm{kN}$.

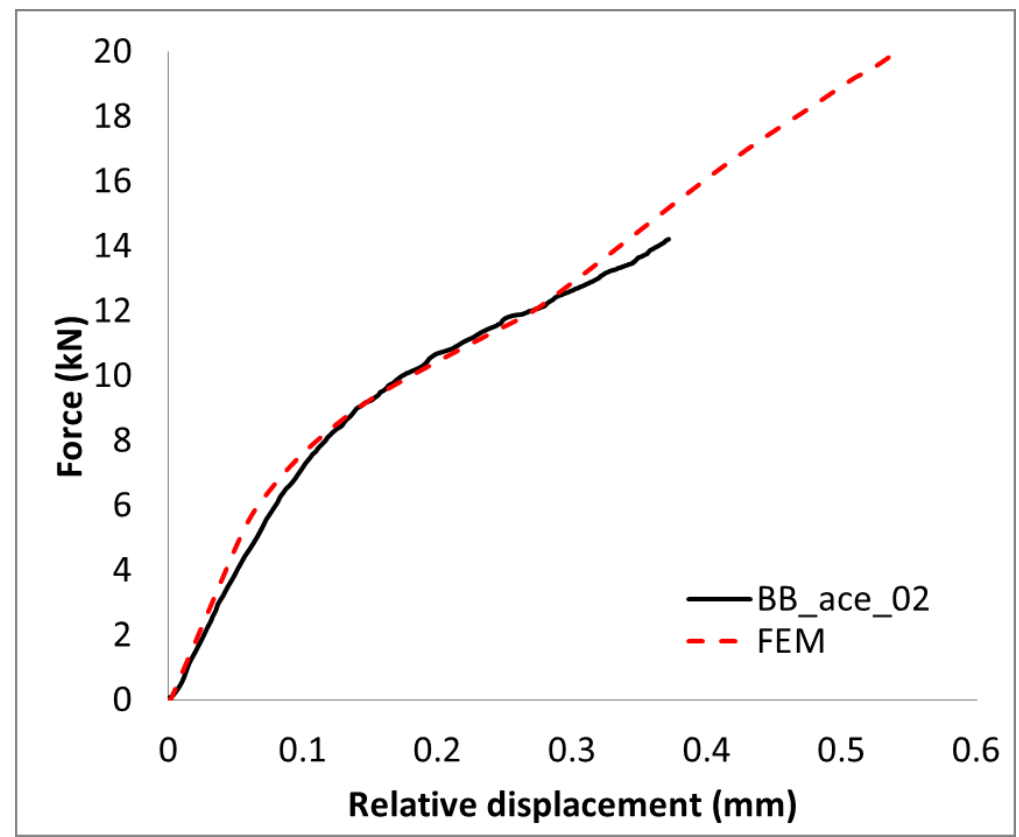

Figure 4.45 Sample ace_02. Force versus relative displacement using $35 \mathrm{~mm}$ reference length

Unfortunately, due to the poor contrast in the sample ace_01, the shear strain data was scattered and the agreement with the FEM analysis was not good enough. Because of the interest to measure this parameter accurately in the joints with EA9361 adhesive, a repetition test was carried out for this treatment. This test was also used to prove that the procedure to subtract the displacement due to the machine test compliance was correct. 
The force versus displacement for this repetition test is shown in Figure 4.46; the maximum strength, $20.96 \mathrm{kN}$, was about $8 \%$ higher than the average values from the previous tested specimens. However, the displacement at this maximum strength was 3.1 $\mathrm{mm}$, very close from the average corrected value, $3.31 \mathrm{~mm}$, from the coupons ace_01, ace_02 and ace_03. That difference is $6.7 \%$, proving that the procedure to remove the rigid motion was accurate.

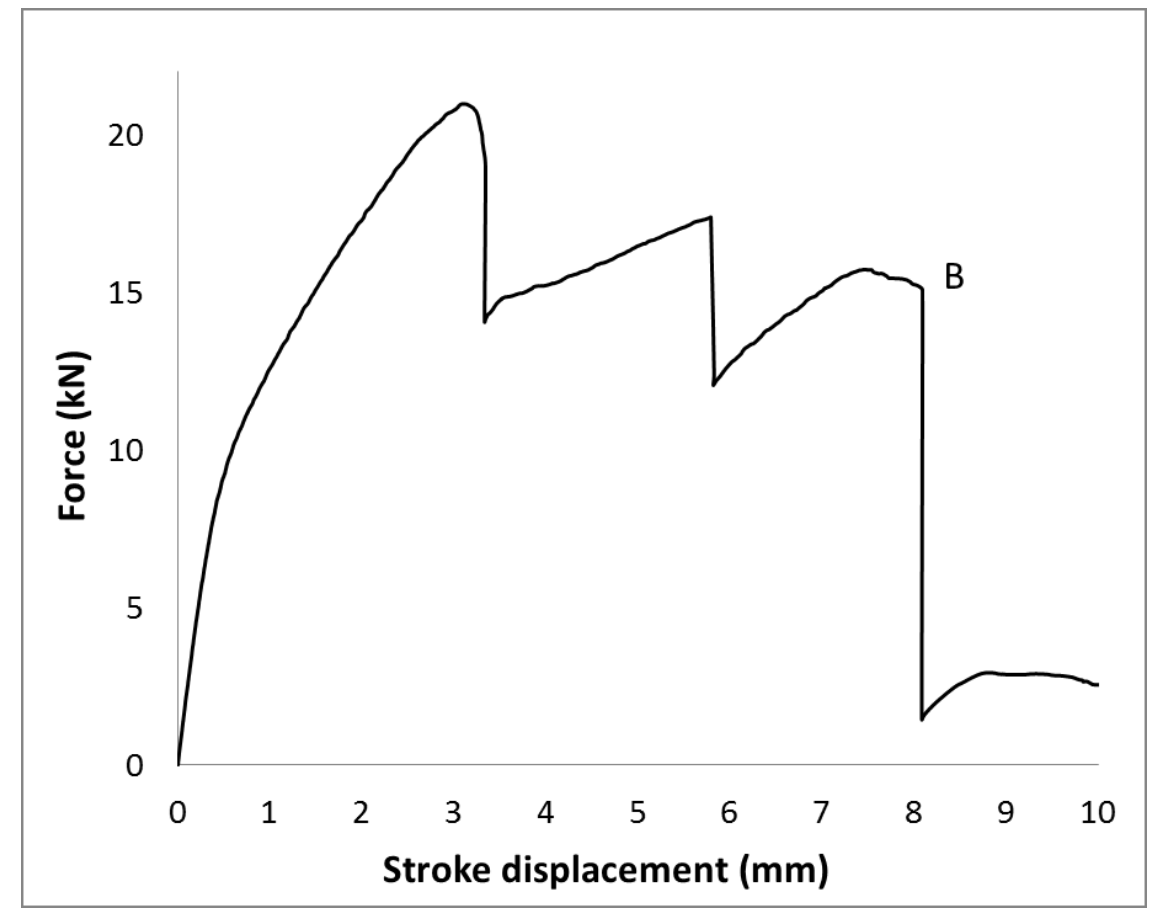

Figure 4.46 Repetition test, treatment ace_04. Force versus displacement results.

The shear strains at several distances from the notch are shown in Figure 4.47. It can be observed that after approximately $16.8 \mathrm{kN}$, the shear strain cannot be measured. This could be due to the crack initiation in the joint, and therefore, producing some jumps in the displacement measured values. 


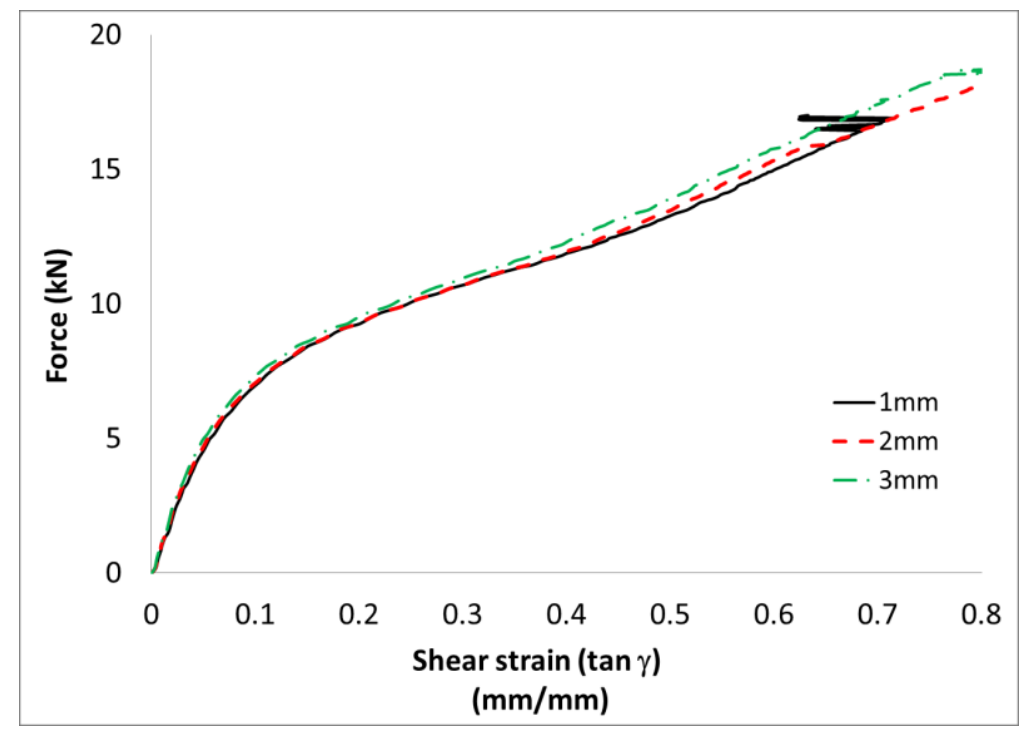

Figure 4.47 Sample ace_rep_01. Adhesive shear strain at different distances from the notch

Similarly to the previous treatments with EA9361 adhesive, the failure in the bolted/bonded joints started before the maximum strength was reached. At a force of $17.73 \mathrm{kN}$, label $a$ in the Figure 4.48, a flaw near to the notch can be observed, this flaw is barely detectable using the DIC images. However, this can be detected easily when the displacements and shear strains are computed. At a force of $16.8 \mathrm{kN}$, the displacement field presents scattered results, a clear sign that the fracture initiation in the adhesive have started. This fracture grows slowly until $19.84 \mathrm{kN}$ and, after this point, the crack grow rate increases rapidly until $20.69 \mathrm{kN}$. After this point, the strength starts to decrease until a sudden failure is reached at $19.48 \mathrm{kN}$. At this point the stroke displacement has reached $3.3 \mathrm{~mm}$. After this point, it seems that the two joining elements are still working together until a displacement of $5.74 \mathrm{~mm}$ when the adhesive seems to have another sudden crack grow and the force has reached a value of $12.19 \mathrm{kN}$. The strength, again, increases steadily until it reaches a maximum value of $15.7 \mathrm{kN}$ and finally, the bolt fails. 


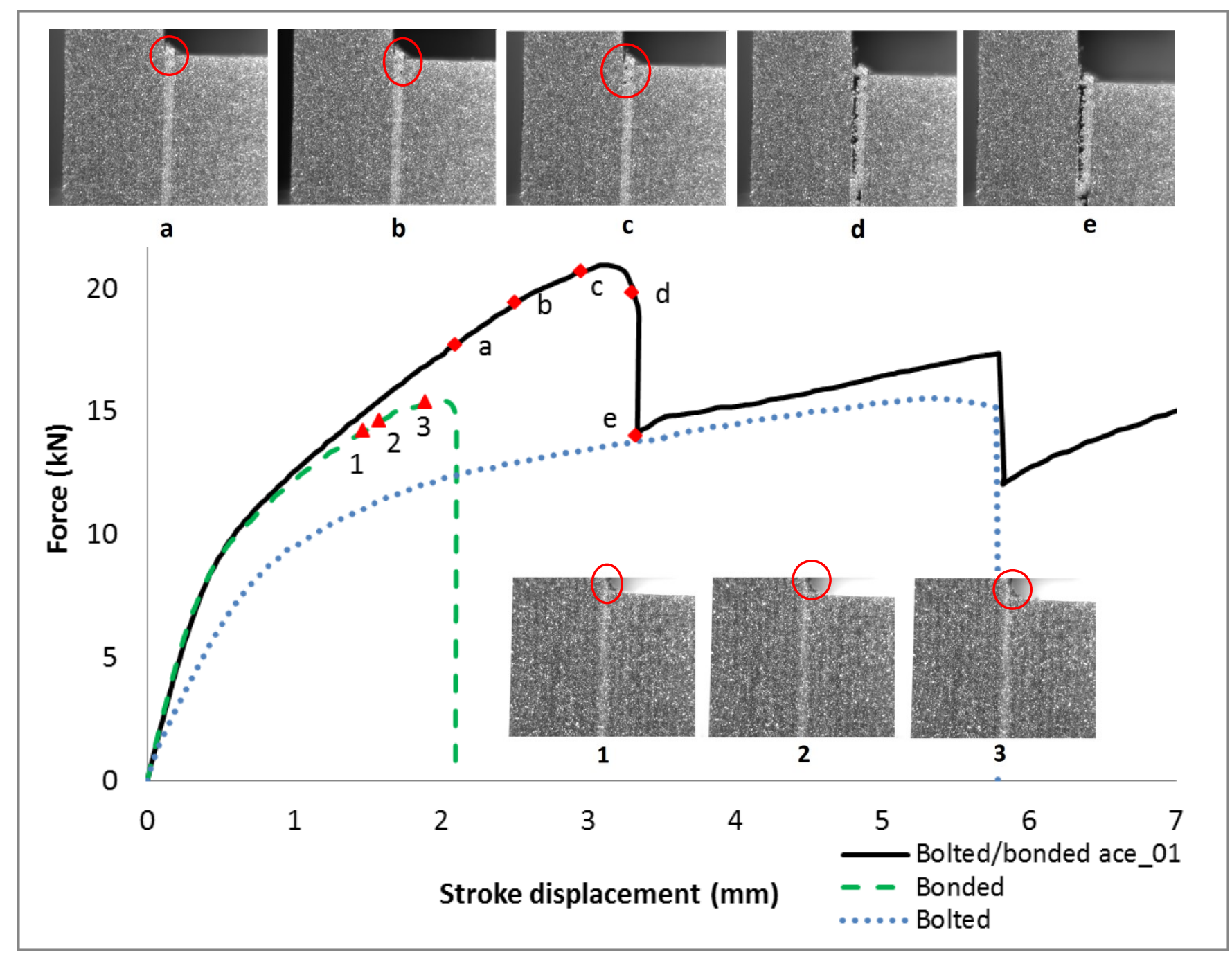

Figure 4.48 Joint failure sequence, treatment ace 
Compared with bonded joints alone, the advantage of the hybridization is that the fracture process in the adhesive layer is delayed, and the strength of the joint is improved. Moreover, a improvement in displacement is also achieved.

\subsubsection{Summary of Results for Joint with EA9361 Adhesive}

Similarly to the joints with FM300-2M, the bolted/bonded joints with EA9361adhesive with adherend thickness of $5.48 \mathrm{~mm}$ were stronger; averaging $20.35 \mathrm{kN}$ and $19.34 \mathrm{kN}$ for the treatments $a$ and ace. The joints with adherend thickness of 3.29 $\mathrm{mm}$ averaged $14.44 \mathrm{kN}$ and $17.93 \mathrm{kN}$ for treatments $d e$ and $c d$ respectively.

The effect of the adhesive's mechanical properties was evident in the failure mode of the adhesive. The damage was progressive without sudden drops in the strength, compared with the joints with FM300-2M adhesive. However, from the DIC images it was found that all the joint presented an initial damage before the adhesive's total failure.

The failure modes were cohesive in the adhesive region followed for shearout or cleavage for the joints with adherend thickness of $3.29 \mathrm{~mm}$. For the joint with adherend thickness of $5.48 \mathrm{~mm}$, the failure was cohesive followed by the failure in the bolt due to shear.

From the DIC analysis, shear strains at several positions using the notch as reference were measured. These results were used to infer the load sharing. A full analysis and discussion of this is described in chapter 6 . 


\subsection{ANOVA Analysis}

An ANOVA analysis was carried out to identify the effect of the analyzed factors on the joint strength. The chosen data was the load versus stroke and the selected response variables were the strength at first failure point and the maximum strength. In addition to this, both comparisons were carried out using the total strength as well as a normalized strength by weight. The weight of the coupons with adherend thickness of $3.29 \mathrm{~mm}$ was $0.077 \mathrm{~kg}$. On the other hand, the weight of the coupons with adherend thickness of $5.48 \mathrm{~mm}$ was $0.122 \mathrm{~kg}, 58 \%$ heavier than the thinner coupons.

In the joints with EA9361 adhesive, the first failure point was the same as the maximum strength point. However, after the analysis using the pictures taken from the DIC, it was found that, due to the ductile nature of the adhesive, the failure was not sudden; the adhesive started the fracture process before total failure. However, it was difficult to determine the exact point on which the adhesive was initially damaged. Therefore, it was decided for these joints, to assume that the first failure point was the same as the maximum strength point.

For analysis purposes in this section, the summarized effect of each factor as well as the plot of these effects will be shown. The full ANOVA results for each response is shown in Appendix C.

Figure 4.49 shows the total strength at the first failure point as well as the normalized results by weight. It can be observed that treatments $a$,abd,ace and abcde, with the thicker adhered show a substantial drop in their performance in the normalized results. It is clear that the gain in strength in these coupons is greatly counteracted by the increase in the weight in the normalized results. Therefore, it should be expected a huge 
negative effect of the adherend thickness from the ANOVA results for the normalized response.

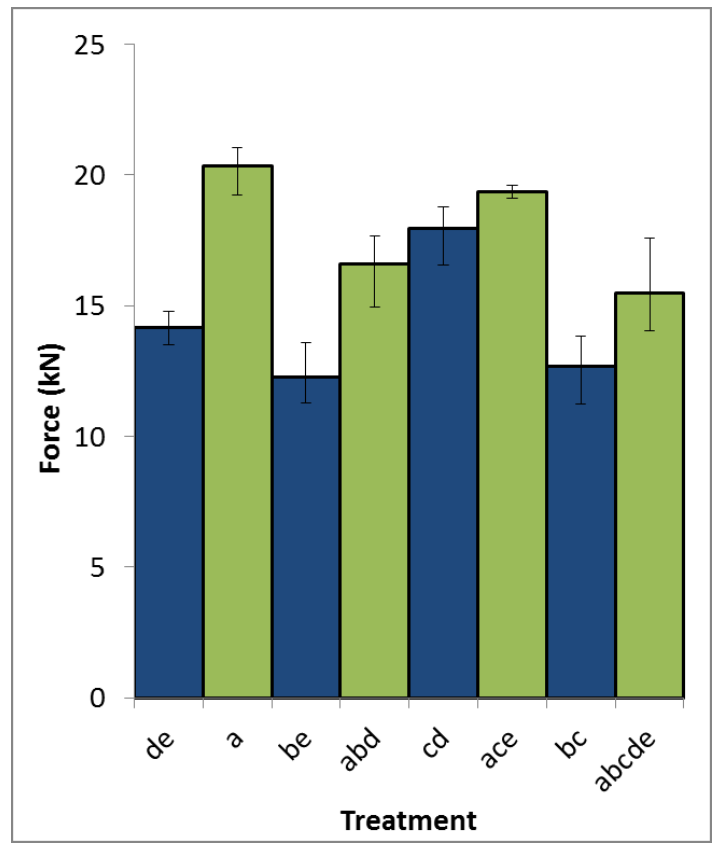

a)

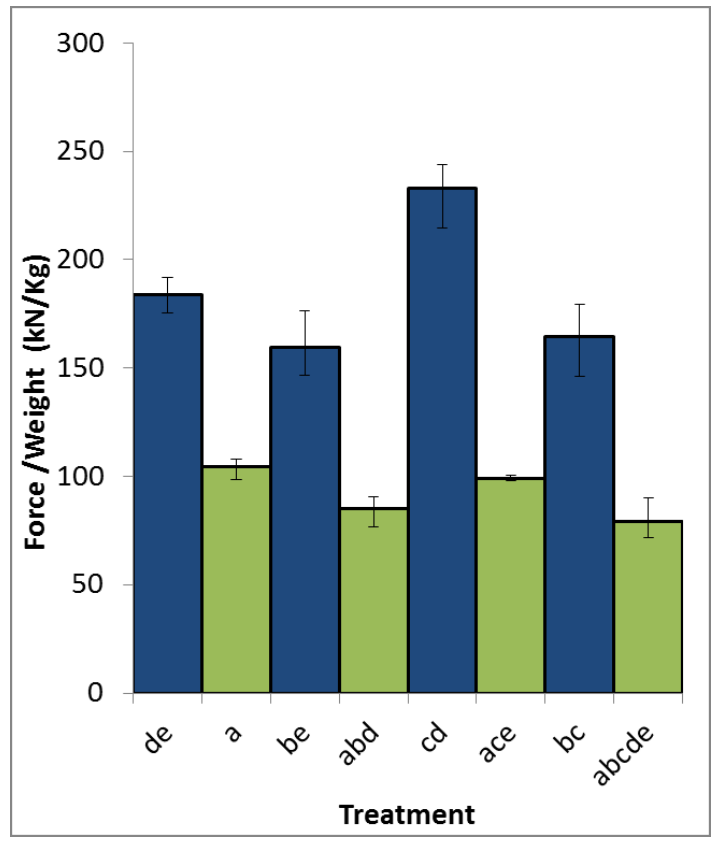

b)

Figure 4.49 Experimental results, stength at first failure point. a) Total force; b) Total force per weight

From the ANOVA results, the effect of each factor for both analyses are shown in Figure 4.50 and the results are summarized in Table 4.11.

The numbers before the results are to identify the nature of the effect, positive or negative in the response, and not for summation purposes. The model's p-value was zero in both cases; therefore, it is concluded that the ANOVA is significant. That means that the factors have a strong effect in the response, and $10 \%$ and $3.02 \%$ respectively, is due to error. 


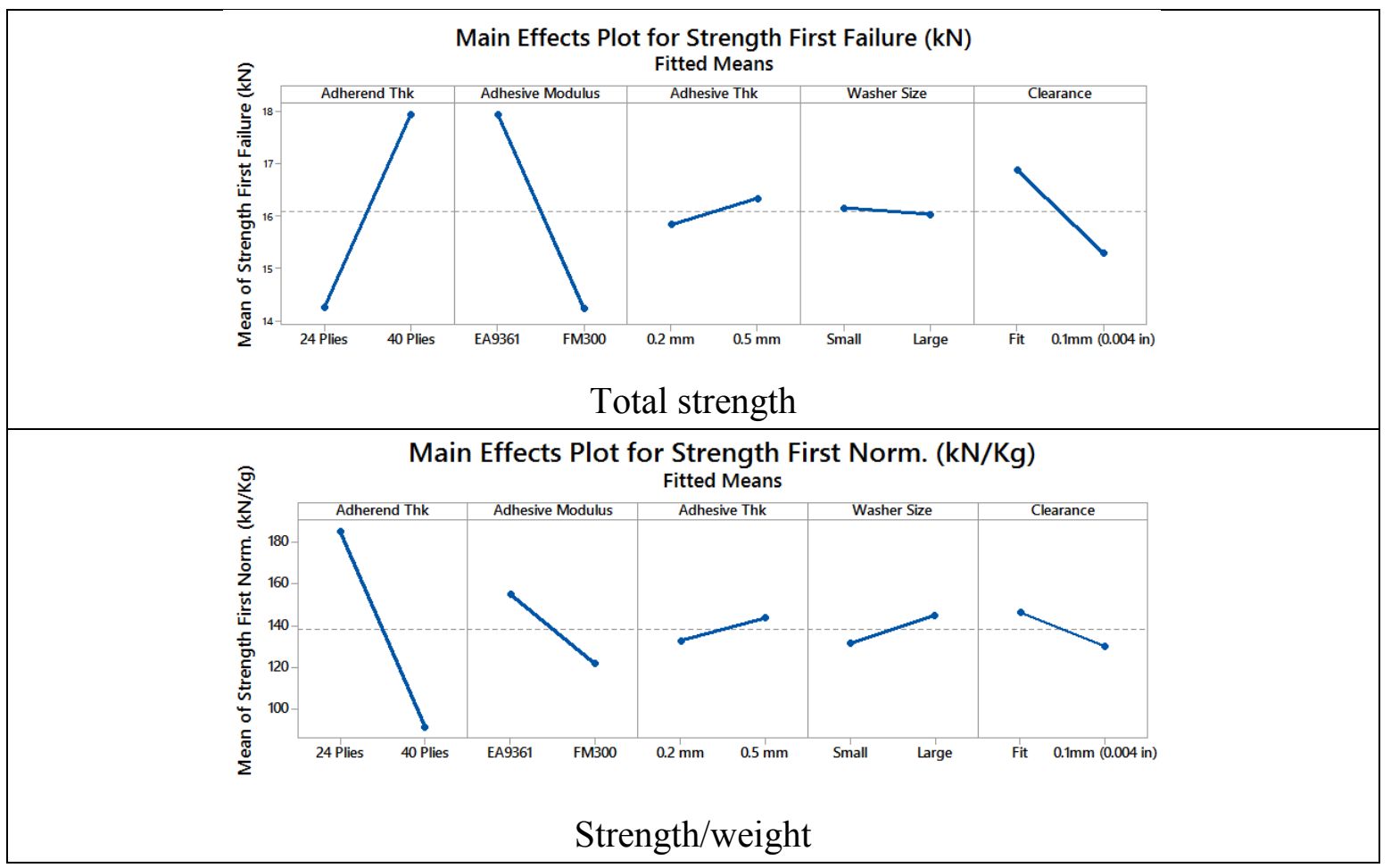

Figure 4.50 ANOVA results, main factors on the strength at first failure point. a) Total force;

b) Total force per weight

Table 4.11 Effect of each factor in the joint strength. First failure point, total strength vs normalized by weight

\begin{tabular}{|c|c|c|}
\hline \multirow{2}{*}{ Factor } & \multicolumn{2}{|c|}{ Effect (\%) } \\
\cline { 2 - 3 } & Total & Normalized \\
\hline Adherend thickness & $(+) 38.3$ & $(-) 79.7$ \\
\hline Adhesive modulus & $(-) 38.8$ & $(-) 9.95$ \\
\hline Hole clearance & $(-) 7.01$ & $(-) 2.4$ \\
\hline Adhesive thickness & $(+) 0.72$ & $(+) 1.06$ \\
\hline Washer size & $(-) 0.04$ & $(+) 1.65$ \\
\hline Adhesive modulus * adhesive \\
thickness & $(-) 2.18$ & $(-) 1.14$ \\
\hline Adhesive modulus * hole clearance & $(-) 1.89$ & $(-) 1.08$ \\
\hline Error & 10.6 & 3.02 \\
\hline
\end{tabular}


It is clear that a total positive variation in the strength at first failure point is $38 \%$ due to the adherend thickness. On the other hand, it is notable the negative effect of adhesive modulus, with a $38.8 \%$ negative influence in the total variation.

The second negative effect is the hole clearance, with an effect of $7 \%$ in the response. The washer size and adhesive thickness have an effect nearly to zero. The combined effect between the adhesive modulus and adhesive thickness account for a $2 \%$ variation and, finally, the adhesive modulus combined with the hole clearance account for only $1.8 \%$ variation.

From the normalized response, it is clear that the increase in the total strength is severely penalized by the increase in weight, and the variation in the normalized strength is explained almost $80 \%$ by the effect of the adherend thickness. An additional $9.95 \%$ negative effect is due to the adhesive modulus. The remaining factors have a marginal effect in the joint strength at the first failure point.

Similarly, the ANOVA was computed using the maximum strength as well as maximum strength per weight as response variables. The total strengths are shown in Figure 4.51 and the effect of each factor is shown in Figure 4.52. The summarized results are shown in Table 4.12.

It can be observed a similar trend in the effect of each factor. The adherend thickness accounts for a positive effect of $42 \%$ in the variation using the total values. However, this positive effect is severely diminished by the increase in the joint's weight. 


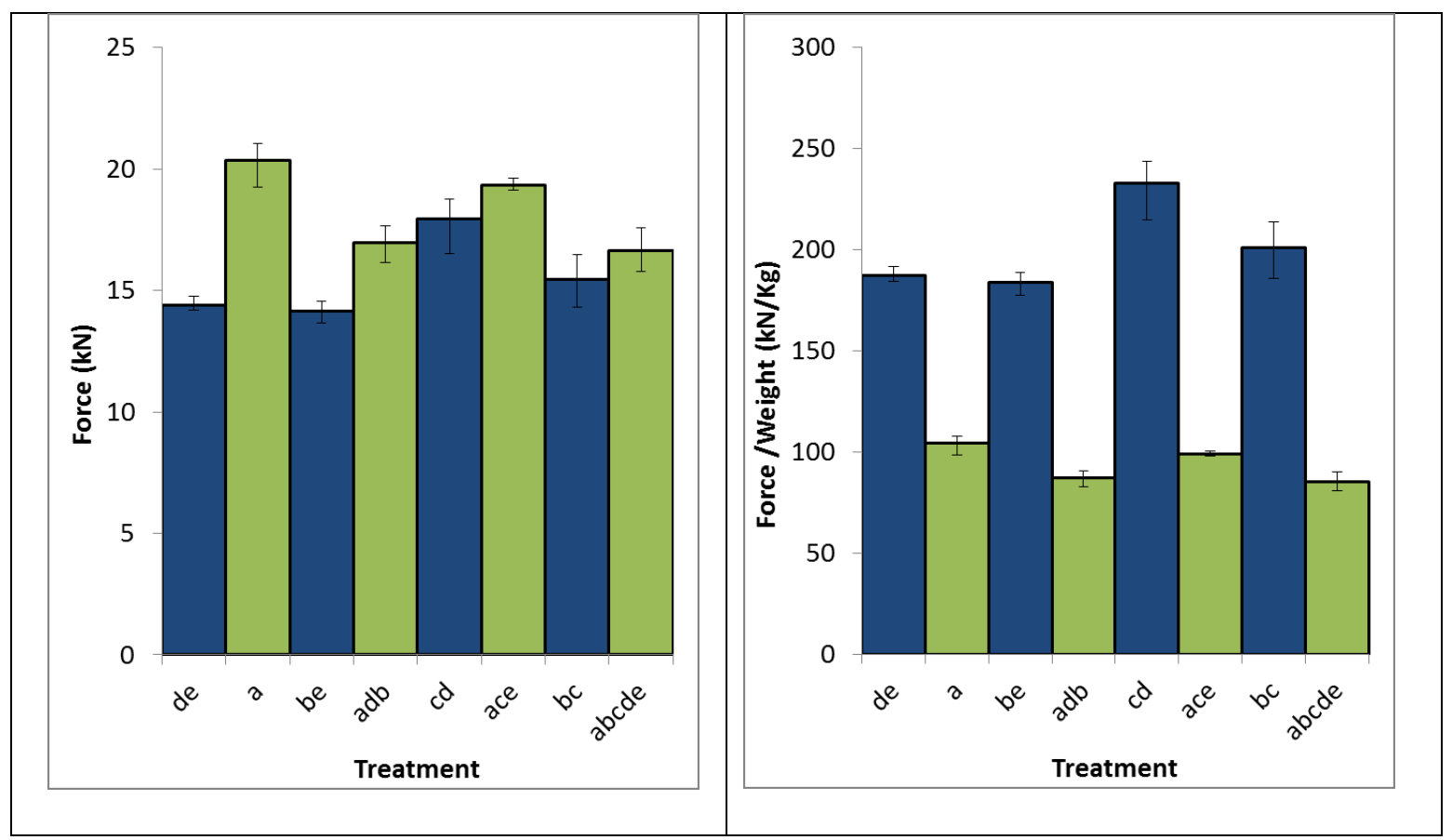

Figure 4.51 Experimental results, maximum strength. a) Total force; b) Total force per weight

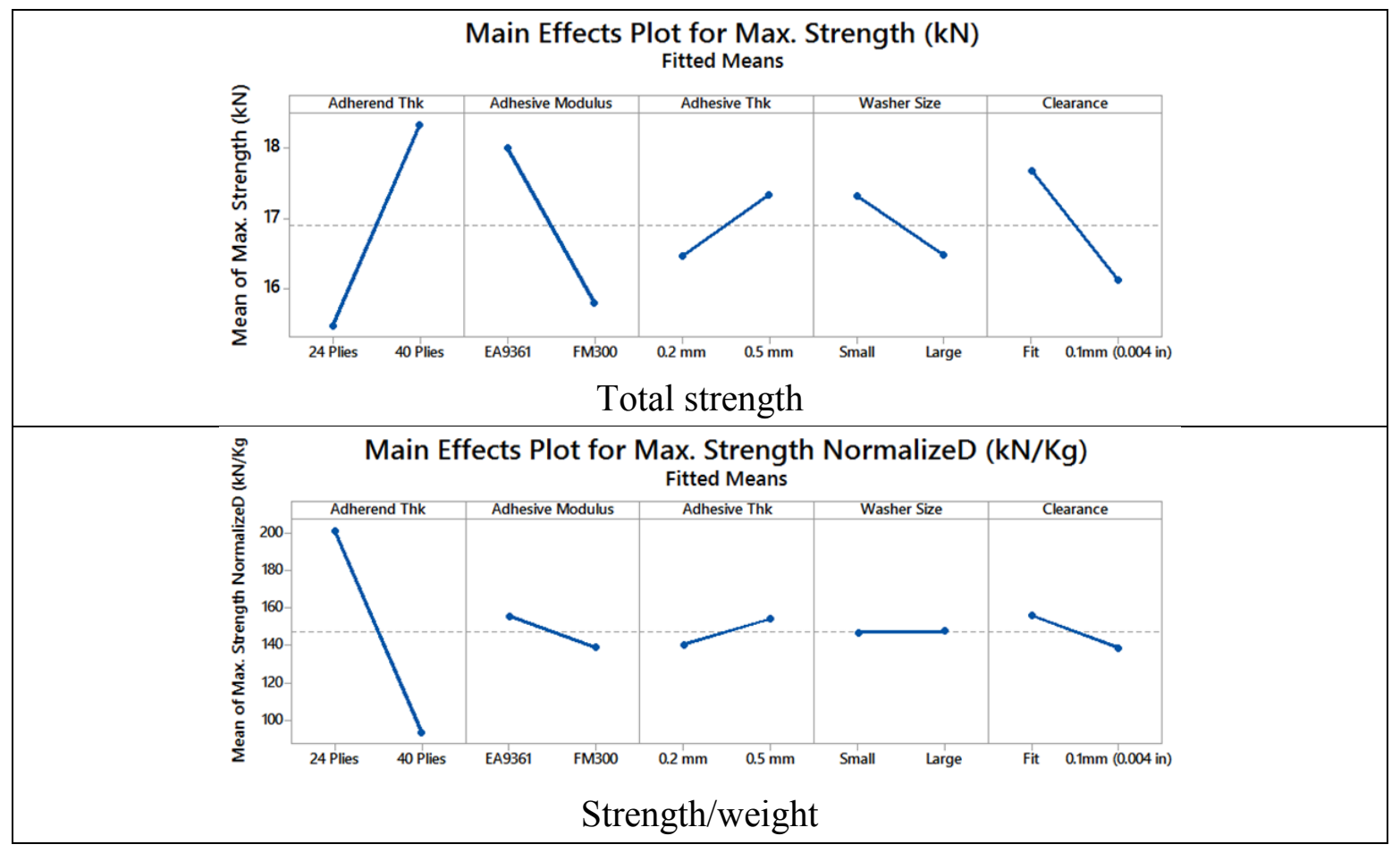

Figure 4.52 ANOVA results, main factors effect in the total maximum strength. a) Total force;

b) Total force per weight 
Table 4.12 Effect of each factor in the maximum strength. Total strength vs normalized by weight

\begin{tabular}{|l|c|c|}
\hline \multirow{2}{*}{\multicolumn{1}{c|}{ Factor }} & \multicolumn{2}{c|}{ Effect (\%) } \\
\cline { 2 - 3 } & Total & Normalized \\
\hline Adherend thickness & $(+) 42.1$ & $(-) 91.5$ \\
\hline Adhesive modulus & $(-) 25.0$ & $(-) 2.18$ \\
\hline Hole clearance & $(-) 12.4$ & $(-) 2.40$ \\
\hline Adhesive thickness & $(+) 3.95$ & $(+) 1.65$ \\
\hline Washer size & $(-) 3.64$ & $(-) 0.01$ \\
\hline $\begin{array}{l}\text { Adhesive modulus * adhesive } \\
\text { thickness }\end{array}$ & 0.79 & 0.32 \\
\hline Adhesive modulus * hole clearance & 2.76 & 0.52 \\
\hline Error & 8.65 & 1.29 \\
\hline
\end{tabular}

The interactions between factors were almost negligible in this experiment. The maximum interactions were found between the adhesive modulus and adhesive thickness with $0.79 \%$ effect, and adhesive modulus with hole clearance with $2.76 \%$ effect. More interactions were not detected or computed with the ANOVA.

In summary, according to the ANOVA results, the strength in the joint is mainly driven by the adherend thickness and adhesive modulus. A comparison between the ANOVA results with the load versus stroke performance for the bolted/bonded joints is discussed in Chapter 7.

Finally, typical failure modes pictures for all the treatments are shown in Appendix F. 


\section{Chapter 5: Finite Element Analysis of Bolted/Bonded Composite Joints}

The commercial finite element modelling software Abaqus ${ }^{\circledR}$ was used to analyze the bolted/bonded joints. The models were created using $\mathrm{AbaqusCAE}^{\circledR} \mathrm{v} 6.13$ and the input files were transferred to a computer cluster at Carleton running Community Enterprise Operating System (CentOS). The assigned server to run the models was an HP DL160 G5 with dual quad core Intel(R) Xeon(R) CPU L5410@ @.33GHz and 16GB ECC RAM. A User Defined Field (USFDL) subroutine was written in Fortran and linked to the FEM software to analyze the models with progressive failure in the composite material. The subroutine was written following the instructions from the Abaqus ${ }^{\circledR}$ user manual [83] and previous research work [124-126].

\subsection{Material Properties}

\subsubsection{Carbon Fibre Unidirectional Prepreg Properties}

The composite material, Cytec Cycom 5320 unidirectional prepreg, was modeled as a layered solid and the mechanical properties were defined using lamina elastic constants. The lamina elastic constants in $0^{\circ}$ and $90^{\circ}$ directions were taken from the manufacturer's datasheet and their values are shown in Table 5.1. The unidirectional lamina can be approximated as a transversely isotropic material [127]; therefore, the remaining elastic constants can be computed as follows: $E_{33}=E_{22} ; G_{13}=G_{12} ; v_{13}=v_{12}$; and

$$
G_{23}=\frac{E_{33}}{2\left(1+v_{23}\right)}
$$


Table 5.1 Cycom 5320 elastic constants [120]

\begin{tabular}{|c|c|c|c|}
\hline $\mathrm{E}_{11}(\mathrm{GPa})$ & $\mathrm{E}_{22}(\mathrm{GPa})$ & $\mathrm{G}_{12}$ & $v_{12}$ \\
\hline 141 & 9.7 & 5.2 & 0.34 \\
\hline
\end{tabular}

In order to compute $G_{23}$, the value of $v_{23}$ is needed. This value, $v_{23}$, can be computed from the Poisson's ratio in the matrix of the composite material [128]. Unfortunately, this information was not available for this material system. However, from the literature review a value of $v_{23}=0.44$ was reported for the Cytec 5320 unidirectional prepreg [72]. Finally, the full set of 9 elastic constants are computed and shown in Table 5.2 .

Table 5.2 Cycom 5320 elastic constants. Adapted from [120]

\begin{tabular}{|c|c|c|c|c|c|c|c|c|}
\hline $\begin{array}{c}\mathrm{E}_{11} \\
(\mathrm{GPa})\end{array}$ & $\begin{array}{c}\mathrm{E}_{22} \\
(\mathrm{GPa})\end{array}$ & $\begin{array}{c}\mathrm{E}_{33} \\
(\mathrm{GPa})\end{array}$ & $\begin{array}{c}\mathrm{G}_{12} \\
(\mathrm{GPa})\end{array}$ & $\begin{array}{c}\mathrm{G}_{13} \\
(\mathrm{GPa})\end{array}$ & $\begin{array}{c}\mathrm{G}_{23} \\
(\mathrm{GPa})\end{array}$ & $v_{12}$ & $v_{13}$ & $v_{23}$ \\
\hline 141 & 9.7 & 9.7 & 5.2 & 5.2 & 3.4 & 0.34 & 0.34 & 0.44 \\
\hline
\end{tabular}

The strength properties, required to apply the failure criteria using progressive failure damage are shown in Table 5.3.

Table 5.3 Cytec Cycom 5320 strengths [120]

\begin{tabular}{|c|c|}
\hline $\begin{array}{c}\text { Fiber Direction } \\
\text { Direction }\end{array}$ & $\begin{array}{c}\text { Strength } \\
(\mathbf{M P a})\end{array}$ \\
\hline $0^{\circ}$ Tension & 2703 \\
\hline $0^{\circ}$ Compression & 1737 \\
\hline $90^{\circ}$ Tension & 81 \\
\hline $90^{\circ}$ Compression & 312 \\
\hline
\end{tabular}


Finally, the elastic constants with degraded values according to the degradation rules proposed by McCarthy et al [104] are shown in Table 5.4.

Table 5.4 Degraded elastic constants

Properties (E and G MPa)

( $v$, unitless)

\begin{tabular}{|c|cc|c|c|c|c|c|c|c|c|c|}
\hline \multicolumn{2}{|c}{ Failure mode } & $\mathbf{E}_{\mathbf{1}}$ & $\mathbf{E}_{\mathbf{2}}$ & $\mathbf{E}_{\mathbf{3}}$ & $\mathbf{v}_{\mathbf{1 2}}$ & $\mathbf{v}_{\mathbf{2 3}}$ & $\mathbf{v}_{\mathbf{1 3}}$ & $\mathbf{G}_{\mathbf{1 2}}$ & $\mathbf{G}_{\mathbf{1 3}}$ & $\mathbf{G}_{\mathbf{2 3}}$ & FV \\
\hline & No degradation & 141000 & 9700 & 9700 & 0.34 & 0.34 & 0.44 & 5200 & 5200 & 3400 & N.A. \\
\hline 1 & Matrix tensile & 141000 & 97 & 97 & 0 & 0 & 0 & 52 & 52 & 34 & 1 \\
\hline 2 & Matrix compressive & 141000 & 97 & 97 & 0 & 0 & 0 & 52 & 52 & 34 & 2 \\
\hline 3 & Fibre tensile & 1410 & 9700 & 9700 & 0 & 0 & 0 & 52 & 52 & 3400 & 3 \\
\hline 4 & Fibre compressive & 1410 & 9700 & 9700 & 0 & 0 & 0 & 52 & 52 & 3400 & 4 \\
\hline
\end{tabular}

Abaqus

field

variable

The bonded joints were modelled in 2D assuming that the adherends behaved as an isotropic material and the elastic modulus as well as the Poisson's ratio were computed from the lamina elastic constants. The computed values were $E=54.5 \mathrm{MPa}$, and $v=0.313$. These values were confirmed experimentally by testing the parent laminate in tension as described below. Strain gauges were installed on the surface longitudinally and transversely to compute Young's modulus as well as Poisson's ratio. The results were $E=52.33 \mathrm{GPa}$, and $v=0.317$ respectively as shown in Figure 5.1, these results were in very good agreement with the computed values. 


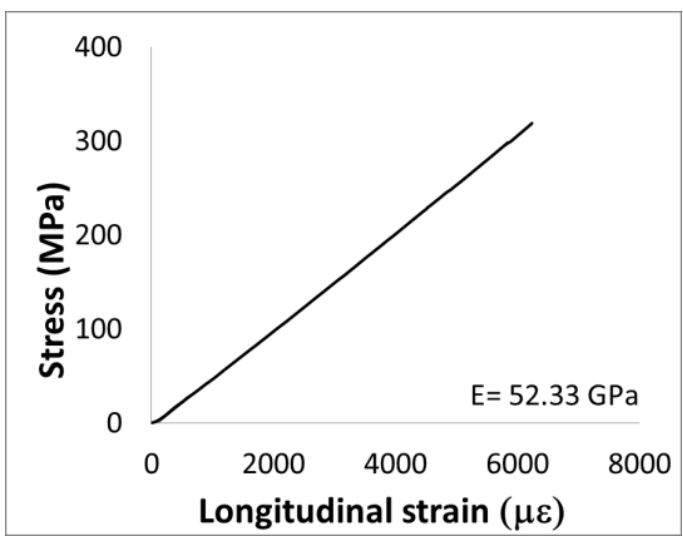

a)

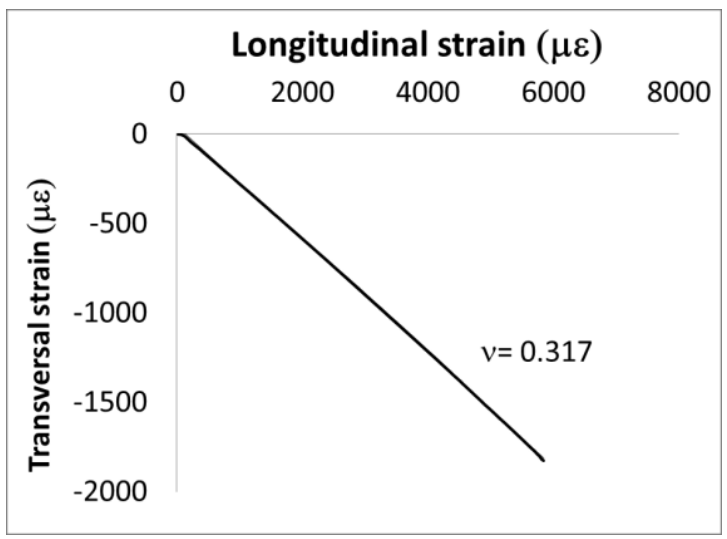

b)

Figure 5.1 Laminate quasi-isotropic Young's modulus and Poisson's ratio. Experimental results

\subsubsection{Adhesive Elastoplastic Properties}

The EA9361 and FM300-2M adhesives were characterized experimentally to obtain the mechanical properties in situ rather than in bulk. The experimental characterization was carried out jointly with the CRAIQ COMP 506 partners [129]. The testing method selected was the ASTM D638-02a (Standard Test Method for Tensile Properties of Plastics). In the case of the FM300-2M adhesive, the results were compared with the manufacturer's datasheet. From Table 5.5 it can be observed that the results are in very good agreement excepting for the ultimate shear strain. On the other hand, there is no shear stain versus shear stress data available in the literature for the EA9361 adhesive. The TAST testing results for this adhesive is shown in Table 5.6.

From the results, it can be observed that the FM300-2M adhesive has a shear modulus 3.66 times the EA9361 adhesive modulus. Conversely, the FM300-2M adhesive can reach one third of the EA9361 adhesive shear strain. 
The results from the tests are shown in Figure 5.2, and the summarized data compared against the data from the manufacturer for the FM300-2M adhesive is shown in Table 5.5.

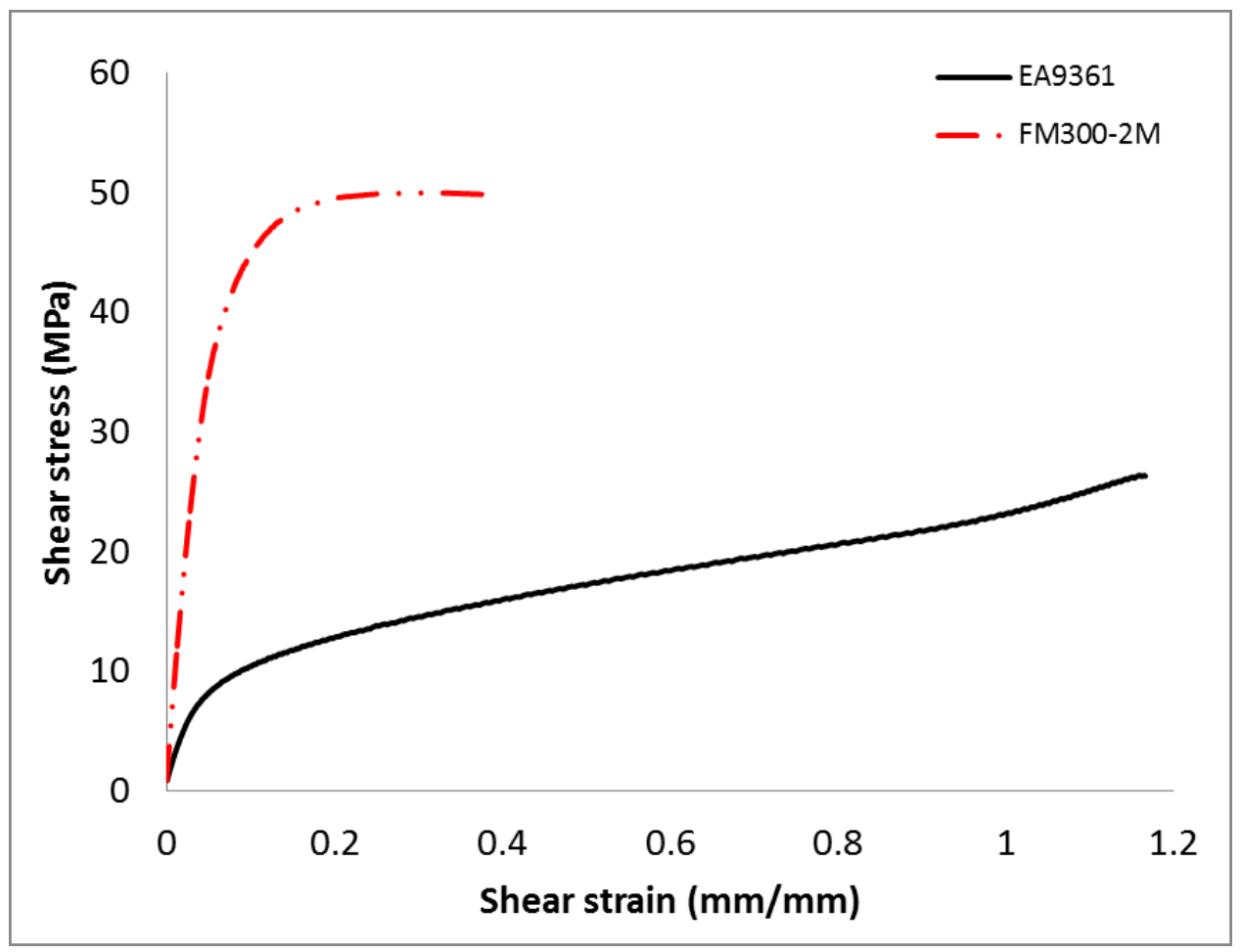

Figure 5.2 Shear stress versus strain data from TAST for EA9361 and FM300-2 adhesives

Table 5.5 Comparison between mechanical properties FM300 and FM300-2M adhesives.

The TAST results are average values

\begin{tabular}{|c|c|c|c|c|c|c|c|}
\hline & \multicolumn{3}{|c|}{$\begin{array}{c}\text { Linear Limit } \\
(\mathrm{LL})\end{array}$} & \multicolumn{2}{c|}{$\begin{array}{c}\text { Knee } \\
(\mathrm{KN})\end{array}$} & \multicolumn{2}{c|}{$\begin{array}{c}\text { Ultimate } \\
\text { Failure (UL) }\end{array}$} \\
\hline & $\begin{array}{c}\text { Shear } \\
\text { Stress } \\
(\mathrm{MPa})\end{array}$ & $\begin{array}{c}\text { Shear } \\
\text { Strain } \\
(\mathrm{mm} / \mathrm{mm})\end{array}$ & $\begin{array}{c}\text { Shear } \\
\text { Modulus } \\
(\mathrm{MPa})\end{array}$ & $\begin{array}{c}\text { Shear } \\
\text { Stress } \\
(\mathrm{MPa})\end{array}$ & $\begin{array}{c}\text { Shear } \\
\text { Strain } \\
(\mathrm{mm} / \mathrm{mm})\end{array}$ & $\begin{array}{c}\text { Shear } \\
\text { Stress } \\
(\mathrm{MPa})\end{array}$ & $\begin{array}{c}\text { Shear } \\
\text { Strain } \\
(\mathrm{mm} / \mathrm{mm})\end{array}$ \\
\hline $\begin{array}{c}\text { Datasheet } \\
\text { FM300 }\end{array}$ & 14.2 & 0.0156 & 907.5 & 42.1 & 0.0932 & 49.8 & 0.5446 \\
\hline $\begin{array}{c}\text { TAST } \\
\text { FM300-2M }\end{array}$ & 14.7 & 0.015 & 881 & 43.5 & .0883 & 50 & 0.376 \\
\hline$\%$ difference & 3.52 & 4 & 3 & 3.21 & 5.54 & 0.4 & 44.84 \\
\hline
\end{tabular}


Table 5.6 TAST data EA9361 adhesive. Average values

\begin{tabular}{|c|c|c|c|c|c|c|c|}
\hline & \multicolumn{3}{|c|}{$\begin{array}{c}\text { Linear Limit } \\
(\mathrm{LL})\end{array}$} & \multicolumn{2}{c|}{$\begin{array}{c}\text { Knee } \\
(\mathrm{KN})\end{array}$} & \multicolumn{2}{c|}{$\begin{array}{c}\text { Ultimate } \\
\text { Failure (UL) }\end{array}$} \\
\hline & $\begin{array}{c}\text { Shear } \\
\text { Stress } \\
(\mathrm{MPa})\end{array}$ & $\begin{array}{c}\text { Shear } \\
\text { Strain } \\
(\mathrm{mm} / \mathrm{mm})\end{array}$ & $\begin{array}{c}\text { Shear } \\
\text { Modulus } \\
(\mathrm{MPa})\end{array}$ & $\begin{array}{c}\text { Shear } \\
\text { Stress } \\
(\mathrm{MPa})\end{array}$ & $\begin{array}{c}\text { Shear } \\
\text { Strain } \\
(\mathrm{mm} / \mathrm{mm})\end{array}$ & $\begin{array}{c}\text { Shear } \\
\text { Stress } \\
(\mathrm{MPa})\end{array}$ & $\begin{array}{c}\text { Shear } \\
\text { Strain } \\
(\mathrm{mm} / \mathrm{mm})\end{array}$ \\
\hline $\begin{array}{c}\text { EA9361 } \\
\text { TAST }\end{array}$ & 5.5 & 0.022 & 189 & 11.7 & 0.1022 & 26.3 & 1.16 \\
\hline
\end{tabular}

The input data in the FE software has to be given in tensile form. Therefore, the TAST results have to be used to determine the tensile properties using an appropriate correlation. In the linear elastic range, the shear modulus, $G$, can be related to the tensile modulus, $E$, using the following equation:

$$
G=\frac{E}{2(1+v)}
$$

where $v$ is the Poisson's ratio. The next step is to correlate the stresses and strains in the plastic regime; this can be carried out by using the procedure proposed by Dolev et al. [130], in which , the bulk properties are related to the adhesive tested in shear according to the von Mises criterion. The stress and strains are correlated as follows:

$$
\begin{gathered}
\tau=\frac{\sigma}{\sqrt{3}} \\
\gamma=\frac{2 \varepsilon(1+v)}{\sqrt{3}}
\end{gathered}
$$

These equations are valid for all the stress-strain values when the effect of the volumetric component of the stress is negligible [131]. However, the simulation results can be improved with ductile adhesives when the volumetric is included [93]. This situation could affect the accuracy in the converted data for the EA9361 adhesive. However, due to the lack of appropriate and reliable data to correlate the stresses and 
strains by taking into account the effect of the volumetric component; the previously described correlation proposed by Dolev et al [130] using the von Mises criterion was applied to correlate the tensile stresses for the two selected adhesives. Figure 5.3 and Figure 5.4 shows the original shear strain data and the converted data to tensile using the aforementioned method for the selected adhesives.

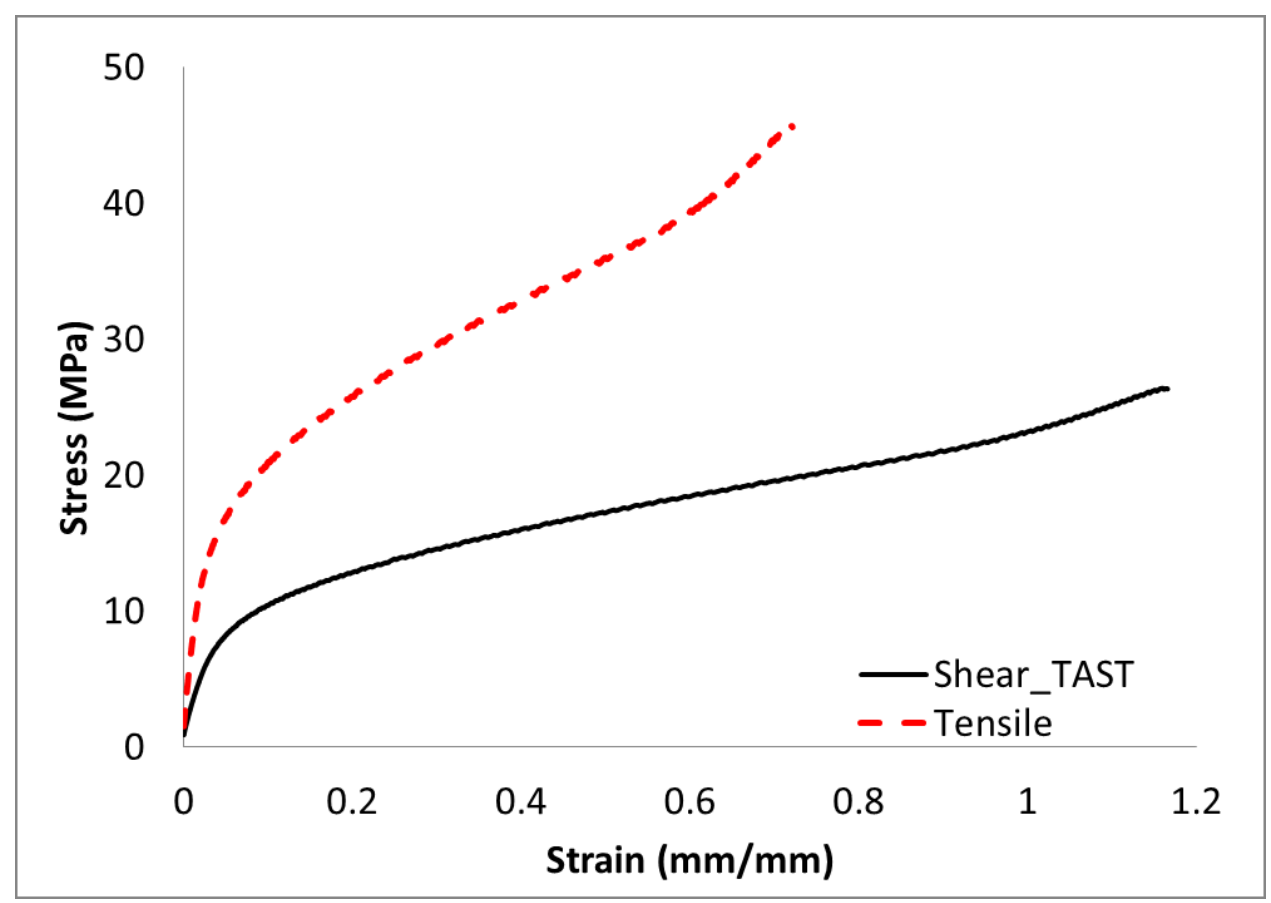

Figure 5.3 EA9361 adhesive. Shear strain-stress properties from TAST, and converted tensile strain-stress properties 


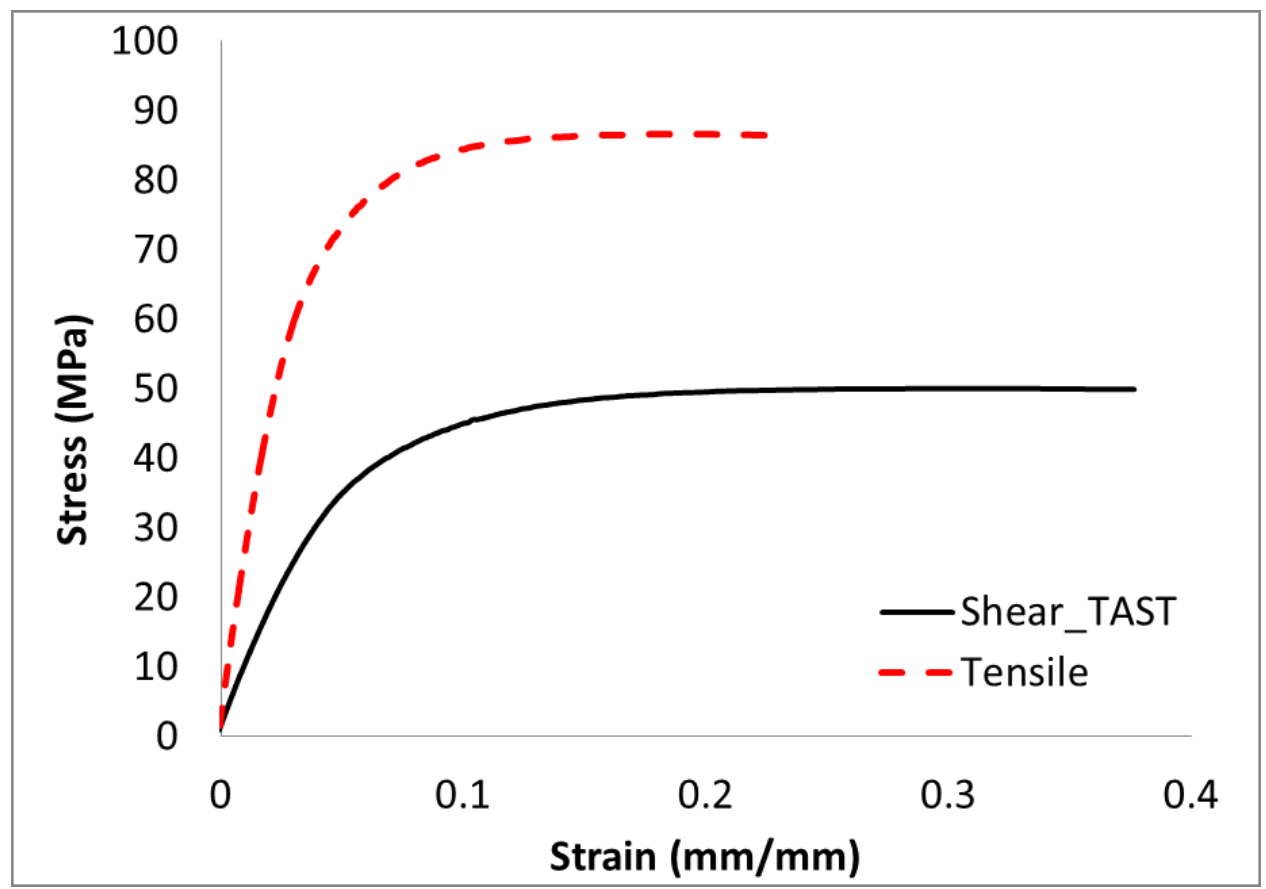

Figure 5.4 FM300-2M adhesive. Shear strain-stress properties from TAST, and converted tensile strain-stress properties

\subsection{Adhesives CZM Material Properties}

Having appropriate CZM material properties is a fundamental requirement to achieve accurate solutions. In addition, these parameters greatly affect the convergence during the numerical computations. The variability of the available data in the literature is another source of noise to perform accurate modelling. Therefore, a good choice on these properties is a fundamental before conducting the modelling.

The required data for a CZM analysis are the nominal tractions separation stresses, $t_{n}$ and $t_{s}$, nominal stiffness, $k_{n}, k_{s}$, and critical energy release rates $G_{I}$ and $G_{I I}$.

The nominal traction stresses are commonly the maximum strengths from the tested adhesive in tensile or shear [92, 132-134]. On the other hand, the critical energy release rates have to be obtained from standardized tests methods, such as the ENF and 
DCB test. Finally, depending on the CZM approach selected, continuum or local, the initial stiffnesses, $k_{n}$ and $k_{s}$ can be defined using the adhesive's Young's modulus as $E$ or $E / t$ respectively, where $t$ is the adhesive thickness. The mode II stiffness can be approximated as $G$ or $G / t$ respectively.

\subsubsection{FM 300-2M Adhesive CZM Properties}

Very few resources about the FM300-2M adhesive CZM parameters are available in the literature. Dzenis [135] characterized FM300-2M adhesive using DCB, ENF and Arcan test method. The details can be found in the aforementioned references. The mode I critical strain energy release values depends on the chosen test method and the adherend material during the test. For composites with unidirectional adherends, $G_{I C}$ was found to be $0.71,0.82$ and $1.13 \mathrm{~kJ} / \mathrm{m}^{2}$ from the $\mathrm{DBC}, \mathrm{ENF}$ and Arcan methods respectively. $G_{I I C}$ was found to be $0.59,0.76$ and $0.98 \mathrm{~J} / \mathrm{m}^{2}$.

Ridha [133] analyzed a composite bonded scarf repair with FM300 adhesive using several traction separation laws, triangular with linear propagation, triangular with exponential propagation and trapezoidal. Quadratic damage initiation and linear energy release for damage propagation was selected for the study. The CZM analysis was carried using $3 \mathrm{D}$ continuum cohesive elements with the following properties: $k_{n}=E / t=3100$ $\mathrm{MPa} / \mathrm{t} ; \mathrm{k}_{\mathrm{s}}=\mathrm{kt}=\mathrm{G} / \mathrm{t}=1250 \mathrm{MPa} / \mathrm{t}$. The nominal stresses were defined from adhesive bulk test data with $t_{\mathrm{n}}=66 \mathrm{MPa}$ and $t_{s}$ assumed to be equal to $53 \mathrm{MPa}$. Finally, the energy release rates were taken from [136] with values $\mathrm{G}_{\mathrm{IC}}=\mathrm{G}_{\mathrm{IIC}}=0.9 \mathrm{~J} / \mathrm{mm}$.

Yan et al [137] analyzed the effect of the a substrate material on fracture toughness. The energy release rates were measure experimentally for FM300 adhesive 
using the ASTM standard D3433-93. The test was carried out using steel and aluminum substrates. From the experimental results, the average critical fracture energy was 695 and $1029 \mathrm{~J} / \mathrm{m}^{2}$ for joints with steel and aluminum adherends respectively.

Donough [138] performed a failure criteria assessment on bonded joints. Several adhesive systems were analyzed including FM300-2K adhesive. The CZM analysis was carried out using the following adhesive properties: $\mathrm{E}=2400 \mathrm{MPa}, \mathrm{G}=840 \mathrm{MPa}$ and $\mathrm{v}=$ 0.4. The energy release rates were obtained from literature with $\mathrm{G}_{\mathrm{IC}}=1.3 \mathrm{~kJ} / \mathrm{m}^{2}$ and $\mathrm{G}_{\mathrm{IIC}}=1.3 \mathrm{~kJ} / \mathrm{m}^{2}$. According to the author, the strengths were obtained from TAST and the maximum shear strength was $54.4 \mathrm{MPa}$. Finally, the ultimate tensile strength of the adhesive was calculated using the von Mises yield criterion, with $\mathrm{X}_{\mathrm{t}}=94.2 \mathrm{MPa}$.

\subsubsection{EA9361 Adhesive CZM Properties}

Using the French standards NFT 76-142, da Silva et al [93]characterized the bulk properties of the EA9361 adhesive. From the testing results, the adhesive properties were: $\mathrm{E}=670 \mathrm{MPa}, \mathrm{v}=0.4, \mathrm{Sy}=4.23 \mathrm{MPa}, \mathrm{Su}=7.99 \mathrm{MPa}$, and failure strain of $44 \%$. The strain energy releases rate were not measured experimentally, instead, typical values from [139] were used with $\mathrm{G}_{\mathrm{IC}}=2.61 \mathrm{~kJ} / \mathrm{m}^{2}$ and $\mathrm{G}_{\mathrm{IIC}}$ was assumed to be equal to $5.22 \mathrm{~kJ} / \mathrm{m}^{2}$.

Using the adhesive properties from [93], Xu and Wei [140] analytically determined the EA9361 adhesive properties dependent on the adhesive thickness. The authors pointed out that the plastic energy has to be taken into account in the values of the mixed mode energy. This energy was incorporated in the author's proposed method to compute the thickness-dependent CZM parameters. The results for the EA9361 adhesive are shown in Figure 5.5 

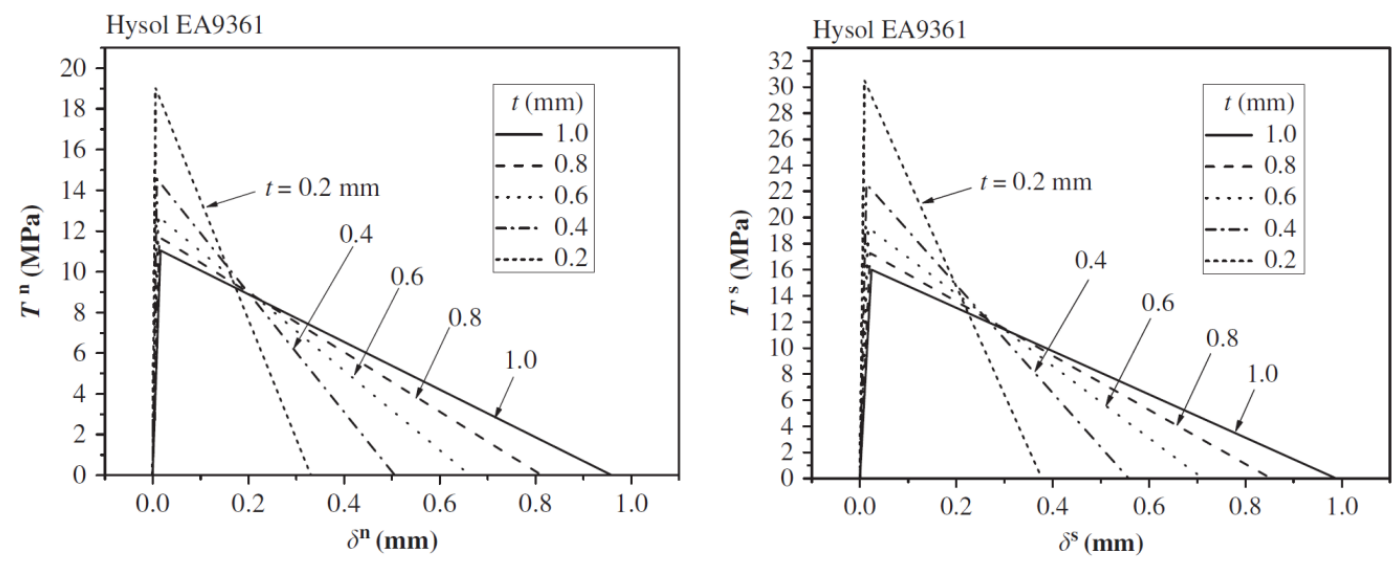

Figure 5.5 EA9361 adhesive. Thickness-dependent cohesive parameters; a) In tension; b) In Shear ( Reprinted from [140] with permission from Elsevier)

The authors carried out a parametric numerical analysis using the proposed thickness-dependent CZM parameters on single lap bonded joints, and the results were in good agreement with previous experimental work.

Using the EA9361 adhesive and the thickness-dependent CZM parameters from [140], Liao et al [88] performed a numerical failure analysis on bonded scarf joints. The adhesive layer was modeled as a single layer of four node cohesive elements sharing nodes with the steel adherends. The cohesive zone law chosen was triangular with quadratic traction damage initiation and linear energetic damage propagation.

Comparing the numerical results with previous experimental work, the authors concluded that the numerical analysis was effective. 


\subsubsection{Summary of CZM Parameters for FM300-2M and EA9361 Adhesives}

From the previous section, it is clear that the CZM properties for the selected adhesives presented variability. A summary of the data for the FM300 and FM300-2 adhesive is given in Table 5.7.

Table 5.7 Summary of CZM data for FM300 adhesive from literature review

\begin{tabular}{|c|c|c|c|c|c|c|c|c|}
\hline & $\begin{array}{c}\mathbf{t}_{\mathbf{n}} \\
(\mathbf{M P a})\end{array}$ & $\begin{array}{c}\mathrm{t}_{\mathbf{s}} \\
(\mathrm{MPa})\end{array}$ & $\begin{array}{c}\mathbf{E} \\
(\mathbf{M P a})\end{array}$ & $\begin{array}{c}\mathrm{G} \\
(\mathrm{MPa})\end{array}$ & $\begin{array}{c}\mathbf{k}_{\mathbf{n}} \\
(\mathrm{MPa})\end{array}$ & $\begin{array}{c}\mathbf{k}_{\mathrm{s}} \\
(\mathrm{MPa})\end{array}$ & $\mathbf{G}_{\mathrm{IC}}$ & $\mathbf{G}_{I I C}$ \\
\hline $\begin{array}{c}\text { Ridha [133] } \\
\text { FM300 }\end{array}$ & 66 & 53 & 3100 & 1250 & $\mathrm{E} / \mathrm{t}$ & $\mathrm{G} / \mathrm{t}$ & $\begin{array}{c}0.9 \\
\mathrm{~J} / \mathrm{mm}\end{array}$ & $\begin{array}{c}0.9 \\
\mathrm{~J} / \mathrm{mm}\end{array}$ \\
\hline $\begin{array}{c}\text { Dzenis [135] } \\
\text { FM300-2M }\end{array}$ & & & & & & & $\begin{array}{c}0.71 \\
0.82 \\
\text { and } \\
1.13 \\
\mathrm{~kJ} / \mathrm{m}^{2}\end{array}$ & $\begin{array}{c}0.59 \\
0.76 \\
\text { and } \\
0.98 \\
\mathrm{~kJ} / \mathrm{m}^{2}\end{array}$ \\
\hline $\begin{array}{c}\text { Yan et al } \\
\text { [137] }\end{array}$ & & & & & & & $\begin{array}{l}695 \\
\mathrm{~J} / \mathrm{m}^{2}\end{array}$ & $\begin{array}{l}1029 \\
\mathrm{~J} / \mathrm{m}^{2}\end{array}$ \\
\hline $\begin{array}{c}\text { Donough } \\
{[138]} \\
\text { FM300-2 }\end{array}$ & 54.4 & 94.2 & 2400 & 840 & & & $\begin{array}{c}1.3 \mathrm{~kJ} / \\
\mathrm{m}^{2}\end{array}$ & $\begin{array}{c}5 \\
\mathrm{~kJ} / \mathrm{m}^{2}\end{array}$ \\
\hline
\end{tabular}

The available data for the EA9361 adhesive is limited to that given by manufacturer's datasheet and the test from da Silva et al [93] as shown in Table 5.8.

Table 5.8 Summary of CZM data for EA9361 adhesive from literature review

\begin{tabular}{|c|c|c|c|c|c|c|c|c|}
\hline & $\begin{array}{c}\mathbf{t}_{\mathbf{n}} \\
(\mathbf{M P a})\end{array}$ & $\begin{array}{c}\mathbf{t}_{\mathrm{s}} \\
(\mathrm{MPa})\end{array}$ & $\begin{array}{c}\mathbf{E} \\
(\mathbf{M P a})\end{array}$ & $\begin{array}{c}\mathrm{G} \\
(\mathrm{MPa})\end{array}$ & $\begin{array}{c}\mathrm{k}_{\mathrm{n}} \\
(\mathrm{MPa})\end{array}$ & $\begin{array}{c}\mathbf{k}_{\mathbf{s}} \\
(\mathrm{MPa})\end{array}$ & $\mathbf{G}_{\mathbf{I C}}$ & $\mathbf{G}_{\text {IIC }}$ \\
\hline da Silva [93] & $\begin{array}{l}4.23 \\
\text { Yield } \\
\text { Stress }\end{array}$ & N.A. & & 670 & & & $\begin{array}{c}2.61 \\
\mathrm{~N} / \mathrm{mm}\end{array}$ & $\begin{array}{c}5.22 \\
\mathrm{~N} / \mathrm{mm}\end{array}$ \\
\hline $\begin{array}{c}\text { Xu and Wei } \\
{[140]}\end{array}$ & \multicolumn{2}{|c|}{$\begin{array}{l}\text { Thickness } \\
\text { dependent } \\
\text { ( Figure 5.5) }\end{array}$} & & 670 & $E / t$ & $\mathrm{G} / \mathrm{t}$ & \multicolumn{2}{|c|}{$\begin{array}{c}\text { Thickness } \\
\text { dependent } \\
\text { (Figure 5.5) }\end{array}$} \\
\hline
\end{tabular}


The CZM law selected for the present study was the triangular law with quadratic traction damage initiation criteria and energetic linear criteria for damage propagation. For the models with EA9361 adhesive and the CZM continuum approach, it was decided to use tractions and stiffnesses values from the CRIAQ TAST data. The reason is the tremendous discrepancy found in the literature review for the $t_{n}$ and $t_{s}$ values. The energy release rates were defined from [93] and [133] for the EA9361 and FM300-2M adhesives respectively. Table 5.9 summarizes the CZM data for the two aforementioned adhesives.

Table 5.9 FM300-2M and EA9361 adhesives. CZM parameters used in the cohesive zone models.

\begin{tabular}{|c|c|c|c|c|c|c|c|c|c|}
\hline \multirow{2}{*}{ Adhesive } & $\mathbf{t}_{\mathbf{n}}$ & $t_{s}$ & $\mathbf{E}$ & G & $\mathbf{k}_{\mathrm{n}}$ & $\mathbf{k}_{\mathrm{s}}$ & \multirow{2}{*}{$\mathbf{G}_{\mathrm{IC}}$} & \multirow{2}{*}{$\mathbf{G}_{\text {IIC }}$} & \multirow{2}{*}{$\begin{array}{c}\text { CZM } \\
\text { approach }\end{array}$} \\
\hline & \multicolumn{6}{|c|}{ (MPa) } & & & \\
\hline $\begin{array}{c}\text { FM300- } \\
2 \mathrm{M}\end{array}$ & 86 & 50 & 2400 & 840 & $2400 / \mathrm{t}$ & $840 / \mathrm{t}$ & $\begin{array}{c}0.9 \\
\mathrm{~N} / \mathrm{mm}\end{array}$ & $\begin{array}{c}0.9 \\
\mathrm{~N} / \mathrm{mm}\end{array}$ & Continuum \\
\hline $\begin{array}{c}\text { EA9361 } \\
0.5 \mathrm{~mm}\end{array}$ & 45.5 & 26 & 529 & 189 & $529 / \mathrm{t}$ & $189 / \mathrm{t}$ & $\begin{array}{c}2.61 \\
\mathrm{~N} / \mathrm{mm} \\
* 3.96\end{array}$ & $\begin{array}{c}5.22 \\
\mathrm{~N} / \mathrm{mm} \\
* 6.57\end{array}$ & $\begin{array}{c}\text { Continuum } \\
\text { Local }\end{array}$ \\
\hline $\begin{array}{c}\text { EA9361 } \\
0.2 \mathrm{~mm}\end{array}$ & 45.5 & 26 & 529 & 189 & $529 / \mathrm{t}$ & $189 / \mathrm{t}$ & $\begin{array}{c}2.61 \\
\mathrm{~N} / \mathrm{mm} \\
* 3.04\end{array}$ & $\begin{array}{c}5.22 \\
\mathrm{~N} / \mathrm{mm} \\
* 5.7\end{array}$ & $\begin{array}{c}\text { Continuum } \\
\text { Local }\end{array}$ \\
\hline
\end{tabular}

* Thickness dependent

\subsection{Fastener and Washer}

The bolt material was 6 Al-4V as per AMS4928 or AMS4967 with minimum strengths of $620 \mathrm{MPa}(95,000 \mathrm{psi})$ and $1100 \mathrm{MPa}(160,000 \mathrm{psi})$ in shear and tensile, 
respectively [121]. However, the strain versus stress data is not available from the manufacturer's specifications. From [141] it was found that elongation at break was $14 \%$ and a yield strength of $880 \mathrm{MPa}$. Therefore, the bolt was modeled as an elastoplastic material with $\mathrm{E}=110,000 \mathrm{MPa}, \mathrm{v}=0.34$, yield strength of $880 \mathrm{MPa}$, maximum strength of $1100 \mathrm{MPa}$ and maximum strain of $0.14 \mathrm{~mm} / \mathrm{mm}$.

The steel washer was modeled as linear elastic material with E=200,000 MPa, and $\mathrm{v}=0.33$.

\subsection{FEM Models}

The model geometry varies according the treatment from the DOE. However, the total length, width and bondline length were constants. Figure 5.6 shows the model geometry.
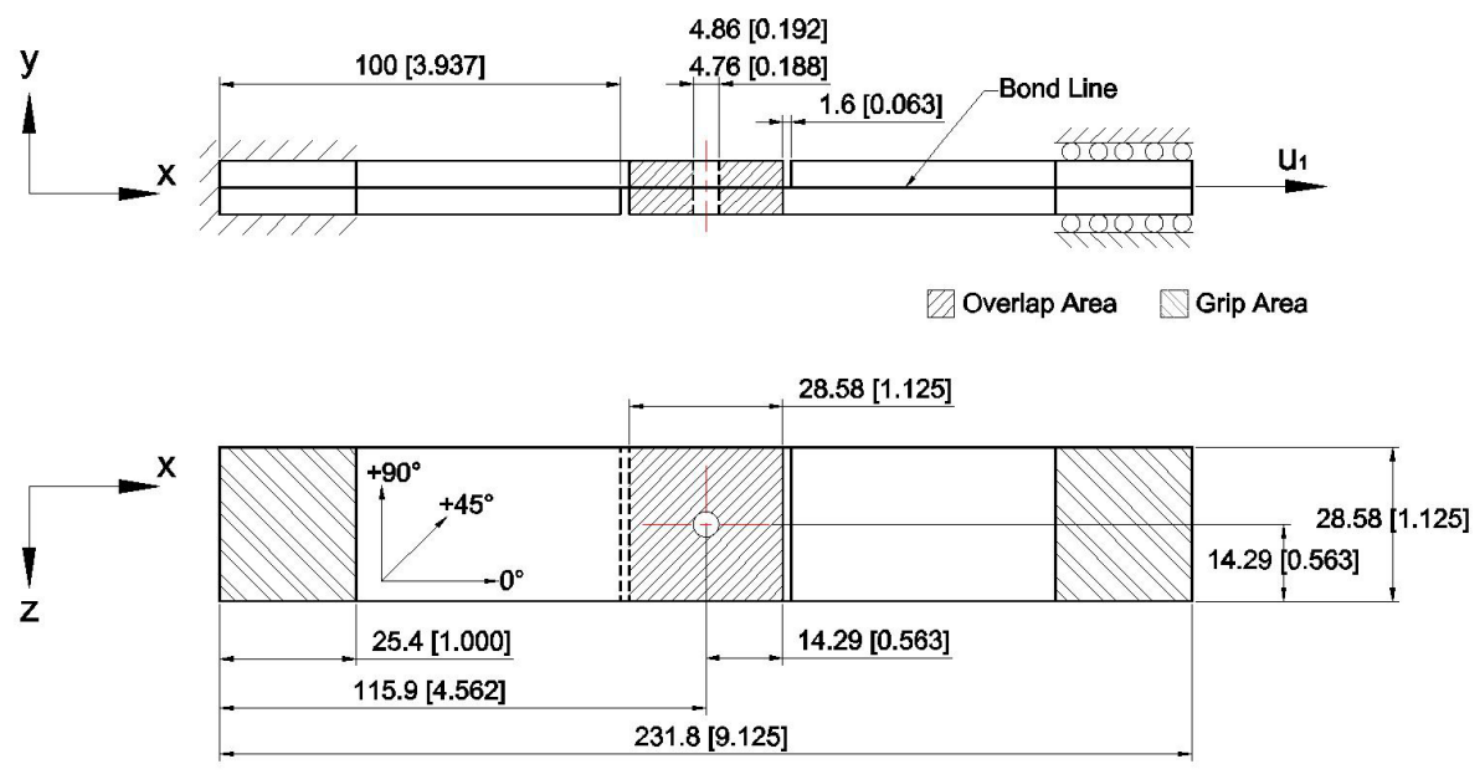

\section{UNITS mm [inch]}

Figure 5.6 FEM model geometry 
The adherend material was modeled as a layered composite by dividing the volume in four cells in the thickness direction. Each cell was defined to have four layers of composite material with stack sequence [0/45/90/-45] for the cells above the mid-plane and $[-45 / 90 / 45 / 0]$ for the cells below the mid-plane. The total number of plies was 24 or 40 depending on the adherend thickness defined in the FE model as shown in Figure 5.7 and Figure 5.8. In addition to this, the adherends were also divided in two cells along the longitudinal direction to separate the bondline region to create a refined mesh near to the hole. The cell in the bondline was meshed with 3D C3D20R elements; the remaining volume was meshed using 3D C3D8 elements. In total, the adherends with $3.29 \mathrm{~mm}$ thickness were meshed with 1326 C3D8 and 4062 C3D20R and the total number of nodes was 21258 . On the other hand, the adherend in the models with adherend thickness of $5.48 \mathrm{~mm}$ were meshed with 2470 C3D8 and 6150 C3D20R elements respectively, and the total number of nodes was 31408 .

The top and bottom doublers were also modeled as layered composites. A single cell of elements with 24 plies was defined for the adherends with 3.29 thickness and two cells of elements with 20 plies each was defined for the adherends with $5.48 \mathrm{~mm}$ thickness. 


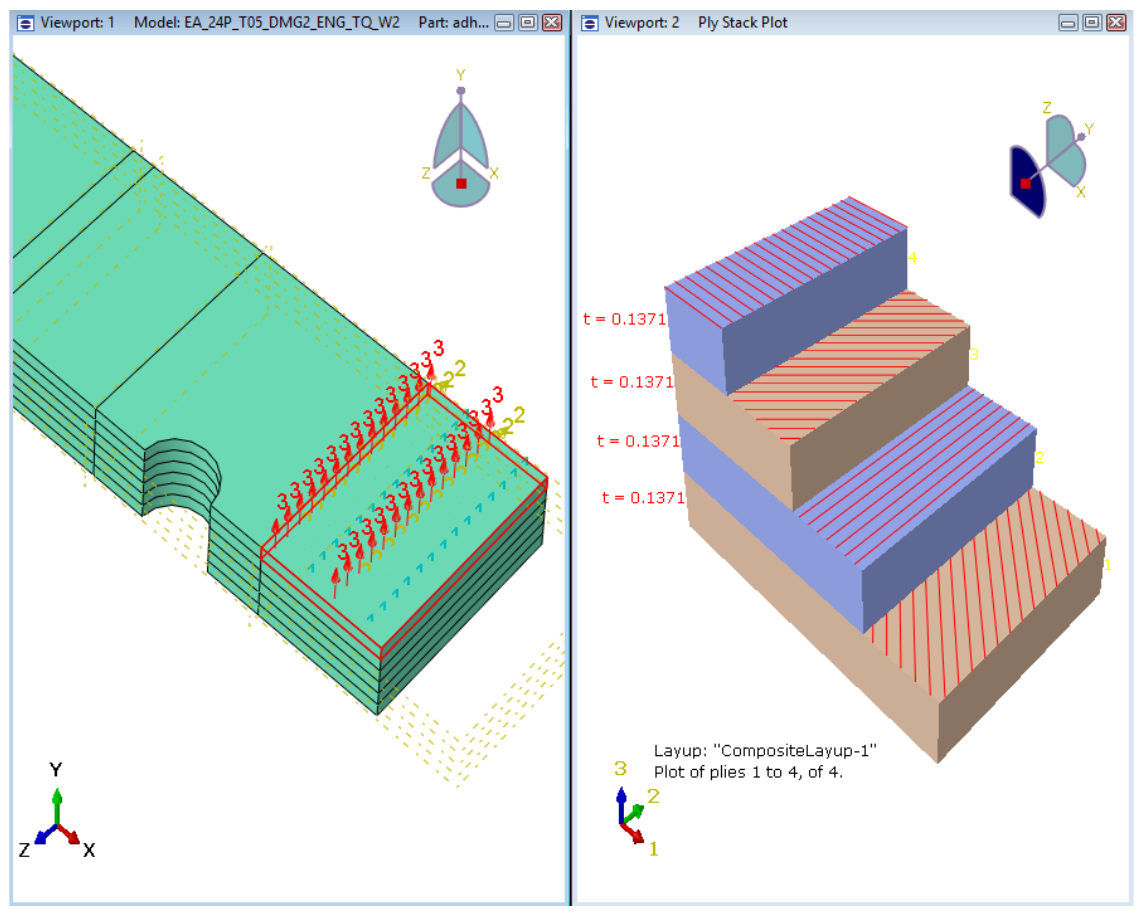

Figure 5.7 Laminate with 24 plies, $3.29 \mathrm{~mm}$ thickness and stacking sequence $[0 / 45 / 90 /-45]_{3 \mathrm{~s}}$

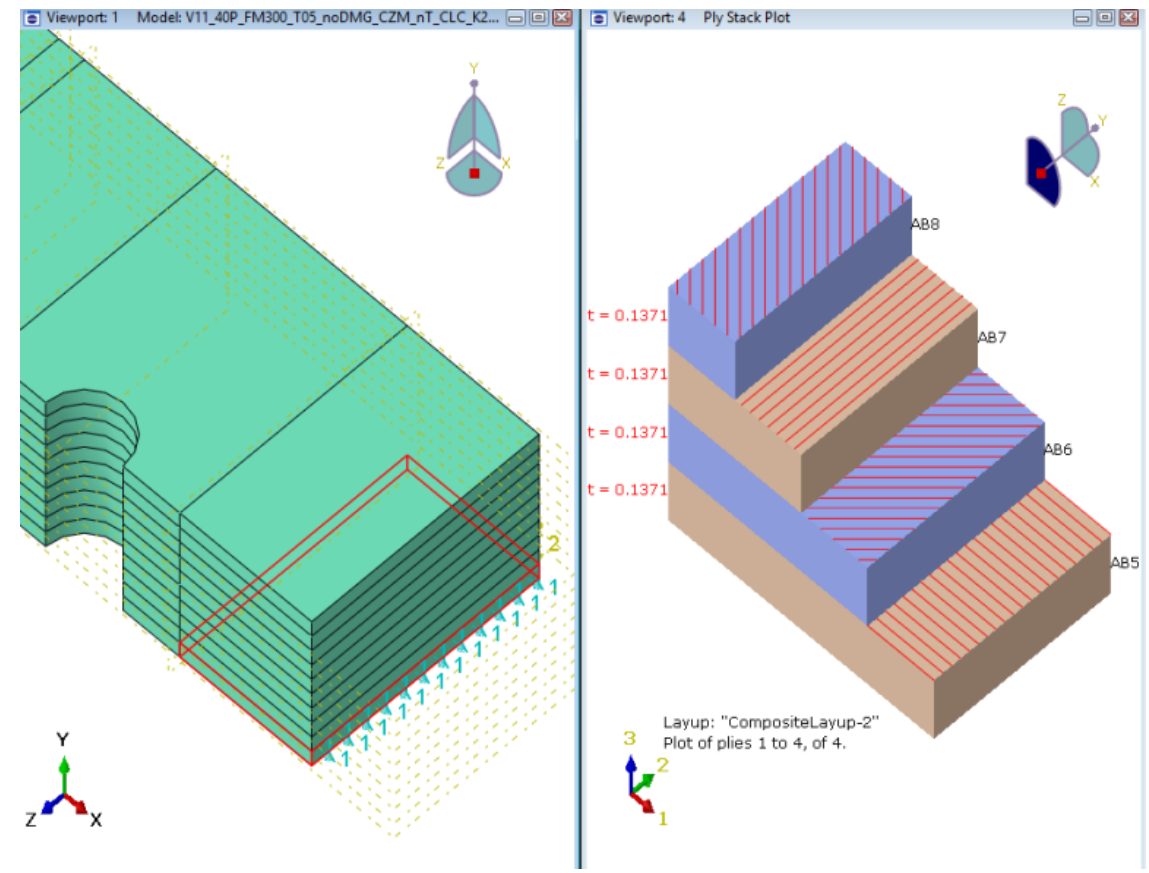

Figure 5.8 Laminate with 40 plies, $5.48 \mathrm{~mm}$ thickness and stacking sequence $[0 / 45 / 90 /-45]_{5 \mathrm{~s}}$

The adhesive was meshed using 12796 C3D20R elements and 71815 nodes for the joints with $0.5 \mathrm{~mm}$ adhesive thickness. The joints with $0.2 \mathrm{~mm}$ adhesive thickness 
were meshed with 27844 C3D20R elements and 155539 nodes. Figure 5.9 shows the FEM models near to the bondline region.

The bolt was meshed using a combination of linear and quadratic wedge and hexahedral elements. The number of elements for joints with thicker adherends was 5040 with 13410 nodes. For the joints with thinner adherends, the number of elements were 9124 with 9214 nodes.

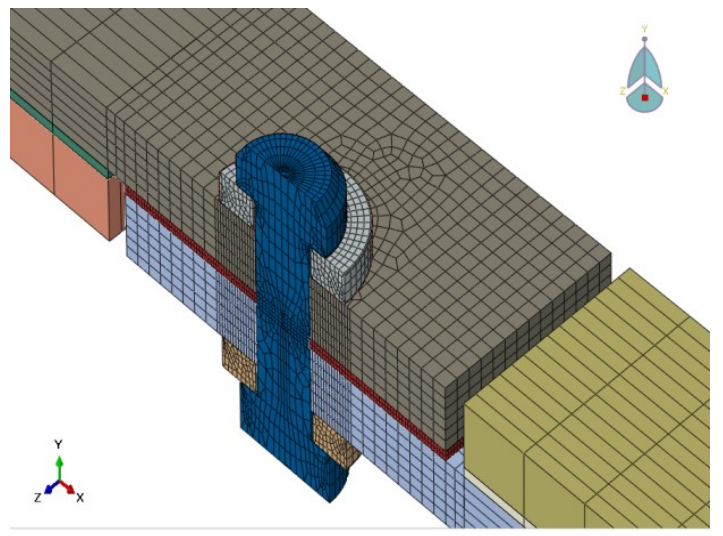

a)

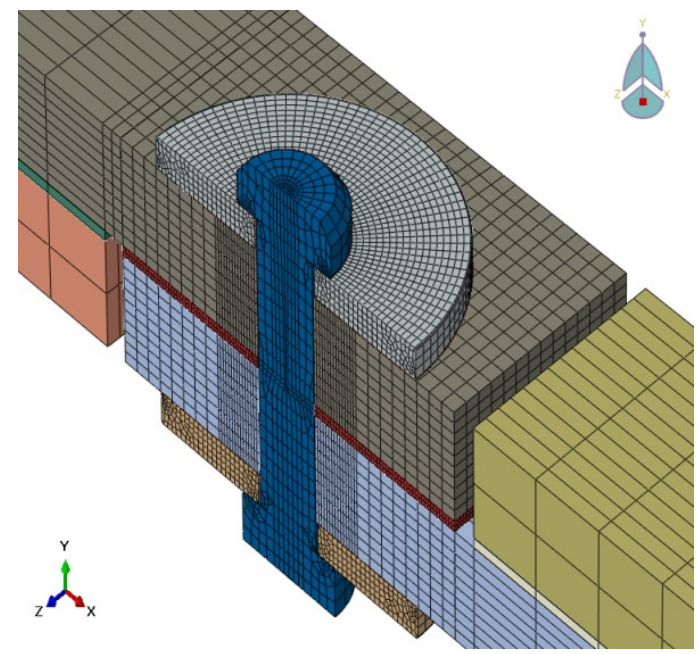

b)

Figure 5.9 Finite element models. a) Adherend thickness $3.29 \mathrm{~mm}$, adhesive thickness 0.5 $\mathrm{mm}$, small washer; b) Adherend thickness $5.48 \mathrm{~mm}$, adhesive thickness $0.5 \mathrm{~mm}$, large washer The washer was meshed using C3D8R linear hexahedral elements. The large washer was meshed with 7102 elements and 8802 nodes, and the small washer with 1888 elements and 2442 nodes.

A friction coefficient of 0.1 was assumed in all the contact surfaces between the bolt and adherend as well as the bolt with the washers. 
The same models were used to carry out the analysis using CZM. The main difference is that the adhesive layer was meshed using a single layer of 3D COH3D8 cohesive elements with thickness equal to the adhesive layer thickness. The number of elements and nodes were $1663 \mathrm{COH} 3 \mathrm{D} 8$ elements and 3512 nodes for 0.5 adhesive thickness, and $9937 \mathrm{COH} 3 \mathrm{D} 8$ elements and 20320 nodes for $0.2 \mathrm{~mm}$ adhesive thickness.

The boundary conditions are shown in Figure 5.6; the joint was fixed on the left end, and a displacement $\mathrm{u}_{1}$, in the $\mathrm{x}$-direction was applied at the right end. In addition, just a half of the joint was modeled using half-symmetry boundary condition along the width.

The preload was defined using the bolt load feature available in the software. The clamping force was computed using the relationship between the torque and force:

$$
T=K F d
$$

where $T$ is the applied torque, $F$ is the clamping force, and $K$ is the friction coefficient between the thread and the nut. Assuming a friction coefficient of 0.2 , the clamping force was computed to be $2961 \mathrm{~N}$ for a torque value of $2819 \mathrm{~N}-\mathrm{mm}$ (25 lb-in).

\subsection{Bonded Joints Cohesive Zone Models}

The aim of the analysis of bonded joint using CZM was to assess the variability of the input parameters, traction forces, energy release rates and stfiffness in the joint performance. As pointed out in the previous section, parametric studies are commonly carried out in bonded joints by varying the cohesive parameters to assess the variation in the joint strength. These parametric studies were carried out due to the variability of the available CZM data. 
Unfortunately, a parametric study in bolted/bonded joints using cohesive zone modelling is out of the aim of this research. However, to test the veracity of the CZM mechanical properties, a preliminay set of analyses using bonded joints was carried out. The reason for this analysis was to save computational time by analyzing bonded joints using simplified 2D models, prior to the the full implementation in bolted/bonded joints models.

The geometry of the 2D bonded joints was the same geometry as the bolted bonded joints, excepting for the hole in the bondline. Simple 2D models with less than two thousand nodes with plane strain elements were created and analyzed. The adherend material was defined as isotropic and the input values in the FEM model were $\mathrm{E}=54.5$ $\mathrm{MPa}$, and $\nu=0.313$ respectively. For the adhesive, the CZM parameters were defined from Table 5.9.

This approach using quasi-isotropic material properties in the adherend may not capture the out-of-plane behaviour propertly. However, for this initial strage, high accuracy in the model is not required. The aim of this preliminary study using $2 \mathrm{D}$ bonded model is to analyze the influence of the cohesive zone model parameters in the bonded joint behaviour. In addition to this, to identify the best parameters in terms of loading and time-stepping. The utlimate goal is to analyze the bolted/bonded joints using a full 3D model.

Using the CZM continuum approach, the adhesive was meshed with a single layer of COH2D4 cohesive elements. The adhesive joints with EA9361 were also modelled using the local approach using a surface-to-surface contact interaction with cohesive 
behaviour. The surfaces were defined at the two interfaces between the adherend and the adhesive. Finally, the CZM parameters were assigned using the data from Table 5.9.

The displacements were defined in several steps in both, elastoplastic and CZM models. This approach was chosen to define the time steps accordingly, and to capture enough data points to compare with the experimental results. Another reason for this, is to apply these displacements smoothly to minimize convergence issues.

\subsubsection{CZM Bonded Joints With FM300-2 Adhesive}

The joint with FM300-2M adhesive, $3.29 \mathrm{~mm}$ adherend thickness and $0.5 \mathrm{~mm}$ adhesive thickness was meshed with 1930 nodes and 1439 elements. The numerical results were compared with the experimental results as shown in Figure 5.10. The displacements from the experimental result are the MTS stroke displacements, and the displacements from the numerical results are the displacement in the joint. The maximum experimental average strength was $10.4 \mathrm{kN}$ and the maximum predicted strength from the CZM model was $9.65 \mathrm{kN}$, the agreement is good, $91.5 \%$. The numerical failure sequence in the adhesive layer is shown in Figure 5.11. It can be observed that the maximum strength, $9.65 \mathrm{kN}$, is reached at early stages of adhesive failure, approximately when the length of the fracture was $2.5 \mathrm{~mm}$, point $\mathrm{b}$. However, even at lower adhesive damaged lengths, the maximum force is very close to the maximum value, point a, at $9.47 \mathrm{kN}$. The difference is barely $2 \%$.

In order to compare the required computational time, this model was solved using two platforms. Using a PC with an Intel i5-3210M CPU with 4 cores and 16 GB RAM, the required processing time was 2736 seconds ( $45.6 \mathrm{~min}$ ). Running the model in the 
Linux cluster, the processing time was 1164 seconds (19.4 min). The foregoing anticipates the challenges to achieve the analysis of the 3D models, where, the number of elements and nodes increase significantly.

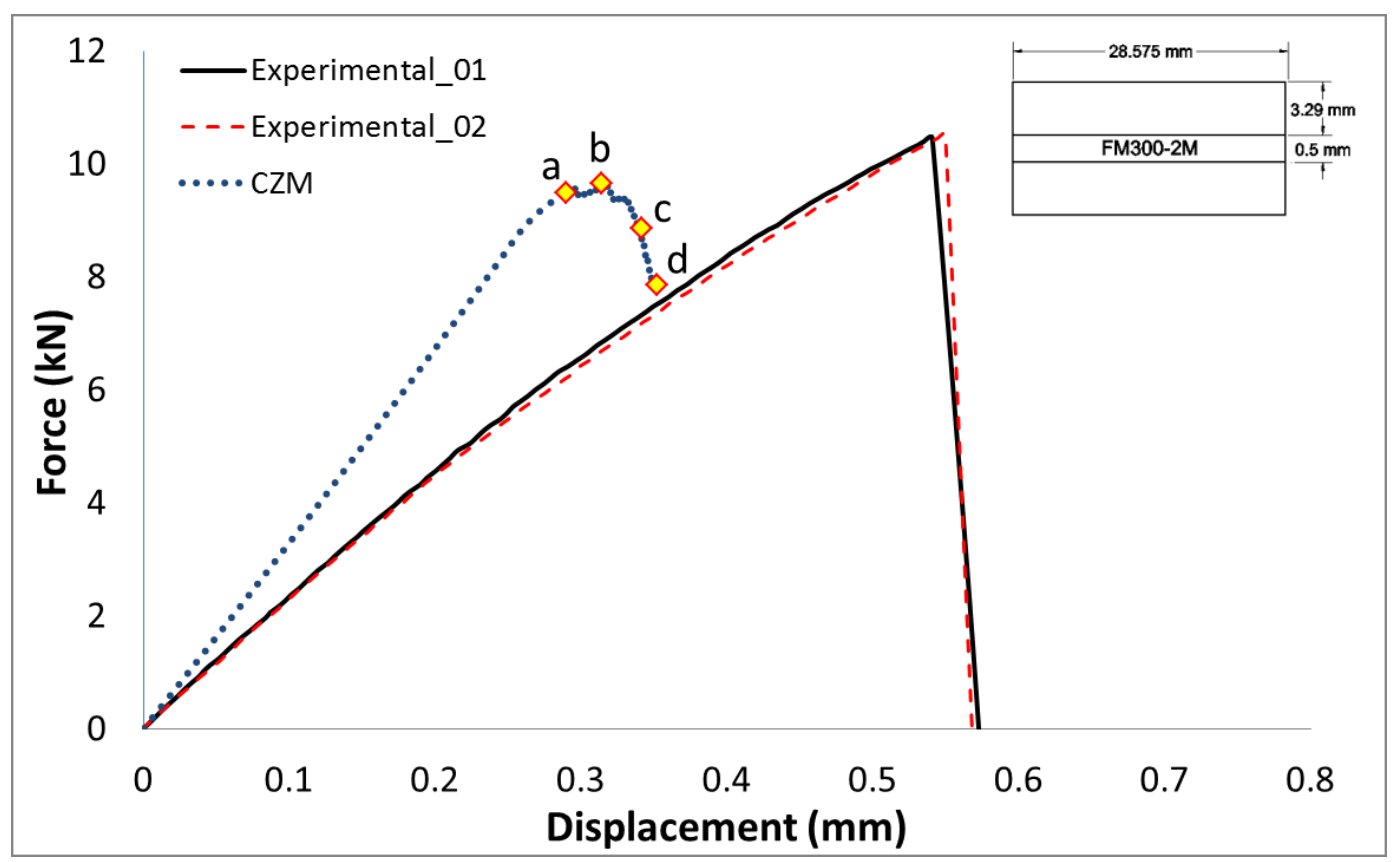

Figure 5.10 Bonded joints with FM300-2M adhesive. Force versus displacement CZM results and comparison with experimental results.

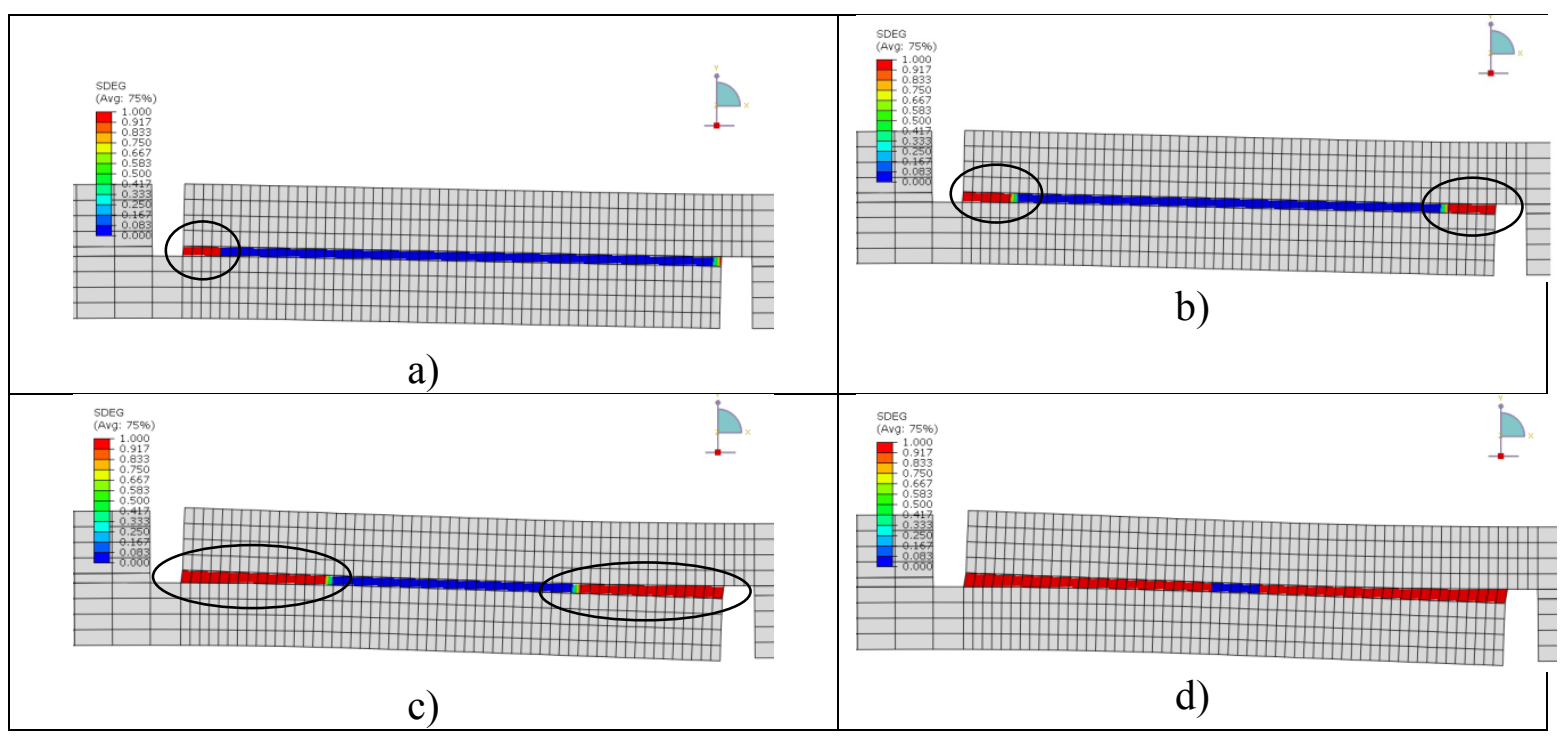

Figure 5.11 Bonded joints with FM300-2M adhesive. CZM results. The adhesive degradation is shown at different force values. 
Although the remaining bonded joint combinations with FM300-2M adhesive were not tested experimentally, these joints were modelled to obtain a reference frame to compare with the bolted bonded joints. The results are shown in Figure 5.12. It can be observed that the strength is not affected significantly by the adhesive thickness. However, the adherend thickness has a positive effect in the maximum strength and stiffness.

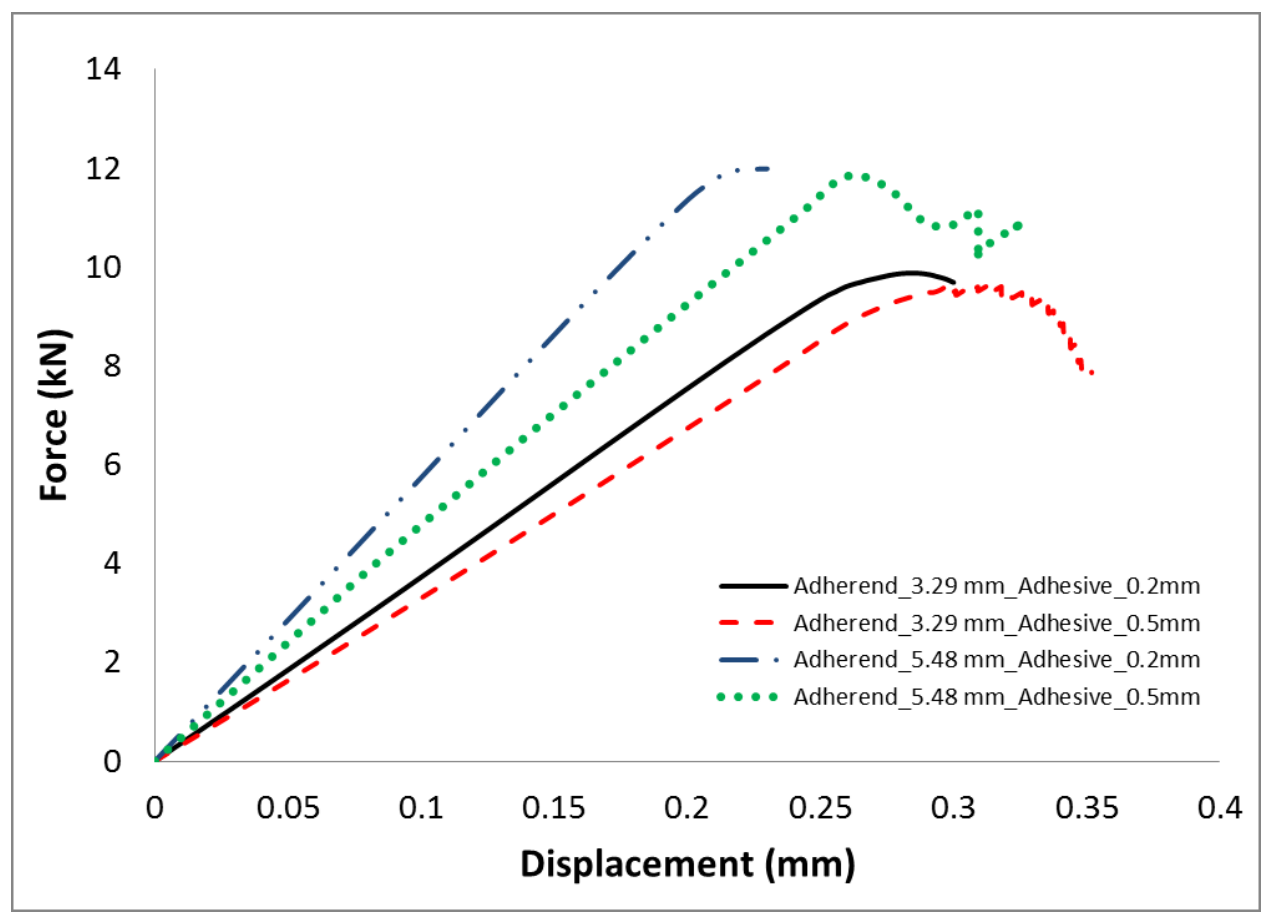

Figure 5.12 Bonded joints with FM300-2M adhesive. Predicted strength using CZM

The numerical results also show that the maximum strengths, in all the joints, were reached once the adhesive at the edge was damaged. For illustration purposes, the adhesive damage in a joint with $5.48 \mathrm{~mm}$ adherend thickness and $0.5 \mathrm{~mm}$ adhesive thickness is shown in Figure 5.13. 


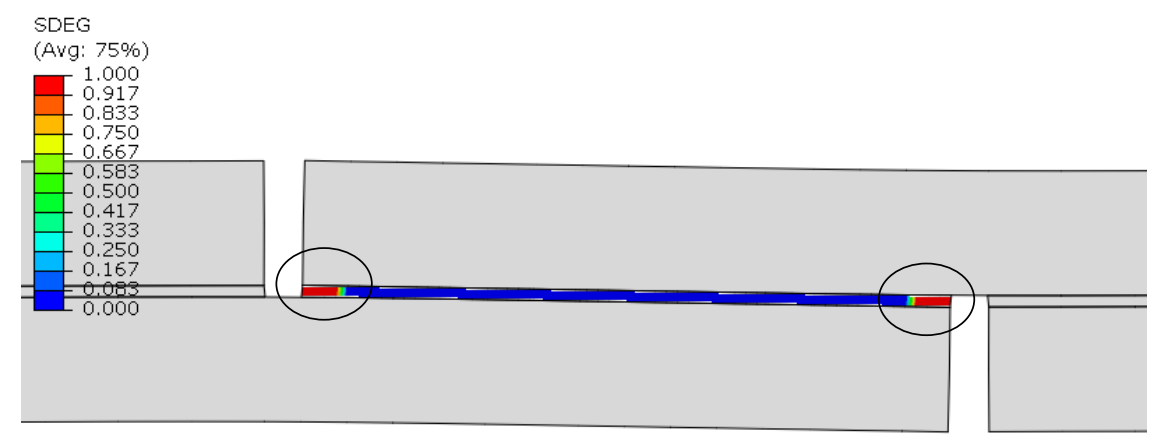

Figure 5.13 Bonded joint with FM300-2M adhesive. CZM results showing the damage in the adhesive at maximum strength of $11.6 \mathrm{kN}$

From the bonded joints modelling results using FM300-2M adhesive, it is concluded that maximum strength is reached once the adhesive near to the edge has reached maximum degradation, and not necessarily the entire adhesive layer.

\subsubsection{CZM Bonded Joints With EA9361 Adhesive}

The CZM results for the joint with adherend thickness of $3.29 \mathrm{~mm}$ and adhesive thickness of $0.2 \mathrm{~mm}$ are shown in Figure 5.14. Two analysis using CZM continuum approach were carried out, the solution labeled $K$ and the end, was solved using the nominal stiffness of the adhesive; the second one, labeled $K / t$, was solved using the stiffness divided by the adhesive thickness. The aim of this analysis was to assess the effect of the stiffness parameter in the results. As observed, the maximum numerical strengths were $17.84 \mathrm{kN}$ and $16.74 \mathrm{kN}$; the $1.1 \mathrm{kN}$ force difference represents $6.6 \%$. From this, it is clear that the effect of the stiffness parameter does not have a great impact in the predicted strength in this configuration. 
Comparing with the maximum experimental strength of $15.32 \mathrm{kN}$, it can be observed that the numerical prediction was slightly higher with a $16 \%$ difference. On the other hand, the agreement in strength is remarkably good compared with the joint bonded_02, whose maximum strength was $16.64 \mathrm{kN}$.

The displacements are not comparable, because the experimental displacements are stroke results. Conversely, the displacements from the numerical results correspond to the joint only.

The maximum predicted strength using the local approach was $17.74 \mathrm{kN}$, almost the same as the result from the continuum approach using the stiffness value of $k / t$.

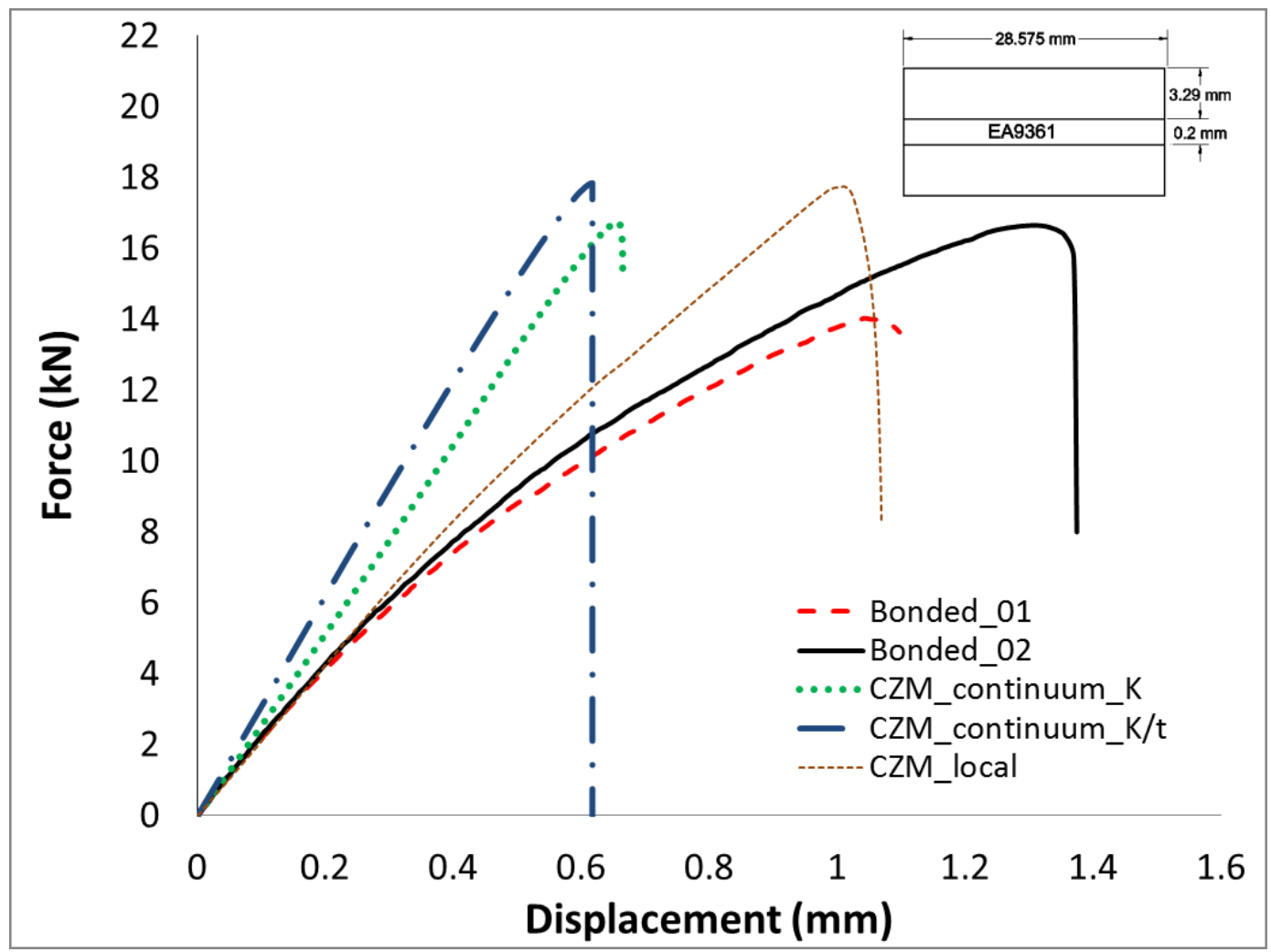

Figure 5.14 Bonded joint with EA9361 adhesive. Load versus displacement response from cohesive zone modelling results. Adherend thickness $3.29 \mathrm{~mm}$ and adhesive thickness 0.2 
Figure 5.15 shows the damage in the adhesive at maximum strength. At this point, the adhesive is damaged almost completely, with damage values from 0.913 to 1 . When these results are compared with the model with local approach, it can be observed that at maximum strength, the adhesive has failed $100 \%$ near to the edge; however, after approximately $1 \mathrm{~mm}$ distance from the notch, the damage value decreased near to zero. After the maximum strength value, the damage in the interface elements grows until total adhesive failure. Figure 5.16 depicts this process - the debond due to damage in the adhesive can be seen.

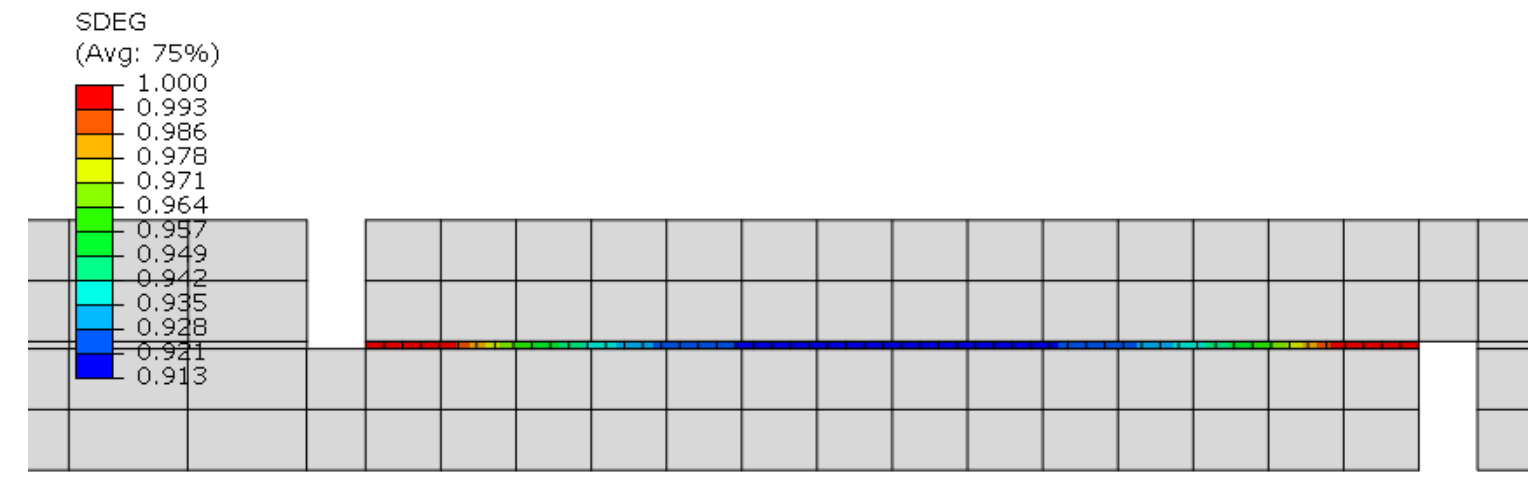

Figure 5.15 Bonded joint with EA9361 adhesive. Damage in the adhesive at maximum strength of $17.84 \mathrm{kN}$ using CZM continuum approach. Adherend thickness $3.29 \mathrm{~mm}$ and adhesive thickness $0.2 \mathrm{~mm}$ 


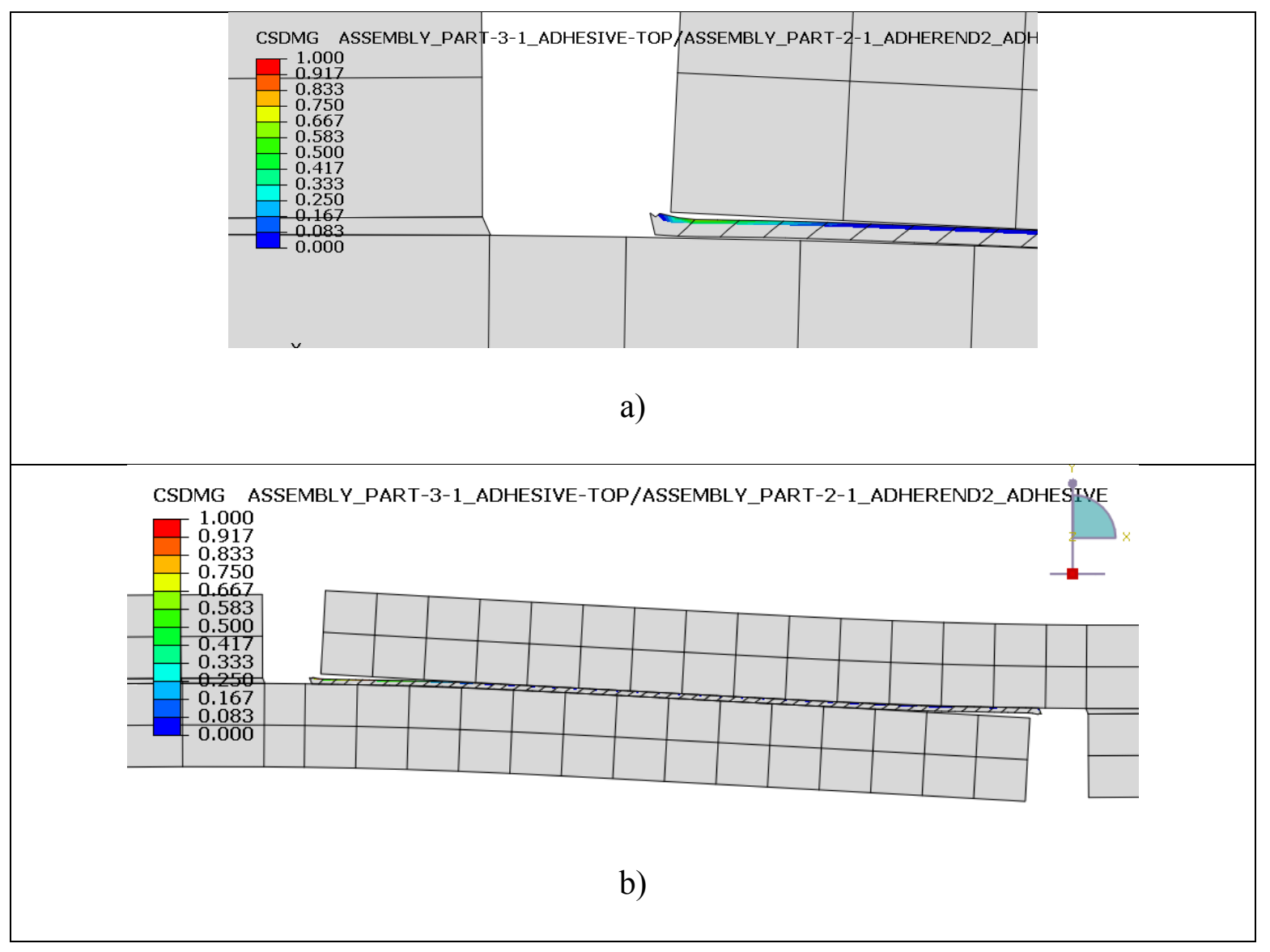

Figure 5.16 Bonded joint with EA9361 adhesive. Damage progression in the adhesive using CZM local approach: a) at maximum strength of $17.84 \mathrm{kN}, \mathrm{b}$ ) at $15.52 \mathrm{kN}$, after the maximum strength. Adherend thickness $3.29 \mathrm{~mm}$ and adhesive thickness $0.2 \mathrm{~mm}$

Figure 5.17 shows the CZM results for a bonded joint with $5.48 \mathrm{~mm}$ adherend thickness and adhesive thickness of $0.2 \mathrm{~mm}$. Due to the lack of material to manufacture more bonded joints with this configuration, only one repetition was tested. The experimental result shows a maximum strength of $16.76 \mathrm{kN}$. The maximum numerical strengths were $18.2 \mathrm{kN}$ and $17.78 \mathrm{kN}$ for the models with continuum approach, and 17.17 $\mathrm{kN}$ for the model with local elements. This represents agreements between numerical and experimental results of $91.4,93.9$ and $97.5 \%$ respectively. 


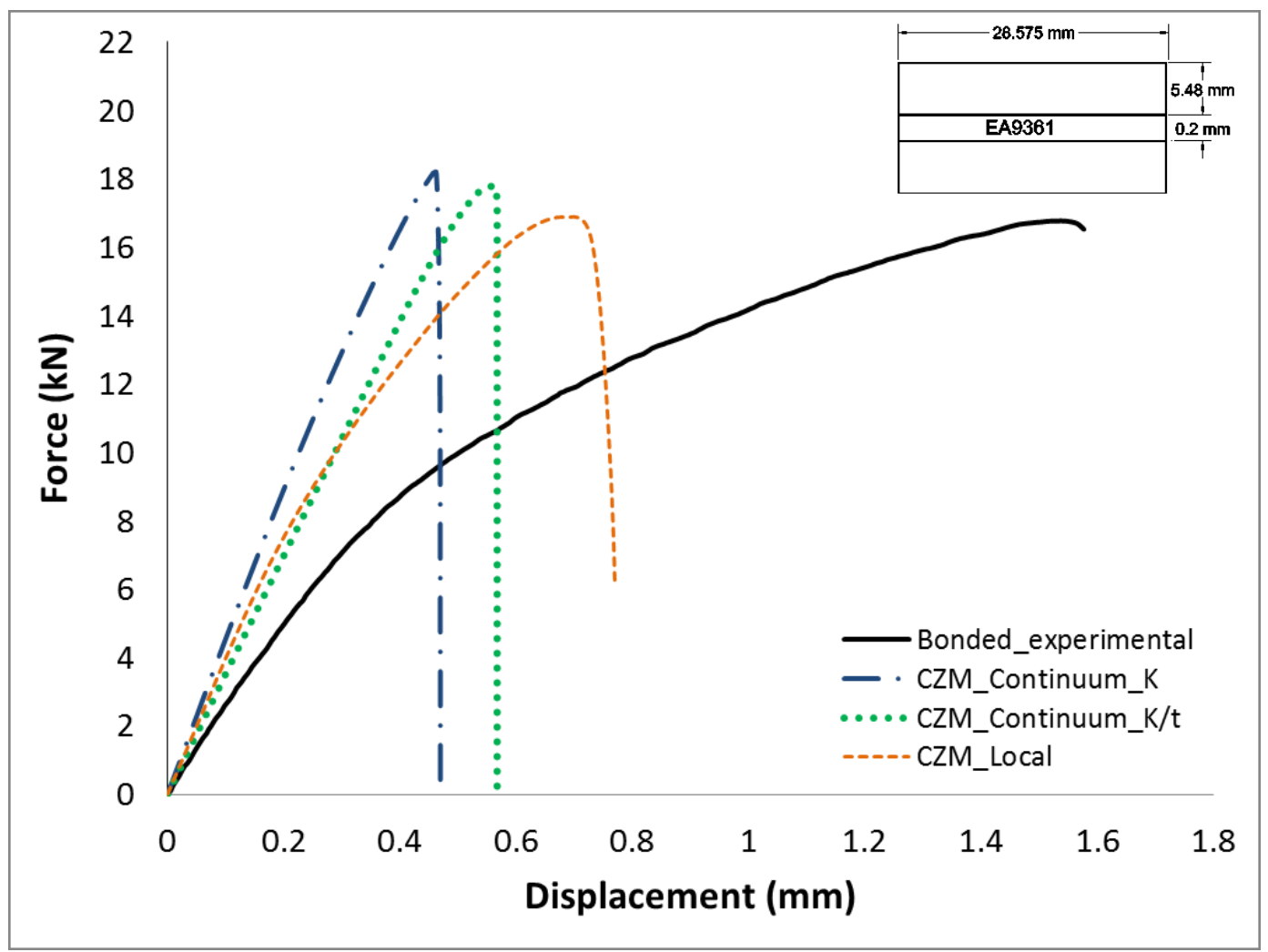

Figure 5.17 Bonded joint with EA9361 adhesive. Load versus displacement from cohesive zone modelling results. Adherend thickness $5.48 \mathrm{~mm}$ and adhesive thickness $0.2 \mathrm{~mm}$

Figure 5.18 shows the strength for the bonded joints with $3.29 \mathrm{~mm}$ adherend thickness and adhesive thickness of $0.5 \mathrm{~mm}$. In this case, four numerical runs were compared with two experimental results. Continuum and local approach were chosen to investigate the effect of the stiffness in the joint performance. The average maximum experimental strength was $15.5 \mathrm{kN}$. It can be observed that the maximum strengths from the CZM models with continuum approach were very similar, with forces equal to 16.04 $\mathrm{kN}$ and $16.34 \mathrm{kN}$ respectively, representing an agreement of $94.6 \%$ and $95 \%$ respectively. From this, it can be also concluded that the effect of the stiffness has very low significance in the predicted strength. When comparing the results from the local 
$\mathrm{CZM}$, the agreement is also very good, with maximum strength of $16.27 \mathrm{kN}$, representing an agreement of $95 \%$.

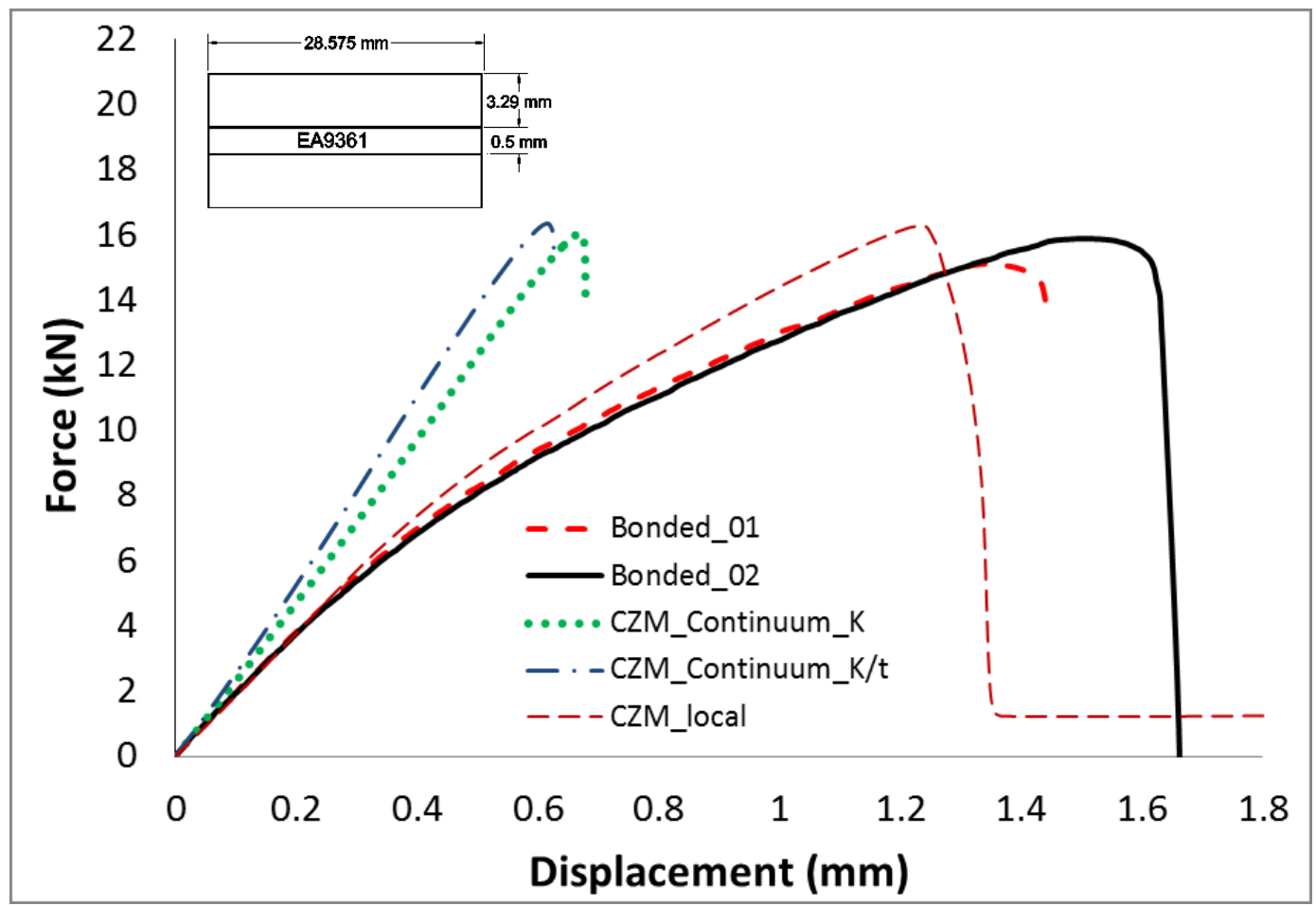

Figure 5.18 Bonded joint with EA9361 adhesive. Load versus displacement from cohesive zone modelling results. Adherend thickness $3.29 \mathrm{~mm}$ and adhesive thickness $0.5 \mathrm{~mm}$

From this preliminary analysis with bonded joints using CZM analysis, it is concluded that the chosen data to carry out the analysis is accurate. The effect of the thickness-dependent stiffness in the cohesive parameters is less than $5 \%$ in the predicted strengths. Finally, the predicted strengths with CZM local approach were in very good agreement, above $90 \%$, and the effect of the adhesive plasticity in the joint displacement was captured. 


\subsection{Modelling Results Bolted/bonded Joints}

The models with elastoplastic properties were developed to compute the strain at the adhesive edge and compare against the experimental results to compute the load in the bolts. In addition to this, the relative displacements or local stiffness can be extracted from the numerical analysis. On the other hand, the models with CZM were developed to predict the joint strength throughout the entire load carrying process. During the process, convergence issues were found. Some issues were solved using the recommendations from the software user manual such as increasing viscous regularization, and using automatic stabilization. After several trials, the best value for the viscous coefficient was found to be $0.001 \mathrm{~N}-\mathrm{s} / \mathrm{mm}$. In addition to this, the minimum element size was $0.5 \mathrm{~mm}$, in accordance with the minimum required element size discussed in section 2.4.

Similarly to the experimental results, the numerical results are discussed by the adhesive type. To avoid confusion during the data analysis, the treatments and their factors are summarized again in Table 5.10.

In addition to this, to avoid confusion with the displayed data, the experimental results are labeled with the treatment coded number followed by test number. The numerical models with elastoplastic adhesive properties are labeled as FE, and the numerical model with CZM are labeled as CZM. After these labels, the treatment code number is added. 
Table 5.10 Experimental treatments with FM300-2 adhesive

\begin{tabular}{|c|c|c|c|c|c|c|}
\hline \multirow{3}{*}{\multicolumn{2}{|c|}{$\begin{array}{c}\text { Treatment } \\
\text { number }\end{array}$}} & \multicolumn{5}{|c|}{ FACTORS } \\
\hline & & \multirow{2}{*}{$\begin{array}{c}\text { A } \\
\text { Adherend } \\
\text { thickness } \\
\text { (mm) }\end{array}$} & B & C & D & $E$ \\
\hline & & & Adhesive & $\begin{array}{c}\text { Adhesive } \\
\text { thickness (mm) }\end{array}$ & $\begin{array}{c}\text { Clamped } \\
\text { area } \\
\left(\mathrm{mm}^{2}\right)\end{array}$ & $\begin{array}{c}\text { Clearance } \\
\mathrm{mm}\end{array}$ \\
\hline 3 & be & 3.29 & FM300-2M & 0.2 & 97 & 0.1 \\
\hline 4 & $\overline{a b d}$ & 5.48 & FM300-2M & 0.2 & 387 & 0 \\
\hline 7 & $\mathrm{bc}$ & 3.29 & FM300-2M & 0.5 & 97 & 0 \\
\hline 8 & abcde & 5.48 & FM300-2M & 0.5 & 387 & 0.1 \\
\hline
\end{tabular}

The first displayed calculation is the relative stiffness using a $35 \mathrm{~mm}$ distance from the bondline, as specified in 3.6. The results are shown in Figure 5.19.

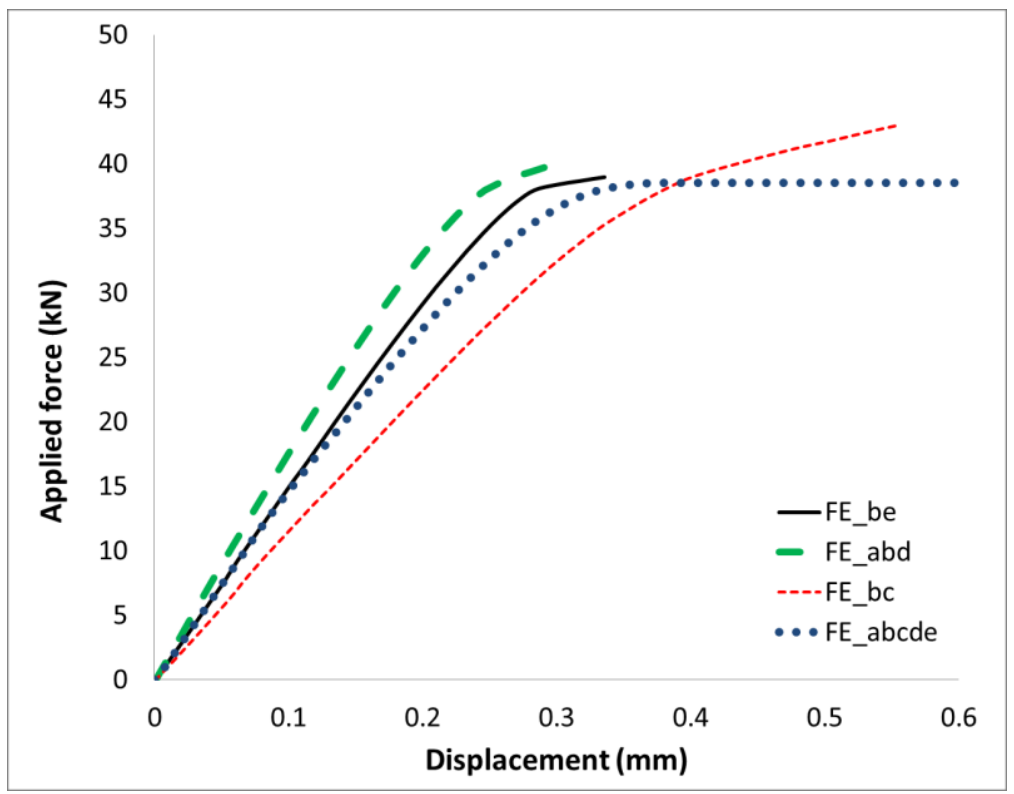

Figure 5.19 Bolted/bonded joints with FM300-2M adhesive. Force versus displacement finite element results

Linear force-displacement behaviour is observed in all the joints until approximately $35-40 \mathrm{kN}$. This is consistent due to the fact that the average shear strain, $\tau_{\mathrm{avg}}=\mathrm{F} / \mathrm{A}$, is $49 \mathrm{MPa}$ at $40 \mathrm{kN}$; where $\mathrm{A}$ is the area in the bondline, $816 \mathrm{~mm}^{2}$. This result 
is very close to the adhesive maximum shear strength of $50 \mathrm{MPa}$. However, these force levels were not reached experimentally.

Using the CZM analysis, the strengths were computed and compared with the experimental results. Due to time-stepping issues, the CZM_abcde model was not computed. The agreement between the predicted values and the experimental results were between $77 \%$ and $80 \%$. Table 5.11 shows the summary of the results. Beyond these failure strengths, the models with FM300-2M adhesive were not able to continue running due to convergence issues.

It can be observed that the treatments with adherend thickness of $3.29 \mathrm{~mm}$, be and $b c$, have almost the same strength at the first adhesive failure point.

Table 5.11 Bolted/bonded joints. Predicted failure load using CZM and comparison with experimental results

\begin{tabular}{|c|c|c|c|}
\hline Treatment & $\begin{array}{c}\text { First failure load } \\
\mathrm{kN}\end{array}$ & $\begin{array}{c}\text { Predicted failure load } \\
\mathrm{kN}\end{array}$ & $\%$ error \\
\hline be & $12.28 \pm 1.18$ & 14.64 & 20 \\
\hline abd & $16.58 \pm 1.15$ & 20.29 & 23 \\
\hline bc & $12.66 \pm 1.31$ & 15.19 & 20 \\
\hline abcde & $15.46 \pm 1.89$ & N.A. & N.A. \\
\hline
\end{tabular}

The plots of applied load versus displacement and the comparison with the results from the elastoplastic models are shown in Figure 5.20. It can be observed that there is good agreement in the stiffness between the elastoplastic and cohesive zone models. 


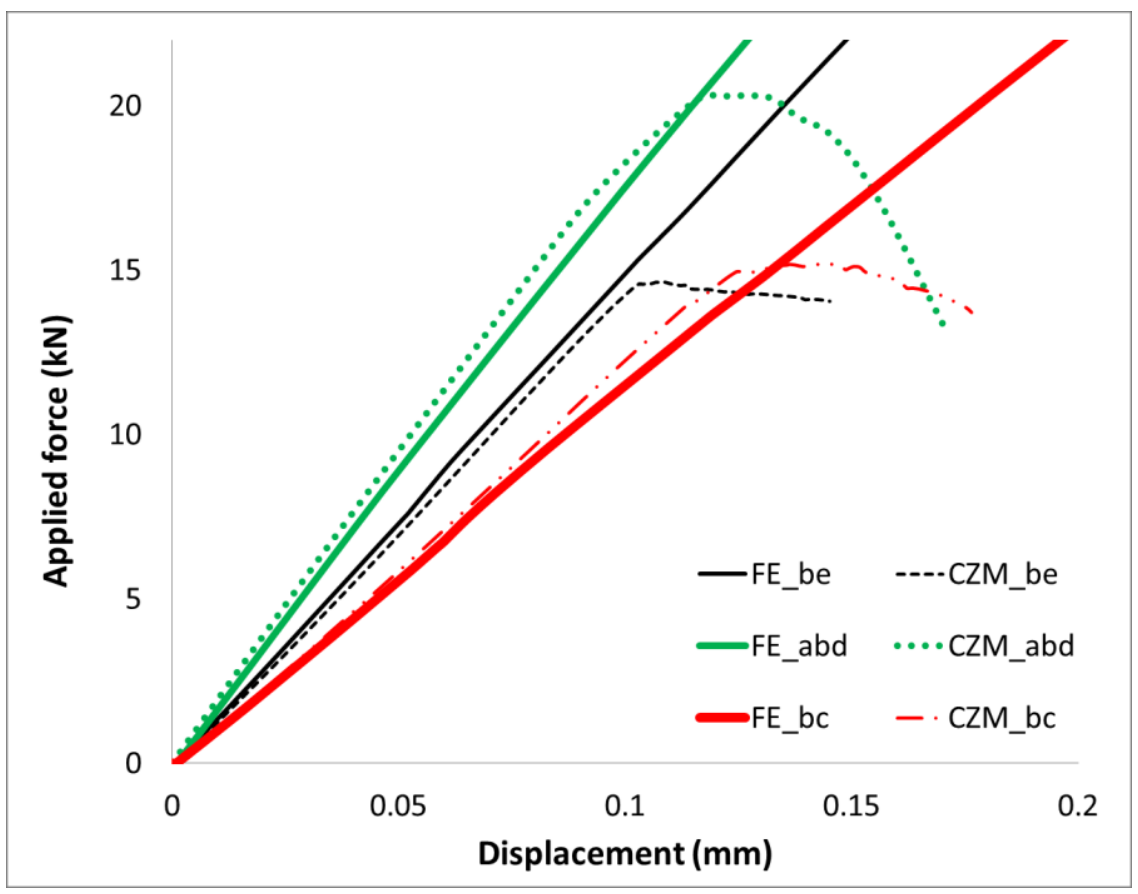

Figure 5.20 Bolted/bonded joints with FM300-2M adhesive. CZM strength prediction and comparison with elastoplastic models

A typical predicted damage progression in the adhesive layer is shown in Figure 5.21. The sequence corresponds to the treatment $b c$. Again, it can be observed that the maximum strength is reached when the edge in the adhesive has reached a damage state.

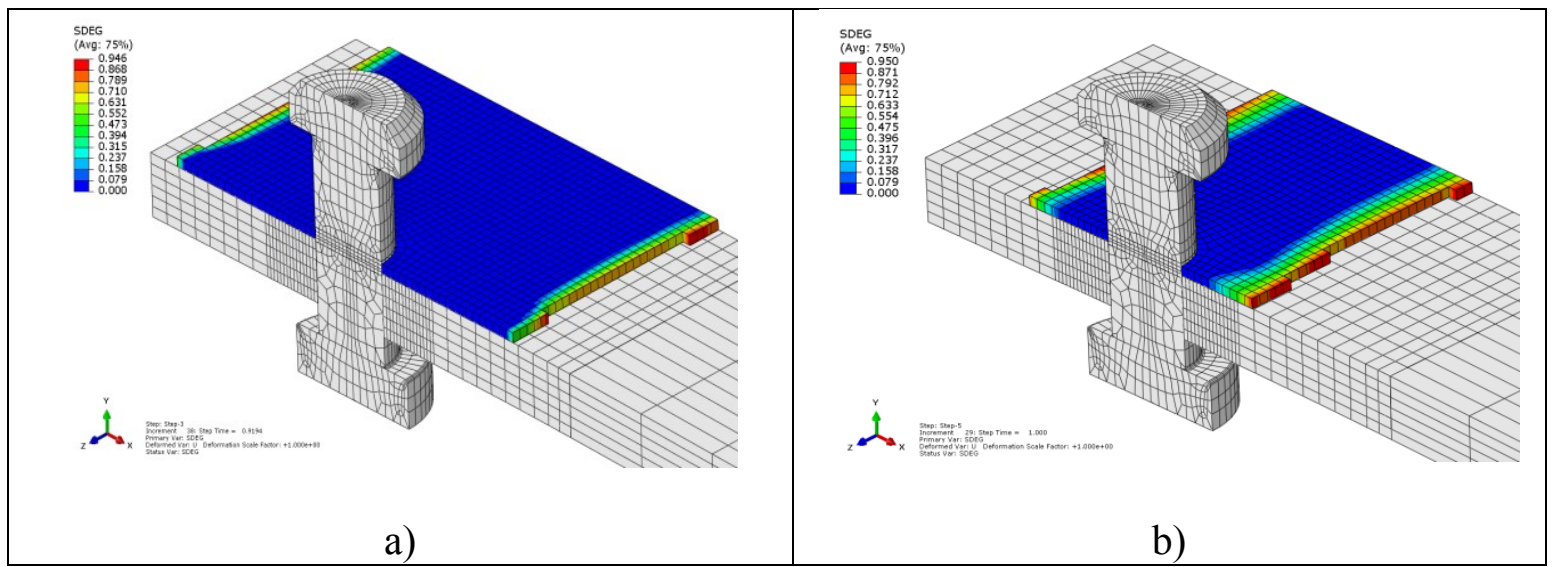

Figure 5.21 Bolted/Bonded joint,combination $b c$. Failure progress in the adhesive layer using CZM analysis. a) Maximum force of $15.2 \mathrm{kN}$, b) After maximum force, at $13.7 \mathrm{kN}$ 
From the elastoplastic models, the load taken by the bolt was computed. The values were considerably low, less than $3 \%$ of the load is taken by the bolt at maximum strength before the adhesive failure, as shown in Figure 5.22. This force represents less than $0.2 \mathrm{kN}$ for the model $F E \_b c$, and the forces in the bolt are considerably less for the remaining FE models. The most critical case is for the combination $b$ e, in which the predicted load taken by the bolts was zero until $16 \mathrm{kN}$, and barely $0.050 \mathrm{kN}$ at an applied force of $30 \mathrm{kN}$ approximately.
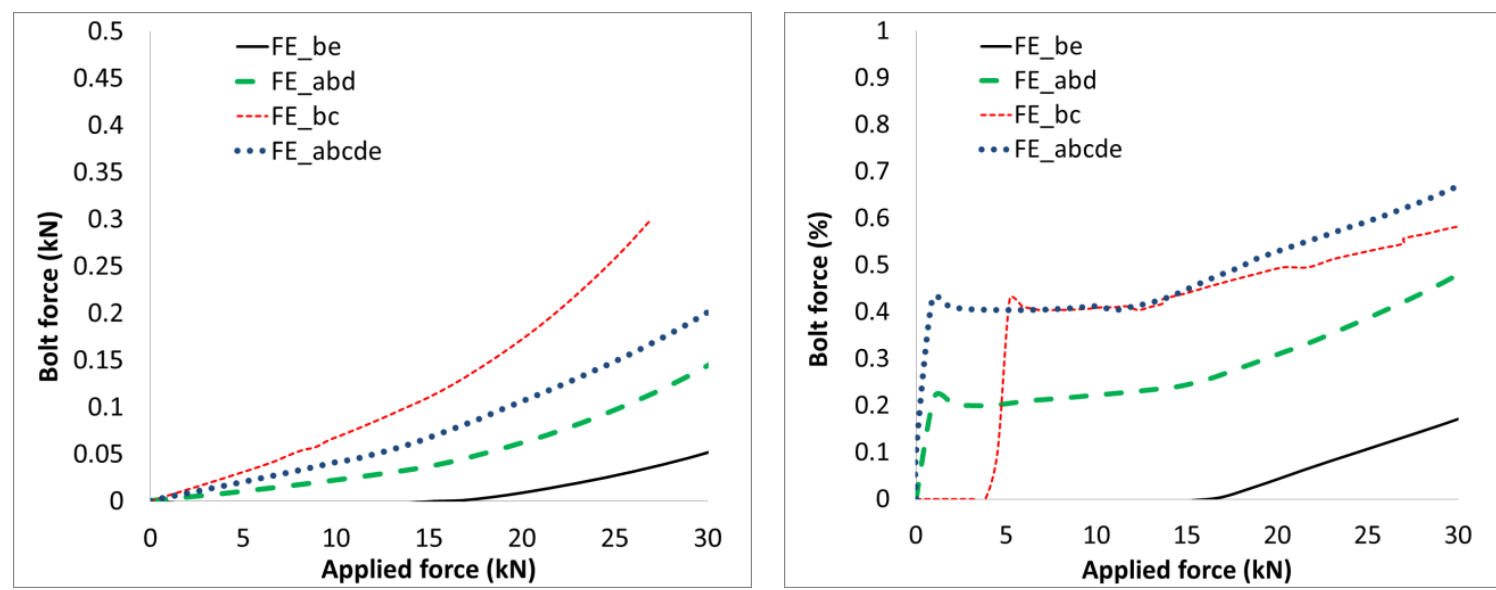

Figure 5.22 Bolted/bonded joints with FM300-2M adhesive. Predicted load at the bolts from finite element analysis

As discussed in section 2.8.2, the proposed method to compute the load taken by the bolt is by measuring the shear strains at the adhesive edge and then comparing the results with the numerical predictions. The predicted shear stains were extracted at 1,2 and $3 \mathrm{~mm}$ distance from the notch as shown in Figure 5.23. 


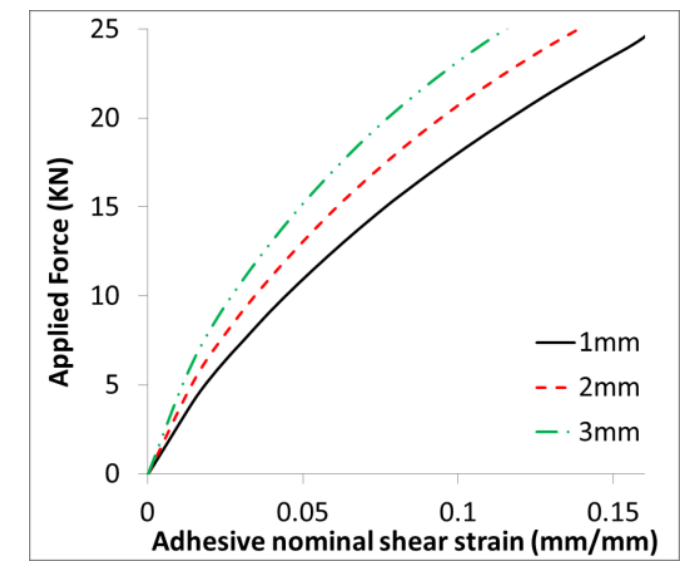

FE_be

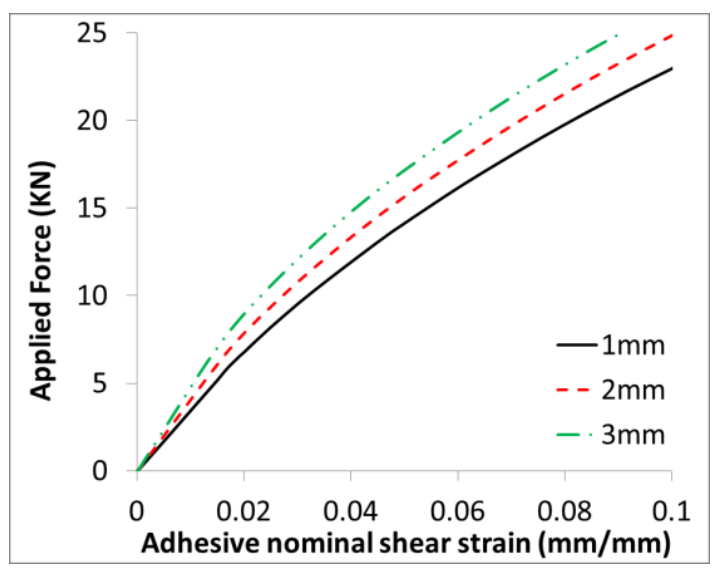

$F E \_b c$

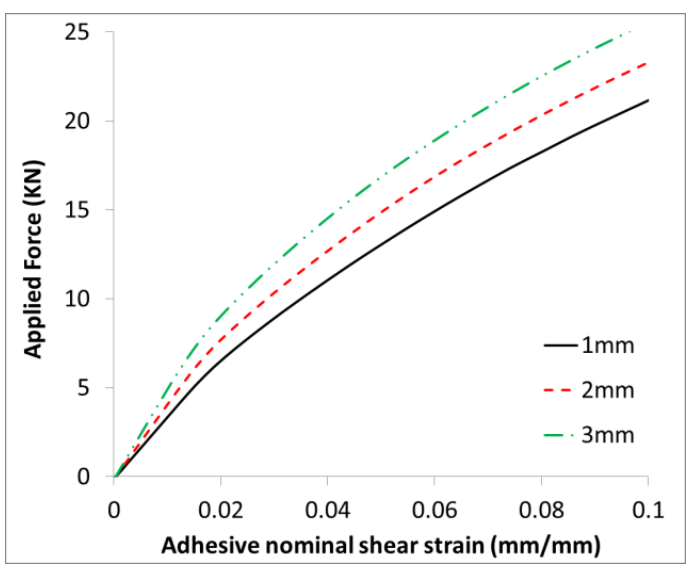

$F E \_a b d$

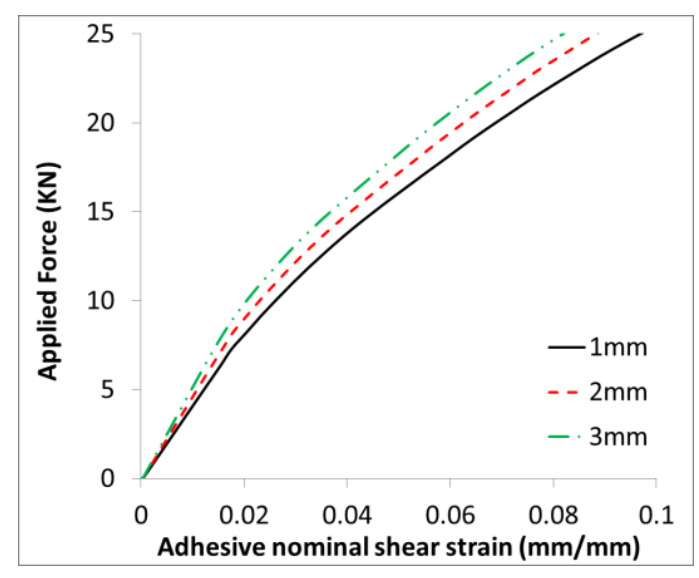

FE_abcde

Figure 5.23 Bolted/bonded joints with FM300-2M adhesive. Predicted adhesive shear strain from finite element analysis

FE models with progressive failure damage were developed to compute the effect of the composite laminate in the joint performance. However, it was found no damage at maximum strength in all the models. Therefore, the effect of the composite in the shear strain as well as the local displacement is negligible.

Figure 5.24 shows the adhesive shear strain at $3 \mathrm{~mm}$ from the notch from the model $b c$. It can be observed that the two plots are exactly the same. Thus, confirming the negligible effect of the laminate in the joint performance at maximum adhesive strength. 


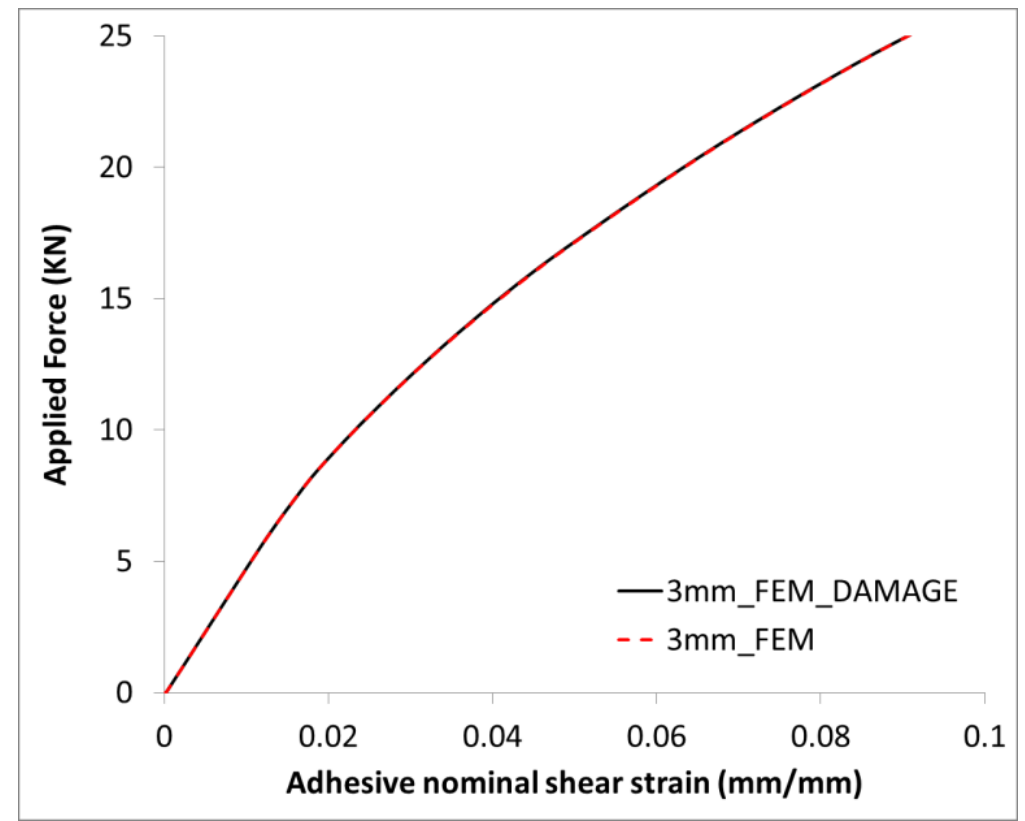

Figure 5.24 Bolted/bonded joint, model bc. Predicted shear strain comparison. Model with progressive failure in the composite vs model without damage in the composite

The same FEM analysis was carried out in the models with EA9361 adhesive.

The treatments data is shown in Table 5.12.

Table 5.12 Experimental Treatments with EA9361 Adhesive

\begin{tabular}{|c|c|c|c|c|c|c|}
\hline \multirow{2}{*}{\multicolumn{2}{|c|}{ Treatment }} & \multicolumn{5}{|c|}{ FACTORS } \\
\hline & & A & B & C & D & E \\
\hline \multicolumn{2}{|c|}{ number } & Adherend & A dhotiv & Adhesive & Clamped & Clearance \\
\hline 1 & de & 3.29 & EA9361 & 0.2 & 387 & 0.1 \\
\hline 2 & $\mathrm{a}$ & 5.48 & EA9361 & 0.2 & 97 & 0 \\
\hline 5 & $\mathrm{~cd}$ & 3.29 & EA9361 & 0.5 & 387 & 0 \\
\hline 6 & ace & 5.48 & EA9361 & 0.5 & 97 & 0.1 \\
\hline
\end{tabular}


Figure 5.25 shows the load versus displacement from the models with elastoplastic properties in the adhesive. In can be observed that the stiffer joints are those with the thinner adhesive layer of $0.2 \mathrm{~mm}, F E \_d e$ and $F E \_a$. The stiffness in the models $F E \_c d$ and FE_ace, with adhesive thickness of $0.5 \mathrm{~mm}$, differ up to $12 \mathrm{kN}$ of applied load. At this point, the load paths converged and behaved similarly. For the models $F E \_d e$ and $F E \_a c e$, it can also be observed a sudden change in the displacement rate versus force at $15.6 \mathrm{kN}$ and $11.5 \mathrm{kN}$ respectively. Because the adhesive ductility, an initial explanation for this pattern could be that the adhesive has reached hyperelastic behaviour; however, after a further analysis, it was found that this change was produced by the activation of the bolt. At these applied forces, the bolt started to carry a relative higher load at and increasing rate, as show in Figure 5.26. The delayed action of the bolt can be explained due to the clearance effect. The two aforementioned models were modelled and tested with a clearance of $0.1 \mathrm{~mm}$.

When compared with the remaining models, $F E \_a$ and $F E \_c d$, it can be observed a continuous path in the displacement versus force, without sudden changes. However, the activation of the bolt is not instantaneous, the model $F E_{-} c d$ was activated at approximately $6 \mathrm{kN}$ of applied force, and the model $F E \_a$ was activated at $8 \mathrm{kN}$. The activation is mainly driven by the adhesive thickness.

A clear indication of the activation loads is observed using percentage values as shown in Figure 5.27. The activation values were those discussed in the previous paragraph. The load sharing rate increases dramatically higher when the adhesive thickness is higher, $0.5 \mathrm{~mm}$ versus $0.2 \mathrm{~mm}$. It can also observed that, once the bolt load 
transfer is activated, there is no significant advantage of having a thicker adherend, FE_ace.

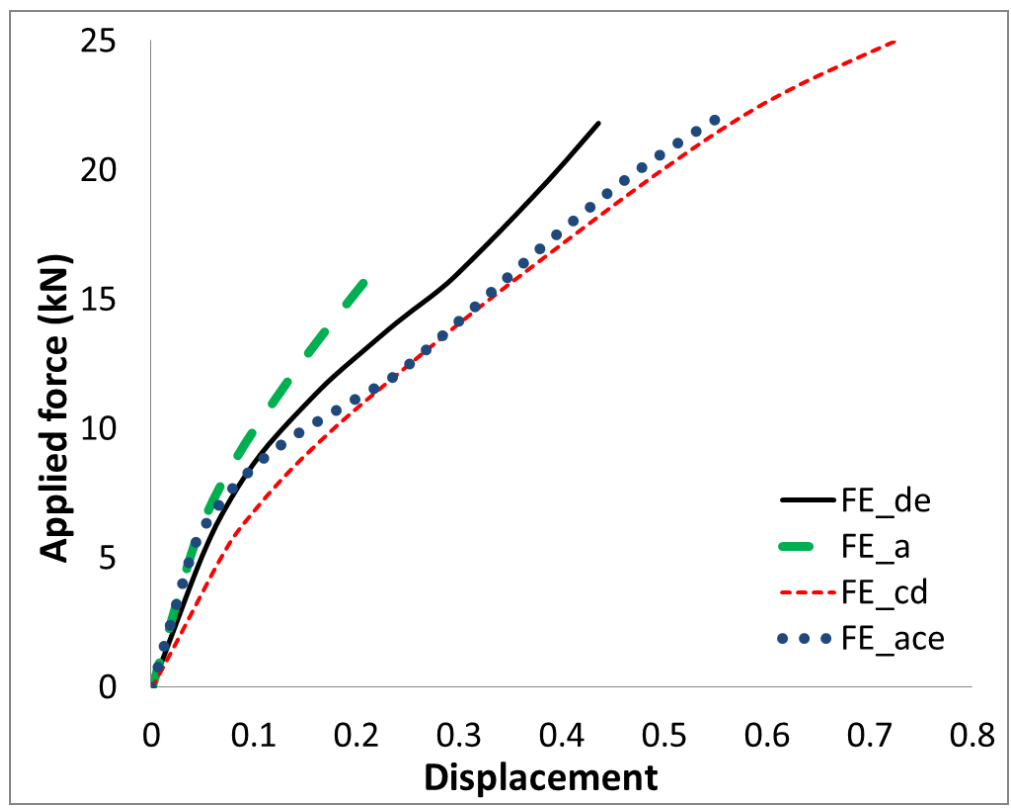

Figure 5.25 Bolted/bonded joints with EA9361 adhesive. Force versus displacement finite element results

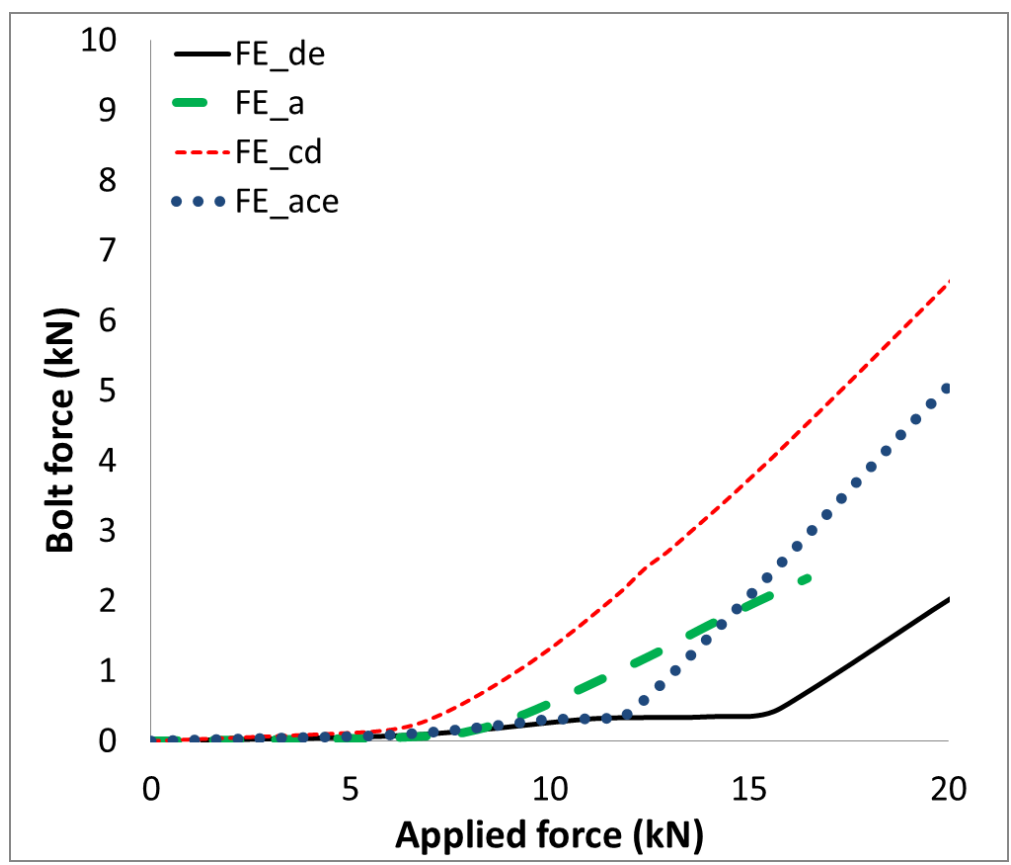

Figure 5.26 Bolted/bonded joints with EA9361 adhesive. Total predicted load at the bolts from finite element analysis 


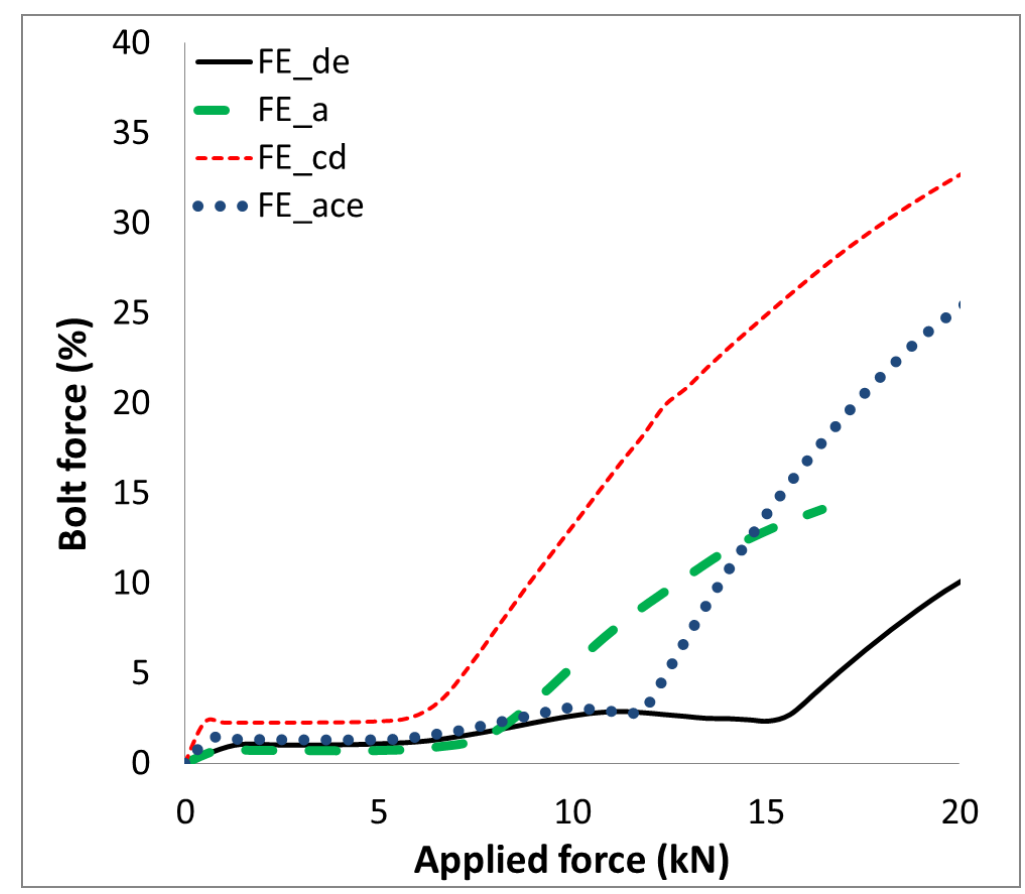

Figure 5.27 Bolted/bonded joints with EA9361 adhesive. Percent of load at the bolts from finite element analysis

The shear strains were also computed at several distances from the notch for every model as shown in Figure 5.28. In addition, a comparison in the shear strain values between all the models is shown in Figure 5.29.

In this plot, the linear elastic limit as well as the knee point are also shown. It can be observed that the maximum elastic strain was reached at the same force of $2.5 \mathrm{kN}$ approximately in all the joints. From Figure 5.26, at this strain value, the load taken by the bolt is barely zero. Therefore, there is no advantage of using a hybrid joint if the adhesive is required to remain in the linear elastic regime.

The shear strain at the adhesive knee point is also reached at the same force values for all the models. After this point, the shear strain versus force values follow different paths depending of the joint treatment. The shear strain versus force rates are lower in the models with net fit assembly, $F E_{-} a$ and $F E_{-} c d$. On the other hand, in the 
models with clearance assembly, the shear strain versus force rate in higher until the bolt is activated, and the shear strain rate decreases considerably after this point. The rate is nearly the same as that of models with neat fit.

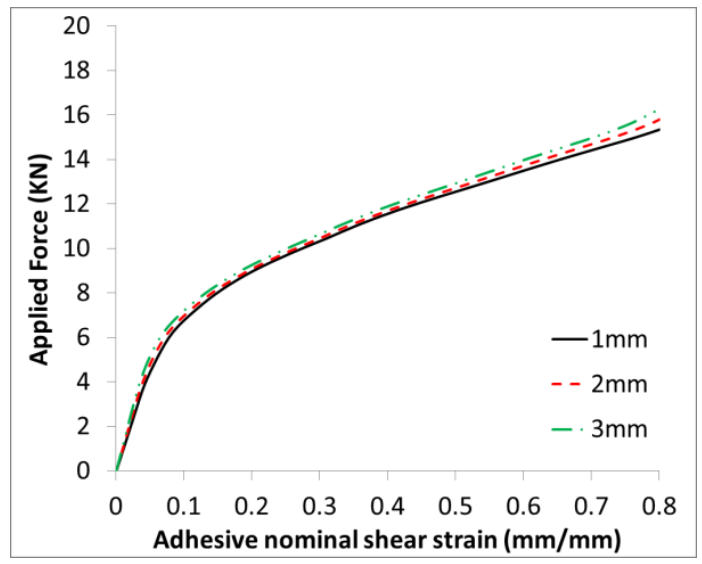

FE_de

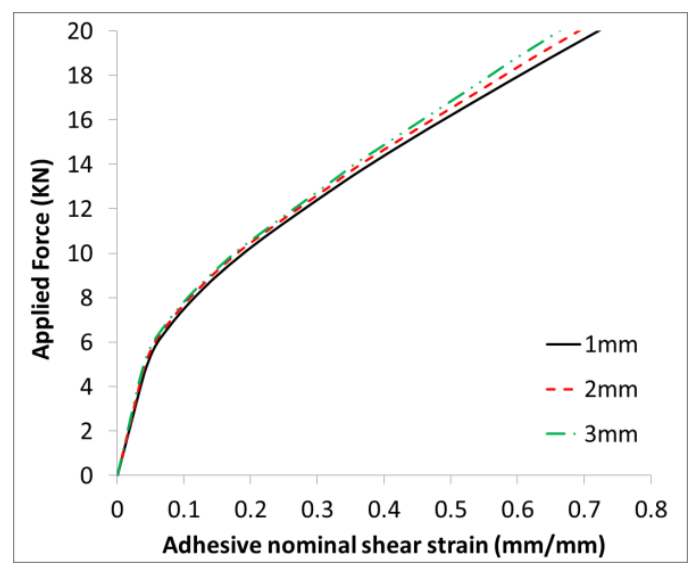

FE_cd

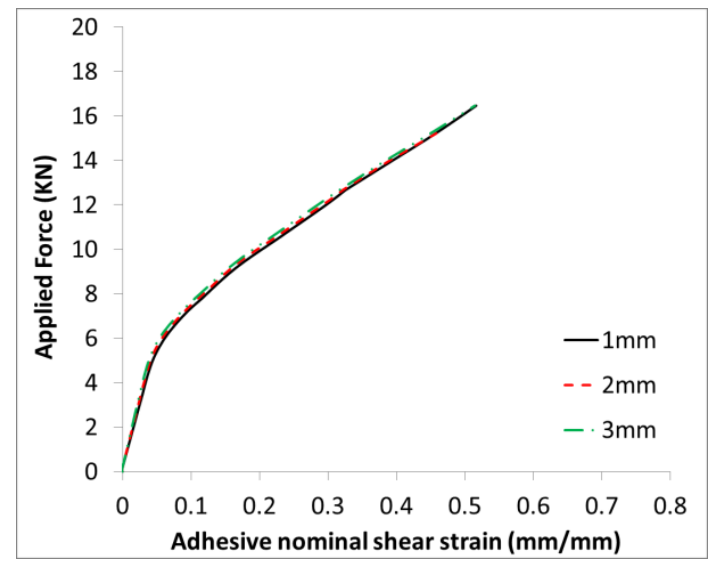

$F E \_a$

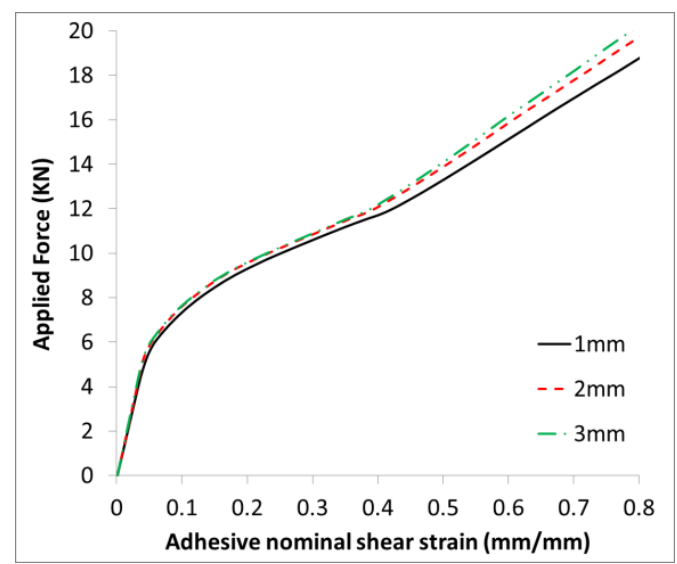

FE_ace

Figure 5.28 Bolted/bonded joints with EA9361 adhesive. Predicted adhesive shear strain from finite element analysis 


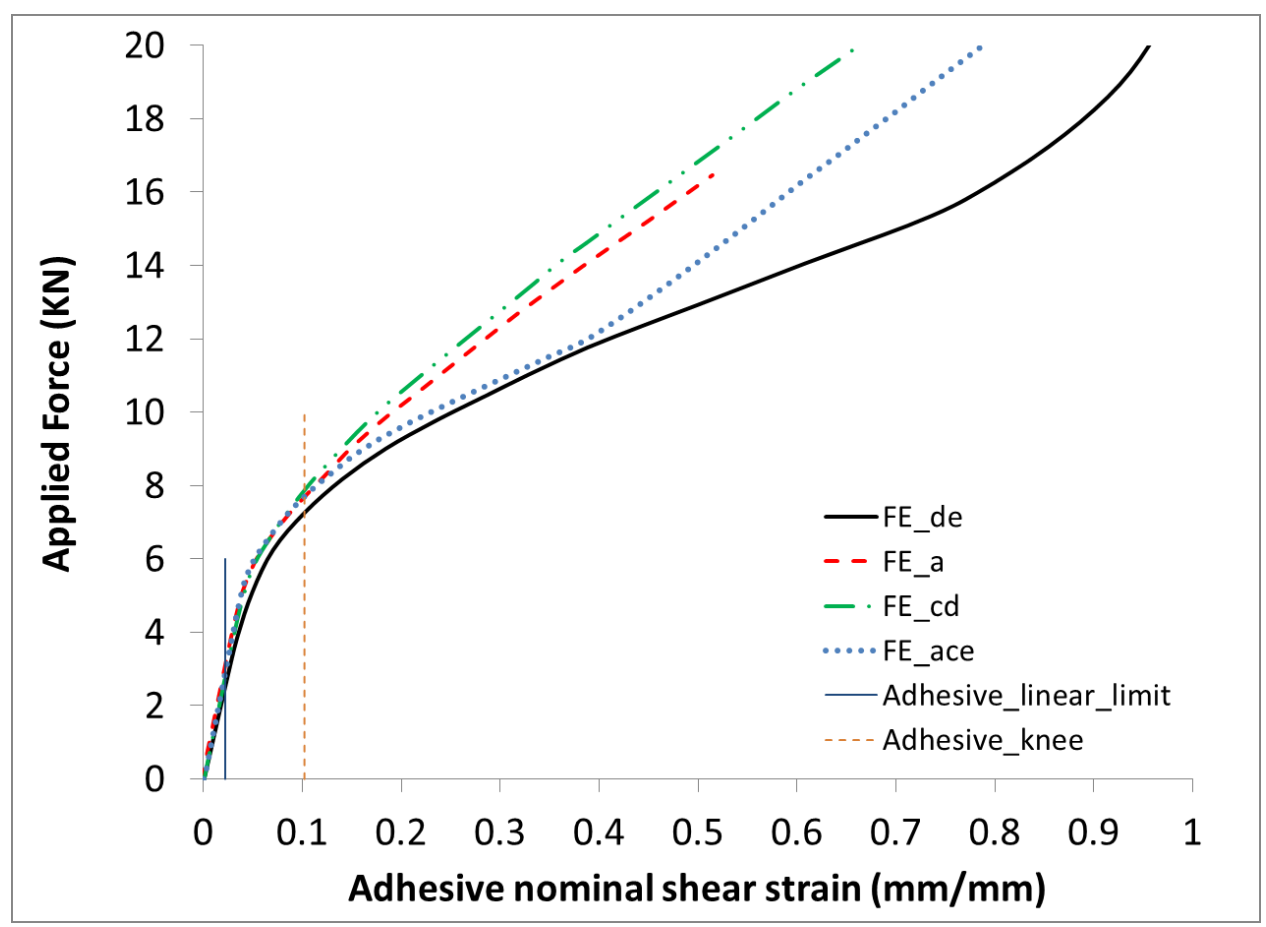

Figure 5.29 Bolted/bonded joints with EA9361 adhesive. Adhesive shear strain comparison. Shear strain at $3 \mathrm{~mm}$ distance from the notch

The effect of the composite in the joint strength was analyzed using a model with progressive failure damage in the composite. All the models were analyzed using the Hashin's failure criteria [98] implemented with the USDFLD subroutine using the elastic constants with the degraded values from Table 5.4.

It was found a negligible effect on the joint performance until failure load. That effect is depicted in Figure 5.30 for the treatment $c d$. At $18 \mathrm{kN}$ of applied force, the difference in the load taken by the bolt is approximately $0.2 \mathrm{kN}$, this represents $1 \%$ of the total applied load. The same effect is also depicted in the shear strain results, with similar percentages.

Therefore, it can be concluded that the damage in the composite material has no effect in the joint performance until maximum strength. 

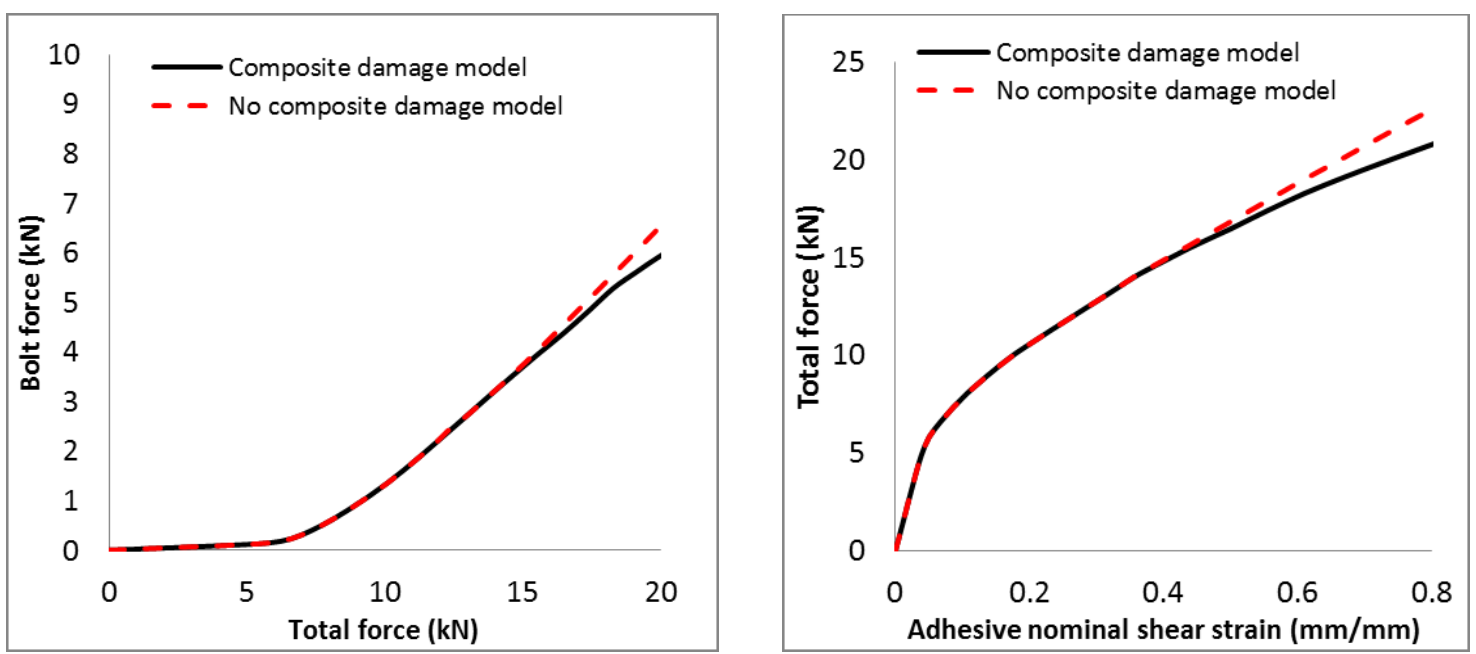

Figure 5.30 Bolted/bonded joint, model FE_cd. Effect of the composite damage in the joint performance. a) Load taken by the bolt; b) Adhesive shear strain

Figure 5.31 shows the predicted failure states presented in the joint at $18 \mathrm{kN}$. The matrix tensile failure is located at the edge of the joint, the behaviour is consistent with the peel stress at the edge of the joint. In the hole area, damage due to matrix compression and fiber tension was found . 


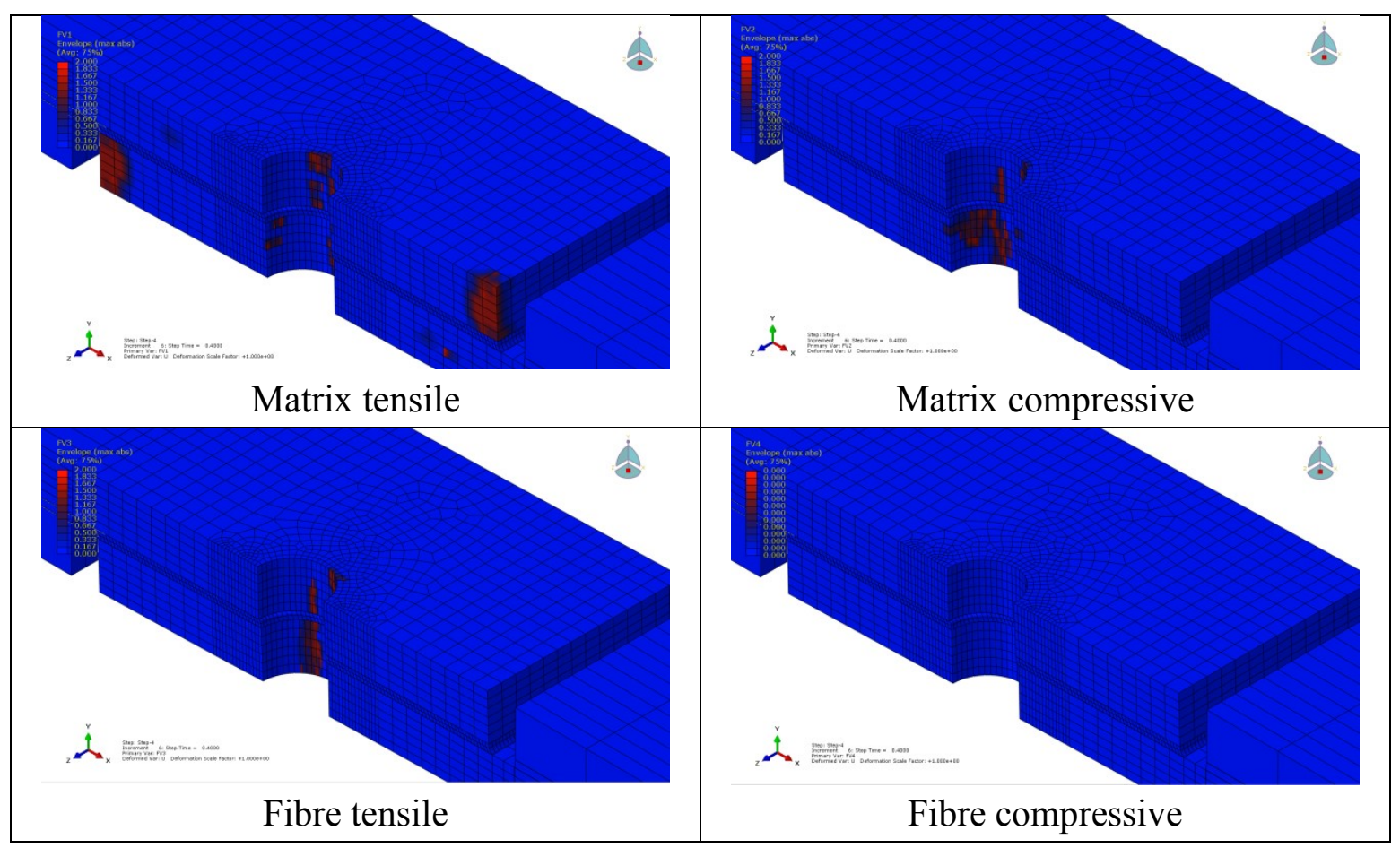

Figure 5.31 Bolted/bonded joint, model $F E_{-} c d$. Predicted failure modes in the composite at $18 \mathrm{kN}$ of applied force

The joint strength was predicted using CZM. However, convergence issues to achieve a solution were found; therefore, only two out of four treatments were analyzed. The geometry and material properties were the same as the FE models with elastoplastic properties. The difference is the formulation in the adhesive material, on which, cohesive properties were defined. The results are shown in terms of force versus displacement. However, it has to be pointed out that the displacement in the experimental results corresponds to the stroke displacement, and the displacement in the CZM models are total displacement in the joint only. The results from the model $C Z M \_c d$, equivalent to treatment $c d$ are plotted in Figure 5.32, and compared against the load versus stroke results from the experimental results. 


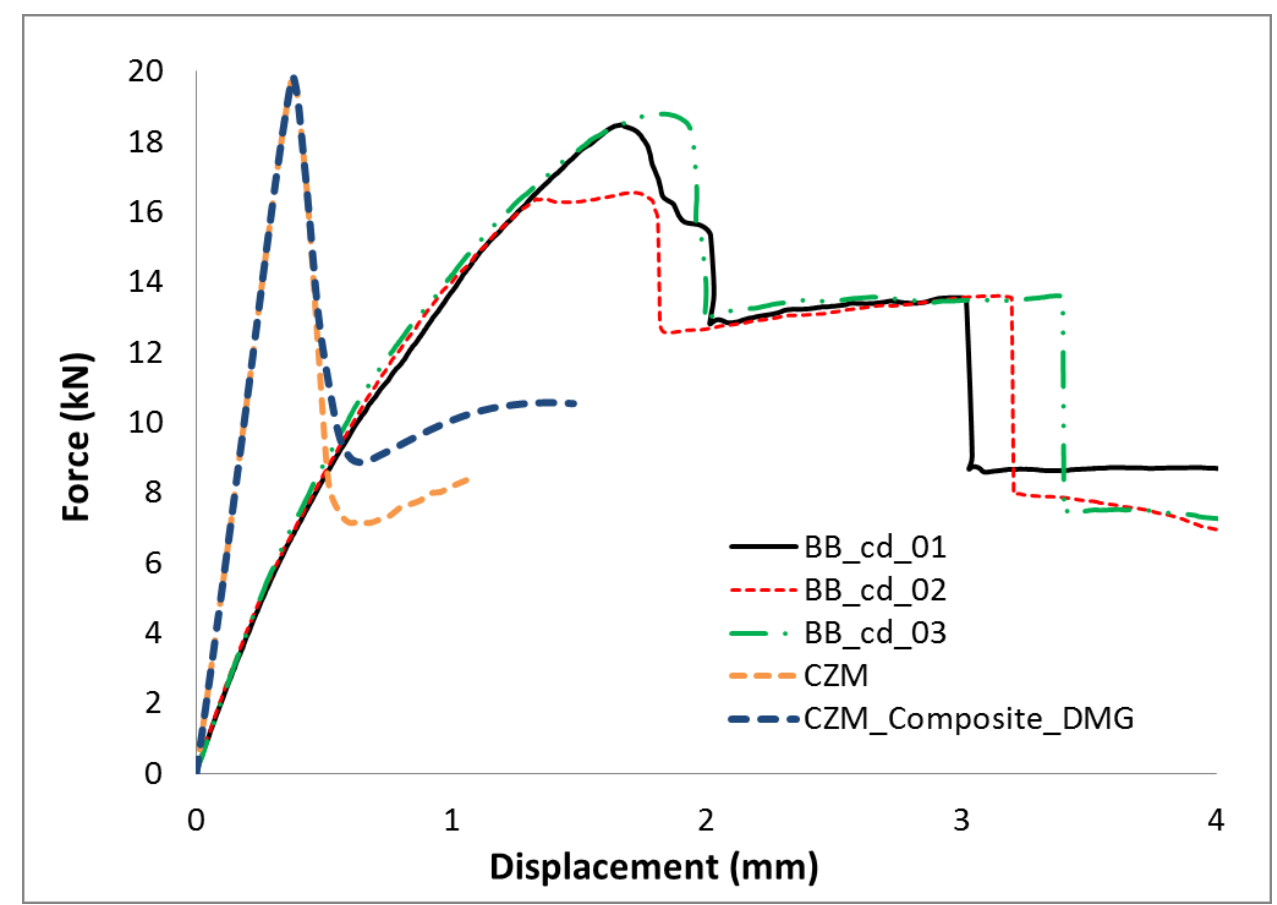

Figure 5.32 Bolted/bonded joint, model $c d$ with EA9361 adhesive. CZM strength prediction

The maximum predicted strength was $19.83 \mathrm{kN}$. This value is $10.5 \%$ higher than the average experimental value, $17.93 \mathrm{kN}$. In addition to this, the plot shows the models with composite degradation formulation, labeled composite_DMG. It can be observed that the predicted maximum force is the same in both CZM models. This can lead to the conclusion that the composite material does not reach failure in any mode before the adhesive failure. This is supported by the experimental results, the DIC images did not shown any sign of composite failure, at least near to the adhesive edge. Failure in the hole is not detectable.

On the other hand, the force after the adhesive has fail completely, is slightly lower when the damage in the composite is modelled. In both cases, the remaining strength computed values are in very good agreement when compared with the strength after the adhesive failure from the experimental results, whose average value is $7.8 \mathrm{kN}$. 
The CZM without degradation in the composite predicts an strength of $8.9 \mathrm{kN}$ after adhesive failure and the model with degradation predicts $7.14 \mathrm{kN}$ after adhesive failure.

The adhesive failure sequence is depicted in Figure 5.33. Similarly to the bonded joints models, the maximum strength is reached when the adhesive has reached degradation at the edge; in this case the adhesive shows a degraded parameter of 0.71 at maximum strength. After this point, the adhesive continues with the degradation process with the subsequent decrease in the joint strength.

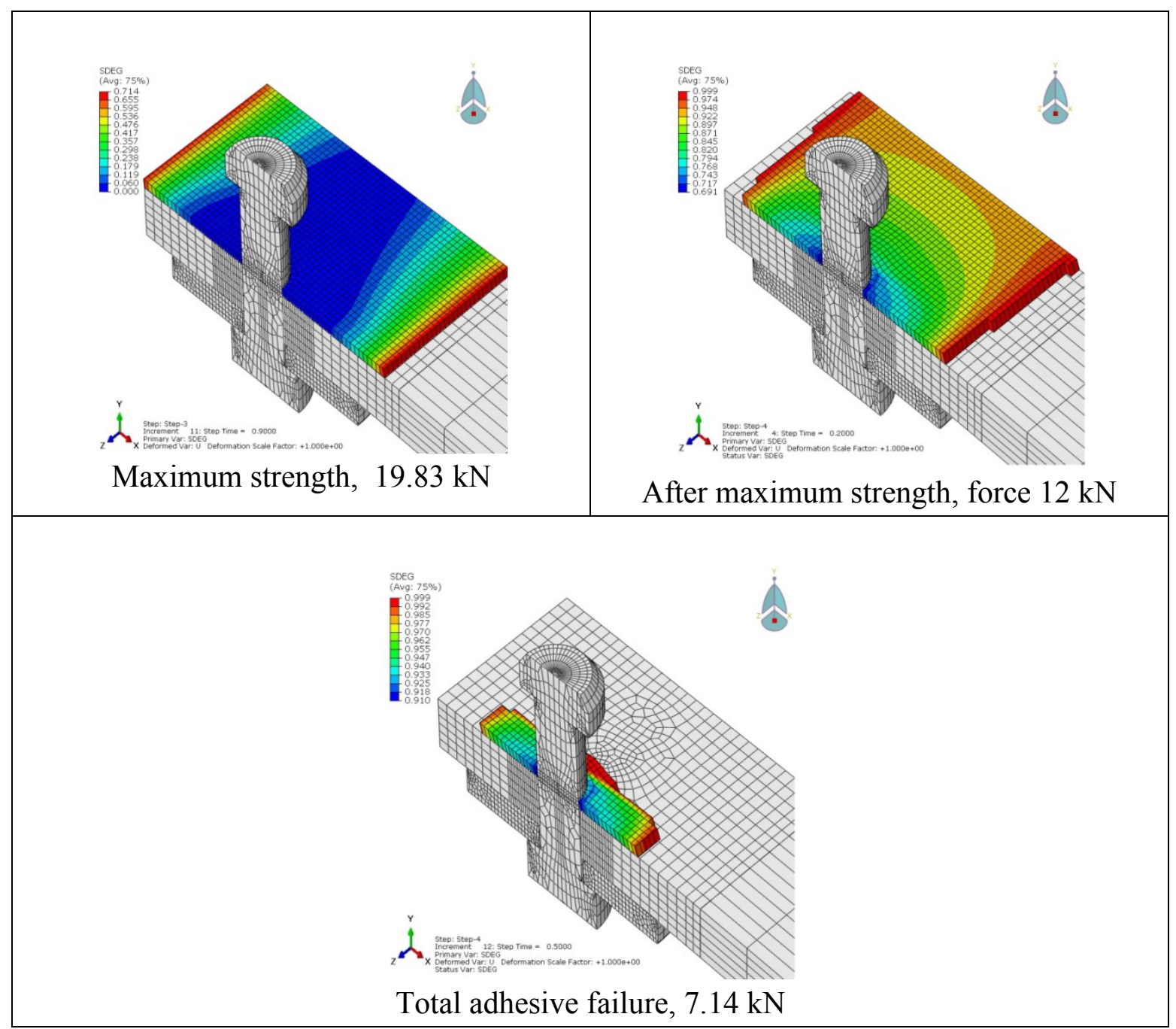

Figure 5.33 Bolted/Bonded joint, combination $c d$. Failure progress in the adhesive layer using CZM analysis 
When the adhesive reached the damage state, the composite material near to the bolt began to experience damage due to different failure modes, increasing according to the progression of damage in the adhesive.

Figure 5.34 depicts the predicted failure in the composite laminate once the adhesive failed completely. An extended discussion about the progression of the damage of the composite material is not part of the scope of this research. The aim, was to quantify the effect of the composite damage in the joint strength including several possible failure modes in the joint.

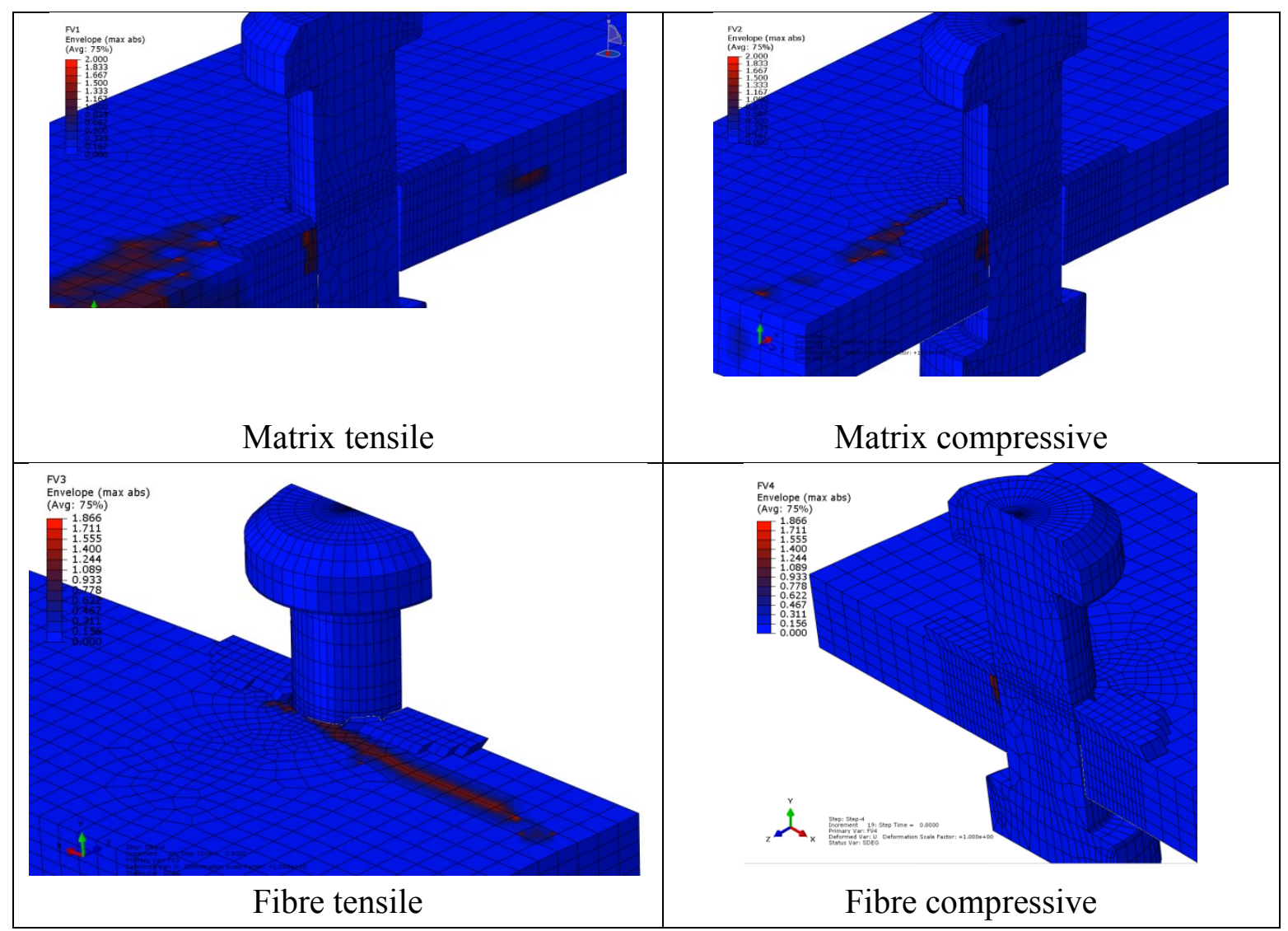

Figure 5.34 Bolted/Bonded joint, combination cd. Predicted composite damage after adhesive failure using CZM 
It can be inferred from the force versus displacement plot that once the adhesive starts its degradation process, the load taken by the bolt increases accordingly. This is depicted in in Figure 5.35. The load transfer seems to be very sudden in the CZM model. This sudden load transfer is due to the fact that the adhesive properties remain linear elastic until the degradation process begins; until this point, the load taken by the bolt is minimal. However, once the adhesive reached some degradation near to the edges, the load transfer process is accelerated. In contrast, the load transfer process in the elastoplastic model is smooth and starts, as discussed previously, when the adhesive shear strain reached the shear strain knee value approximately.

As analyzed from the experimental study and depicted in Figure 4.42, the adhesive starts its fracture and damage progression well after the maximum force is reached. Therefore, there must be a change in the load transfer rate once the adhesive begins the aforementioned degradation process. This behaviour can only be captured numerically by combining the elastoplastic model with a CZM layer in the adhesive region. 


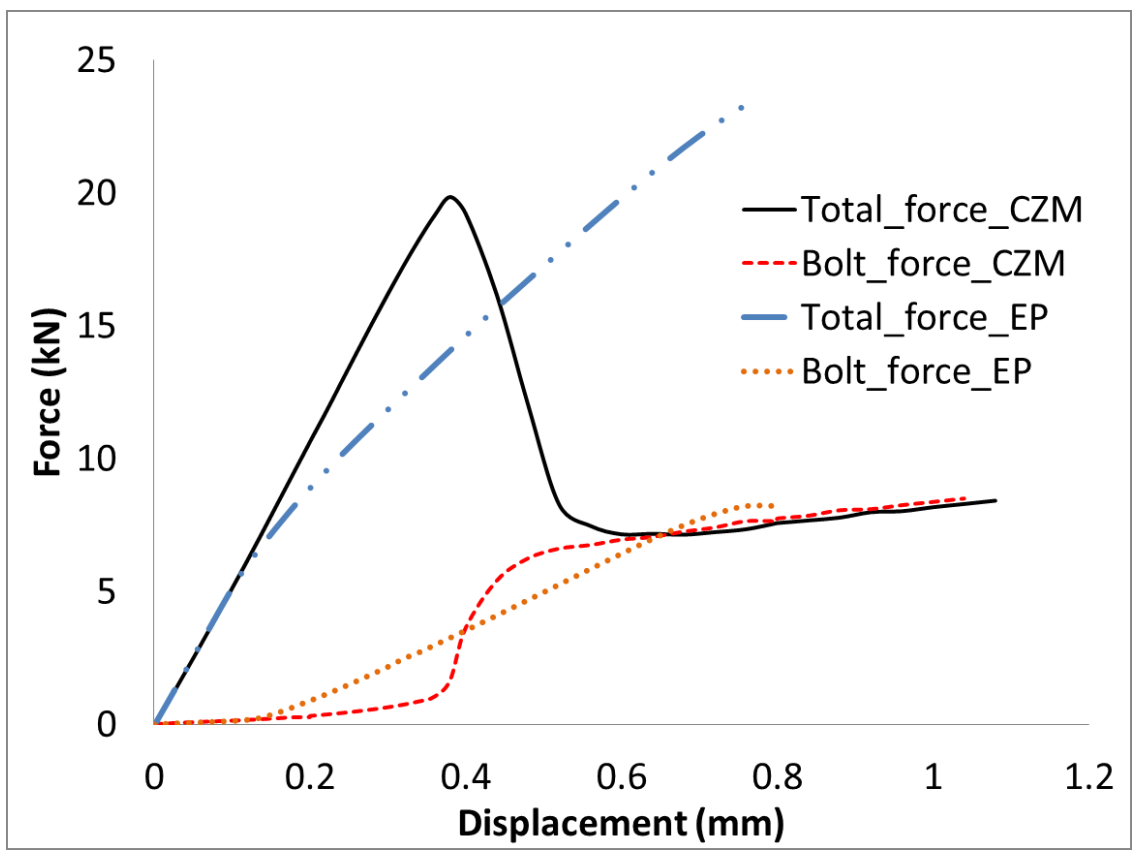

Figure 5.35 Bolted/bonded joint, model $c d$. Load transfer comparison CZM vs FEM elastoplastic

The CZM model results for the treatment acd are shown in Figure 5.36. For this combination, specifically the test ace_02, it was possible to measure the relative displacement using the $35 \mathrm{~mm}$ distance outside the bondline as reference. To point out the importance of the mechanical properties in the model, the plot also includes a second model using the traction forces, $t_{n}$ and $t_{s}$, from [93].

Since the data from the experimental results and numerical results were extracted locally, the displacements are comparable. Unfortunately, the experimental results are partially complete due to the issue of test machine compliance discussed in section 3.5. However, the maximum average strength, $19.34 \mathrm{kN}$, is used for comparative purposes. Analyzing the models with CZM continuum approach, the maximum strength using the adhesive properties from the TAST data was $21.9 \mathrm{kN}$. This contrasts severely 
with the results using the data from the aforementioned reference, the maximum strength for this model was $13.95 \mathrm{kN}$. The difference is $57 \%$ between the two models. When compared with the maximum average experimental strength, $19.34 \mathrm{kN}$, the agreements are $87 \%$ and $61.3 \%$ respectively. In addition to this, when the CZM results were compared with the maximum strength from the repetition test, treatment ace_rep_01, whose maximum strength was $20.96 \mathrm{kN}$, the agreement is remarkable for the model with the TAST data. This is another point to claim that the adhesive properties from the inhouse testing using the TAST method were very important for this research.

Regarding the local displacements, it can be observed, from the experimental results and the CZM_TAST model, the very good agreement in the linear portion of the response. After this point the models diverge, this is reasonable due to the elastic nature of the formulation into the adhesive using the chosen CZM model.

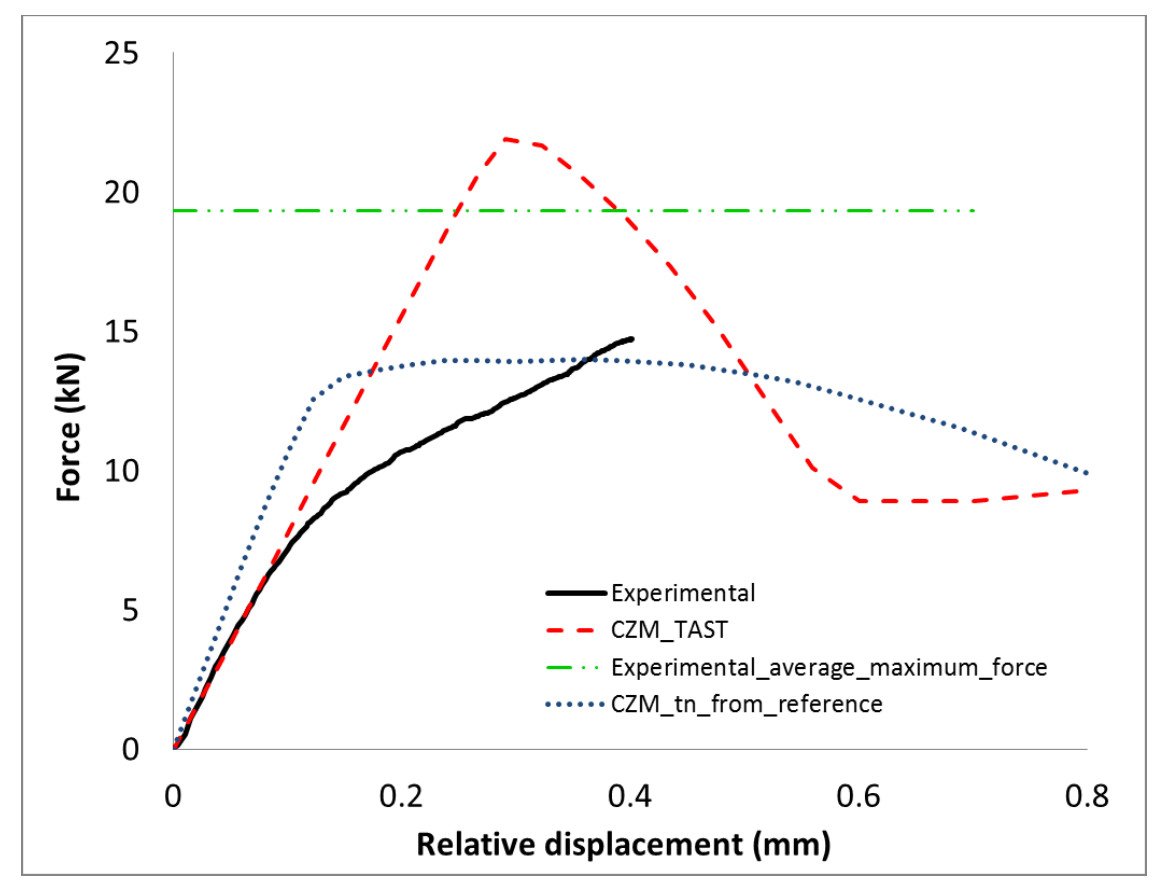

Figure 5.36 Bolted/bonded joint, model ace with EA9361 adhesive. CZM strength prediction 
An attempt to carry out a combined analysis using the elastoplastic properties in the adhesive and inserting local cohesive elements in the interface between the adherend and adhesive was made for this model. However, it was found that, due to convergence issues, that generating such that model required to remove the torque and clearance features. Once these features were removed, the model still required a lot of computational time to run a single step. To achieve the results shown in Figure 5.37, the computational time required was 11710 minutes (195 hours) using with 4 processors. Unfortunately, the complete solution was not achieved.

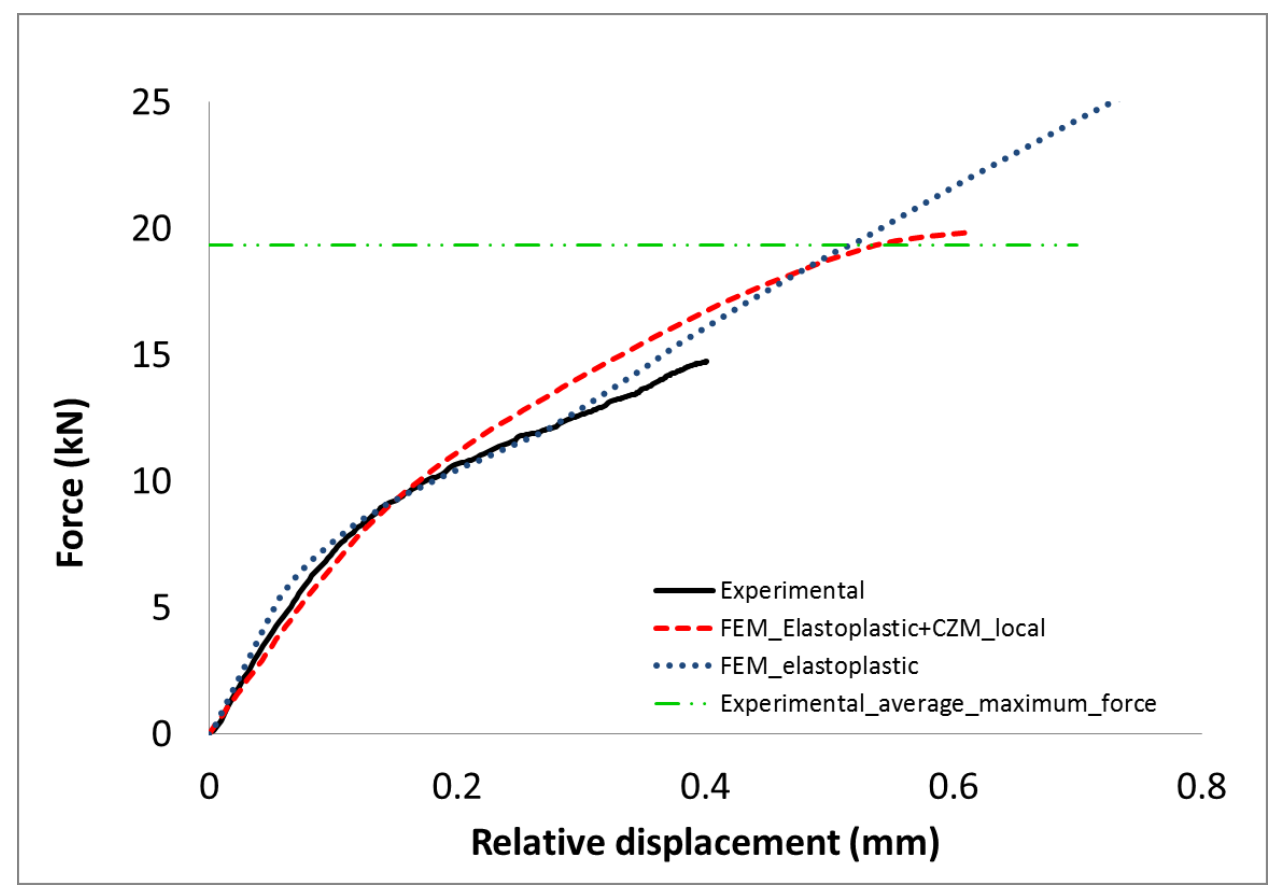

Figure 5.37 Bolted/bonded joints, model ace. Strength prediction using CZM local approach and comparison with experimental results and FEM elastoplastic

The result were compared with the experimental results as well as the FE model with elastoplastic properties with torque and clearance. The elastoplastic model has better agreement because it captures the point at which the bolt is activated due to 
diameter clearance between the bolt and the hole, the inflexion point is located at $12 \mathrm{kN}$ approximately. In contrast, the CZM does not capture that point because it does not have the clearance feature.

On the other hand, the elastoplastic model continues the load path beyond the maximum average experimental strength. Conversely, the CZM model presents a plateau region once the $20 \mathrm{kN}$ of applied force is reached. This maximum force is very close to the maximum value from the experimental results, $19.34 \mathrm{kN}$. At this point, the adhesive reached a considerable amount of damage, mainly near to the edge as shown in Figure 5.38. As demonstrated from the previous analysis, once the adhesive is damaged near to the edge, the joint has reached its maximum strength. Therefore, it can be concluded that the maximum strength in the bolted/bonded joint, using the CZM local approach has been reached at this point.

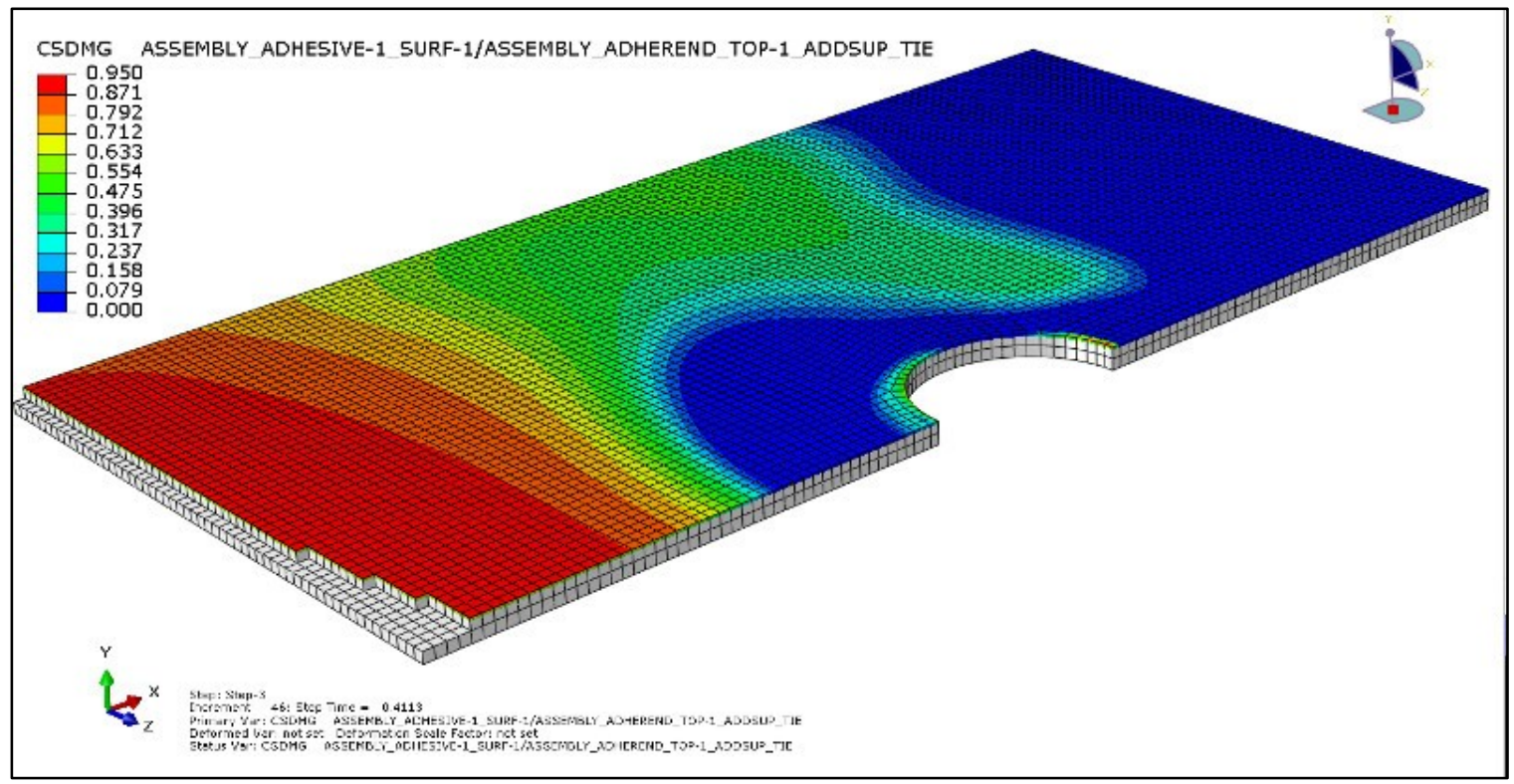

Figure 5.38 Bolted/bonded joint, model ace. Adhesive damage at $20 \mathrm{kN}$ of applied force at the surface of the cohesive elements located at the top interface between the adhesive and the composite adherend 
Several attempts to re-start the analysis to analyze the load sharing behaviour beyond this point were carried out with unsatisfactory results.

\subsection{Chapter Summary}

In this chapter, the procedures, results and discussion of finite element analysis of bolted/bonded joints was presented. Firstly, the discussion about the material properties was addressed in sections 5.1 to 5.3. For the adhesive, the elastoplastic as well as cohesive zone model parameters were discussed.

In section 5.4. , the model was described, including the geometry, elements, number of nodes, interactions and boundary conditions.

In order to assess the cohesive zone parameters, the bonded joints were analyzed and discussed in section 5.5. The experimental results compared with the numerical solution were in good agreement, ranging from $90 \%$ to $97 \%$

A full 3D analysis using FE elastoplastic models to compute the load sharing and strains was carried out. Also, the strength in the joints were computed using cohesive zone modelling with agreements above $77 \%$ between the experimental and numerical results for the models with FM300-2M adhesive and above $87 \%$ for the models with EA9361 adhesive.

The load sharing was computed numerically and it was found that the load taken by the bolts was less than $1 \%$ for load before adhesive failure in the models with FM3002M adhesive. On the other hand, the load taken by the bolt in the models with EA9361 adhesive can be above $25 \%$. However, it was also found that the activation of the bolt is significantly affected by the amount of clearance between the bolt and hole. 
The influence of the progressive damage of the composite material on the load sharing was negligible.

Using cohesive zone modeling, it was found that load transfer mechanism using elastoplastics and cohesive zone model differ. The elastoplastic model predicts a smooth transition in the load transfer when the cohesive zone model predicts a sudden transition.

Finally, a model combining elastoplastic elements with local cohesive elements in the interface between the adherends and adhesive predicted the onset of force versus displacement with good accuracy. However, the computational time required to achieve this partial solution was dramatically extended. 


\section{Chapter 6: Load Sharing Analysis}

\subsection{Introduction}

So far, as discussed in section 2.3.3, the load sharing measurement of the hybrid bolted/bonded joints described in literature has been carried out using instrumented bolts. In this present study, the proposed method to determine the load sharing is by measuring the shear strain in the adhesive layer to infer the load taken by the bolt. By using the instrumented bolt technique, the force is directly computed from strain gauges installed directly on the bolt. Conversely, the method proposed in this study is an indirect measurement technique to measure the distribution of force between the bolt and the adhesive.

First, the strain distribution at the bondline must be known in order to infer the applied force in the adhesive. That strain distribution depends on the joint configuration and materials involved. Therefore, a reference shear strain pattern must be available to compare with the strain from the bolted/bonded joint. There are two possible options to obtain that reference shear strain profile to compare against the measured strain from the bolted/bonded joints.

Experimentally, the reference shear strain must be extracted from a bonded joint with the same geometry and material as the bolted/bonded joint. Once the shear strain profiles from the bonded and bolted/bonded joint are extracted, the force in the bolt can be inferred by computing the difference between the bonded and bolted/bonded joints. This assumption is based on the fact that the bonded joint has exactly the same geometry and parameters as the bolted/bonded joint; therefore, the strain distribution at certain 
applied force must be the same, unless another element were carrying some part of the applied load. This assumption can lead to small errors because the adhesive's shear area in the bonded joint is slightly higher than that of the hybrid joint due to the presence of the hole. The tested coupons have a nominal shear area equal to $816.5 \mathrm{~mm}^{2}$. Due to the drilling process, the adhesive shear area is reduced to $798.7 \mathrm{~mm}^{2}$, this represents a difference of $2.18 \%$.

It could be assumed that this variation is minimal and should not affect the measurements; however, this could be significant, especially in joints with stiffer adhesives where the predicted load sharing is less than $3 \%$. On the other hand, as predicted numerically, the clearance effect delays the contact between the bolt and the adherend; thus, theoretically, the bonded joint should be stronger than the bolted bonded joint by $2.18 \%$ at the beginning of the loading process. Finally, the position at which the shear strain is measured must be the same for both the bonded and bolted/bonded joint. Numerically, a very accurate model with the bolted/bonded joint configuration must be developed to compute the shear strains and compare the results with the shear strains from the experimental results. The challenging part of this option is the necessity of reliable data to be input in the FE models.

Another advantage of using FE analysis is that the comparison against experimental results is not limited to the shear strain. Depending on the experimental setup, any other response factor can be defined to be compared; for instance, local stiffness. The stiffness response can be calculated from displacements measured experimentally using extensometers or DIC. Similarly to the shear strain analysis, the results can be comparable to assess the load transfer. 
The load sharing was assessed using local displacements or shear strains depending on DIC setup and magnification. For joints with EA9361 adhesive, the measurement of the shear strains was carried out.

The proposed method from [115] to compute the shear strains using DIC was compared with measurements using a KGR extensometer with very good agreement. Therefore, we can assume that the shear stains computed using the same method would give accurate and correct results. However, to corroborate the method numerically, using the FEA results, the shear strains extracted directly from the nodes and compared with the shear strains computed using aforementioned method using displacements. The assessment was carried out using two FE models with EA9361 adhesive and the adherends with $3.29 \mathrm{~mm}$ thickness. The results from the first model, with a $0.5 \mathrm{~mm}$ bondline thickness and neat fit between the bolt and the hole are shown in Figure 6.1. The second model correspond to the treatment $d e$. This model included $0.2 \mathrm{~mm}$ bondline thickness and $0.1 \mathrm{~mm}$ clearance between the bolt and the hole. Figure 6.2 shows the results for this model. It can be observed the very good agreement in shear strain results using the displacement method and the strains extracted from the nodes. Thus, the strain measurement using the three points method is accurate and reliable, at least, using the numerical results. 


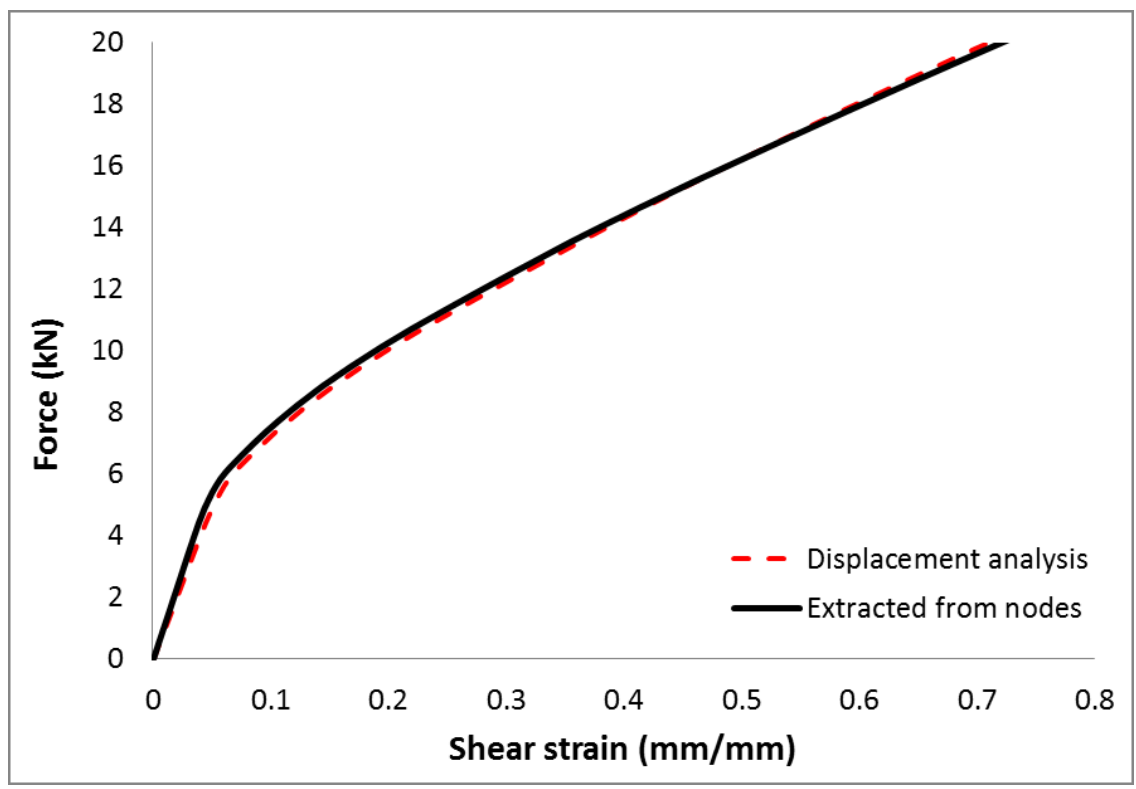

Figure 6.1 Bolted/bonded joint, FE model $c d$. Comparison between the extracted shear strain from the nodes and the shear strain computed using the 3 point method. Distance $1 \mathrm{~mm}$ from the notch

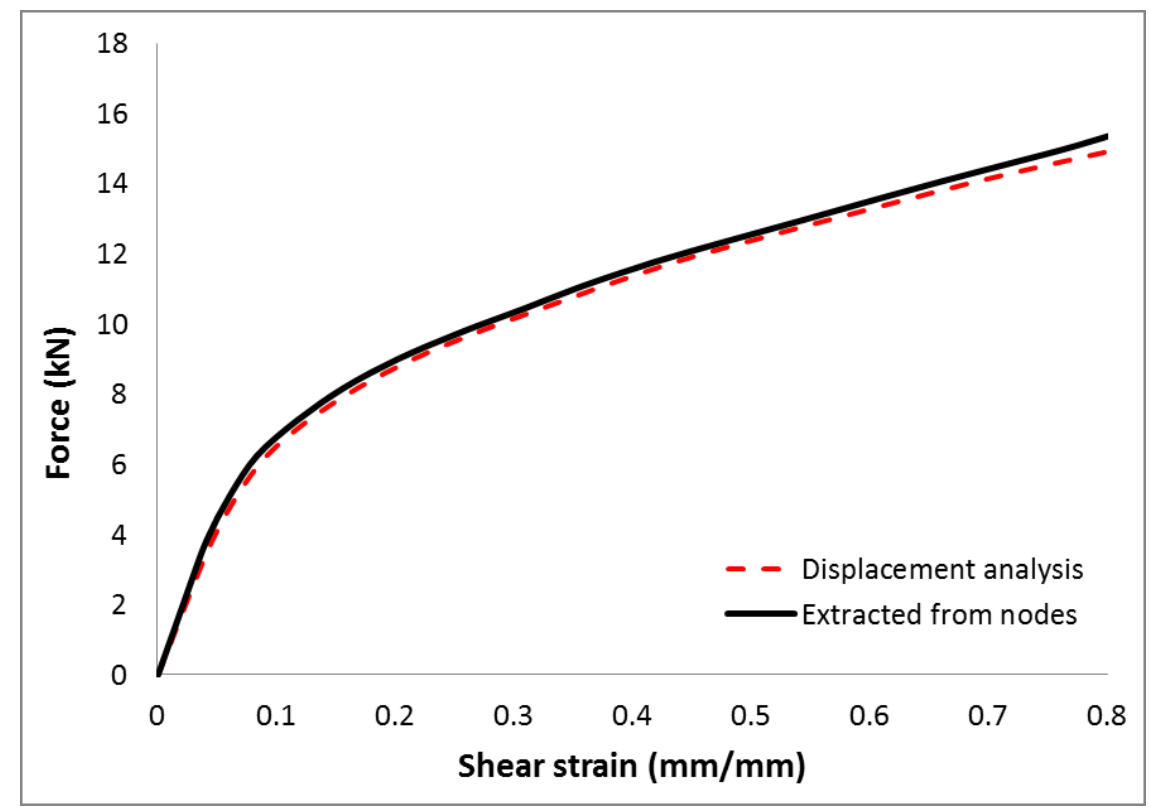

Figure 6.2 Bolted/bonded joint, FE model de. Comparison between the extracted shear strain from the nodes and the shear strain computed using the 3 point method. Distance $1 \mathrm{~mm}$ from the notch 


\subsection{Load Sharing Analysis in Joints with FM300-2M Adhesive}

The load sharing for treatment be was analyzed from the results shown in Figure 6.3. The shear strain at $1 \mathrm{~mm}$ distance from the notch using the test be02 was extracted and compared with the shear strains from the FE model. The predicted bolt force values were very low compared with the applied force; therefore, a secondary axis was used to plot this value.

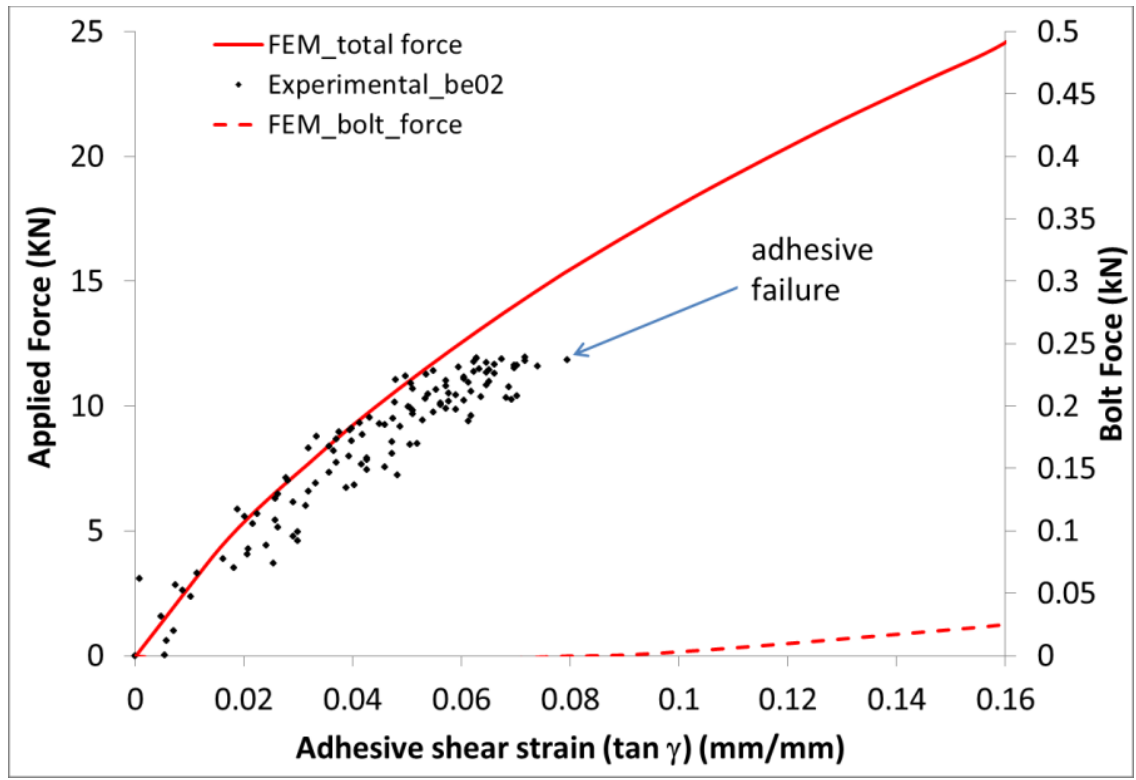

Figure 6.3 Bolted/bonded joint, treatment be_02 with FM300-2M adhesive. Predicted force in the bolt comparing experimental and numerical adhesive shear strains

Firstly, we can notice that the experimental and numerical results were in good agreement. However, the numerical results deviates from the adhesive failure force by approximately $20 \%$. Even though, the predicted load taken by the bolt is barely zero. It is not clear if the adhesive stiffness or the assembly clearance prevents the bolt from touching the adherend hole wall, with the consequent increase in the bolt load at approximately $15 \mathrm{kN}$ of applied force. However, the force in the bolt is still minimal 
compared with the total force. For instance, at $25 \mathrm{kN}$ of applied force, the bolt barely takes $0.025 \mathrm{kN}$.

The same trend should be expected by using the displacement measurement as shown in Figure 6.4. It can be observed the good agreement between the FEM, CZM and experimental results. The slopes are nearly identical, excepting for the offset discrepancy. Moreover, the agreement with the CZM models in both stiffness and strength is remarkable. This comparison was discussed in the previous chapter. The possible explanation about the offset discrepancy between the numerical and experimental results could be due to an initial slippage from the grip wedges. However, this assumption was not confirmed.

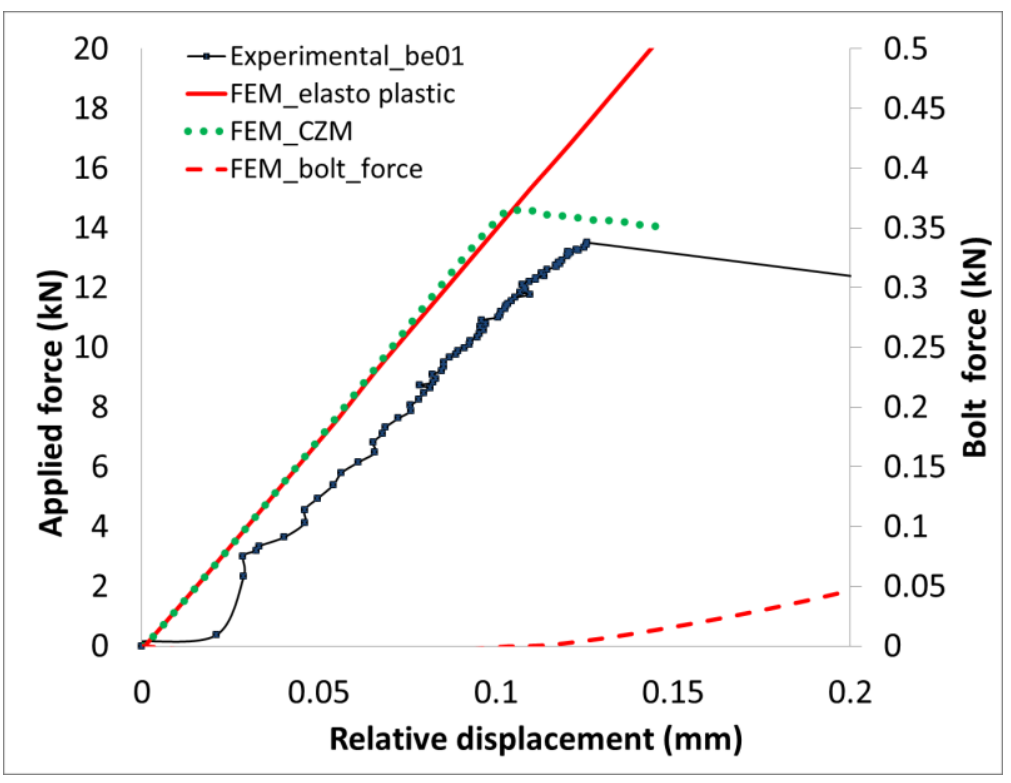

Figure 6.4 Bolted/bonded joint, treatment be_01 with FM300-2M adhesive. Predicted force in the bolt comparing experimental and numerical relative displacements

The same analysis using shear strain measurements as well as relative displacements was carried out for the remaining treatment with FM300-2M adhesive. 
However, it was found that, at maximum strength, the load taken by the bolt barely reached $0.4 \mathrm{kN}$. The results are shown in Table 6.1 .

Table 6.1 Predicted force in the bolt. Treatments with FM300-2M adhesive

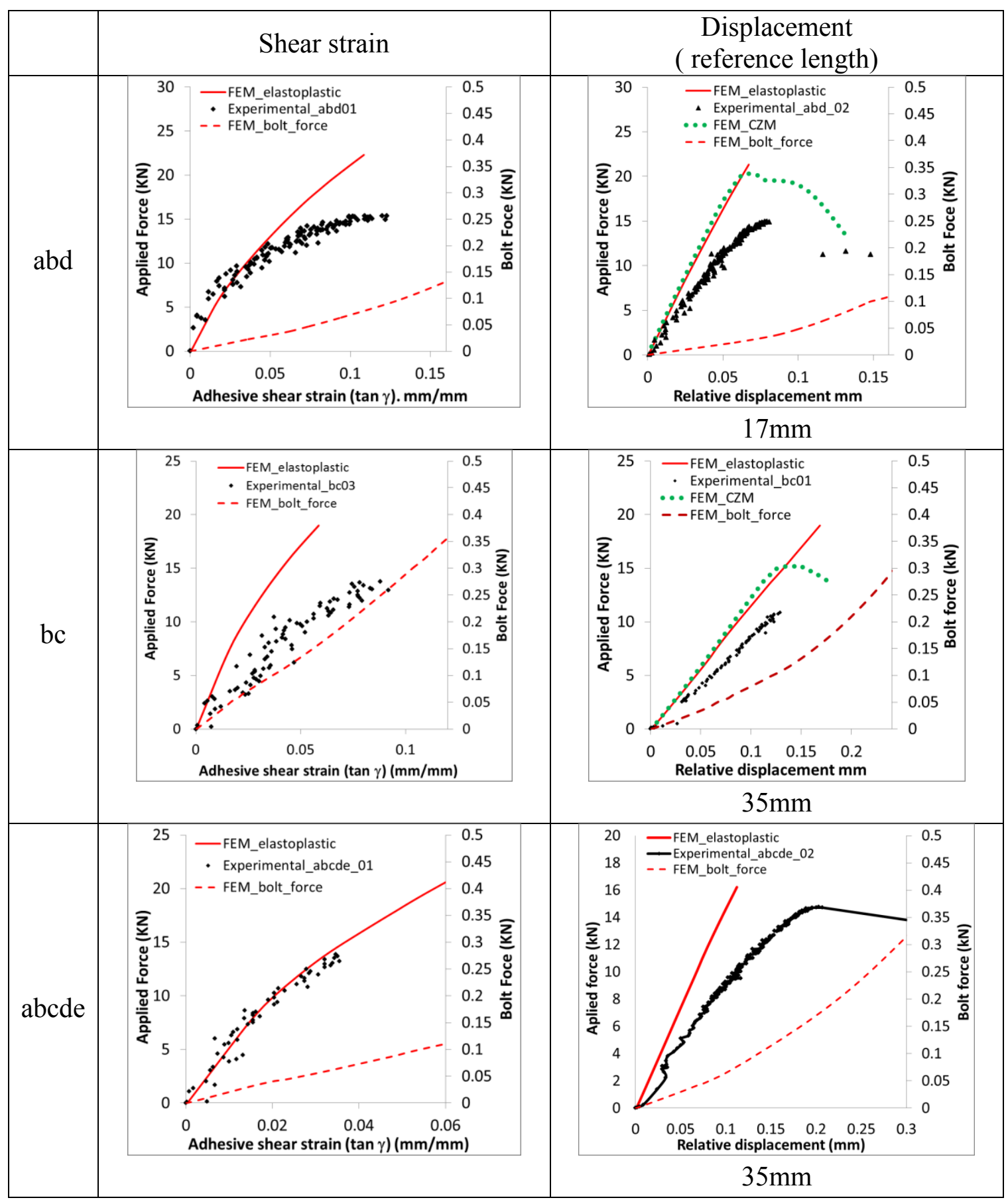


From the results from Table 6.1 , it can be observed that the variation in agreements with the modelling results is wide. In some cases, the agreements is higher than $95 \%$ (shear strain treatment $a b c d e$ ) and, in other tests, the agreement is as low as $50 \%$ (shear strain treatment $b c$ ). In addition to this, all the results from the FEM models under predict the shear strains and displacements compared with the experimental results.

In spite of this variability in the agreement between the FEM and experimental results, the predicted loads taken by the bolts is still very limited in the bolted/bonded joint with FM300-2M adhesive.

From the bolted/bonded joints experimental results, the adhesive showed a point on which the edge of the adhesive layer fails. However, the adhesive did not fail completely. From the test on bonded joints, the failure was catastrophic without delaying the crack grown in the adhesive. This strongly suggests that, after this failure point, the bolt starts carrying a considerable amount of load and; therefore, the failure in the adhesive is delayed.

Unfortunately, this process was not completely captured for the joints with FM300-2M adhesive using CZM analysis.

From the previous numerical and experimental comparison, we can conclude that the bolted bonded joints with FM300-WM adhesive behaves as a bonded joint before the first adhesive failure point, and the load shared with the bolt is barely zero.

\subsection{Load Sharing Joint with EA9361 Adhesive}

The original DIC setup was aimed to measure the local displacements as well as the shear strain in the bondline. With this setup, a speckle pattern using regular spray 
paint was too coarse to measure the shear strain in the bondline. Therefore, it was decided to test the coupons without speckle pattern assuming that the surface roughness contrast was sufficient for the DIC analysis. Unfortunately, some coupons did not have enough roughness and the contrast was not satisfactory for the DIC measurements. As a result, the computed displacements and strains presented some degree of scatter. This is evident from the results shown in Figure 6.3 and Table 6.1.

Therefore, in order to detect the displacements near the interface between the adhesive and adherends and compute the strains more accurately, it was decided to change the setup for the remaining coupons with the EA9361 adhesive. A finer speckle pattern was applied by using an airbrush. In addition to this, the magnification was increased to achieve resolutions up to 6 microns per pixel $(0.006 \mathrm{~mm} / \mathrm{pixel})$, and the region of interest was reduced to the a small area very close to the adhesive layer. The disadvantage of this new setup is that the local displacement in bondline region cannot be measured. The setup was also improved by increasing the number of frames per second to $4 \mathrm{~Hz}$. to capture the strains smoothly.

With this new setup, the scatter in the data was eliminated completely and the agreement with the FE models was excellent as shown in Figures 6.5 to 6.9.

The procedure to measure the load taken by the bolt was by comparing the strains in the bonded and bolted/bonded joint configuration and computing the force difference. Similarly to the results from FM300-2M adhesive, a secondary axis in the plots was incorporated to enhance the visualization of the load taken by the bolt. The secondary axis scale is half of the total applied force. Lower scale in the secondary axis was not chosen to avoid a misinterpretation of the results. 
Figure 6.5 shows the comparison between the experimental results and FE model of load sharing for treatment $d e$. The shear strains are very similar in the bonded and bolted/bonded joint along the full load path. There is a small shifting region where the bolted/bonded joint seems to be stronger, but the difference is barely $4 \%$. It is also remarkable the very good agreement in the shear strain between the experimental results from the bolted/bonded joint and the results from the numerical analysis. These results are also congruent with the load stroke values, where the bonded joint and the bolted/bonded joints seem to behave similarly.

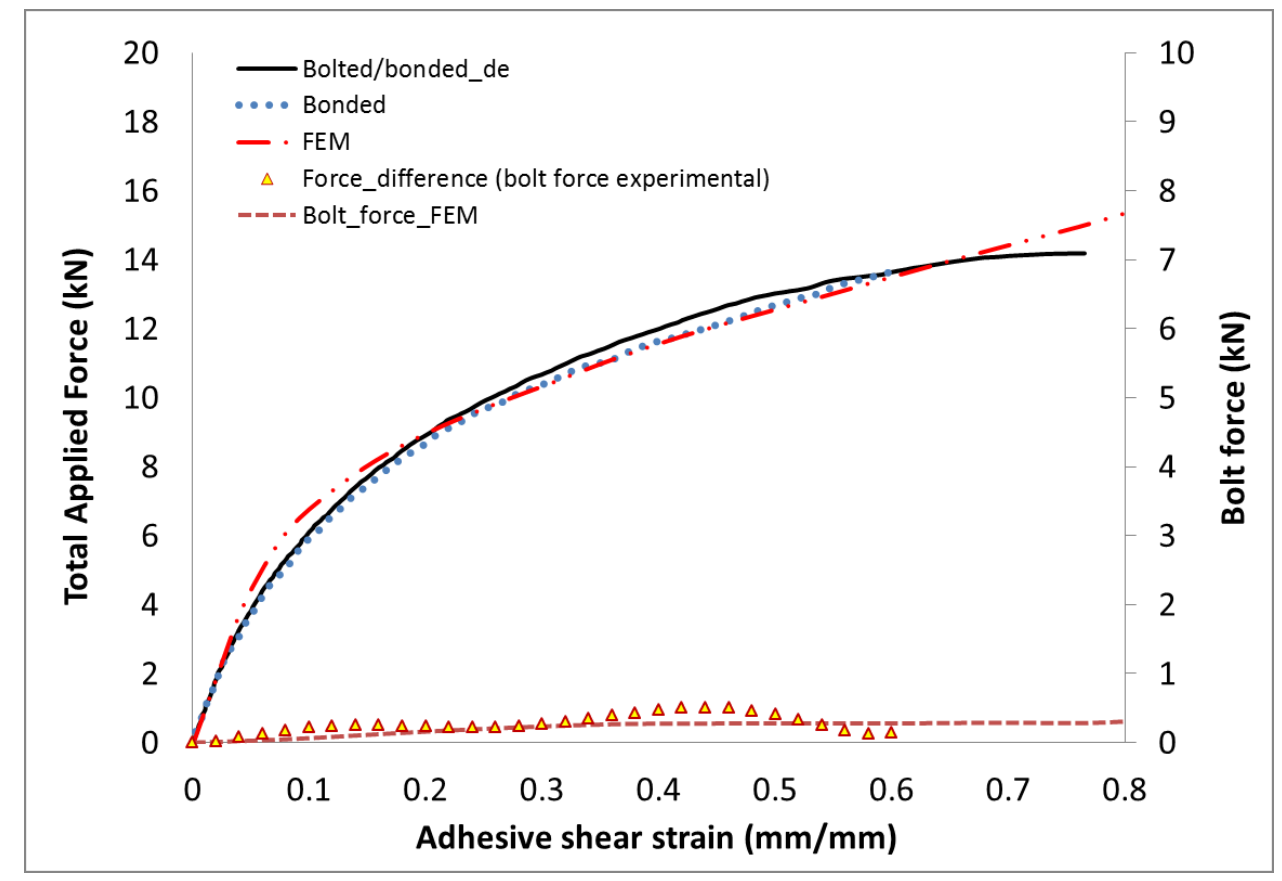

Figure 6.5 Bolted/bonded joint, treatment $d e$. Bolt force analysis using shear strains at $1 \mathrm{~mm}$ form the notch.

By computing the difference in force between both joints, the inferred values of load taken by the bolt is very low. Therefore, it can be deduced that the bolt is never activated when the adhesive layer is still undamaged. The load is carried almost entirely 
by the adhesive. This in agreement with the FE results, where the maximum predicted load taken by the bolt is $0.32 \mathrm{kN}$ at $14 \mathrm{kN}$ of applied force, barely $2 \%$ of the total value. The former contrast severely when it is compared with a similar joint without clearance. From the numerical results using a bolted/bonded joint configuration with neat fit assembly is shown in Figure 6.6, the load taken by the bolt is less than $0.2 \mathrm{kN}$ until 8 $\mathrm{kN}$ of applied force. From this point, the force in the bolt increases steadily until $1.7 \mathrm{kN}$, $12 \%$ of the total applied force of $14.2 \mathrm{kN}$. This force is equivalent to the last point extracted from the experimental result from the joint with clearance. Finally, at maximum shear strain of $0.76 \mathrm{~mm} / \mathrm{mm}$, the numerical analysis predicts, for the same joint with fit assembly, a load taken by the bolt of $2.87 \mathrm{kN}$ for a total applied force of $18 \mathrm{kN}$. This represents $16 \%$ load taken by the bolt.

From this analysis is clear that assembling the joint without clearance is a key factor to increase the load taken by the bolt and also activate it in early the stages of loading with a subsequent increment in the strength, and minimizing the adhesive damage at early stages of loading. 


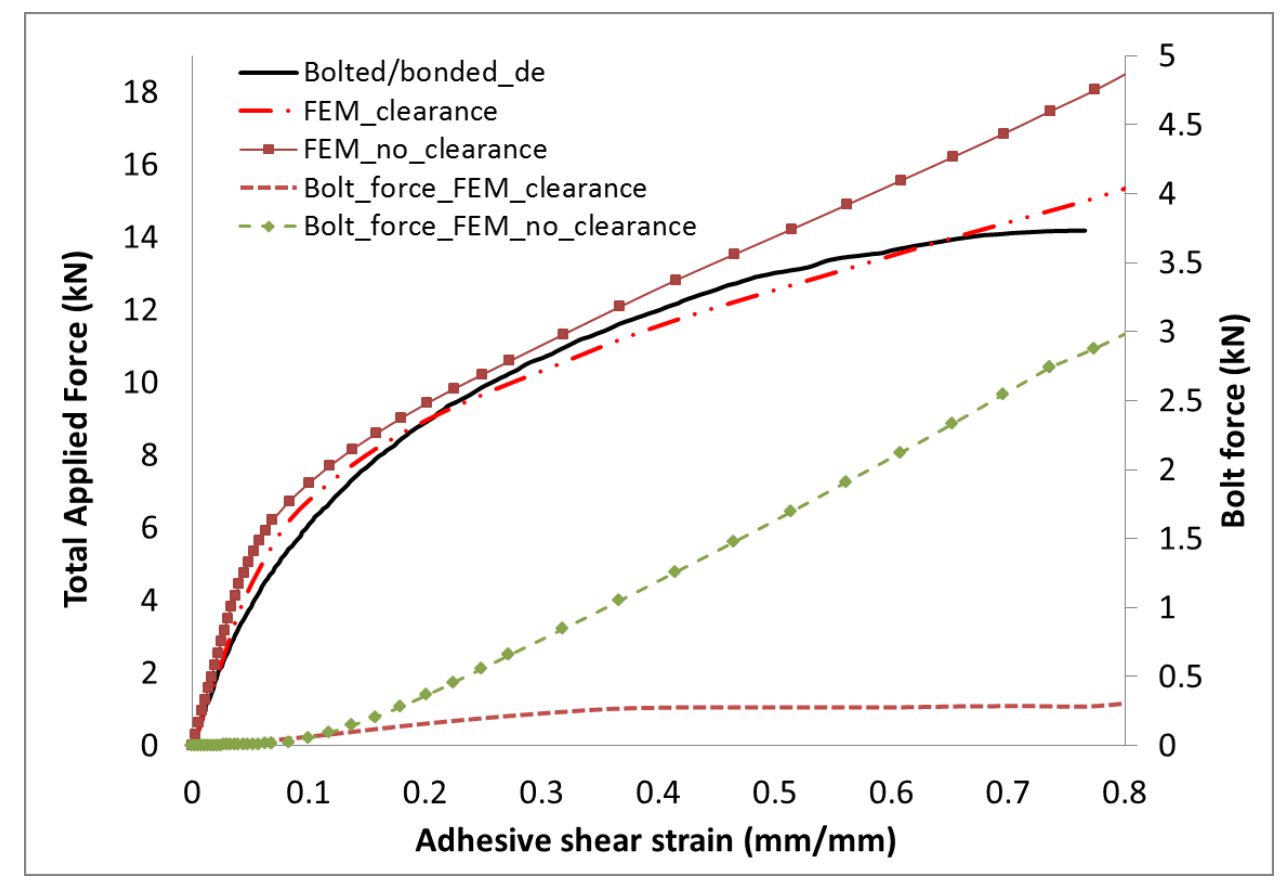

Figure 6.6 Bolted/bonded joint, treatment de. Bolt force analysis and comparison with a joint with neat fit assembly

Figure 6.7 shows the comparison between the experimental results and FE model on load sharing for treatment $a$. Similarly to the previous results, a secondary axis was used to enhance the visualization of the results on load taken by the bolts. The experimental shear strains in the bonded and bolted/bonded joint are nearly identical until approximately $6 \mathrm{kN}$ of applied force. From this point and forward, the capacity to carry load at same shear strain is higher in the bolted/bonded joint. The load path, compared with the FEM results is also in very good agreement. 


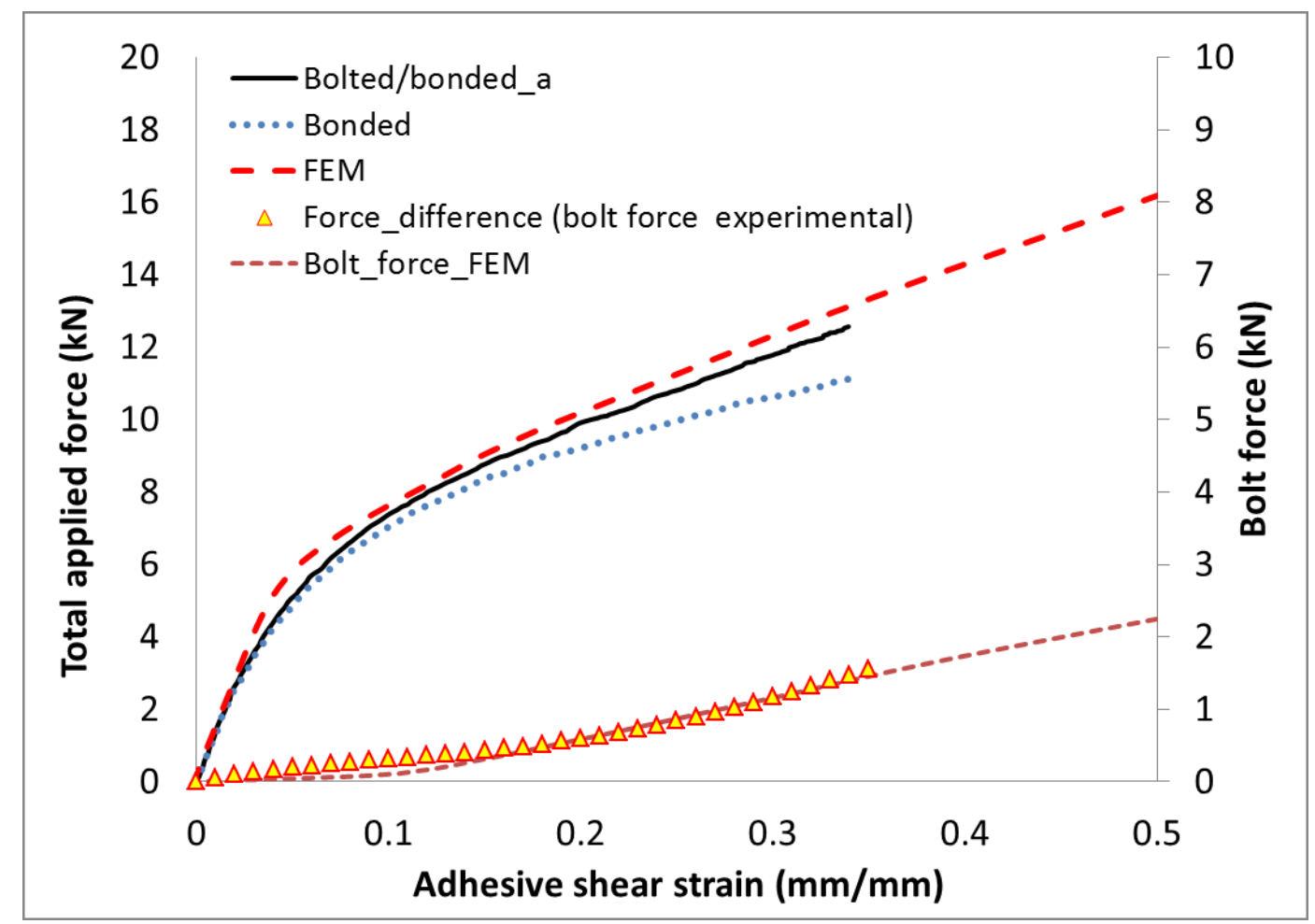

Figure 6.7 Bolted/Bonded joint, treatment a. Bolt force analysis using shear strains at a distance $3 \mathrm{~mm}$ from the notch

Experimentally, it is evident that the bolt is activated almost immediately. However, at lower values of applied force, the load shared is still limited. At $8 \mathrm{kN}$, the difference is $0.4 \mathrm{kN}$, which represents $5 \%$ of the total force. This point of applied force, represents, according to the FEA model, the inflexion point in which the load taken by the bolt starts to increase at a higher rate. The model predicts a shared load of $0.18 \mathrm{kN}$, which is half of the experimental value. However, such a force difference is too small to be captured experimentally with high precision using this method. After this point, the agreement in load sharing between the experimental results and numerical calculation increases dramatically. Unfortunately, the measurement of shear strain beyond 0.35 $\mathrm{mm} / \mathrm{mm}$ presented high levels of scatter in the data due to the fracture initiation in the 
adhesive, and the comparison against the experimental results, after this point could not be carried out. At this strain value, $0.35 \mathrm{~mm} / \mathrm{mm}$, the experimental results showed a load in the bolt of $1.56 \mathrm{kN}$, representing $11.7 \%$ of the total applied force. The numerical results does not have an exact match at this strain values due to the time stepping; however, at $0.33 \mathrm{~mm} / \mathrm{mm}$ of shear strain, the predicted force in the bolt is $1.26 \mathrm{kN}$, and at $0.39 \mathrm{~mm} / \mathrm{mm}$ of shear strain, the bolt force is $1.64 \mathrm{kN}$, that is $10.2 \%$ of the total force. This agreement can be observed in the plots of bolt force versus shear strain.

The load sharing results from this treatment compared with the treatment $d e$, reinforced the statement previously discussed, where the effect of the clearance delays the activation of the bolt dramatically.

The next treatment to be analyzed is $c d$. This treatment had a neat fit assembly in the bolt. Figure 6.8 shows the strain-force results from experimental measurements and FEM model. In this case, it was decided to use the same scale for all the results because the predicted load taken by the bolt was considerably higher. From the experimental results it is evident that the shear strain in the adhesive from bonded and bolted bonded joints behave similarly until approximately $6 \mathrm{kN}$, at this point these values start to diverge dramatically, reaching a maximum difference at a shear strain of $0.42 \mathrm{~mm} / \mathrm{mm}$. After this point, the shear strain measurements were scattered due to fracture in the adhesive layer; therefore an accurate comparison was not possible. The experimental shear strain in the bolted/bonded joint compared with FE results were in good agreement. At early force values, between 4 and $8 \mathrm{kN}$, there is a small discrepancy; however, from 8 $\mathrm{kN}$ until $15.6 \mathrm{kN}$ the agreement is very good. After this point, the predicted FE results still increases steadily, contrasting with the experimental results. However, from the DIC 
pictures it was found that the adhesive started a fracture process, creating an inflexion point in the shear strain values versus applied load. Therefore, the comparison against FE was not possible after this point.

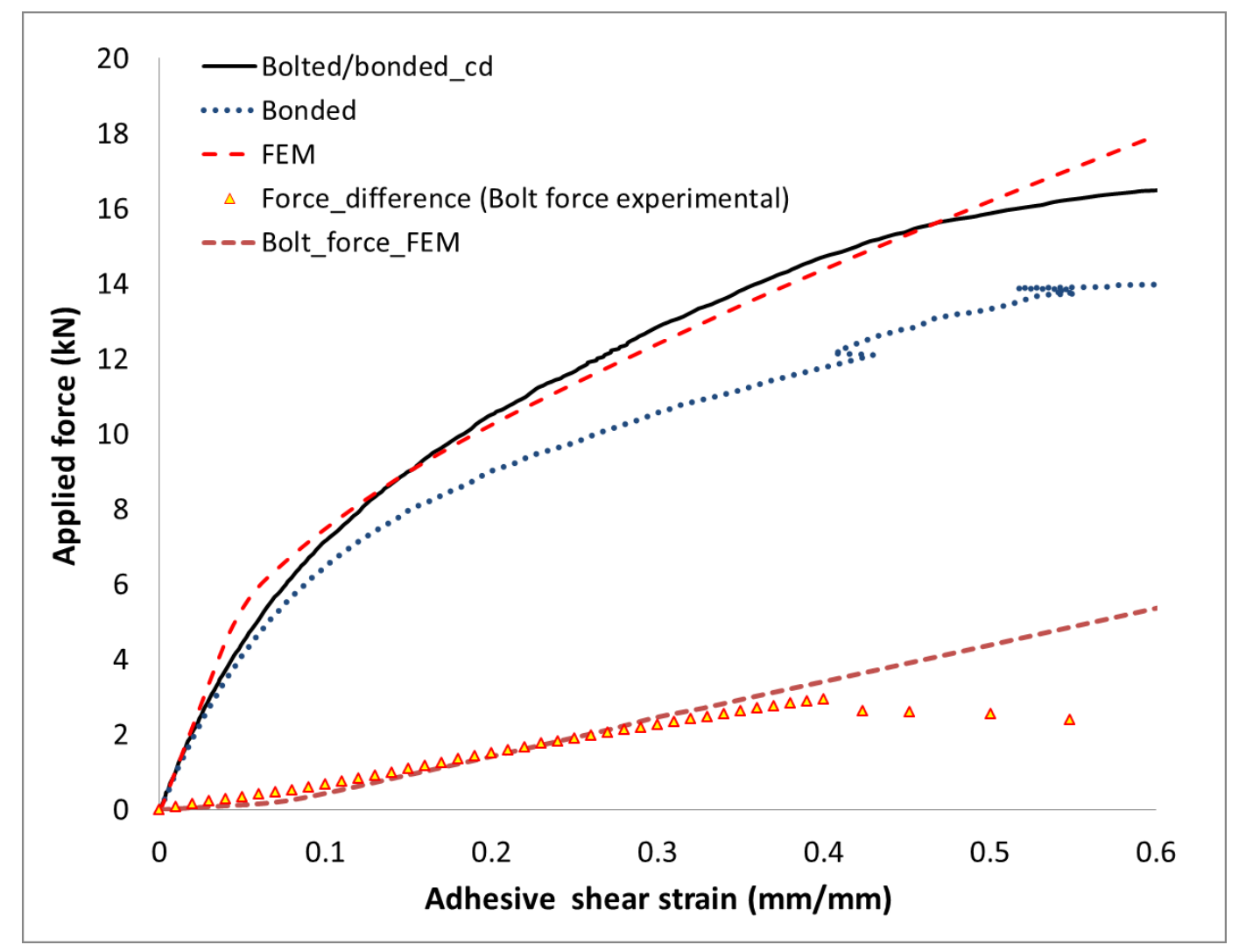

Figure 6.8 Bolted/bonded joint, treatment cd. Bolt force analysis using shear strains at a distance $1 \mathrm{~mm}$ from the notch

Experimentally, the load taken by the bolt shows an inflection point at approximately $6 \mathrm{kN}$ of applied force; until this value, the experimental force in the bolt is $0.6 \mathrm{kN}, 10 \%$ of the applied load. Conversely, at the same load value, the FEM model predicts a bolt force of $0.23 \mathrm{kN}$ and, after this inflection point, the increases steadily until $2.9 \mathrm{kN}$ at an applied force of $14.7 \mathrm{kN}$. This represents $20 \%$ of the applied force. The FEM result shows a very good agreement until approximately $12 \mathrm{kN}$ of applied force. 
From this point, there is a slight difference between the predicted and measured values. However, the agreement is very good until $14.7 \mathrm{kN}$ of applied force, where the experimental results dropped significantly. At this point, the shear strain in both joints, bolted and bolted/bonded is $0.4 \mathrm{~mm} / \mathrm{mm}$.

The sudden discrepancy is produced by the fracture in the adhesive in the bonded joint; therefore, the comparison against the bolted/bonded joints is not possible after this value of shear strain. The shear strain in the bolted/bonded joint was measured until 0.68 $\mathrm{mm} / \mathrm{mm}$ at $16.7 \mathrm{kN}$ of applied force. After this point, again, the fracture process in the adhesive made impossible to measure the strains. The numerical results show, at this applied load, the load taken by the bolt is approximately $27 \%$ of the total applied force, a promising result.

The load sharing analysis for the last treatment ace was carried out using the results from the repetition test $a c e \_r e p \_01$ instead the original tested coupons. This coupon was tested using the improved DIC setup. The shear strain extracted from the experimental test was compared with the computed strains from the FEM analysis. Figure 6.9 shows the results. It can be observed that the agreement in very well along all the loading path; therefore, it can be claimed that the load taken by the bolt computed numerically corresponds to the experimental value.

It can be observed that the load taken by the bolt barely reached $0.32 \mathrm{kN}$ at a shear strain of $0.42 \mathrm{~mm} / \mathrm{mm}$ and $11.96 \mathrm{kN}$ of applied force. At this point, the load in the bolt increases dramatically until $16.8 \mathrm{kN}$ of applied force and $0.70 \mathrm{~mm} / \mathrm{mm}$ of shear strain. This corresponds to $18 \%$ of the total applied force. 
This applied force of $16.8 \mathrm{kN}$ does not corresponds to the maximum strength for this joint, $20.96 \mathrm{kN}$. The reason was the same as the previous treatments, the initiation of the fracture process in the adhesive, thus, preventing an accurate measurement of the shear strains.

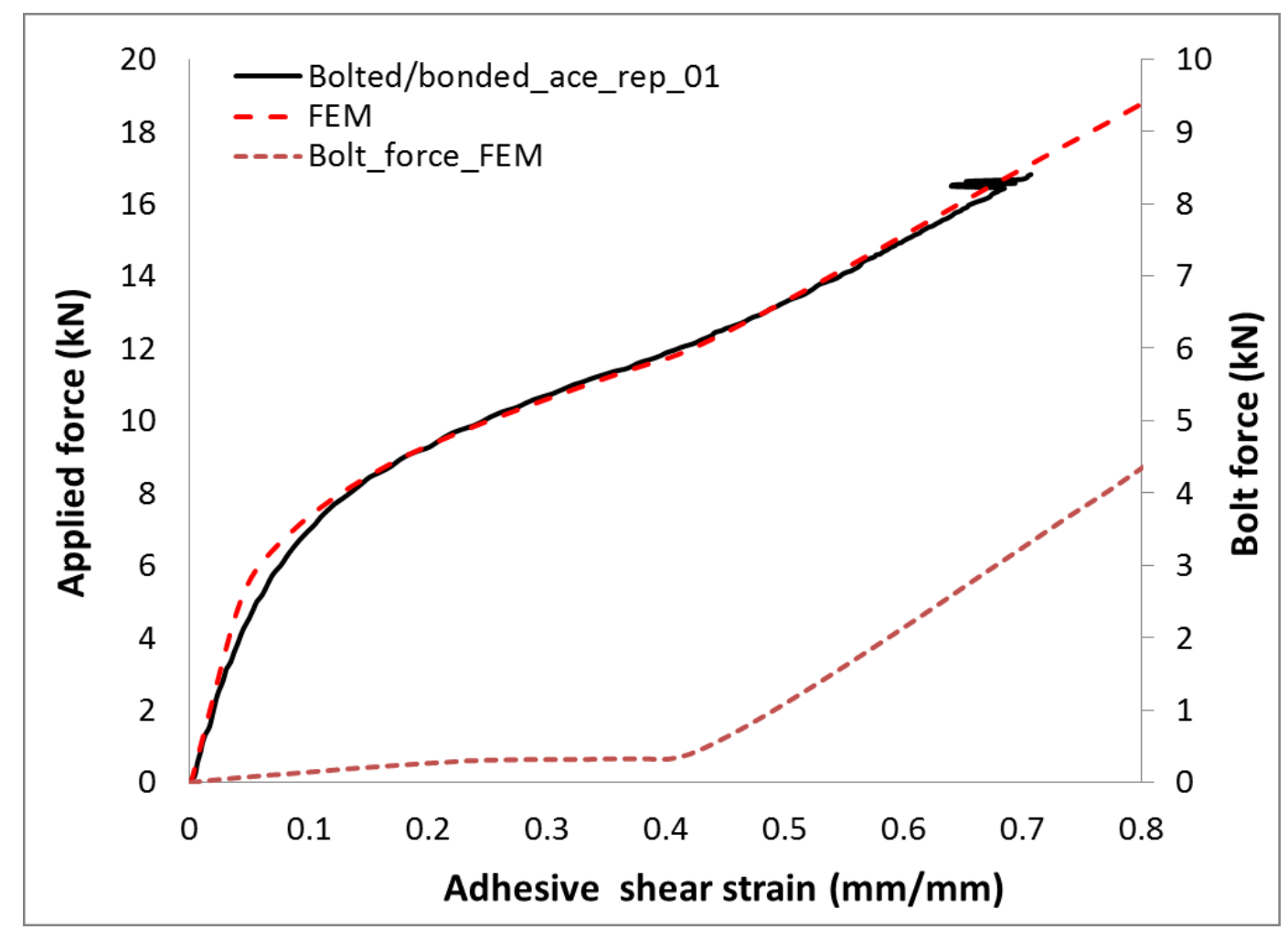

Figure 6.9 Bolted/bonded joint, treatment ace_rep_01. Bolt force analysis using shear strains at a distance $1 \mathrm{~mm}$ from the notch

The delay in the bolt activation demonstrates the negative effect of the clearance between the bolt and the hole in the load sharing. The contact between the bolt shank and the hole surfaces is delayed due to the gap between the bolt and the hole. Therefore, the activation of the bolt is carried out after the rotation of the bolt is large enough to allow a force contact between the aforementioned surfaces. Unfortunately, this process is reached after a relatively high force value. 
According to the ANOVA results, the washer has a negative effect in the maximum strength. Conversely, as pointed out in the literature review [56], the washer size has a positive effect in the joint strength. However, from the load sharing analysis, there is no evidence of the effect of the washer in the joint performance because the maximum force was not reached during the shear strain measurement.

On the other hand, the ANOVA indicates that the clearance has a negative impact of $7.01 \%$ at first failure point and $12.4 \%$ at maximum strength. This effect was detected in the load sharing analysis. The load taken by the bolt from the treatments de and ace presented a well-defined inflection point that suggested that the activation of the bolt was delayed due to the clearance effect.

\subsection{Bolted/bonded Joints with Interference Fit Assembly}

It has to be pointed out that the results from the ANOVA are only valid within the limits of the DOE study. According to the results, the strength in the bolted/bonded joint is mainly driven by the bonded joint part, the adhesive strength and the adherend thickness with combined effect of $77.1 \%$ and individual effects of $38.3 \%$ and $38.8 \%$ at first failure point respectively. Therefore, to improve the joint performance, using the factors from the DOE, the only option is by varying the clearance, washer size and adhesive thickness. From these factors, the most relevant was the clearance.

Since the neat fit and clearance was already explored, the obvious choice is to explore the interference fit. The aim of this test was to explore the possibility to activate the bolt in the early stages of the loading due to the interference assembly between the bolt and the hole, and to assess any improvement in the joint performance. 
Two coupons with EA9361 and FM300-2M adhesives were tested with the configuration shown in Table 6.2.

Table 6.2 Interference fit joints

\begin{tabular}{|c|c|c|c|c|}
\hline $\begin{array}{c}\text { Adherend } \\
\text { thickness } \\
(\mathrm{mm})\end{array}$ & Adhesive & $\begin{array}{c}\text { Adhesive } \\
\text { thickness }(\mathrm{mm})\end{array}$ & $\begin{array}{c}\text { Clamped } \\
\text { area }\left(\mathrm{mm}^{2}\right)\end{array}$ & $\begin{array}{c}\text { Clearance } \\
\mathbf{m m}\end{array}$ \\
\hline 3.29 & EA9361 & 0.5 & 97 & Interference \\
\hline 3.29 & FM300-2M & 0.5 & 97 & Interference \\
\hline
\end{tabular}

For the joints with EA9361 adhesive, the joint configuration was similar to the treatment $c d$, excepting for the washer size. Therefore, the results were compared with the treatment $c d$.

The experimental load versus stroke results for the joints with EA9361 adhesive are shown in Figure 6.10. It can be observed that there is no a visible improvement in the initial stage of the loading or maximum strength. The maximum average strength for the interference fit joints were $18.56 \mathrm{kN}$. When compared with the average maximum strength from the combination test $c d$, whose maximum average strength was $17.93 \mathrm{kN}$, the difference is $3.5 \%$.

The results of the shear strain in the joints with interference fit at its comparison against the treatment $b c$ is shown in Figure 6.11. It is evident that bolted/bonded joint with interference fit assembly behaved similarly as the joints with neat fit assembly. Therefore, it can be concluded that there is no improvement in the load taken by the bolt. 


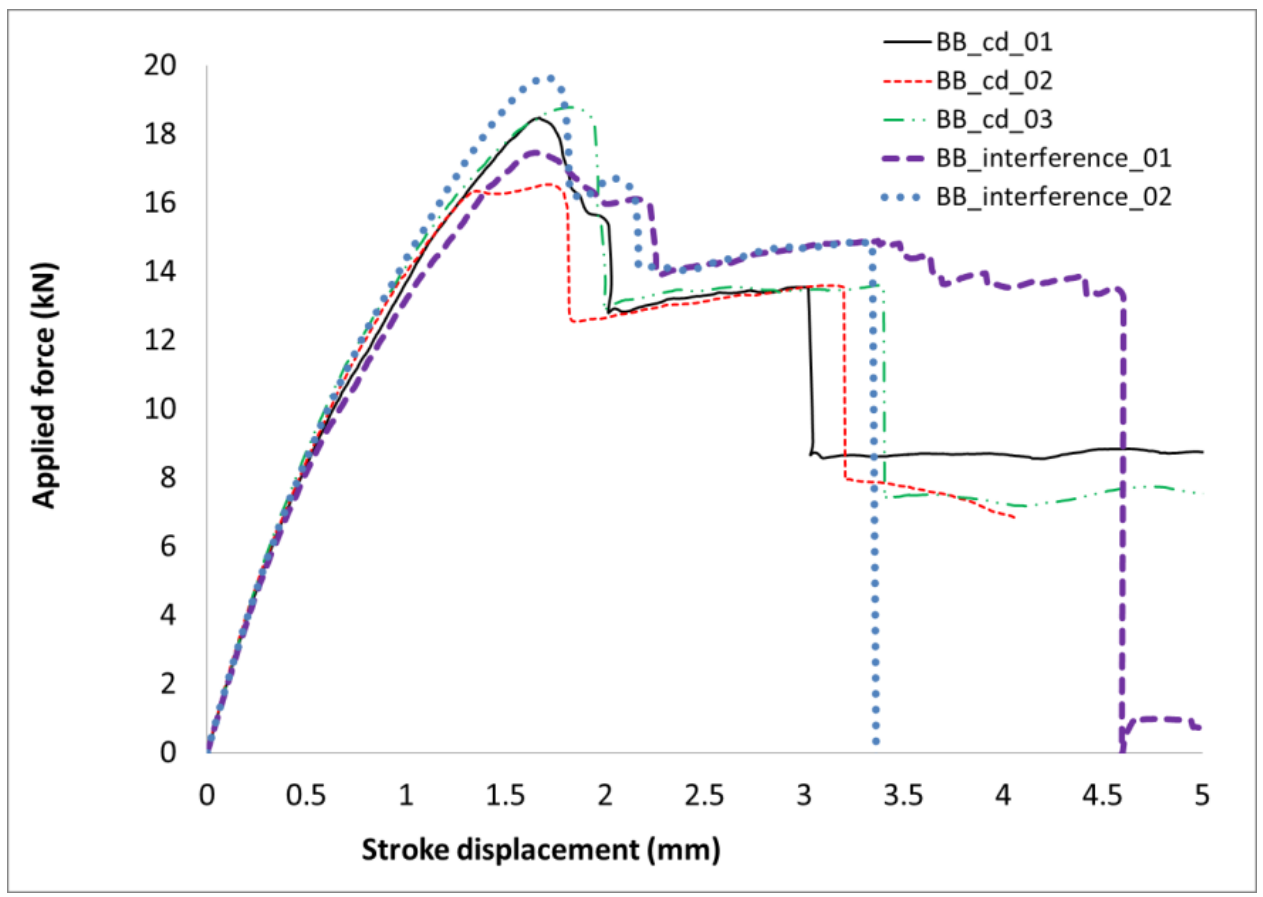

Figure 6.10 Bolted/bonded joints with EA9361 adhesive and interference fit. Force versus displacement results

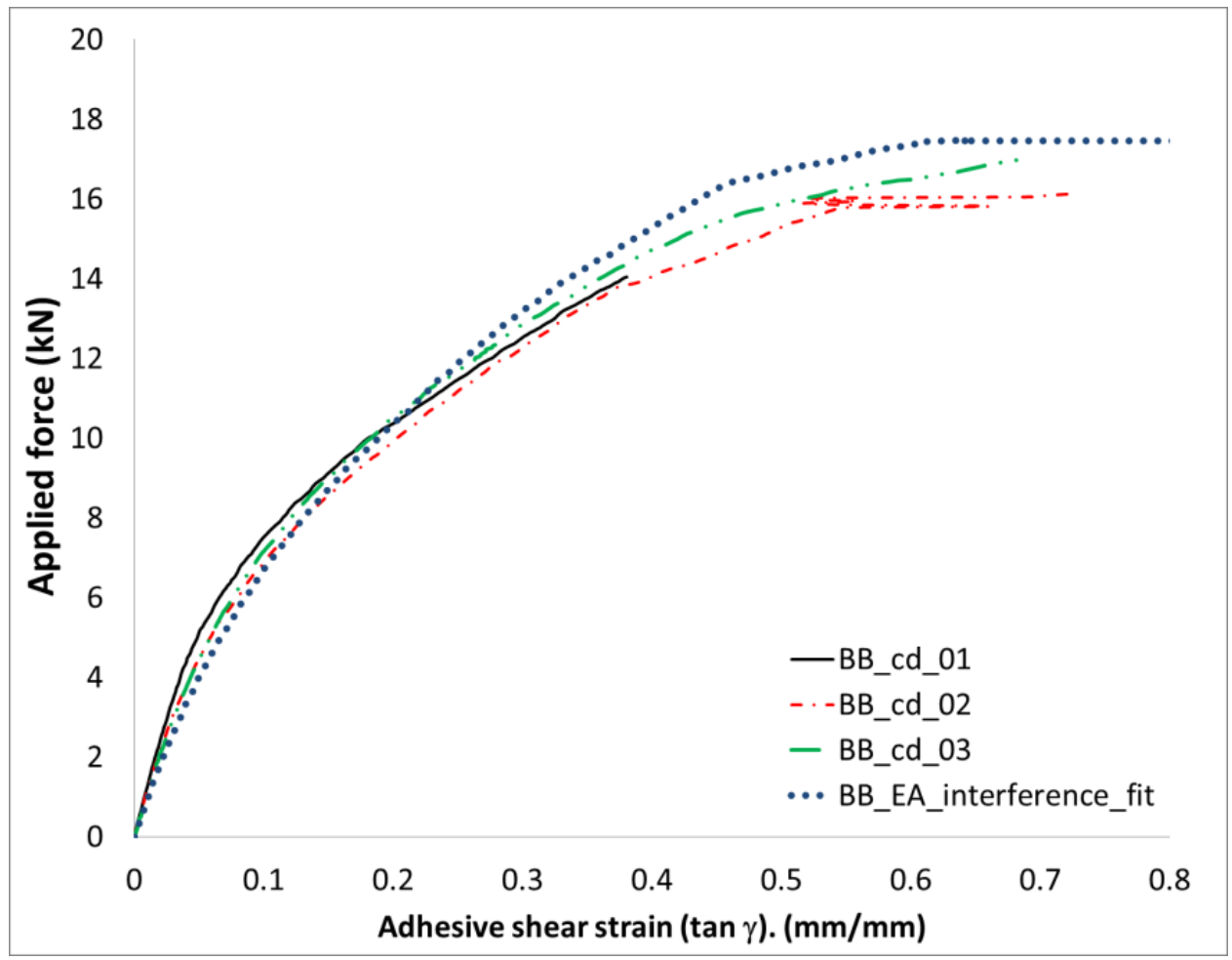

Figure 6.11. Bolted/bonded joint with EA9361 adhesive and interference fit assembly. Adhesive shear strain at $1 \mathrm{~mm}$ distance from the notch 
For the joints with FM300-2M adhesive, the results are shown in Figure 6.12 along with the results from bonded specimens. It is clear that the bolted/bonded joints behaved as bonded joints until the first failure point. The load path is exactly the same; however, the failure is catastrophic for the bonded joints. The bolted/bonded joint the adhesive failed in stages, similarly to the coupons with FM300-2M from the original DOE.

The average maximum strength in the bolted/bonded joints was $10.91 \mathrm{kN}$, and for the bonded joints was $10.50 \mathrm{kN}$. A marginal improvement of $0.41 \mathrm{kN}$, representing $3.9 \%$.

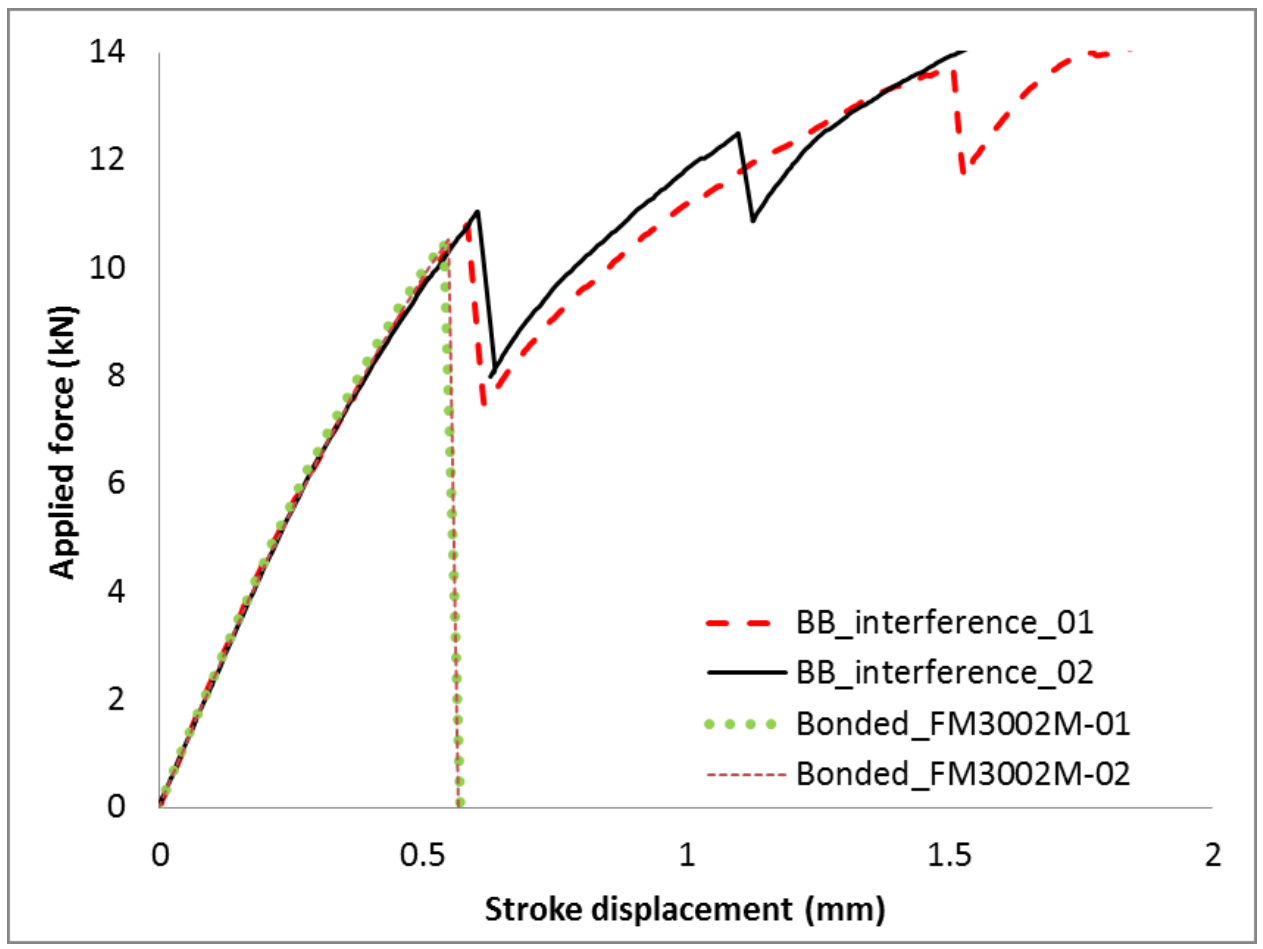

Figure 6.12 Bolted/bonded joints with FM300-2M adhesive and interference fit. Force versus displacement results

The force versus shear strain measurements shown in Figure 6.13 confirm that the bolted/bonded joint behaviour is the same as for a bonded joint before the first failure in 
the adhesive. The force versus strain paths are identical; therefore, it is deducted that the load in the bolt is nearly zero.

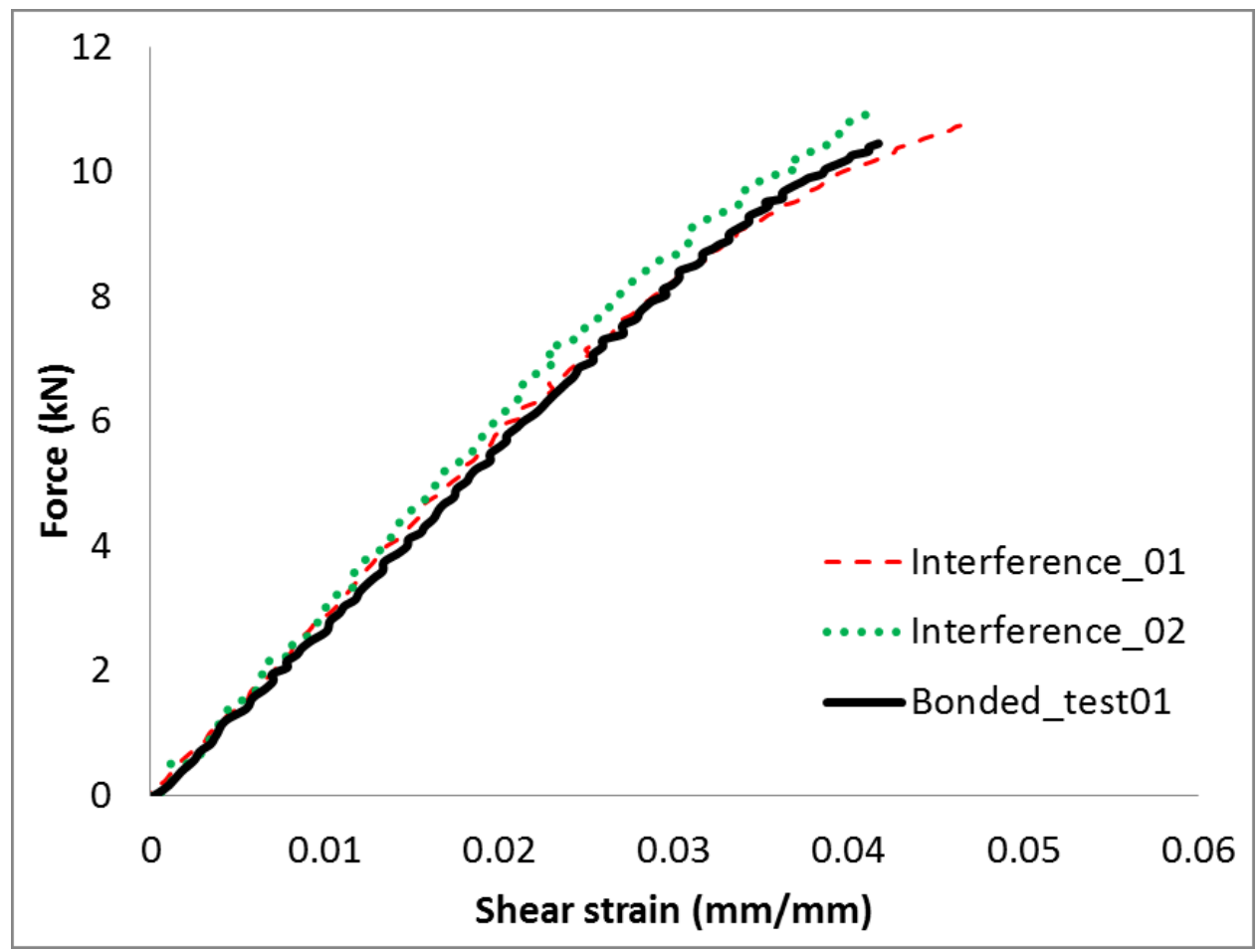

Figure 6.13 Bolted/bonded joint with FM300-2M adhesive and interference fit assembly. Adhesive shear strain at $1 \mathrm{~mm}$ distance from the notch

Notwithstanding this, the advantage of the bolted/bonded joint is the arresting of the adhesive's crack grow along the bondline, with a subsequent increase in the joint strength and displacement.

Finally, it is concluded that the joints with interference fit behave similarly as the joints with neat fit assembly. 


\subsection{Limitations of the DIC Technique}

The proposed DIC measurement technique to assess the load sharing has the following advantages compared to instrumented bolts:

- There is no limitation in the joint configuration

○ The instrumented bolt is limited by the bolt size, clearance and torqueing

- Local displacements and strains can be measured

- Instrumented bolt only measures the shear strain in the bolt

- The experimental setup does not interfere with the joint preparation

- The instrumented bolt requires careful installation of the bolt to align the strain gauges in the midline of the joint

- The installation can damage the strain gauge circuitry

- Visually detects the adhesive damage and failure

Instrumented bolt does not detect adhesive damage and failure

On the other hand, some limitations can be observed:

- Requires the shear strain from a bonded joint as a reference

- The measurements are limited until a load when the adhesive starts the damage process

- Requires extra hardware (camera)

- Requires software to post-process the data

- A clear view of the bondline is required 
Previous research work using instrumented bolts have reported $32 \%$ load sharing out of $8 \mathrm{kN}$ of applied force [58] and 36\% out of $10 \mathrm{kN}$ of applied force [73] - in both cases using ductile adhesives. However, the argument to use these load levels was to avoid the damage of the instrumented bolt. In addition to this, compared with the present study, there is no information about the strain in the adhesive and possible adhesive damage during the loading process.

\subsection{Chapter summary}

In this chapter, the load sharing measurement was carried out using the proposed method of measuring local displacement in the joint and shear strains in the adhesive layer instead measuring the strain in the bolt.

The measurements using local displacements were carried out in the joints with FM300-2M adhesive. There were found systematic errors due to the DIC setup and some degree of scatter in the data. The agreement between experimental and numerical results was on the order of $50 \%$ to $95 \%$ depending on the setup and model. However, the predicted load in the bolts before adhesive failure was less than $0.4 \mathrm{kN}$

On the other hand, using an improved setup and measuring the shear strains in the adhesive was found to give very good agreement experimentally and numerically, above $95 \%$ along the load path for all the treatments. The experimental procedure was carried out by measuring the strains in bonded joints and comparing the performance with similar bolted/bonded joints.

It was also found that the clearance has an adverse effect on the activation of the bolt. 
In order to analyze the opposite effect of the clearance, bolted/bonded joints with interference fit were tested; however, it was found that they behave similarly as the joints with neat fit assembly. 


\section{Chapter 7: Discussion}

From the experimental results and the analysis of variance (ANOVA) it was found that the main factors in the strength of the bolted/bonded joints are the adhesive modulus and adherend thickness. The adherend thickness has a positive effect in the total strength with $38.3 \%$ and $42.1 \%$ at first failure point and maximum strength respectively. However, the gain in total strength using thicker adherends is severely penalized in the strength/weight ratio, and the effect becomes severely negative. The results were $-79.7 \%$ and $-91.5 \%$ respectively. On the other hand, the effect of the adhesive modulus in the total strength was negative in both cases with $-38.8 \%$ and $-25 \%$ respectively.

From the finite element modelling, it was found that the bolted/bonded joints behaved similarly to a bonded joint at lower applied forces. The load carrying capacity of the bolt is very limited. This was confirmed experimentally by measuring the shear strain at the edge of the joint and comparing the strain profile with a bonded joint with the same configuration and materials.

On the other hand, it was found that the total load carrying capacity and the ability of sustain higher deformations is improved using a bolted/bonded joint configuration.

With stiffer adhesives, the bolts were acting as bondline crack arresters, delaying the sudden propagation of the crack along the bondline. However, this effect was observed only after partial adhesive failure at lower force values. Therefore, the synergistic effect between the adhesive and bolt is present at a damaged state in the joint. The positive effect is an increase in the strength as well as the capability to sustain larger displacements, as shown in Figure 7.1. The bonded, bolted, and bolted/bonded joints 
were compared. The arrows show the improvement in displacement and strength in the bolted/bonded joint compared with the bonded and bolted joint configuration.

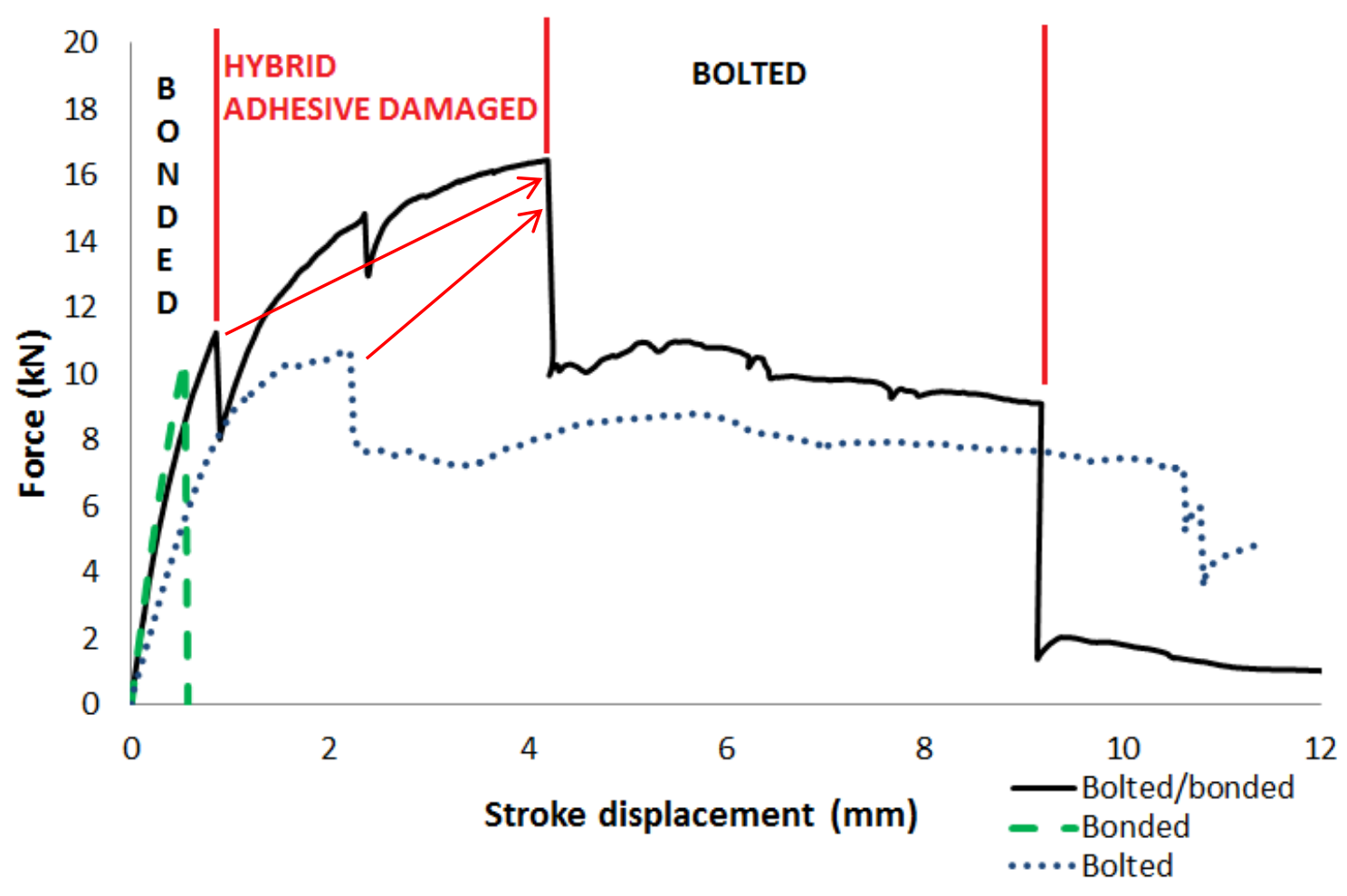

Figure 7.1 Bonded, bolted and bolted bonded joint comparison with stiff adhesive FM300$2 \mathrm{M}$.

The same behaviour was found with the ductile adhesive EA9361; however, the joint did not show sudden failure with a subsequent arresting in the crack. Instead, the damaged was progressive and smooth until final failure. This was depicted in Figures $4.33,4.38,4.43$ and 4.49 .

In general, the behaviour of a bolted/bonded joint with ductile adhesive and within the DOE levels, can be explained using the Figure 7.2. At lower values of force, less than $6 \mathrm{kN}$, the joint behave similarly as a bonded joint, the load sharing in this stage is less than $5 \%$. This also was corroborated by analyzing the shear strains as shown in 
Figure 6.7 and Figure 6.8. Moreover, at this force, the shear strain at the measured points was beyond the linear elastic range.

After this point, the effect of the hybridization is visible due to increment in the strength in the bolted/bonded joint. However, before total adhesive failure, there is a point in which the adhesive begins the failure process. Between these two points, the load taken by the bolt, depending on the joint design and, according to the numerical and experimental results, can be between 5 and $30 \%$.

After this point, the joint contained a considerable amount of damage in the adhesive. This was also detected during the shear strain analysis. Nevertheless, the strength and displacement capability is improved, as shown with the arrows. This region behaves as a crack arrestor, similar to the joint with FM300-2M adhesive. Finally, after the adhesive failure, the load is taken by the bolt.

According to the Federal Aviation Regulations [22], the limit load is the expected load in service and the ultimate load is the limit load multiplied by a safety factor (usually 1.5). Since the strength in the joint is improved, the ultimate load capability is also improved. 


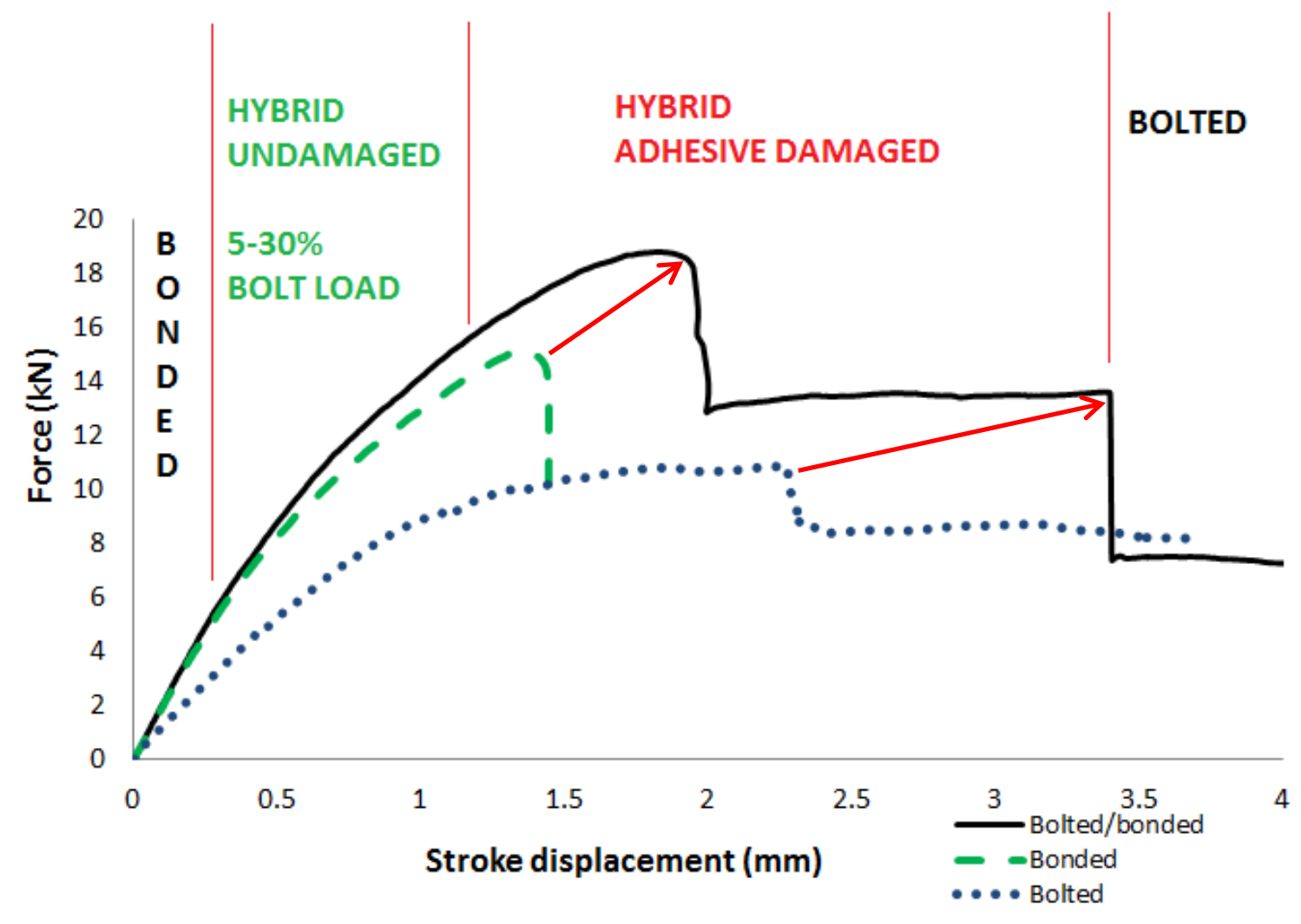

Figure 7.2 Bonded, bolted and bolted bonded joint comparison with ductile adhesive EA9361.

This behaviour observed in the bolted/bonded joints is also supported the ANOVA results. Before the first failure point, it is clear that the joint behaves as a bonded joint. At these points, the strength variability is mainly driven by the adherend thickness and adhesive modulus, with individual effects of $38.3 \%$ and $38.8 \%$ respectively, and a total effect of $77.1 \%$.

At maximum strength, the effect of the adhesive is reduced to $25 \%$ and the effect of the adherend is increased to $42 \%$. This is also consistent with the joint behaviour. At these points, the adhesive has reduced his capability of load carrying because is partially damaged. The load has to be transferred to the bolt and, according to the bolted test 
results, the joint with thicker adherend is stronger; therefore, the effect of the adherend thickness increases.

The load sharing analysis was carried out using the displacement analysis as well as the shear strain analysis in the adhesive. With the proposed procedure, it was possible to capture the strains in the full elastoplastic range. Therefore, compared with the instrumented bolt technique, the region of analysis was extended. However, it was found that the technique is limited due to the adhesive damage. The experimental results, compared with the numerical results were in very good agreement along the load path.

From this analysis a strong adverse effect was found from the clearance on the load sharing process and; therefore in the joint strength. This was also consistent with the ANOVA results.

On the other hand, there was no strong evidence of the effect of the washer size in the load sharing process. This lack of evidence was also found in the numerical results. This is consistent with the ANOVA results for the analysis until first failure point. It should be pointed out that the FM300-2M adhesive failed prematurely; therefore, according to the ANOVA, the effect of the washer is negligible at this point.

On the other hand, the load sharing analysis in the joints with EA9361 was not carried out until the maximum strength due to the adhesive damage. Therefore, the negative effect of the washer, $3.64 \%$ estimated from the from the ANOVA, was not detected. 


\section{Chapter 8: Conclusions and Recommendations}

\subsection{Conclusions}

One major objective of this research was to quantify the load sharing in bolted bonded/joints using the DIC technique. From the results, we can conclude the following:

- The proposed technique was accurate and repeatable;

- Extensive measurement and analysis was carried out on every single coupon; and

- Reliable and accurate material properties in the FEM models are required to obtain good results comparable with the experiments

The proposed numerical model using cohesive zone modelling to predict the strength in the joints was developed progressive failure in the composite. From the results we can conclude the following:

- The model predicts the joint strength with agreement above $77 \%$ for the joints with stiffer adhesives and from $87 \%$ to $97 \%$ for joints with relatively ductile EA9361 adhesive;

- The damage sequence in the adhesive layer and the subsequent load transferred to the bolt was predicted; and

- The effect of the damage progression in the adherend at maximum strength is negligible

The present experimental study was designed using the DOE approach. Within the limits of this design of experiments we can conclude: 
- The effect of five factors in the performance of bolted/bonded composite joints was quantified;

- The major positive factor in the bolted/bonded joint performance is the increase in the adherend thickness $(42 \%)$;

- The major negative factors in the bolted/bonded joint performance are the adhesive type (-25\%) and bolt-hole clearance (-12.4\%);

- No significant effect due to interactions were found, the maximum effect was the combination between the adhesive type and bolt-hole clearance (2.76 \%);

- The effect of the washer size in the strength is almost negligible and first failure point and negative at maximum strength (3.64\%);

- In addition to this, the number of treatments derived from the DOE was used to analyze the load sharing extensively with different joint configurations; and

- The major advantages of hybrid bolted/bonded joints is still the cracking arresting feature in the adhesive and increase in the performance

Lastly, it has to be pointed out that in previous research work, the quantified results were comparisons at maximum strength between hybrid bolted bonded joints versus bonded and versus bolted joints

\subsection{Recommendations and Future Research Work}

By using design of experiments approach and complementing this with robust numerical analysis, the present work has given a further insight into the performance of composite bolted/bonded joints. However, there are some aspects found in this 
investigation and from the literature review that might be worthy to consider for future research:

- The typical load sharing measurement technique using instrumented bolt is limited by the bolt size. In this research, that limitation was surpassed using a new methodology by measuring the shear strain at the adhesive edge. Therefore, further experimentation to investigate the effect of the bolt size can be carried out using this approach

- Similarly, hybrid joints with multi-bolt configurations can be analyzed using this method to assess the distribution of force in the bolts.

- Since the load sharing and strength was improved with ductile adhesives, it is recommended to keep working using DOE with adhesives in three levels of mechanical properties

- It is recommended to carry out experiments with thinner joints that those already tested in this research to find the best strength-to-weight ratio

- It recommended to improve the DIC setup by using cameras with higher resolution and to measure the strain along the full bondline

- It is recommended to improve the CZM models with elastoplastic adhesive and local cohesive elements minimizing the required computational time

- It is recommended to expand the DOE to include fatigue and environmental effects 


\section{Bibliography}

1. Smith, F., The use of composites in aerospace. Royal Aeronautical Society: Available: http://aerosociety.com/Assets/Docs/Events/693/Presentations/(7) Dr Faye Smith.pdf.

2. Kassapoglou, C., Applications of Advanced Composites in Aircraft Structures. Design and Analysis of Composite Structures: With Applications to Aerospace Structures. 2010: John Wiley \& Sons.

3. Bowles, M.D., The Apollo of Aeronautics: NASA's Aircraft Energy Efficiency Program, 1973-1987. 2010: National Aeronautics and Space Administration.

4. Baker, A., Dutton, S., and Kelly, D., Composite Materials for Aircraft Structures (2nd Edition). American Institute of Aeronautics and Astronautics.

5. Hale, J. Boeing 787. From the ground up.

Boeing.com/commercial/aeromagazine, 2006. February 2014]; Available from: http://www.boeing.com/commercial/aeromagazine/articles/qtr_4_06/article 04 _ 2. html.

6. Roeseler, W., Sarh, B., and Kismarton, M., Composite Structures: The first 100 years, in 16th International conference of composite materials. June, 2007:

Kyoto, Japan.

7. Airbus, Intelligent airframe. February 2014]; Available from: http://www.a350xwb.com/advanced/fuselage/.

8. Lee, S.M., Handbook of Composite Reinforcements. 1992: Wiley. 
9. Gardiner, G., Out-of-autoclave prepregs: Hype or revolution? , in Composites World. 2011, Composites World: http://www.compositesworld.com/articles/outof-autoclave-prepregs-hype-or-revolution.

10. Campbell, F.C., Structural Composite Materials. 2010: ASM International.

11. Miller, S., et al., Thermod. Composite airframe temperature prediction tool evaluation, validation, and enhancement with initial steady-state temperature data. 2004, FAA.

12. Kaddour, A. and Hinton, M., Maturity of 3D failure criteria for fibre-reinforced composites: Comparison between theories and experiments: Part B of WWFE-II. Journal of Composite Materials, 2013. 47(6-7): p. 925-966.

13. Soden, P., Kaddour, A., and Hinton, M., Recommendations for designers and researchers resulting from the world-wide failure exercise. Composites Science and Technology, 2004. 64(3-4): p. 589-604.

14. Bunin, B.L., Critical Joints in Large Composite Primary Aircraft Structures. Volume 1: Technical Summary. NASA Contractor Report 3914. 1985.

15. Hart-Smith, L.J., Analysis and design of advanced composite bonded joints. NASA. CR-2218. 1974, National Aeronautics and Space Administration.

16. Hart-Smith, L.J., Bolted Joints in Graphite-epoxy Composites. NASA CR-144899. 1976, Douglas Aircraft Company.

17. Nelson, W.D., Bunin, B.L., and Hart-Smith, L.J., Critical joints in large composite aircraft structure. NASA Contractor Report 3710. 1983, National Aeronautics and Space Administration, Scientific and Technical Information Branch. 
18. Potter, D.L., Primary Adhesively Bonded Structure Technology (PABST): Design Handbook for Adhesive Bonding. 1979, Air Force Flight Dynamics Laboratory.

19. Tan, S.C., Evaluation of Composite Joints. WL-TR-94-4077. 1994.

20. Boltless assembling of primary aerospace composite structures. December, 2015]; Available from: http://www.bopacs.eu/index.html.

21. U.S. Deparment of Transportation. Advisory Circular AC 20-107B. Composite aircraft structure.

22. Code of Federal Regulations, Title 14, Aeronautics and Space: Pt. 1-59, Revised As of January 1 2011. 2011: U.S. Government Printing Office.

23. Gardiner, G., Certification of bonded composite primary structures, in Composites World.2014, Composites World: http://www.compositesworld.com/articles/certification-of-bonded-compositeprimary-structures.

24. Kelly, G., Joining of Carbon Fiber Reinforced Plastics for Automotive Applications. 2004, Royal Institute of Technology, Sweden, PhD Thesis.

25. Ebnesajjad, S., Handbook of Adhesives and Surface Preparation: Technology, Applications and Manufacturing. 2010: Elsevier Science.

26. Cognard, P., Handbook of Adhesives and Sealants. 2005: Elsevier.

27. Volkersen, O., Die Nietkraftverteilung in Zugbeanspruchten Nietverbindungen mit Konstanten Laschenquerschnitten. Luftfahrtforschung, 1938. 15(1/2): p. 4147.

28. Goland, M. and Reissner, S., The Stress in Cemented Joints. J. Appl. Mech, 1944. 11(1): p. 17-27. 
29. da Silva, L.F.M., et al., Analytical models of adhesively bonded joints-Part I:

Literature survey. International Journal of Adhesion and Adhesives, 2009. 29(3): p. 319-330.

30. Ojalvo, I.U. and Eidinoff, H.L., Bond Thickness Effects upon Stresses in SingleLap Adhesive Joints. AIAA Journal, 1978. 16(3): p. 204-211.

31. Hart-Smith, L.J., Adhesive-Bonded Single-Lap Joints, NASA.CR 112236. 1973.

32. Hart-Smith, L.J., Adhesive-Bonded Double-Lap Joints. NASA. CR-112235. 1973.

33. Hart-Smith, L.J., Adhesive-Bonded Scarf and Stepped-Lap Joints. NASACR112237. 1973.

34. da Silva, L.F.M., et al., Analytical models of adhesively bonded joints-Part II: Comparative study. International Journal of Adhesion and Adhesives, 2009. 29(3): p. 331-341.

35. Skorupa, A. and Skorupa, M., Riveted Lap Joints in Aircraft Fuselage: Design, Analysis and Properties. 2012: Springer Netherlands.

36. Tate, M.B., Preliminary investigation of the loads carried by individual bolts in bolted joints. 1946, National advisory committee for aeronautics. Technical note No. 1051 .

37. Barrois, W., Stresses and displacements due to load transfer by fasteners in structural assemblies. Engineering Fracture Mechanics, 1978. 10(1): p. 115-176.

38. Huth, H., Influence of fastener flexibility on the prediction of load transfer and fatigue life for multiple-row joints. Fatigue in mechanically fastened composite and metallic joints, ASTM STP, 1986. 927: p. 221-250. 
39. Swift, T. Development of the fail-safe design features of the DC-10. in Damage Tolerance in Aircraft Structures, ASTM STP 486. 1971. ASTM International.

40. De Jong, T., Stresses Around Pin-Loaded Holes in Elastically Orthotropic or Isotropic Plates. Journal of Composite Materials, 1977. 11(3): p. 313-331.

41. Zhang, K.-D. and Ueng, C.E.S., Stresses Around a Pin-loaded Hole in Orthotropic Plates. Journal of Composite Materials, 1984. 18(5): p. 432-446.

42. Hyer, M.W., Klang, E.C., and Cooper, D.E., The Effects of Pin Elasticity, Clearance, and Friction on the Stresses in a Pin-Loaded Orthotropic Plate. Journal of Composite Materials, 1987. 21(3): p. 190-206.

43. Aluko, O. and Whitworth, H.A., Analysis of stress distribution around pin loaded holes in orthotropic plates. Composite Structures, 2008. 86(4): p. 308-313.

44. Sebaey, T., Bolted Joints in Laminated Composites. 2013: Lap Lambert Academic Publishing GmbH KG.

45. Godwin, E.W. and Matthews, F.L., A review of the strength of joints in fibrereinforced plastics: Part 1. Mechanically fastened joints. Composites, 1980. 11(3): p. 155-160.

46. Military Handbook-MIL-HDBK-17-3F: Composite Materials Handbook, Volume 3 - Polymer Matrix Composites Materials Usage, Design, and Analysis. U.S. Department of Defense.

47. Hart-Smith, L.J., Bonded-bolted composite joints. Journal of Aircraft, 1985. 22(11): p. 993-1000. 
48. Yamaguchi, Y. and Amano, S., Mechanical behaviour of a combined joint composed of mechanical fastening and adhesive bonding. International Journal of Adhesion and Adhesives, 1985. 5(4): p. 193-199.

49. Sawa, T. and Kobayashi, T., The Strength of Joints Combining an Adhesive with a Bolt. The Journal of Adhesion, 1988. 25(4): p. 269-280.

50. Gómez, S., Oñoro, J., and Pecharromán, J., A simple mechanical model of a structural hybrid adhesive/riveted single lap joint. International Journal of Adhesion and Adhesives, 2007. 27(4): p. 263-267.

51. Paroissien, E., et al., Analytical Two-Dimensional Model of a Hybrid (Bolted/Bonded) Single-Lap Joint. Journal of Aircraft, 2007. 44(2): p. 573-582.

52. Erkan, O., et al., Analysis of Bolted-Bonded Composite Lap Joints, in 48th AIAA/ASME/ASCE/AHS/ASC Structures, Structural Dynamics, and Materials Conference. 2007, American Institute of Aeronautics and Astronautics.

53. Bois, C., et al., An analytical model for the strength prediction of hybrid (bolted/bonded) composite joints. Composite Structures, 2013. 97: p. 252-260.

54. Melinda, S., An experimental investigation of composite bonded and/or bolted repairs using single lap joint designs, in 38th Structures, Structural Dynamics, and Materials Conference. 1997, American Institute of Aeronautics and Astronautics.

55. Chan, W.S. and Vedhagiri, S., Analysis of Composite Bonded/Bolted Joints Used in Repairing. Journal of Composite Materials, 2001. 35(12): p. 1045-1061. 
56. Fu, M. and Mallick, P.K., Fatigue of hybrid (adhesive/bolted) joints in SRIM composites. International Journal of Adhesion and Adhesives, 2001. 21(2): p. 145-159.

57. Lees, J.M. and Makarov, G., Mechanical/bonded joints for advanced composite structures. Proceedings of the Institution of Civil Engineers - Structures and Buildings, 2004. 157(1): p. 91-97.

58. Kelly, G., Load transfer in hybrid (bonded/bolted) composite single-lap joints. Composite Structures, 2005. 69(1): p. 35-43.

59. Kelly, G., Quasi-static strength and fatigue life of hybrid (bonded/bolted) composite single-lap joints. Composite Structures, 2006. 72(1): p. 119-129.

60. Ganji, N., Parametric Study of Load Transfer in Two-bolted Single Lap Hybrid (bonded/bolted) Shear Joints. 2007, Wichita State University, MScThesis.

61. Kweon, J.-H., et al., Failure of carbon composite-to-aluminum joints with combined mechanical fastening and adhesive bonding. Composite Structures, 2006. 75(1-4): p. 192-198.

62. Matsuzaki, R., Shibata, M., and Todoroki, A., Improving performance of GFRP/aluminum single lap joints using bolted/co-cured hybrid method. Composites Part A: Applied Science and Manufacturing, 2008. 39(2): p. 154-163.

63. Kumar, B., et al., Adding Additional Load Paths in a Bonded/Bolted Hybrid Joint. Journal of Aircraft, 2010. 47(5): p. 1593-1598.

64. Moroni, F., Pirondi, A., and Kleiner, F., Experimental analysis and comparison of the strength of simple and hybrid structural joints. International Journal of Adhesion and Adhesives, 2010. 30(5): p. 367-379. 
65. Lee, Y.-H., et al., Failure load evaluation and prediction of hybrid composite double lap joints. Composite Structures, 2010. 92(12): p. 2916-2926.

66. Backman, D., Li, G., and Sears, T., Determining the Strain Distribution in Bonded and Bolted/Bonded Composite Butt Joints Using the Digital Image Correlation Technique and Finite Element Methods, in Optical Measurements, Modeling, and Metrology, Volume 5: Proceedings of the 2011 Annual Conference on Experimental and Applied Mechanics, T. Proulx, Editor. 2011, Springer New York: New York, NY.p. 401-406.

67. Hai, N.D. and Mutsuyoshi, H., Structural behavior of double-lap joints of steel splice plates bolted/bonded to pultruded hybrid CFRP/GFRP laminates. Construction and Building Materials, 2012. 30: p. 347-359.

68. Li, G., et al., Static strength of a composite butt joint configuration with different attachments. Composite Structures, 2012. 94(5): p. 1736-1744.

69. Di Franco, G., Fratini, L., and Pasta, A., Analysis of the mechanical performance of hybrid (SPR/bonded) single-lap joints between CFRP panels and aluminum blanks. International Journal of Adhesion and Adhesives, 2013. 41: p. 24-32.

70. Fiore, V., et al., Effect of curing time on the performances of hybrid/mixed joints. Composites Part B: Engineering, 2013. 45(1): p. 911-918.

71. Hoang-Ngoc, C.-T. and Paroissien, E., Simulation of single-lap bonded and hybrid (bolted/bonded) joints with flexible adhesive. International Journal of Adhesion and Adhesives, 2010. 30(3): p. 117-129. 
72. Bodjona, K. and Lessard, L., Load sharing in single-lap bonded/bolted composite joints. Part II: Global sensitivity analysis. Composite Structures, 2015. 129: p. 276-283.

73. Bodjona, K., et al., Load sharing in single-lap bonded/bolted composite joints. Part I: Model development and validation. Composite Structures, 2015. 129: $\mathrm{p}$. $268-275$.

74. Sawyer, J.W. and Rothgeb, T.M., An instrumented fastener for shear force measurements in joints. Experimental Mechanics, 1994. 34(1): p. 16-22.

75. Starikov, R., Mechanically fastened joints: critical testing of single overlap joints. FOI Swedish Defence Research Agency, Scientific Report FOI, 2002.

76. McCarthy, M.A., Lawlor, V.P., and Stanley, W.F., An Experimental Study of Bolt-Hole Clearance Effects in Single-lap, Multibolt Composite Joints. Journal of Composite Materials, 2005. 39(9): p. 799-825.

77. McCarthy, M., et al., Measurement of Bolt Pre-load in Torqued Composite Joints. Strain, 2005. 41(3): p. 109-112.

78. Wymyslowski, A., et al., Molecular Modeling and Multiscaling Issues for Electronic Material Applications. 2014: Springer International Publishing.

79. Dugdale, D.S., Yielding of steel sheets containing slits. Journal of the Mechanics and Physics of Solids, 1960. 8(2): p. 100-104.

80. Barenblatt, G.I., The Mathematical Theory of Equilibrium Cracks in Brittle Fracture, in Advances in Applied Mechanics. 1962, Elsevier. p. 55-129. 
81. Katnam, K.B., et al., The Static Failure of Adhesively Bonded Metal Laminate Structures: A Cohesive Zone Approach. Journal of Adhesion Science and Technology, 2012. 25(10): p. 1131-1157.

82. https://upload.wikimedia.org/wikipedia/commons/e/e7/Fracture modes_v2.svg. [cited 2016 March, 2016].

83. Abaqus 6.13 Analysis User's Guide. Dassault Systemes Simulia Corp. Providence, RI, USA.

84. da Silva, L.F.M. and Campilho, R.D.S.G., Advances in Numerical Modeling of Adhesive Joints. 2011: Springer.

85. Falk, M., L., Needleman, A., and Rice, J., R., A critical evaluation of cohesive zone models of dynamic fractur. Vol. 11. 2001. Pr5-43-Pr5-50.

86. Moës, N. and Belytschko, T., Extended finite element method for cohesive crack growth. Engineering Fracture Mechanics, 2002. 69(7): p. 813-833.

87. Turon, A., et al., An Engineering Solution for using Coarse Meshes in the Simulation of Delamination With Cohesive Zone Models. 2005, NASA. .

88. Liao, L., Huang, C., and Sawa, T., Effect of adhesive thickness, adhesive type and scarf angle on the mechanical properties of scarf adhesive joints. International Journal of Solids and Structures, 2013. 50(25-26): p. 4333-4340.

89. de Moura, M.F.S.F., Application of Cohesive Zone Modeling to Composite Bonded Repairs. The Journal of Adhesion, 2015. 91(1-2): p. 71-94.

90. Gustafson, P.A. and Waas, A.M., The influence of adhesive constitutive parameters in cohesive zone finite element models of adhesively bonded joints. International Journal of Solids and Structures, 2009. 46(10): p. 2201-2215. 
91. Campilho, R.D.S.G., et al., Modelling of Single-Lap Joints Using Cohesive Zone Models: Effect of the Cohesive Parameters on the Output of the Simulations. The Journal of Adhesion, 2012. 88(4-6): p. 513-533.

92. Neto, J.A.B.P., Campilho, R.D.S.G., and da Silva, L.F.M., Parametric study of adhesive joints with composites. International Journal of Adhesion and Adhesives, 2012. 37: p. 96-101.

93. da Silva, L.F.M., et al., Effect of Adhesive Type and Thickness on the Lap Shear Strength. The Journal of Adhesion, 2006. 82(11): p. 1091-1115.

94. Campilho, R.D.S.G., de Moura, M.F.S.F., and Domingues, J.J.M.S., Using a cohesive damage model to predict the tensile behaviour of CFRP single-strap repairs. International Journal of Solids and Structures, 2008. 45(5): p. 1497-1512.

95. Sadowski, T., Golewski, P., and Zarzeka-Raczkowska, E., Damage and failure processes of hybrid joints: Adhesive bonded aluminium plates reinforced by rivets. Computational Materials Science, 2011. 50(4): p. 1256-1262.

96. Campilho, R.D.S.G., et al., Optimization study of hybrid spot-welded/bonded single-lap joints. International Journal of Adhesion and Adhesives, 2012. 37: p. 86-95.

97. Hinton, M. and Kaddour, A., The background to Part B of the Second WorldWide Failure Exercise: Evaluation of theories for predicting failure in polymer composite laminates under three-dimensional states of stress. Journal of Composite Materials, 2013. 47(6-7): p. 643-652.

98. Hashin, Z., Failure criteria for unidirectional fiber composites. Journal of applied mechanics, 1980. 47(2): p. 329-334. 
99. Garnich, M.R. and Akula, V.M.K., Review of Degradation Models for Progressive Failure Analysis of Fiber Reinforced Polymer Composites. Applied Mechanics Reviews, 2009. 62(1): p. 010801.

100. Chang, F.-K. and Chang, K.-Y., A progressive damage model for laminated composites containing stress concentrations. Journal of composite materials, 1987. 21(9): p. 834-855.

101. Tan, S.C., A Progressive Failure Model for Composite Laminates Containing Openings. Journal of Composite Materials, 1991. 25(5): p. 556-577.

102. Camanho, P.P. and Matthews, F.L., A Progressive Damage Model for Mechanically Fastened Joints in Composite Laminates. Journal of Composite Materials, 1999. 33(24): p. 2248-2280.

103. Tserpes, K.I., Papanikos, P., and Kermanidis, T.H., A three-dimensional progressive damage model for bolted joints in composite laminates subjected to tensile loading. Fatigue \& Fracture of Engineering Materials \& Structures, 2001. 24(10): p. 663-675.

104. McCarthy, C.T., McCarthy, M.A., and Lawlor, V.P., Progressive damage analysis of multi-bolt composite joints with variable bolt-hole clearances. Composites Part B: Engineering, 2005. 36(4): p. 290-305.

105. Bing, P., et al., Two-dimensional digital image correlation for in-plane displacement and strain measurement: a review. Measurement Science and Technology, 2009. 20(6): p. 062001. 
106. Zhou, J.-W., et al., Application of Digital Image Correlation to Measurement of Packaging Material Mechanical Properties. Mathematical Problems in Engineering, 2013. 2013: p. 8.

107. Haddadi, H. and Belhabib, S., Use of rigid-body motion for the investigation and estimation of the measurement errors related to digital image correlation technique. Optics and Lasers in Engineering, 2008. 46(2): p. 185-196.

108. Colavito, K., et al., Digital Image Correlation for Adhesive Strains in Bonded Composite Lap Joints, in 49th AIAA/ASME/ASCE/AHS/ASC Structures, Structural Dynamics, and Materials Conference, . 2008, American Institute of Aeronautics and Astronautics.

109. Colavito, K., et al., Refinements in Digital Image Correlation Technique to Extract Adhesive Strains in Lap Joints, in 50th AIAA/ASME/ASCE/AHS/ASC Structures, Structural Dynamics, and Materials Conference. 2009, American Institute of Aeronautics and Astronautics.

110. Kumar, R.V., Bhat, M., and Murthy, C., Experimental analysis of composite single-lap joints using digital image correlation and comparison with theoretical models. Journal of Reinforced Plastics and Composites, 2013. 32(23): p. 18581876.

111. Guo, B., et al., Study on the mechanical behavior of adhesive interface by digital image correlation. Science China Physics, Mechanics and Astronomy, 2011. 54(4): p. 574-580. 
112. Katnam, K.B., et al., Tensile strength of two-part epoxy paste adhesives:

Influence of mixing technique and micro-void formation. International Journal of Adhesion and Adhesives, 2011. 31(7): p. 666-673.

113. Crammond, G., Boyd, S.W., and Dulieu-Barton, J.M., Evaluating the localised through-thickness load transfer and damage initiation in a composite joint using digital image correlation. Composites Part A: Applied Science and Manufacturing, 2014. 61: p. 224-234.

114. Lemmen, H.J.K., et al., The power of Digital Image Correlation for detailed elastic-plastic strain measurements, in WSEAS International Conference on ENGINEERING MECHANICS, STRUCTURES, ENGINEERING GEOLOGY (EMESEG '08). July 22-24, 2008: 2008. Heraklion, Crete Island, Greece.

115. Van Blitterswyk J., et al. Application of Digital Image Correlation to the Thick Adherend Shear Test. in Society of Experimental Mechanics XIII International Congress. 2016. Orlando, Florida USA. Submitted.

116. Ross, P.J., Taguchi Techniques for Quality Engineering: Loss Function, Orthogonal Experiments, Parameter and Tolerance Design. 1996: McGraw-Hill.

117. Montgomery, D.C., Design and Analysis of Experiments, 8th Edition. 2012: John Wiley \& Sons, Incorporated.

118. Mathews, P.G., 4.2 Design of Experiments: Definition, Scope, and Motivation, in Design of Experiments with MINITAB. American Society for Quality (ASQ).

119. NIST/SEMATECH e-Handbook of Statistical Methods. http://www.itl.nist.gov/div898/handbook/.

120. CYCOM® 5320-1 Epoxy Resin System. Cytec Engineered Materials. 
121. Hi-Lite St Pin HST12. LISI Aerospace. http://www.lisiaerospace.com/products/fasteners/externally-threaded/pin/Pages/hi-lite.aspx.

122. ASTM Standard D3165-07, "Standard Test Method for Strength Properties of Adhesives in Shear by Tension Loading of Single-Lap-Joint Laminated Assemblies, ” ASTM International, West Conshohocken, PA, 2007, DOI:10.1520/D3165-07, www.astm.org.

123. ASTM Standard D5961/D5961M-10, "Standard Test Method for Bearing Response of Polymer Matrix Composite Laminates,". ASTM International, West Conshohocken, PA, 2007, DOI:10.1520/D5961_D5961M-10., www.astm.org.

124. Camanho, P., Application of numerical methods to the strength prediction of mechanically fastened joints in composite laminates. 1999, Imperial College London (University of London), PhD Thesis.

125. Winzen, A., Simulation of stringer stiffened CFRP panels in consideration of skin-stringer separation. 2006, Aachen University of Applied Sciences.

126. Maligno, A.R., Finite element investigations on the microstructure of composite materials. 2008, University of Nottingham, PhD Thesis.

127. Voyiadjis, G.Z. and Kattan, P.I., Mechanics of Composite Materials with MATLAB. 2005: Springer Berlin Heidelberg.

128. Zoghi, M., The International Handbook of FRP Composites in Civil Engineering. 2013: Taylor \& Francis.

129. Lopez-Cruz, P., et al., Influence of adhesive properties on load transfer in hybrid bolted/bonded composite joints. Manuscript in review, 2016. 
130. Gali, S., Dolev, E., and Ishai, O., An effective stress/strain concept in the mechanical characterization of structural adhesive bonding. International Journal of Adhesion and Adhesives, 1981. 1(3): p. 135-140.

131. Jeandrau, J.P., Analysis and design data for adhesively bonded joints. International Journal of Adhesion and Adhesives, 1991. 11(2): p. 71-79.

132. De Moura, M.F.S.F., et al., Cohesive and continuum mixed-mode damage models applied to the simulation of the mechanical behaviour of bonded joints. International Journal of Adhesion and Adhesives, 2008. 28(8): p. 419-426.

133. Ridha, M., Tan, V.B.C., and Tay, T.E., Traction-separation laws for progressive failure of bonded scarf repair of composite panel. Composite Structures, 2011. 93(4): p. 1239-1245.

134. Campilho, R.D.S.G., et al., Modelling adhesive joints with cohesive zone models: effect of the cohesive law shape of the adhesive layer. International Journal of Adhesion and Adhesives, 2013. 44: p. 48-56.

135. Dzenis, Y.A., Durability and Intelligent nondestructive Evaluation of adhesive composite joints. 2000, Department of Engineering Mechanics, University of Nebraska.

136. Mall, S.a.J., W.S., Characterization of mode I and mixed-moded failure of adhesive bonds between composite adherends. 1985, NASA.

137. Yan, C., et al., Effects of substrate materials on fracture toughness measurement in adhesive joints. International Journal of Mechanical Sciences, 2001. 43(9): p. 2091-2102. 
138. Donough, M., et al., Critical assessment of failure criteria for adhesively bonded composite repair design, in International Council of the Aeronautical Sciences,

A.D. Prof I. Grant, Editor. 2012, ICAS: Brisbane, Australia. p. 1-11.

139. Tomczyk, A.J., MTS Adhesives Project 2: Report No. 5: Summary Report: Test Methods for Adhesive Fracture Properties. 1997.

140. Wei, X. and Yueguang, W., Influence of adhesive thickness on local interface fracture and overall strength of metallic adhesive bonding structures. International Journal of Adhesion and Adhesives, 2013. 40: p. 158-167.

141. CRP Meccanica. Titanium Ti-6Al-4V (Grade 5), Annealed AMS 4928 AMS 4911. September, 2013]; Available from: http://www.crpmeccanica.com/PDF/titaniumti-6al-4v-annealed-ams-4928.pdf. 


\section{Appendix A: Procedure to Adjust the Stroke Displacement due to the}

\section{Test Machine Compliance}

A reference high stiffness composite coupon taken from a bonded strip without a hole or notch was tested using exactly the same conditions as the tested joint coupons to measure the test machine compliance. The dimensions were $231 \mathrm{~mm} \times 28.575 \mathrm{~mm} \times$ $11.46 \mathrm{~mm}$. After post-processing the results, the displacement due to the test machine compliance was computed as $13.1 \mathrm{~mm}$, and subtracted from the coupons to be adjusted. Figure A.1 shows the results using the treatment abcde_04, and Figure A.2 shows the adjusted load versus stroke for the tested coupon.

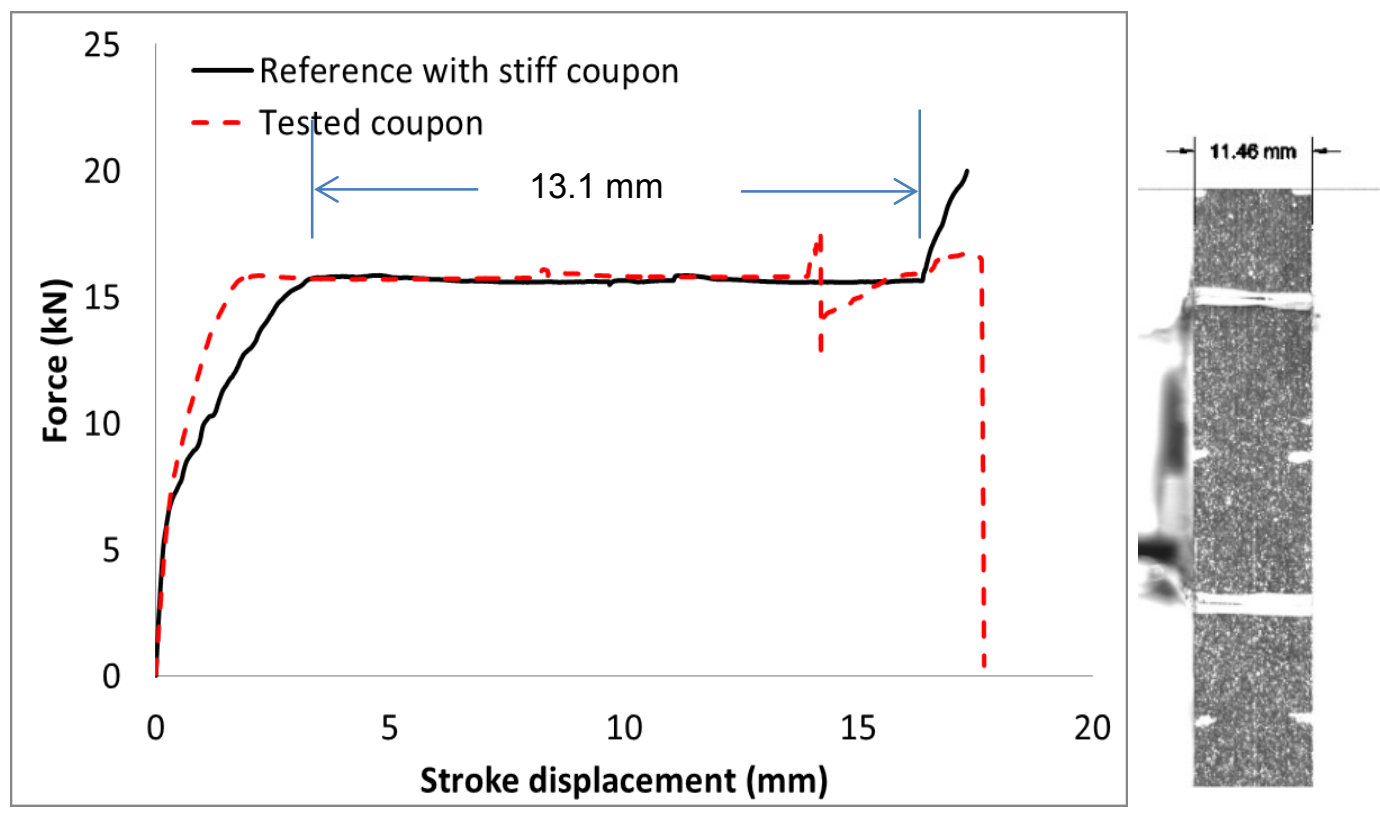

Figure A.1 Test machine compliance displacement 


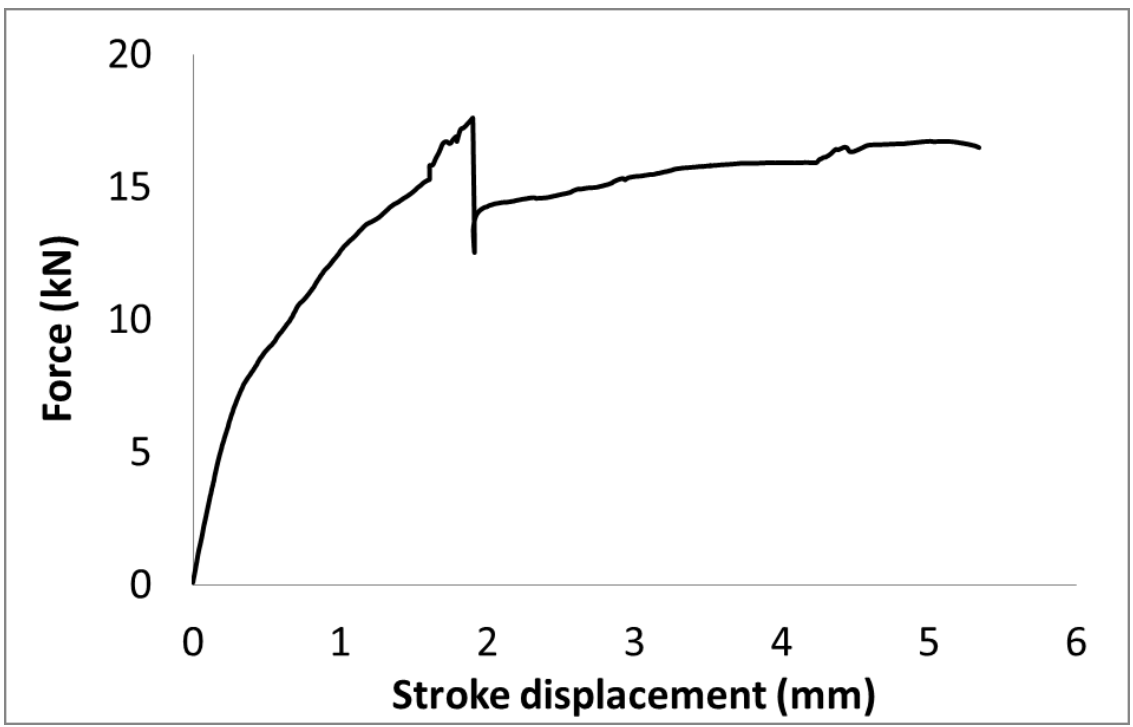

Figure A.2 Adjusted force versus displacement 


\section{Appendix B: Procedure to Measure Shear Strain at the Adhesive Edge}

The procedure is based on that described in [115].

Firstly, the original position of the three points are labeled as $l_{o}, r_{o}$ and $b_{o}$ respectively. After an applied force, the specimen deforms and the region rotates by an angle $\theta$. The extraction points move to the deformed position labeled $l^{\prime}, r^{\prime}$ and $b^{\prime}$ respectively as shown in Figure B.1.

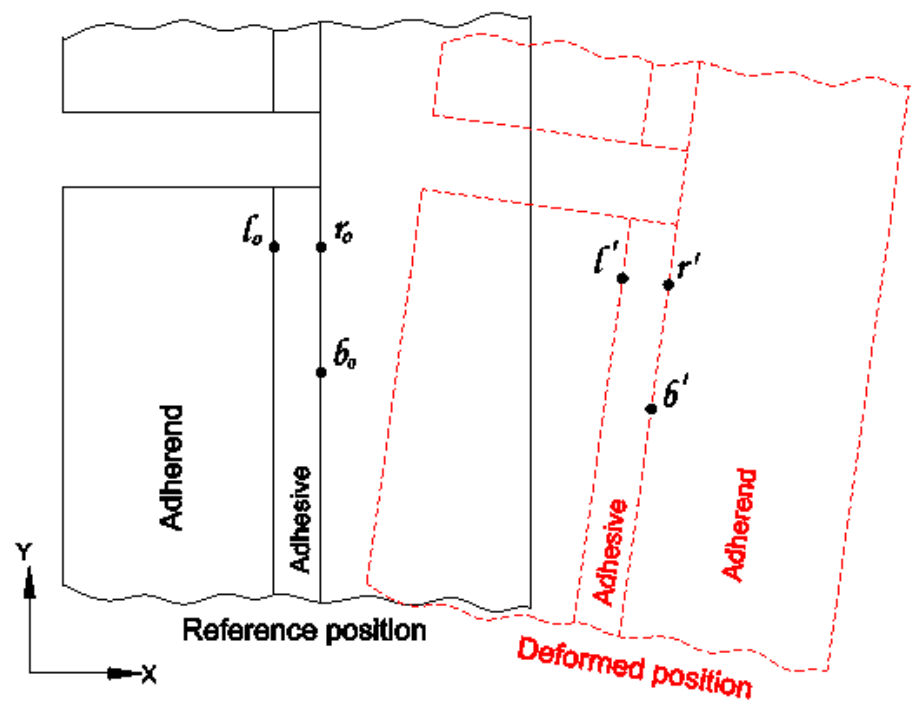

Figure B.1 Adhesive shear strain analysis. Points before and after applied force

The relative shear displacement is imbedded in this motion, with final deformed positions labeled $l,{ }^{\prime}, r$ ', and $b$ "' as shown in Figure B.2. 

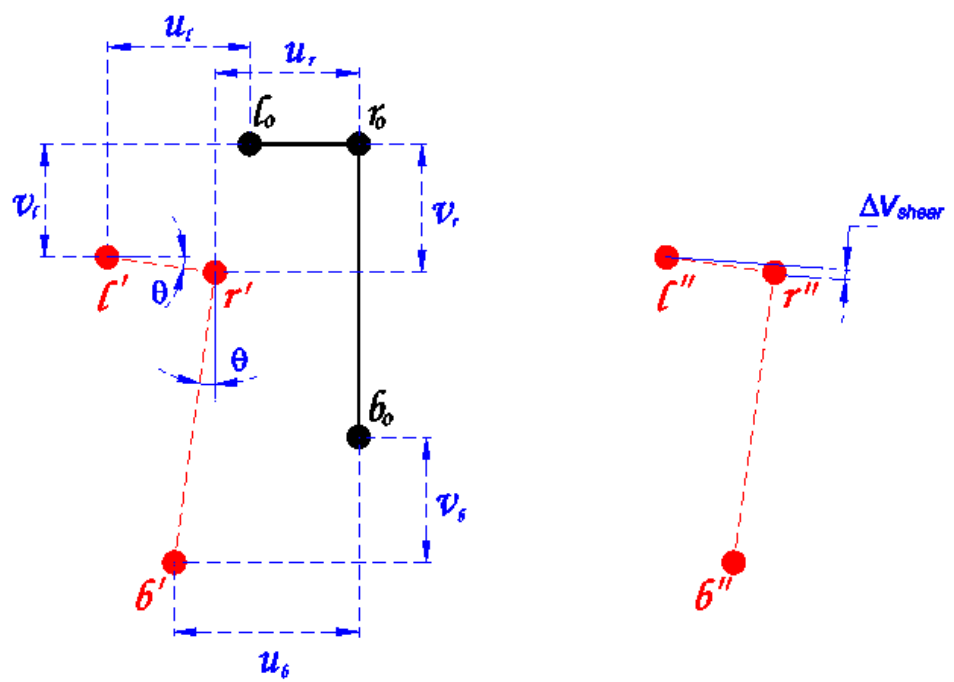

Figure B.2 Adhesive shear strain analysis. Final position of points $I, r$, and $b$

With the relative orientation of the points known, the angle of rotation of the reference frame is calculated as follows:

$$
\theta=\tan ^{-1}\left(u_{b}-u_{r} / h\right)
$$

The procedure is based in the assumption that the relative displacements in the $\mathrm{x}$ direction between the points $l$ and $r$ are negligible. The resultant relative displacement is that produced by pure shear deformation in the adhesive:

$$
\Delta v_{\text {resultant }}=\Delta v_{D I C}-l \tan \theta
$$

Where:

$$
\Delta v_{D I C}=v_{r}-v_{l}
$$

The shear strain $(\gamma)$ is computed using the relative displacement in the adhesive, divided by the adhesive thickness, $t$.

$$
\gamma=\frac{\Delta v_{\text {resultant }}}{t}
$$




\section{Implementation using DIC images}

The following measurement corresponds to the coupon ace_rep_01. The shear strain was measured at $1 \mathrm{~mm}$ distance from the notch and the positions of point $l_{o}, r_{o}$ and $b_{o}$ are in the interface between the adhesive and the adherend as shown in Figure B.3. The reference positions are extracted from the DIC software. After an applied force, the coupon deforms as shown in Figure B.4.

The displacements $u$ and $v$ are extracted from each point, as shown in table B.1. The calculations are performed using the equations B. 1 to B.4 and the shear strain is tabulated (Table B2) and plotted versus the applied force as shown in Figure B.5

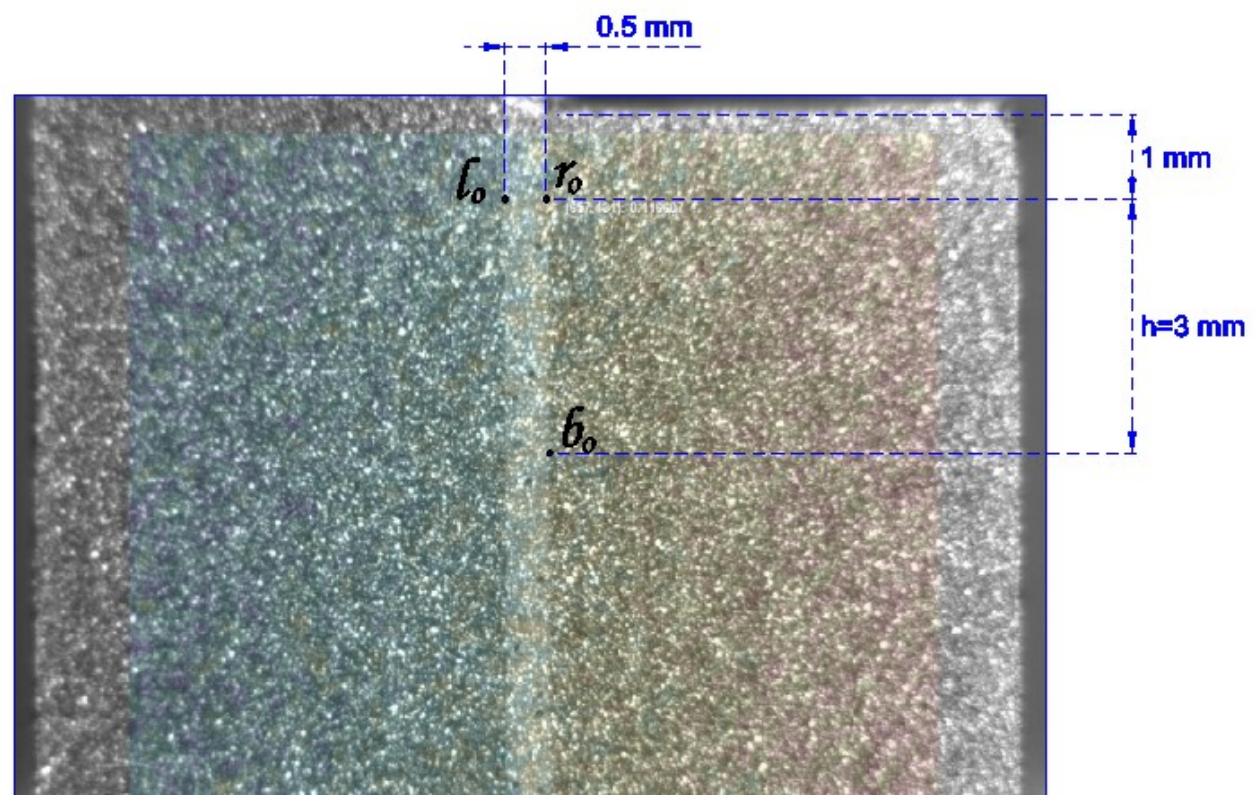

Figure B.3 Adhesive shear strain measurement. Initial image and reference points 


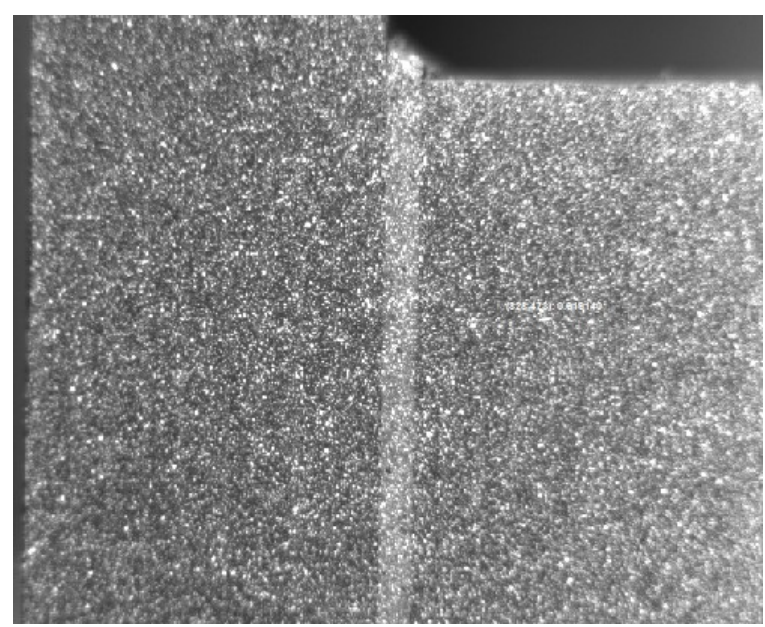

Figure B.4 Adhesive shear strain measurement. Deformed image number 142, time $=70.5$ sec, applied force $15.03 \mathrm{kN}$

Table B.1 Extracted displacements from the DIC software

\begin{tabular}{|l|l|l|l|l|}
\hline Filename & X & Y & U & V \\
\hline BB_aceR1_0002.bmp & 5.777 & 1.264 & 0.001201 & 0.0007544 \\
\hline BB_aceR1_0003.bmp & 5.777 & 1.264 & 0.003915 & 0.002299 \\
\hline BB_aceR1_0004.bmp & 5.777 & 1.264 & 0.01189 & 0.007217 \\
\hline BB_aceR1_0005.bmp & 5.777 & 1.264 & 0.0216 & 0.01318 \\
\hline BB_aceR1_0006.bmp & 5.777 & 1.264 & 0.02799 & 0.0175 \\
\hline BB_aceR1_0007.bmp & 5.777 & 1.264 & 0.03417 & 0.02153 \\
\hline BB_aceR1_0008.bmp & 5.777 & 1.264 & 0.04065 & 0.0249 \\
\hline BB_aceR1_0009.bmp & 5.777 & 1.264 & 0.05237 & 0.03293 \\
\hline BB_aceR1_0010.bmp & 5.777 & 1.264 & 0.06246 & 0.04131 \\
\hline BB_aceR1_0011.bmp & 5.777 & 1.264 & 0.06903 & 0.04423 \\
\hline BB_aceR1_0012.bmp & 5.777 & 1.264 & 0.07333 & 0.04856 \\
\hline BB_aceR1_0013.bmp & 5.777 & 1.264 & 0.08095 & 0.05282 \\
\hline BB_aceR1_0014.bmp & 5.777 & 1.264 & 0.0861 & 0.05634 \\
\hline BB_aceR1_0015.bmp & 5.777 & 1.264 & 0.09092 & 0.05957 \\
\hline BB_aceR1_0016.bmp & 5.777 & 1.264 & 0.09819 & 0.06403 \\
\hline BB_aceR1_0017.bmp & 5.777 & 1.264 & 0.1053 & 0.06941 \\
\hline BB_aceR1_0018.bmp & 5.777 & 1.264 & 0.1131 & 0.07372 \\
\hline BB_aceR1_0019.bmp & 5.777 & 1.264 & 0.1193 & 0.07834 \\
\hline BB_aceR1_0020.bmp & 5.777 & 1.264 & 0.1264 & 0.08197 \\
\hline BB_aceR1_0021.bmp & 5.777 & 1.264 & 0.134 & 0.08768 \\
\hline BB_aceR1_0022.bmp & 5.777 & 1.264 & 0.1403 & 0.09229 \\
\hline BB_aceR1_0023.bmp & 5.777 & 1.264 & 0.1462 & 0.09623 \\
\hline BB_aceR1_0024.bmp & 5.777 & 1.264 & 0.1542 & 0.1006 \\
\hline BB_aceR1_0025.bmp & 5.777 & 1.264 & 0.1625 & 0.1063 \\
\hline BB_aceR1_0026.bmp & 5.777 & 1.264 & 0.1683 & 0.1103 \\
\hline BB aceR100027.bmp & 5.777 & 1.264 & 0.1731 & 0.1133 \\
\hline & & & & \\
\hline & & & \\
\hline
\end{tabular}


Table B.2 Computed adhesive shear strain

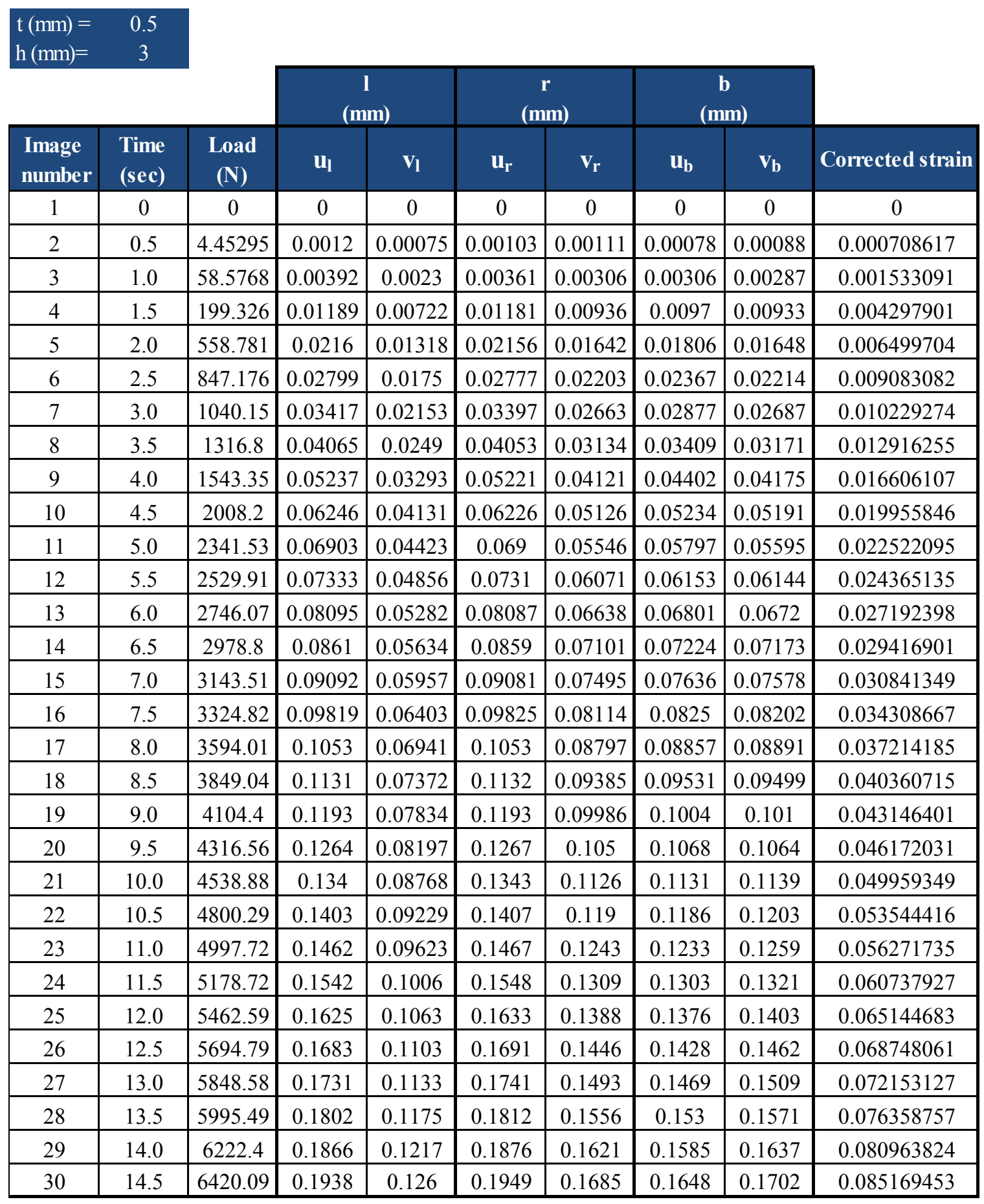




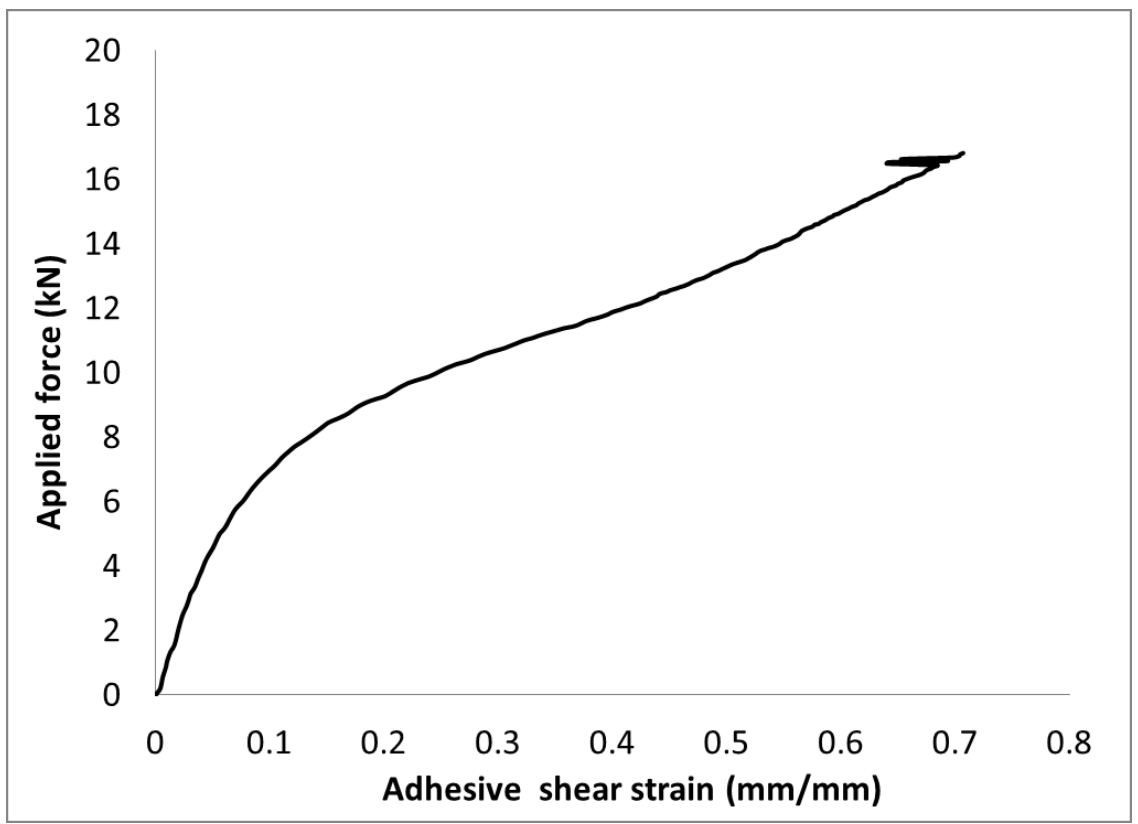

Figure B.5 Shear strain at the adhesive edge 


\section{Appendix C: ANOVA Results}

Table C.1 ANOVA results. First failure point, total strength

\begin{tabular}{|c|c|c|c|c|c|}
\hline \multicolumn{6}{|l|}{ Analysis of Variance } \\
\hline Source & $\mathrm{DF}$ & Adj $S S$ & Adj $\mathrm{MS}$ & F-Value & P-Value \\
\hline Model & 9 & 188.837 & 20.9818 & 13.10 & 0.000 \\
\hline Blocks & 2 & 0.878 & 0.4389 & 0.27 & 0.764 \\
\hline Linear & 5 & 179.343 & 35.8685 & 22.39 & 0.000 \\
\hline Adherend Thk & 1 & 80.909 & 80.9087 & 50.51 & 0.000 \\
\hline Adhesive Modulus & 1 & 81.995 & 81.9946 & 51.19 & 0.000 \\
\hline Adhesive Thk & 1 & 1.524 & 1.5241 & 0.95 & 0.346 \\
\hline Washer Size & 1 & 0.092 & 0.0917 & 0.06 & 0.814 \\
\hline Clearance & 1 & 14.824 & 14.8235 & 9.25 & 0.009 \\
\hline 2-Way Interactions & 2 & 8.616 & 4.3081 & 2.69 & 0.103 \\
\hline Adhesive Modulus*Adhesive Thk & 1 & 4.606 & 4.6059 & 2.88 & 0.112 \\
\hline Adhesive Modulus*Clearance & 1 & 4.010 & 4.0103 & 2.50 & 0.136 \\
\hline Error & 14 & 22.425 & 1.6018 & & \\
\hline Total & 23 & 211.262 & & & \\
\hline
\end{tabular}

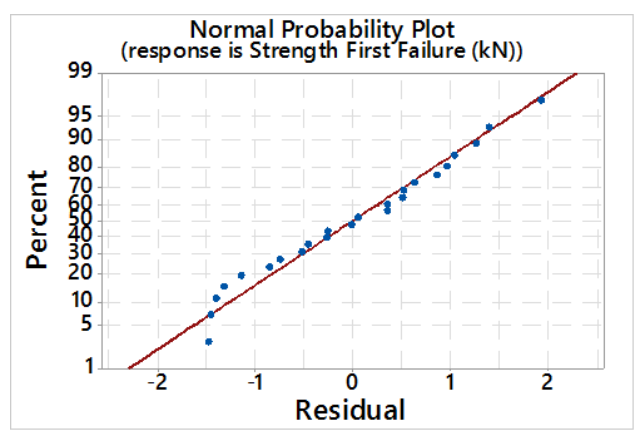

a)

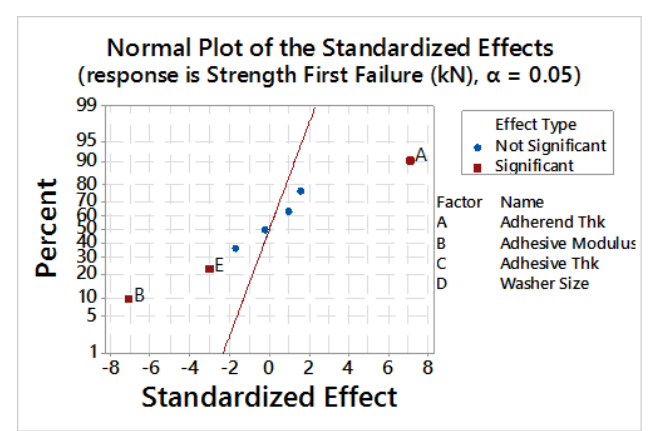

b)

Figure C.1 Strength at first failure point. a) Normal probability plot; b) Normal plot of standardized effect

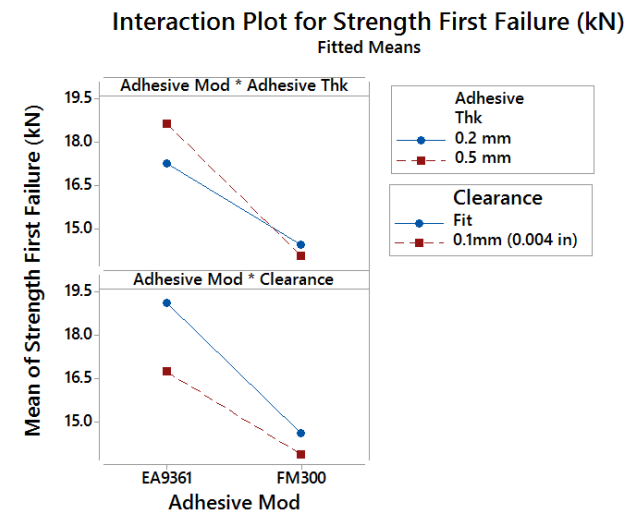

Figure C.2 Interaction plots strength at first failure point 
Table C.2 ANOVA results. First failure point, strength normalized by weight

\begin{tabular}{|c|c|c|c|c|c|}
\hline Source & $\mathrm{DF}$ & Adj $S S$ & Adj MS & F-Value & P-Value \\
\hline Model & 9 & 63654.9 & 7072.8 & 49.93 & 0.000 \\
\hline Blocks & 2 & 44.9 & 22.5 & 0.16 & 0.855 \\
\hline Linear & 5 & 62158.2 & 12431.6 & 87.75 & 0.000 \\
\hline Adherend Thk & 1 & 52278.9 & 52278.9 & 369.03 & 0.000 \\
\hline Adhesive Modulus & 1 & 6529.2 & 6529.2 & 46.09 & 0.000 \\
\hline Adhesive Thk & 1 & 692.6 & 692.6 & 4.89 & 0.044 \\
\hline Washer Size & 1 & 1080.0 & 1080.0 & 7.62 & 0.015 \\
\hline Clearance & 1 & 1577.4 & 1577.4 & 11.13 & 0.005 \\
\hline 2-Way Interactions & 2 & 1451.8 & 725.9 & 5.12 & 0.021 \\
\hline Adhesive Modulus*Adhesive Thk & 1 & 745.7 & 745.7 & 5.26 & 0.038 \\
\hline Adhesive Modulus*Clearance & 1 & 706.1 & 706.1 & 4.98 & 0.042 \\
\hline Error & 14 & 1983.3 & 141.7 & & \\
\hline Total & 23 & 65638.2 & & & \\
\hline
\end{tabular}

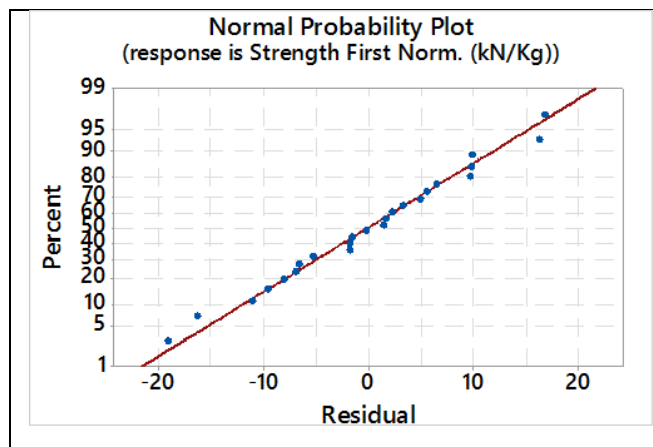

a)

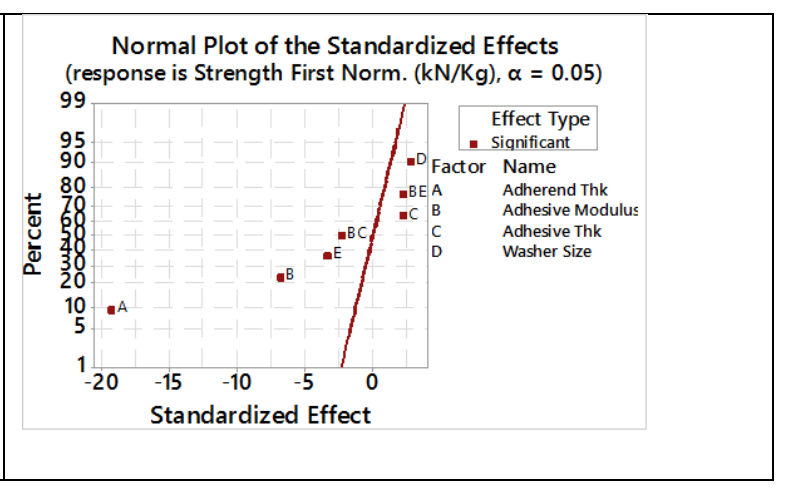

b)

Figure C.3 Strength normalized at first failure point. a) Normal probability plot; b) Normal plot of standardized effect

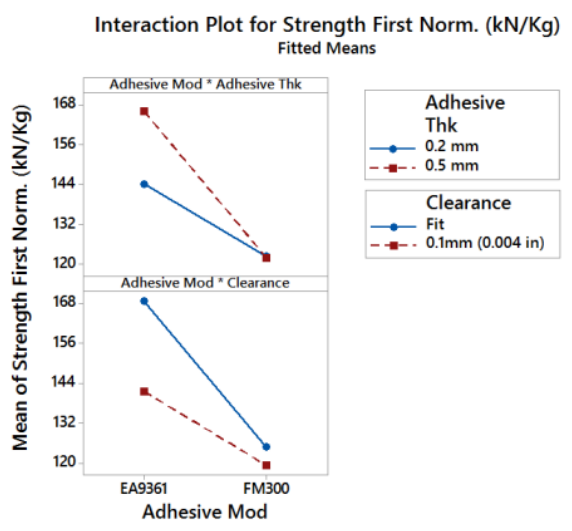

Figure C.4 Interaction plots strength at first failure point. Normalized by weight 
Table C.3 ANOVA results. Maximum strength.

\begin{tabular}{|lrrrrr|}
\hline Analysis of Variance & & & & & \\
& & & & \\
Source & DF & Adj SS & Adj MS & F-Value & P-Value \\
Model & 9 & 105.095 & 11.6772 & 16.43 & 0.000 \\
$\quad$ Blocks & 2 & 0.875 & 0.4375 & 0.62 & 0.554 \\
Linear & 5 & 100.135 & 20.0270 & 28.18 & 0.000 \\
$\quad$ Adherend Thk & 1 & 48.388 & 48.3883 & 68.09 & 0.000 \\
$\quad$ Adhesive Modulus & 1 & 28.763 & 28.7630 & 40.47 & 0.000 \\
$\quad$ Adhesive Thk & 1 & 4.546 & 4.5458 & 6.40 & 0.024 \\
$\quad$ Washer Size & 1 & 4.186 & 4.1865 & 5.89 & 0.029 \\
$\quad$ Clearance & 1 & 14.251 & 14.2514 & 20.05 & 0.001 \\
2-Way Interactions & 2 & 4.085 & 2.0424 & 2.87 & 0.090 \\
$\quad$ Adhesive Modulus*Adhesive Thk & 1 & 0.909 & 0.9085 & 1.28 & 0.277 \\
$\quad$ Adhesive Modulus*Clearance & 1 & 3.176 & 3.1762 & 4.47 & 0.053 \\
Error & 14 & 9.949 & 0.7107 & & \\
Total & 23 & 115.044 & & & \\
\hline
\end{tabular}
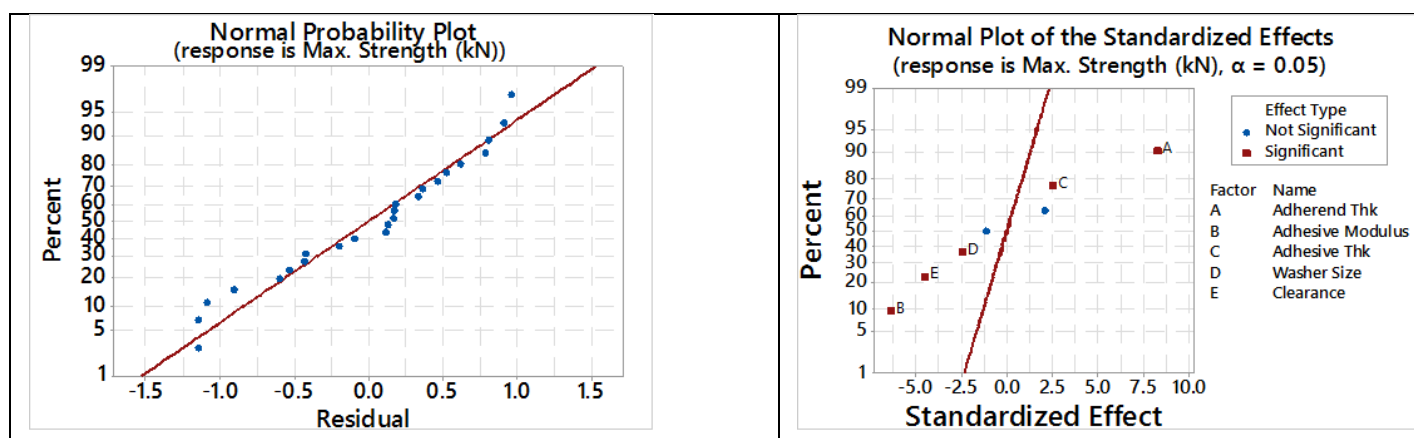

Figure C.5 Maximum strength. a) Normal probability plot; b) Normal plot of standardized effect

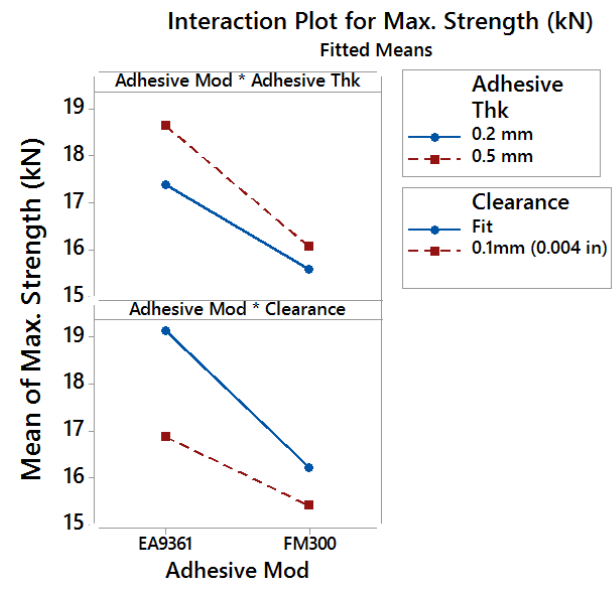

Figure C.6 Interaction plots at maximum strength 
Table C.4 ANOVA results. Maximum strength, normalized by weight

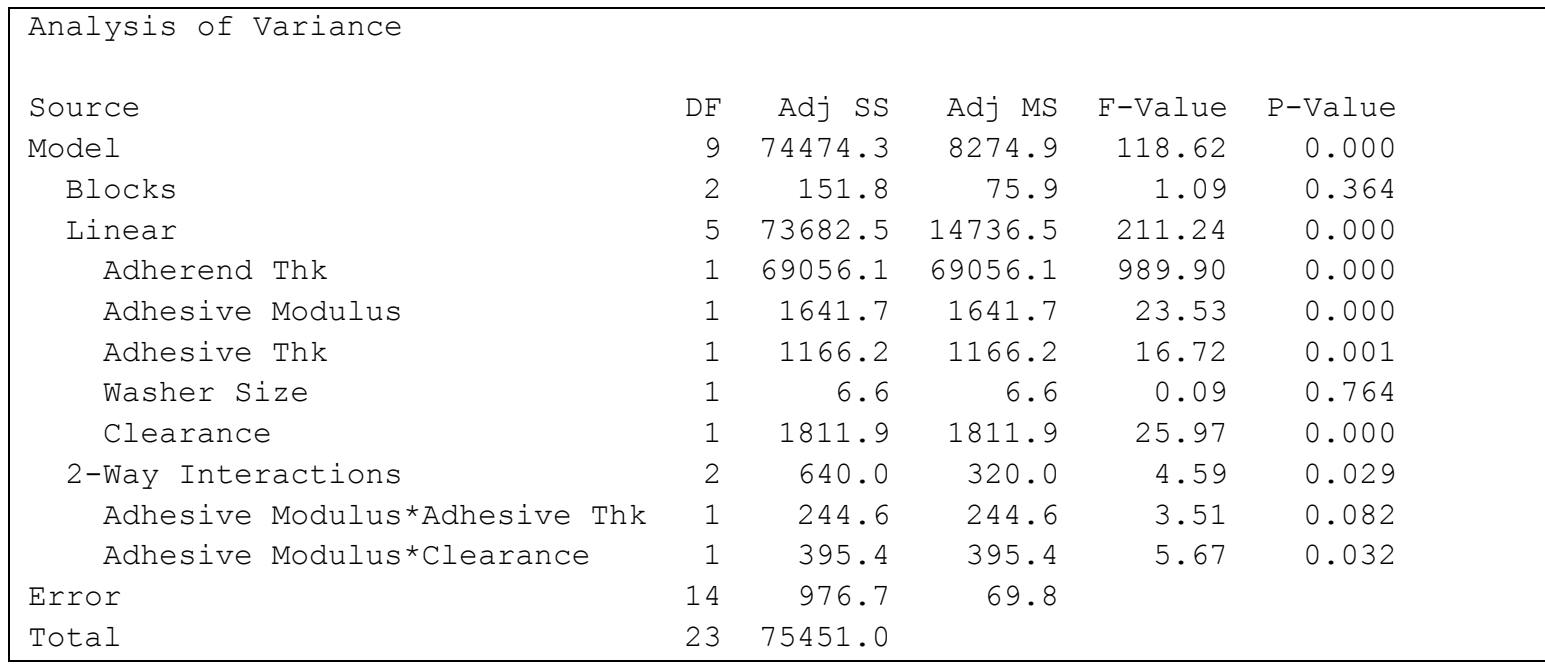
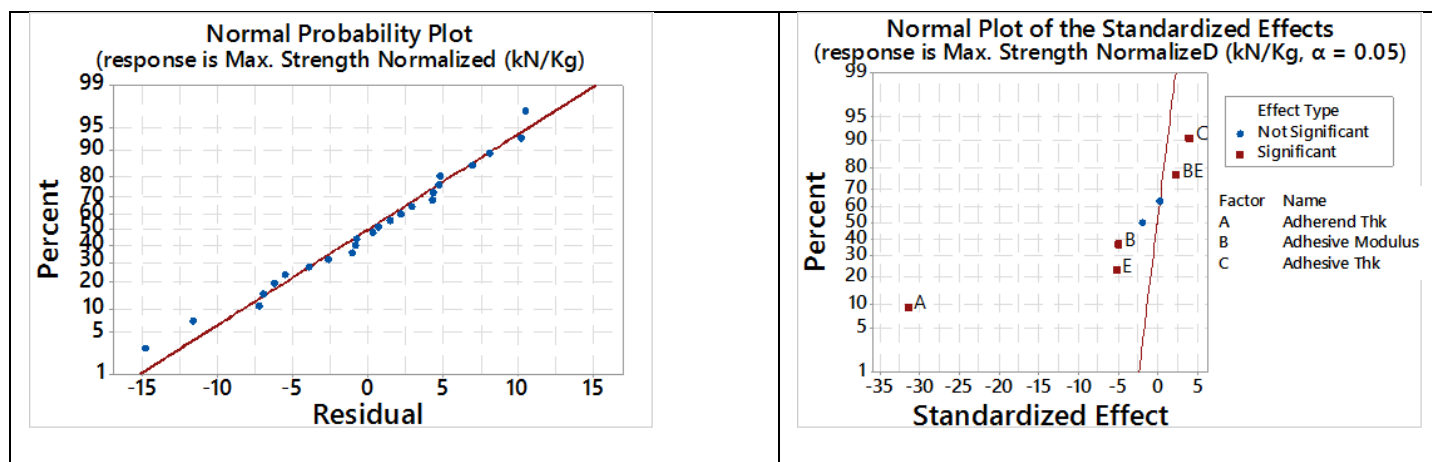

Figure C.7 Maximum strength normalized. a) Normal probability plot; b) Normal plot of standardized effect

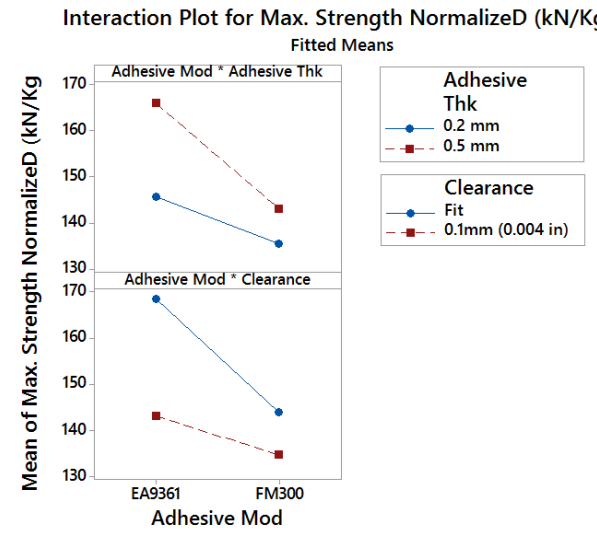

Figure C.8 Interaction plots maximum strength.Normalized by weight 


\section{Appendix D: FORTRAN USDFLD Subroutine.}

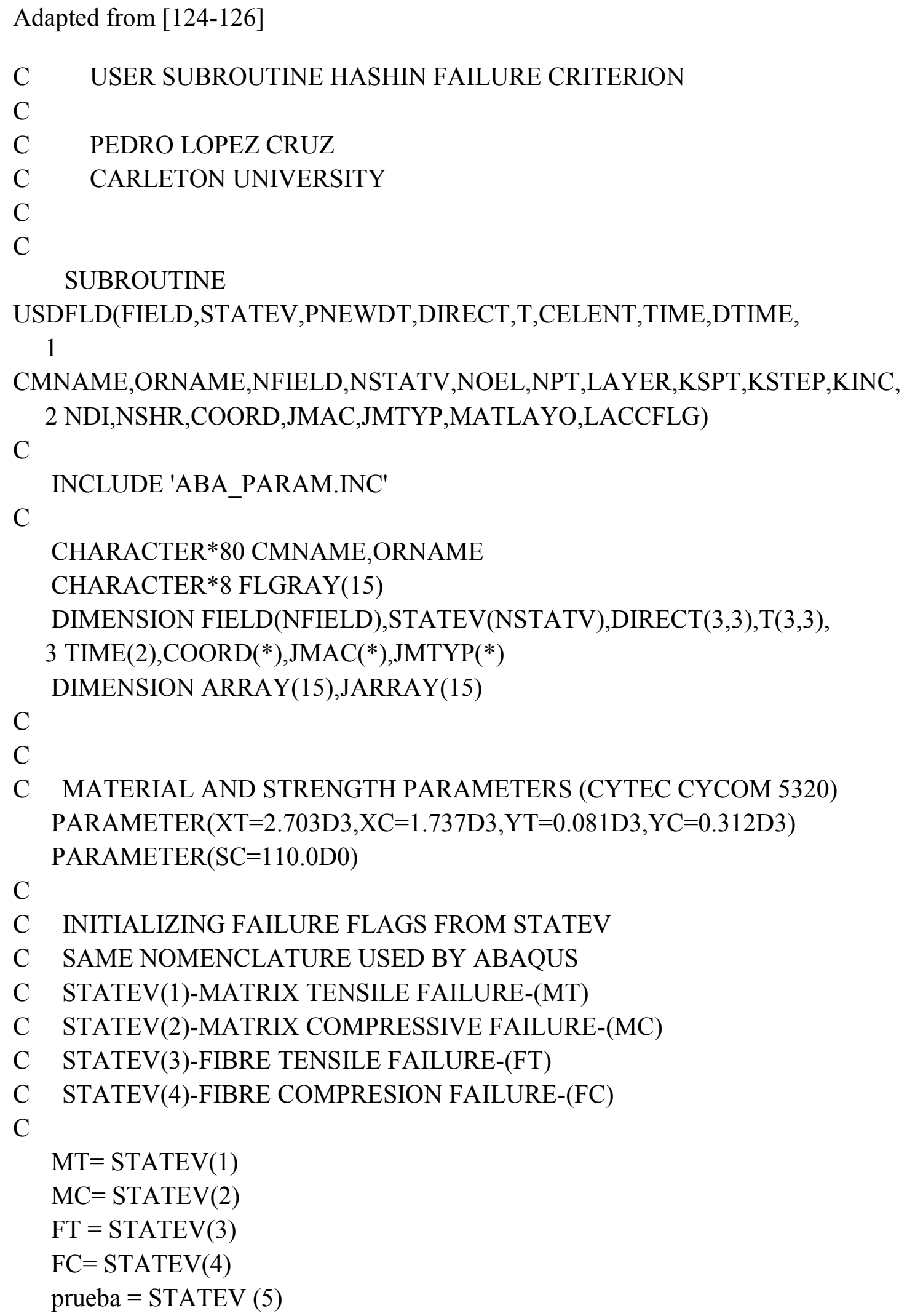




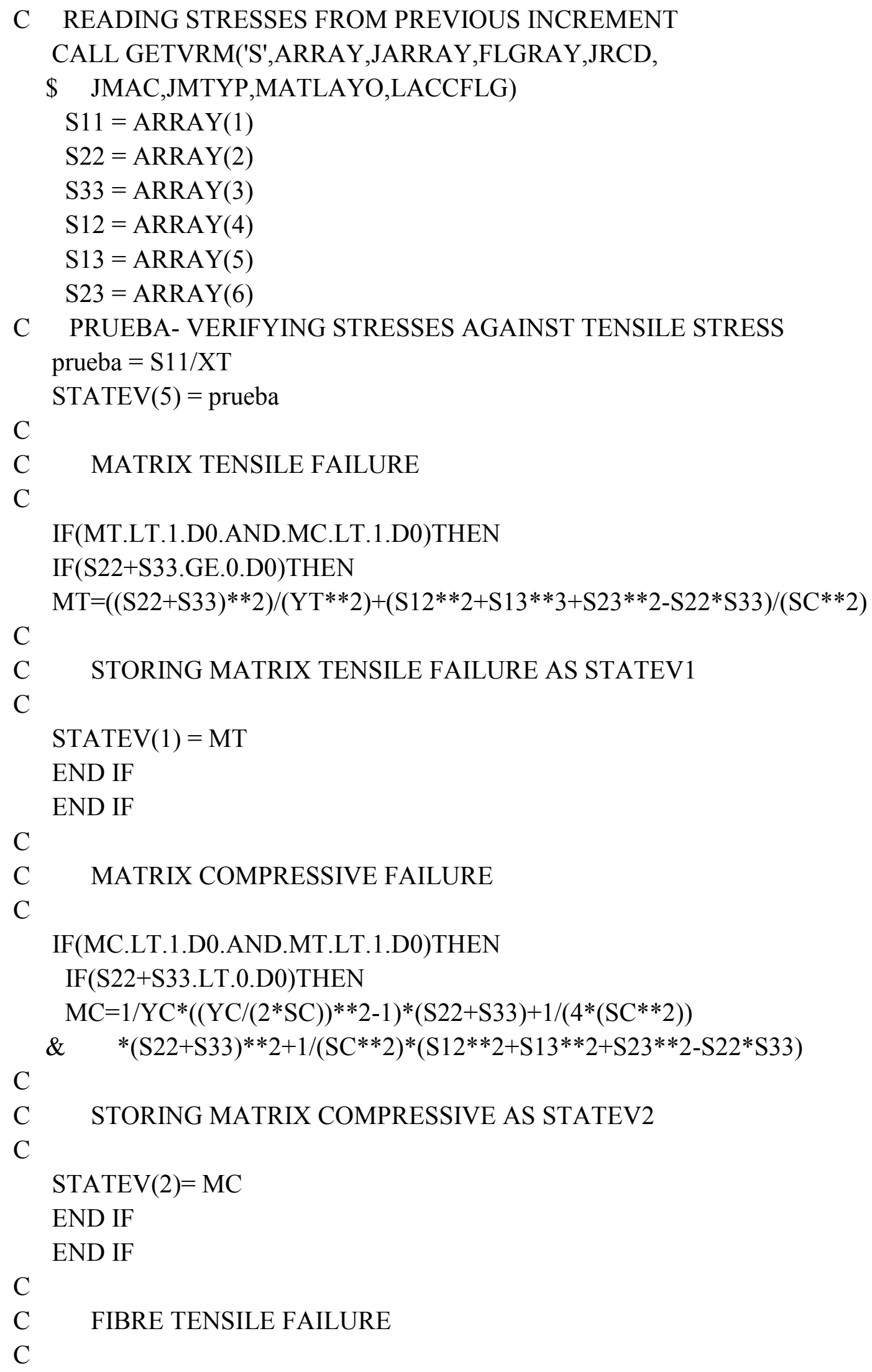




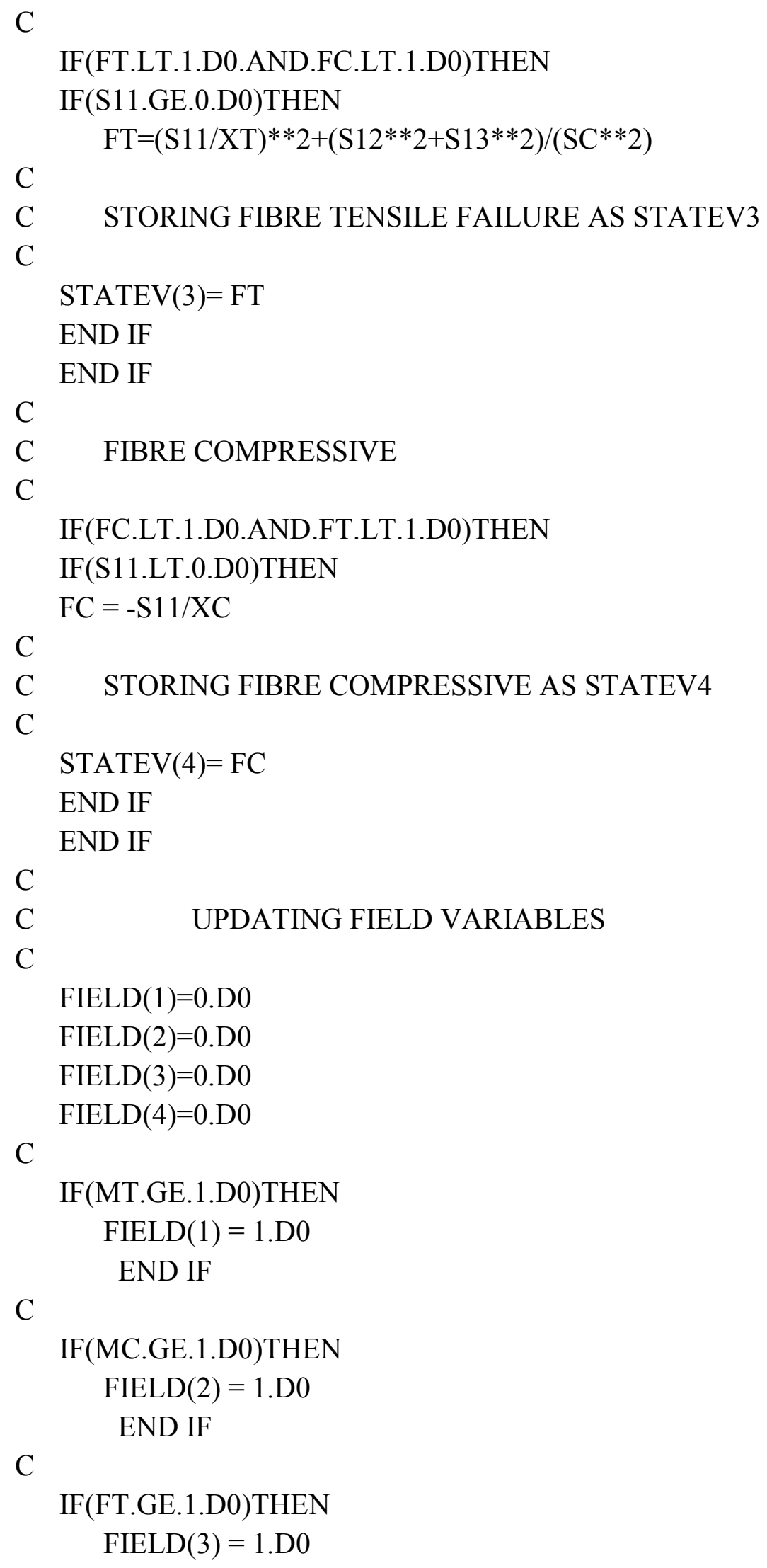




$\begin{array}{cc} & \text { END IF } \\ \text { C } & \\ & \text { IF(FC.GE.1.D0)THEN } \\ & \text { FIELD(4)=1.D0 } \\ & \text { END IF } \\ & \text { END } \\ \text { C } & \end{array}$




\section{Appendix E: Adhesive Thickness. Bolted/bonded joints}

Table E.1 Adhesive thickness in bolted/bonded joints

\begin{tabular}{|c|c|c|c|c|}
\hline Treatment & Sample & $\begin{array}{c}\text { Adhesive } \\
\text { type }\end{array}$ & $\begin{array}{l}\text { Nominal } \\
\text { adhesive } \\
\text { thickness } \\
\text { (mm) }\end{array}$ & $\begin{array}{l}\text { Average adhesive } \\
\text { thickness in the } \\
\text { bondline } \\
\text { (mm) }\end{array}$ \\
\hline \multirow{3}{*}{$\begin{array}{c}1 \\
\text { de }\end{array}$} & de_01 & EA9361 & \multirow{3}{*}{0.2} & 0.22 \\
\hline & de_02 & EA9361 & & 0.215 \\
\hline & de_03 & EA9361 & & 0.215 \\
\hline \multirow{3}{*}{$\begin{array}{l}2 \\
\mathrm{a}\end{array}$} & a_01 & EA9361 & \multirow{3}{*}{0.2} & 0.215 \\
\hline & a_02 & EA9361 & & 0.21 \\
\hline & a_03 & EA9361 & & 0.22 \\
\hline \multirow{3}{*}{$\begin{array}{c}3 \\
\text { be }\end{array}$} & be_01 & FM300-2M & \multirow{3}{*}{0.2} & 0.205 \\
\hline & be_02 & FM300-2M & & 0.21 \\
\hline & be_03 & FM300-2M & & 0.21 \\
\hline \multirow{3}{*}{$\begin{array}{c}4 \\
\mathrm{abd}\end{array}$} & abd_01 & FM300-2M & \multirow{3}{*}{0.2} & 0.21 \\
\hline & abd_02 & FM300-2M & & 0.22 \\
\hline & abd_03 & FM300-2M & & 0.21 \\
\hline \multirow{3}{*}{$\begin{array}{c}5 \\
\mathrm{~cd}\end{array}$} & cd_01 & EA9361 & \multirow{3}{*}{0.5} & 0.52 \\
\hline & cd_02 & EA9361 & & 0.52 \\
\hline & cd_03 & EA9361 & & 0.52 \\
\hline \multirow{4}{*}{$\begin{array}{c}6 \\
\text { ace }\end{array}$} & ace_01 & EA9361 & \multirow{4}{*}{0.5} & 0.46 \\
\hline & ace_02 & EA9361 & & 0.45 \\
\hline & ace_03 & EA9361 & & 0.47 \\
\hline & ace_rep_01 & EA9361 & & 0.5 \\
\hline \multirow{3}{*}{$\begin{array}{c}7 \\
\mathrm{bc}\end{array}$} & bc_01 & FM300-2M & \multirow{3}{*}{0.5} & 0.47 \\
\hline & bc_02 & FM300-2M & & 0.47 \\
\hline & bc_03 & FM300-2M & & 0.47 \\
\hline \multirow{3}{*}{$\begin{array}{c}8 \\
\text { abcde }\end{array}$} & abcde_01 & FM300-2M & \multirow{3}{*}{0.5} & 0.465 \\
\hline & abcde_02 & FM300-2M & & 0.46 \\
\hline & abcde_03 & FM300-2M & & 0.46 \\
\hline
\end{tabular}




\section{Appendix F: Typical Failure Modes in Tested Coupons}

\section{F.1 Bolted/bonded joints with FM300-2M adhesive}
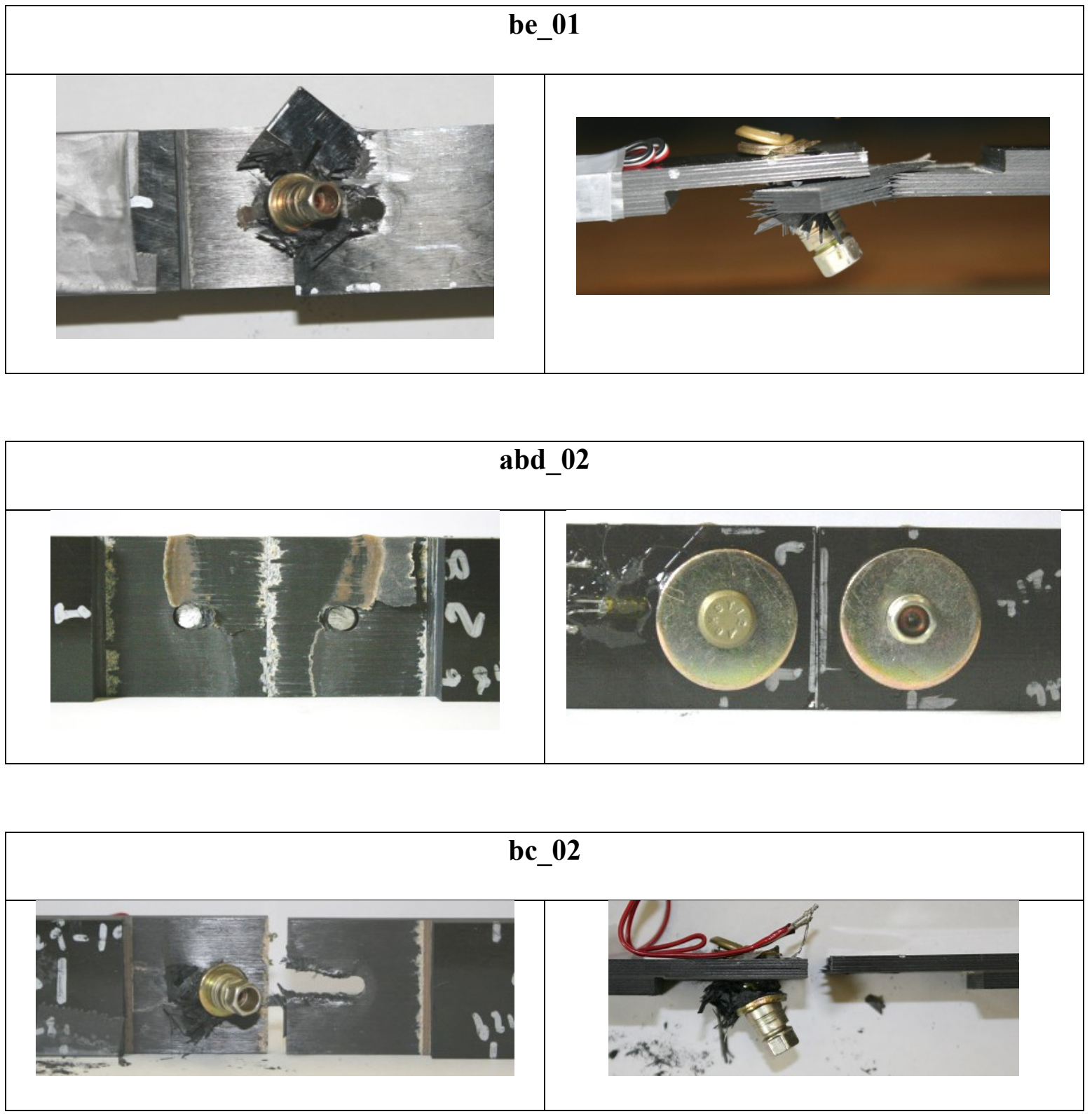


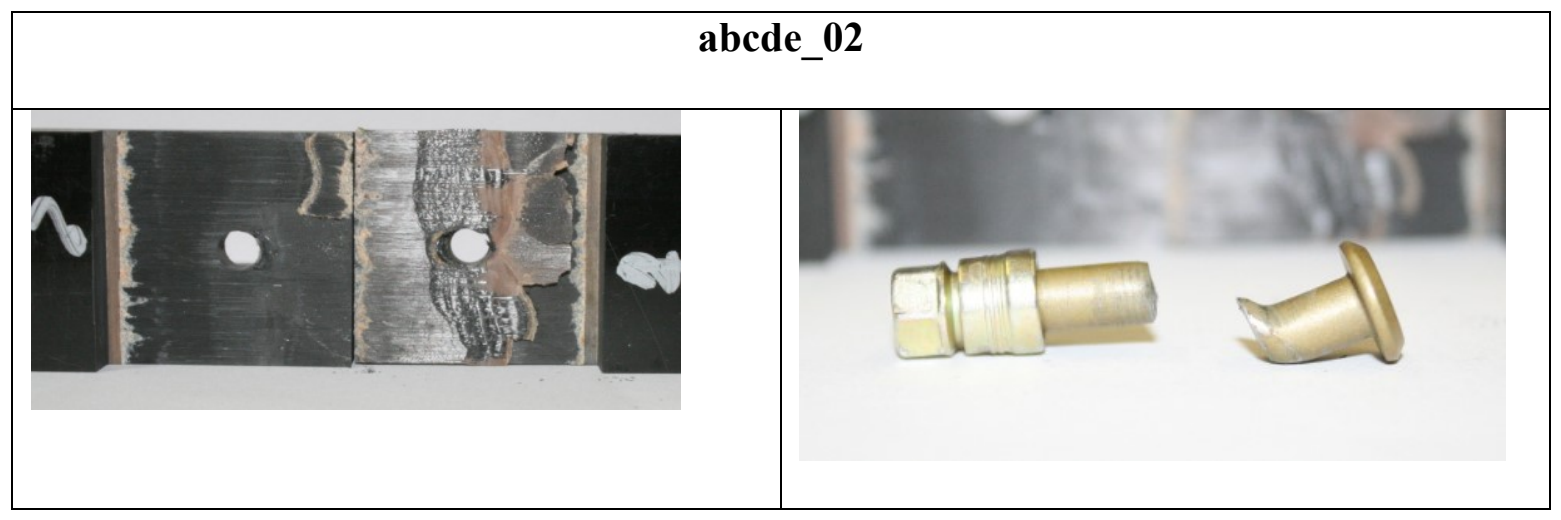

\section{F.2 Bolted/bonded joints with EA9361 adhesive}
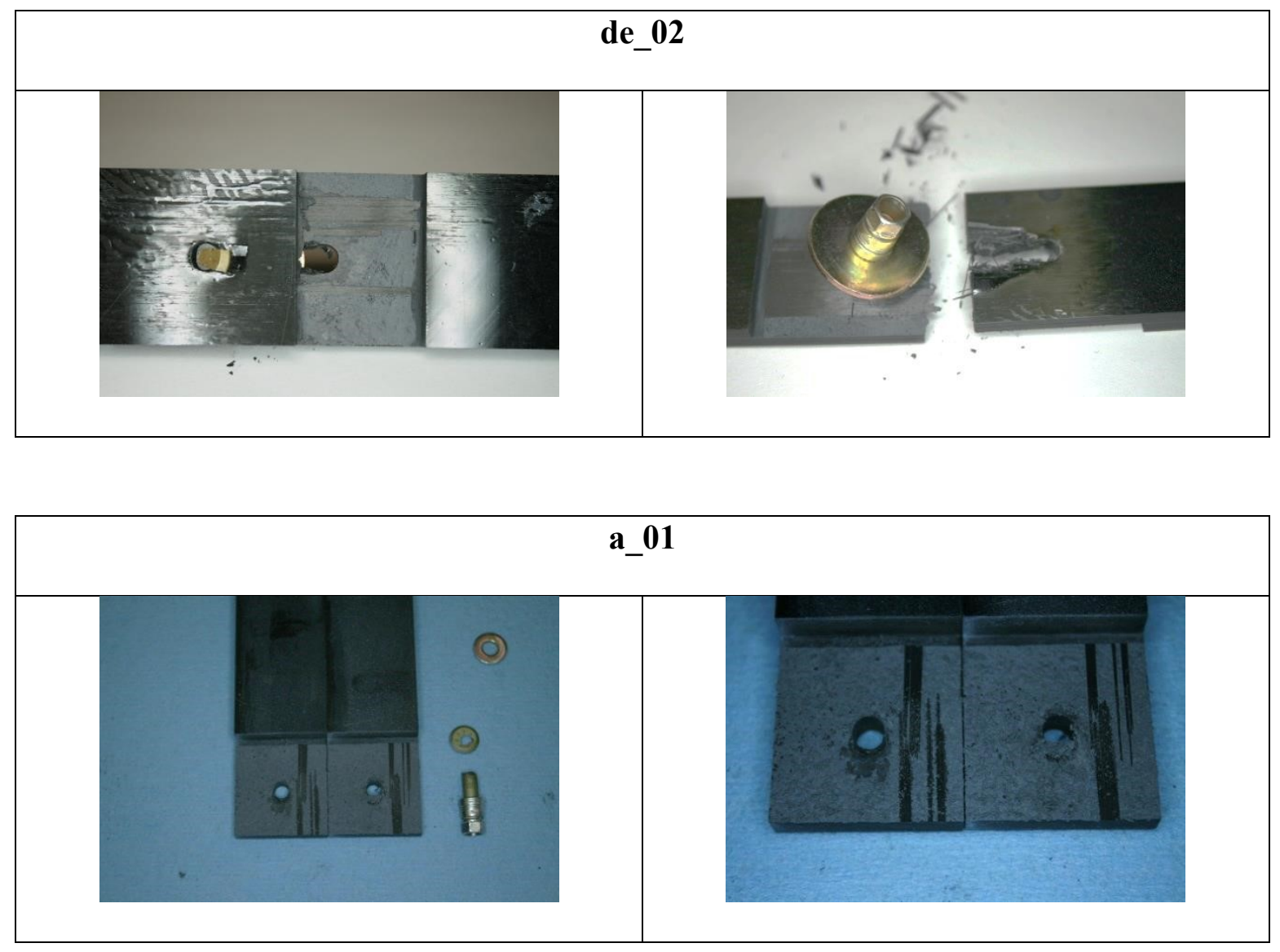

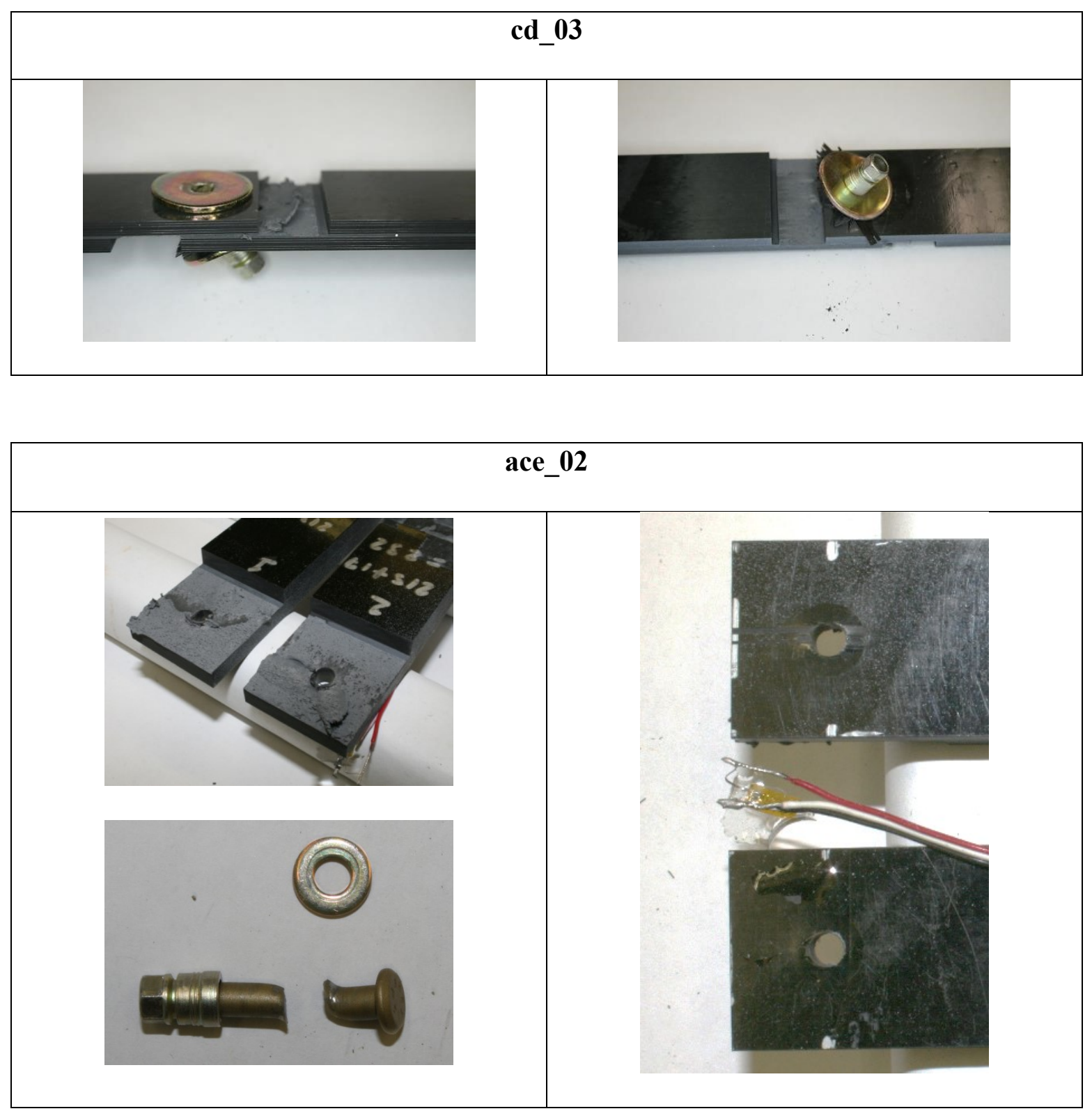


\section{F.3 Bonded Joints}
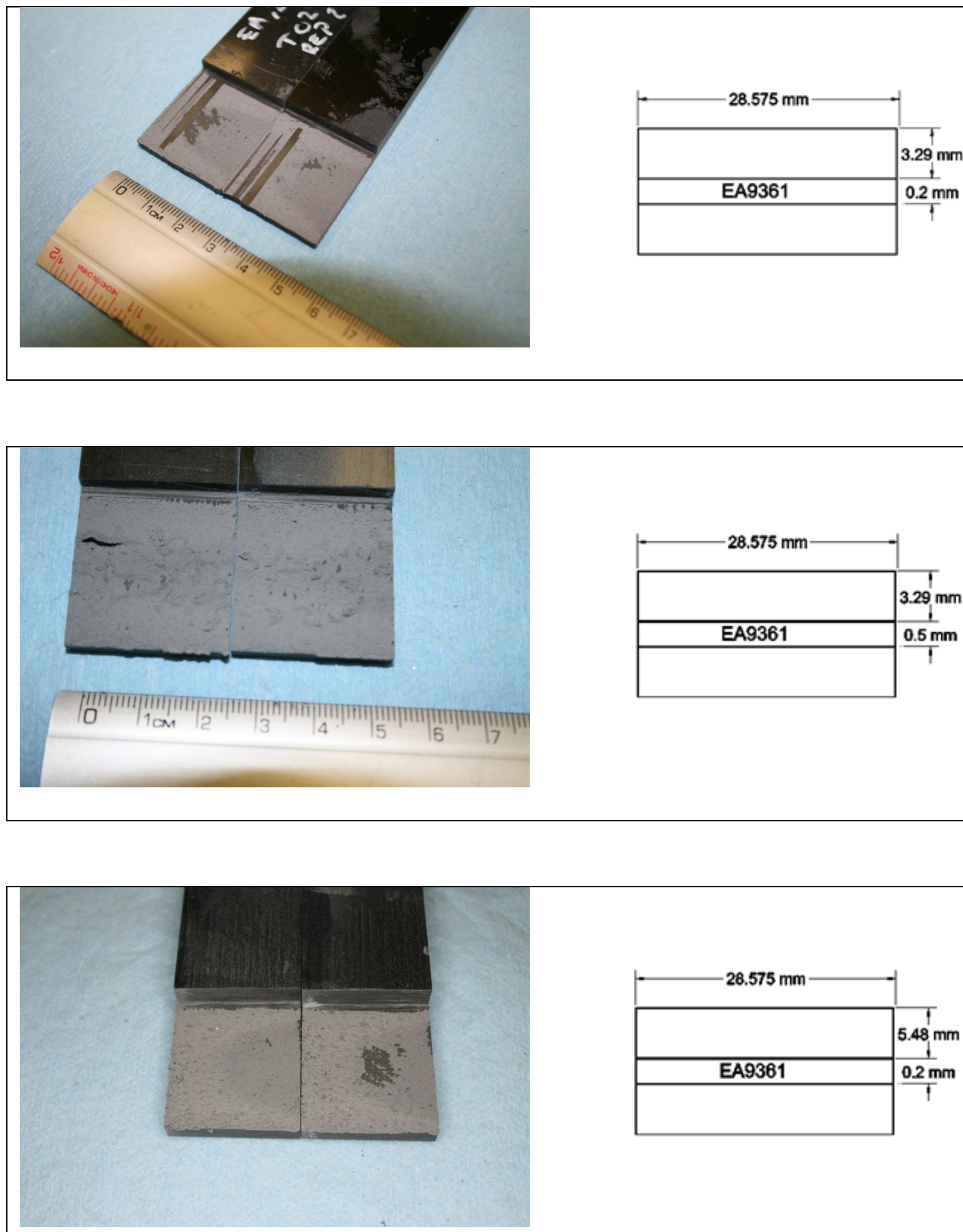


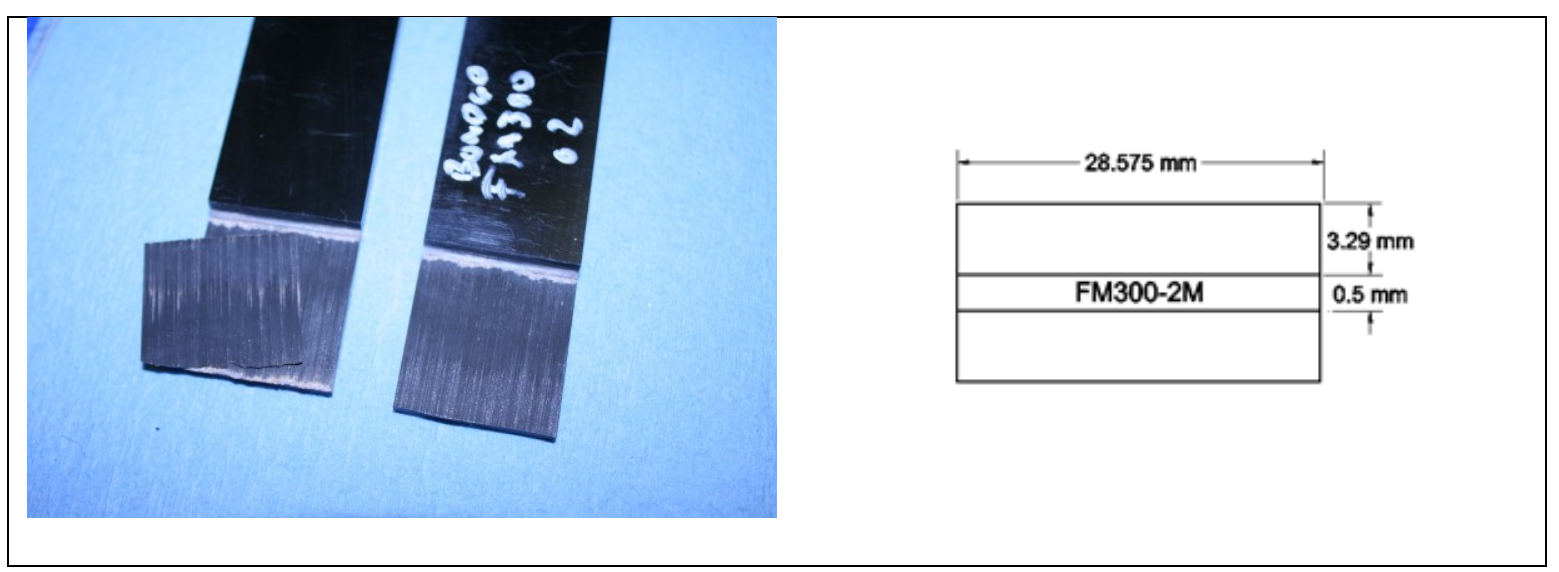

